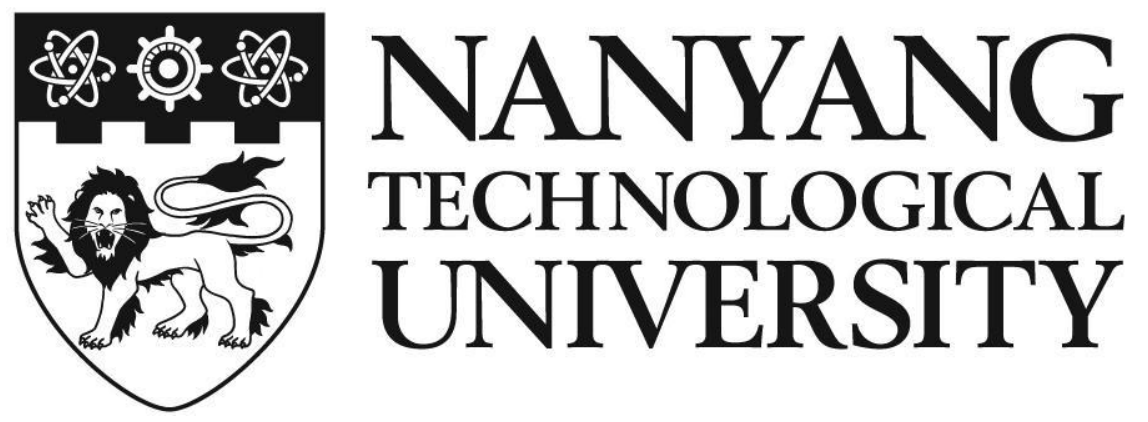

\title{
METAL CARBONYLS FOR BIO-IMAGING AND -SENSING
}

\section{LAM ZHIYONG}

School of Physical and Mathematical Sciences 


\title{
METAL CARBONYLS FOR BIO-IMAGING AND -SENSING
}

\author{
LAM ZHIYONG \\ B.Sc. (Hons.), 2012
}

A thesis submitted to the Nanyang Technological University in partial fulfilment of the requirement for the degree of Doctor of Philosophy

Division of Chemistry and Biological Chemistry, School of Physical and Mathematical Sciences 


\section{Acknowledgement}

I would like to express my heartfelt gratitude to the following people/organization who have contributed one way or another to my journey:

- Prof Leong Weng Kee (supervisor, NTU) - for his guidance, patience and support for the past seven years. I am very thankful for the exposure and education he has given me (from tobacco mosaic virus, to cholesterol and to this thesis), as well as inculcating in us the right attitude towards Science and lab safety.

- Prof Malini Olivo (co-supervisor, SBIC, A*STAR) - for her support and encouragement throughout the course of study. I am grateful for the opportunity given to work with a dynamic team of diverse skills and backgrounds, on various multi-disciplinary projects.

- Dr Kong Kien Voon (mentor) - for his guidance, advice and never-ending inspiration and ideas.

- Holly, Shabnam, Shi Ning, Aaron and Carmen - undergraduate/exchange students who have assisted me over the past four years.

- Colleagues from the EM clusters lab (NTU) and Bio-Optical Imaging Group, (SBIC, A*STAR).

- CBC central lab staff - Dr Rakesh, Dr Li, Ee Ling, Derek, Wen Wei and Joanne.

- Dr Ghayathri (BOIG, SBIC) for the animal work.

- Dr Bahareh and Prof Richard Webster (CBC, NTU) for ICP-MS analysis and CV experiments.

- Joey Lee, Dr Nic and Dr Loo Lit Hsin from the Bioinformatics Institute (BII), A*STAR, for the cell imaging work and the apoptosis studies.

- Family and friends for their care and support.

- My parents, whom may not understand what I am doing in the lab, for their upbringing, unspoken love and care for me for the past 29 years.

- Finally, to my collaborator, playmate, soulmate, best friend and wife-to-be, Joey Lee. Thank you for your love, unwavering support and for standing by me all this while. It's time to move forward!

- A*STAR Graduate Scholarship for financial support. 


\section{Table of Contents}

Acknowledgement II

Table of contents

Abstract $\quad$ VII

$\begin{array}{lll}\text { Abbreviations and conventions } & \text { VIII }\end{array}$

Molecule numbering scheme $\quad X$

$\begin{array}{ll}\text { List of schemes } & \text { XIV }\end{array}$

$\begin{array}{ll}\text { List of figures } & \text { XV }\end{array}$

$\begin{array}{ll}\text { List of tables } & \text { XX }\end{array}$

Chapter 1: Organometallic Complexes for Bio-Imaging and - $\quad 1$ Sensing

1.1 Bioorganometallic chemistry 1

1.2 Electrochemical-based probes 2

$\begin{array}{ll}1.3 \text { Radioisotope-based probes } & 6\end{array}$

$\begin{array}{ll}1.4 \text { Fluorescence-based probes } & 8\end{array}$

1.5 Transition metal carbonyls as mid-infrared (IR) probes 12

1.5.1 Bonding in transition metal carbonyls $\quad 12$

1.5.2 Applications based on CO vibrational intensities 13

- Steroid hormonal assay 13

- Carbonylmetalloimmunoassay (CMIA) 15

- IR probes for cellular imaging $\quad 18$

1.5.3 Sensing based on frequency shifts in CO vibrations 23

$\begin{array}{ll}\text { - } \mathrm{pH} \text { sensing } & 27\end{array}$

- Protein interaction 28

- Solvent environment 29

1.5.4 Applications based on CO vibrational lifetime 30

- Ultrafast vibrational lifetime of metal carbonyls in protein and 30 membrane studies

1.6 Transition metal carbonyls as Raman probes 33

- IR vs Raman characteristics of CO 33

- Metal carbonyls as Raman probes for cellular imaging 35 
- Detection through surface-enhanced Raman spectroscopy 36

1.7 Transition metal carbonyls as photoacoustic probes 38

1.8 Aims and objectives 41

1.9 References 42

Chapter 2: Surface-Enhanced Raman Spectroscopy (SERS) 52 of Metal Carbonyls on Gold Nanospheres

2.1 Metal carbonyl clusters as Raman reporters 53

2.1.1 Synthesis and design of metal carbonyl clusters 53

2.1.2 SERS spectra of metal carbonyl clusters on AuNS 58

2.2 Half-sandwich metal carbonyl complexes as Raman reporters 61

2.3 Effects of aggregation $\quad 66$

$\begin{array}{ll}2.4 \text { Conclusion } & 69\end{array}$

$\begin{array}{ll}2.5 \text { Experimental section } & 70\end{array}$

2.5.1 General methods $\quad 70$

2.5.2 Synthesis of cluster 3b $\quad 71$

2.5.3 Synthesis of cluster $6 \quad 71$

2.5.4 Synthesis of cluster $6 . \mathrm{H}^{+} \quad 72$

2.5.5 Synthesis of cluster $\mathbf{7 b} \quad 72$

2.5.6 Preparation of AuNS conjugates of metal carbonyl clusters 2a- 73 $7 \mathbf{b}$

2.5.7 Preparation of AuNS conjugates of half-sandwich metal 73 carbonyl complexes $\mathbf{8 a - 1 3 a}$

Chapter 3: Metal Carbonyl-Boronic Acid Conjugate - a 77 SERS Probe for Glucose Detection and its Cytotoxicty

3.1 Methods for glucose detection 77

3.2 Concept of selective detection of glucose on SERS planar substrate using $\quad 79$ boronic acid chemistry

$\begin{array}{ll}\text { 3.3 Fabrication of boronic acid functionalized SERS substrate } & 82\end{array}$

3.4 Synthesis and characterization of cluster $\mathbf{2 b}-\mathrm{B}(\mathrm{OH})_{2}$

3.5 Selective detection and quantification of glucose 86

3.6 Cytotoxicity of $\mathbf{2 b}-\mathrm{B}(\mathrm{OH})_{2}$ and analogues towards cancer and non- 88 
cancer cells

3.7 Induction of apoptosis by $\mathbf{2 b}-\mathrm{B}(\mathrm{OH})_{2}$ and $-\mathrm{OH}$

3.7.1 Cell nuclear staining using Hoechst 33342

3.7.2 Phosphatidylserine detection by Annexin V-FITC conjugate 98

3.8 Chemical oxidation of $\mathbf{2} \mathbf{b}-\mathrm{B}(\mathrm{OH})_{2}$ to $\mathbf{2} \mathbf{b}-\mathrm{OH}$ and electrochemical studies $\quad 100$

3.9 Conclusion 105

$\begin{array}{ll}3.10 \text { Experimental section } & 106\end{array}$

3.10.1 General methods 106

3.10.2 Synthesis of cluster 2b-B $(\mathrm{OH})_{2}$

3.10.3 Incubation of $\mathbf{2 b}-\mathrm{B}(\mathrm{OH})_{2}$ with glucose 107

3.10.4 Fabrication of BMFON substrate 107

3.10.5 Immobilization of 4-mercaptophenylboronic acid on BMFON 108

3.10.6 Detection of saccharides (glucose, fructose and galactose) 108

3.10.7 Detection of glucose in clinical urine sample 108

3.10.8 Synthesis of cluster $\mathbf{2 b}-\mathrm{OH}$ and $\mathbf{2 b}-\mathrm{COOH}$

3.10.9 Cell culture 109

3.10.10 Cell proliferation assay 110

3.10.11 Apoptosis assay 110

3.10.12 Image acquisition, segmentation and features extraction 111

3.10.13 Chemical oxidation of $\mathbf{2 b}-\mathrm{B}(\mathrm{OH})_{2} \quad 111$

3.10.14 Cyclic voltammetry 111

3.10.15 X-ray crystallographic analysis of $\mathbf{2 b}$-boroxine, -OH and - 112 $\mathrm{COOH}$

Chapter 4: High Nuclearity Metal Carbonyl Clusters as 121 Near-IR Contrast Agents for Photoacoustic In Vivo Imaging

4.1 Optical properties of group 8 metal carbonyl clusters - low to high nuclearity

4.2 Photoacoustic properties of high nuclearity carbonyl clusters 22-26 125

4.3 Biocompatible sodium salt of cluster $\mathbf{2 6} \quad 126$

4.4 Photoacoustic in vivo imaging and biodistribution of 26

4.5 Development of biocompatible HNCCs via polymer encapsulation 136 
$\begin{array}{ll}4.6 \text { Conclusion } & 140\end{array}$

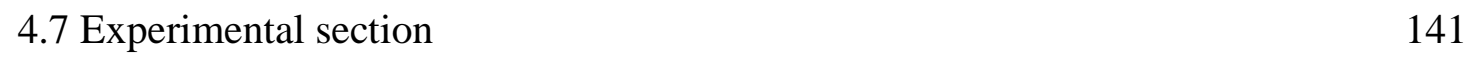

$\begin{array}{ll}\text { 4.7.1 General methods } & 141\end{array}$

$\begin{array}{ll}\text { 4.7.2 Synthesis of cluster } \mathrm{Na}_{2} . \mathbf{2 6} & 142\end{array}$

$\begin{array}{ll}\text { 4.7.3 Cell culture and cell viability assay } & 142\end{array}$

$\begin{array}{ll}\text { 4.7.4 Gel electrophoresis } & 143\end{array}$

4.7.5 Multispectral optoacoustic tomography (MSOT) experimental 143 parameters and protocol

4.7.6 Evaluation of PA activity of clusters 22-26 in phantom model 144

4.7.7 In vivo longitudinal monitoring of probe biodistribution in mouse 144 xenograft model

4.7.8 Image processing and analysis for in vivo experiments 145

4.7.9 Microwave acid digestion and ICP-MS analysis of osmium content of 145 tissue samples

4.7.10 Histopathology of organ tissues of mice $\quad 146$

4.7.11 Attempted polymer encapsulation of cluster $\mathbf{2 3}$ via the 146 thermodynamic approach

4.7.12 Attempted polymer encapsulation of cluster $\mathbf{2 3}$ via the kinetic 147 approach

4.8 References

Chapter 5: Conclusion 


\begin{abstract}
$\underline{\text { Abstract }}$
Metal carbonyls, a class of organometallic complexes that consist of carbon monoxide $(\mathrm{CO})$ ligands coordinated to a metal center, show intense $\mathrm{CO}$ vibrational frequencies in the mid-infrared (IR) $2200-1800 \mathrm{~cm}^{-1}$. This region is unique to the metal carbonyl species and is typically free of interference by other biomolecules. This property can be put to good use for mid-IR bio-sensing, which was first demonstrated by Jaouen and co-workers in the development of IR-based quantitative assays for detecting various biomolecules.

In this thesis, the concept is extended to surface-enhanced Raman spectroscopy (SERS) through the development of metal carbonyl-based Raman reporters. The conjugation of two classes of metal carbonyls, namely, metal carbonyl clusters and mononuclear half-sandwich metal carbonyl complexes, onto colloidal gold nanospheres gave good CO vibrational stretches in the $1800-2200 \mathrm{~cm}^{-1}$ region. Alterations to the ligand sphere and the charge (for cluster complexes) were found to be effective in tuning the $\mathrm{CO}$ vibrational stretches over a wide frequency range. It was also observed that the SERS enhancement was highly dependent on the extent of aggregation of the colloid.

In another extension, a SERS-active planar substrate was utilized in tandem with a triosmium carbonyl cluster-boronic acid conjugate acting as a secondary saccharide probe, in a sensitive and selective SERS-based glucose assay. While investigating extension of this idea to the detection of cancer cells via their surface glycans, thiolato triosmium carbonyl clusters of the type $\mathrm{Os}_{3}(\mu-\mathrm{H})(\mu-\mathrm{SAryl})(\mathrm{CO})_{10}$ were found to be cytotoxic. Cell death was found to be via the induction of apoptosis.

Metal carbonyl clusters were also demonstrated to be good contrast agents for photoacoustic (PA) imaging. The cluster nuclearity was shown to be a useful parameter for fine-tuning the absorption wavelength, as increasing the cluster nuclearity shifted the absorption into the near-IR region. The high nuclearity carbonyl cluster $\left[\mathrm{Os}_{10}\left(\mu_{6}-\mathrm{C}\right)(\mathrm{CO})_{24}\right]^{2-}$ was shown to exhibit good PA properties in the NIR region, and its sodium salt was applied successfully for the whole body PA imaging of live mice.
\end{abstract}




\section{$\underline{\text { Abbreviations and Conventions }}$}

$\begin{array}{ll}\text { ACN } & \text { Acetonitrile } \\ \text { AuNS } & \text { Gold nanospheres } \\ \text { BA } & \text { Boronic acid } \\ \text { BMFON } & \text { Bimetallic film over nanospheres } \\ \text { Cp } & \text { Cyclopentadienyl }\left(\mathrm{C}_{5} \mathrm{H}_{5}\right) \\ \text { Cp* } & \text { Pentamethylcyclopentadienyl }\left(\mathrm{C}_{5} \mathrm{Me}_{5}\right) \\ \text { CV } & \text { Cyclic voltammetry } \\ \text { Cy } & \text { Cyclohexanyl } \\ \text { DCM } & \text { Dichloromethane } \\ \text { DMF } & \text { Dimethylformamide } \\ \text { DMEM } & \text { Dulbecco's Modified Eagle's Medium } \\ \text { DMSO } & \text { Dimethylsulfoxide } \\ \text { EDC } & \text { 1-ethyl-3-(3-dimethylaminopropyl)carbodiimide } \\ \text { ESI } & \text { Electospray ionization } \\ \text { EtOAc } & \text { Ethyl acetate } \\ \text { Fc } & \text { Ferrocene } \\ \text { FITC } & \text { Fluorescein isothiocyanate } \\ \text { HNCC } & \text { High nuclearity carbonyl cluster } \\ \text { HOMO } & \text { Highest occupied molecular orbital } \\ \text { HRMS } & \text { High resolution mass spectroscopy } \\ \text { IC } & \\ \text { IR } & \text { 50\% Inhibitory conentration } \\ \text { LCMS } & \text { Infrared } \\ \text { LUMO } & \text { Liquid chromatography-mass spectrometry } \\ \text { MSOT } & \text { Lowest unoccupied molecular orbital } \\ \text { MTS } & \text { Multi-spectral optoacoustic tomography } \\ & \text { 3-(4,5-dimethylthiazol-2-yl)-5-(3-carboxymethoxyphenyl)-2-(4- } \\ m / z & \text { sulfophenyl)-2H-tetrazolium) } \\ \text { NMR } & \text { Mass-to-charge ratio } \\ \text { PA } & \text { Nuclear magnetic resonance } \\ \text { PEG } & \text { Photoacoustic } \\ \text { Ph } & \text { Polyethylene glycol } \\ \text { PPN } & \text { Phenyl } \\ \text { PS- } b \text {-PAA } & \text { Bis(triphenylphosphine)iminium }\left[\left(\mathrm{PPh}_{3}\right)_{2} \mathrm{~N}^{+}\right. \\ \mathrm{R}_{\mathrm{f}} & \text { Polystyrene-block-poly(acrylic acid }) \\ \text { RT } & \text { Retention factor } \\ \text { SERS } & \text { Room temperature } \\ \text { TEM } & \text { Surface-enhanced Raman spectroscopy } \\ \text { THF } & \text { Transmission electron microscopy } \\ \text { TLC } & \text { Tetrahydrofuran } \\ \text { TMNO } & \text { Trimethylamine N-oxide } \\ \mu & \text { Bridging } \\ \end{array}$




\section{Vibrational spectroscopy (IR and SERS)}

$\begin{array}{ll}v_{\mathrm{CO}} & \text { Stretching frequency in the carbonyl region }\left(2200-1800 \mathrm{~cm}^{-1}\right) \\ \mathrm{vw} & \text { Very weak } \\ \mathrm{w} & \text { Weak } \\ \mathrm{m} & \text { Medium } \\ \mathrm{s} & \text { Strong } \\ \mathrm{vs} & \text { Very strong } \\ \mathrm{br} & \text { Broad } \\ \mathrm{sh} & \text { Shoulder }\end{array}$

\section{Nuclear magnetic resonance (NMR) spectroscopy}

$\begin{array}{ll}\delta & \text { Chemical shift } \\ \text { ppm } & \text { Parts per million } \\ \left\{{ }^{1} \mathrm{H}\right\} & \text { Protons decoupled } \\ \mathrm{s} & \text { Singlet } \\ \mathrm{d} & \text { Doublet } \\ \mathrm{dt} & \text { Doublet of triplet } \\ \mathrm{t} & \text { Triplet } \\ \mathrm{m} & \text { Multiplet } \\ \text { COSY } & \text { Correlation spectroscopy }\end{array}$




\section{Molecule Numbering Scheme}

The metallic frameworks of all molecular structures are drawn in outline only. The short line extending from the metal atom (in the molecular structure diagrams) represents a coordinate bond from carbon monoxide to the metal center (M-CO). This is illustrated in the example below:
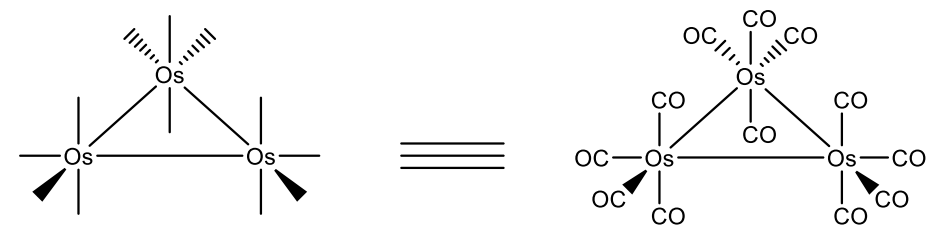

$\mathrm{Os}_{3}(\mathrm{CO})_{12}$

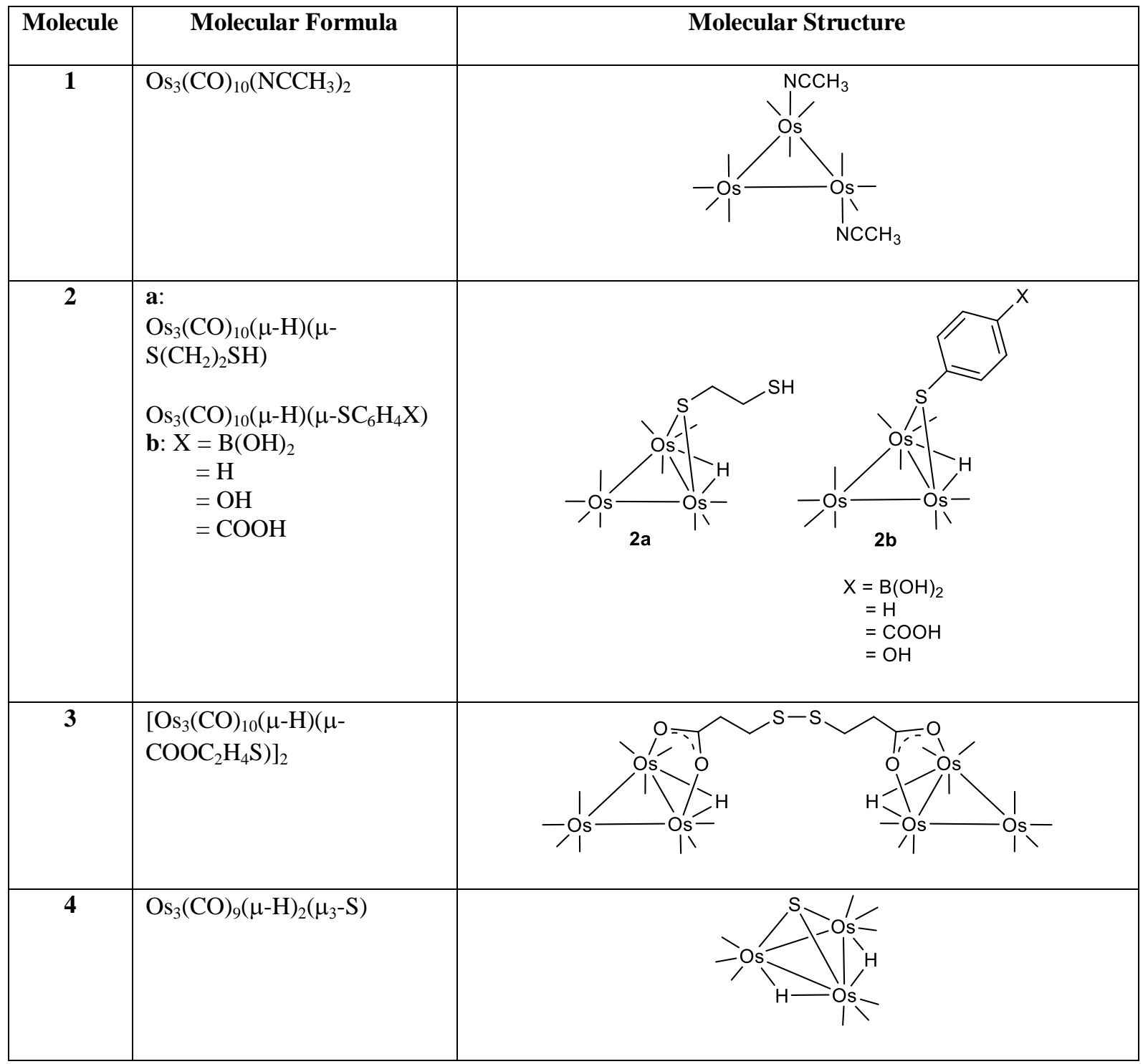




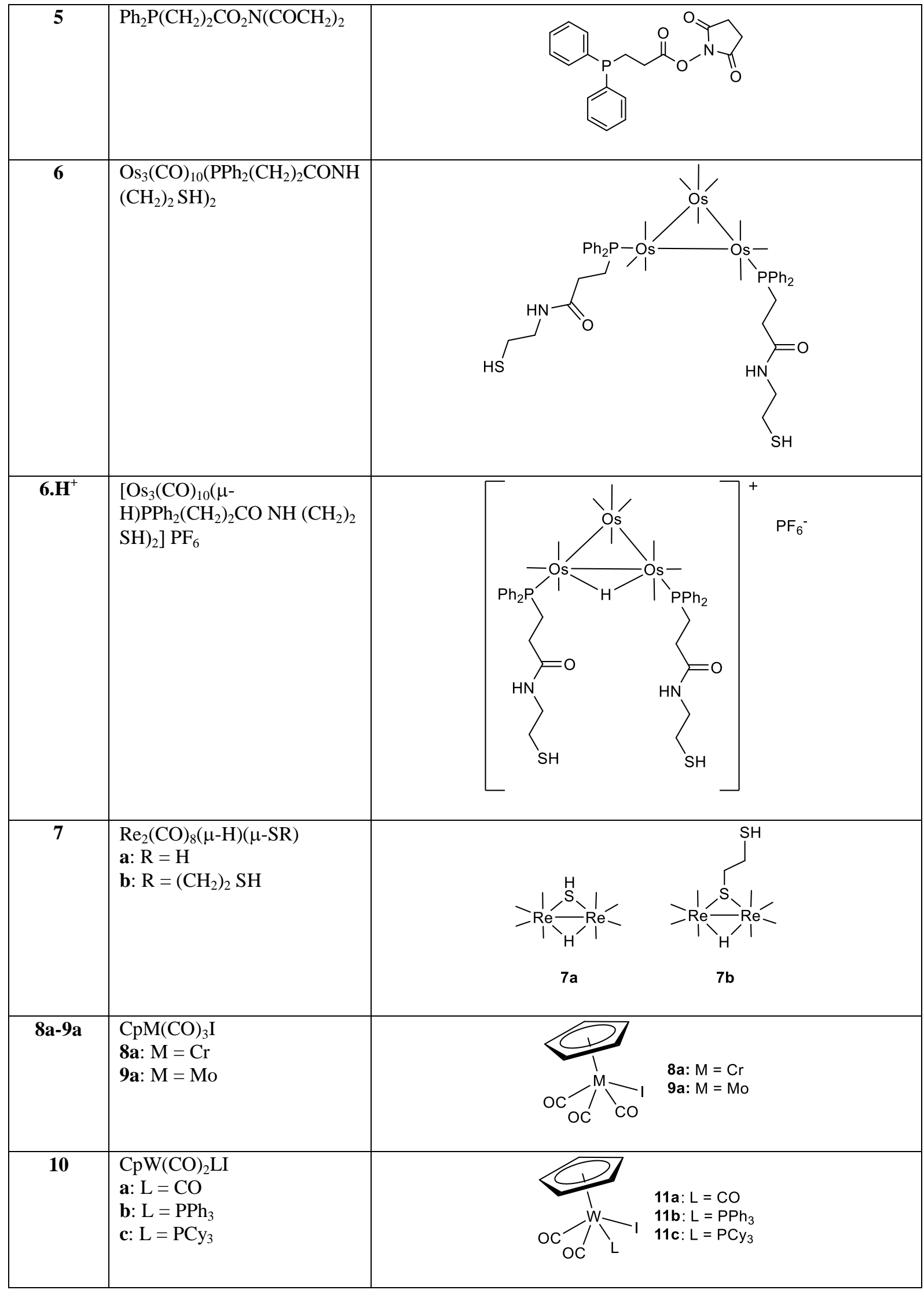




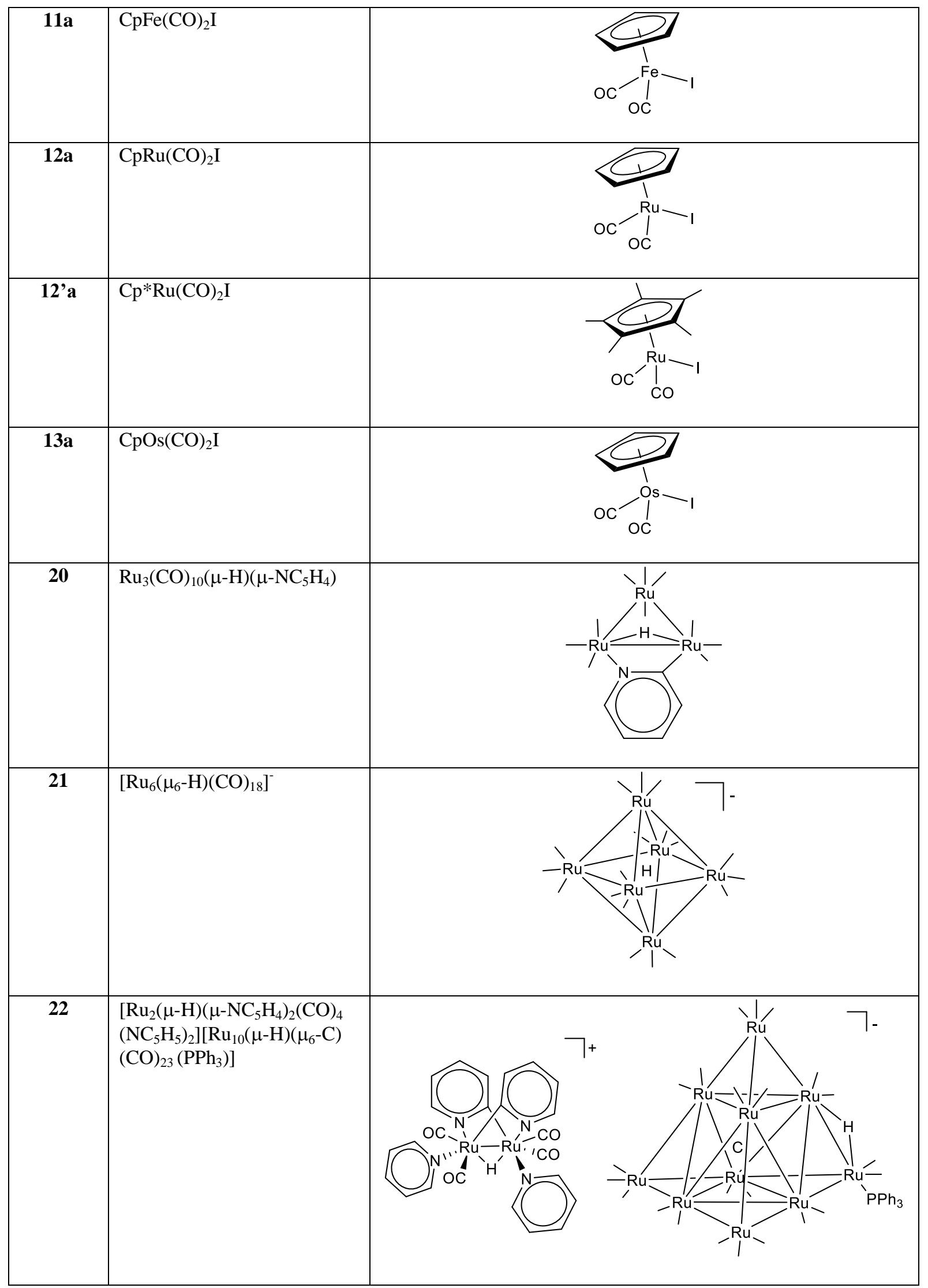




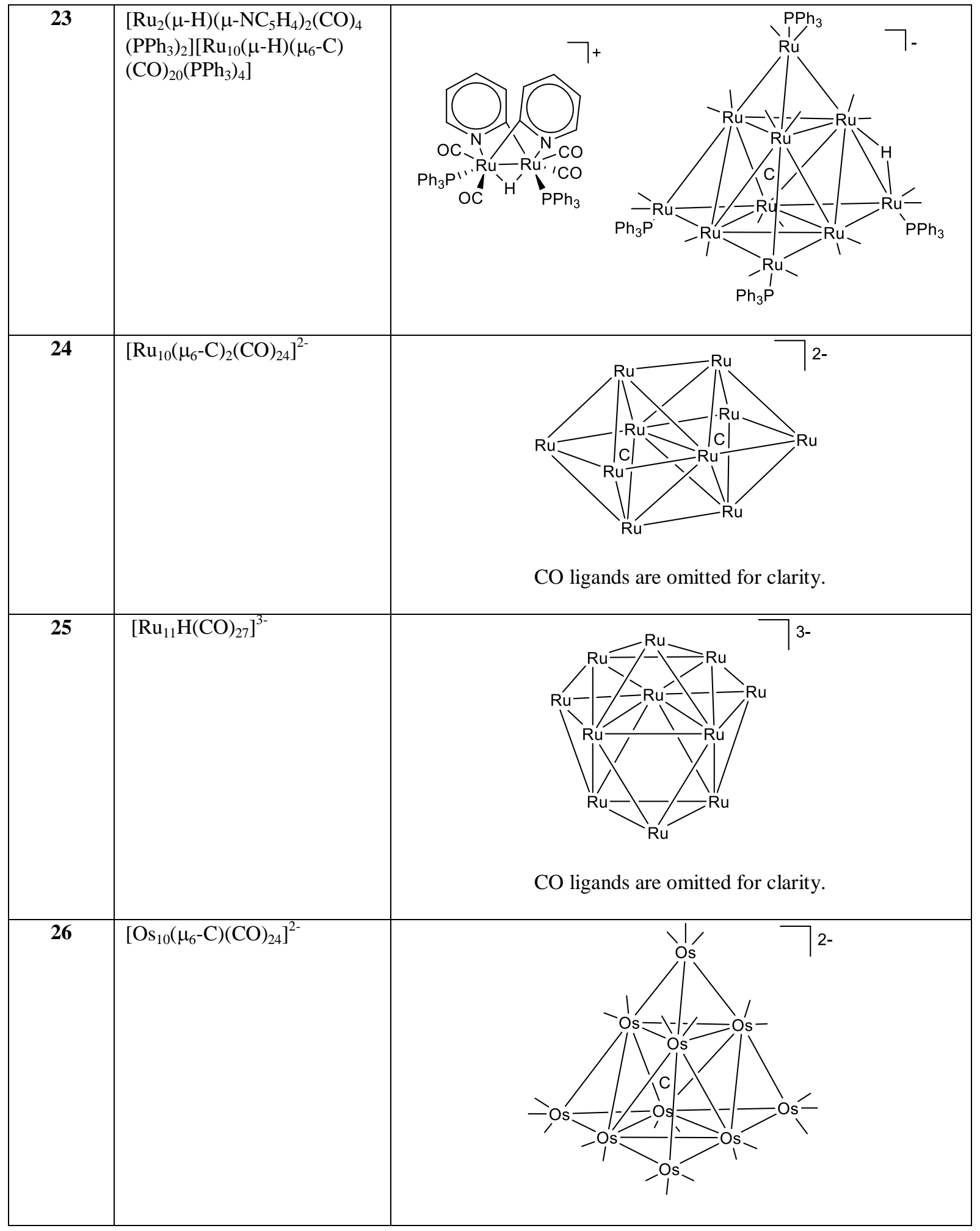




\section{List of Schemes}

\section{Content Pg}

Scheme 1.1. Synthesis of $\left[{ }^{99 \mathrm{~m}} \mathrm{Tc}\left(\mathrm{OH}_{2}\right)_{3}(\mathrm{CO})_{3}\right]^{+}$and some examples of conjugates to biomolecules

Scheme 1.2. Synthesis of $\left[{ }^{99 \mathrm{~m}} \mathrm{Tc}\left(\mathrm{OH}_{2}\right)_{3}(\mathrm{CO})_{3}\right]^{+}$and some examples of conjugates to

biomolecules

Scheme 2.1. Synthesis of clusters $2 a, 3 b$ and 4 .

Scheme 2.2. Synthesis of clusters 6 and $6 . \mathrm{H}^{+}$.

Scheme 2.3. Synthesis of clusters $7 \mathbf{a}$ and $7 \mathbf{b}$.

Scheme 3.1. Interaction of the phenylboronate anion with 1,2- and 1,3-diols to form boronate esters complexes with five- and six-membered rings, respectively

Scheme 3.2. Synthesis of $\mathbf{2 b}-\mathrm{B}(\mathrm{OH})_{2}$ and its reaction with glucose. 84

Scheme 3.3. Formation of $\mathbf{2 b}$-boroxine from $\mathbf{2 b}-\mathrm{B}(\mathrm{OH})_{2}$. 85

Scheme 3.4. Synthesis of cluster $\mathbf{2 b}-\mathrm{H}$, $-\mathrm{OH}$ and $-\mathrm{COOH}$. 89

$\begin{array}{ll}\text { Scheme 3.5. Oxidation of boronic acid or boronate ester by } \mathrm{H}_{2} \mathrm{O}_{2} & 100\end{array}$

Scheme 3.6. a) Proposed mechanism for the oxidation and formation of quinone methides 103 from ferrocenyl phenol

Scheme 4.1. Microwave-assisted synthesis of cluster $\mathrm{Na}_{2} .26$. 


\section{List of Figures}

\section{Content $\quad$ Pg}

Figure 1.1. a) Schematic representation for the detection of matrix metalloproteinase: selfassembly of electrochemical Fc-peptide conjugate on the gold substrate, followed by cleavage of the peptide in the presence of MMP-7. b) Cyclic voltammetry and c) square wave voltammetry of the Fc-peptide-Au electrode.

Figure 1.2. a) Structural representation of PNA backbone and side chains $(A, C, G, T=$ nucleobase). b) Structural representation of PNA/DNA hybrid

Figure 1.3. a) Molecular structure of the Fc-PNA conjugate. b) Working principle of a gene sensor based on the conformational flexibility change in the probe structure induced by hybridization of PNA with DNA.

Figure 1.4. a) Immobilization of $\mathrm{Fc}$ on $\mathrm{IgG}$ using carbodiimide chemistry. b) Schematic diagram of an electrochemical capillary flow immunoassay system and the working principle.

Figure 1.5. Some commercially available ${ }^{99 \mathrm{~m}} \mathrm{Tc}$-based radiopharmaceuticals

Figure 1.6. Jablonski diagram (top), and the absorption and emission spectra (bottom) of the common fluorophore fluorescein isothiocyanate (FITC).

Figure 1.7. Molecular structures of selected cyclometalated $\left[\operatorname{Ir}(\mathrm{C}-\mathrm{N})_{2}(\mathrm{~N}-\mathrm{N})\right]$ complexes

Figure 1.8. a) Molecular structure of the complexes $[\mathrm{Pt}(\mathrm{N}-\mathrm{C}-\mathrm{N}) \mathrm{Cl}]$. b) Confocal micrographs of human dermal fibroblasts, C8161 human melanoma and $\mathrm{CHO}$ cells incubated with $[\mathrm{Pt}(\mathrm{N}-\mathrm{C}-\mathrm{N}) \mathrm{Cl}]-\mathbf{a}$.

Figure 1.9. a) Molecular structure of the dinuclear $\mathrm{Au}(\mathrm{I}) \mathrm{NHC}$ complexes and cellular distribution of the complex $(200 \mu \mathrm{M})$ in RAW264.7 cells after incubation for $15 \mathrm{~h}$ at $37^{\circ} \mathrm{C}$. b) Molecular structure of the trinuclear $\mathrm{Au}(\mathrm{I})$ alkynyl containing the PTA ligands and cellular distribution of the complex in A2780 cells after incubation for $6 \mathrm{~h}$ at $37{ }^{\circ} \mathrm{C}$.

Figure 1.10. a) Orbital diagram showing the bonding between carbon monoxide with a transition metal centre. b) Different bonding modes of $\mathrm{CO}$ with different number of metal centers. c) IR spectra recorded in absorbance mode of untreated mucosa cells (bottom), cells treated with a triosmium carbonyl cluster (middle and top).

Figure 1.11. a) FT-IR ( $v C O)$ spectrum of precipitated lamb uterine cytosol incubated with $\eta^{6}$ - $\mathrm{Cr}(\mathrm{CO})_{3}$-estradiol $\alpha$-derivative, prepared in CsI pellet. b) Correlation between the weight of the pellet and the area of the $\mathrm{A}_{1}(\mathrm{vCO})$ peak at $1957 \mathrm{~cm}^{-1}$.

Figure 1.12. Molecular structures of metal carbonyl-steroid derivatives used for steroidal hormonal assay.

Figure 1.13. Schematic representation of the working principles of CMIA.

Figure 1.14. Molecular structures of metal carbonyl derivatives used as tracers in CMIA.

Figure 1.15. a) Synthesis of $\mathrm{CpFe}(\mathrm{CO})_{2}$-gold nanoparticle conjugates and immobilization of goat anti-mouse IgG antibody. b) Synthesis of $\mathrm{CpFe}(\mathrm{CO})_{2}-\mathrm{MNP}$ conjugates and immobilization of IgG antibody.

Figure 1.16. a) Molecular structure of $\left[\mathrm{Os}_{3}(\mathrm{CO})_{10}(\mu-\mathrm{H})\left(\mu-\mathrm{S}\left(\mathrm{CH}_{2}\right)_{10} \mathrm{COO}\right)\right] \mathrm{Na}$ and the phosphatidylcholine analogue. b) Normal optical image and c) false-color IR image of oral mucosa cells treated with $\left[\mathrm{Os}_{3}(\mathrm{CO})_{10}(\mu-\mathrm{H})\left(\mu-\mathrm{S}\left(\mathrm{CH}_{2}\right)_{10} \mathrm{COO}\right)\right] \mathrm{Na}(32 \mu \mathrm{M})$, taken at 2013 $\mathrm{cm}^{-1}$, one of the carbonyl vibration band.

Figure 1.17. a) Molecular structure of the vitamin $B_{12}-M n$ CORM conjugate and optical images of a 3 T3 fibroblast after incubation with the conjugate $(1 \mathrm{mM})$. The top and bottom inserts show images reconstructed from integration of the intensity of the carbonyl 
stretching vibrations.

Figure 1.18. a) Molecular structure of $\operatorname{ReCl}(\mathrm{CO})_{3}-$ pyta- $\mathrm{C}_{12} \mathrm{~N}_{3}$. b) Bright field, SR-FTIR mapping and epifluorescence images of MDA-MB-231 cell incubated with $\operatorname{ReCl}(\mathrm{CO})_{3^{-}}$ pyta- $\mathrm{C}_{12} \mathrm{~N}_{3}(10 \mu \mathrm{M}, 1 \mathrm{~h})$.

Figure 1.19. a) Molecular structure of rhenium carbonyl-peptide conjugate. b) IR mapping, bright field and luminescence images of skin slice after a 24 h-exposure to a $2 \times 10^{-2} \mathrm{M}$ solution of conjugate in water.

Figure 1.20. Molecular structure of $\mathrm{CpRe}(\mathrm{CO})_{3}$-hydroxytamoxifen conjugate and images of MDA-MB-231 cells (AFM topography and PTIR mappings at different wavelengths).

Figure 1.21. a) Molecular structures of $\eta^{6}$-(1-carboxybenzene $) \mathrm{Cr}(\mathrm{CO})_{3}$ and $\eta^{4}$-1-carboxy1,3-pentadiene $) \mathrm{Fe}(\mathrm{CO})_{3}$ and their $\mathrm{pH}$ sensing capability. b) FTIR spectra in the 2050-1800 $\mathrm{cm}^{-1}$ region of $\eta^{6}-(1$-carboxybenzene $) \mathrm{Cr}(\mathrm{CO})_{3}$ in buffer solutions over the $\mathrm{pH}$ range 2.1-6.0

Figure 1.22. a) Reaction of $\left(\eta^{4}\right.$-diene)- $\mathrm{Fe}(\mathrm{CO})_{3}$ with amine functionality in proteins. b) (a-f) FTIR spectra of $\left(\eta^{4} \text {-diene)-Fe(CO) }\right)_{3}$ in $\mathrm{pH} 7.5$ buffer solution containing $\alpha$-chymotrypsin recorded at intervals over $3 \mathrm{~h}$. (g) Component bands resulting from decomposition of spectrum c using a scale expansion of 2. (h) FTIR spectrum of $\left[\mathrm{Fe}(\mathrm{CO})_{3}\left(\mathrm{C}_{6} \mathrm{H}_{7} \mathrm{NH}_{2} \mathrm{Pr}^{\mathrm{i}}\right)\right]\left(\mathrm{PF}_{6}\right)$ in buffer.

Figure 1.23. a) Molecular structures of various $\left(\eta^{4}\right.$-cyclohexadiene $)-\mathrm{Fe}(\mathrm{CO})_{3}$ complexes used for solvent environment sensing. b) Symmetric and antisymmetric stretching frequencies of $\mathrm{Fe}(\mathrm{CO})_{3}-(\mathrm{a})$ in various solvents. c) Variation of $v_{\mathrm{sym}}$, and $v_{\text {asym }}, \mathrm{of} \mathrm{Fe}(\mathrm{CO})_{3}-$ (a) in binary mixtures of ethyl acetate and dimethyl acetamide.

Figure 1.24. a) Molecular structure of ruthenium carbonyl (RC) protein label on HEWL and HuLys. b) Crystal structures of HEWL-RC (PDB code 2XJW) overlaid with the crystal structure of native HuLys (PDB code 2ZIJ). c) Linear FTIR spectra, 2D-IR rephrasing spectra and vibrational lifetime of HEWL-RC and HuLys-RC in $\mathrm{D}_{2} \mathrm{O}$ and $\mathrm{H}_{2} \mathrm{O}$ respectively.

Figure 1.25. a) Incorporation of $\mathrm{CpRe}(\mathrm{CO})_{3}$ with an alkylated methanethiosulfonate onto thiol functionality on proteins. b) Bioorthogonal incorporation of a $\mathrm{CpRe}(\mathrm{CO})_{3}$ alkynl derivative onto residue 109 of the protein CTL9, located in a loop, using the "click" reaction.

Figure 1.26. a) Incorporation of $\mathrm{Cr}(\mathrm{CO})_{3}$ labelled cholesterol into membrane constructs. bi) Rapidly acquired spectral diffusion (RASD) measures the FFCF of the conjugate in bulk water and at the bicelle-water interface. (ii) Rephasing decay for the bicelle sample showing the full data set and the biexponential fit.

Figure 1.27. a) Jablonski diagram representing the energy transition for Rayleigh, Raman scattering and IR absorption. b) A schematic Raman spectrum showing the placement of the Stokes and anti-Stokes shift in relation to the Rayleigh line.

Figure 1.28. Molecular structure of $\left[\mathrm{Mn}(\mathrm{tpm})(\mathrm{CO})_{3}\right] \mathrm{Cl}$ and its use for Raman imaging in HT29 human colon cancer cell.

Figure 1.29. i) Preparation of OM-NP-L conjugates, using the strong interactions of $\mathrm{Os}_{3}(\mathrm{CO})_{10}(\mu-\mathrm{H})_{2}$ with gold nanoparticles, and further functionalized with PEG and antibodies to give conjugate OM-NP-L conjugates. ii) Bright-field, dark-field, and SERS mapping images of OSCC cells and SKOV cells treated with OM-NP-L.

Figure 1.30. Illustration of the photoacoustic (PA) mechanism.

Figure 1.31. Molecular structure of the water-soluble triosmium carbonyl cluster and its use for in vivo photoacoustic imaging of the rat cerebral cortex vasculature.

Figure 2.1. a) Components of a metal carbonyl cluster as Raman reporters. b) Binding of the metal carbonyl clusters onto AuNS via the sulfur groups.

Figure 2.2. Overlay of IR spectra ( $v C O)$ of cluster $\mathbf{2 a}, \mathbf{3 b}$ and $\mathbf{4}$ in DCM. 
Figure 2.3. Overlay of IR spectra ( $v C O)$ of cluster 6 and $6 . \mathrm{H}^{+}$in DCM.

Figure 2.4. SERS spectra (vCO) of cluster $\mathbf{2 a}, \mathbf{3 b}$ and $\mathbf{4}$ incubated with AuNS.

Figure 2.5. SERS spectra ( $v C O)$ of cluster $2 \mathbf{a}, 6$ and $6 . \mathrm{H}^{+}$incubated with AuNS.

Figure 2.6. SERS spectra ( $v \mathrm{CO})$ of clusters $\mathbf{7 a}$ and $\mathbf{7 b}$ incubated with AuNS.

60

Figure 2.7. a) The CO vibrational frequency of a half-sandwich metal carbonyl complex can be tuned via the $\mathrm{Cp}$ ring, metal center and the ligands. b) Molecular structures of halfsandwich metal carbonyl complexes used in the study.

Figure 2.8. SERS spectra (vCO) of 9a-13a incubated with AuNS.

Figure 2.9. Proposed interaction of the half-sandwich metal complexes with AuNS.

Figure 2.10. SERS spectra ( $v C O)$ of $\mathbf{2 a}$ incubated with AuNS with centrifugation at low and high speed respectively.

Figure 2.11. a) Optical absorption spectra and b) TEM images of 9a-10a and 12a-13a incubated with AuNS.

Figure 3.1. Sequence of reactions that occur in glucose oxidase-mediated biosensor.

66

Figure 3.2. Schematic showing the partitioning of glucose by the (1-mercaptoundeca-11yl)tri(ethylene glycol) partition layer on the metallic SERS substrate.

Figure 3.3. a) Interaction of a fluorescent diboronic acids anthracene conjugate with a glucose molecule. b) Illustration of glucose-bidendate boronic acid complex while other saccharides form a monodendate complex.

Figure 3.4. Schematic for the selective detection of glucose on the SERS-active substrate using boronic acid chemistry.

Figure 3.5. (a) Bright field image of BMFOM. SERS mapping images at $1580 \mathrm{~cm}^{-1}$ before (b) and after (c) immobilization of BA. Stability test of BA-functionalized BMFON in PBS solution - SERS mapping images at $1580 \mathrm{~cm}^{-1}$ on (c) day 1, (d) day 2 and (e) day 3. (f) SERS spectra of BA-functionalized BMFON from days 1 - 3.

Figure 3.6. ORTEP plot of the molecular structure of $\mathbf{2 b}$-boroxine.

Figure 3.7. The Raman responses for BA-functionalized BMFON incubated with (a) glucose, (b) fructose, (c) galactose and (d) control, prior to incubation with $\mathbf{2 b}-\mathrm{B}(\mathrm{OH})_{2}$.

Figure 3.8. (a) Plot represents the intensity of $\mathrm{CO}$ stretching frequency versus different concentrations of glucose, fructose and galactose, with the interpolated value for the spiked urine sample shown in red. (b) SERS spectrum of $\mathbf{2 b}-\mathrm{B}(\mathrm{OH})_{2}$ obtained from different concentrations of glucose. (c) SERS spectrum of $\mathbf{2} \mathbf{b}-\mathrm{B}(\mathrm{OH})_{2}$ after BMFON has been incubated with a urine sample spiked with glucose.

Figure 3.9. Molecular structures of sialic acid and glycan epitopes commonly found in cancer cells.

Figure 3.10. ORTEP plot of the molecular structure of $\mathbf{2 b - O H}$ (top) and $\mathbf{2 b}-\mathrm{COOH}$ (bottom). Thermal ellipsoids are drawn at the $50 \%$ probability level.

Figure 3.11. Comparison of the antiproliferative activity among the clusters $(5 \mu \mathrm{M})$ and ligand BA $(50 \mu \mathrm{M})$ on MDA-MB-231 cells, using 1 as the positive control. Control: DMSO $(0.5 \%, \mathrm{v} / \mathrm{v})$.

Figure 3.12. Comparison of the $\mathrm{IC}_{50}$ of $1,2 \mathbf{b}-\mathrm{B}(\mathrm{OH})_{2}$ and $-\mathrm{OH}$ on $\mathrm{MDA}-\mathrm{MB}-231$ and MCF-7 cells under serum and serum-free conditions.

Figure 3.13. Bright-field images of MDA-MB-231, MCF-7 and MCF-10A cells, in serumfree condition, after $24 \mathrm{~h}$ incubation with $10 \mu \mathrm{M}$ solutions of $\mathbf{1}, \mathbf{2 b}-\mathrm{B}(\mathrm{OH})_{2}$ and $\mathbf{2 b}-\mathrm{OH}$. 
Figure 3.14. Molecular structure of Hoechst 33342.

Figure 3.15. Fluorescence images of MCF-7 cells stained with Hoechst 33342 after $20 \mathrm{~h}$ 96 treatment with: a) negative control, $0.5 \% \operatorname{DMSO}(\mathrm{v} / \mathrm{v})$, b) $\mathbf{1}(4 \mu \mathrm{M})$, c) $\mathbf{2 b}-\mathrm{B}(\mathrm{OH})_{2}(6 \mu \mathrm{M})$ and d) $\mathbf{2 b}-\mathrm{OH}(4 \mu \mathrm{M})$. The arrows indicate apoptotic cells.

Figure 3.16. Contour-scatter plots showing the nuclear area vs total DNA fluorescence intensity of single MCF-7 cells after $20 \mathrm{~h}$ treatment: a) negative control, $0.5 \% \mathrm{DMSO}(\mathrm{v} / \mathrm{v})$, b-c) $\mathbf{2 b}-\mathrm{B}(\mathrm{OH})_{2}$ (3 and $6 \mu \mathrm{M}$ respectively) and d-e) $\mathbf{2 b}-\mathrm{OH}$ (2 and $4 \mu \mathrm{M}$ respectively). The dots represent single-cell measurements quantified from the images.

Figure 3.17. Externalization of phosphartidylserine at the plasma membrane of apoptotic cells and detection by Annexin V-FITC conjugate.

Figure 3.18. Fluorescence images of MCF-7 cells stained with Hoechst 33342 (blue) and Annexin V-FITC (green) after $20 \mathrm{~h}$ treatment with: a) negative control, 0.5\% DMSO (v/v), b-c) $\mathbf{2 b}-\mathrm{B}(\mathrm{OH})_{2}$ (3 and $6 \mu \mathrm{M}$ respectively), d-e) $\mathbf{2 b}-\mathrm{OH}$ (2 and $4 \mu \mathrm{M}$ respectively) and f) $\mathbf{1}$ $(4 \mu \mathrm{M})$.

Figure 3.19. Image-based quantification of total fluorescence intensity of Annexin V-FITC from MCF-7 cells treated with various clusters. Negative control, 0.5\% DMSO (v/v).

Figure 3.20. a) Overlays of IR spectra of $2 \mathbf{b}-\mathrm{B}(\mathrm{OH})_{2}$ incubated with 5 eq. of $\mathrm{H}_{2} \mathrm{O}_{2}$ and 1 eq. of imidazole in $\mathrm{H}_{2} \mathrm{O}: \mathrm{MeOH}\left(1: 3\right.$, v/v) over time. Authentic spectra of $\mathbf{2 b}-\mathrm{B}(\mathrm{OH})_{2}$ and $-\mathrm{OH}$ were overlaid for comparison. All spectra were taken in $\mathrm{MeOH}$. b-d) Expanded regions of various bands in the spectra.

Figure 3.21. ESI-HRMS of $\mathbf{2 b}-\mathrm{B}(\mathrm{OH})_{2}$ incubated with 5 eq. of $\mathrm{H}_{2} \mathrm{O}_{2}$ and 1 eq. of imidazole in $\mathrm{H}_{2} \mathrm{O}: \mathrm{MeOH}(1: 3, \mathrm{v} / \mathrm{v})$ taken at a) $1 \mathrm{~h}$ and b) $3 \mathrm{~h}$. c) ESI-HRMS of $\mathbf{2 b}-\mathrm{B}(\mathrm{OH})_{2}$ incubated with 1 eq. imidazole but without the addition of $\mathrm{H}_{2} \mathrm{O}_{2}$. The $\mathrm{m} / \mathrm{z}$ isotopic pattern corresponding to $\mathbf{2} \mathbf{b}-\mathbf{B}(\mathrm{OH})_{2}$ was highlight in blue, while the $\mathbf{2} \mathbf{b}-\mathrm{OH}$ was in red.

Figure 3.22. Cyclic voltammograms of $2 \mathrm{mM}$ of $\mathbf{2 b}-\mathrm{OH}, \mathbf{2 b}$ and $\mathbf{2 b}-\mathrm{COH}$ in $\mathrm{CH}_{3} \mathrm{CN}$ at $22 \pm$ $0.2{ }^{\circ} \mathrm{C}$ with $0.1 \mathrm{M} \mathrm{nBu} \mathrm{NPF}_{6}$, recorded at a $1 \mathrm{~mm}$ diameter planar glassy carbon electrode at a scan rate of $0.1 \mathrm{~V} \mathrm{~s}^{-1}$.

Figure 4.1. a) Molecular structures of low nuclearity ruthenium complexes 12'a, 20 and 21. Counter cation has been omitted for clarity. b) Optical absorption spectra of the complexesin DMSO at their respective concentrations.

Figure 4.2. a) Molecular structures of HNCC 22-26. Counter cations and CO ligands in the HNCCs have been omitted for clarity. b) Optical absorption spectra of HNCCs in DMSO at $200 \mu \mathrm{M}$. Boxed area represents the NIR region of interest.

Figure 4.3. i) PA (red) and optical absorption (black) spectra of a) cluster 24 and b) 26. c) Combined plot of PA amplitude as a function of concentrations of 22-26 in DMSO.

Figure 4.4. Cell viability (MTS) assay of a) $[\mathrm{PPN}] \mathrm{Cl}$ and b) $\mathrm{Na}_{2} .26$ on OSCC cells, using $1 \%$ DMSO as vehicle.

Figure 4.5 a) Optical absorption (black) and PA spectra (red) of $\mathrm{Na}_{2} .26(200 \mu \mathrm{M}$, $10 \%$ DMSO in modified DMEM). b) PA response of $\mathrm{Na}_{2} .26$ as a function of concentration in three different solvent compositions. c) Single wavelength PA image of the phantom at $760 \mathrm{~nm}$. The right channel contains a solution of $\mathrm{Na}_{2} .26(200 \mu \mathrm{M}$, $10 \%$ DMSO in modified DMEM), while the left channel contains only the DMSO in medium.

Figure 4.6. Time course study of a solution of $\mathrm{Na}_{2} . \mathbf{2 6}(200 \mu \mathrm{M})$ in $10 \%$ DMSO in modified DMEM, over a period of 7 days: a) FTIR spectra ( $v C O)$ obtained from the dried samples, b) integrals of the $v \mathrm{CO}$ absorbance, c) optical absorption spectra, d) electrophoresis gel.

Figure 4.7. Time course studies of $\mathrm{Na}_{2} .26$ and ICG over 120 min with irradiation at the respective $\lambda_{\max }$ : a) PA amplitude, b) optical absorbance as a function of time. 
Figure 4.8. In vivo PA imaging of mouse OSCC xenograft model using $\mathrm{Na}_{2} . \mathbf{2 6}(200 \mu \mathrm{L}$, $500 \mu \mathrm{M})$ as a contrast agent.

Figure 4.9. In vivo PA imaging of mouse OSCC xenograft model using $\mathrm{Na}_{2} . \mathbf{2 6}(200 \mu \mathrm{L}$, $500 \mu \mathrm{M})$ as a contrast agent. a) Transverse sections of the mouse abdomen, at $2 \mathrm{~mm}$ interval, before and at $1 \mathrm{~h}$ after the injection of $\mathrm{Na}_{2} .26$. b) Fold change in the PA intensity in the liver, spleen and kidneys over that at $\mathrm{t}=0$.

Figure 4.10. ICP-MS analysis of the amount of Os per tissue mass in various major organs of mice, $24 \mathrm{~h}$ and $96 \mathrm{~h}$ after injection of $\mathrm{Na}_{2} . \mathbf{2 6}$, with that of control samples.

Figure 4.11. Histopathology ( $\mathrm{H} \& \mathrm{E}$ staining) of liver and spleen tissues of mice, $24 \mathrm{~h}$ and $96 \mathrm{~h}$ after intravenous injection of $\mathrm{Na}_{2} . \mathbf{2 6}$, with that of control samples. Scale bar $=100 \mu \mathrm{m}$.

Figure 4.12. Graphical representation of the encapsulation of HNCC by PS- $b$-PAA via the thermodynamic and kinetic approach.

Figure 4.13. TEM images of $\mathrm{PS}_{154}-b-\mathrm{PAA}_{49}$ encapsulation of 23 heated at $110{ }^{\circ} \mathrm{C}$ for $5 \mathrm{~min}$

Figure 4.14. ATR-FTIR spectra of polymer bound cluster 23 heated at $110{ }^{\circ} \mathrm{C}$ for $5 \mathrm{~min}$ (red) and 15 min (blue) respectively, in comparison with cluster 23 (black).

Figure 4.15. Photograph of cluster 23 incubated with various amount of polymer $(0.48$, $0.40,0.24,0.16$ and $0.08 \mathrm{mg}$ from samples 1 to 5 respectively). Control sample contains no polymer.

Figure 4.16. TEM images of (a-c) samples 1, 2 and 5, and d) control sample.

Figure 4.17. IR spectra of cluster 23 (red) and the cluster with polymer (black).

Figure 5.1. Summary of the work investigated in this thesis.

Figure 5.2. Future work for translating the glucose assay to the detection of other biomolecules and for the use of cheaper transition metals as SERS probes.

Figure 5.3. Future work for the validation of the role of the $R$ group in modulating the cytotoxicity of the phenylthiolatio clusters $\mathbf{2 b}$ and bioconjugation of $\mathbf{2} \mathbf{b}$ to cancer-targeting ligands via the $\mathrm{X}$ group. 


\section{List of Tables}

\section{Content Pg}

Table 1.1. Use of various metal carbonyls for sensing applications via frequency shifts in $\mathrm{CO}$ vibrations.

Table 1.2. Summary and comparison between IR and Raman spectroscopy.

Table 2.1. IR frequencies of $\mathbf{2 a}$ to $\mathbf{7 b}$ and the respective SERS frequencies of the AuNS conjugates.

Table 2.2. IR frequencies of 9a to 13a and the respective SERS frequencies of the AuNS conjugates.

Table 3.1. Inhibition of cell growth on MDA-MB-231 cells under serum-free conditions, after $24 \mathrm{~h}$, as determined by MTS assay.

Table 3.2. Inhibition of cell growth on breast cell lines, after $24 \mathrm{~h}$ in serum and serum-free condition, as determined by MTS assay.

Table 3.3. Crystallographic data for $\mathbf{2 b}$-boroxine, $-\mathrm{OH}$ and $-\mathrm{COOH}$.

Table 4.1. Optical absorption and PA properties of clusters 22-26 and indocyanine green (ICG). 


\section{Chapter 1: Organometallic Complexes for Bio- Imaging and -Sensing}

\subsection{Bioorganometallic chemistry}

Organometallic chemistry, which lies at the intersection between organic and inorganic chemistry, involves the study of compounds with metal-carbon bond(s). Early interests were driven by its relevance to catalysis, and their industrial applications. Some important examples of industrial catalyst systems are those for the Monsanto and Cativa processes for the production of acetic acid, and Ziegler-Natta catalyst for the synthesis of polymers. ${ }^{1-3}$ Generally perceived as air- and -moisture sensitive as a consequence of research in the early days, the relevance of organometallic compounds to biology was largely neglected. The term "bioorganometallics", first coined in 1985, refers to the study and synthesis of organometallic species of biological and medical interest and has attracted much attention since then. ${ }^{4,5}$ This is especially so after the first International Symposium on Bioorganometallic Chemistry (ISBOMC) which was held in 2002. Current research interest is wide-ranging and includes applications in pharmaceuticals, bio-catalysis, bio-imaging and -sensing.

The interest in organometallic complexes as biosensors is of great relevance, especially in an era with higher demand for better healthcare and disease diagnosis than before. Biosensors can be defined as analytical devices incorporated with a biological sensing element which allows them to have specificity and sensitivity for the identification of targets of interest in biological systems; they are usually coupled with physiochemical transducers to deliver complex bioanalytical measurements for quantitative or qualitative detection. ${ }^{6,7}$ In bio-imaging, a visual output or quantitative measurement is produced which can be processed to give a visual image or map.

The interest in organometallic complexes for bio-sensing mainly lies in the unique properties (electrochemical, radiological or optical) that arise from the metal centers, as well as the ligand-metal interactions, which are not present in organic compounds. The metal centers exhibit diverse coordination numbers and geometry and hence can provide multiple conjugation sites for the development of multi-functional probes. The 
latter have enabled various sensing modalities, in the form of electrochemical, radioisotopic and optical-based probes. A brief survey of the first two modalities will be followed by a more in-depth survey of optical modality; the latter can be described as the study of light and its interaction with matter, and includes fluorescence, vibrational spectroscopy and photoacoustic imaging.

\subsection{Electrochemical-based probes}

Ferrocene $(\mathrm{Fc})$, which consists of a $\mathrm{Fe}^{2+}$ bound between two cyclopentadienyl rings in a sandwich manner, is the classic representative organometallic compound. Since its discovery more than 60 years ago, ${ }^{8,9} \mathrm{Fc}$ has contributed to the development of bioorganometallic chemistry tremendously, especially in the area of pharmaceutical and electrochemical sensing. ${ }^{10-12}$ An important example is its incorporation into Fcmediated amperometric glucose sensors, which has been made available commercially since 1987 , allowing diabetic patients to monitor their conditions with ease. ${ }^{6,13}$ The application of $\mathrm{Fc}$ in electrochemical sensing has been largely driven by its intrinsic robust and fully reversible reduction/oxidation properties, as well as good aqueous stability. The ease with which Fc can be functionalized has also allowed it to be utilized as a building scaffold for bioconjugation to a wide range of biomolecules, thus allowing it to be developed into an efficient and selective electrochemical sensor. ${ }^{14}$

In one example, a helical peptide with the sequence Arg-Pro-Leu-Ala-Leu-TrpArg-Ser-Cys conjugated to $\mathrm{Fc}$ at the N-terminal was used to detect the activity of matrix metalloproteinase-7 (MMP-7) (figure 1.1). ${ }^{15}$ MMPs are a class of extracellular, zinc-dependent proteinases known to be involved in many important biological processes, and they have been identified as important biomarkers of diseases, inflammation and infection. ${ }^{16,17}$ The Fc-peptide conjugate was self-assembled onto a gold electrode surface, followed by the incubation with MMP-7. The conjugate was anchored onto the gold surface via the thiol moiety at the Cys residue and, in the presence of MMP-7, the peptide is cleaved leading to loss of the Fc moiety. The substantial decrease in the redox current, detected by cyclic voltammetry (CV) and square wave voltammetry (SWV), was used for quantification. The specificity of the cleavage by MMP-7 was achieved at a much faster rate, in comparison to other subtypes of the enzyme (MMP-2 and MMP-3), with a low detection limit of 3.4 pM. 

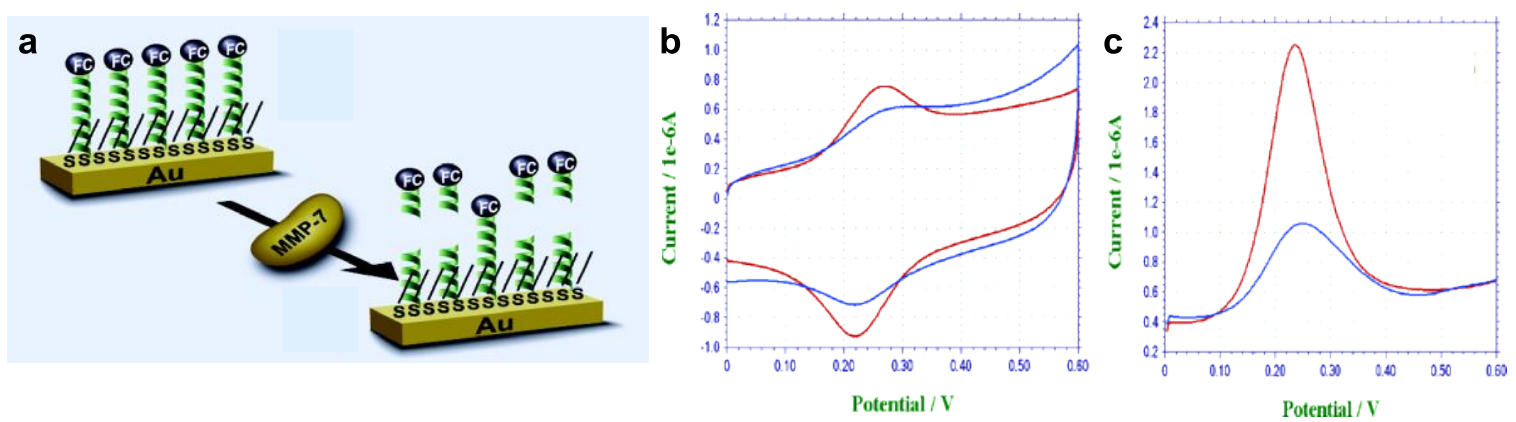

Figure 1.1. a) Schematic representation for the detection of matrix metalloproteinase: selfassembly of electrochemical Fc-peptide conjugate on the gold substrate, followed by cleavage of the peptide in the presence of MMP-7. b) CV of the Fc-peptide-Au electrode in $0.6 \mathrm{M}$ $\mathrm{NaClO}_{4}$ solution before (red curve) and after (blue curve) cleavage with $2 \mathrm{ng} \mathrm{mL}{ }^{-1}$ MMP-7. c) Corresponding SWV of Fc-peptide-Au electrode. All potentials are referred to an $\mathrm{Ag} / \mathrm{AgCl}$ reference electrode. Adapted from ref. 15 with permission from the American Chemical Society.

Other than peptides, the conjugation of Fc to peptide nucleic acid (PNA) for use as electrochemical probes has also been widely reported. ${ }^{18-20} \mathrm{PNA}$, which is analogous to DNA, is a polymer consisting of a backbone formed from repeating $N$-(2-aminoethyl) glycine units linked by peptide bonds (figure 1.2a). ${ }^{21}$ Various purine and pyrimidine nucleobases, which are linked to the backbone via methylene carbonyl bonds, can be hydrogen-bonded to complementary bases of DNA, forming hybrid PNA/DNA, similar to that of double stranded DNA (figure 1.2b).

Aoki and co-workers reported the anchorage of Fc-PNA conjugates onto gold electrode for detection of a specific DNA strand. ${ }^{18}$ The detection mechanism is based on hybridization of the conjugate with a complementary target DNA which leads to the formation of a rigid duplex structure that restricts the Fc moiety from approaching the surface. This led to a decrease in current which can be used to quantify the concentration of the target DNA (figure 1.3). 
a

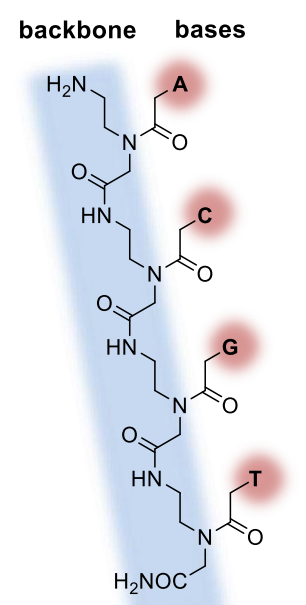

b

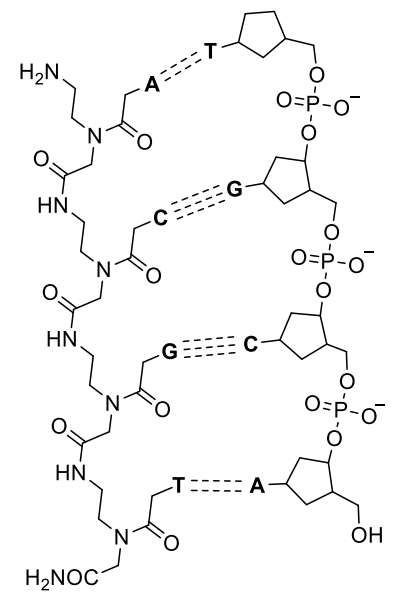

Figure 1.2. a) Structural representation of PNA backbone and side chains $(A, C, G, T=$ nucleobase). b) Structural representation of PNA/DNA hybrid. ${ }^{12}$ Adapted from ref. 12 with permission from the Royal Society of Chemistry.

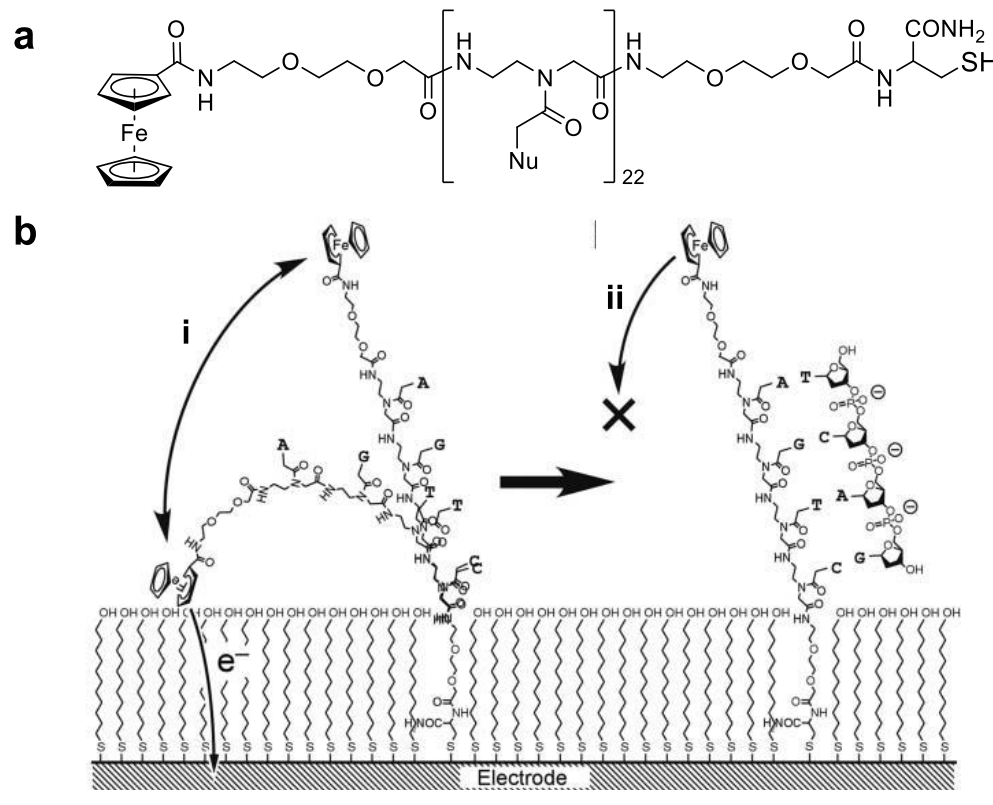

Figure 1.3. a) Molecular structure of the Fc-PNA conjugate. Nu represents a nucleobase (A, T, $\mathrm{G}$, or C). b) Working principle of a gene sensor based on the conformational flexibility change in the probe structure induced by hybridization, where the pair of sequences in PNA and DNA is represented as an example: (i) before hybridization, and (ii) after hybridization, the formation of duplexes makes the conjugate structure more rigid, access of the Fc to the surface is restricted, and consequently the electron transfer is inhibited. Adapted from ref. 18 with permission from the Royal Society of Chemistry. 
Fc-antibody conjugates have also been constructed to utilize their specific interactions with antigens to detect and study binding events. In many instances, selective binding of the conjugate to the antigen of choice is followed by separation from the free conjugate and detection of the Fc-antibody-antigen electrochemically. ${ }^{22-}$ ${ }^{24}$ For the detection of human chorionic gonadotropin (HCG), Fc carboxylic acid conjugated to anti-HCG immunoglobulin $\mathrm{G}$ ( $\mathrm{Ig} \mathrm{G}$ ) was prepared via carbodiimide coupling chemistry, and used as a detection tag in tandem with electrochemical capillary flow immunoassay (figure 1.4). ${ }^{22}$ Increasing HCG concentration leads to an increase in current which is used for quantification.

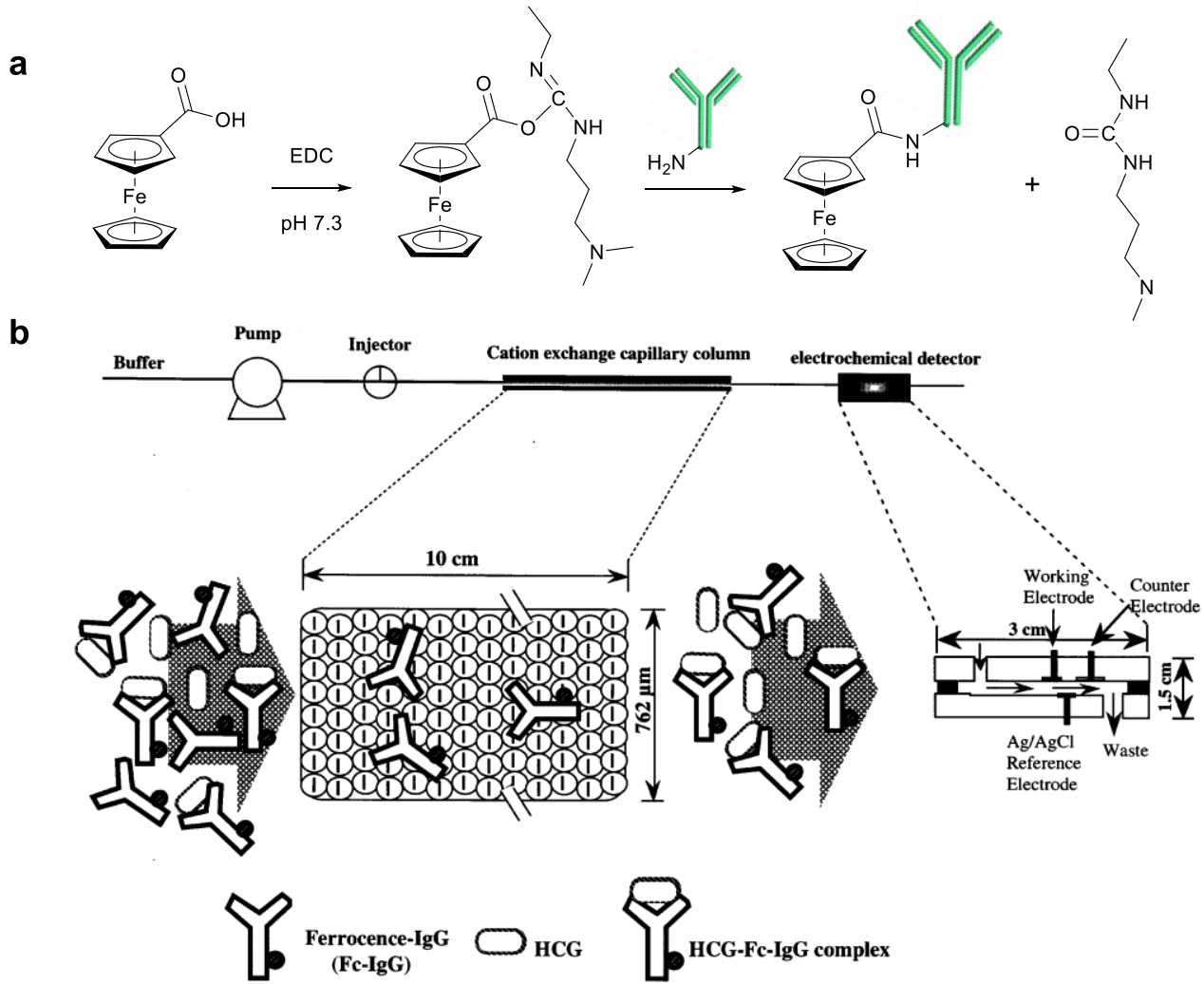

Figure 1.4. a) Immobilization of $\mathrm{Fc}$ on $\mathrm{IgG}$ using carbodiimide coupling chemistry. b) Schematic diagram of an electrochemical capillary flow immunoassay system (upper) and the working principle (lower). The reaction mixture consists of HCG, Fc-IgG and HCG-Fc-IgG complex. The mixture is passed through a capillary column that selectively traps Fc-IgG. The eluted species passes through the flow cell, and the current associated with the HCG-Fc-IgG antibody complex is monitored. Applied potential: $395 \mathrm{mV}$ versus $\mathrm{Ag} / \mathrm{AgCl}$. Adapted from ref. 22 with permission from Elsevier. 


\subsection{Radioisotope-based probes}

Positron emission tomography (PET) and single photon emission computed tomography (SPECT) represent two important diagnostic tools for the visualization of organ functions. ${ }^{25,26}$ Both techniques provide good sensitivity, and utilize the detection of gamma emission produced by exogenously administered radioactive probes. The demand for more efficient and safer probes continues to drive development in radiopharmaceuticals. One radionuclide of choice for this is technetium-99m $\left({ }^{99 \mathrm{~m}} \mathrm{Tc}\right)$.

The artificial element technetium (Tc) was first prepared by deuteron bombardment of molybdenum in $1937 .^{27}$ All the Tc isotopes are radioactive, with the metastable nuclear isomer $\left({ }^{99 \mathrm{~m}} \mathrm{Tc}\right)$ being the most applicable in nuclear medicine. ${ }^{28}$ The pivotal role that ${ }^{99 \mathrm{~m}}$ Tc plays in this field owes much to the ease of production (using the ${ }^{99} \mathrm{Mo} /{ }^{99 \mathrm{~m}} \mathrm{Tc}$ generator), ${ }^{28,29}$ low cost (in comparison to other radionuclides), biocompatibility and, most importantly, its ideal decay characteristics; the monoenergetic gamma ray of $140 \mathrm{keV}$ is of sufficient energy for deep tissue imaging, and the short half-life of $6 \mathrm{~h}$ is sufficient for diagnostic procedure and minimizes radiation exposure. ${ }^{30}$

There are many ${ }^{99 \mathrm{~m}} \mathrm{Tc}$-based radiopharmaceuticals available on the market, with only one (Cardiolite ${ }^{\circledR}$ ) being an organometallic complex; the rest are coordination complexes with non-carbon atoms (figure 1.5). ${ }^{31}$ Nevertheless, extension of the chemistry of technetium to its organometallic compounds has revealed many exciting avenues for their development into pharmaceuticals for targeted imaging. Most of those work has been devoted to developing synthetic strategies, as $\left[{ }^{99 m} \mathrm{TcO}_{4}\right]^{-}$is generally the only source of ${ }^{99 \mathrm{~m}} \mathrm{Tc}$ available in hospitals, in the form of an aqueous solution.

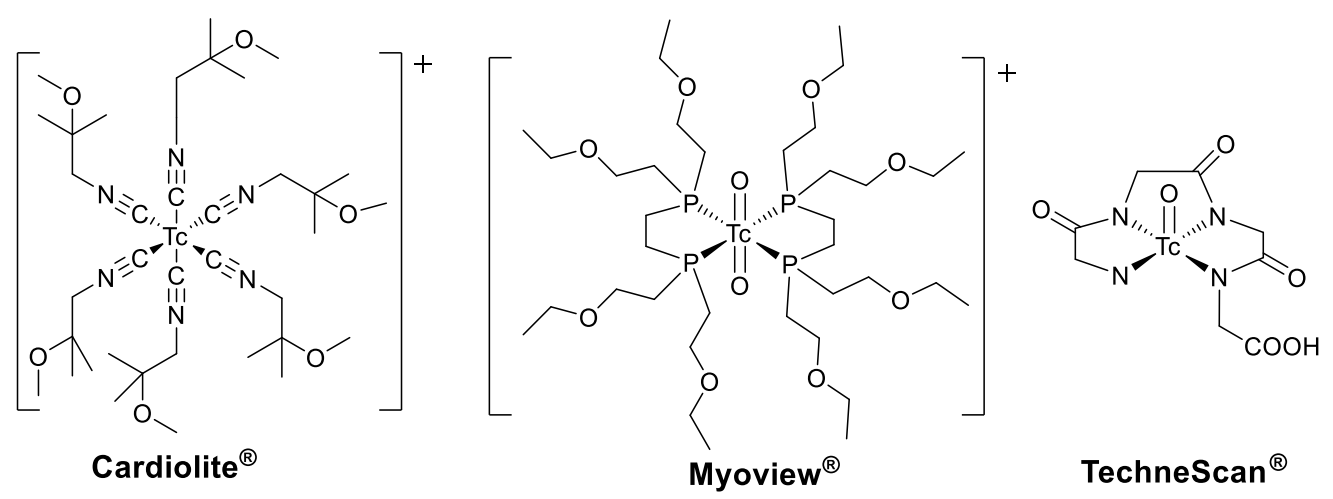

Figure 1.5. Some commercially available ${ }^{99 \mathrm{~m}} \mathrm{Tc}$-based radiopharmaceuticals. 
Metal carbonyls, which consist of one or more carbon monoxide (CO) ligands bound to the metal center, are common in organometallic chemistry and more details will be described in section 1.6. To explore the use of carbon monoxide (CO) as possible ligands for coordination to metal center, $\left[{ }^{99 \mathrm{~m}} \mathrm{Tc}\left(\mathrm{OH}_{2}\right)_{3}(\mathrm{CO})_{3}\right]^{+}$was synthesized in excellent yield using $\mathrm{CO}$ gas and $\mathrm{NaBH}_{4}$ (Scheme 1). ${ }^{32}$ For practical reasons, boranecarbonate became a preferred $\mathrm{CO}$ source as it enabled routine synthesis to be carried out clinically (scheme 1). ${ }^{33}$ The synthesis of $\left[{ }^{99 m} \mathrm{Tc}\left(\mathrm{OH}_{2}\right)_{3}(\mathrm{CO})_{3}\right]^{+}$ expanded the synthetic versatility of Tc as the aquo ligands could be easily replaced, leading to the development of many Tc-carbonyl complexes containing other ligands or biomolecules of choice. ${ }^{34,35}$ Some examples include conjugation to amino acid, ${ }^{36}$ annexin $\mathrm{A} 5,^{37}$ acridine-based nuclear targeting agent and specific cell-receptor binding peptides bombesin $(\mathrm{BBN}) .^{38}$

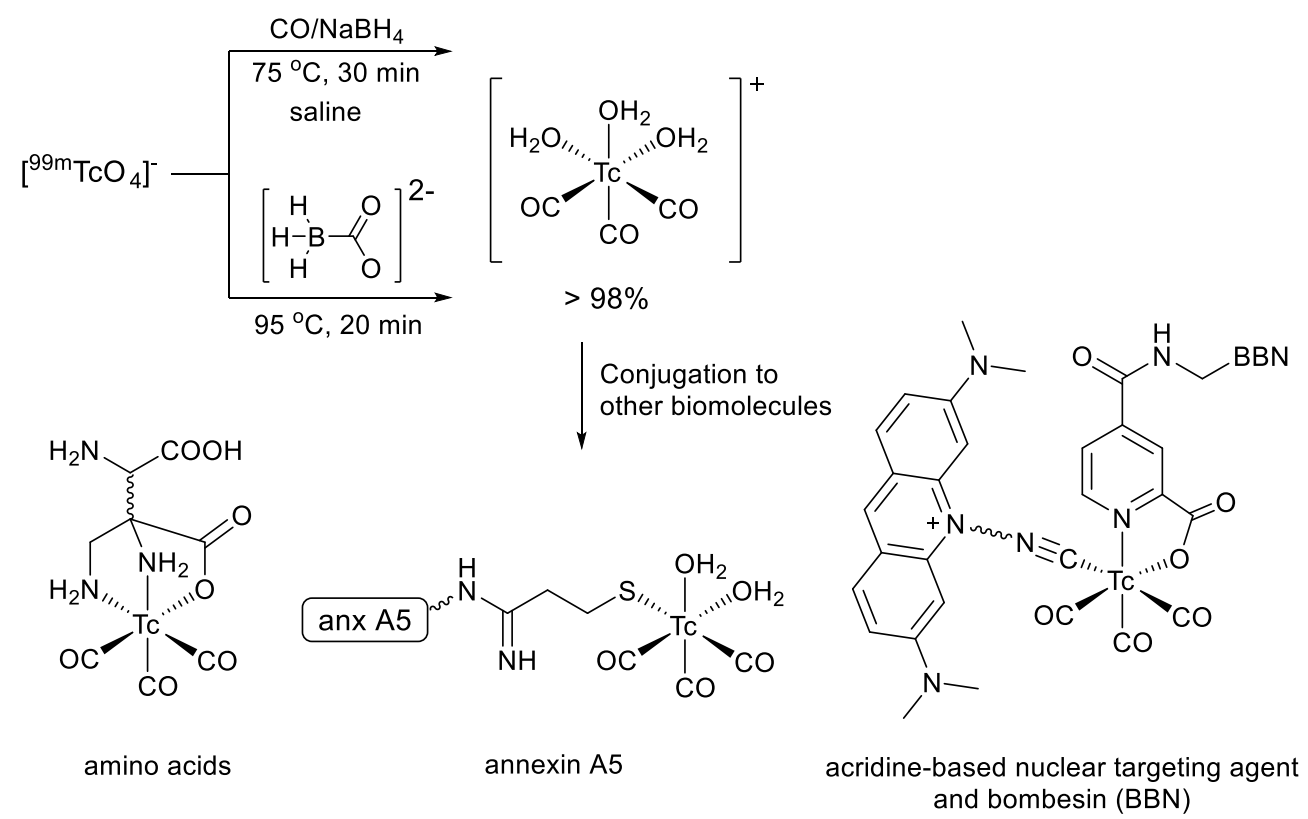

Scheme 1.1. Synthesis of $\left[{ }^{99 \mathrm{~m}} \mathrm{Tc}\left(\mathrm{OH}_{2}\right)_{3}(\mathrm{CO})_{3}\right]^{+}$and some examples of conjugates to biomolecules.

The coordination of classical organometallic ligands like cyclopentadienyl $(\mathrm{Cp})$ to ${ }^{99 \mathrm{~m}}$ Tc can be achieved using water-soluble acetyl Cp derivatives. ${ }^{39,40}$ An aqueous onepot synthesis of $\mathrm{CpTc}(\mathrm{CO})_{3}$ from $\left[{ }^{99 m} \mathrm{TcO}_{4}\right]^{-}$was demonstrated, which likely proceeded sequentially as shown (Scheme 1.2). ${ }^{39}$ More recently, facile syntheses of ${ }^{99 \mathrm{~m}} \mathrm{Tc}$ (arene) $)_{2}$ from $\left[{ }^{99 \mathrm{~m}} \mathrm{TcO}_{4}\right]^{-}$was achieved, allowing functionalized arenes to be used as building blocks. ${ }^{41}$ 


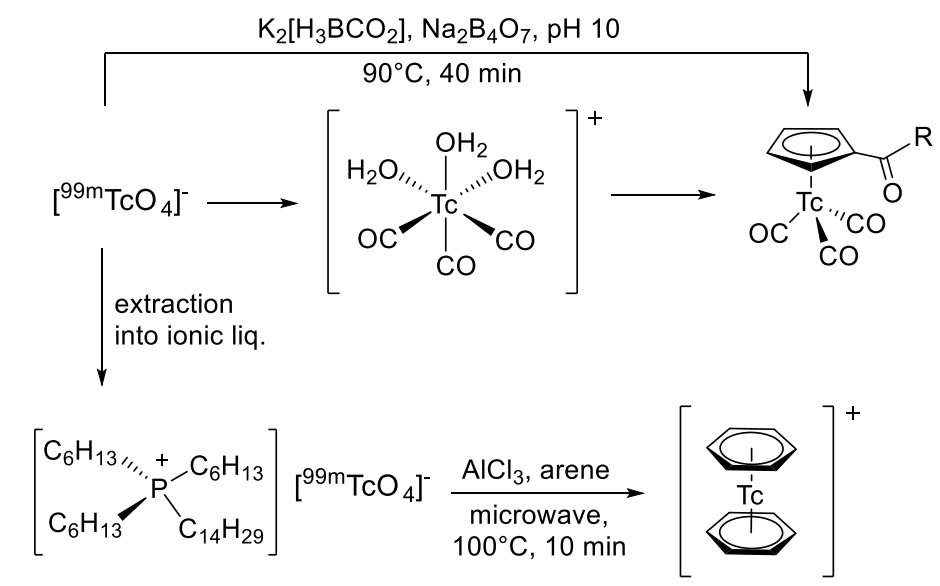

Scheme 1.2. Synthesis of $\left[{ }^{99 \mathrm{~m}} \mathrm{Tc}\left(\mathrm{OH}_{2}\right)_{3}(\mathrm{CO})_{3}\right]^{+}$and some examples of conjugates to biomolecules.

\subsection{Fluorescence-based probes}

Optical imaging is an emerging technology for biomedical imaging. Unlike modalities such as X-ray and positron emission tomography (PET), the use of nonionizing radiation in optical imaging is safer for both the user and the subject. Valuable physical and chemical properties, useful for diagnostics, may also be obtained when molecules absorb and scatter different wavelengths. ${ }^{42,}{ }^{43}$ One important example is fluorescence microscopy which has become an essential tool in biology. The development of fluorescence probes from green fluorescence protein, first isolated from the Aequorea jellyfish, ${ }^{44-46}$ to various fluorescence small organic molecules, ${ }^{47}$ provides a useful labelling tool for studying biological systems ranging from the cellular to single molecule level. ${ }^{48-50}$

The principle of fluorescence microscopy is dependent on light emission from the site of interest to improve contrast from the background. Fluorescence involves the emission of light upon absorption of incident light (typically of shorter wavelength) from an external source, or the natural emission from the sample. ${ }^{51,52}$ The higher wavelength emitted light can be detected while the excitation light is filtered off; the difference in wavelength between the excitation and emitted light is known as Stokes shift (figure 1.6). Excellent contrast can be obtained between the area of interest and areas detected via normal methods (reflection, transmission).

Besides organic fluorophores, fluorescent organometallic complexes have also been reported. These are mainly based on three classes: cyclometalated metal 
complexes, gold-based complexes and rhenium tricarbonyl polypyridyl complexes; some examples of the first two classes will be presented here. ${ }^{52}$

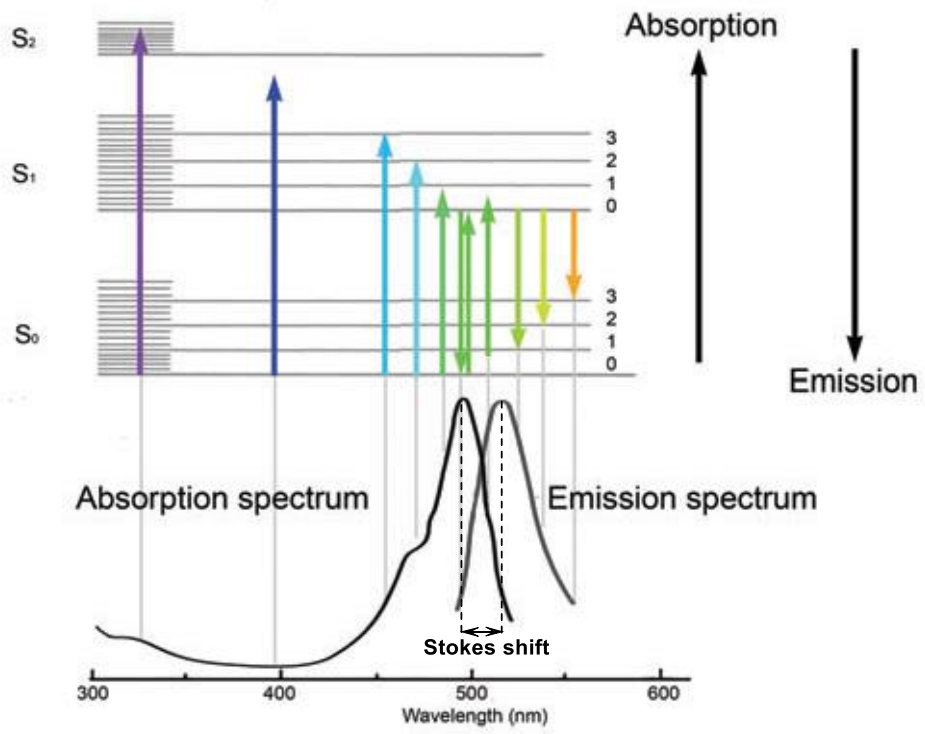

Figure 1.6. Jablonski diagram (top), and the absorption and emission spectra (bottom) of the common fluorophore fluorescein isothiocyanate (FITC). Each vertical gray line aligns the spectra with the energy of the absorbed (arrows pointing up) or emitted (arrows pointing down) photons. The arrows are colored to represent the wavelength of the photons. For example, the purple arrow to the left represents the energy of an ultraviolet photon that can cause the molecule to transition from the ground state to the second singlet excited state. The orange arrow on the right represents the lowest energy photon that can be emitted by this molecule as it drops back from the lowest energy state $S_{1}$ to a high vibrational state of $S_{0}$. Adapted from ref. 51 with permission from Macmillan Publishers Ltd: Nat. Methods.

Luminescent complexes can be formed via the cyclometalation of 2phenylpyridine with group 9 metals like iridium and rhodium. Many examples of such complexes, $\left[\mathrm{M}(\mathrm{C}-\mathrm{N})_{2}(\mathrm{~N}-\mathrm{N})\right]^{+}$(where $\mathrm{M}=\mathrm{Ir}$ or $\mathrm{Rh}$ ), have been reported and they generally show good cellular uptake and photophysical properties (figure 1.7). ${ }^{53-56}$ For example, Ir complexes coupled with $\mathrm{pH}$-responsive functionalities and photosensitizers have been employed in $\mathrm{pH}$ sensing and photodynamic therapy. ${ }^{57,58}$ 

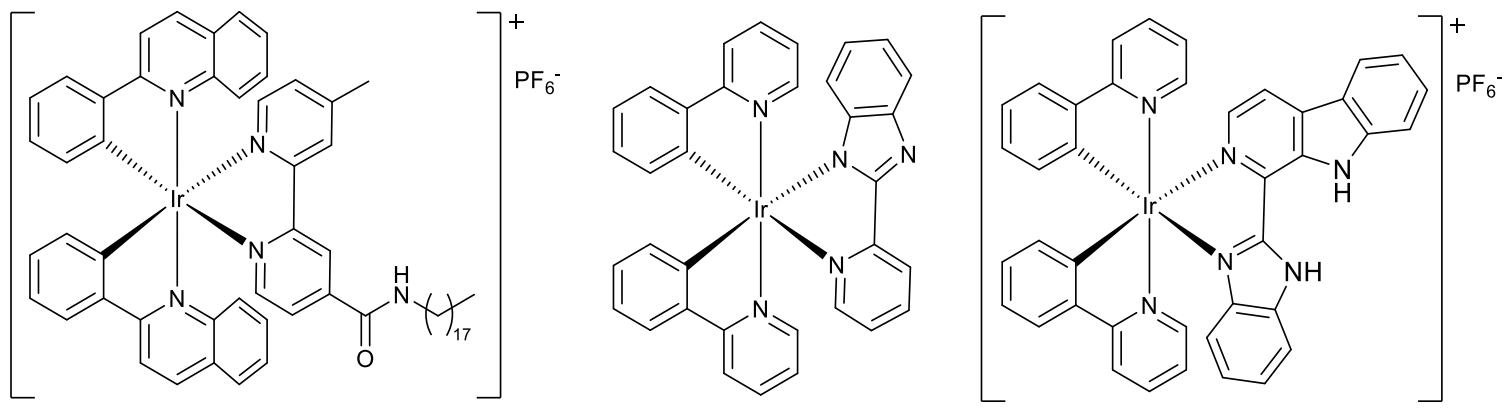

Figure 1.7. Molecular structures of selected cyclometalated $\left[\operatorname{Ir}(\mathrm{C}-\mathrm{N})_{2}(\mathrm{~N}-\mathrm{N})\right]$ complexes. ${ }^{53,55,58}$

Platinum cyclometalates also possess robust luminescent properties. An example is the use of $\mathrm{Pt}(\mathrm{II})$ complexes with cyclometalated 1,3-di(2-pyridyl) benzene and derivatives in time-resolved and two-photon emission imaging microscopy of live cells (figure 1.8). ${ }^{59}$ These complexes are highly tunable, via the substituent $\mathrm{R}$, over a spectral range from blue-green to orange. ${ }^{60}$ The complex $[\mathrm{Pt}(\mathrm{N}-\mathrm{C}-\mathrm{N}) \mathrm{Cl}]$-a possesses high emission, good photo-stability and low toxicity. It shows good cellular accumulation, via passive uptake, within five minutes of incubation and is preferentially localized onto nuclei acids.

a

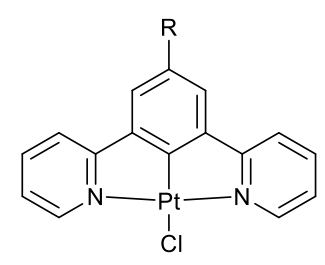

$[\mathrm{Pt}(\mathrm{N}-\mathrm{C}-\mathrm{N}) \mathrm{Cl}]$

a: $\mathrm{R}=\mathrm{H}$

b: $\mathrm{R}=\mathrm{C}(\mathrm{O}) \mathrm{OCH}_{3}$ c: $\mathrm{R}=\mathrm{CH}_{3}$

d: $\mathrm{R}=\mathrm{C}_{6} \mathrm{H}_{4}-\mathrm{N}\left(\mathrm{CH}_{3}\right)_{2}$ b
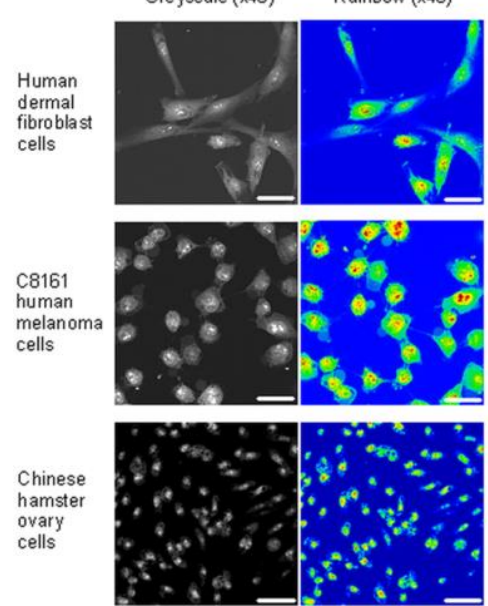

Greyscale (x63) Rainbow (x63)
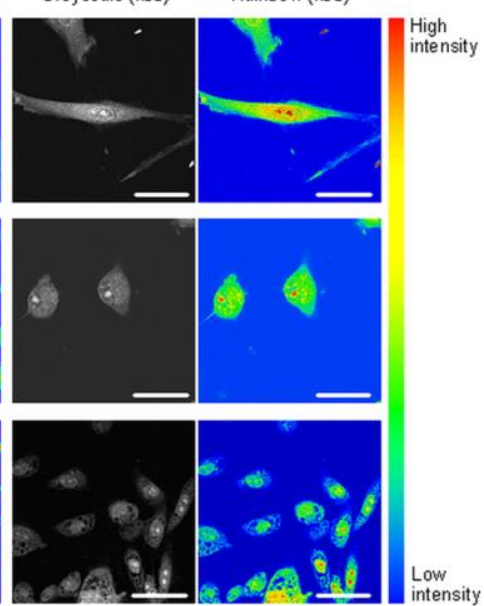

Figure 1.8. a) Molecular structure of the complexes $[\mathrm{Pt}(\mathrm{N}-\mathrm{C}-\mathrm{N}) \mathrm{Cl}]$. b) Confocal micrographs of human dermal fibroblasts, C8161 human melanoma, and CHO cells. Cells were incubated, washed with PBS and imaged by using an excitation wavelength of $488 \mathrm{~nm}$. Paired images show grayscale and rainbow intensities respectively, identifying regions of $[\mathrm{Pt}(\mathrm{N}-\mathrm{C}-\mathrm{N}) \mathrm{Cl}]-\mathbf{a}$ accumulation (Scale bar: $10 \mu \mathrm{m}$ ). Adapted from ref. 59.

The use of luminescent $\mathrm{Au}(\mathrm{I})$ complexes for fluorescence imaging, though rare, has also been reported. There is a report on a dinuclear $\mathrm{Au}(\mathrm{I})$ complex with a bidentate 
$\mathrm{N}$-heterocyclic carbene (NHC) ligand separated by methylene bridging groups; this is believed to provide a stronger support for an $\mathrm{Au} \cdots \mathrm{Au}$ interaction in comparison to the use of benzyl as the bridging group. ${ }^{61}$ This was largely motivated by the desire to tune the aurophilicity (the $\mathrm{Au} \cdots \mathrm{Au}$ interaction) and hence, the luminescent profile of the $\mathrm{Au}(\mathrm{I})$ complex (red-shifted); complexes with longer $\mathrm{Au} \cdots \mathrm{Au}$ separations are nonemissive. ${ }^{62,63}$ The as-synthesized Au(I) complex was introduced into RAW264.7 cells (mouse macrophage cancer cell line) and was found to localize within the lysozymes instead of the mitochondria (figure 1.9a). In another example, Dyson and co-workers synthesized a series of $\mathrm{Au}(\mathrm{I})$ alkynyl complexes containing water-soluble 1,3,5-triaza7-phosphaadamantane (PTA), which showed anticancer and luminescent properties (figure 1.9b). ${ }^{64}$ These trinuclear $\mathrm{Au}(\mathrm{I})$ alkynyl complexes showed good $\mathrm{IC}_{50}$ values of $3.3 \pm 0.2 \mu \mathrm{M}$ with A2780 ovarian cancer cells, and cellular uptake was confirmed by luminescence which was detected throughout the cells.
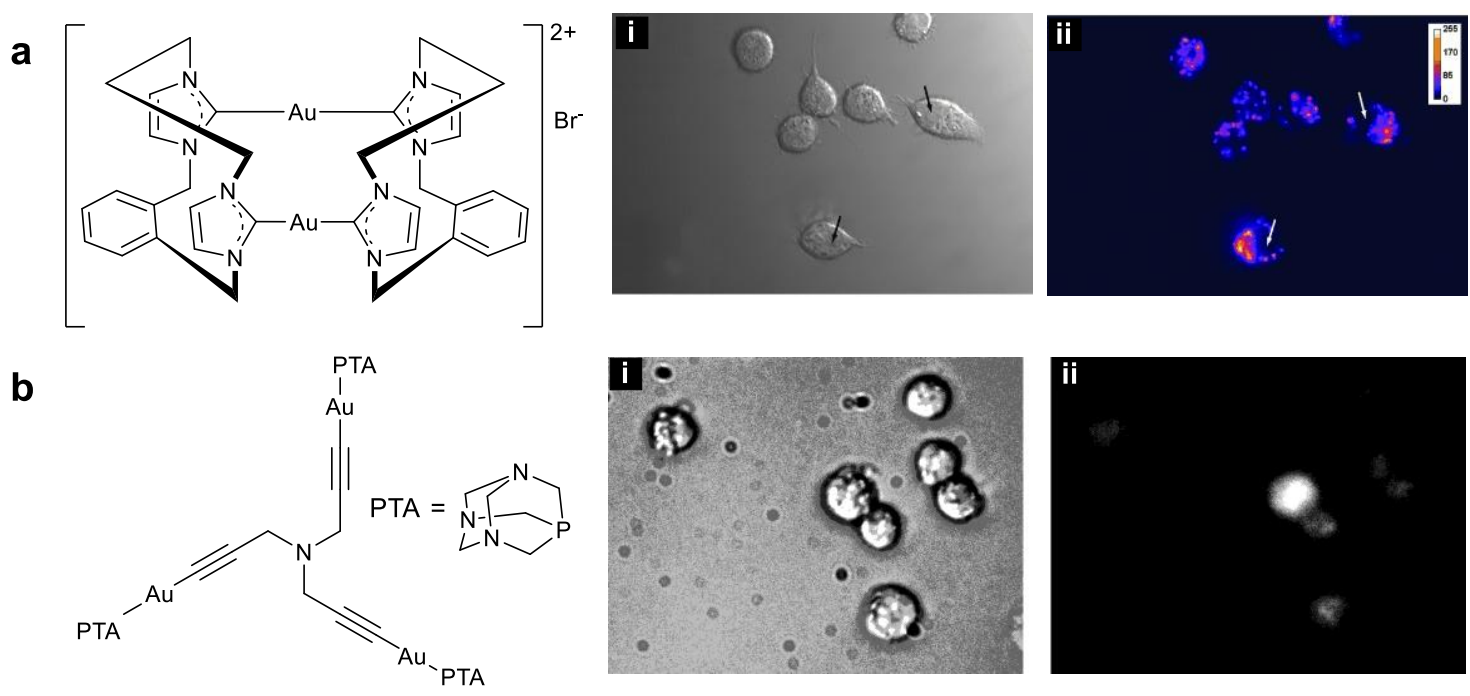

Figure 1.9. a) Molecular structure of the dinuclear $\mathrm{Au}(\mathrm{I}) \mathrm{NHC}$ complexes and cellular distribution of the complex $(200 \mu \mathrm{M})$ in RAW264.7 cells after incubation for $15 \mathrm{~h}$ at $37{ }^{\circ} \mathrm{C}$; i) brightfield image and ii) luminescence image $\left(\lambda_{\mathrm{ex}} 351 \mathrm{~nm}\right)$. The black and white arrows mark the positions of the nuclei. Adapted from ref. 61 with permission from John Wiley and Sons. b) Molecular structure of the trinuclear Au(I) alkynyl containing the PTA ligands and cellular distribution of the complex in A2780 cells after incubation for $6 \mathrm{~h}$ at $37{ }^{\circ} \mathrm{C}$; i) bright field image and ii) luminescence image. Adapted from ref. 64 with permission from the American Chemical Society. 


\subsection{Transition metal carbonyls as mid-infrared (IR) probes}

As mentioned earlier, a class of organometallic compounds which has found wide applications in biology is the transition metal carbonyl complexes, i.e., complexes with CO ligand(s) coordinated to the metal center. The earliest example of a metal carbonyl, $\mathrm{Ni}(\mathrm{CO})_{4}$, was discovered by Ludwig Mond in 1890, which subsequently led to the Mond process which uses $\mathrm{CO}$ gas to purify and extract nickel from its ores in the form of $\mathrm{Ni}(\mathrm{CO})_{4}{ }^{65}$

\subsubsection{Bonding in transition metal carbonyls}

$\mathrm{CO}$ is an unsaturated ligand due to the $\mathrm{C}-\mathrm{O}$ multiple (triple) bond. Hence, it can be regarded as a soft ligand with $\pi$-accepting property. Its interaction with a metal center involves the donation of a lone pair of electrons from the carbon atom to the metal, and back donation from the metal to an anti-bonding orbital of the $\mathrm{C}-\mathrm{O}$ bond (figure 1.10a). The extent of $\pi$-back donation is usually the main factor affecting the $\mathrm{C}$-O bond order, which can be affected indirectly by the effective charge on, and ligands coordinated to, the metal. This in turn affects the vibrational frequency. Bonding to a metal center generally reduces the $\mathrm{C}-\mathrm{O}$ bond order, and the different bonding modes that a $\mathrm{CO}$ ligand can adopt, ranging from triply-bridging to terminal, leads to characteristic vibrational frequencies in the range of $1650-2200 \mathrm{~cm}^{-1}$ (figure $1.10 \mathrm{~b}-\mathrm{c}$ ). ${ }^{66}$ Hence, the $\mathrm{CO}$ vibrational frequencies of metal carbonyls, situated in a region which is relatively devoid of interference from other functional groups, are ideal for sensing applications. 


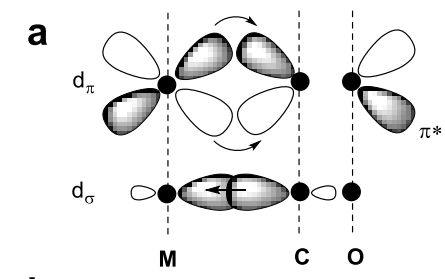

b

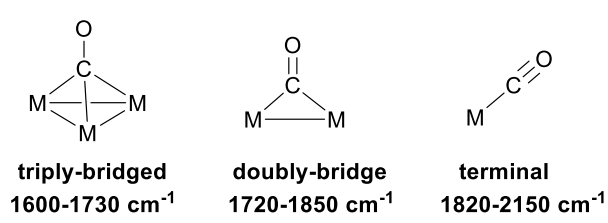

$\pi$ back donation from $\mathrm{M} \mathrm{d}$ to $\mathrm{C}-\mathrm{O} \pi^{*}$ orbital

$\sigma$ donation from $\mathrm{C}$ lone pair electrons to $M d_{\sigma}$ orbital

$1820-2150 \mathrm{~cm}^{-1}$

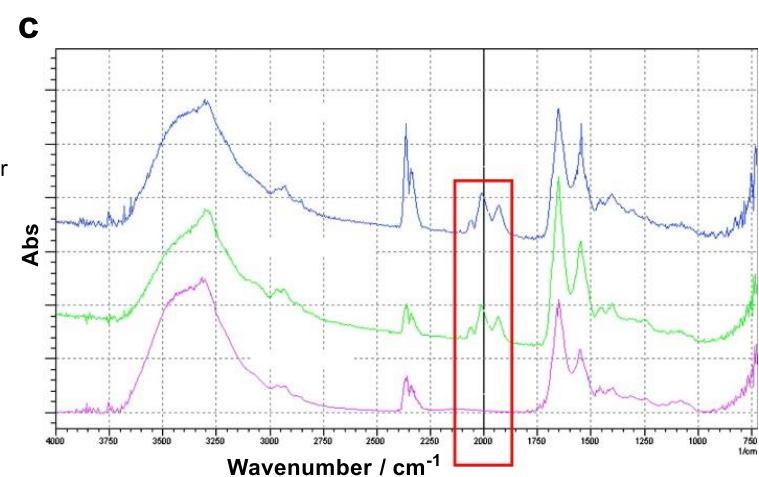

Figure 1.10. a) Orbital diagram showing the bonding between carbon monoxide with a transition metal centre. b) Different bonding modes of $\mathrm{CO}$ with different number of metal centers, forming the triply-bridge to terminal $\mathrm{CO}$ with vibrational frequencies in the range of $1650-2200 \mathrm{~cm}^{-1}$. c) IR spectra recorded in absorbance mode of untreated mucosa cells (bottom), cells treated with a triosmium carbonyl cluster (middle and top). Boxed region shows carbonyl (CO) stretching vibrations of the organometallic fragment. Adapted from ref. ${ }^{67}$ with permission from the American Chemical Society.

\subsubsection{Applications based on $\mathrm{CO}$ vibrational intensities}

The absorption intensity of metal carbonyls can be applied for quantitative sensing. Similar to fluorescence spectroscopy, the absorbance of the CO vibrational frequencies follows the Beer-Lambert law: $A=\varepsilon b c$, where $A$ is the absorbance, $\varepsilon$ is the molar absorptivity coefficient $\left(\mathrm{M}^{-1} \mathrm{~cm}^{-1}\right)$, b is the path length $(\mathrm{cm})$ and $\mathrm{c}$ is the analyte concentration (M). The viability of such quantification has allowed the development of mid-IR assays based on metal carbonyl, and was first realized by the group of Jaouen in a steroid hormonal assay to study the hormonal receptors on breast cancer cells. ${ }^{5,68}$

\section{- Steroidal hormonal assay}

This assay was driven by the need for an alternative to the hazardous and costly radio-isotopic method. The concept was demonstrated with the use of an $\eta^{6}-\mathrm{Cr}(\mathrm{CO})_{3^{-}}$ estradiol derivative which binds onto estrogen receptors in lamb uterus cytosol. ${ }^{5,68}$ The precipitated protein, incubated with the $\alpha$-derivative, was then prepared in the form of a CsI pellet and quantified by FT-IR spectroscopy; the area of the $A_{1}\left(v_{C O}\right)$ peak to the mass of the pellet gave a linear plot (figure 1.11). 

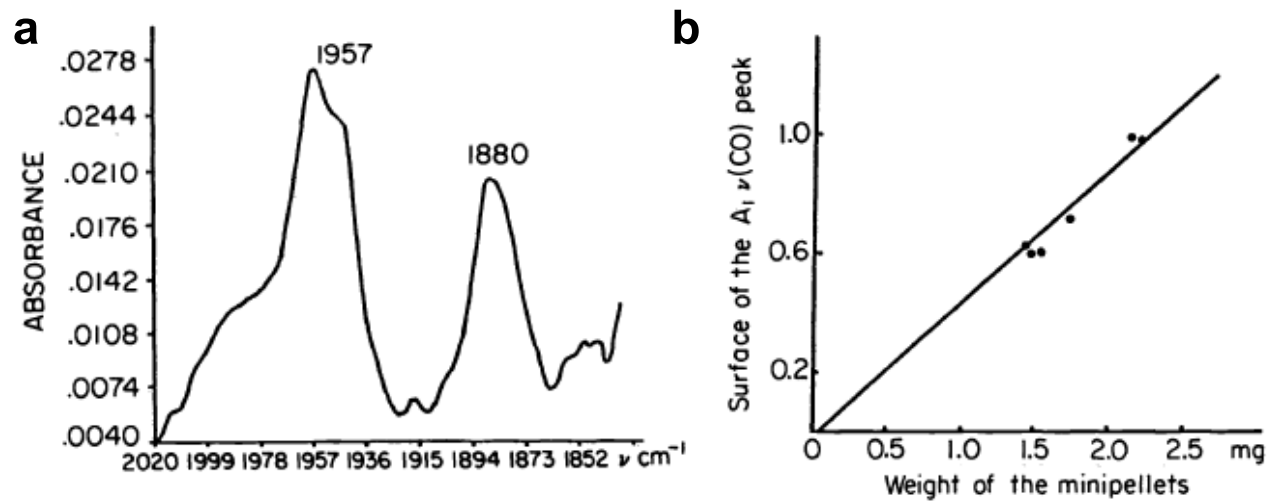

Figure 1.11. a) FT-IR ( $v C O)$ spectrum of precipitated lamb uterine cytosol incubated with $\eta^{6}-\mathrm{Cr}(\mathrm{CO})_{3}$-estradiol $\alpha$-derivative, prepared in CsI pellet. b) Correlation between the weight of the pellet and the area of the $\mathrm{A}_{1}(\mathrm{vCO})$ peak at $1957 \mathrm{~cm}^{-1}$. Adapted from ref. 5.

The conjugation to estradiol was subsequently extended to a derivative with an alkyne functionality at the $\mathrm{C} 17 \alpha$ - position. This allowed for complexation with di- and tri-nuclear metal carbonyl clusters like $\left[\mathrm{CpMo}(\mathrm{CO})_{2}\right]_{2},\left[\mathrm{Co}_{2}(\mathrm{CO})_{6}\right]$ and $\left[\mathrm{Os}_{3}(\mathrm{CO})_{10}\right]$ respectively (figure 1.12). ${ }^{69,70}$ Conjugation of metal carbonyls to other receptor binding molecules have also been reported, such as, mifepristone and hexestrol, with the former used for progesterone and glucocorticoid receptor assays, and the latter for estrogen receptor assays. ${ }^{71,72}$ 

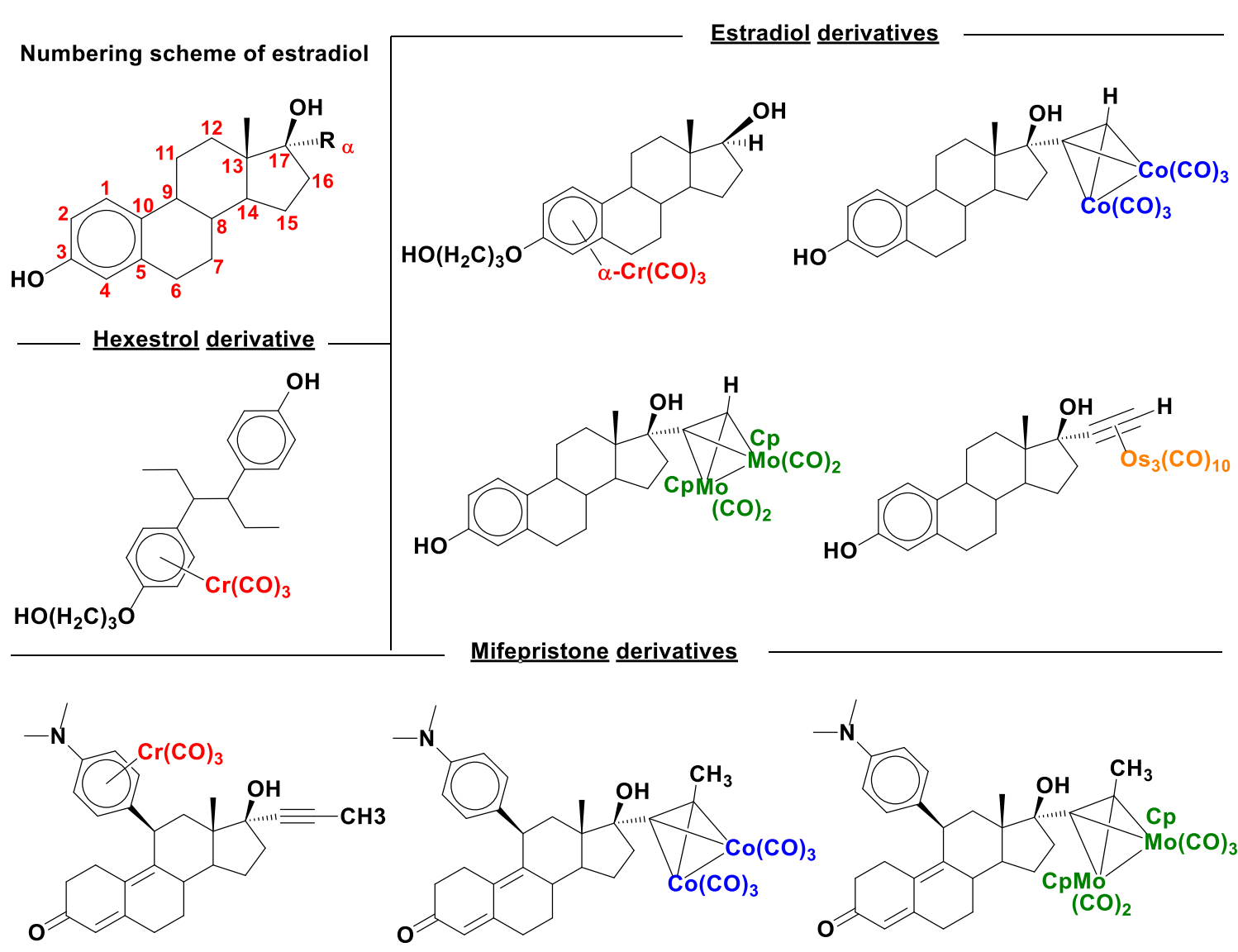

Figure 1.12. Molecular structures of metal carbonyl-steroid derivatives used for steroidal hormonal assay.

\section{- Carbonylmetalloimmunoassay (CMIA)}

Another quantitative assay subsequently developed was the carbonylmetalloimmuno-assay (CMIA) which utilized analytes labelled with metal carbonyls for mid-IR quantification down to femtomolar concentrations. ${ }^{73}$ The analyte to be detected was first conjugated to a stable metal carbonyl fragment; this conjugate can be referred to as the tracer. In this competition-type immunoassay, a known amount of the tracer was incubated with the antibodies (the binding proteins) and unknown amounts of the analyte to be detected, leading to the competition between the tracer and the analyte for binding to the limited amount of antibody. The unbound tracer was then extracted with organic solvents and quantified by FT-IR spectroscopy, allowing indirect determination of the amount of the analyte present (figure 1.13). ${ }^{74,75}$ 


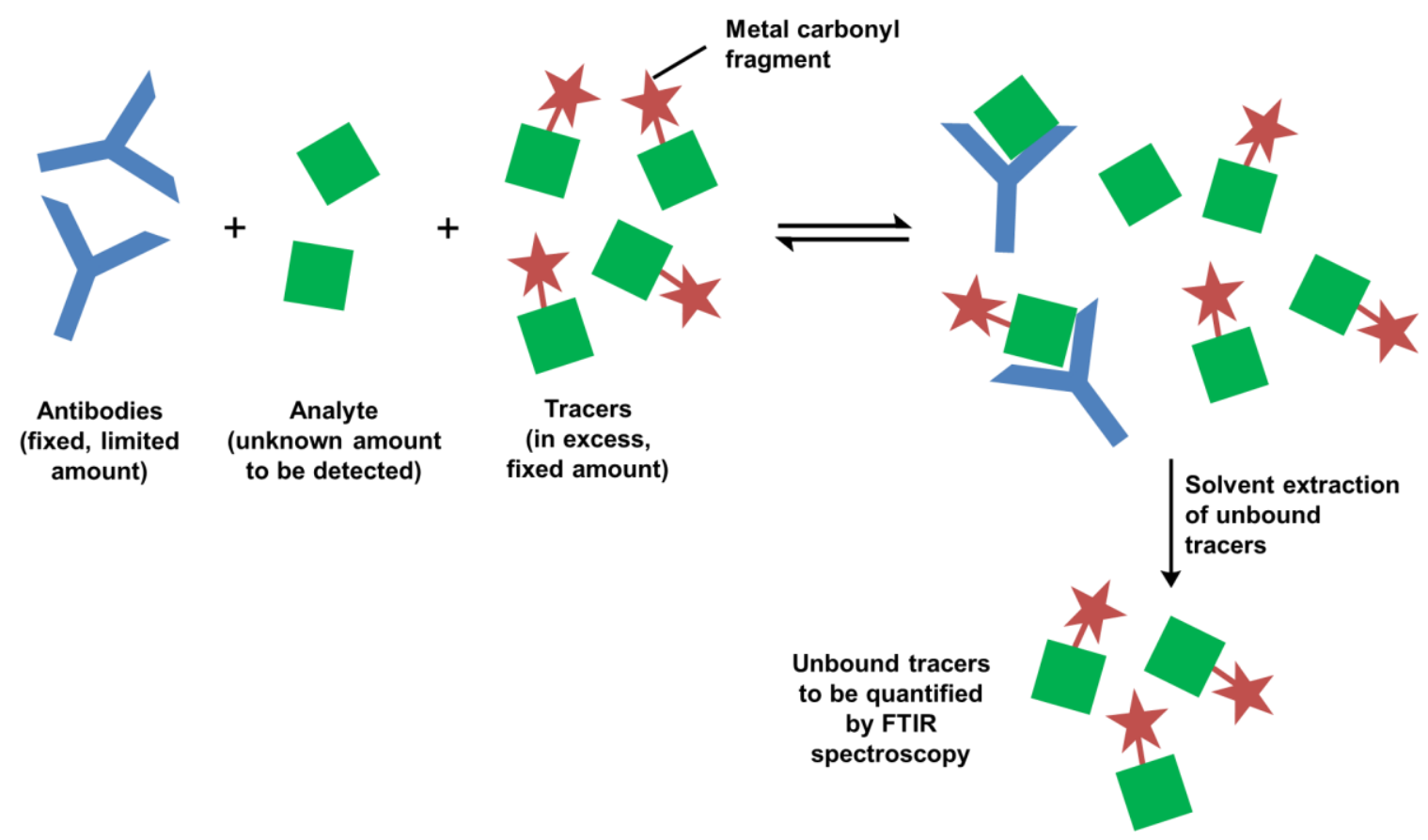

Figure 1.13. Schematic representation of the working principles of CMIA. Adapted from ref. 74 with permission from the Royal Society of Chemistry.

This assay has been utilized for the detection of anti-epileptic drugs like diphenylhydantoin, carbamazepine, phenobarbital, diphenylhydantoin, the antidepressant nortriptyline and cortisol, with the use of the corresponding metal carbonyl conjugates as tracers (figure 1.14) ${ }^{73,76-79}$ The simultaneous detection of two or more targets, also known as multiplex detection, can be incorporated into CMIA. FT-IR spectroscopy is suitable for multiplex detection owing to the narrow bandwidths of the vibrational bands, and is advantageous for achieving minimal or non-overlapping of the signals. Duplex and triplex detection of antiepileptic drugs like carbamazepine, phenobarbital and diphenylhydantoin were achieved, using a different metal carbonyl for each target. ${ }^{80,81}$ 


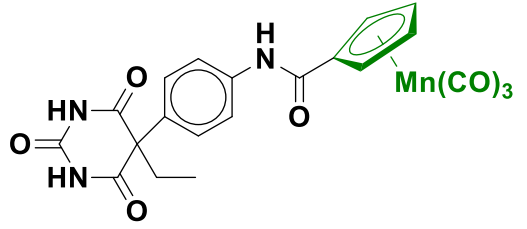

5,5'-diphenvlhydantoin derivative

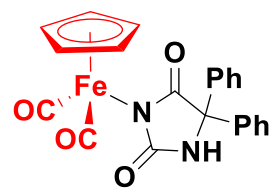

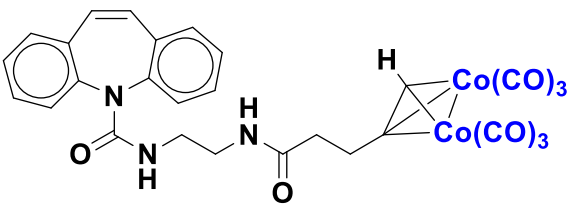

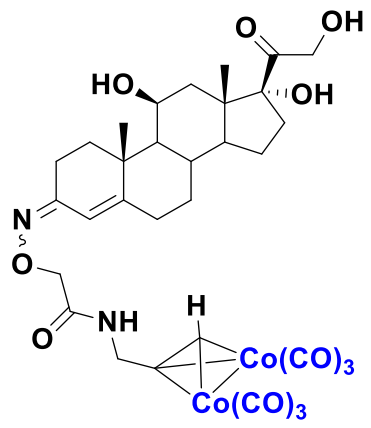

Figure 1.14. Molecular structures of metal carbonyl derivatives used as tracers in CMIA.

Enhancements to the CMIA sensing platform have also been made over the years. This includes the development of solid-phase CMIA, which improves on the purification step and minimizes the interference from water. For example, streptavidin-coated microtiter plates and nitrocellulose membrane allow for immobilization of metal carbonyl dendrimer conjugates. ${ }^{82-84}$ Nanoparticle-based platforms have also been demonstrated with the use of gold nanoparticles to anchor disulfide-linked metal carbonyls onto the surface via strong Au-S interactions (figure 1.15a). Further functionalization with antibodies using carbodiimide coupling chemistry turns it into a secondary label to detect mouse monoclonal anti-staphylococcal enterotoxin A (SEA) antibody via polarization modulation infrared reflection-absorption spectroscopy (PM-IRAAS) and atomic force microscopy (AFM). ${ }^{85,86}$

More recently, $\mathrm{CpFe}(\mathrm{CO})_{2}(\mathrm{~N}$-maleimidato $)$ was immobilized onto amine or thiol-functionalized magnetic nanoparticles (MNP) followed by conjugation to antibodies (figure 1.15b). ${ }^{87}$ The nanoparticles-based platform is attractive as it allows the metal carbonyl-ligand ratio to be increased, thereby enhancing the CO vibrational signal, as well as providing a mean to easily modify the support without having to functionalize the metal carbonyl directly. 

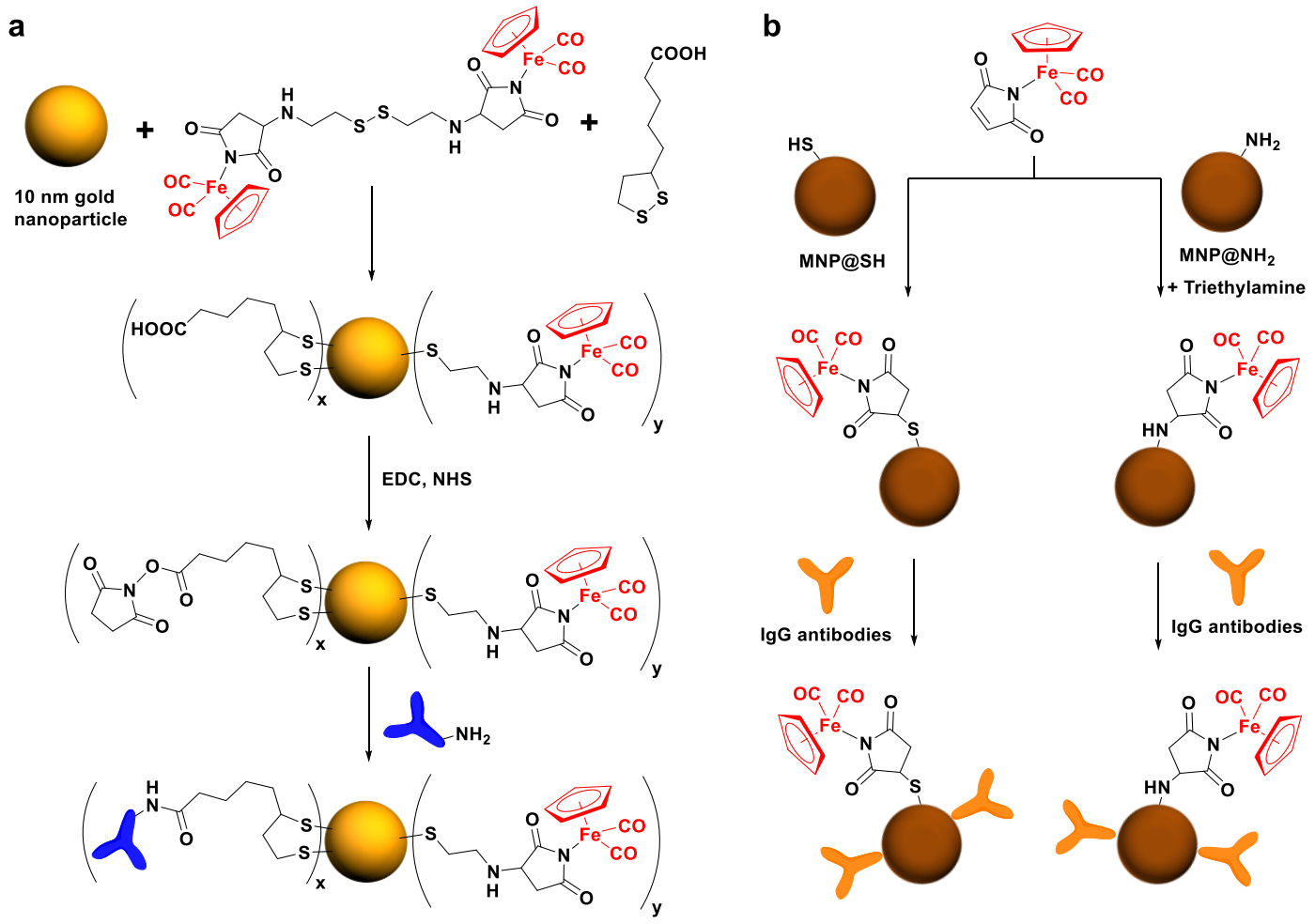

Figure 1.15. a) Synthesis of $\mathrm{CpFe}(\mathrm{CO})_{2}$-gold nanoparticle conjugates and immobilization of goat anti-mouse IgG antibody. Adapted from ref. 85 with permission from Elsevier. b) Synthesis of $\mathrm{CpFe}(\mathrm{CO})_{2}$-MNP conjugates and immobilization of $\mathrm{IgG}$ antibody. Adapted from ref. 87 with permission from Elsevier.

\section{- IR probes for cellular imaging}

The CO vibrational bands of metal carbonyls can also be utilized as a spectroscopic handle for cellular imaging. Such applications rest on qualitative rather than quantitative measurements, largely due to the uneven topology of the cells. Accurate quantification can be complicated and requires determination of the optical path length which, in one case, was measured via 3D reconstructed IR images, with the absorption coefficient pre-determined from a similar setup of the IR microscope. ${ }^{88}$ Quantification has also been carried out indirectly by measuring the absorbance from a large number of cells incubated with the complex deposited on a nitrocellulose disk and air-dried, to obtain a calibration curve. $^{89}$

The first application of metal carbonyls for mid-IR cellular imaging was reported by Leong and co-workers, using the water-soluble and stable $\left[\mathrm{Os}_{3}(\mathrm{CO})_{10}(\mu-\mathrm{H})\left(\mu-\mathrm{S}\left(\mathrm{CH}_{2}\right)_{10} \mathrm{COO}\right)\right] \mathrm{Na}$, an inorganic salt of a fatty acid 
conjugated to a metal carbonyl cluster, and its phosphatidylcholine analogue, as the probe (figure 1.16). ${ }^{67}$ Chemical mapping at $2013 \mathrm{~cm}^{-1}$, which corresponded to one of the $\mathrm{CO}$ vibration bands of the cluster, of oral mucosa cells was carried out with an IR microscope fitted with a 64 x 64 pixel image plate detector. The IR images correlated well to the optical image, indicating internalization of the compound into the cells.
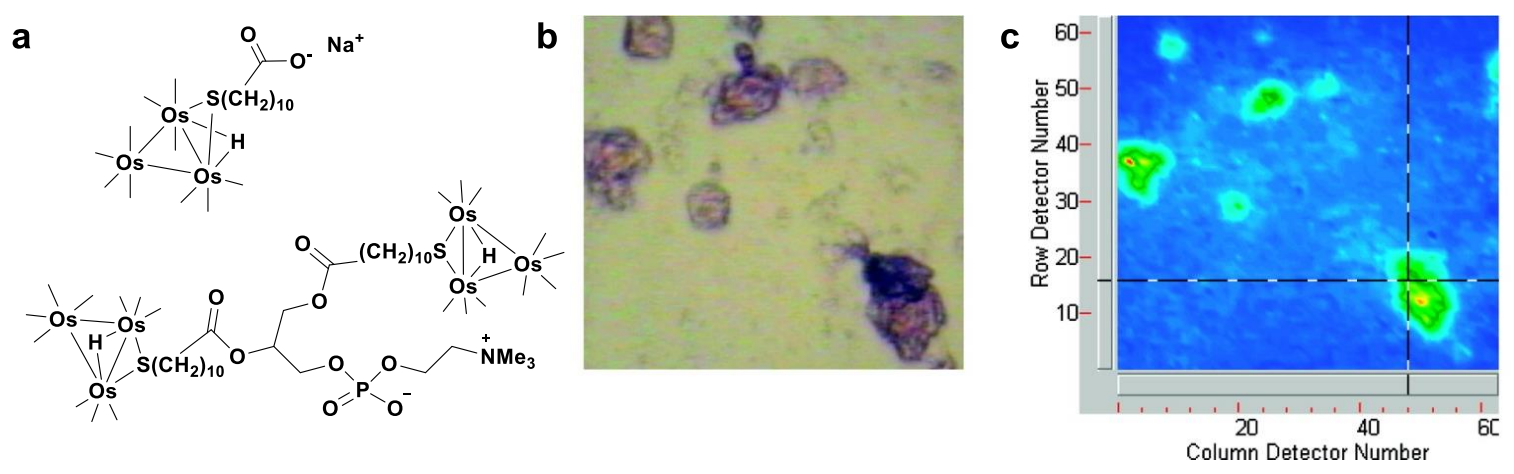

Figure 1.16. a) Molecular structure of $\left[\mathrm{Os}_{3}(\mathrm{CO})_{10}(\mu-\mathrm{H})\left(\mu-\mathrm{S}\left(\mathrm{CH}_{2}\right)_{10} \mathrm{COO}\right)\right] \mathrm{Na}$ and the phosphatidylcholine analogue. The short lines extending from Os represent CO ligands. b) Normal optical image and c) false-color IR image of oral mucosa cells treated with $\left[\mathrm{Os}_{3}(\mathrm{CO})_{10}(\mu-\mathrm{H})\left(\mu-\mathrm{S}\left(\mathrm{CH}_{2}\right)_{10} \mathrm{COO}\right)\right] \mathrm{Na}(32 \mu \mathrm{M})$, taken at $2013 \mathrm{~cm}^{-1}$, one of the carbonyl vibration band. Adapted from ref. 67 with permission from the American Chemical Society.

The interest in metal carbonyl compounds for biological applications has partly been spurred on by the discovery that carbon monoxide $(\mathrm{CO})$ is produced endogenously through heme catabolism and is a vital cell signaling mediator and regulator, ${ }^{90,91}$ and reports that it induces vasorelaxation and exhibits antiinflammatory and cytoprotective effects against disease pathology. ${ }^{91-94}$ As carriers for carbon monoxide, metal carbonyls are potential CO-releasing molecules (CORMs) for controlled and site-specific delivery. ${ }^{95,96}$ Release of CO from the complexes can be triggered by various external factors which include light; the term "photoCORMs" is used to describe such complexes. ${ }^{97,} 98$ Zobi and Quaroni, et. al., reported the synthesis of a manganese photoCORM conjugated to the 5 ' $-\mathrm{OH}$ of the ribose sugar of vitamin $\mathrm{B}_{12}$ and its delivery into live 3 T3 fibroblasts. ${ }^{99}$ The cellular uptake and distribution of the $\mathrm{B}_{12}$-MnCORM 
was monitored by synchrotron FTIR spectromicroscopy in the live cells (figure 1.17). The moderate spatial resolution, however, only allowed the nucleus and cytoplasm to be distinguished and accumulation of the compound in the nucleus was supported by atomic absorption spectrophotometric measurements on the cell fractions.
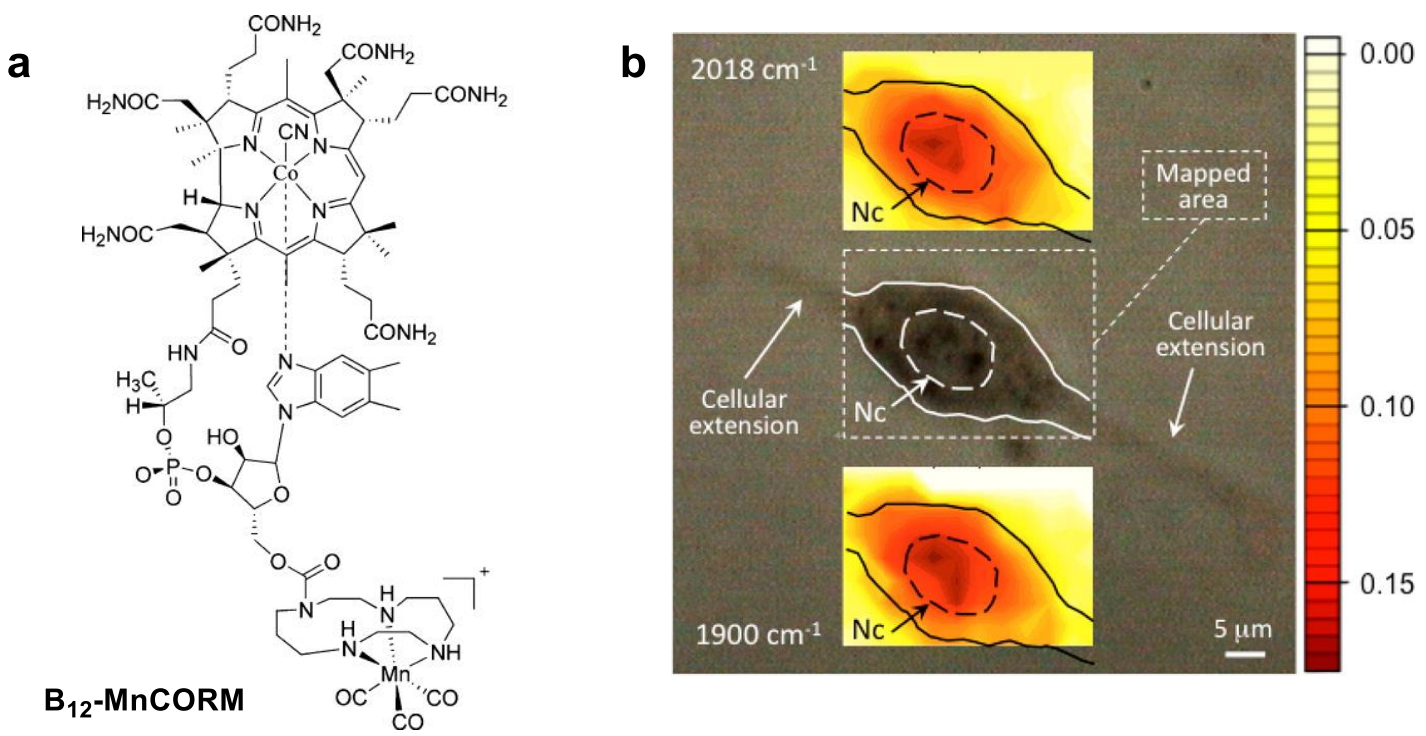

Figure 1.17. a) Molecular structure of the vitamin $B_{12}-M n$ CORM conjugate and optical images of a $3 \mathrm{~T} 3$ fibroblast after incubation with the conjugate $(1 \mathrm{mM})$. The top and bottom inserts show images reconstructed from integration of the intensity of the carbonyl stretching vibrations at 2018 and $1900 \mathrm{~cm}^{-1}$, respectively. The scale represents units of integrated band area in an absorbance vs wavenumber spectrum. Nc = cell nuclear area. Adapted from ref. 99 with permission from the American Chemical Society.

Another growing interest is the development of multimodal imaging probes for correlative and more precise detection. There have been a number of reports on rhenium tris-carbonyl derivatives, which display IR and luminescent properties, as single-core, multimodal probes to image breast cancer cells. ${ }^{100-102}$ In one example, MDA-MB-231 cells incubated with the rhenium tris-carbonyl derivative, $\mathrm{ReCl}(\mathrm{CO})_{3}$-pyta- $\mathrm{C}_{12} \mathrm{~N}_{3}$ (pyta = 2-pyridyl-1,2,3-triazole), was imaged by synchrotron radiation FT-IR spectromicroscopy (SR-FTIR-SM), to produce chemical mapping of the complexes within the cell (figure 1.18). ${ }^{100}$ The IR images were correlated with the fluorescent image of the cells, which showed 
luminescence due to the metal-to-ligand charge transfer (MLCT) of the complex.

Although the IR and fluorescence bimodal probes can facilitate correlation studies between IR and fluorescence imaging, the former technique remains the more reliable for the quantification of cellular uptake. ${ }^{102}$ This is because the quantum yield of a fluorescent probe is highly susceptible to degradation through photo-bleaching and quenching, and it is also highly dependent on the environment, including the temperature and solvent. ${ }^{102,103}$ On the other hand, IR vibrations tend to be more stable and easily quantified via a calibration curve. Although the probe environment can induce frequency shifts and/or broadening, the peak area remains relatively consistent, making quantification more reliable.
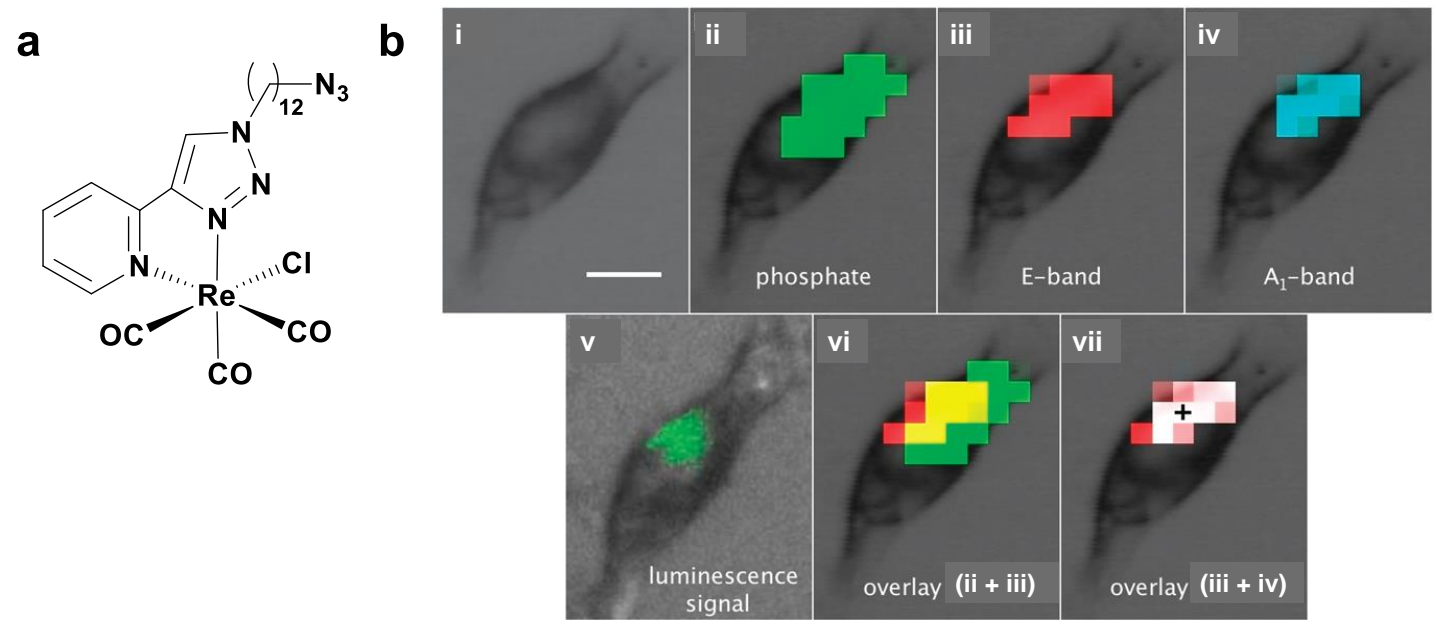

Figure 1.18. a) Molecular structure of $\mathrm{ReCl}(\mathrm{CO})_{3}-$ pyta- $\mathrm{C}_{12} \mathrm{~N}_{3}$. b) MDA-MB-231 cell incubated with $\operatorname{ReCl}(\mathrm{CO})_{3}$-pyta- $\mathrm{C}_{12} \mathrm{~N}_{3}(10 \mu \mathrm{M}, 1 \mathrm{~h})$. (i) Bright field image (scale bar 10 $\mu \mathrm{m}$ ). (ii-iv) SR-FTIR mappings, pixel size: $3 \times 3 \mu \mathrm{m}^{2}$ ): (ii) phosphate asymmetric stretching (green), (iii) E-band (red), (iv) $\mathrm{A}_{1}$-band (cyan). (v) Epifluorescence image, localization of the complex (green). (vi-vii) Overlays of SR-FTIR hot spots: (vi) overlay (yellow) of ii (green) and iii (red), (vii) overlay (white) of iii (red) and iv (cyan). Adapted from ref. 100 with permission from the Royal Society of Chemistry. 
The same rhenium chemistry was also recently extended to tissue imaging. ${ }^{104}$ Policar and co-workers used a fluorescence and IR bimodal rhenium derivative conjugated to a nona-arginine peptide to study transdermal delivery into the skin (figure 1.19). ${ }^{105}$ The topical translocation of the conjugates through the extracellular matrix was monitored by IR and fluorescence imaging of skin slices over time. They observed that the conjugate was localized in the stratum corneum after $6 \mathrm{~h}$, and penetrated into the epidermis after $24 \mathrm{~h}$.
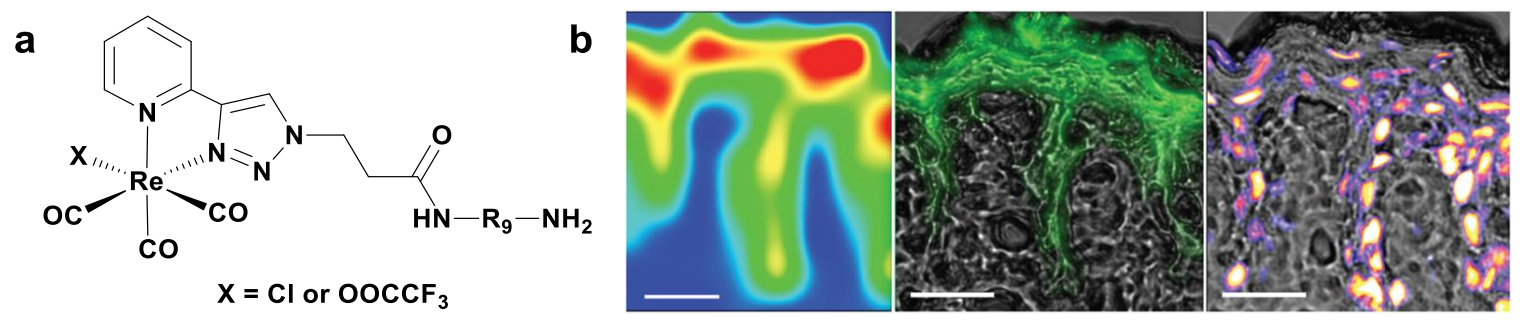

Figure 1.19. a) Molecular structure of rhenium carbonyl-peptide conjugate. b) Skin slice after a $24 \mathrm{~h}$-exposure to a $2 \times 10^{-2} \mathrm{M}$ solution of conjugate in water. Left: mapping of the integral of the absorbance of the $\mathrm{A}_{1}$-band (2040-2000 $\mathrm{cm}^{-1}$ ). Middle: bright field image merged with the luminescence signal of the conjugate. Right: bright field image merged with the staining of nuclei by DAPI. Scale bar $20 \mu \mathrm{m}$. Adapted from ref. 105 with permission from the Royal Society of Chemistry.

A fundamental limitation associated with IR imaging lies with its spatial resolution (in the micrometer range) which is insufficient for subcellular imaging. A technical breakthrough was the use of photothermally induced resonance (PTIR) for the subcellular IR imaging of a metal carbonyl; localization of the $\mathrm{CpRe}(\mathrm{CO})_{3}$-hydroxytamoxifen analogue in the cell nucleus of MDA-MB-231 cells was demonstrated (figure 1.20). ${ }^{89}$ The technique, patented by Dazzi, et. al., comprised an atomic force microscope (AFM) coupled with a tunable pulsed infrared laser which allowed cellular mapping to be carried out with the AFM tip. The temperature rise in a sample as a result of absorption of the laser pulse leads to local thermal deformation, causing the AFM cantilever to oscillate with an amplitude that is correlated to the absorbance. This allowed a high spatial resolution of $20-50 \mathrm{~nm}$, similar to that for AFM, to be achieved. 

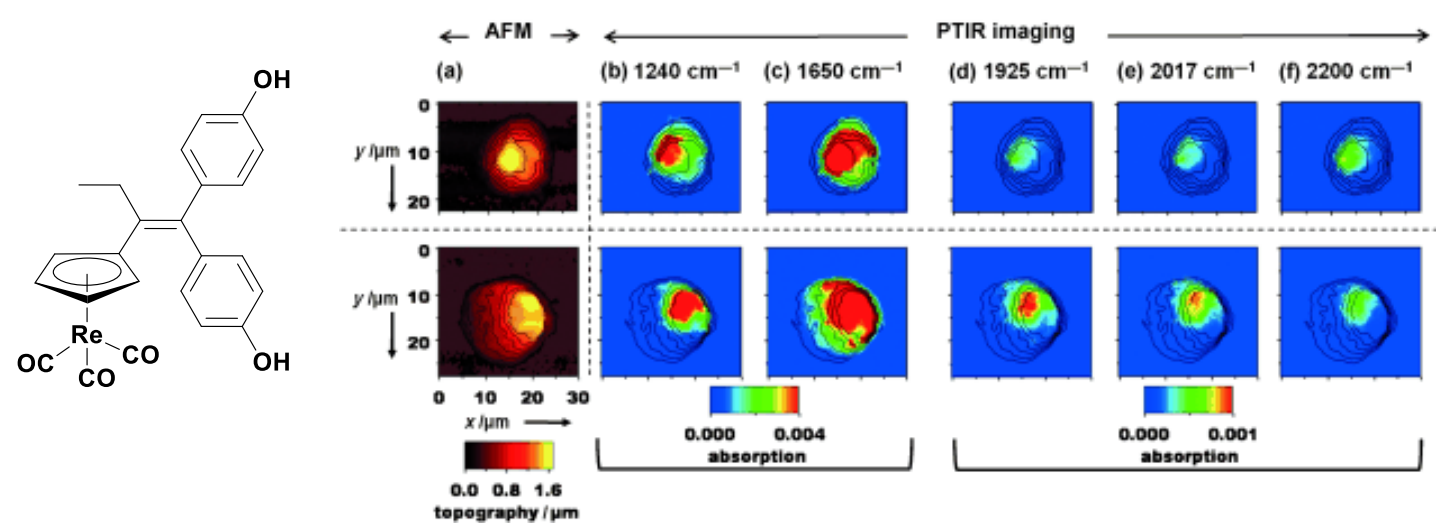

Figure 1.20. Molecular structure of $\mathrm{CpRe}(\mathrm{CO})_{3}$-hydroxytamoxifen conjugate and images of MDA-MB-231 cells. Top row: control cells; bottom row: cells treated with a $10 \mu \mathrm{M}$ solution of the conjugate for $1 \mathrm{~h}$ at $37^{\circ} \mathrm{C}$. a) AFM topography; b-f) PTIR mappings at different wavelengths, with the AFM contours superimposed as black lines; the wavelengths correspond to the following bands: phosphate (b), amide (c), tricarbonyl (d and e), and out of band (f). Adapted from ref. 89 with permission from John Wiley and Sons.

\subsubsection{Sensing based on frequency shifts in $\mathrm{CO}$ vibrations}

Besides the use of intensity for quantitation, qualitative shift in frequency of the $\mathrm{CO}$ vibrations has also proven to be useful for sensing applications. The frequency of the $\mathrm{CO}$ vibration is largely governed by the extent of $\pi$-back donation from the metal to the CO ligand. Hence, ligands in the coordination sphere of the metal can easily affect the electron density of the metal, giving rise to changes in the $\mathrm{CO}$ vibrational frequency. The design and application of metal carbonyl "bioprobes" that give a spectroscopic response upon interaction with the target was first discussed by Stephenson. ${ }^{106}$ The term "bioprobes" refers to molecular structures that can provide information about biological systems and are required to possess functions such as recognition, transduction and response. Much work has been carried out, and these are summarized in table 1.1. Detailed discussions will be made on the applications in sensing of $\mathrm{pH}$, protein interaction and solvent environment. 
Table 1.1. Use of various metal carbonyls for sensing applications via frequency shifts in CO vibrations.

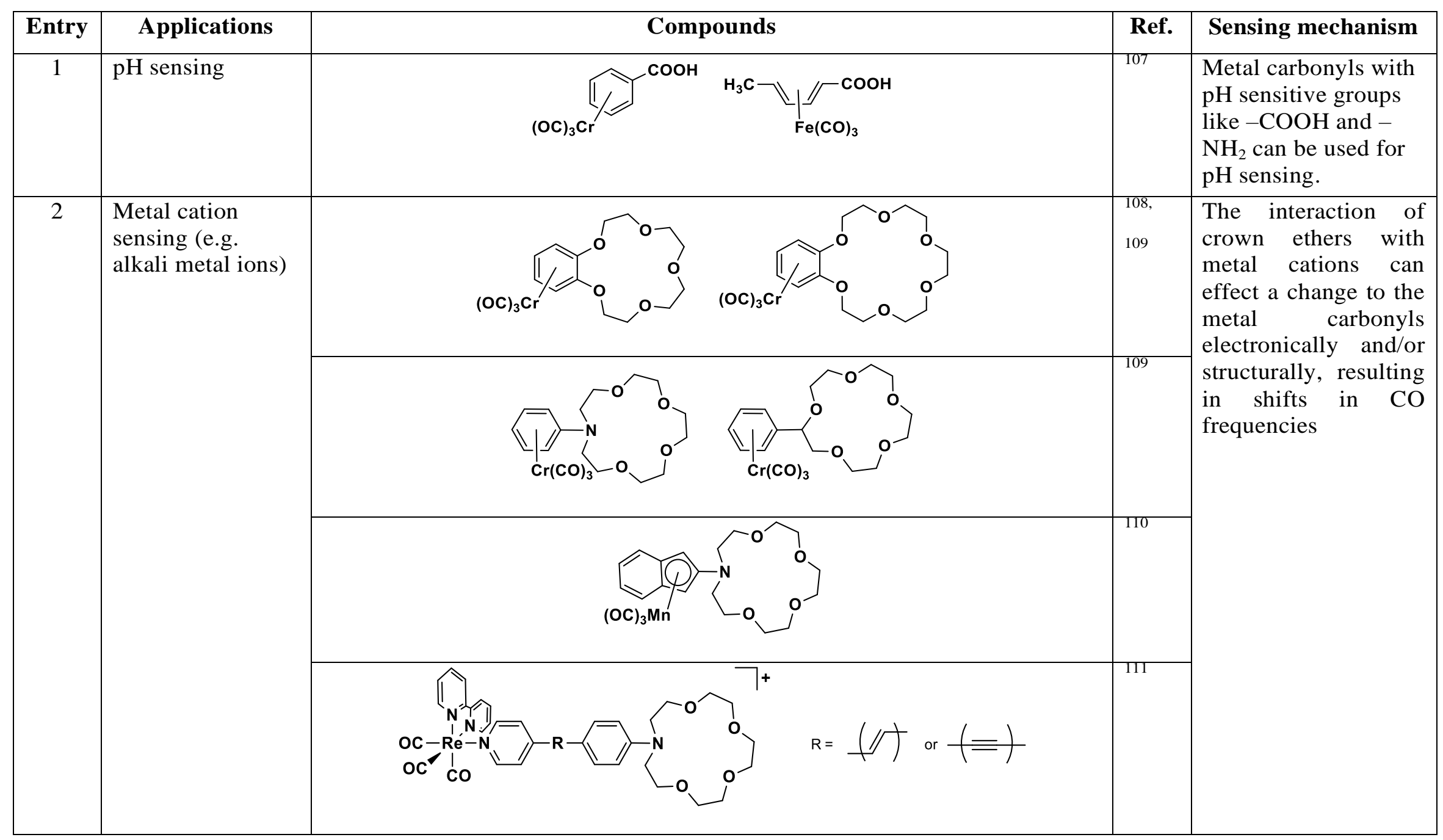




\begin{tabular}{|c|c|c|c|c|}
\hline \multirow[t]{2}{*}{3} & $\begin{array}{l}\text { Anion sensing } \\
\text { (e.g. carboxylate } \\
\text { and fluoride) }\end{array}$ & (OC) $)_{3} \mathrm{Cr}=\mathrm{C}_{6} \mathrm{H}_{5}$ or $\mathrm{CH}_{3}$ & 112 & \multirow{2}{*}{$\begin{array}{l}\text { Metal carbonyls } \\
\text { coordinated to } \\
\text { ligands with free - } \\
\text { NH or -OH groups } \\
\text { can act as hydrogen } \\
\text { bond donors for } \\
\text { interaction with } \\
\text { anions via hydrogen } \\
\text { bonding }\end{array}$} \\
\hline & & $\overbrace{M i n}$ & 113 & \\
\hline 4 & $\begin{array}{l}\text { Hydrogen bond } \\
\text { sensing (e.g. } \\
\text { indole, } \\
\text { diphenylamine } \\
\text { and } 2,4,6- \\
\text { trimethylphenol) }\end{array}$ & 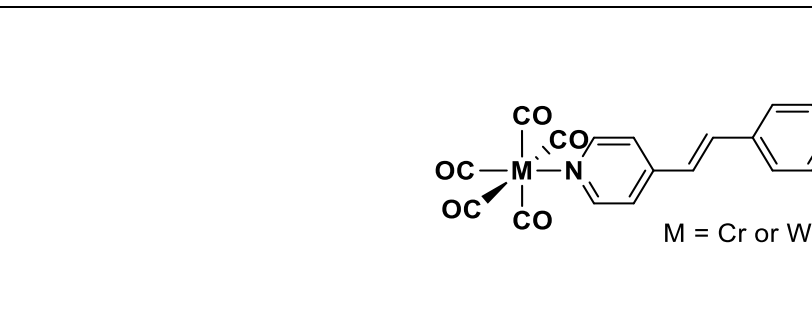 & 114 & $\begin{array}{l}\text { Sensing of hydrogen } \\
\text { bond donors was } \\
\text { achieved via the } \\
\text { nitrogen lone pair of } \\
\text { dipyridylethene } \\
\text { ligand coordinated to } \\
\mathrm{M}(\mathrm{CO})_{5}\end{array}$ \\
\hline 5 & $\begin{array}{l}\pi \text {-interaction } \\
\text { sensing (e.g. 1, 3, } \\
\text { 5-trimethoxy- } \\
\text { benzene and 1, 2- } \\
\text { dimethoxy- } \\
\text { benzene) }\end{array}$ & 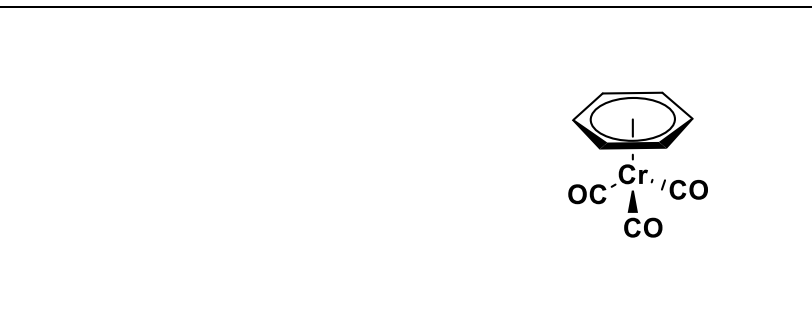 & 115 & $\begin{array}{l}\pi \text {-stacking interaction } \\
\text { between the arene } \\
\text { ring of Cr carbonyl } \\
\text { with aromatic } \\
\text { analytes can lead to } \\
\text { changes in the CO } \\
\text { frequencies. }\end{array}$ \\
\hline
\end{tabular}




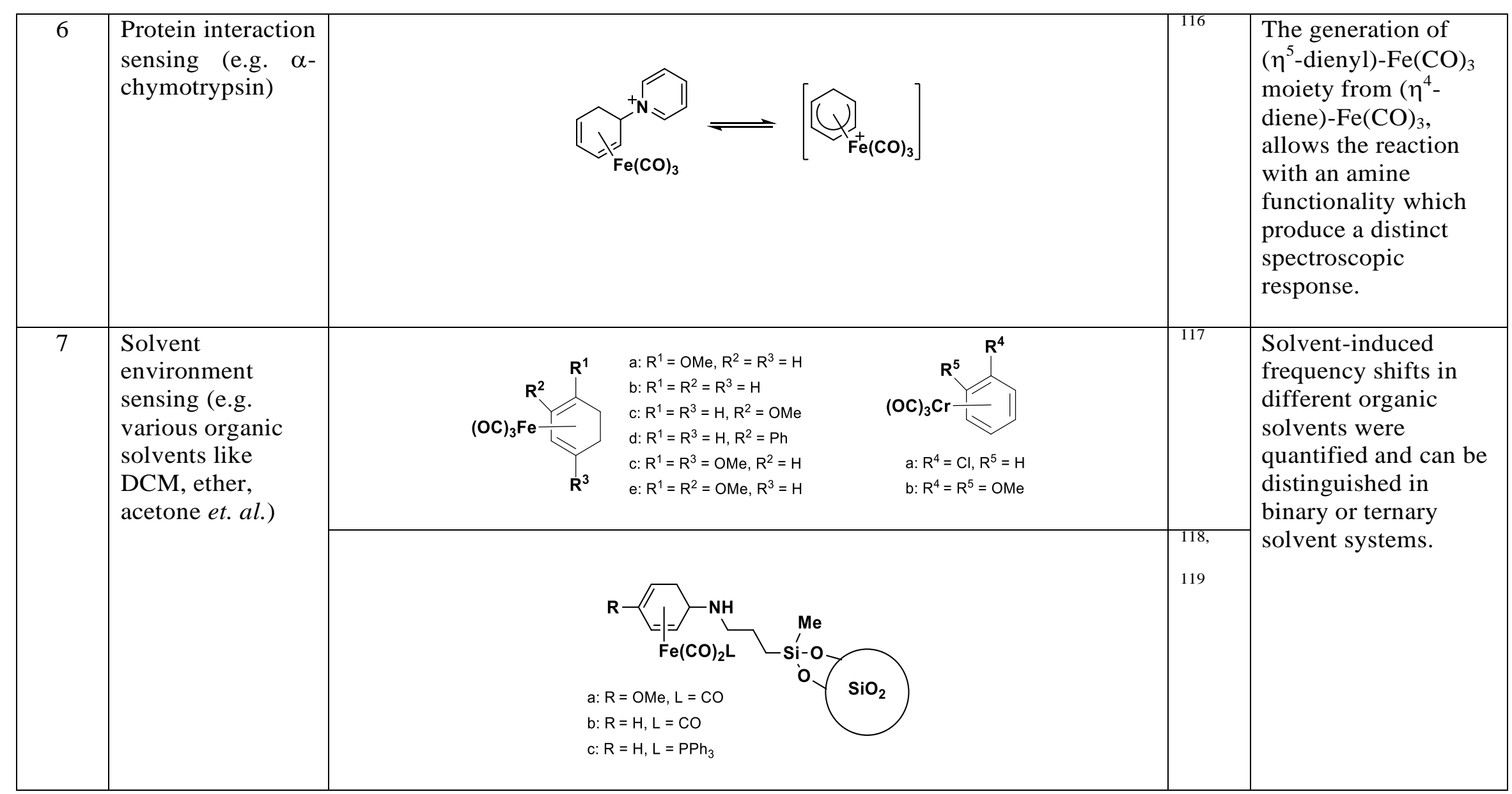




\section{- pH sensing}

Metal carbonyl $\pi$-complexes containing $\mathrm{pH}$-sensitive groups like carboxylic acids and amines, can be utilized as pH-sensitive IR probes (Figure 1.21). ${ }^{107}$ Examples include $\eta^{6}$-(1-carboxybenzene $) \operatorname{Cr}(\mathrm{CO})_{3}$ and $\eta^{4}$-1-carboxy-1,3pentadiene $) \mathrm{Fe}(\mathrm{CO})_{3}$. Protonation/ deprotonation of these groups affect the $v_{\text {sym }}(\mathrm{CO})$ and $v_{\text {asym }}(\mathrm{CO})$ vibrational modes; deprotonation of the carboxylic acid group increases the electron density of the $\pi$-conjugated system, thereby increasing $\pi$-back donation from the metal to $\mathrm{C}-\mathrm{O} \pi^{*}$ anti-bonding orbital, which is translated into a shift to lower wavenumber of the $\mathrm{CO}$ vibrations.
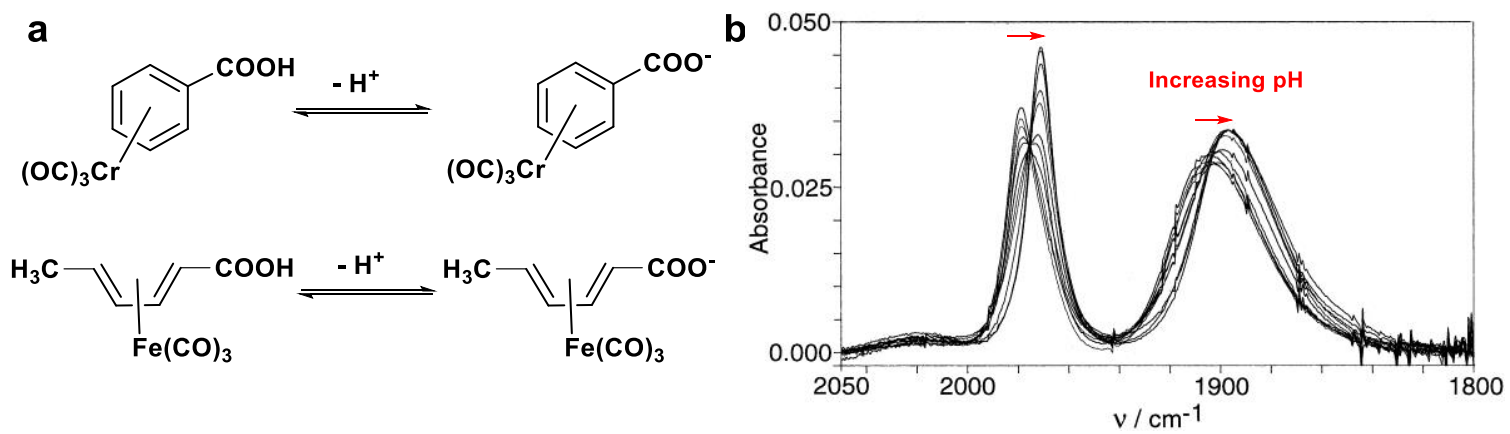

Figure 1.21. a) Molecular structures of $\eta^{6}-(1$-carboxybenzene $) \operatorname{Cr}(\mathrm{CO})_{3}$ and $\eta^{4}-1$ carboxy-1,3-pentadiene $) \mathrm{Fe}(\mathrm{CO})_{3}$ and their $\mathrm{pH}$ sensing capability. b) FTIR spectra in the 2050-1800 $\mathrm{cm}^{-1}$ region of $\eta^{6}-(1$-carboxybenzene $) \mathrm{Cr}(\mathrm{CO})_{3}$ in buffer solutions over the $\mathrm{pH}$ range 2.1-6.0. Adapted from ref. 107 with permission from the American Chemical Society.

The response to $\mathrm{pH}$ can also be adjusted through the use of other $\mathrm{pH}$ sensitive groups like hydroxyl or amine groups, or through different substituents on the $\pi$-system. A combination of the two above-mentioned $\mathrm{pH}$-active probes with non-overlapping $\mathrm{CO}$ frequencies can act effectively as a "dual-sensor" which allows simultaneous but independent measurements to be made. Since the probes have different pKa $\left(2.9\right.$ and 4.2 for the $\mathrm{Cr}(\mathrm{CO})_{3}$ and $\mathrm{Fe}(\mathrm{CO})_{3}$ derivatives, respectively), they respond to different $\mathrm{pH}$ and hence, allow a wider $\mathrm{pH}$ range that can be probed. 


\section{- Protein interaction sensing}

A responsive metal carbonyl which gives a spectral change upon reaction with a functional group may be advantageous for reaction monitoring, especially in cases where excess probes are difficult to remove. The complex ( $\eta^{4}$-diene)$\mathrm{Fe}(\mathrm{CO})_{3}$, which bears a labile pyridinium group, can be deprotonated to form the $\left(\eta^{5}\right.$-dienyl $)-\mathrm{Fe}(\mathrm{CO})_{3}$ moiety, which can then react readily with an amine functionality (figure 1.22). ${ }^{116}$
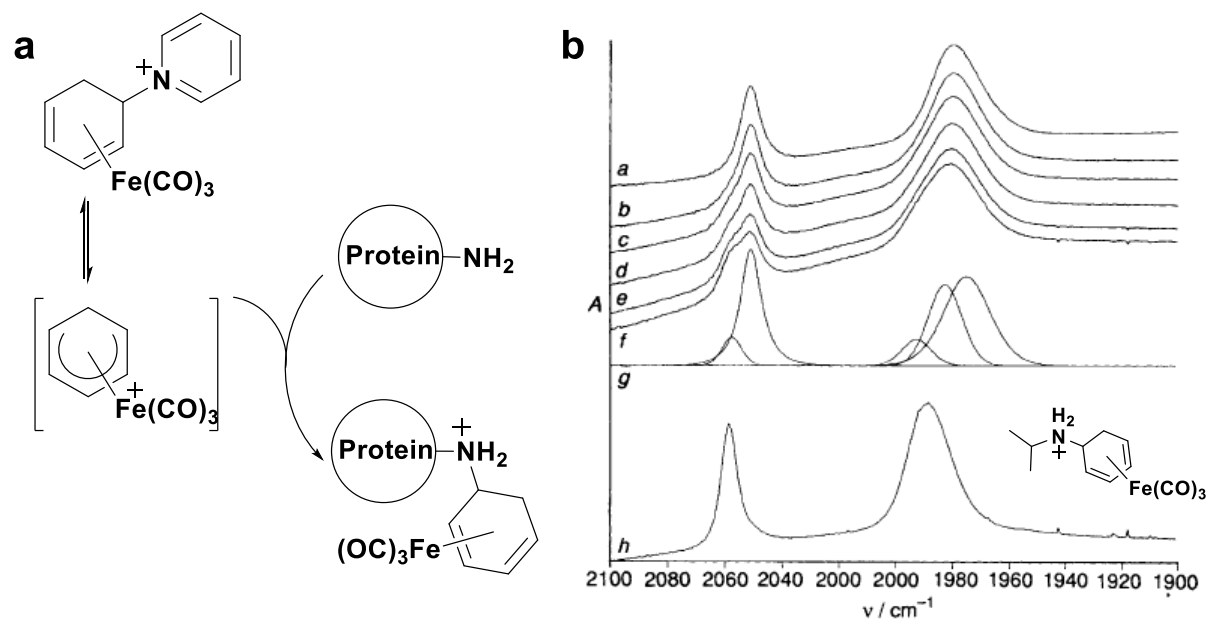

Figure 1.22. a) Reaction of $\left(\eta^{4}\right.$-diene $)-\mathrm{Fe}(\mathrm{CO})_{3}$ with amine functionality in proteins. $\left.b\right)$ (a-f) FTIR spectra of $\left(\eta^{4} \text {-diene)-Fe(CO) }\right)_{3}$ in $\mathrm{pH} 7.5$ buffer solution containing $\alpha$ chymotrypsin recorded at intervals over $3 \mathrm{~h}$. (g) Component bands resulting from decomposition of spectrum (c) using a scale expansion of 2. (h) FTIR spectrum of $\left[\mathrm{Fe}(\mathrm{CO})_{3}\left(\mathrm{C}_{6} \mathrm{H}_{7} \mathrm{NH}_{2} \mathrm{Pr}^{\mathrm{i}}\right)\right]\left(\mathrm{PF}_{6}\right)$ in buffer. Adapted from ref. 116 with permission from the Royal Society of Chemistry.

Incubation of the protein $\alpha$-chymotrypsin with excess of the complex led to the emergence of high frequency shoulders on the $v_{\text {sym }}$ and $v_{\text {asymm }}$ stretches. Curve-fitting analysis resolved the spectrum of the bound complex from that of the excess complex, and identified to be the isolated species $\left[\mathrm{Fe}(\mathrm{CO})_{3}\left(\mathrm{C}_{6} \mathrm{H}_{7} \mathrm{NH}_{2} \mathrm{Pr}^{\mathrm{i}}\right)\right]\left(\mathrm{PF}_{6}\right)$. The interaction of $\left(\eta^{4}\right.$-diene $)-\mathrm{Fe}(\mathrm{CO})_{3}$ with various amino acids and poly(lysine) also afforded species exhibiting similar spectra, and the study was extended to other enzymes like ribonuclease A, alkaline phosphatase and triacylglycerol lipase. ${ }^{120}$ With a change in $\mathrm{pH}$, which results in deprotonation/protonation of the amine group, the spectrum of the 
enzyme-bound complex undergo further shifts, thus demonstrating its dualfunction as a tag and $\mathrm{pH}$ sensor on the protein surface.

\section{- Solvent environment}

The observation of solvent-induced frequency shifts in metal carbonyls was made as early as in the 1960 s by R. S. Nyholm and co-workers. ${ }^{121}$ Typically, CO vibrational frequencies are shifted and broadened in the presence of polar solvents. Quantitative interpretation of the shifts was first carried out by Stephenson et. al. using a series of $\left(\eta^{6}\right.$-arene)- $\mathrm{Cr}(\mathrm{CO})_{3}$ and $\left(\eta^{4}\right.$-cyclohexadiene $)-\mathrm{Fe}(\mathrm{CO})_{3}$ complexes (figure 1.23$) .{ }^{117}$

a

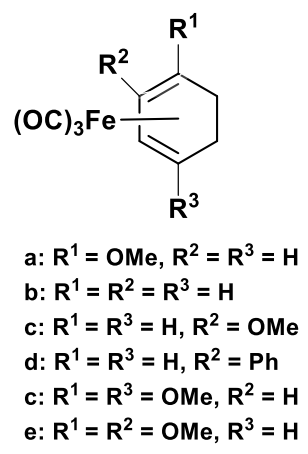

b

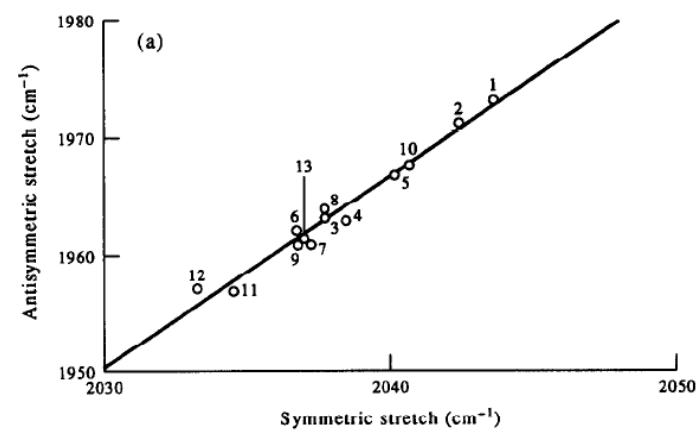

C

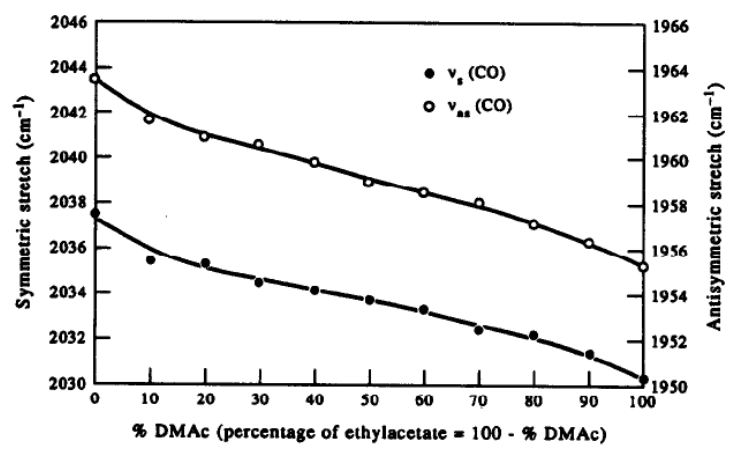

Figure 1.23. a) Molecular structures of various $\left(\eta^{4}\right.$-cyclohexadiene) $-\mathrm{Fe}(\mathrm{CO})_{3}$ complexes used for solvent environment sensing. b) Symmetric and antisymmetric stretching frequencies of $\mathrm{Fe}(\mathrm{CO})_{3}-$ (a) in various solvents: 1 , hexane; 2 , cyclohexane; 3 , toluene; 4, dichloromethane; 5 , diethyl ether; 6 , tetrahydrofuran; 7, acetonitrile; 8, ethyl acetate; 9, acetone; 10, 1-octanol; 11, dimethyl formamide; 12, dimethyl acetamide; 13, acetic anhydride. c) Variation of $v_{\text {sym }}$, and $v_{\text {asym }}$, of $\mathrm{Fe}(\mathrm{CO})_{3}-$ (a) in binary mixtures of ethyl acetate and dimethyl acetamide. Reproduced from ref. 117 with permission from Elsevier.

Bellamy-type plots of $v_{\text {sym }}$ against $v_{\text {asymm }}$ stretches of $\mathrm{Fe}(\mathrm{CO})_{3}-(\mathrm{a})$ in various solvents showed that the frequency shifts gave a better correlation with the solvent environment than the band broadening (figure 1.23b). The vibrational frequencies of $\mathrm{Fe}(\mathrm{CO})_{3}-(\mathrm{a})$ in a binary solvent mixture of ethyl acetate and 
dimethyl acetamide showed good quantitative correlation with the composition, and the correlation for a ternary solvent mixture could be resolved with principal components analysis. Extension of the idea to metal carbonyl grafted onto a solid support for sensing binary to ternary solvent mixtures was demonstrated with $\left(\eta^{4}\right.$-cyclohexadiene)-Fe(CO) $2(\mathrm{~L})$, where $\mathrm{L}=\mathrm{CO}$ or $\mathrm{PPh}_{3}$, covalently attached onto silica particles via a 3-(diethoxymethylsilyl)propyl linker. ${ }^{118,119}$

\subsubsection{Applications based on CO vibrational lifetime}

The vibrational lifetime of metal carbonyls is another feature which can be used for sensing applications. The vibrational lifetime of metal carbonyls in organic solvents was first reported in 1987. ${ }^{122}$ Applications of this for sensing only surfaced recently and are generally used in protein and membrane studies.

\section{- Ultrafast vibrational lifetime of metal carbonyls in proteins and membrane studies}

The vibrational lifetime of metal carbonyl in water was first measured by Kubarych and co-workers in their study on the water-soluble $\left[\mathrm{RuCl}_{2}(\mathrm{CO})_{2}\right]_{2}$, also known as CORM-2. ${ }^{123}$ The vibrational relaxation was probed by twodimensional (2D) IR spectroscopy, and showed that there was a great contrast between the relaxation time in water $(3.12 \pm 0.29 \mathrm{ps})$ compared to that in a polar organic solvent like methanol $(42.25 \pm 3$ ps $)$. This property was subsequently utilized in a study to probe the hydration environment at the protein-water interface (figure 1.24). ${ }^{124}$ The study employed metal carbonyls as a site-specific IR label on globular proteins, such as hen egg white lysozyme (HEWL) and human lysozyme (HuLys). The proteins were labelled by incubation with tricarbonylchloro(glycinato) ruthenium(II) (CORM-3); while HEWL was labelled at the His15 residue to form $\left[(\mathrm{His} 15) \mathrm{Ru}(\mathrm{CO})_{2}\left(\mathrm{H}_{2} \mathrm{O}\right)_{3}\right]^{2+}$, HuLys was labelled at the His 78 residue. ${ }^{125}$ Vibrational relaxation times of the ruthenium carbonyl residue measured in $\mathrm{H}_{2} \mathrm{O}, \mathrm{D}_{2} \mathrm{O}$, and in an amphiphilic alcohol cosolvent of $\mathrm{D}_{2} \mathrm{O}$-trifluroethanol to mimic a hydrophobic environment, suggested that the residue on HEWL experienced dynamically constrained water in a largely hydrophobic surface of the protein, while that on HuLys was more exposed to solvent and experienced bulk-like hydration dynamics. The 
ruthenium carbonyl-labelled HEWL was also used to investigate hydration and protein dynamics using a glycerol/ $/ \mathrm{D}_{2} \mathrm{O}$ mixture. ${ }^{126}$

a

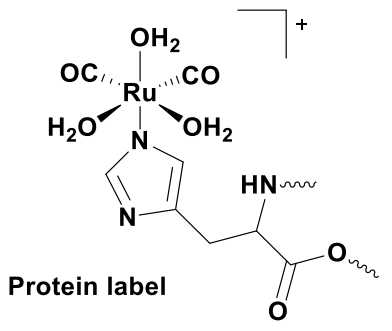

b

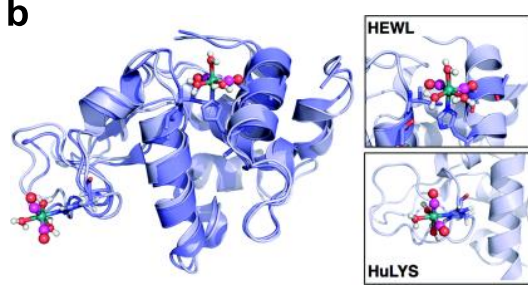

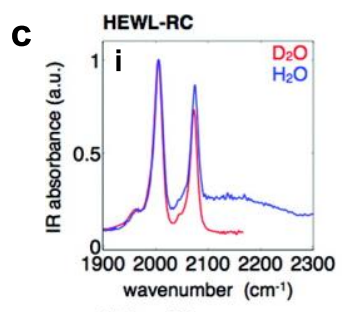
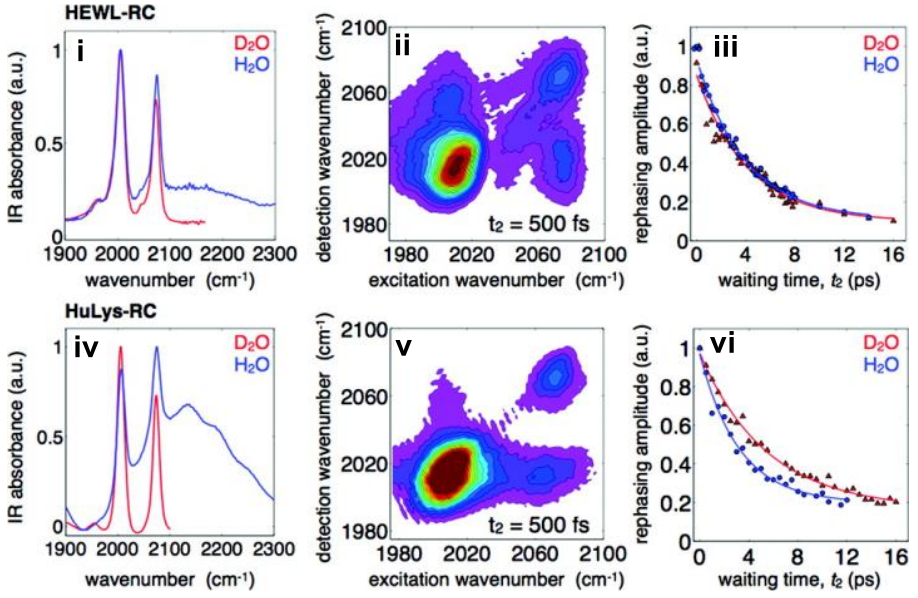

Figure 1.24. a) Molecular structure of ruthenium carbonyl (RC) protein label on HEWL and HuLys. b) Crystal structures of HEWL-RC (PDB code 2XJW) overlaid with the crystal structure of native HuLys (PDB code 2ZIJ). While no crystallographic data are available for the HuLys-RC complex, the binding location is proposed by comparison with the HEWL-RC complex. c) Linear FTIR spectra of HEWL-RC (i) and HuLys-RC (iv) in $\mathrm{D}_{2} \mathrm{O}$ and $\mathrm{H}_{2} \mathrm{O}$. The 2D-IR rephasing spectra for HEWL-RC (ii) and HuLys-RC (v) in $\mathrm{D}_{2} \mathrm{O}$ are shown for a waiting time of $\mathrm{t}_{2}=500 \mathrm{fs}$. Monitoring the amplitude of the $2004 \mathrm{~cm}^{-1}$ peak as a function of waiting time, $t_{2}$, provides the vibrational lifetime of the mode. For HEWL-RC there is no observable isotope effect in the vibrational relaxation between $\mathrm{D}_{2} \mathrm{O}$ and $\mathrm{H}_{2} \mathrm{O}$ (iii), whereas HuLys-RC shows a very clear isotope effect (vi). Adapted from ref. 124 with permission from the American Chemical Society.

A similar dependence of the $\mathrm{CO}$ frequencies and lifetime on the solvent environment was also demonstrated with a series of $\mathrm{CpRe}(\mathrm{CO})_{3}$ with linkers to methanethiosulfonate to tag cysteine residues (figure 1.25a). ${ }^{127} \mathrm{The} \mathrm{CpRe}(\mathrm{CO})_{3}$ moiety was also bioconjugated to the ribosomal protein CTL9 through an alkynyl linkage which was then coupled to an azidohomoalanine residue on the $\mathrm{C}$-terminal domain of the protein via a "click" reaction (figure 1.25b). ${ }^{128}$ The vibrational lifetimes of the metal carbonyl probe in $\mathrm{H}_{2} \mathrm{O}$ and $\mathrm{D}_{2} \mathrm{O}$ were found to be different, showing accessibility to the solvent. 


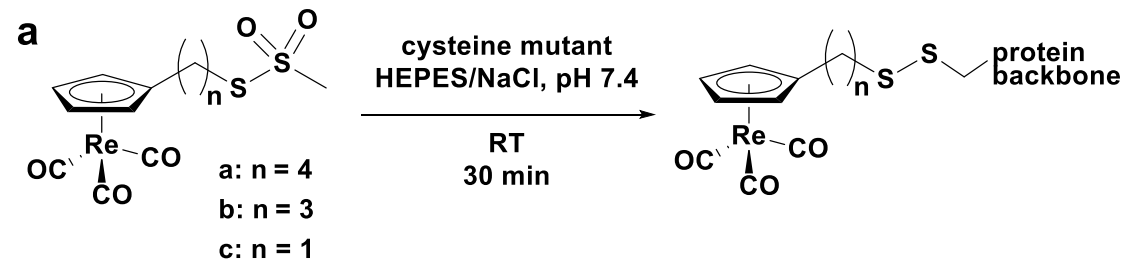

b

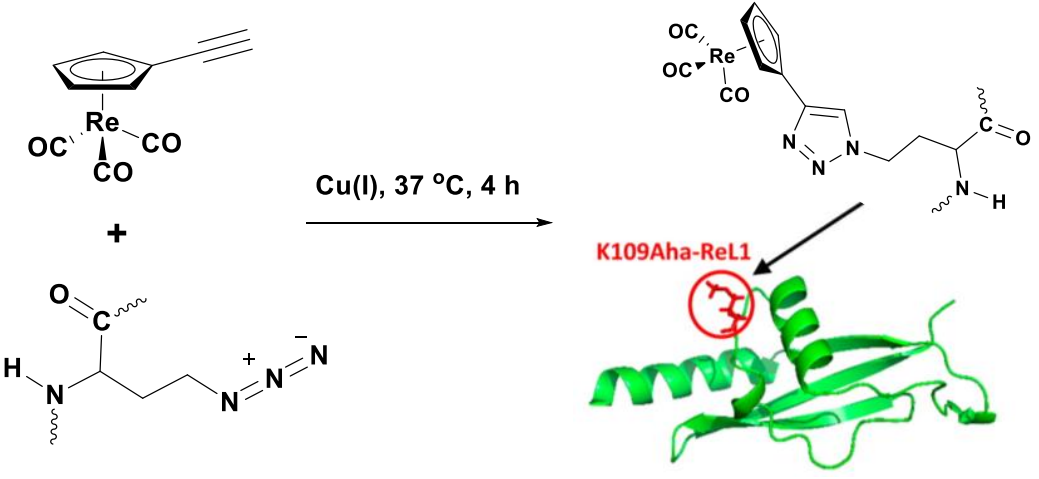

Figure 1.25. a) Incorporation of $\mathrm{CpRe}(\mathrm{CO})_{3}$ with an alkylated methanethiosulfonate onto thiol functionality on proteins. b) Bioorthogonal incorporation of a $\mathrm{CpRe}(\mathrm{CO})_{3}$ alkynyl derivative onto residue 109 of the protein CTL9, located in a loop, using the "click" reaction. Adapted from ref. 128 with permission from the American Chemical Society.

The water-membrane interface in a model lipid membrane has also been probed with a chromium tricarbonyl-labelled cholesterol (figure 1.26). ${ }^{129}$ The frequencyfrequency correlation function (FFCF) at the water-membrane interface showed a distinct three-fold slowdown compared to that in bulk water. A similar incorporation of $\mathrm{W}(\mathrm{CO})_{6}$ into the alkane interior of the membrane bilayer allowed sensing of its environment through the $\mathrm{CO}$ vibrations, and probing of the internal structural dynamics via $2 \mathrm{D}$-IR measurements. ${ }^{130}$ It was found that the addition of cholesterol induced a slowdown in the structural dynamics, and an abrupt change occurred when the concentration reached 35\%; this change was also observed in the FT-IR absorption spectra and ascribed to a phase change. 

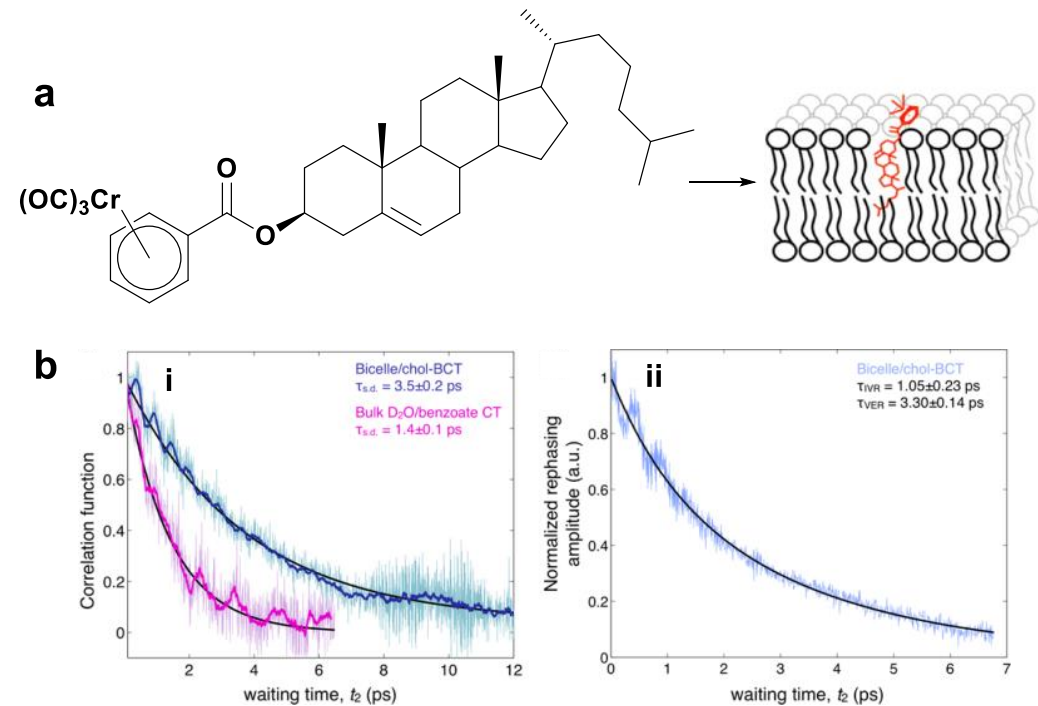

Figure 1.26. a) Incorporation of $\mathrm{Cr}(\mathrm{CO})_{3}$ labelled cholesterol into membrane constructs such as bicelles or unilamellar vesicles. bi) Rapidly acquired spectral diffusion (RASD) measures the FFCF of the conjugate in bulk water and at the bicelle-water interface. The faint lines are the full data set; the thick colored lines are the result of a $100 \mathrm{fs}$ windowed moving average. The fits are shown as black lines. (ii) Rephasing decay for the bicelle sample showing the full data set (blue) and the biexponential fit. The sub-5-ps lifetime is indicative of water exposure. Adapted from ref. 129 with permission from the American Chemical Society.

\subsection{Transition metal carbonyls as Raman probes}

Despite the demonstrated success of metal carbonyls for mid-IR sensing and imaging, there are also several limitations associated with this technique. First, the strong water absorption in living cells or biological media can overlap and interfere with the CO stretches. Secondly, IR microscopy has a spatial resolution (for CO vibrations) of about $6 \mu \mathrm{m}$ which is generally inadequate for sub-cellular imaging. Though this can be circumvented by techniques such as PTIR and SR-FTIR, sophisticated instrumental set-ups are required for these.

\section{- IR vs Raman characteristics of CO}

The inelastic scattering of a photon was first observed experimentally by C.V. Raman in 1928. ${ }^{131}$ It involves a change in the polarizability of the molecule (with respect to the vibrational motion) induced by the oscillating electric field of the incident light. Light scattered from a molecule contains both elastic Rayleigh and inelastic Raman scattering. Rayleigh scattering corresponds to the scattering at the same frequency of the incident light, while Raman scattering involves a shift in the 
frequency of the incident light, which can be to a lower frequency (Stokes, $v_{0}-v_{j}$ ) or to a higher frequency (anti-Stokes, $v_{0}+v_{j}$ ); the shifts are associated with vibrational modes of the molecule (figure 1.27). ${ }^{132}$

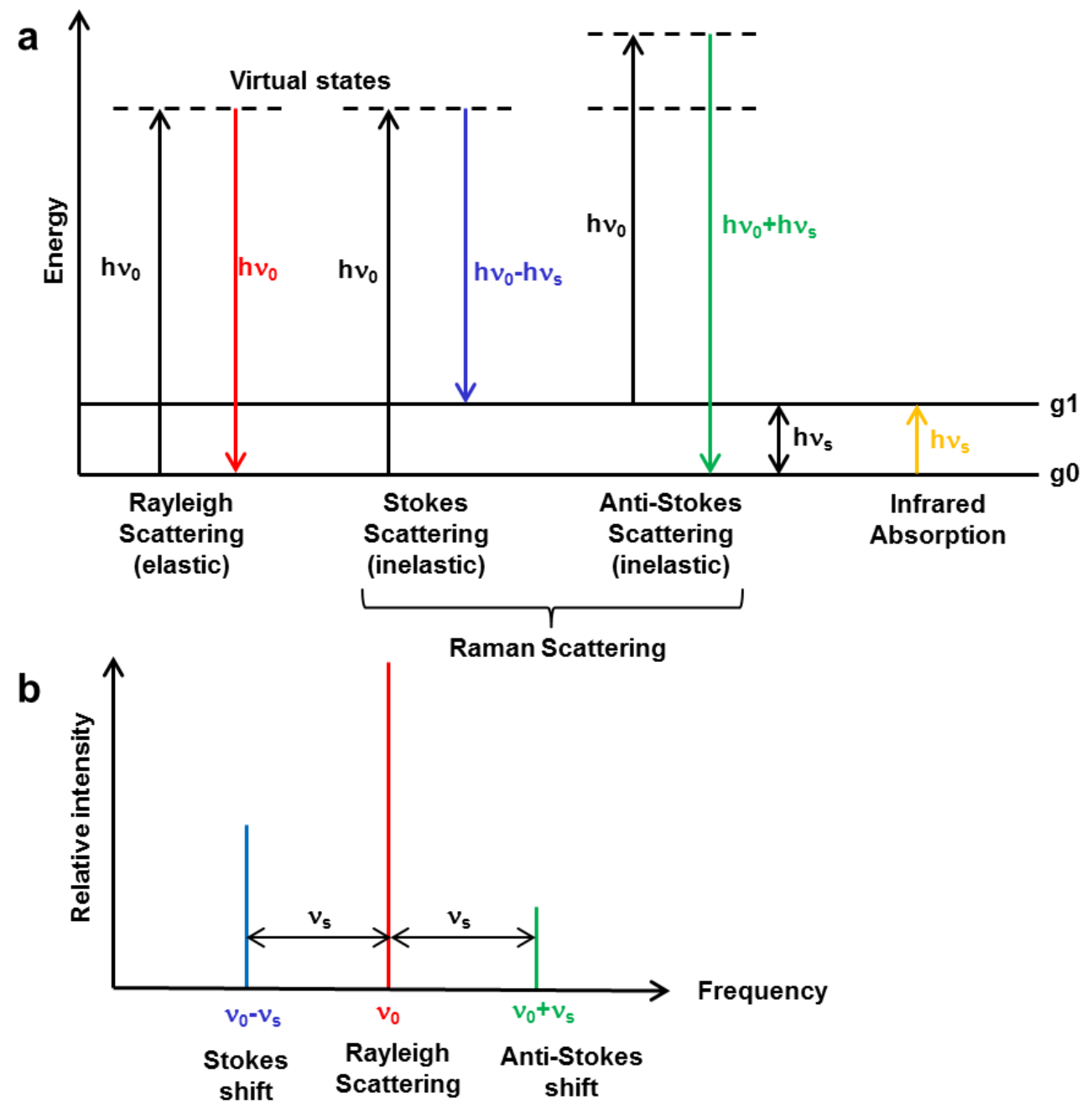

Figure 1.27. a) Jablonski diagram representing the energy transition for Rayleigh, Raman scattering and IR absorption. b) A schematic Raman spectrum showing the placement of the Stokes and anti-Stokes shift in relation to the Rayleigh line. Adapted from ref. 74 with permission from the Royal Society of Chemistry.

Rayleigh scattering is of much greater intensity than Raman scattering (a factor of $\sim 10^{6}-10^{8}$ ) and by the Boltzmann distribution, more molecules will be in the vibrational ground state than the excited state at ambient conditions and hence, Stokes scattering is of higher intensity than anti-Stokes scattering. The Stokes scattering is what is usually recorded as the Raman shift with respect to the Rayleigh scattering. The difference between IR and Raman spectroscopy are summarized in table 1.2. 
Table 1.2. Summary and comparison between IR and Raman spectroscopy

\begin{tabular}{|c|c|c|}
\hline $\begin{array}{c}\text { Excitation } \\
\text { wavelength }\end{array}$ & $\begin{array}{c}\text { Mid-IR region from } 2500 \mathrm{~nm} \text { to } \\
25000 \mathrm{~nm}\left(4000 \text { to } 400 \mathrm{~cm}^{-1}\right)\end{array}$ & $\begin{array}{c}\text { Visible-near IR region from } \\
532 \mathrm{to} 1064 \mathrm{~nm}\end{array}$ \\
\hline Nature of techniques & Light absorption & Inelastic light scattering \\
\hline Mechanism & Requires a dipole moment change & $\begin{array}{c}\text { Requires a change in } \\
\text { polarizability }\end{array}$ \\
\hline $\begin{array}{c}\text { Spatial resolution at } \\
\text { the diffraction limit }\end{array}$ & $\sim 1.3$ to $12.5 \mu \mathrm{M}$ & $\sim 266 \mathrm{~nm}$ to $532 \mathrm{~nm}$ \\
\hline Limitations & $\begin{array}{c}\text { Low spatial resolution and prone to } \\
\text { interference by water }\end{array}$ & $\begin{array}{c}\text { Weak signals for CO } \\
\text { vibrational frequencies. }\end{array}$ \\
\hline
\end{tabular}

The intensity of a Raman signal is inherently weak, and its molecular determinant is termed the Raman cross-section $(\beta)$; it is analogous to molar extinction in absorption spectroscopy. In general, $\beta$ is a function of the chemical bonds and other electronic factors. ${ }^{133}$ For instance, molecules with extended $\pi$ systems such as benzene, naphthalene and anthracene, in which the electrons are easily polarized, have large $\beta$. In contrast, small molecules without electron-rich atoms, like $\mathrm{H}_{2}$ and $\mathrm{CO}$, have small $\beta$ and hence produce weak Raman scattering. The CO ligand in a complex will likewise have a small Raman cross-section. For applications in biological systems, ${ }^{134-137}$ Raman spectroscopy has two advantages over IR spectroscopy - the relatively weak Raman scattering of water, and sub-cellular resolution with simple instrumentation. The latter derives from the fact that the incident light for Raman excitation is in the visible to near-IR range and since spatial resolution scales as $\sim \lambda / 2$, it provides $\mathrm{nm}$ as opposed to $\mu \mathrm{m}$ resolution for IR spectroscopy.

\section{- Metal carbonyls as Raman probes for cellular imaging}

Despite the fundamental limitation of weak Raman scattering in metal carbonyls, uptake of the manganese complex $\left[\mathrm{Mn}(\mathrm{tpm})(\mathrm{CO})_{3}\right] \mathrm{Cl}(\mathrm{tpm}=\operatorname{tris}(1-$ pyrazolyl $)$ methane $)$ in HT29 human colon cancer cells was demonstrated with 3D Raman imaging; the complex was determined to be localized in the nuclear membrane and nucleolus (figure 1.28). ${ }^{138,139}$ 

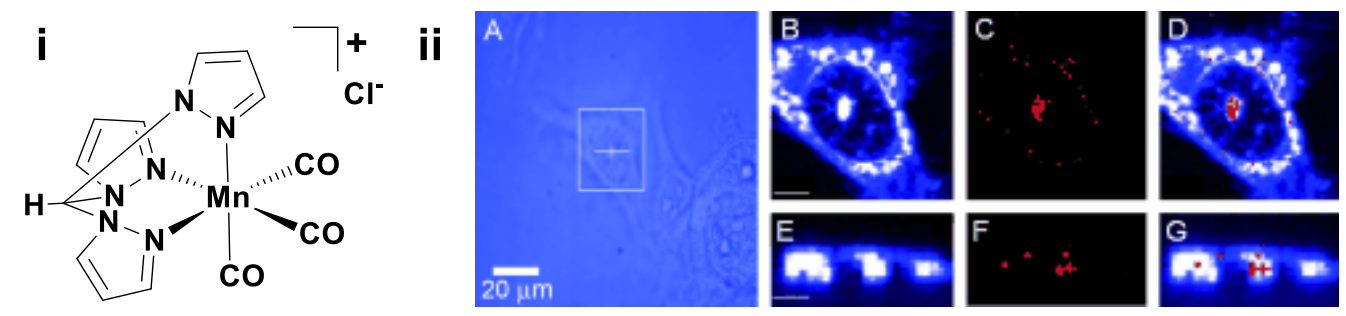

Figure 1.28. i) Molecular structure of $\left[\mathrm{Mn}(\mathrm{tpm})(\mathrm{CO})_{3}\right] \mathrm{Cl}$. ii) (A) Optical image of a HT29 human colon cancer cell incubated with an aqueous solution $(2 \mathrm{mM})$ of $\left[\mathrm{Mn}(\mathrm{tpm})(\mathrm{CO})_{3}\right] \mathrm{Cl}$ for 3 h. $(B, C)$ Raman images reconstructed from integrating the intensities of the $\mathrm{C}-\mathrm{H}$ and $\mathrm{C} \equiv \mathrm{O}$ stretching peaks. The integration range was $2800-3050( \pm 2) \mathrm{cm}^{-1}$ for $(\mathrm{B})$ and $1945-1965( \pm 2)$ $\mathrm{cm}^{-1}$ for (C). (D) Overlaid image of panels (B) and (C). (E-G) Cross-section Raman images along the $\mathrm{x}, \mathrm{z}$-direction of the same cell. Scanning positions are indicated by the white bar in the optical image. The scale bar for the Raman images is $6 \mu \mathrm{m}$. Reproduced from ref. 138 with permission from John Wiley and Sons.

\section{- Detection through surface-enhanced Raman spectroscopy (SERS)}

A paradigm shift occurred with the discovery of Raman intensity enhancement by Fleischmann and co-workers, and the subsequent investigation by Jeanmaire and Van Duyne. ${ }^{140,141}$ The phenomenon refers to the increase in the Raman intensity of a molecule on the surface of a metallic nanostructure; enhancement factors of $10^{6}$ to $10^{9}$ have been reported. It led to the development of surface enhanced Raman scattering (SERS), and widespread applications followed. ${ }^{137,142-145}$ The mechanism of SERS has been studied over the last few decades, ${ }^{146-148}$ and it is now widely accepted that the enhancement is the product of two mechanisms: electromagnetic and chemical enhancement. Electromagnetic enhancement refers to the resonance interaction of light with the localized surface plasmon of the metallic surface, generating enhanced local electric field, which is responsible for the increase in Raman signals; the surface plasmon is the collective electron cloud at the surface of the metallic nanostructure. ${ }^{147}$, ${ }^{149}$ Chemical enhancement refers to a metal-to-adsorbate charge-transfer that can occur with chemisorbed species.

SERS application requires the use of highly reproducible substrates to enhance the $\mathrm{CO}$ vibrations and is generally based on two types of substrate - colloidal and planar substrates. Colloidal substrates, which include plasmonic nanoparticles suspended in liquid phase, are frequently used in the formulation of SERS tags for used in biological systems. These tags typically consist of Raman-active molecules that act as reporters adsorbed onto the surface of plasmon-resonant gold or silver nanoparticles protected 
with a coating, and bio-functionalized with targeting biomolecules. ${ }^{150}$ Even though silver gives a stronger SERS enhancement than gold, the latter is preferred due to their better biocompatibility, size distribution and long-term stability. ${ }^{151,152}$

A number of works, including a review, on SERS tags that comprise organic molecules as reporters for SERS imaging have appeared, ${ }^{150,153,154}$ but the first report on the use of metal carbonyls as Raman reporters in a SERS tag for live cell imaging was more recent, and comprised the triosmium carbonyl cluster $\mathrm{Os}_{3}(\mu-\mathrm{H})_{2}(\mathrm{CO})_{10}$ conjugated onto gold nanoparticles. ${ }^{155}$ This organometallic-nanoparticle (OM-NP) conjugate showed strong Raman signals in the CO vibration region (1800-2200 $\left.\mathrm{cm}^{-1}\right)$, with an enhancement factor estimated at $\sim 15,000$. The OM-NP conjugates were nontoxic, showed good aqueous dispersibility and stability, and could be easily functionalized with the desired biomolecule (L) to give OM-NP-L conjugate for targeted delivery; in this case, they were biofunctionalized with an antibody against epidermal growth factor receptors (EGFR), which are over-expressed in oral squamous cell carcinoma (OSCC) (figure 1.29).

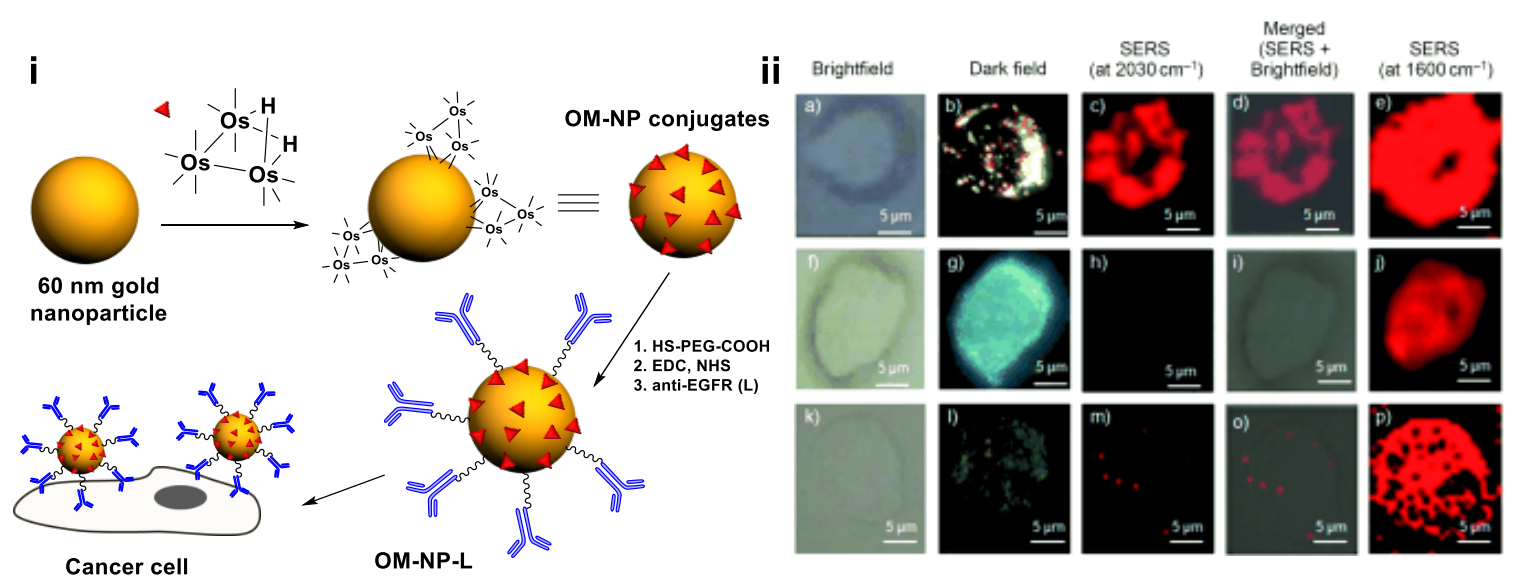

Figure 1.29. i) Preparation of OM-NP-L conjugates using the strong interactions of $\mathrm{Os}_{3}(\mathrm{CO})_{10}(\mu-\mathrm{H})_{2}$ with gold nanoparticles. OM-NP conjugates are then functionalized with PEG and antibodies to give conjugate OM-NP-L conjugates. Ii) Bright-field, dark-field, and SERS mapping images of (a-e) OSCC cells, ( $\mathrm{f}-\mathrm{j})$ SKOV cells treated with OM-NP-L, and ( $\mathrm{k}-$ p) OSCC cells treated with anti-EGFR prior to incubation with OM-NP-L. All SERS mapping images of CO $\left(2030 \mathrm{~cm}^{-1}\right)$ and protein $\left(1600 \mathrm{~cm}^{-1}\right)$ were taken at an interval of $1 \mu \mathrm{m}(633 \mathrm{~nm}$ excitation). Adapted from ref. 155 with permission from John Wiley and Sons.

The essential components of these tags are the metal carbonyl and the SERS-active substrate. Furthering their application will require examination of these two 
components. For example, it will be of interest to explore the tunability of the $\mathrm{CO}$ vibrational frequencies of such conjugates. This will enable a new class of Raman reporters that can provide non-overlapping signals in the $2200-1800 \mathrm{~cm}^{-1}$ region, thus allowing the use of more than one conjugates in multiplex applications. To extend the practicality of this system, it will also be desirable to examine SERS-active substrates in other forms apart from the colloidal form. One is the SERS planar substrate, which comprises a plasmonic nanomaterial immobilized onto a flat surface. Such planar substrates are known to give excellent reproducibility and SERS performance, and can potentially be used to develop chip-based sensors.

\subsection{Transition metal carbonyl as photoacoustic probe}

Pure optical imaging modalities like multiphoton microscopy, confocal microscopy and optical coherence tomography often suffer from poor depth penetration (restricted to the optical transport mean free path in biological tissue) as a result of the strong scattering by soft tissues. ${ }^{156-158}$ To circumvent the fundamental depth limitation, a hybrid modality, photoacoustic imaging, was developed and its first functional imaging application was demonstrated in 2003. ${ }^{159}$ This emerging imaging modality effectively combines the strengths of both optical and acoustic imaging, i.e., strong optical contrast with high spatial resolution, and is thus being recognized as an attractive, non-invasive method for in vivo imaging. ${ }^{160-162}$

Photoacoustic imaging is based on the photoacoustic effect, first described by Alexander Graham Bell in 1880. ${ }^{163}$ The effect refers to the generation of acoustic waves resulting from thermoelastic expansion following the absorption of light (figure 1.30). ${ }^{162,} 164,165$ The ultrasound waves as detected by the transducers can be reconstructed to generate an image based on algorithms in photoacoustic computed tomography or the detection in point-by-point raster scanning in the case of photoacoustic microscopy. ${ }^{160}$ The optical contrast is determined by the absorption of light by the object of interest, while the resolution is derived from the detected ultrasonic emission. Since biological tissue scattered ultrasound wave less as compared to light, reconstructed images of higher spatial resolution can be obtained. Photoacoustic imaging has allowed a wide range of in vivo studies, including probing dynamic changes in the hemoglobin oxygen saturation of skin, brain hemodynamic changes and tumor angiogenesis. ${ }^{159,166,167}$ 


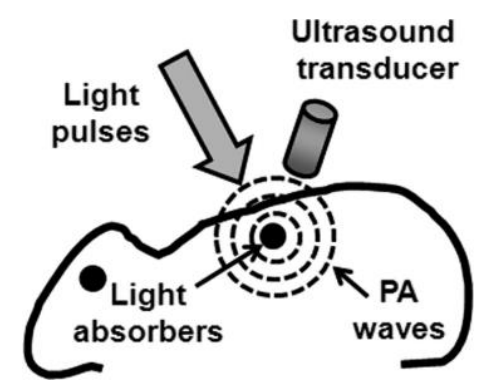

Figure 1.30. Illustration of the photoacoustic (PA) mechanism. When a target absorbs light, photoacoustic waves are generated via thermo-elastic expansion. The photoacoustic waves propagate and are detected by an ultrasonic transducer. Adapted from ref. 160 with permission from the American Chemical Society.

Although photoacoustic imaging can be used to visualize the distribution of endogenous molecules like oxy- and deoxy-hemoglobin, exogenous contrast agents are, nevertheless, often introduced into biological systems for site-specific imaging. Various contrast agents have been developed, including single-walled carbon nanotubes, ${ }^{168}$ gold nanostructures, ${ }^{169,}{ }^{170}$ fluorescent proteins, ${ }^{171}$ and cyanine dyes. ${ }^{172}$ More recently, Leong and Olivo et. al. have demonstrated the use of a water-soluble triosmium carbonyl cluster for in vivo imaging of the rat cerebral cortex vasculature (figure 1.31). ${ }^{173}$ This highly stable contrast agent allows the brain vasculature, especially the superior sagittal sinus (SSS), to exhibit higher photoacoustic contrast as compared to their intrinsic contrast and the use of carbon nanotubes. Although the photoacoustic contrast of the triosmium carbonyl cluster is high, a major drawback lies in the incident wavelength of $410 \mathrm{~nm}$; tissue penetration is low at this wavelength and thus an invasive procedure is required to carry out the imaging. It is therefore of interest to identify metal carbonyls that absorb in the near-IR (NIR) range (680 to 1000 $\mathrm{nm}$ ) which can allow the use of NIR light for deeper tissue penetration and less scattering; such metal carbonyls can potentially be used for whole body in vivo imaging. 
a
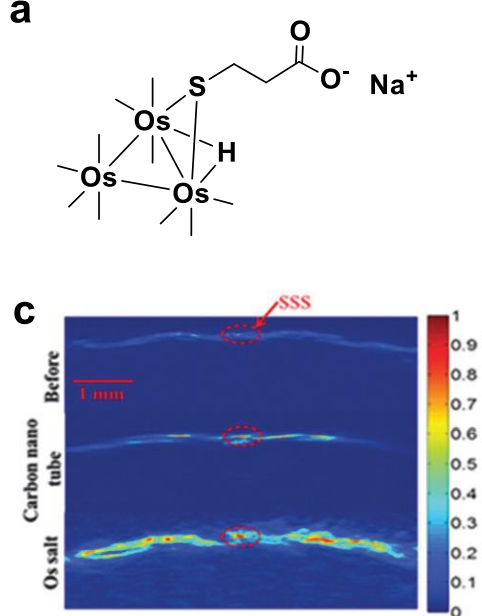
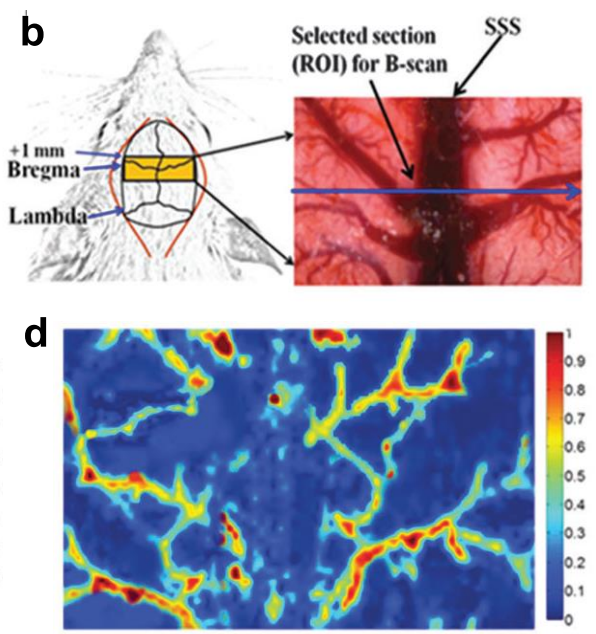

Figure 1.31. a) Molecular structure of the water-soluble triosmium carbonyl cluster. a) Photograph of the rat cortical blood vessels, with the superior sagittal sinus (SSS) indicated. The ROI cross-section location is indicated by a blue arrow. b) Photoacoustic B-scan images acquired before, and $20 \mathrm{~min}$ after, the administration of a $30 \mu \mathrm{M}$ aqueous solution of the cluster or carbon nanotubes. The SSS is highlighted with a red oval. d) The brain vasculature shows up clearly in the photoacoustic C-scan. Adapted from ref. 173 with permission from the Royal Society of Chemistry. 


\subsection{Aim and objectives}

From the foregoing survey and analysis, it is clear that the application of metal carbonyls in bioimaging has come some way with current development lies with SERS and photoacoustic imaging. The aim of this project is therefore to explore the use of metal carbonyls as SERS- and photoacoustic-based reporters for various bio-imaging and-sensing applications.

Specific objectives:

1. Investigate different classes of metal carbonyls as Raman reporters with colloidal gold nanospheres in SERS-based applications.

2. Demonstrate the use of metal carbonyl clusters with SERS planar substrates in SERS-based bioassays.

3. Develop metal carbonyls as near-IR reporters for whole body in vivo photoacoustic imaging. 


\subsection{References}

1. F. E. Paulik and J. F. Roth, Chem. Commun., 1968, 1578a-1578a.

2. G. J. Sunley and D. J. Watson, Catal. Today, 2000, 58, 293-307.

3. G. Natta and I. Pasquon, in Advances in Catalysis, eds. P. W. S. D.D. Eley and B. W. Paul, Academic Press, 1959, vol. Volume 11, pp. 1-66.

4. S. Top, G. Jaouen, A. Vessieres, J. P. Abjean, D. Davoust, C. A. Rodger, B. G. Sayer and M. J. McGlinchey, Organometallics, 1985, 4, 2143-2150.

5. G. Jaouen and A. Vessieres, Pure Appl. Chem., 1985, 57, 1865-1874.

6. A. P. F. Turner, Chem. Soc. Rev., 2013, 42, 3184-3196.

7. A. P. F. Turner, I. Karube and G. S. Wilson, Biosensors: Fundamentals and Applications, Oxford University Press, NY, 1987.

8. T. J. Kealy and P. L. Pauson, Nature, 1951, 168, 1039-1040.

9. S. A. Miller, J. A. Tebboth and J. F. Tremaine, J. Chem. Soc., 1952, 632-635.

10. G. Jaouen, A. Vessieres and S. Top, Chem. Soc. Rev., 2015, 44, 8802-8817.

11. C. Roux and C. Biot, Future Med. Chem., 2012, 4, 783-797.

12. S. Martic, M. Labib, P. O. Shipman and H.-B. Kraatz, Dalton Trans., 2011, 40, 7264-7290.

13. A. E. G. Cass, G. Davis, G. D. Francis, H. A. O. Hill, W. J. Aston, I. J. Higgins, E. V. Plotkin, L. D. L. Scott and A. P. F. Turner, Anal. Chem., 1984, 56, 667671.

14. D. R. van Staveren and N. Metzler-Nolte, Chem. Rev., 2004, 104, 5931-5986.

15. G. Liu, J. Wang, D. S. Wunschel and Y. Lin, J. Am. Chem. Soc., 2006, 128, 12382-12383.

16. J. F. Woessner, FASEB J., 1991, 5, 2145-2154.

17. R. Visse and H. Nagase, Circul. Res., 2003, 92, 827-839.

18. H. Aoki and H. Tao, Analyst, 2007, 132, 784-791.

19. N. Hüsken, M. Gębala, W. Schuhmann and N. Metzler-Nolte, ChemBioChem, 2010, 11, 1754-1761.

20. X. Luo, T. M.-H. Lee and I. M. Hsing, Anal. Chem., 2008, 80, 7341-7346.

21. P. Nielsen, M. Egholm, R. Berg and O. Buchardt, Science, 1991, 254, $1497-$ 1500.

22. T.-K. Lim and T. Matsunaga, Biosens. Bioelectron., 2001, 16, 1063-1069.

23. T.-K. Lim, H. Ohta and T. Matsunaga, Anal. Chem., 2003, 75, 3316-3321. 
24. T. Tanaka, S. Tsukube, K. Izawa, M. Okochi, T.-K. Lim, S. Watanabe, M. Harada and T. Matsunaga, Biosens. Bioelectron., 2007, 22, 2051-2056.

25. M. M. Khalil, J. L. Tremoleda, T. B. Bayomy and W. Gsell, Int. J. Mol. Imaging, 2011, 2011.

26. M. E. Phelps, J. Nucl. Med., 2000, 41, 661-681.

27. C. Perrier and E. Segrè, J. Chem. Phys., 1937, 5, 712-716.

28. P. Richards, W. D. Tucker and S. C. Srivastava, Int. J. Appl. Radiat. Isot., 1982, 33, 793-799.

29. V. J. Molinski, Int. J. Appl. Radiat. Isot., 1982, 33, 811-819.

30. E. M. Smith, J. Nucl. Med., 1964, 5, 871-882.

31. R. Alberto, Top. Curr. Chem., 2005, 252, 1-44.

32. R. Alberto, R. Schibli, A. Egli, A. P. Schubiger, U. Abram and T. A. Kaden, J. Am. Chem. Soc., 1998, 120, 7987-7988.

33. R. Alberto, K. Ortner, N. Wheatley, R. Schibli and A. P. Schubiger, J. Am. Chem. Soc., 2001, 123, 3135-3136.

34. T. L. Mindt, H. Struthers, L. Brans, T. Anguelov, C. Schweinsberg, V. Maes, D. Tourwé and R. Schibli, J. Am. Chem. Soc., 2006, 128, 15096-15097.

35. R. Alberto, Eur. J. Inorg. Chem., 2009, 2009, 21-31.

36. Y. Liu, J. K. Pak, P. Schmutz, M. Bauwens, J. Mertens, H. Knight and R. Alberto, J. Am. Chem. Soc., 2006, 128, 15996-15997.

37. M.-L. Biechlin, A. Bonmartin, F.-N. Gilly, M. Fraysse and A. du Moulinet d'Hardemare, Nucl. Med. Biol., 2008, 35, 679-687.

38. N. Agorastos, L. Borsig, A. Renard, P. Antoni, G. Viola, B. Spingler, P. Kurz and R. Alberto, Chem. - Eur. J., 2007, 13, 3842-3852.

39. J. Wald, R. Alberto, K. Ortner and L. Candreia, Angew. Chem. Int. Ed., 2001, 40, 3062-3066.

40. J. Bernard, K. Ortner, B. Spingler, H. J. Pietzsch and R. Alberto, Inorg. Chem., 2003, 42, 1014-1022.

41. M. Benz, H. Braband, P. Schmutz, J. Halter and R. Alberto, Chem. Sci., 2015, 6, 165-169.

42. G. W. Faris, V. Ntziachristos, D. M. Rector and L. Wang, Appl. Opt., 2007, 46, 1595-1596.

43. L. V. Wang and H.-I. Wu, in Biomedical Optics: Principles and Imaging, John Wiley \& Sons, Inc., Hoboken, NJ, USA, 2009, pp. 1-15. 
44. O. Shimomura, F. H. Johnson and Y. Saiga, Journal of Cellular and Comparative Physiology, 1962, 59, 223-239.

45. R. Y. Tsien, Annu. Rev. Biochem, 1998, 67, 509-544.

46. T. H. Ward and J. Lippincott-Schwartz, in Green Fluorescent Protein: Properties, Applications, and Protocols, eds. M. Chalfie and S. R. Kain, John Wiley \& Sons, Inc., Hoboken, NJ, 2005, pp. 305-337.

47. The Molecular Probes Handbook-A Guide to Fluorescent Probes and Labeling Technologies, 11th edition, https://www.thermofisher.com/sg/en/home/references/molecular-probes-thehandbook.html, Accessed 9 March, 2016.

48. T. Terai and T. Nagano, Curr. Opin. Chem. Biol., 2008, 12, 515-521.

49. M. Fernandez-Suarez and A. Y. Ting, Nat. Rev. Mol. Cell Biol., 2008, 9, 929943.

50. T. Xia, N. Li and X. Fang, Annu. Rev. Phys. Chem., 2013, 64, 459-480.

51. J. W. Lichtman and J.-A. Conchello, Nat. Methods, 2005, 2, 910-919.

52. F. L. Thorp-Greenwood, R. G. Balasingham and M. P. Coogan, J. Organomet. Chem., 2012, 714, 12-21.

53. K. K.-W. Lo, P.-K. Lee and J. S.-Y. Lau, Organometallics, 2008, 27, 29983006.

54. K. Y. Zhang and K. K.-W. Lo, Inorg. Chem., 2009, 48, 6011-6025.

55. L. Murphy, A. Congreve, L.-O. Palsson and J. A. G. Williams, Chem. Commun., 2010, 46, 8743-8745.

56. K. Y. Zhang, S. P.-Y. Li, N. Zhu, I. W.-S. Or, M. S.-H. Cheung, Y.-W. Lam and K. K.-W. Lo, Inorg. Chem., 2010, 49, 2530-2540.

57. A. Kando, Y. Hisamatsu, H. Ohwada, T. Itoh, S. Moromizato, M. Kohno and S. Aoki, Inorg. Chem., 2015, 54, 5342-5357.

58. L. He, Y. Li, C.-P. Tan, R.-R. Ye, M.-H. Chen, J.-J. Cao, L.-N. Ji and Z.-W. Mao, Chem. Sci., 2015, 6, 5409-5418.

59. S. W. Botchway, M. Charnley, J. W. Haycock, A. W. Parker, D. L. Rochester, J. A. Weinstein and J. A. G. Williams, Proc. Natl. Acad. Sci. U. S. A., 2008, 105, 16071-16076.

60. S. J. Farley, D. L. Rochester, A. L. Thompson, J. A. K. Howard and J. A. G. Williams, Inorg. Chem., 2005, 44, 9690-9703. 
61. P. J. Barnard, L. E. Wedlock, M. V. Baker, S. J. Berners-Price, D. A. Joyce, B. W. Skelton and J. H. Steer, Angew. Chem. Int. Ed., 2006, 45, 5966-5970.

62. N. L. Coker, J. A. Krause Bauer and R. C. Elder, J. Am. Chem. Soc., 2004, 126, 12-13.

63. P. J. Barnard, M. V. Baker, S. J. Berners-Price, B. W. Skelton and A. H. White, Dalton Trans., 2004, 1038-1047.

64. E. Vergara, E. Cerrada, A. Casini, O. Zava, M. Laguna and P. J. Dyson, Organometallics, 2010, 29, 2596-2603.

65. L. Mond, C. Langer and F. Quincke, J. Chem. Soc., Trans., 1890, 57, 749-753.

66. R. H. Crabtree, in The Organometallic Chemistry of the Transition Metals, John Wiley \& Sons, Inc., Hoboken, NJ, USA, 2005, pp. 87-124.

67. K. V. Kong, W. Chew, L. H. K. Lim, W. Y. Fan and W. K. Leong, Bioconjugate Chem., 2007, 18, 1370-1374.

68. G. Jaouen, A. Vessières, S. Top, A. A. Ismail and I. S. Butler, J. Am. Chem. Soc., 1985, 107, 4778-4780.

69. G. Jaouen, A. Vessieres, S. Top, M. Savignac, A. A. Ismail and I. S. Butler, Organometallics, 1987, 6, 1985-1987.

70. A. Vessieres, G. Jaouen, M. Gruselle, J. L. Rossignol, M. Savignac, S. Top and S. Greenfield, J. Steroid Biochem, 1988, 30, 301-305.

71. S. Tondu, S. Top, A. Vessieres and G. Jaouen, J. Chem. Soc., Chem. Commun., $1985,326-328$.

72. A. Vessieres, S. Tondu, G. Jaouen, S. Top, A. A. Ismail, G. Teutsch and M. Moguilewsky, Inorg. Chem., 1988, 27, 1850-1852.

73. M. Salmain, A. Vessières, P. Brossier, I. S. Butler and G. Jaouen, J. Immunol. Methods, 1992, 148, 65-75.

74. Z. Lam, K. V. Kong, M. Olivo and W. K. Leong, Analyst, 2016, 141, 15691586.

75. M. Salmain and A. Vessières, in Bioorganometallics, ed. G. Jaouen, WileyVCH Verlag GmbH \& Co. KGaA, 2006, pp. 263-302.

76. A. A. Ismail, G. Jaouen, P. Cheret and P. Brossier, Clin. Biochem., 1989, 22, 297-299.

77. M. Salmain, A. Vessieres, I. S. Butler and G. Jaouen, Bioconjugate Chem., 1991, 2, 13-15. 
78. A. Vessières, K. Kowalski, J. Zakrzewski, A. Stepien, M. Grabowski and G. Jaouen, Bioconjugate Chem., 1999, 10, 379-385.

79. V. Philomin, A. Vessières and G. Jaouen, J. Immunol. Methods, 1994, 171, 201-210.

80. M. Salmain, A. Vessieres, P. Brossier and G. Jaouen, Anal. Biochem., 1993, 208, $117-120$.

81. M. Salmain, A. Vessières, A. Varenne, P. Brossier and G. Jaouen, J. Organomet. Chem., 1999, 589, 92-97.

82. M. Salmain, N. Fischer-Durand, L. Cavalier, B. Rudolf, J. Zakrzewski and G. Jaouen, Bioconjugate Chem., 2002, 13, 693-698.

83. N. Fischer-Durand, M. Salmain, B. Rudolf, A. Vessières, J. Zakrzewski and G. Jaouen, ChemBioChem, 2004, 5, 519-525.

84. N. Fischer-Durand, M. Salmain, B. Rudolf, L. Dai, L. Jugé, V. Guérineau, O. Laprévote, A. Vessières and G. Jaouen, Anal. Biochem., 2010, 407, 211-219.

85. B. Rudolf, M. Salmain, J. Grobelny, G. Celichowski and M. Cichomski, J. Organomet. Chem., 2013, 734, 32-37.

86. B. Rudolf, M. Salmain, J. Grobelny, G. Celichowski and E. Tomaszewska, Colloids Surf., A, 2011, 385, 241-248.

87. B. Rudolf, M. Salmain, A. Z. Wilczewska, A. Kubicka, I. Misztalewska and N. Fischer-Durand, Colloids Surf., A, 2014, 457, 142-151.

88. L. Quaroni, M. Obst, M. Nowak and F. Zobi, Angew. Chem. Int. Ed., 2015, 54, 318-322.

89. C. Policar, J. B. Waern, M.-A. Plamont, S. Clède, C. Mayet, R. Prazeres, J.-M. Ortega, A. Vessières and A. Dazzi, Angew. Chem. Int. Ed., 2011, 50, 860-864.

90. R. Tenhunen, H. S. Marver and R. Schmid, Proc. Natl. Acad. Sci. U. S. A., 1968, 61, 748-755.

91. R. Motterlini and L. E. Otterbein, Nat. Rev. Drug Discovery, 2010, 9, 728-743.

92. R. Wang, Z. Wang and L. Wu, Br. J. Pharmacol., 1997, 121, 927-934.

93. L. E. Otterbein, Antioxid. Redox Signaling, 2002, 4, 309-319.

94. J. Boczkowski, J. J. Poderoso and R. Motterlini, Trends Biochem. Sci, 2006, 31, 614-621.

95. C. C. Romão and H. L. A. Vieira, in Advances in Organometallic Chemistry and Catalysis, ed. A. J. L. Pombeiro, John Wiley \& Sons, Inc., 2013, pp. 543561. 
96. U. Schatzschneider, Br. J. Pharmacol., 2015, 172, 1638-1650.

97. U. Schatzschneider, Inorg. Chim. Acta, 2011, 374, 19-23.

98. I. Chakraborty, S. J. Carrington and P. K. Mascharak, Acc. Chem. Res., 2014, 47, 2603-2611.

99. F. Zobi, L. Quaroni, G. Santoro, T. Zlateva, O. Blacque, B. Sarafimov, M. C. Schaub and A. Y. Bogdanova, J. Med. Chem., 2013, 56, 6719-6731.

100. S. Clede, F. Lambert, C. Sandt, Z. Gueroui, M. Refregiers, M.-A. Plamont, P. Dumas, A. Vessieres and C. Policar, Chem. Commun., 2012, 48, 7729-7731.

101. S. Clede, F. Lambert, C. Sandt, S. Kascakova, M. Unger, E. Harte, M.-A. Plamont, R. Saint-Fort, A. Deniset-Besseau, Z. Gueroui, C. Hirschmugl, S. Lecomte, A. Dazzi, A. Vessieres and C. Policar, Analyst, 2013, 138, 5627-5638.

102. S. Clède, F. Lambert, R. Saint-Fort, M.-A. Plamont, H. Bertrand, A. Vessières and C. Policar, Chem. - Eur. J., 2014, 20, 8714-8722.

103. I. Kitanovic, S. Can, H. Alborzinia, A. Kitanovic, V. Pierroz, A. Leonidova, A. Pinto, B. Spingler, S. Ferrari, R. Molteni, A. Steffen, N. Metzler-Nolte, S. Wölfl and G. Gasser, Chem. - Eur. J., 2014, 20, 2496-2507.

104. E. Fernández, G. Rodríguez, S. Hostachy, S. Clède, M. Cócera, C. Sandt, F. Lambert, A. de la Maza, C. Policar and O. López, Colloids Surf., B, 2015, 131, 102-107.

105. S. Clede, N. Delsuc, C. Laugel, F. Lambert, C. Sandt, A. Baillet-Guffroy and C. Policar, Chem. Commun., 2015, 51, 2687-2689.

106. G. R. Stephenson, in Bioorganometallics, ed. G. Jaouen, Wiley-VCH Verlag GmbH \& Co. KGaA, 2006, pp. 215-262.

107. C. E. Anson, T. J. Baldwin, C. S. Creaser, M. A. Fey and G. R. Stephenson, Organometallics, 1996, 15, 1451-1456.

108. C. E. Anson, C. S. Creaser and G. R. Stephenson, J. Chem. Soc., Chem. Commun., 1994, 2175-2176.

109. G. R. Stephenson, C. E. Anson, C. S. Creaser and C. A. Daul, Eur. J. Inorg. Chem., 2011, 2011, 2086-2097.

110. H. Plenio and D. Burth, Organometallics, 1996, 15, 1151-1156.

111. J. D. Lewis and J. N. Moore, Phys. Chem. Chem. Phys., 2004, 6, 4595-4606.

112. X. Tian, L. Song, Y. Yuan and J. Lin, Organometallics, 2010, 29, 509-511.

113. J. W. Kee and W. Y. Fan, J. Organomet. Chem., 2013, 729, 14-19. 
114. E. Peris, J. A. Mata and V. Moliner, J. Chem. Soc., Dalton Trans., 1999, 38933898.

115. C. E. Anson, C. S. Creaser and G. Richard Stephenson, Spectrochim. Acta, Part A, 1996, 52, 1183-1191.

116. C. E. Anson, C. S. Creaser, O. Egyed, M. A. Fey and G. R. Stephenson, J. Chem. Soc., Chem. Commun., 1994, 39-40.

117. C. S. Creaser, M. A. Fey and G. R. Stephenson, Spectrochim. Acta, Part A, 1994, 50, 1295-1299.

118. C. S. Creaser, W. E. Hutchinson and R. G. Stephenson, Appl. Spectrosc., 2000, 54, 1624-1628.

119. C. S. Creaser, W. E. Hutchinson and G. R. Stephenson, Analyst, 2001, 126, 647-651.

120. C. E. Anson, C. S. Creaser, O. Egyed and G. R. Stephenson, Spectrochim. Acta, Part A, 1997, 53, 1867-1877.

121. C. C. Barraclough, J. Lewis and R. S. Nyholm, J. Chem. Soc., 1961, 2582.

122. E. J. Heilweil, R. R. Cavanagh and J. C. Stephenson, Chem. Phys. Lett., 1987, 134, 181-188.

123. J. T. King, M. R. Ross and K. J. Kubarych, J. Phys. Chem. B, 2012, 116, 37543759.

124. J. T. King, E. J. Arthur, C. L. Brooks and K. J. Kubarych, J. Phys. Chem. B, 2012, 116, 5604-5611.

125. T. Santos-Silva, A. Mukhopadhyay, J. D. Seixas, G. J. L. Bernardes, C. C. Romão and M. J. Romão, J. Am. Chem. Soc., 2011, 133, 1192-1195.

126. J. T. King and K. J. Kubarych, J. Am. Chem. Soc., 2012, 134, 18705-18712.

127. A. M. Woys, S. S. Mukherjee, D. R. Skoff, S. D. Moran and M. T. Zanni, J. Phys. Chem. B, 2013, 117, 5009-5018.

128. I. Peran, T. Oudenhoven, A. M. Woys, M. D. Watson, T. O. Zhang, I. Carrico, M. T. Zanni and D. P. Raleigh, J. Phys. Chem. B, 2014, 118, 7946-7953.

129. D. G. Osborne, J. A. Dunbar, J. G. Lapping, A. M. White and K. J. Kubarych, J. Phys. Chem. B, 2013, 117, 15407-15414.

130. O. Kel, A. Tamimi, M. C. Thielges and M. D. Fayer, J. Am. Chem. Soc., 2013, 135, 11063-11074.

131. C. V. Raman and K. S. Krishnan, Nature, 1928, 122, 169. 
132. L. A. Nafie, in Handbook of Raman Spectroscopy, eds. I. R. Lewis and H. G. M. Edwards, CRC Press, Boca Raton, FL, USA, 2001.

133. R. L. McCreery, in Raman Spectroscopy for Chemical Analysis, John Wiley \& Sons, Inc., Hoboken, NJ, USA, 2005, pp. 15-33.

134. T. W. Collette and T. L. Williams, J. Environ. Monit., 2002, 4, 27-34.

135. H. Yamakoshi, K. Dodo, A. Palonpon, J. Ando, K. Fujita, S. Kawata and M. Sodeoka, J. Am. Chem. Soc., 2012, 134, 20681-20689.

136. K. Klein, Alexander M. Gigler, T. Aschenbrenner, R. Monetti, W. Bunk, F. Jamitzky, G. Morfill, Robert W. Stark and J. Schlegel, Biophys. J., 2012, 102, 360-368.

137. Q. Tu and C. Chang, Nanomedicine: NBM, 2012, 8, 545-558.

138. K. Meister, J. Niesel, U. Schatzschneider, N. Metzler-Nolte, D. A. Schmidt and M. Havenith, Angew. Chem. Int. Ed., 2010, 49, 3310-3312.

139. P. Hildebrandt, Angew. Chem. Int. Ed., 2010, 49, 4540-4541.

140. M. Fleischmann, P. J. Hendra and A. J. McQuillan, Chem. Phys. Lett., 1974, 26, 163-166.

141. D. L. Jeanmaire and R. P. Van Duyne, J. Electroanal. Chem., 1977, 84, 1-20.

142. B. Sharma, R. R. Frontiera, A. I. Henry, E. Ringe and R. P. Van Duyne, Mater. Today, 2012, 15, 16-25.

143. W. Xie and S. Schlucker, Phys. Chem. Chem. Phys., 2013, 15, 5329-5344.

144. K. Kneipp, H. Kneipp, I. Itzkan, R. R. Dasari and M. S. Feld, Chem. Rev., 1999, 99, 2957-2976.

145. K. Saha, S. S. Agasti, C. Kim, X. Li and V. M. Rotello, Chem. Rev., 2012, 112, 2739-2779.

146. A. Campion and P. Kambhampati, Chem. Soc. Rev., 1998, 27, 241-250.

147. P. L. Stiles, J. A. Dieringer, N. C. Shah and R. P. Van Duyne, Annu. Rev. Anal. Chem., 2008, 1, 601-626.

148. M. Moskovits, Phys. Chem. Chem. Phys., 2013, 15, 5301-5311.

149. S. Schlücker, Angew. Chem. Int. Ed., 2014, 53, 4756-4795.

150. Y. Wang, B. Yan and L. Chen, Chem. Rev., 2012, 113, 1391-1428.

151. S. Lee, H. Chon, M. Lee, J. Choo, S. Y. Shin, Y. H. Lee, I. J. Rhyu, S. W. Son and C. H. Oh, Biosens. Bioelectron., 2009, 24, 2260-2263. 
152. S. Abalde-Cela, P. Aldeanueva-Potel, C. Mateo-Mateo, L. Rodríguez-Lorenzo, R. A. Alvarez-Puebla and L. M. Liz-Marzán, J. R. Soc., Interface, 2010, 7, S435-S450.

153. K. K. Maiti, U. S. Dinish, A. Samanta, M. Vendrell, K.-S. Soh, S.-J. Park, M. Olivo and Y.-T. Chang, Nano Today, 2012, 7, 85-93.

154. A. Samanta, K. K. Maiti, K.-S. Soh, X. Liao, M. Vendrell, U. S. Dinish, S.-W. Yun, R. Bhuvaneswari, H. Kim, S. Rautela, J. Chung, M. Olivo and Y.-T. Chang, Angew. Chem. Int. Ed., 2011, 50, 6089-6092.

155. K. V. Kong, Z. Lam, W. D. Goh, W. K. Leong and M. Olivo, Angew. Chem. Int. Ed., 2012, 51, 9796-9799.

156. R. H. Webb, in Methods Enzymol., ed. P. M. Conn, Academic Press, San Diego, 1999, vol. 307, pp. 3-20.

157. F. Helmchen and W. Denk, Nat. Methods, 2005, 2, 932-940.

158. E. M. Frohman, J. G. Fujimoto, T. C. Frohman, P. A. Calabresi, G. Cutter and L. J. Balcer, Nat. Clin. Pract. Neurol., 2008, 4, 664-675.

159. X. Wang, Y. Pang, G. Ku, X. Xie, G. Stoica and L. V. Wang, Nat. Biotechnol., 2003, 21, 803-806.

160. C. Kim, C. Favazza and L. V. Wang, Chem. Rev., 2010, 110, 2756-2782.

161. V. Ntziachristos and D. Razansky, Chem. Rev., 2010, 110, 2783-2794.

162. L. V. Wang and H.-I. Wu, in Biomedical Optics: Principles and Imaging, John Wiley \& Sons, Inc., Hoboken, NJ, USA, 2009, pp. 283-321.

163. A. G. Bell, Am. J. Sci., 1880, 20, 305-324.

164. J. Xia, J. Yao and L. V. Wang, Prog. Electromagn. Res., 2014, 147, 1-22.

165. M. Xu and L. V. Wang, Rev. Sci. Instrum., 2006, 77, 041101.

166. H. F. Zhang, K. Maslov, G. Stoica and L. V. Wang, Nat. Biotechnol., 2006, 24, 848-851.

167. G. Ku, X. Wang, X. Xie, G. Stoica and L. V. Wang, Appl. Opt., 2005, 44, 770775.

168. A. De La Zerda, C. Zavaleta, S. Keren, S. Vaithilingam, S. Bodapati, Z. Liu, J. Levi, B. R. Smith, T.-J. Ma, O. Oralkan, Z. Cheng, X. Chen, H. Dai, B. T. Khuri-Yakub and S. S. Gambhir, Nat. Nanotechnol. , 2008, 3, 557-562.

169. X. Yang, S. E. Skrabalak, Z.-Y. Li, Y. Xia and L. V. Wang, Nano Lett., 2007, 7, 3798-3802. 
170. M. Eghtedari, A. Oraevsky, J. A. Copland, N. A. Kotov, A. Conjusteau and M. Motamedi, Nano Lett., 2007, 7, 1914-1918.

171. D. Razansky, M. Distel, C. Vinegoni, R. Ma, N. Perrimon, R. W. Koster and V. Ntziachristos, Nat. Photonics, 2009, 3, 412-417.

172. T. Temma, S. Onoe, K. Kanazaki, M. Ono and H. Saji, J. Biomed. Opt., 2014, 19, 090501-090501.

173. K. V. Kong, L.-D. Liao, Z. Lam, N. V. Thakor, W. K. Leong and M. Olivo, Chem. Commun., 2014, 50, 2601-2603. 


\section{Chapter 2: Surface-Enhanced Raman Spectroscopy (SERS) of Metal Carbonyls on Gold Nanospheres}

Surface-enhanced Raman spectroscopy (SERS) has garnered much attention as an important and robust optical technique for bio-sensing. Most SERS applications involve the sensing of various molecules or analytes that are close to the surface of the plasmonic metallic substrate, giving valuable chemical information for qualitative or quantitative analysis. This technique has been utilized to develop optical probes, commonly known as "SERS tags", which are essentially plasmonic nanoparticles with Raman reporters attached onto the surface. Such SERS tags, akin to fluorescent probes, can be functionalized and applied for sensitive SERS-based sensing and imaging. ${ }^{1-5}$ The components of SERS tags, the plasmonic nanoparticle and the Raman reporter, are two important considerations for the development of effective and functional tags. Over the past few decades, most efforts have been dedicated to the design of novel metallic nanoparticles with high plasmonic activity for better SERS enhancement. ${ }^{2}$ Some examples include gold nanostars, ${ }^{6}$ gold nanoflowers, ${ }^{7}$ gold-silver bimetallic particles, ${ }^{8}$ and gold aggregates. ${ }^{9}$ On the other hand, the development of highly sensitive Raman reporters with unique spectral characteristics remains less explored. An example is the work by Olivo and Chang who reported the screening of an 80member tricarbocyanine library for the selection of lead compounds with strong signals for in vitro and in vivo SERS detection. ${ }^{10,11}$ The study and design of Raman reporters to allow for a library of compound with non-overlapping signals remain important as it will allow the use of multiple SERS tags for multiplex detection - the simultaneous detection of more than one target. ${ }^{11,12}$

The Raman reporters used thus far are all organic-based, with the exception of an organometallic-based reporter, a triosmium carbonyl cluster, conjugated onto gold nanoparticles for the SERS detection of cancer cells. ${ }^{13}$ The advantage of using a metal carbonyl as the Raman reporter lies in the CO vibrational stretch in the region of 2200$1800 \mathrm{~cm}^{-1}$, which is free from interference by other biomolecules, thereby offering a 
clear spectral window for sensing applications. This chapter details the investigation into the SERS effect of various metal carbonyls on gold nanospheres (AuNS), in an attempt to identify compounds with unique spectral characteristics that can potentially be used for multiplex applications.

\subsection{Metal carbonyl clusters as Raman reporters}

\subsubsection{Synthesis and design of metal carbonyl clusters}

The first example of metal carbonyl-gold nanoparticle conjugates involved the use of an electron-deficient triosmium carbonyl cluster, $\mathrm{Os}_{3}(\mathrm{CO})_{10}(\mu-\mathrm{H})_{2}$, as the Raman reporter. ${ }^{14}$ The interaction between the gold nanoparticle with the cluster was deduced to be direct bonding between the gold atoms and the three-center two-electron Os $(\mu$ $\mathrm{H}) \mathrm{Os}$ moiety. In order to have tunability of the $\mathrm{CO}$ vibrational frequencies and yet have strong binding to the gold surface, however, a general design comprising a metal carbonyl cluster, a linker and a sulfur-containing functionality was envisaged (figure 2.1).

\section{a}
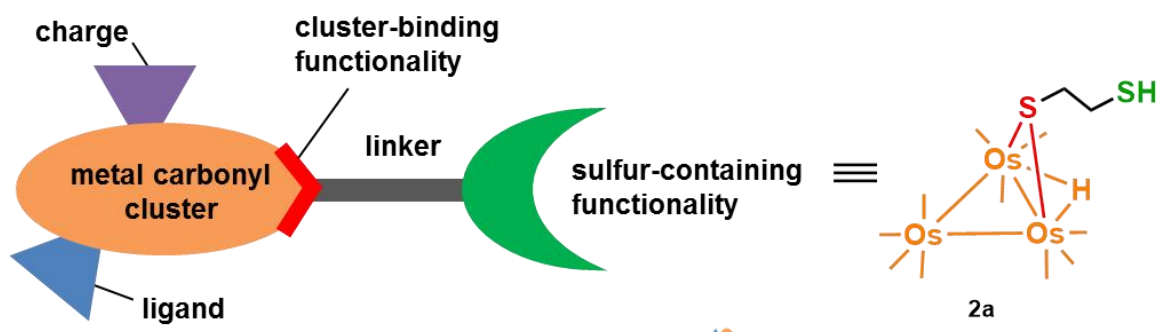

b

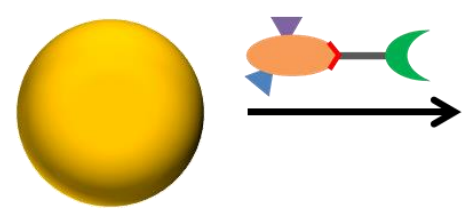

$60 \mathrm{~nm}$ AuNS

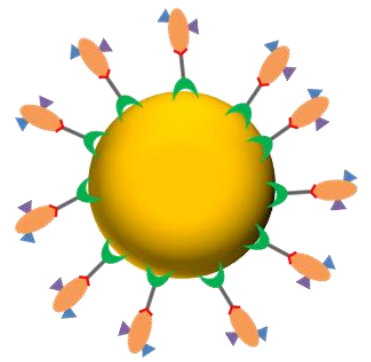

Figure 2.1. a) Components of a metal carbonyl cluster as Raman reporters. b) Binding of the metal carbonyl clusters onto AuNS via the sulfur groups.

The cluster-binding functionality, ligand and charge form an integral part of the cluster and have direct effect in tuning the $\mathrm{CO}$ vibrational frequencies. The linker modulates the distance between the cluster and the sulfur functionality, thereby affecting the AuNS-cluster distance. Lastly, the sulfur functionality is needed to anchor the metal carbonyl clusters onto the AuNS surface, as sulfur compounds can bind covalently with gold nanoparticles via an Au-S bond. This is not limited to the 
more commonly used thiols, but can include disulfides and thioethers as well. ${ }^{10,15-19}$ In this study, $60 \mathrm{~nm}$ AuNS was chosen as the substrate for investigating the SERS activity of the metal carbonyls as it has better biocompatibility, a narrow size distribution, and is less prone to oxidation compared to the silver counterpart. The size of around $60 \mathrm{~nm}$ is also regarded as a good size for both in vitro applications and for SERS enhancement. ${ }^{2,10,20-22}$

A simple model that fits the requirements is $\mathbf{2 a}$. It is essentially a thiolato-bridged $(\mu-\mathrm{S})$ triosmium carbonyl cluster, with a two-carbon linker and a free thiol functionality on the other end. Given the high reactivity of the precursor, cluster $\mathbf{1}$, towards thiols to form stable $\mu$-S clusters, 2a was synthesized from the reaction of $\mathbf{1}$ with 1,2-ethanedithiol according to a reported method (scheme 2.1$){ }^{23-25}$

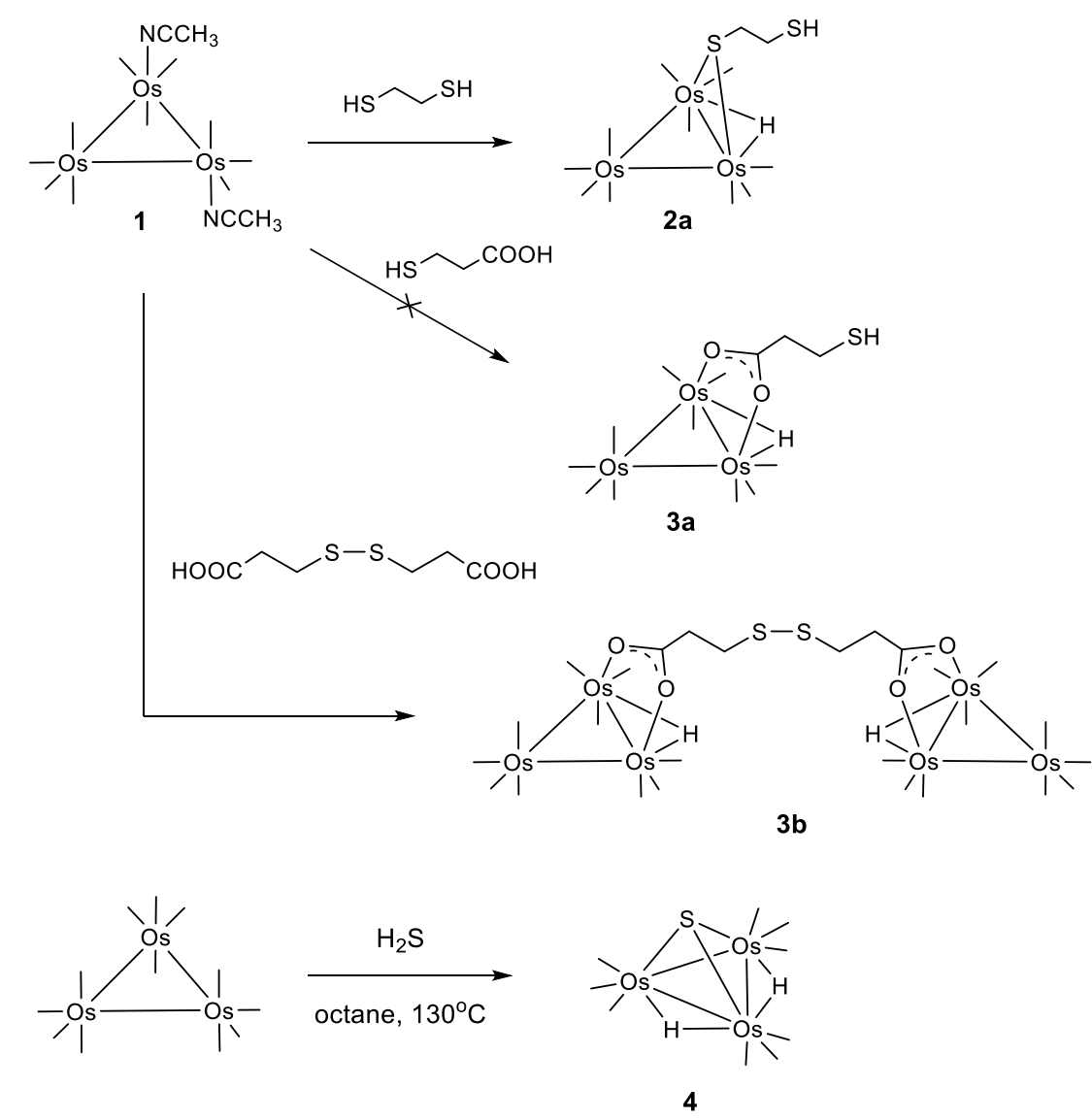

Scheme 2.1. Synthesis of clusters $2 a, 3 b$ and 4 .

The cluster-binding $\mu-\mathrm{S}$ functionality could also be replaced by a $\mu, \kappa^{2}-\mathrm{O}, \mathrm{O}$, functionality, viz., cluster 3a, which can be synthesized from the same precursor 1; this would modulate the $\mathrm{CO}$ vibrational frequencies. However, the higher reactivity of thiol 
over carboxylic acid with triosmium clusters made it difficult to obtain 3a from the reaction of 1 with 3-mercaptopropionic acid as the soft $\mathrm{SH}$ group reacted more readily and strongly with the soft Lewis acidic metal center than the hard $\mathrm{COOH}$ group. ${ }^{26,27}$ Instead, cluster 1 was reacted with 3,3'-dithiodipropionic acid to give the carboxylatebridged cluster $\mathbf{3 b}$ as the disulfide was much less reactive than free thiol. Cluster $\mathbf{3 b}$ is expected to react with AuNS via cleavage of the S-S bond to form similar species as that of 3a. ${ }^{17}$ Another cluster which was synthesized was the sulfur-capped $\left(\mu_{3}-\mathrm{S}\right)$ cluster $4 .^{28}$ The sulfur atom in $\mathbf{4}$ could act as the cluster-binding functionality, linking group, as well as the anchor onto the AuNS.

The IR spectra of $\mathbf{2 a}, \mathbf{3 b}$ and $\mathbf{4}$ showed distinct $\mathrm{CO}$ vibrational frequencies (figure 2.2); their symmetric stretch are at 2113,2109 and $2121 \mathrm{~cm}^{-1}$, respectively. ${ }^{29-31}$ The intensity of this stretch is usually the weakest in the IR spectrum but shows up strongly in the Raman spectrum.

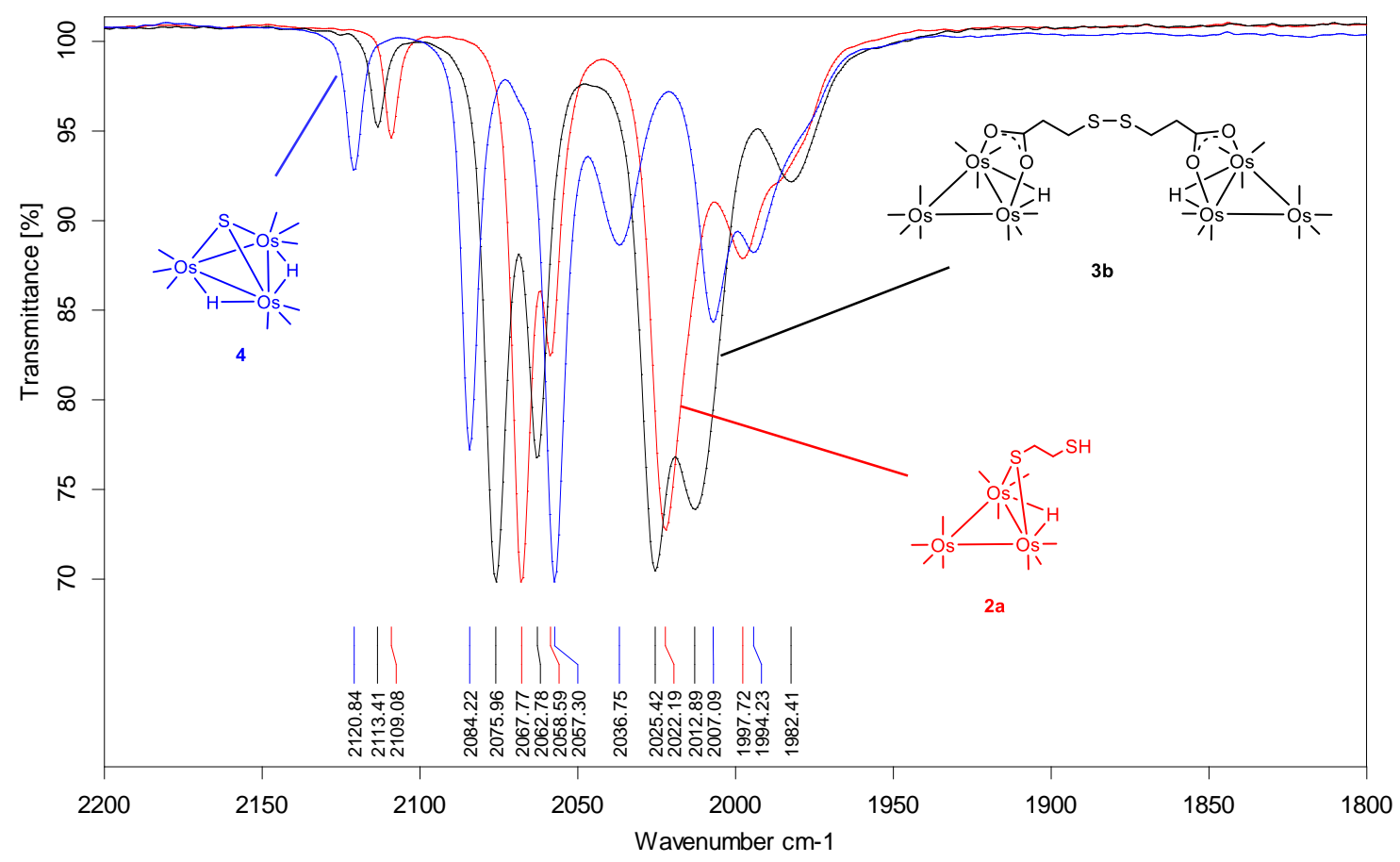

Figure 2.2. Overlay of IR spectra ( $v C O)$ of cluster $\mathbf{2 a}, \mathbf{3 b}$ and $\mathbf{4}$ in DCM.

The CO vibrational frequency can also be tuned through modification of the ligand sphere or by introducing a charge on the cluster. As mentioned in section 1.5.1, an electron-donating phosphine can increase the electron density on the metal center, thereby increasing $\pi$-back donation to the $\pi^{*}$ orbitals of $\mathrm{C}-\mathrm{O}$, and shifting the $\mathrm{CO}$ vibrations to lower frequencies. On the other hand, the $\mathrm{CO}$ vibrations for a cationic 
cluster are shifted to higher frequencies because of the decrease in $\pi$-back donation to the $\pi^{*}$ orbitals of $\mathrm{C}-\mathrm{O}$.

Tuning the $\mathrm{CO}$ vibrational frequency through these two modifications was made with cluster $\mathbf{6}$, which was obtained from the reaction of $\mathbf{5}$ with $\mathbf{1}$, followed by cysteamine (scheme 2.2).

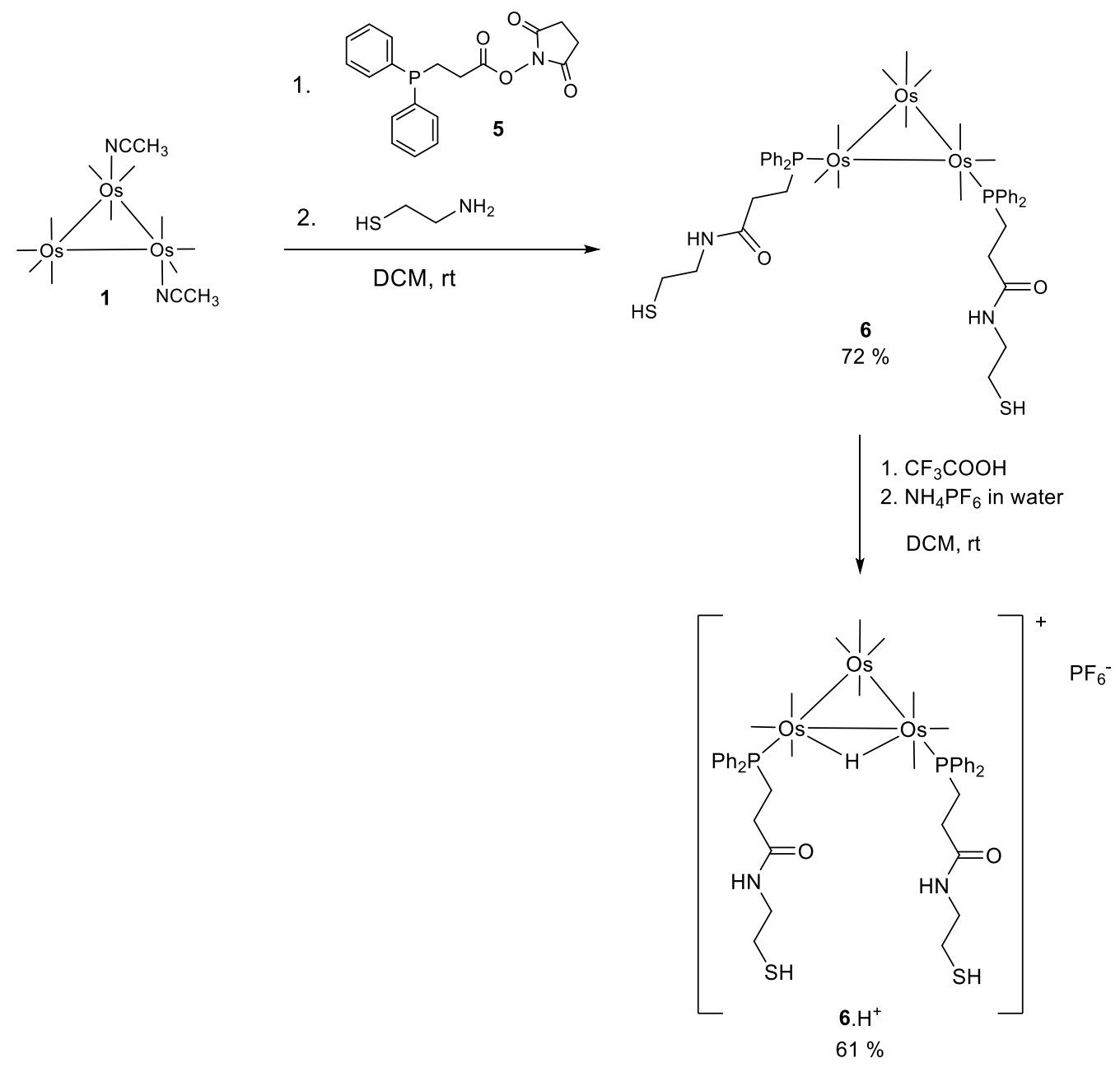

Scheme 2.2. Synthesis of clusters 6 and $6 . \mathrm{H}^{+}$.

The phosphine 5, with an N-hydroxy succinide (NHS) ester group, was synthesized according to a reported method. ${ }^{32}$ The orthogonality of $\mathbf{5}$ allowed the phosphine to coordinate to the osmium, while the N-hydroxy succinimide (NHS) ester was selective towards the amine functionality of cysteamine. Cluster 6 has both di-phosphine substitution and a thiol functionality, with the phosphines acting as both the ligand and cluster-binding functionality. The cluster was purified and characterized by IR, HRMS, ${ }^{1} \mathrm{H},{ }^{31} \mathrm{P}\left\{{ }^{1} \mathrm{H}\right\}$ and ${ }^{1} \mathrm{H}\left\{{ }^{31} \mathrm{P}\right\}$ NMR spectroscopy, and a COSY experiment. The ${ }^{31} \mathrm{P}\left\{{ }^{1} \mathrm{H}\right\}$ NMR spectrum showed three signals which can be attributed to the presence of trans- 
trans and cis-trans isomers, a common observation for diphosphine-substituted triosmium carbonyl clusters. ${ }^{33}$

Protonation of 6 with $\mathrm{CF}_{3} \mathrm{COOH}$ afforded the cationic cluster $6 . \mathrm{H}^{+}$, which could be isolated in moderate yield after anion exchange using $\mathrm{NH}_{4} \mathrm{PF}_{6}$. Successful protonation of 6 is evident from the colour change from orange to yellow. Cluster $\mathbf{6} \cdot \mathrm{H}^{+}$was characterized fully by IR, ${ }^{1} \mathrm{H},{ }^{1} \mathrm{H}\left\{{ }^{31} \mathrm{P}\right\},{ }^{31} \mathrm{P}\left\{{ }^{1} \mathrm{H}\right\}$ NMR spectroscopy, and a COSY experiment. As expected, the $\mathrm{CO}$ vibrations for $\mathbf{6} . \mathrm{H}^{+}$were shifted to higher frequency with respect to those of $\mathbf{6}$ (figure 2.3).

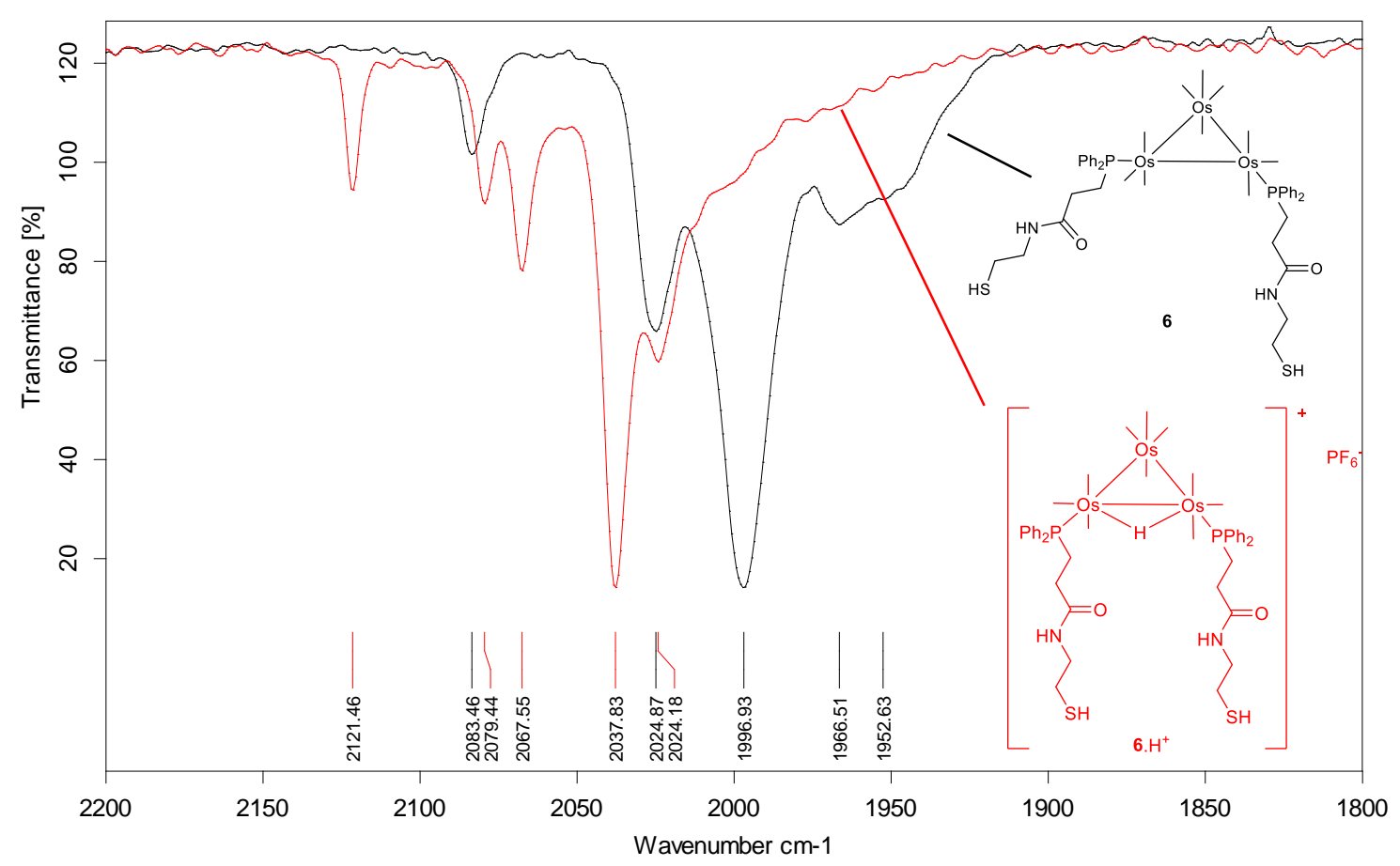

Figure 2.3. Overlay of IR spectra ( $v C O)$ of cluster 6 and $\mathbf{6} \cdot \mathrm{H}^{+}$in DCM.

The effect of changing the metal centres was also investigated with the rhenium clusters $\mathbf{7 a}$ and $\mathbf{7 b}$, which contain a free $\mathrm{SH}$ functionality; they were synthesized following a reported method (scheme 2.3). ${ }^{34}$ 


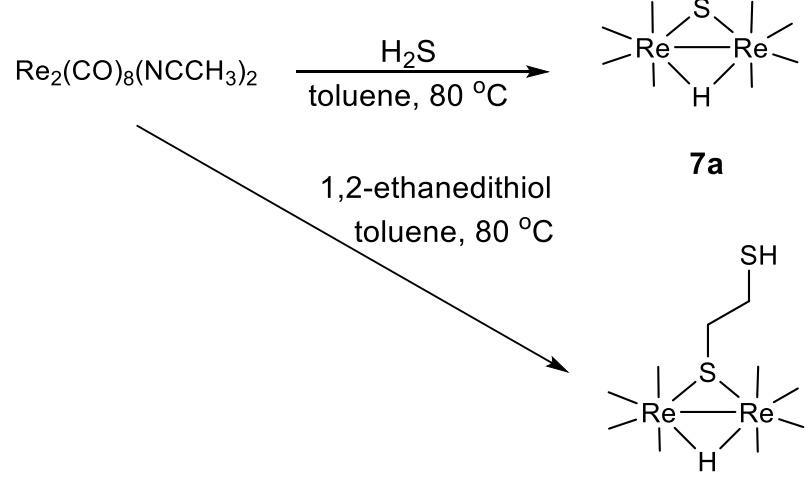

$7 b$

Scheme 2.3. Synthesis of clusters $7 \mathbf{a}$ and $7 \mathbf{b}$.

\subsubsection{SERS spectra of metal carbonyl clusters on AuNS}

The as-synthesized clusters were incubated with $60 \mathrm{~nm}$ AuNS, followed by centrifugation and re-dispersion in water. The SERS spectra for $\mathbf{2 a}, \mathbf{3 b}$ and $\mathbf{4}$ in the $1800-2200 \mathrm{~cm}^{-1}$ region are shown in figure 2.4 .

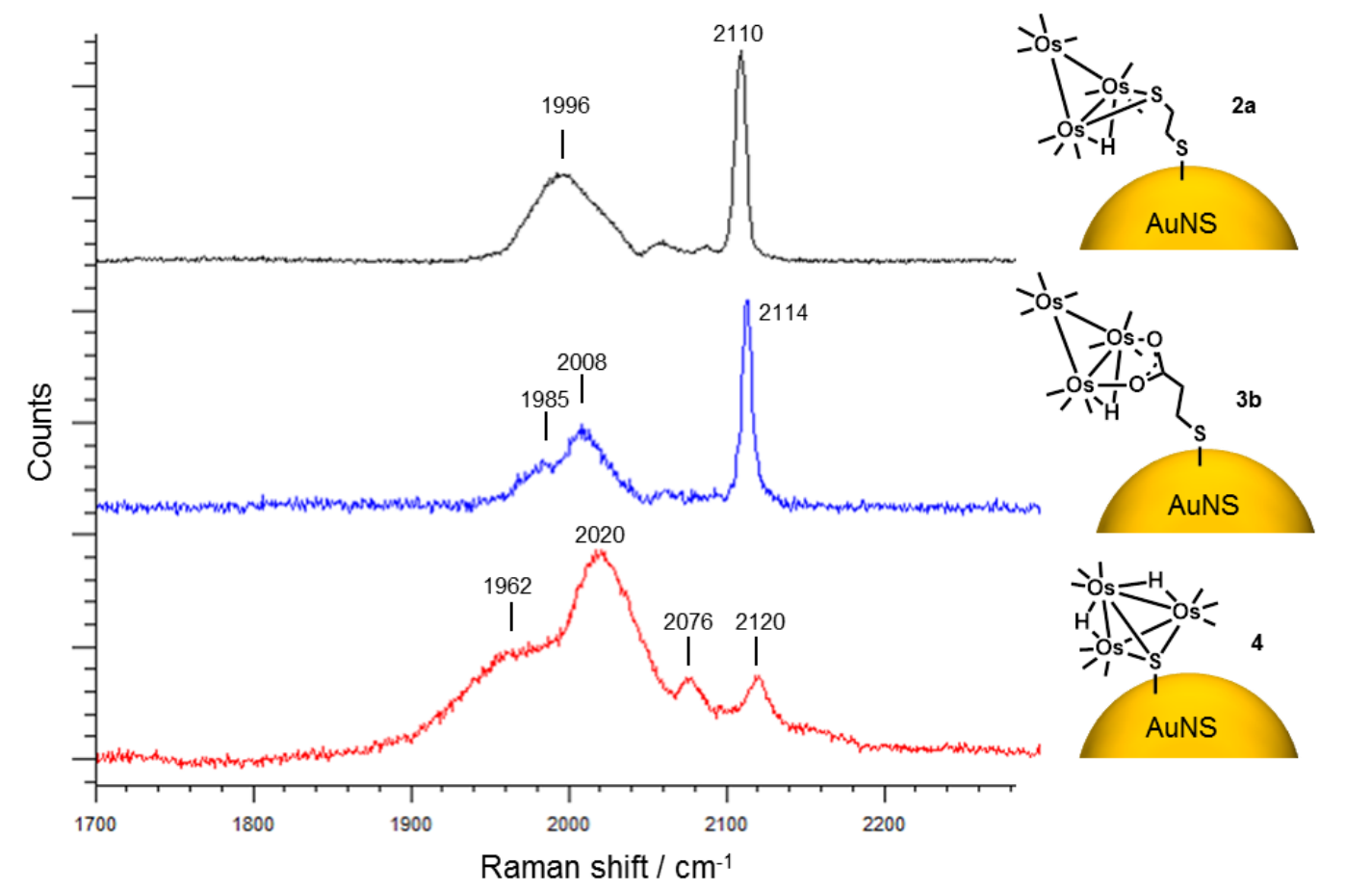

Figure 2.4. SERS spectra ( $v C O)$ of cluster $\mathbf{2 a}, \mathbf{3 b}$ and $\mathbf{4}$ incubated with AuNS.

The spectra for $\mathbf{2} \mathbf{a}$ and $\mathbf{3 b}$ were similar in the pattern, with a broad peak at 2000 $\mathrm{cm}^{-1}$ and a sharp peak at higher frequency at 2110 and $2114 \mathrm{~cm}^{-1}$ for $\mathbf{2 a}$ and $\mathbf{3 b}$, respectively, and the shift to higher wavenumber for $\mathbf{3 b}$ is due to the more electron withdrawing carboxylate group. These strong signals can therefore be useful for SERS 
detection. On the other hand, the SERS spectrum for 4 did not show a strong symmetric stretch (at $2121 \mathrm{~cm}^{-1}$ ) but showed broad bands from 1962 to $2076 \mathrm{~cm}^{-1}$. This could be due to the close proximity of the binding group (sulfur atom) to the osmium core, resulting in direct interaction of the gold atoms with the triosmium core; gold compounds are reported to have a high affinity for the osmium core. ${ }^{35-39}$ The simple SERS spectra of conjugates $\mathbf{2 a}$ and $\mathbf{3 b}$ appear to be derived from a single structural moiety of the clusters near the AuNS surface, while the broader and more complex spectra of conjugate 4 could be due to the different bonding interactions between the cluster and AuNS.

The attempts at conjugating $6 . \mathrm{H}^{+}$to AuNS led to irreversible aggregation; the AuNS changed colour from red to purple upon the addition of $6 . \mathrm{H}^{+}$. The cationic nature of $6 . \mathrm{H}^{+}$probably disrupted the mutual repulsion of the negatively charged nanoparticles. Nevertheless, the SERS spectrum of the conjugate showed signals in the $1800-2200 \mathrm{~cm}^{-1}$ region; the symmetric stretch for the conjugates of $\mathbf{6}$ and $\mathbf{6} \cdot \mathrm{H}^{+}$shows up strongly at 2082 and $2121 \mathrm{~cm}^{-1}$, respectively, and do not overlap with that for $\mathbf{2 a}$ (figure 2.5).

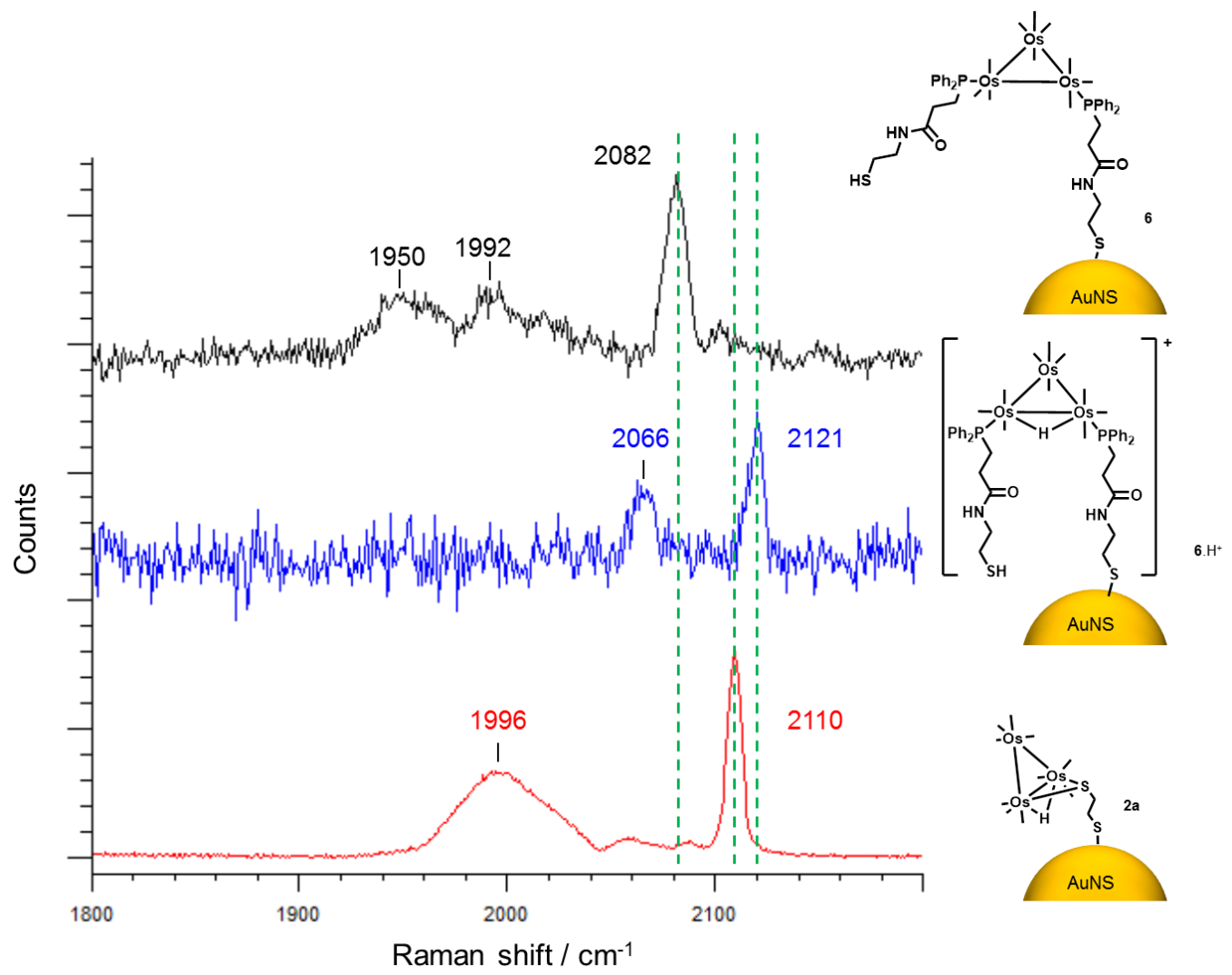

Figure 2.5. SERS spectra (vCO) of cluster $\mathbf{2 a}, \mathbf{6}$ and $\mathbf{6} \cdot \mathrm{H}^{+}$incubated with AuNS. 
The AuNS conjugates of cluster $\mathbf{7 a}$ and $\mathbf{7 b}$ showed drastically different SERS spectra, even though the clusters had similar $\mathrm{CO}$ vibrational frequencies in their IR spectra (figure 2.6). The SERS spectrum for the AuNS conjugate of 7a showed broad bands at 1939 and $2009 \mathrm{~cm}^{-1}$, with weak signals at higher wavenumber. On the other hand that for the AuNS conjugate of $\mathbf{7 b}$ showed a distinct, strong peak at $2114 \mathrm{~cm}^{-1}$ which corresponded to the symmetric stretch. Taken together with the SERS spectra of the conjugates of $\mathbf{2 a}$ and $\mathbf{4}$, it is clear that the linker length does have an effect on the SERS spectrum. The linker modulates the distance and hence the interaction between the gold surface and the cluster core, producing SERS spectra with different features.

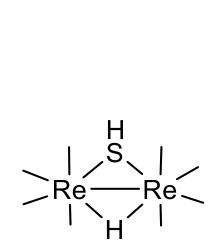

$7 a$

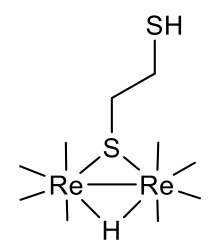

$7 \mathrm{~b}$

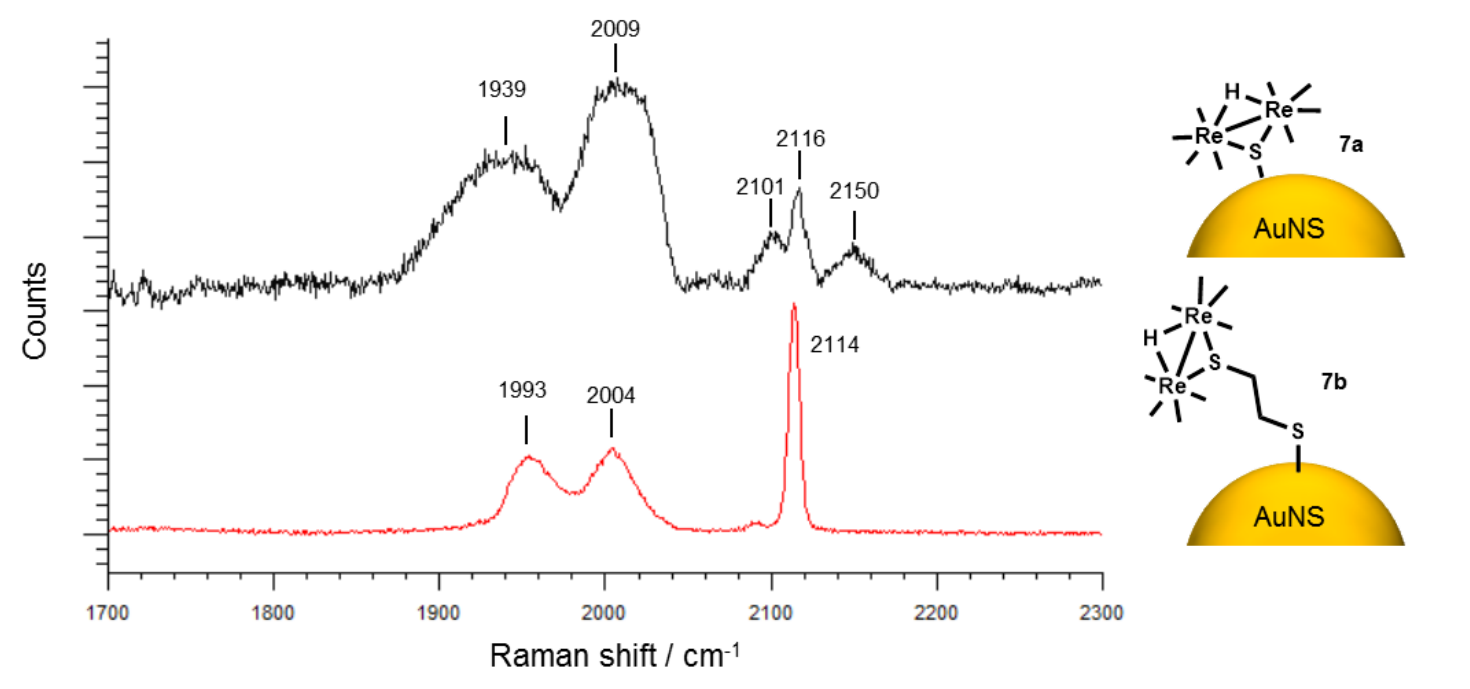

Figure 2.6. SERS spectra ( $(\mathrm{CO})$ of clusters $\mathbf{7 a}$ and $\mathbf{7 b}$ incubated with AuNS.

A comparison of the $\mathrm{CO}$ stretching frequencies for the clusters with the SERS frequencies of their AuNS conjugates is given in table 2.1. A few points can be noted:

1) The symmetric stretch shows up sharply in the SERS spectra.

2) There is good correlation between the symmetric stretch in the IR spectra of the clusters with the SERS spectra of the AuNS conjugates, with minimal frequency shifts. 
3) Changing the ligands or charge is effective for tuning the $\mathrm{CO}$ vibrational frequency. Changing to a different cluster binding functionality or transition metal is less effective.

4) The length of the linker has a direct effect on the SERS spectra; too short a linker (one atom between the cluster and AuNS) leads to broad signals while a longer linker gives a more defined spectrum with a sharp symmetric stretch.

Table 2.1. IR frequencies of $\mathbf{2 a}$ to $\mathbf{7 b}$ and the respective SERS frequencies of the AuNS conjugates.

\begin{tabular}{|c|c|c|}
\hline Compounds & ${ }^{\mathrm{a}} \mathrm{IR}\left(\mathrm{vCO}, \mathrm{cm}^{-1}\right)$ & SERS $\left(v C O, \mathrm{~cm}^{-1}\right)$ \\
\hline $2 \mathbf{2 a}$ & $\begin{array}{l}\text { 2109(w), 2068(s), 2059(m), 2022(s), } \\
\text { 1998(m), 1986(m, sh) }\end{array}$ & 2110(s), 1996(m, br) \\
\hline $\mathbf{3 b}$ & $\begin{array}{l}\text { 2113(w), 2076(s), 2063(m), 2025(s), } \\
\text { 2013(s), 1982(w) }\end{array}$ & 2114(s), 2008(m, br) 1985(m, sh) \\
\hline 4 & $\begin{array}{l}\text { 2121(w), 2084(s), 2057(s), 2037(m), } \\
\text { 2007(m), 1994(m) }\end{array}$ & $\begin{array}{l}2120(w), 2076(w), 2020(s, b r) \\
1962(m, b r)\end{array}$ \\
\hline 6 & 2084(w), 2025(s), 1997(vs), 1969(m, br) & 2082(s), 1992(m, br) 1950(m, br) \\
\hline 6.H+ & $\begin{array}{l}\text { 2121(w), 2080(w), 2067(m), 2038(vs), } \\
\text { 2025(m) }\end{array}$ & 2121(s), 2066(m) \\
\hline $7 \mathbf{a}$ & $\begin{array}{l}\text { 2117(w), 2093(m), 2019(s), 1999(m), } \\
1965(\mathrm{~m})\end{array}$ & $\begin{array}{l}2150(\mathrm{w}, \mathrm{br}), 2116(\mathrm{~m}), 2101(\mathrm{w}), \\
2009(\mathrm{~s}, \mathrm{br}), 1939(\mathrm{~m}, \mathrm{br})\end{array}$ \\
\hline $7 b$ & $\begin{array}{l}\text { 2115(w), 2091(m), 2017(vs), 1999(s), } \\
\text { 1964(s) }\end{array}$ & 2114(s), 2004(m, br), 1993(m, br) \\
\hline
\end{tabular}

${ }^{\mathrm{a}}$ In DCM.

\subsection{Half-sandwich metal carbonyl complexes as Raman reporters}

In an attempt to expand the scope of metal carbonyl-based Raman reporters, halfsandwich carbonyl complexes of the group 6 and 8 metals were investigated. Halfsandwich or piano-stool metal carbonyl complexes are organometallic complexes with a cyclic polyhapto ligand bound to an metal center with CO ligands. ${ }^{40}$ Arene and cyclopentadienyl groups often serve as reliable spectator ligands in forming $\eta^{6}$-arene and $\eta^{5}$-cyclopentadienyl $(\mathrm{Cp})$ complexes, respectively. In comparison to metal carbonyl clusters, mononuclear metal carbonyl complexes have a number of advantages. Firstly, the number of $\mathrm{CO}$ vibrational bands is reduced, giving a simpler 
spectum; this reduces the possibility of overlapping bands when more than one tag is used. Secondly, the synthesis and chemistry of half-sandwich complexes are very well-established and can provide many synthetic routes and opportunities for derivatization. ${ }^{41-44}$ Lastly, the use of less metal can reduce the cost and complexity of the system.

Similar to the metal carbonyl clusters, the $\mathrm{CO}$ vibrations of the half-sandwich metal carbonyls were tuned through the use of different transition metals and ligand set. To allow the anchorage of the complexes onto AuNS, complexes with a good-leaving group (iodide) were used; it is envisaged that the electon-rich gold metal atoms can react with the metal center via nuclephilic substitution of the iodide. The halfsandwich complexes 8-13 were synthesized according to reported methods (figure 2.7), with the exception of $\mathbf{1 1 a}$ which was obtained commercially. ${ }^{45-51}$

a

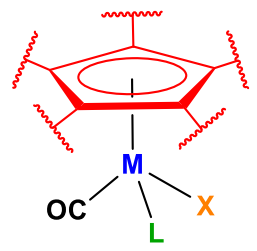

$\mathbf{M}=$ metal

$\mathrm{X}=$ good leaving group

$\mathrm{L}=$ ligand

b
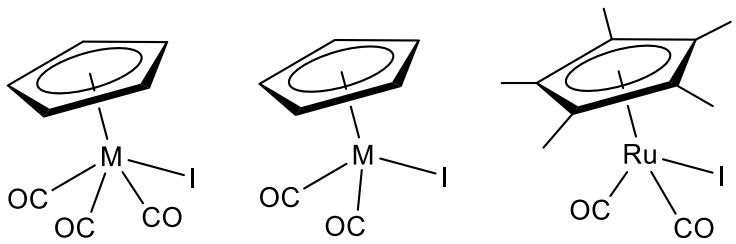

8a: $\mathrm{M}=\mathrm{Cr}$

9a: $M=M o$

10a: $M=W$

11a: $M=F e$

12a: $M=R u$

13a: $M=$ Os

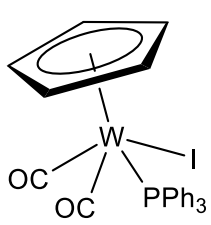

$9 b$

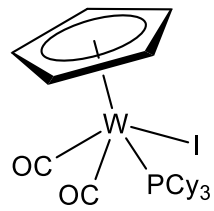

9c

Figure 2.7. a) The $\mathrm{CO}$ vibrational frequency of a half-sandwich metal carbonyl complex can be tuned via the $\mathrm{Cp}$ ring, metal center and the ligands. b) Molecular structures of halfsandwich metal carbonyl complexes used in the study.

The complexes were conjugated to AuNS by incubation, and they generally showed good CO stretching vibrations in the $1800-2200 \mathrm{~cm}^{-1}$ region (figure 2.8). Attempts at conjugating the chromium (8a) and iron (11a) analogues were futile, however; no Raman signal in the 1800-2200 $\mathrm{cm}^{-1}$ was observed, and the AuNS showed signs of irreversible aggregation. Presumably, the first row transition metal complexes were not sufficiently stable for such conjugation. A comparison of the SERS spectra of the conjugates of 9a-10a and 12a-13a, which differ in their metal 
center, show that the two peaks for all the four complexes fall within a narrow range (1943 to $2046 \mathrm{~cm}^{-1}$ ). This would pose problems when the conjugates are utilized for multiplex detection and quantification. On the other hand, the SERS spectra of the conjugates of 12'a, $\mathbf{1 0 b}$ and $\mathbf{1 0 c}$ are shifted to lower frequency compared to those of 12a and 10a, respectively. This is consistent with the presence of more electrondonating ligands in the former. The SERS spectra for all conjugates showed similar pattern as their respective IR spectra, indicating that the geometry of the complexes on the AuNS remained relatively the same as the free complexes. 

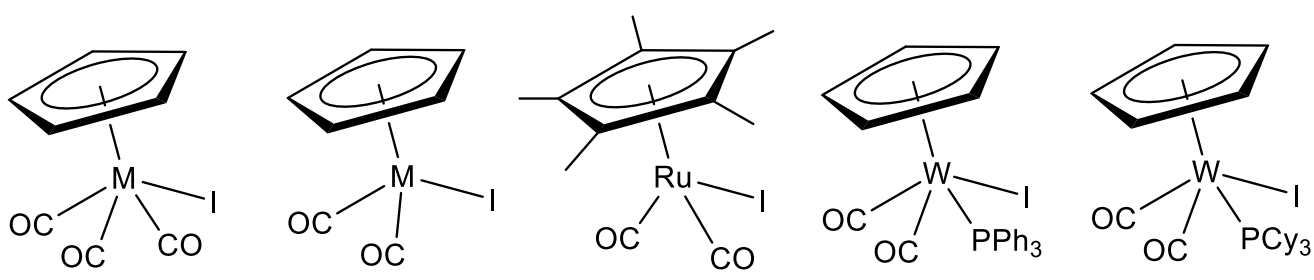

8a: $M=C r$

11a: $M=F e$

12 'a

10b

10c

9a: $M=M o$

12a: $M=R u$

10a: $M=W$

13a: $M=$ Os

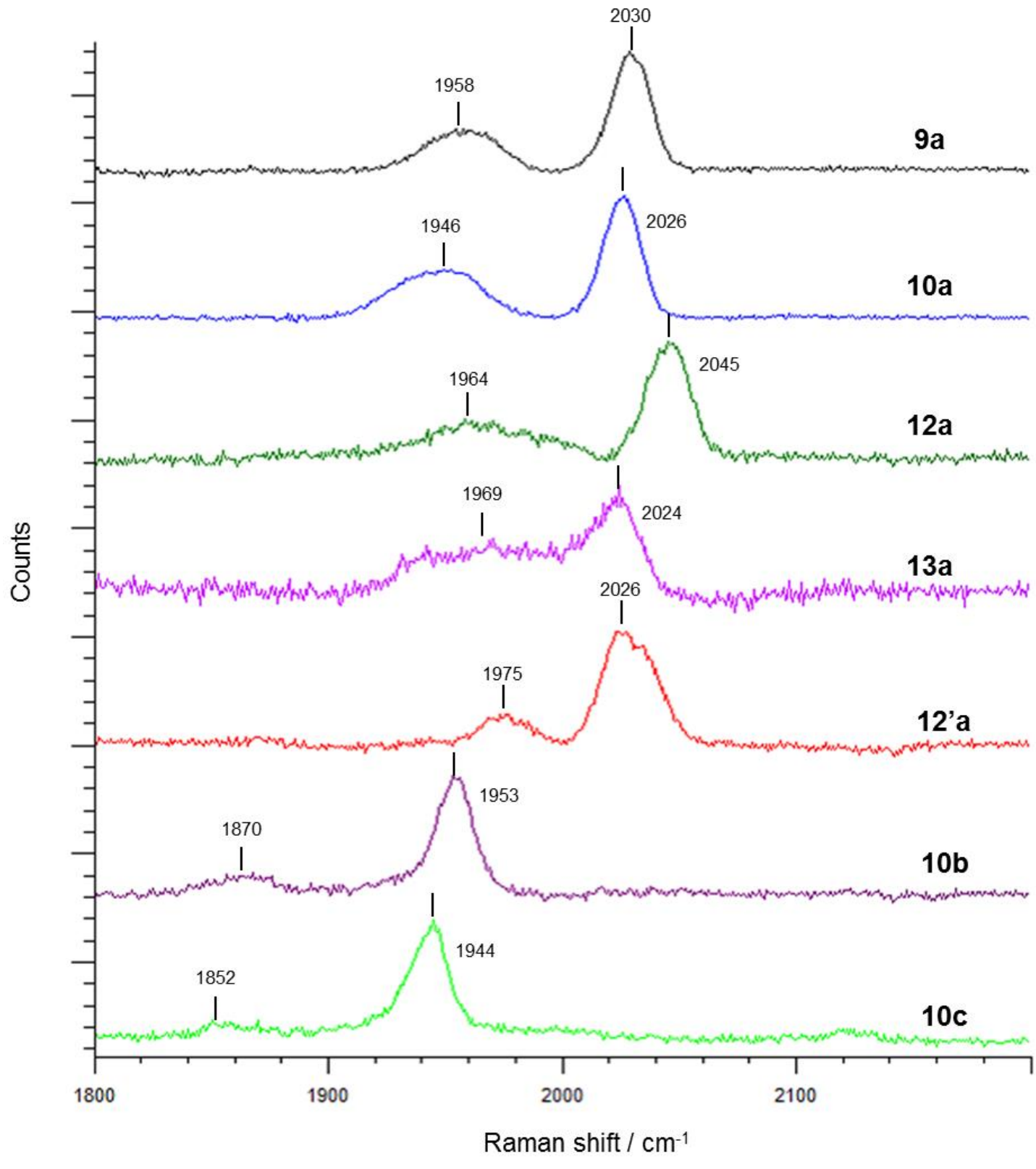

Figure 2.8. SERS spectra ( $v C O)$ of 9a-13a incubated with AuNS. 
Table 2.2. IR frequencies of 9a to 13a and the respective SERS frequencies of the AuNS conjugates.

\begin{tabular}{|c|c|c|}
\hline Compounds & ${ }^{\mathrm{a}} \mathbf{I R}\left(\mathbf{v C O}, \mathbf{c m}^{-\mathbf{1}}\right)$ & SERS $\left(\mathbf{} \mathbf{C O}, \mathbf{~ c m}^{-\mathbf{1}}\right)$ \\
\hline $\mathbf{9 a}$ & $2043(\mathrm{~m}), 1967(\mathrm{~s}, \mathrm{br})$ & $2030(\mathrm{~s}), 1958(\mathrm{~m}, \mathrm{br})$ \\
\hline $\mathbf{1 0 a}$ & $2037(\mathrm{~m}), 1952(\mathrm{~s}, \mathrm{br})$ & $2026(\mathrm{~s}), 1946(\mathrm{~m}, \mathrm{br})$ \\
\hline $\mathbf{1 2 a}$ & $2049(\mathrm{~s}), 1999(\mathrm{~s})$ & $2045(\mathrm{~s}), 1964(\mathrm{~m}, \mathrm{br})$ \\
\hline $\mathbf{1 3 a}$ & $2034(\mathrm{~m}), 1978(\mathrm{~s})$ & $2024(\mathrm{~s}), 1969(\mathrm{~m}, \mathrm{br})$ \\
\hline 12'a & $2028(\mathrm{~s}), 1977(\mathrm{~s})$ & $2026(\mathrm{~s}), 1975(\mathrm{~m})$ \\
\hline $\mathbf{1 0 b}$ & ${ }^{\mathrm{b}} 1955(\mathrm{~m}), 1876(\mathrm{~s})$ & $1953(\mathrm{~s}), 1870(\mathrm{~m})$ \\
\hline $\mathbf{1 0 c}$ & ${ }^{\mathrm{b}} 1942(\mathrm{~s}), 1859(\mathrm{~m})$ & $1944(\mathrm{~s}), 1852(\mathrm{w})$ \\
\hline
\end{tabular}

${ }^{\mathrm{a}}$ In DCM, unless otherwise stated. ${ }^{\mathrm{b}} \mathrm{In}$ THF.

Unlike the case in the cluster-based metal carbonyls which showed little or no frequency shifts in the symmetric stretch on conjugation to the AuNS (section 2.2), the SERS spectra $(\mathrm{vCO})$ of the AuNS conjugates of some of the half-sandwich metal carbonyl complexes showed significant shifts to lower frequency on conjugation (table 2.2). This is indicative of the increase in electron density at the metal center in the conjugates, which can be attributed to the Au-M interaction; such an interaction is lacking in the cluster conjugates where the cluster is seperated from the AuNS by a linker. This is consistent with the expectation that there is direct bonding interaction between the half-sandwich complexes and the AuNS, via elimination of the iodide (figure 2.9).

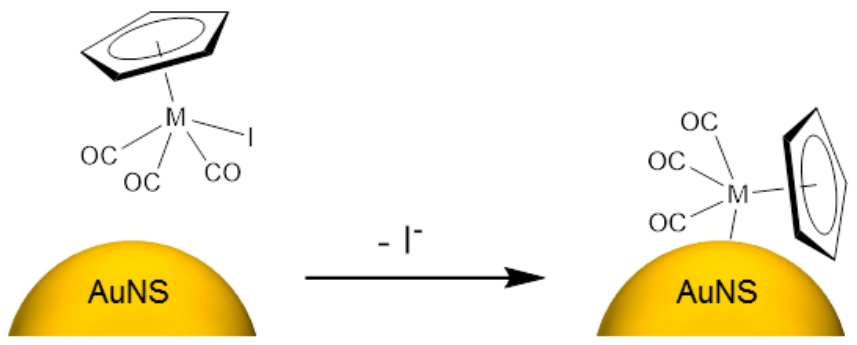

Figure 2.9. Proposed interaction of the half-sandwich metal complexes with AuNS.

A separate study using the bromide and chloride analogues of 10a incubated with AuNS did not show any CO vibrational frequency in the SERS spectra (appendix A2.27). This can probably be attributed to the lower reactivity of the bromide and chloride analogues as compared to $\mathbf{1 0 a}$, thus the conjugate did not form. 


\subsection{Effect of aggregation}

During the course of the above studies, it was observed that while the AuNS played a critical role in producing detectable Raman signals (no signal was observed in the absence of AuNS), obtaining consistency in the SERS intensity was a problem. We suspected that this was due to variation in the extent of aggregation across different samples. Gold colloids are known to be very temperamental, and in our case, can be difficult to control. Aggregation can be easily induced during the washing steps where high centrifugal force is applied. ${ }^{52}$ A highly ionic environment and low $\mathrm{pH}$ can also reduce the negative charges on the particle surface. This reduces mutual repulsion, resulting in aggregation. ${ }^{52-54}$

To understand the origin of the SERS enhancement in the metal carbonyl-AuNS conjugates, the AuNS conjugate of cluster 2a was prepared and subjected to centrifugation at low speed $(1200 \mathrm{~g}$ for $10 \mathrm{~min})$ to minimize aggregation. Aliquots of the conjugates were scanned under the Raman microscope, and weak signals were detected (figure 2.10). The conjugates were then topped up with water and spun down at a much higher speed (10 000g for $2 \mathrm{~min})$. Aggregation was observed, with some irreversible precipitation. Aliquots of these were scanned and, this time, stronger signals were observed.

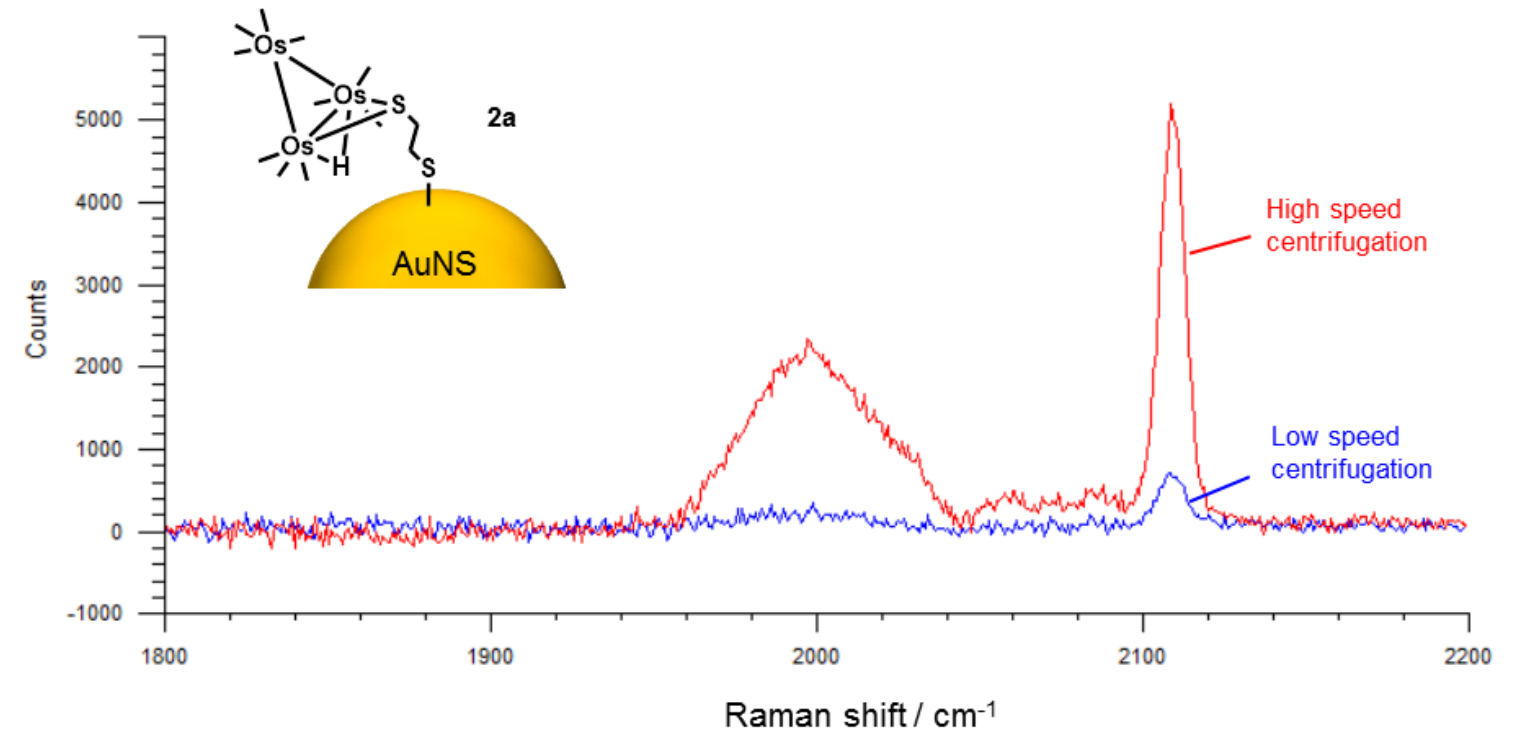

Figure 2.10. SERS spectra ( $\mathrm{vCO}$ ) of 2a incubated with AuNS with centrifugation at low and high speed respectively. 
In another study, the AuNS conjugates of 8a-11a, after centrifugation and redispersion in water, were characterized by UV-vis spectroscopy to assess the state of aggregation of the conjugates. The conjugates showed similar optical spectra as that for the non-conjugated $60 \mathrm{~nm}$ gold nanoparticles, except for a broad shoulder above $600 \mathrm{~nm}$, which is indicative of aggregation (figure 2.11a). ${ }^{53,55}$ Transmission electron microscopy (TEM) images of the conjugates showed spherical Au particles in contact with neighboring particles (figure 2.11b). However, this could be due to aggregation during sample preparation on the TEM grid and under the high vacuum conditions in the TEM chamber. Hence, TEM characterization is not conclusive for evaluation of the state of aggregation.

Other workers have reported that SERS enhancement is particularly strong in the gaps between plasmon-coupled metal nanoparticles, commonly referred to as "hotspots". ${ }^{56,57}$ A recent study has shown that monomers of Ag and AuNS do not show SERS enhancement and suggested that the observed SERS signals reported in many earlier work could have originated from aggregation of the colloids; the close interparticle distance in those aggregates can generate hot-spots for SERS enhancement. ${ }^{52}$ That study clarified the misrepresentation of Ag and AuNS as highly SERS-active substrates, and was well-supported by theoretical simulations. ${ }^{52,} 58$ In another work, Chen and co-workers described the SERS scattering in the hot-spots of colloidal nanoparticles, and reported that dimers and trimers gave 16 and 87 times enhancements, respectively, in comparison to the intensity from monomers. ${ }^{53}$ Hence, a small amount of aggregation in a sample can effectively generate a strong SERS signal, but often erroneously regarded to be from the monomers. Given the low Raman crosssection of the CO ligand and the low SERS-activity of AuNS, it is likely that the observed SERS spectra for the various metal carbonyls resulted from aggregation of the colloid during sample preparation, and not from the AuNS monomer. 

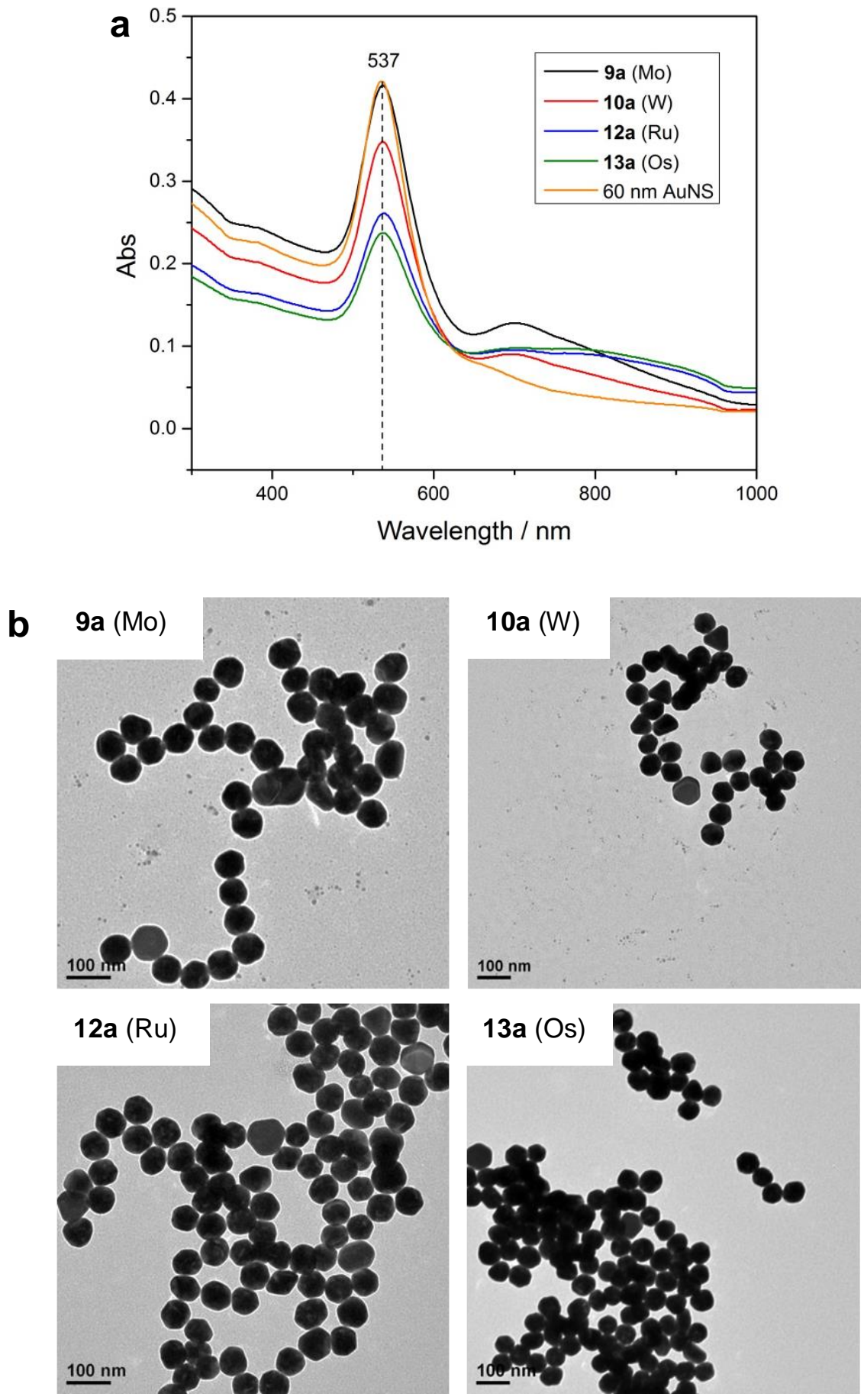

Figure 2.11. a) Optical absorption spectra and b) TEM images of 9a-10a and 12a-13a incubated with AuNS. 


\subsection{Conclusion}

A qualitative survey of two classes of metal carbonyls, namely, metal carbonyl clusters and half-sandwich metal carbonyl complexes, as Raman reporters was carried out. The cluster design (cluster-linker-anchor) serves as a good model for the tuning of the CO vibrational frequency. Tunability over a larger frequency range was achieved with the use of ligands and charge on the cluster core, while variations in the binding functionality and the use of different transition metals were less effective. The linker, which modulates the distance, hence, the interaction between the cluster and AuNS, has little effect in tuning the $\mathrm{CO}$ frequency but played an important role in altering the SERS spectral feature. Lastly, the sulfur-containing anchor is important for strong interaction with AuNS.

The extension of the study to half-sandwich metal carbonyl complexes of group 6 and 8 metals gives the advantages of simpler spectra and easier modification with the more established chemistry. Similar to the clusters, tunability of the CO vibrational stretches can be obtained with the use of various metals and ligands ( $\mathrm{Cp}$ ring and phosphines). Direct interaction between the AuNS and the metal centers with the elimination of iodide as the leaving group was deduced to be the mode of interaction; this can do away with the need for sulfur-containing functionality for anchorage onto AuNS. However, the use of other halides as leaving group can have different result and first row transition metal complexes $(\mathrm{Cr}$ and $\mathrm{Fe}$ ) were found to be not sufficiently stable for such conjugation.

Lastly, the SERS enhancement was deduced to be from the aggregation of the AuNS during the sample preparation and not from the AuNS monomer. Rational design and tuning of the SERS signal on metal carbonyls are feasible and can potentially contribute to the current library of organic Raman reporters. Taken together, these findings serve as important considerations for design and use of metal carbonyls for SERS applications. 


\subsection{Experimental section}

\subsubsection{General methods}

All reactions and manipulations were carried out under an argon atmosphere using standard Schlenk techniques. Solvents that were used for reaction were distilled over the appropriate drying agents under argon before use. Purification of compounds was generally carried out by column chromatography on silica gel, or by preparative thinlayer chromatography (TLC) using $20 \mathrm{~cm}$ x $20 \mathrm{~cm}$ plates pre-coated with silica gel 60 $\mathrm{F}_{254}$ Infrared (IR) spectra were recorded on a Bruker Alpha FT-IR spectrometer. Solution spectra were recorded in DCM solution, unless otherwise stated, in a solution IR cell with $\mathrm{NaCl}$ windows and a path length of $0.1 \mathrm{~mm}$, at a resolution of $2 \mathrm{~cm}^{-1}$. For compounds in THF solution, a solution IR cell with $\mathrm{CaF}_{2}$ windows and a path length of $0.1 \mathrm{~mm}$ was used. Optical absorption spectra were recorded using a Hitachi U-2900 double-beam spectrophotometer using quartz cuvette with path length of $10 \mathrm{~mm} .{ }^{1} \mathrm{H}$, ${ }^{13} \mathrm{C}\left\{{ }^{1} \mathrm{H}\right\},{ }^{31} \mathrm{P}\left\{{ }^{1} \mathrm{H}\right\},{ }^{1} \mathrm{H}\left\{{ }^{31} \mathrm{P}\right\}$ and COSY NMR spectra were recorded on a JEOL ECA 400 or ECA 400SL (400 MHz) spectrometer, in $\mathrm{CDCl}_{3} \cdot{ }^{1} \mathrm{H},{ }^{13} \mathrm{C}\left\{{ }^{1} \mathrm{H}\right\}$ chemical shifts are referenced to the residual proton resonance of the solvent HRMS were recorded in ESI mode on a Waters UPLC-Q-Tof MS mass spectrometer. The Raman spectral measurements were carried out using a Renishaw InVia Raman (UK) microscope with a Peltier cooled CCD detector and an excitation wavelength at $785 \mathrm{~nm}$, where the laser beam was directed to the sample through a 50x objective lens, which was used to excite the sample and also to collect the return Raman signal. All Raman spectra were processed with the WiRE3.4 software. The laser power at the sample was measured to be $20.8 \mathrm{~mW}$ and the exposure time was set at $10 \mathrm{~s}$, throughout the measurements. Prior to each measurement, the instrument was calibrated against a silicon standard with a Raman peak centered at $520 \mathrm{~cm}^{-1}$.

Copper specimen grids (300 mesh) with formvar/carbon support film were purchased from Beijing XXBR Technology Co. TEM images were collected from a JEM-1400 (JEOL) Transmission Electron Microscopy operated at $100 \mathrm{kV}$. TEM grids were treated with oxygen plasma in a Harrick® plasma cleaner/sterilizer for 1 min to improve the surface hydrophilicity. A sample solution $(10 \mu \mathrm{L})$ was dropped onto the hydrophilic side of the TEM grid. Filter paper was used to wick off the excess solution on the TEM grid, which was then left to dry in air for $5 \mathrm{~min}$. 
Clusters $\mathbf{1},{ }^{59} \mathbf{2} \mathbf{a},{ }^{23} \mathbf{4},{ }^{28} \mathbf{7 a},{ }^{34}$ and phosphine $\mathbf{5},{ }^{32}$ were prepared according to reported procedures with slight modifications. Half-sandwich complexes $\mathbf{8 a - 1 0 a},{ }^{45-47} \mathbf{1 0 b}-\mathbf{c},{ }^{51}$ $\mathbf{1 2 a},{ }^{48} \mathbf{1 2} \mathbf{a}^{60}{ }^{60}$ and $\mathbf{1 3 a},{ }^{49}$ were also synthesized according to reported procedures with slight modifications. Gold nanospheres $(60 \mathrm{~nm})$ were purchased from BBI Solutions. $\mathrm{Os}_{3}(\mathrm{CO})_{12}$ was purchased from Oxkem Ltd, and all other reagents were purchased from other commercial sources and used as supplied without further purification.

\subsubsection{Synthesis of cluster $3 b$}

Cluster 1 (57 mg, $0.061 \mathrm{mmol}$ ) and 3,3'-dithiodipropionic acid (7.7 mg, $0.037 \mathrm{mmol}$ ) were dissolved in THF ( $8 \mathrm{~mL}$ ) and left to stir overnight at room temperature. Solvents and volatiles were removed in vacuo and the yellow residue was purified by TLC using DCM:hexane (1:1, v/v) as eluent to yield cluster $\mathbf{3 b}$.

Yield: $9 \mathrm{mg}(15 \%)$

$\mathrm{R}_{\mathrm{f}}=0.56$

IR (DCM, $\left.\mathrm{cm}^{-1}\right)$ : 2113(w), 2076(s), 2063(m), 2025(s), 2013(s), 1982(w).

${ }^{1} \mathrm{H}$ NMR (400 MHz, $\mathrm{CDCl}_{3}$ ): $\delta$ 2.65-2.58 (m, 8H, $\underline{\mathrm{C}}_{2} \mathrm{C}_{2}$ ), -10.46 (s, 2H, OsHOs).

${ }^{13} \mathrm{C}\left\{{ }^{1} \mathrm{H}\right\}$ NMR $\left(100.46 \mathrm{MHz}, \mathrm{CDCl}_{3}\right): \delta$ 189.50, 183.61, 181.67, 175.97, 174.40, $174.29,37.09,34.20$.

\subsubsection{Synthesis of cluster 6}

Cluster 1 (30 mg, $0.032 \mathrm{mmol})$ was dissolved in DCM (6 mL) and stirred under argon. Phosphine 5 (25 mg, $0.071 \mathrm{mmol}$ ) was added and the mixture left to stir for $30 \mathrm{~min}$. The solution turned from yellow to orange, and monitoring by IR spectroscopy showed the presence of the di-substituted phosphine cluster. Cysteamine ( $6 \mathrm{mg}, 0.071 \mathrm{mmol})$ was then added and the reaction mixture was left to stir for $2 \mathrm{~h}$. Solvents and volatiles were removed in vacuo and the residue was purified by flash column chromatography and TLC. However, it was noted that purification by flash column chromatography and silica TLC led to tailing and the excess phosphine could not be separated from the product. Hence, the residue was purified by reversed-phase TLC using ethyl acetate:hexane $(1: 1, \mathrm{v} / \mathrm{v})$ as eluant to yield the product as a yellow-orange solid.

Yield: $35 \mathrm{mg}(72 \%)$

$\mathrm{R}_{\mathrm{f}}=0.53$

IR (DCM, cm ${ }^{-1}$ ): 2084(w), 2025(s), 1997(vs), 1969(m, br). 
${ }^{1} \mathrm{H}$ NMR (400 MHz, $\left.\mathrm{CDCl}_{3}\right): \delta$ 7.48-7.42 (m, 20H, Ar), $5.75(\mathrm{br}, 2 \mathrm{H}, \mathrm{NH}), 3.33(\mathrm{dt}$, $4 \mathrm{H}, J=6.3,6.3, \mathrm{NHCH}_{2} \mathrm{CH}_{2} \mathrm{SH}$ ), 3.03 (br, 4H, $\mathrm{PCH}_{2} \mathrm{CH}_{2} \mathrm{CO}$ ), 2.58 (dt, 4H, $J=6.6$, 8.7, $\mathrm{NHCH}_{2} \underline{\mathrm{C}}_{2} \mathrm{SH}$ ), 2.12-2.06 (m, 4H, $\left.\mathrm{PC}_{2} \mathrm{CH}_{2} \mathrm{CO}\right), 1.30$ (t, $2 \mathrm{H}, J=8.7, \mathrm{~S} \underline{\mathrm{H}}$ ). ${ }^{31} \mathrm{P}\left\{{ }^{1} \mathrm{H}\right\} \mathrm{NMR}\left(160.26 \mathrm{MHz}, \mathrm{CDCl}_{3}\right): \delta-8.76,-10.45,-13.21$.

HRMS-ESI m/z calculated for $\mathrm{C}_{44} \mathrm{H}_{41} \mathrm{~N}_{2} \mathrm{O}_{12} \mathrm{P}_{2} \mathrm{~S}_{2}{ }^{186} \mathrm{Oss}^{190} \mathrm{Oss}^{192} \mathrm{Os}[\mathrm{M}+\mathrm{H}]^{+}$1483.0314, found 1483.0278 .

\subsubsection{Synthesis of cluster $6 . \mathrm{H}^{+}$}

Cluster 6 (40 mg, $0.027 \mathrm{mmol})$ was dissolved in DCM $(2 \mathrm{~mL})$, followed by the addition of $\mathrm{CF}_{3} \mathrm{COOH}(60 \mu \mathrm{L}, 0.78 \mathrm{mmol})$. The reaction turned from orange to yellow immediately and was left to stir for $15 \mathrm{~min}$. $\mathrm{NH}_{4} \mathrm{PF}_{6}(2 \mathrm{mg}, 0.21 \mathrm{mmol})$ dissolved in deionized water $(2 \mathrm{~mL})$ was added and the biphasic mixture was stirred vigorously for $2 \mathrm{~h}$. The organic layer was extracted, washed with deionized water $(3 \times 2 \mathrm{~mL})$ and dried over anhydrous $\mathrm{MgSO}_{4}$. Solvent was removed in vacuo and the yellow residue was purified by column chromatography, using MeOH:DCM (1:500 to 6:500, v/v) as eluent with gradual increase of eluent strength, to yield the product as a yellow solid.

Yield: $27 \mathrm{mg}(61 \%)$

IR (DCM, cm ${ }^{-1}$ ): 2121w, 2080w, 2067m, 2038vs, $2025 \mathrm{~m}$.

${ }^{1} \mathrm{H}$ NMR (400 MHz, $\mathrm{CDCl}_{3}$ ): $\delta$ 7.62-7.47 (m, 20H, Ar), $7.01(\mathrm{t}, 2 \mathrm{H}, J=5.5, \mathrm{NH}), 3.29$ (dt, $4 \mathrm{H}, J=6.1,6.1, \mathrm{NHC}_{2} \mathrm{CH}_{2} \mathrm{SH}$ ), 3.09-3.04 (m, 4H, $\left.\mathrm{PCH}_{2} \underline{\mathrm{C}}_{2} \mathrm{CO}\right), 2.54$ (dt, 4H, $J$ $\left.=7.3,7.3, \mathrm{NHCH}_{2} \mathrm{C}_{2} \mathrm{SH}\right), 2.24-2.18\left(\mathrm{~m}, 4 \mathrm{H}, \mathrm{PC}_{2} \mathrm{CH}_{2} \mathrm{CO}\right), 1.35$ (t, 2H, $J=8.3, \underline{\mathrm{H}}$ ),

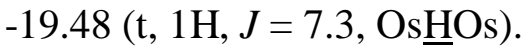

${ }^{31} \mathrm{P}\left\{{ }^{1} \mathrm{H}\right\}$ NMR $\left(160.26 \mathrm{MHz}, \mathrm{CDCl}_{3}\right): \delta-12.03(\mathrm{OsP}),-143.69\left(\mathrm{PF}_{6}\right)$.

HRMS-ESI $\mathrm{m} / \mathrm{z}$ calculated for $\mathrm{C}_{44} \mathrm{H}_{41} \mathrm{~N}_{2} \mathrm{O}_{12} \mathrm{P}_{2} \mathrm{~S}_{2}{ }^{187} \mathrm{Os}^{190} \mathrm{Oss}^{192} \mathrm{Os}[\mathrm{M}]^{+}$1484.0333, found 1484.0366 .

\subsubsection{Synthesis of cluster $7 \mathrm{~b}$}

Cluster $\mathrm{Re}_{2}(\mathrm{CO})_{8}\left(\mathrm{NCCH}_{3}\right)_{2}(104 \mathrm{mg}, 0.153 \mathrm{mmol})$, synthesized from a reported method, ${ }^{34}$ and 1,2-ethanedithiol $(129 \mu \mathrm{L}, 1.53 \mathrm{mmol})$ were dissolved in toluene $(5 \mathrm{~mL})$ and heated at $80{ }^{\circ} \mathrm{C}$ for $30 \mathrm{~min}$. Solvents and volatiles were removed in vacuo and the residue was purified by TLC using DCM:hexane $(1: 1, \mathrm{v} / \mathrm{v})$ as eluant to yield cluster $\mathbf{7 b}$ as a colourless oil.

Yield: $3 \mathrm{mg}(3 \%)$ 
$\mathrm{R}_{\mathrm{f}}=0.61$

IR (DCM, $\left.\mathrm{cm}^{-1}\right): 2115 \mathrm{w}, 2091 \mathrm{~m}, 2017 \mathrm{vs}, 1999 \mathrm{~s}, 1964 \mathrm{~s}$.

${ }^{1} \mathrm{H}$ NMR $\left(400 \mathrm{MHz}, \mathrm{CDCl}_{3}\right): \delta 3.01\left(\mathrm{t}, 2 \mathrm{H}, J=7.3, \mathrm{SC}_{2} \mathrm{CH}_{2} \mathrm{SH}\right), 2.86(\mathrm{dt}, 2 \mathrm{H}, J=$

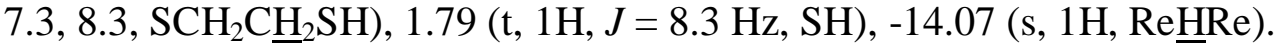

LCMS-ESI $m / z: 660.29$ [M-H-CO]', 632.83 [M-H-2CO]'.

\subsubsection{Preparation of AuNS conjugates of metal carbonyl clusters 2a-7b}

In a typical reaction, $60 \mathrm{~nm}$ AuNS $\left(3 \mathrm{~mL}, 2.6 \times 10^{10}\right.$ particles $\left./ \mathrm{mL}\right)$ was centrifuged and re-dispersed in deionized water till about $990 \mu \mathrm{L}$. Freshly prepared solutions of the clusters in DMSO $(10 \mu \mathrm{L}, 10 \mathrm{mM})^{*}$ were added and allowed to incubate at room temperature for $2 \mathrm{~h}$. Excess complexes were removed by centrifugation $(1200 \mathrm{~g}, 20$ min), and the conjugate pellets were resuspended in deionised water (100 $\mu \mathrm{L})$. An aliquot $(20 \mu \mathrm{L})$ of the solution was dropped onto a glass slide and scanned under the Raman microscope. An excitation wavelength of $785 \mathrm{~nm}$, with exposure time of $10 \mathrm{~s}$ and three accumulations was used throughout the measurement.

*For clusters 6 and $6 . \mathrm{H}^{+}, 10 \mu \mathrm{L}$ of $5 \mathrm{mM}$ DMSO solution was added instead.

\subsubsection{Preparation of AuNS conjugates of half-sandwich metal carbonyl complexes 8a-13a}

In a typical reaction, $60 \mathrm{~nm}$ AuNS $\left(3 \mathrm{~mL}, 2.6 \times 10^{10}\right.$ particles $\left./ \mathrm{mL}\right)$ was centrifuged and re-dispersed in ethanol till about $990 \mu \mathrm{L}$. Freshly prepared solutions of 8a-11a in ethanol $(10 \mu \mathrm{L}, 10 \mathrm{mM})$ were added and allowed to incubate at room temperature for 2 h. Excess complexes were removed by centrifugation $(1200 \mathrm{~g}, 20 \mathrm{~min})$, and the conjugate pellets were resuspended in deionised water $(100 \mu \mathrm{L})$. An aliquot $(20 \mu \mathrm{L})$ of the solution was dropped onto a glass slide and scanned under the Raman microscope. An excitation wavelength of $785 \mathrm{~nm}$, with exposure time of $10 \mathrm{~s}$ and three accumulations was used throughout the measurement. 


\subsection{References}

1. Y. Zhang, J. Qian, D. Wang, Y. Wang and S. He, Angew. Chem. Int. Ed., 2013, 52, 1148-1151.

2. Y. Wang, B. Yan and L. Chen, Chem. Rev., 2012, 113, 1391-1428.

3. L. Guerrini, E. Pazos, C. Penas, M. E. Vázquez, J. L. Mascareñas and R. A. Alvarez-Puebla, J. Am. Chem. Soc., 2013, 135, 10314-10317.

4. N. Guarrotxena and G. C. Bazan, Chem. Commun., 2011, 47, 8784-8786.

5. A. Samanta, S. Jana, R. K. Das and Y. T. Chang, Nanomedicine, 2014, 9, 523535.

6. H. Yuan, A. M. Fales, C. G. Khoury, J. Liu and T. Vo-Dinh, J. Raman Spectrosc., 2013, 44, 234-239.

7. J. Xie, Q. Zhang, J. Y. Lee and D. I. C. Wang, ACS Nano, 2008, 2, 2473-2480.

8. A. Shen, L. Chen, W. Xie, J. Hu, A. Zeng, R. Richards and J. Hu, Adv. Funct. Mater., 2010, 20, 969-975.

9. P.-J. Huang, L.-K. Chau, T.-S. Yang, L.-L. Tay and T.-T. Lin, Adv. Funct. Mater., 2009, 19, 242-248.

10. A. Samanta, K. K. Maiti, K.-S. Soh, X. Liao, M. Vendrell, U. S. Dinish, S.-W. Yun, R. Bhuvaneswari, H. Kim, S. Rautela, J. Chung, M. Olivo and Y.-T. Chang, Angew. Chem. Int. Ed., 2011, 50, 6089-6092.

11. K. K. Maiti, U. S. Dinish, A. Samanta, M. Vendrell, K.-S. Soh, S.-J. Park, M. Olivo and Y.-T. Chang, Nano Today, 2012, 7, 85-93.

12. C. L. Zavaleta, B. R. Smith, I. Walton, W. Doering, G. Davis, B. Shojaei, M. J. Natan and S. S. Gambhir, Proc. Natl. Acad. Sci. USA, 2009, 106, 13511-13516.

13. K. V. Kong, Z. Lam, W. D. Goh, W. K. Leong and M. Olivo, Angew. Chem., 2012, 124, 9934-9937.

14. K. V. Kong, Z. Lam, W. D. Goh, W. K. Leong and M. Olivo, Angew. Chem. Int. Ed., 2012, 51, 9796-9799.

15. D.-H. Tsai, F. W. DelRio, R. I. MacCuspie, T. J. Cho, M. R. Zachariah and V. A. Hackley, Langmuir, 2010, 26, 10325-10333.

16. J. Gao, X. Huang, H. Liu, F. Zan and J. Ren, Langmuir, 2012, 28, 4464-4471.

17. L. A. Porter, D. Ji, S. L. Westcott, M. Graupe, R. S. Czernuszewicz, N. J. Halas and T. R. Lee, Langmuir, 1998, 14, 7378-7386.

18. T. Peterle, A. Leifert, J. Timper, A. Sologubenko, U. Simon and M. Mayor, Chem. Commun., 2008, 3438-3440. 
19. X.-M. Li, M. R. d. Jong, K. Inoue, S. Shinkai, J. Huskens and D. N. Reinhoudt, J. Mater. Chem., 2001, 11, 1919-1923.

20. B. D. Chithrani, A. A. Ghazani and W. C. W. Chan, Nano Lett., 2006, 6, 662668.

21. P. N. Njoki, I. I. S. Lim, D. Mott, H.-Y. Park, B. Khan, S. Mishra, R. Sujakumar, J. Luo and C.-J. Zhong, J. Phys. Chem. C, 2007, 111, 14664-14669.

22. S. Hong and X. Li, Journal of Nanomaterials, 2013, 2013, 9.

23. K. M. Hanif, S. E. Kabir, M. A. Mottalib, M. B. Hursthouse, K. M. A. Malik and E. Rosenberg, Polyhedron, 2000, 19, 1073-1080.

24. E. W. Ainscough, A. M. Brodie, R. K. Coll, A. J. A. Mair and J. M. Waters, J. Organomet. Chem., 1996, 509, 259-264.

25. C. Li and W. K. Leong, J. Organomet. Chem., 2008, 693, 1292-1300.

26. R. G. Pearson, J. Am. Chem. Soc., 1963, 85, 3533-3539.

27. R. G. Pearson, J. Chem. Educ., 1968, 45, 581.

28. A. J. Deeming and M. Underhill, J. Organomet. Chem., 1972, 42, C60-C62.

29. G. Bor, Spectrochim. Acta, 1963, 19, 1209-1224.

30. G. Bor and K. Noack, J. Organomet. Chem., 1974, 64, 367-372.

31. B. V. Lokshin, Z. S. Klemenkova and Y. V. Makarov, Spectrochim. Acta, Pt. A: Mol. Spectrosc., 1972, 28, 2209-2215.

32. E. L. Myers and R. T. Raines, Angew. Chem. Int. Ed., 2009, 48, 2359-2363.

33. W. K. Leong and Y. Liu, J. Organomet. Chem., 1999, 584, 174-178.

34. H. Egold, D. Schwarze and U. Florke, J. Chem. Soc., Dalton Trans., 1999, 3203-3207.

35. S. R. Drake, B. F. G. Johnson and J. Lewis, J. Chem. Soc., Dalton Trans., 1989, 505-510.

36. B. F. G. Johnson, R. Khattar, J. Lewis and P. R. Raithby, J. Chem. Soc., Dalton Trans., 1989, 1421-1426.

37. Z. Akhter, J. F. Gallagher, J. Lewis, P. R. Raithby and G. P. Shields, J. Organomet. Chem., 2000, 614-615, 231-237.

38. Y. Li and W.-T. Wong, Eur. J. Inorg. Chem., 2003, 2003, 2651-2662.

39. J. Lewis and P. R. Raithby, in Metal Clusters in Chemistry, Wiley-VCH Verlag GmbH, 2008, pp. 348-380.

40. C. Elschenbroich, Organometallics, 3rd Ed., Wiley-VCH Verlag GmbH \& Co. KGaA, Weinheim, 2006. 
41. G. Consiglio and F. Morandini, Chem. Rev., 1987, 87, 761-778.

42. N. J. Coville, K. E. du Plooy and W. Pickl, Coord. Chem. Rev., 1992, 116, 1267.

43. C. Ganter, Chem. Soc. Rev., 2003, 32, 130-138.

44. J. Liu, X. Wu, J. A. Iggo and J. Xiao, Coord. Chem. Rev., 2008, 252, 782-809.

45. E. W. Abel, A. Singh and G. Wilkinson, J. Chem. Soc., 1960, 1321-1324.

46. J. C. T. R. Burckett-St. Laurent, J. S. Field, R. J. Haines and M. McMahon, J. Organomet. Chem., 1979, 181, 117-130.

47. L. Verdonck, A. R. Bossuyt, A. Praet, F. Verpoort, J. Geeraert, D. Van de Vondel and G. P. Van der Kelen, J. Mol. Catal., 1992, 76, 319-325.

48. A. Munyaneza, M. D. Bala and N. J. Coville, S. Afr. J. Chem, 2009, 62, 14-19.

49. S. Rosenberg, A. W. Herlinger, W. S. Mahoney and G. L. Geoffroy, Inorg. Synth., 1989, 25, 187-192.

50. T. Arliguie, C. Border, B. Chaudret, J. Devillers and R. Poilblanc, Organometallics, 1989, 8, 1308-1314.

51. C. Moreno, M.-J. Macazaga, R.-M. Medina, D. H. Farrar and S. Delgado, Organometallics, 1998, 17, 3733-3738.

52. Y. Zhang, B. Walkenfort, J. H. Yoon, S. Schlucker and W. Xie, Phys. Chem. Chem. Phys., 2015, 17, 21120-21126.

53. G. Chen, Y. Wang, M. Yang, J. Xu, S. J. Goh, M. Pan and H. Chen, J. Am. Chem. Soc., 2010, 132, 3644-3645.

54. S. K. Ghosh and T. Pal, Chem. Rev., 2007, 107, 4797-4862.

55. J. R. G. Navarro, M. Plugge, M. Loumaigne, A. Sanchez-Gonzalez, B. Mennucci, A. Debarre, A. M. Brouwer and M. H. V. Werts, Photochem. Photobiol. Sci., 2010, 9, 1042-1054.

56. H. Xu, E. J. Bjerneld, M. Käll and L. Börjesson, Phys. Rev. Lett., 1999, 83, 4357-4360.

57. J. P. Camden, J. A. Dieringer, J. Zhao and R. P. Van Duyne, Acc. Chem. Res., 2008, 41, 1653-1661.

58. C. E. Talley, J. B. Jackson, C. Oubre, N. K. Grady, C. W. Hollars, S. M. Lane, T. R. Huser, P. Nordlander and N. J. Halas, Nano Lett., 2005, 5, 1569-1574.

59. J. N. Nicholls, M. D. Vargas, A. J. Deeming and S. E. Kabir, Inorg. Synth., 1990, 28, 232.

60. G. O. Nelson and C. E. Sumner, Organometallics, 1986, 5, 1983-1990. 


\section{Chapter 3: Metal Carbonyl-Boronic Acid Conjugate - a SERS Probe for Glucose Detection and its Cytotoxicty}

According to a report by the World Health Organization, 422 million people worldwide have diabetes in 2014, a drastic rise from 108 million in $1980 .{ }^{1}$ Diabetes is a chronic metabolic disease characterized by the pancreas not being able to produce enough insulin, or the body not being able to utilize the insulin produced effectively. ${ }^{2,3}$ Type two diabetes, which is the major contributor to the rise in this disease, is largely caused by obesity - a product of rising living standard worldwide. Over the past decade, the occurrence of diabetes has increased faster in low- and middle-income countries than in high-income countries. ${ }^{1}$ A person is normally diagnosed with diabetes via symptoms like hyperglycaemia, which is characterized by a fasting blood glucose level of $\geq 7.0 \mathrm{mmol} \mathrm{L}{ }^{-1}$. ${ }^{3}$ Hence, tight glycemic control is important for diabetes management which requires strict dietary control as well as frequent blood glucose measurement. Quantification of glucose using various platforms, thus, remains important and relevant. This chapter details the concept of coupling boronic acid to a metal carbonyl and a SERS planar substrate to develop a selective SERS-based glucose assay, and an attempt to extend it to an in vivo application.

\subsection{Methods for glucose detection}

Portable hand held glucose meter for home use was first available in the 1980s based on the ferrocene-mediated electrochemistry of glucose oxidase (GOx). ${ }^{4-6}$ The working principle involves the oxidation of glucose by glucose oxidase (GOx), followed by electron transfer facilitated by the mediator (ferrocene) to the electrode (figure 3.1). ${ }^{4,8}$ The protein layer surrounding the flavin adenine dinucleotide (FAD) redox center of GOx hinders direct electron transfer to the electrode, hence the need for a mediator. ${ }^{8}$ The method was subsequently modified to a ferricyanide-mediated version and since then, the method of detection has remained unchanged. 


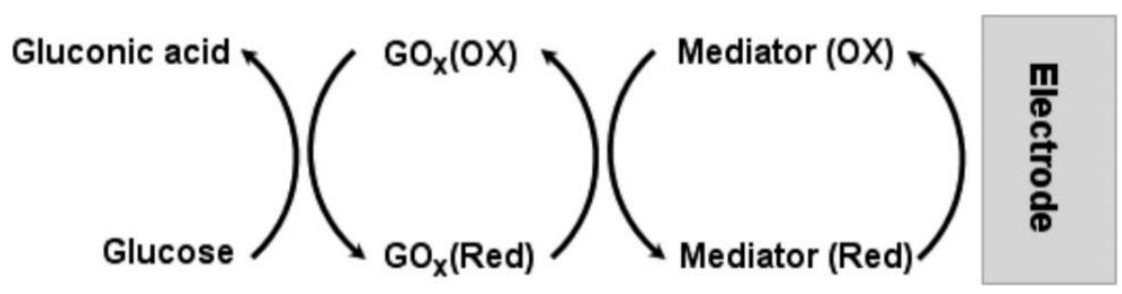

Figure 3.1. Sequence of reactions that occur in glucose oxidase-mediated biosensor. Adapted from ref. 8 with permission from the American Chemical Society.

In general, a drop of biofluid (in most cases, blood) is applied onto an enzymatic test strip and inserted into the device, which comprises a transducer-detector unit for electrochemical detection and quantification. ${ }^{6}$ Though the enzymatic reaction is specific, the electrochemical reaction can be interfered with by redox-active species present in the fluid. Another drawback is the pain associated with the frequent drawing of blood samples.

Progress has also been made on the use of optical tools for direct glucose detection. Polarimetric detection of glucose in the aqueous humor of the eye is a potentially useful non-invasive method which is built upon the chirality of glucose rotating the plane of polarization of incident light. ${ }^{9-11}$ However, the presence of other chiral molecules in the biofluid interferes with the detection, resulting in a lack of selectivity. Another optical technique is Raman spectroscopy, which has been used for the direct in-vitro detection of glucose in blood and simulated aqueous humour, with quantification by partial least squares analysis. ${ }^{12-15}$ This spectroscopic method provides valuable structural and functional information, and samples in aqueous media can be used due to the low Raman scattering cross section of water. Unfortunately, high laser power and long data acquisition times are required due to the intrinsic low Raman scattering cross section of the glucose molecule.

Efforts have therefore been made to develop SERS substrate-based glucose sensors; the advantage of using SERS is the improved Raman signal which can lead to sensitive detection in clinical samples. This was pioneered by Van Duyne and co-workers in the development of silver or gold Metallic Film Over Nanospheres (MFON) substrates functionalized with self-assembled monolayer of alkanethiol or alkanethiolate tri(ethylene glycol) for partitioning glucose from the aqueous media for SERS sensing (figure 3.2). ${ }^{16-21}$ This was also subsequently demonstrated with SERS-active nanogap substrates functionalized with mixed monolayers. ${ }^{22}$ 

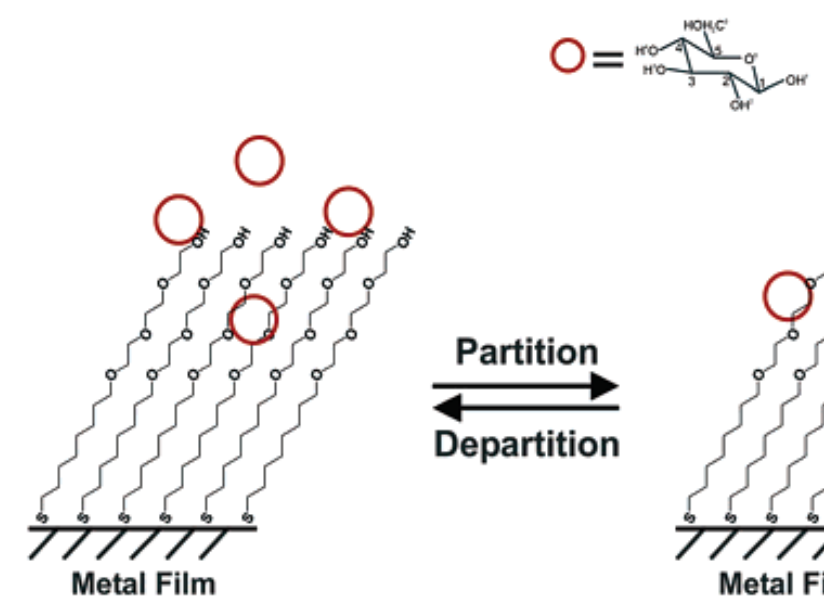

Metal Film
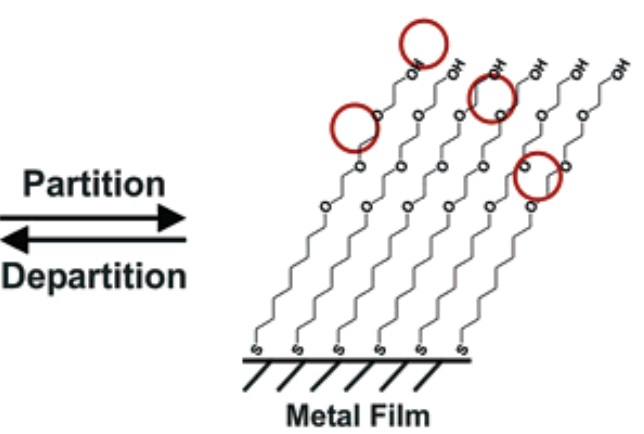

Figure 3.2. Schematic showing the partitioning of glucose by the (1-mercaptoundeca-11yl)tri(ethylene glycol) partition layer on the metallic SERS substrate. Adapted from ref. 17 with permission from the American Chemical Society.

\subsection{Concept of selective detection of glucose on SERS planar substrate using boronic acid chemistry}

Besides the use of nanoparticles, SERS enhancement can also be achieved on planar substrates. Gold nanoparticles are used extensively for the cellular detection of biomarkers but they are susceptible to aggregation and hence require the need for methods to stabilize them. Planar substrates, on the other hand, comprising patterned metallic surfaces immobilized onto a platform, are chemically more stable and resistant to aggregation. This makes them suitable for both in-vivo and in-vitro SERS biosensing. ${ }^{17,21}$ Some of the patterned metallic substrates developed include sphere template films, ${ }^{23}$ nanoparticle-anchored surfaces, ${ }^{24}$ biomimetic substrates, ${ }^{25}$ substrates with 3D Raman active volume, ${ }^{26}$ and metallic film over nanosphere (MFON). ${ }^{17}$ All of these substrates, though differing in their physical appearance and performance, serve to enhance the Raman signals of molecules adsorbed onto their surface, with enhancement factors ranging from $10^{6}$ to $10^{8} .^{27}$ As a result, ultrasensitive detection can be achieved and they have been employed in the detection of a wide array of biological and chemical molecules. ${ }^{27-36}$

There are various glucose-responsive systems which employ glucose oxidase, lectin and boronic acid chemistry for the detection of glucose. ${ }^{37}$ In this study, we utilized boronic acid (BA) chemistry to selectively target glucose in a SERS-based assay. The small but robust boronic acid functional group $\left(\mathrm{R}-\mathrm{B}(\mathrm{OH})_{2}\right)$ is simple and can be easily functionalized via the $\mathrm{R}$ group. The interaction of boronic acid with diols 
has been well-studied; they are known to bind covalently and reversibly to diols to form boronate esters in aqueous media (scheme 3.1)..$^{38-40}$
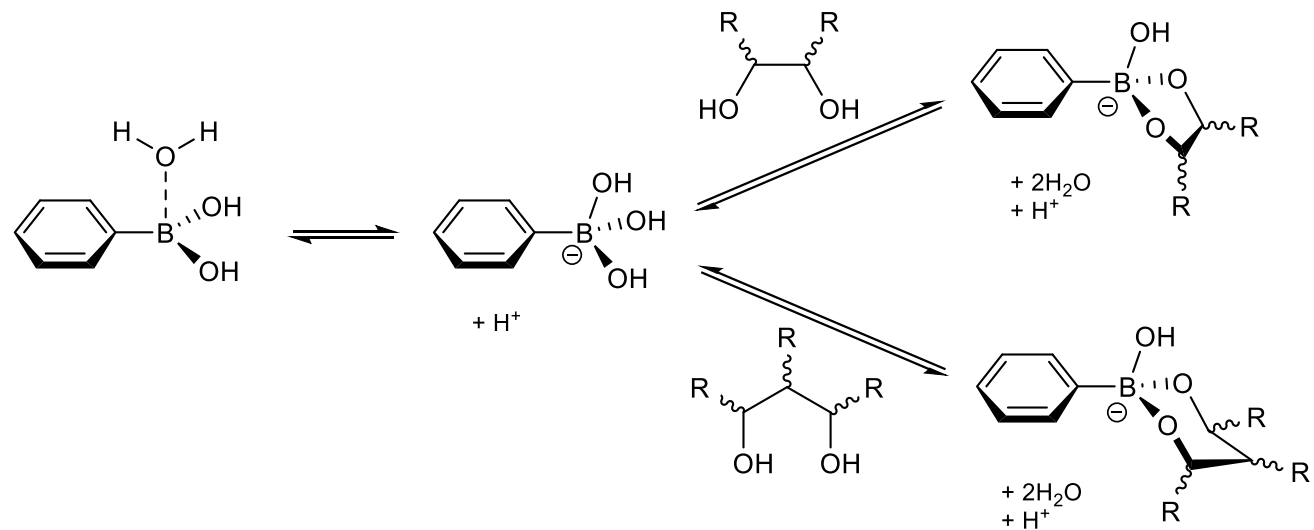

Scheme 3.1. Interaction of the phenylboronate anion with 1,2- and 1,3-diols to form boronate esters complexes with five- and six-membered rings, respectively. ${ }^{40}$

Saccharides contain many hydroxyl groups which can interact with the Lewis acidic boronic acid. This has formed the basis for fluorescence-based probes for saccharides sensing, whereby the fluorescence emission can be modulated with the binding of saccharides to boronic acid. ${ }^{41,}{ }^{42}$ In most cases, selective detection of glucose over other saccharides can be achieved with specially designed systems that employed diboronic acid moieties. ${ }^{39,43-54}$ The principle behind this is the formation of a stable boronic acid-diol complex which requires the presence of syn-periplanar hydroxyl groups for preferential binding; these diboronic acids are designed to straddle the two sets of syn-periplanar diol groups present in the $\alpha$-D-glucofuranose ring (figure 3.3a). ${ }^{40,44-46,55}$ This results in a higher tendency for the glucose molecule to form a bidentate boronic acid complex; the other physiologically relevant saccharides form monodentate complexes (figure 3.3b). ${ }^{46,55}$ 


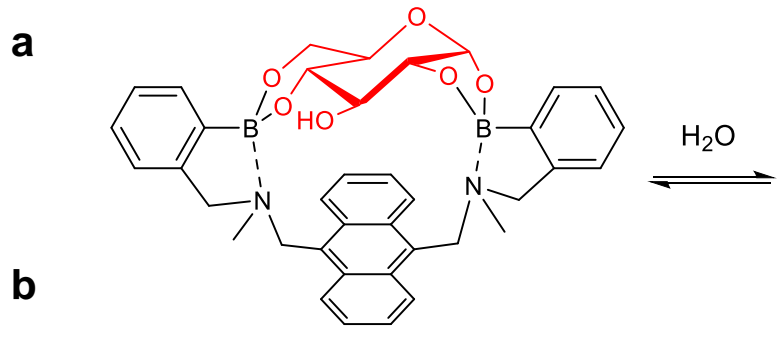

With $\alpha$-D-glucopyranose

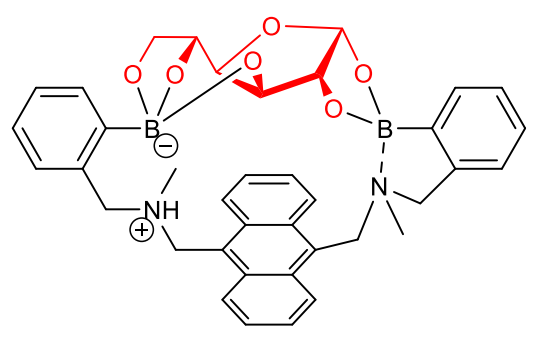

With $\alpha$-D-glucofuranose
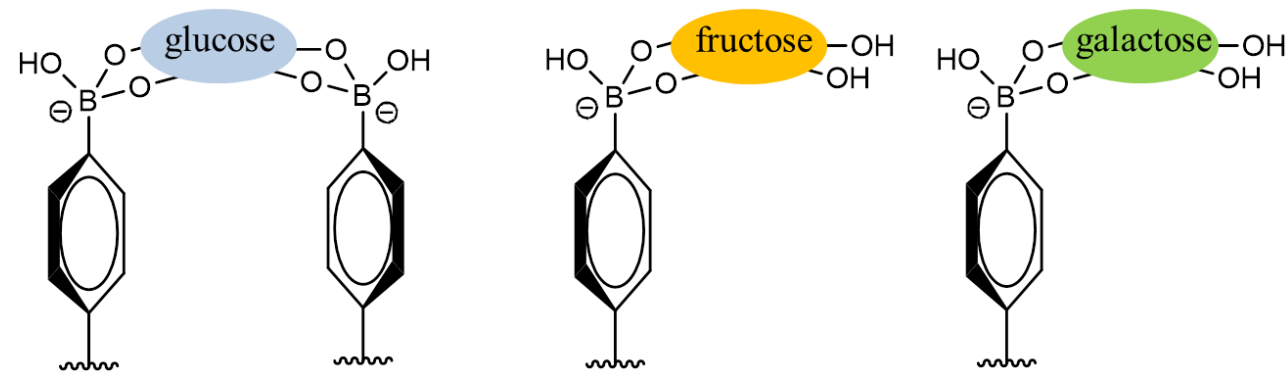

Figure 3.3. a) Interaction of a fluorescent diboronic acids anthracene conjugate with a glucose molecule. ${ }^{44,46,55}$ b) Illustration of glucose-bidendate boronic acid complex while other saccharides form a monodendate complex.

Building on this concept, the strategy was to develop a selective glucose assay analogous to that of the sandwich-based enzyme-linked immunosorbent assay (ELISA), with a metal carbonyl-boronic acid conjugate as the label. ${ }^{56,57}$ Two saccharide receptors were used; the first (primary) receptor comprised 4mercaptophenylboronic acid (BA) attached onto a SERS substrate, and the second (secondary) receptor was a metal carbonyl-boronic acid conjugate. Glucose was first captured by the primary saccharide receptor, followed by labeling by the conjugate (figure 3.4). This allowed selective quantification of the glucose via the $\mathrm{CO}$ stretching vibrations of the metal carbonyl, enhanced by the SERS substrate. 


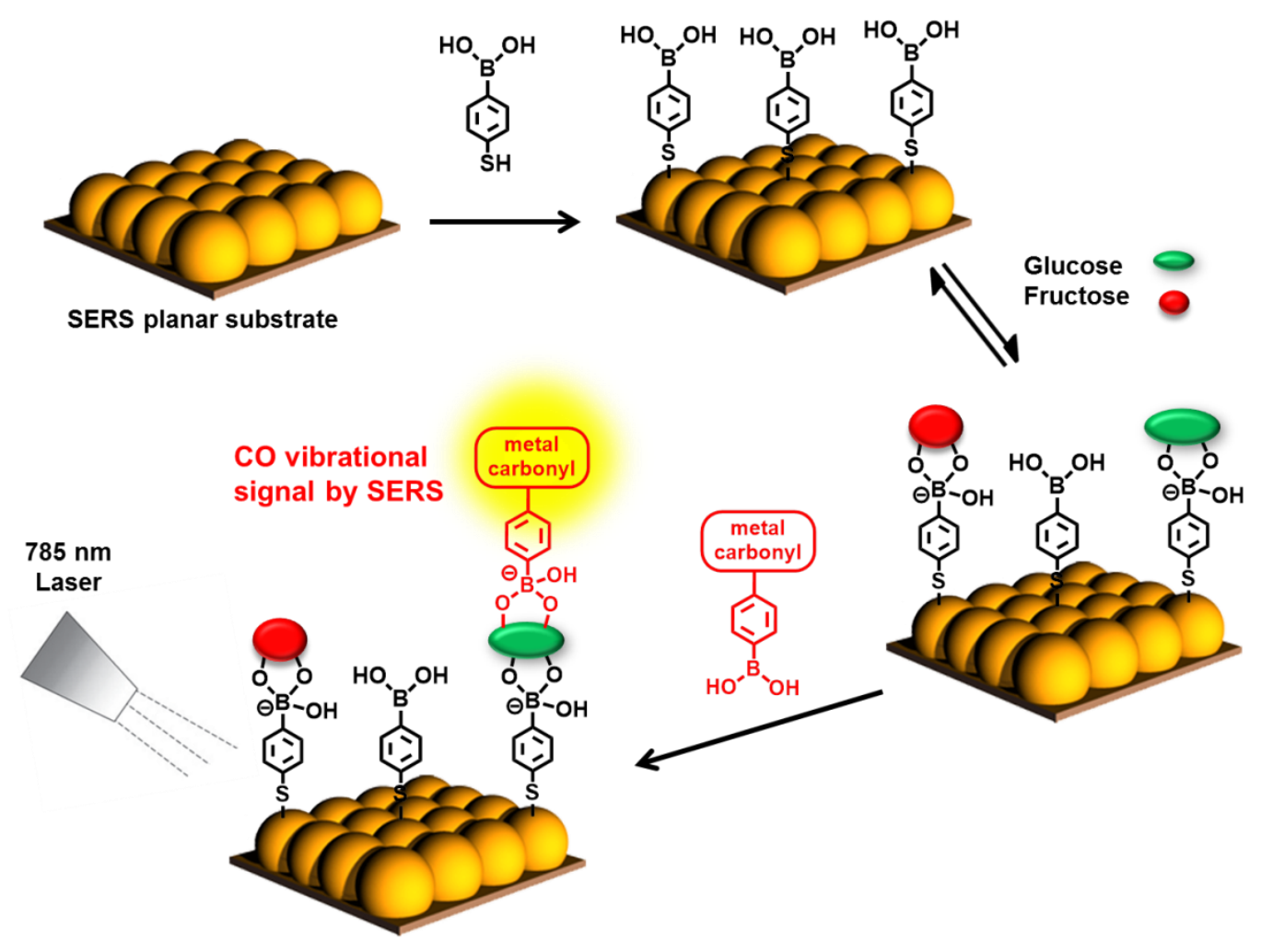

Figure 3.4. Schematic for the selective detection of glucose on the SERS-active substrate. The presence of a glucose molecule brings the metal carbonyl-boronic acid conjugate to the substrate via formation of a bidentate complex.

\subsection{Fabrication of boronic acid functionalized SERS substrate}

The SERS-active substrate used in this study was Bimetallic Film Over Nanospheres (BMFON). ${ }^{58-61}$ This is a substrate, fabricated in-house, that does not require clean room environment or sophisticated instruments for fabrication, and it can give excellent Raman signal enhancement with an enhancement factor of $2.0 \times 10^{7}$; it has been employed in the detection of biomolecules within an extremely low sample volume $(\sim 20 \mu \mathrm{L})^{58,59,61}$ The BA was immobilised onto BMFON via its thiol (-SH) group, with its boronic acid group $-\mathrm{B}(\mathrm{OH})_{2}$ serving as the saccharide receptor. This eliminated the need to introduce the saccharide receptor $\left(-\mathrm{B}(\mathrm{OH})_{2}\right)$ and the substratebinding moiety (-SH) separately. The distribution of BA prior to incubation with glucose was first examined by SERS mapping, using an absorption peak of the phenyl group at $1580 \mathrm{~cm}^{-1}$ which showed an even distribution of BA on BMFON (figure 3.5). The BA layer was very stable as the substrate soaked in PBS showed only slight variations in the signals and distribution of BA over a period of three days. 

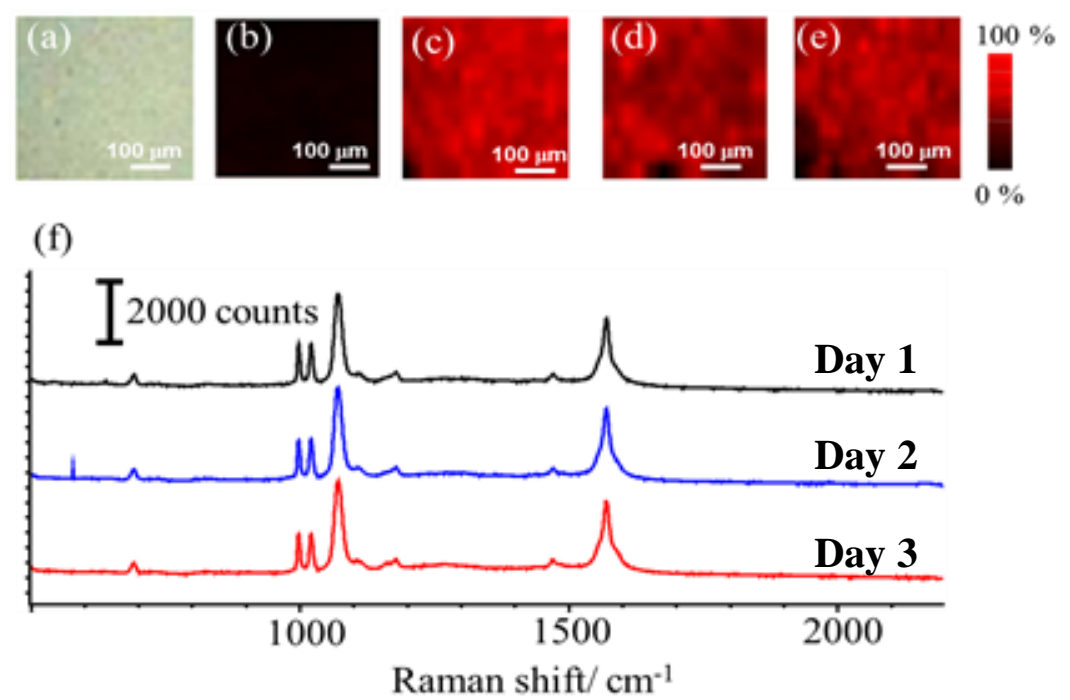

Figure 3.5. (a) Bright field image of BMFOM. SERS mapping images at $1580 \mathrm{~cm}^{-1}$ before (b) and after (c) immobilization of BA. Stability test of BA-functionalized BMFON in PBS solution - SERS mapping images at $1580 \mathrm{~cm}^{-1}$ on (c) day 1, (d) day 2 and (e) day 3. (f) SERS spectra of BA-functionalized BMFON from days $1-3$.

\subsection{Synthesis and characterization of cluster $2 \mathrm{~b}-\mathrm{B}(\mathrm{OH})_{2}$}

The secondary saccharide $\mathbf{2} \mathbf{b}-\mathbf{B}(\mathrm{OH})_{2}$ is a triosmium carbonyl cluster-boronic acid conjugate. Osmium carbonyl clusters are often air- and moisture-stable, and they have been shown to be sufficiently robust for bio-imaging applications. ${ }^{62}$ Cluster $\mathbf{2 b}-$ $\mathrm{B}(\mathrm{OH})_{2}$ was prepared by the reaction of $\mathrm{Os}_{3}(\mathrm{CO})_{10}\left(\mathrm{NCCH}_{3}\right)_{2}$, 1, with 4mercaptophenylboronic acid to form the $\mu, \kappa S$-thiolate bridged cluster (scheme 3.2). It has been completely characterized spectroscopically by IR, HR-MS, ${ }^{1} \mathrm{H}$ and ${ }^{13} \mathrm{C} \mathrm{NMR}$ (appendix A3.1-5). A sample of $\mathbf{2} \mathbf{b}-\mathrm{B}(\mathrm{OH})_{2}$ incubated with an excess of glucose at $\mathrm{pH}$ 9.0 showed the presence of an adduct of $2 \mathbf{b}-\mathrm{B}(\mathrm{OH})_{2}$ with glucose in the HR-MS, demonstrating its ability to bind glucose (appendix A3.6) . 


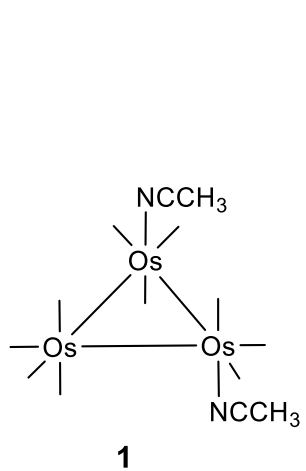

1

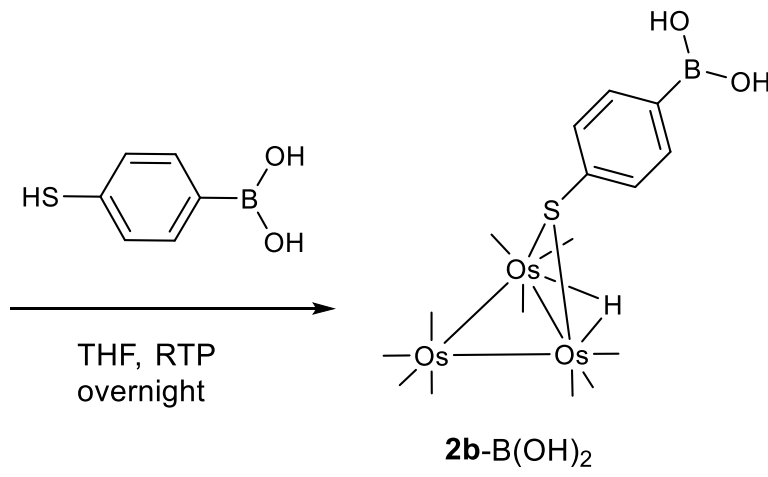

$63 \%$
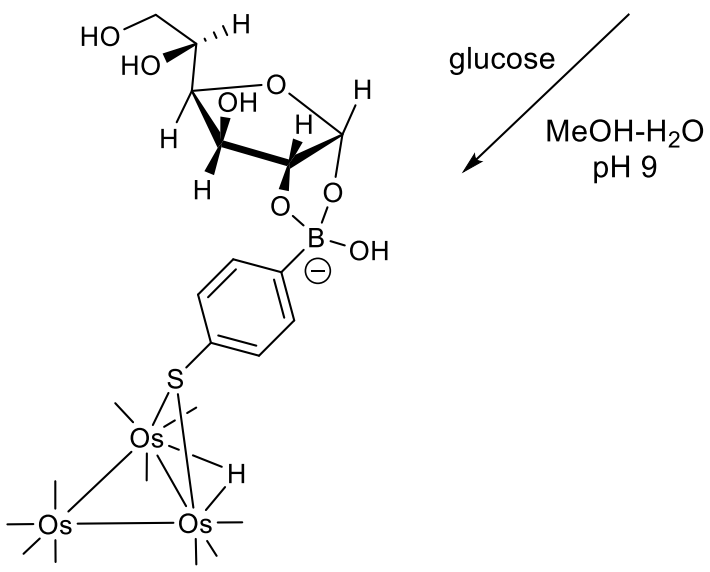

$\mathrm{pH} 9$

Scheme 3.2. Synthesis of $\mathbf{2 b}-\mathrm{B}(\mathrm{OH})_{2}$ and its reaction with glucose.

The structure of $\mathbf{2 b}-\mathrm{B}(\mathrm{OH})_{2}$ was also confirmed by a single-crystal X-ray crystallographic study which showed it to be a condensation trimer, 2b-boroxine, comprising a six-membered cluster-decorated boroxine ring (figure 3.6); this is commonly observed in solid-state characterization of boronic acid moieties. ${ }^{63}$ Boroxine is an important precursor for the synthesis of covalent organic frameworks and polymeric materials. ${ }^{63-65}$ Organometallic-decorated boroxines containing a triply ferrocene-bridged boroxine cyclophane has been reported, ${ }^{66}$ but to the best of our knowledge, this is the first example of a metal carbonyl-boroxine. 


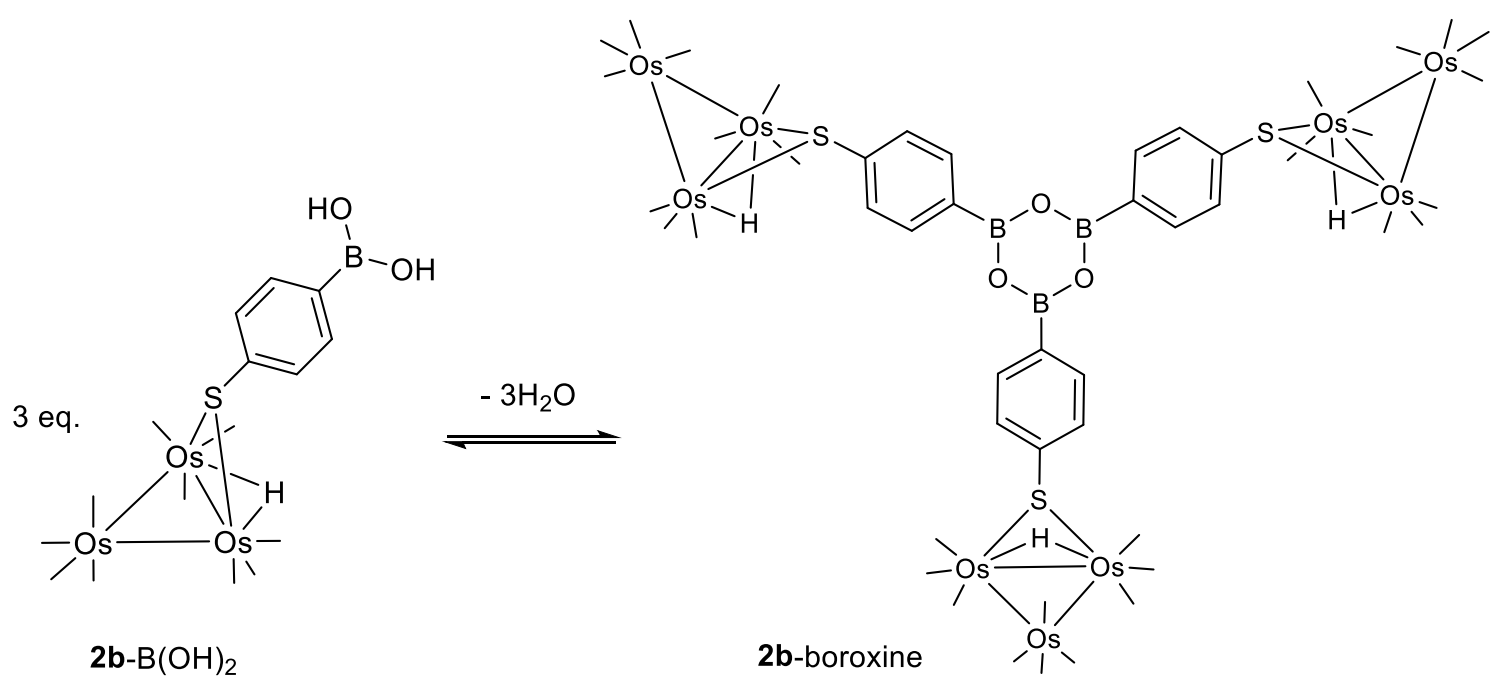

Scheme 3.3. Formation of $\mathbf{2 b}$-boroxine from $\mathbf{2 b}-\mathrm{B}(\mathrm{OH})_{2}$.

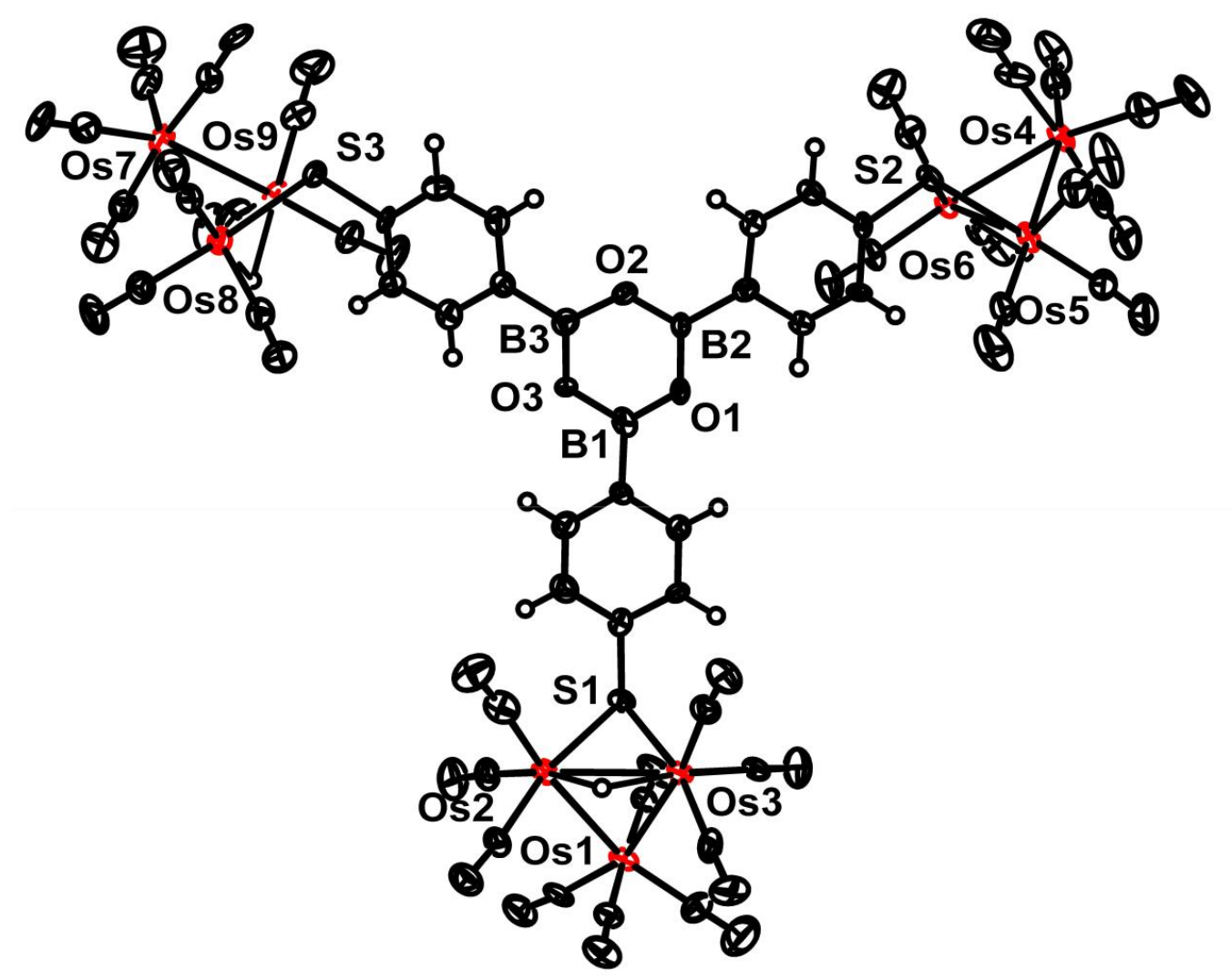

Figure 3.6 ORTEP plot of the molecular structure of $\mathbf{2 b}$-boroxine. Thermal ellipsoids are drawn at the $50 \%$ probability level. 


\subsection{Selective detection and quantification of glucose}

Fructose and galactose are the most abundant monosaccharides after glucose. It is thus useful to examine if the assay could discriminate glucose in the presence of these other monosaccharides. ${ }^{67-69}$ Indeed, BA-functionalized BMFON substrate incubated with a small amount ( $20 \mu \mathrm{L}, 20 \mathrm{mM})$ of a solution of glucose, fructose or galactose prior to treatment with $\mathbf{2 b}-\mathrm{B}(\mathrm{OH})_{2}$ showed significant $\mathrm{CO}$ stretching vibrations at $\sim 2000 \mathrm{~cm}^{-1}$ only with glucose; it was very modest with fructose or galactose (figure $3.7)$.

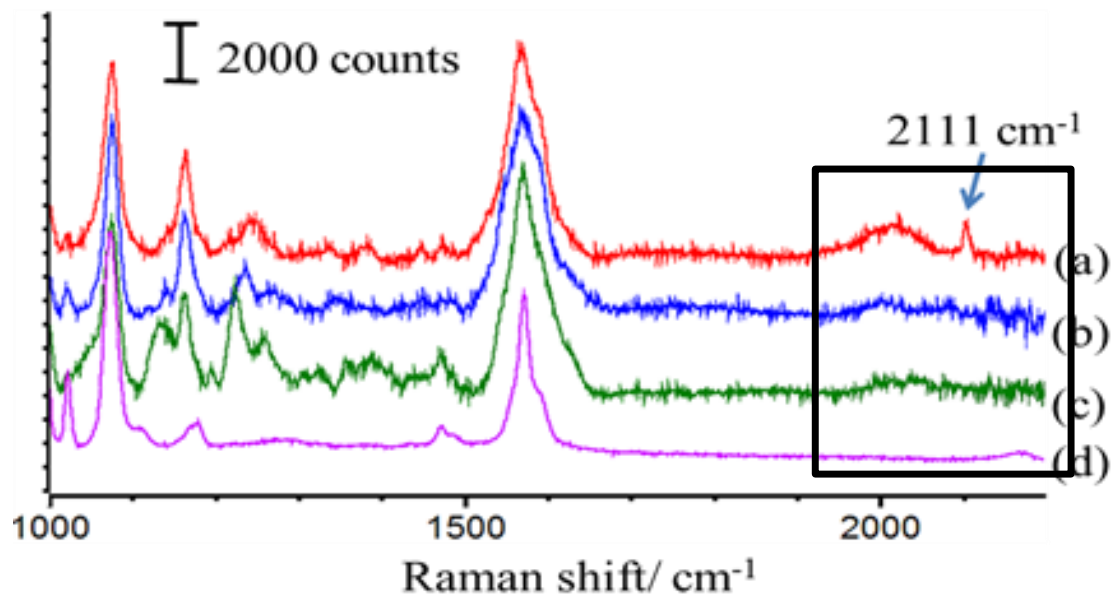

Figure 3.7. The Raman responses for BA-functionalized BMFON incubated with (a) glucose, (b) fructose, (c) galactose and (d) control, prior to incubation with 6. Boxed region shows $\mathrm{CO}$ stretching vibrations of $\mathbf{2} \mathbf{b}-\mathrm{B}(\mathrm{OH})_{2}$ which is free of interference from other functional groups.

The intensity of the sharp peak at $2111 \mathrm{~cm}^{-1}$ as a function of incubation with varying concentrations of the three saccharides showed an increase in the SERS signal intensity as a function of the concentration of glucose (figure 3.7a-b). The limit of detection was estimated at $0.1 \mathrm{mM}$, with a detection range $(0.1-10 \mathrm{mM})$ that covered the physiological concentration $(5 \mathrm{mM})$. The responses for fructose and galactose were, in contrast, much lower. The greater affinity of $\mathbf{2} \mathbf{b}-\mathbf{B}(\mathrm{OH})_{2}$ for glucose may be attributed to the higher tendency of glucose to form a bidentate complex with boronic acid. This is reminiscent of a recent report which utilized the greater affinity of glucose for binding to boronic acids to form 1:2 (glucose:boronic acid) adducts, while fructose only forms 1:1 adducts. ${ }^{54}$ Presumably, all three saccharides interacted with the BAfunctionalized BMFON, but there was preferential binding of $\mathbf{2} \mathbf{b}-\mathrm{B}(\mathrm{OH})_{2}$ with the surface-bound glucose. 
Glucose monitoring for diabetes is usually carried out on urine or blood samples; the former is not a substitute but rather, an alternative or complement, which can provide very valuable information where blood glucose monitoring is not accessible, affordable, or desirable. To demonstrate the practicality of our platform, we determined glucose in urine samples doped with a standard glucose solution (figure $3.8 \mathrm{a}, \mathrm{c})$. The concentration of glucose in the urine sample was determined to be 5.1 $\mathrm{mM}$, in good agreement with the amount of glucose added (5.0 mM).
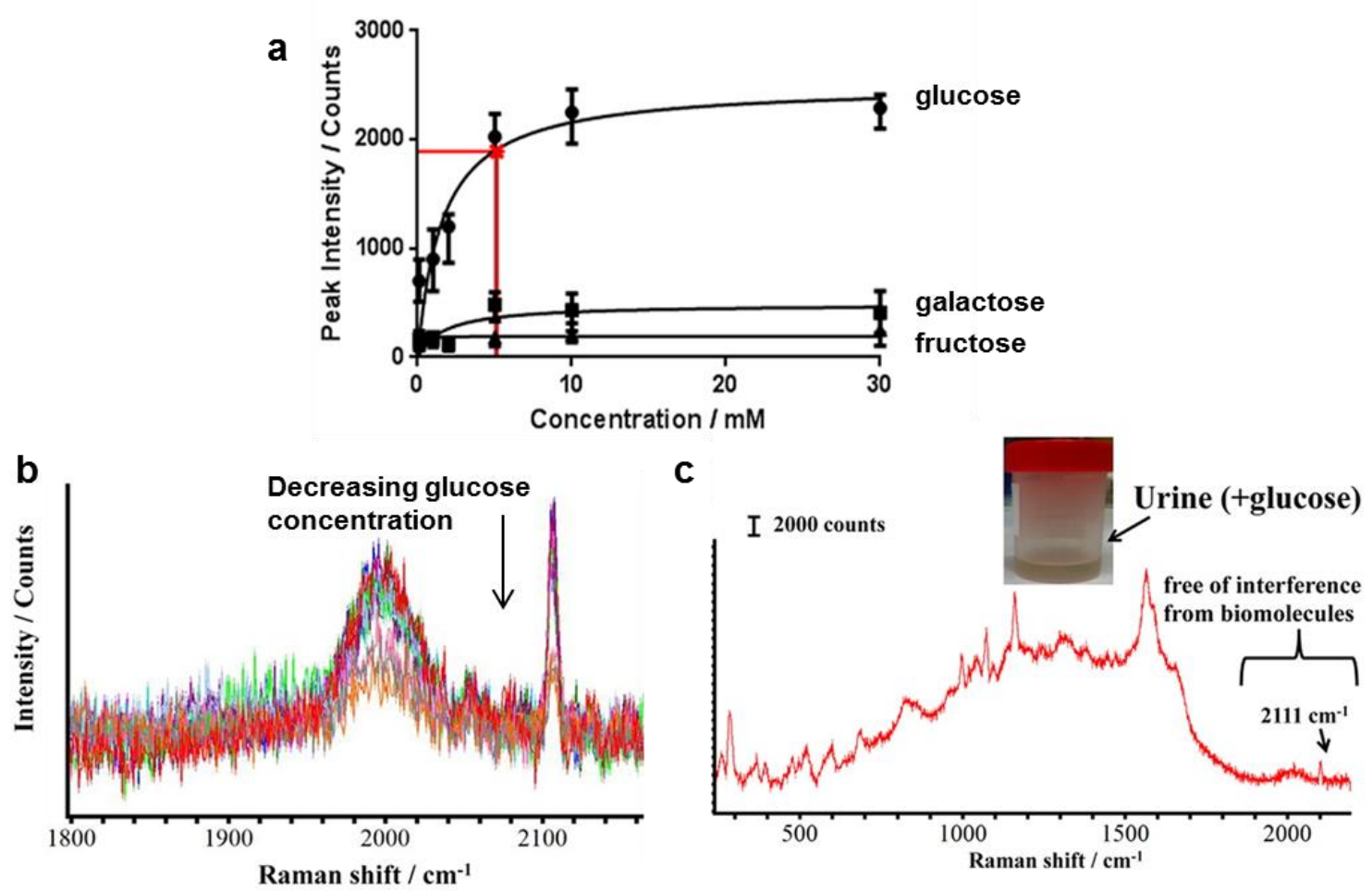

Figure 3.8. (a) Plot represents the intensity of $\mathrm{CO}$ stretching frequency versus different concentrations of $(\bullet)$ glucose, $(\boldsymbol{\Delta})$ fructose, and ( $\bullet$ galactose, with the interpolated value for the spiked urine sample shown in red. The first data points are at $0.1 \mathrm{mM}$. (b) SERS spectrum of $\mathbf{2} \mathbf{b}-\mathrm{B}(\mathrm{OH})_{2}$ obtained from different concentrations of glucose. (c) SERS spectrum of $\mathbf{2} \mathbf{b}-$ $\mathrm{B}(\mathrm{OH})_{2}$ after BMFON has been incubated with a urine sample spiked with glucose. 


\subsection{Cytotoxicity of $2 \mathrm{~b}-\mathrm{B}(\mathrm{OH})_{2}$ and analogues towards cancer and non-cancer cells}

Sugars play an essential role, not just as the energy source, but also as an integral part of the cell in the form of glycans - oligo- or polysaccharides linked to proteins and lipids via glycosylation. ${ }^{70,71}$ Alterations in glycan expression is a hallmark of malignant transformation, with the expression of sialic acid on the cell surface being the most widely reported. ${ }^{72,73}$ Sialic acid is a class of $N$ - or $O$-substituted neuraminic acid (nine-carbon monosaccharide) and some common examples found on the terminal ends of glycans are Neu5Ac and Kdn (figure 3.9). ${ }^{74}$ Overexpression of the enzymes, $\mathrm{N}$-acetylglucosaminyltransferase $\mathrm{V}$ and glycosyltransferase, in cancer cells can lead to increased branching of the glycans and glycosylation at the terminal ends, respectively, resulting in an overall increase in sialylation. ${ }^{75,76}$ Examples of glycan epitopes (parts of a proteins for antibodies recognition) commonly found in cancer cells include sialyl Lewis $\mathrm{x}\left(\mathrm{sLe}^{\mathrm{x}}\right)$ and polysialic acid (PSA) (figure 3.9$){ }^{77}$

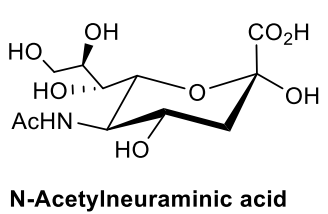

N-Acetylneuraminic acid
(Neu5Ac) Neu5Ac)

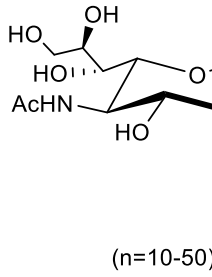

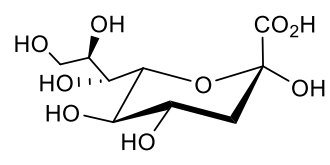
2-Keto-3-deoxynononic acid
(Kdn)

\begin{abstract}
cosicis
\end{abstract}

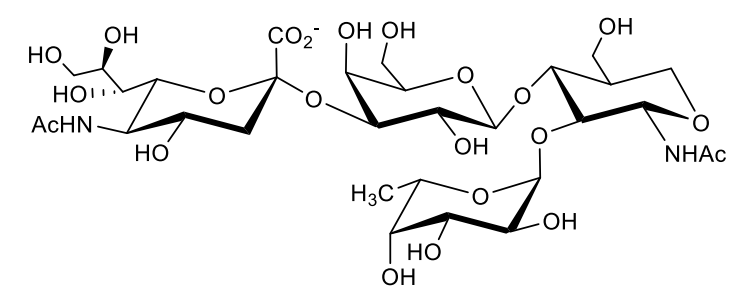

Sialyl Lewis $x\left(s L e^{x}\right)$

Figure 3.9. Molecular structures of sialic acid and glycan epitopes commonly found in cancer cells.

Other than having a strong affinity for saccharides, boronic acid is reported to interact with Neu5Ac at physiological pH (7.4), with a significantly higher binding constant than glucose. ${ }^{78,79}$ From this has spawned applications that utilized boronic acid to target cell surface glycans. An interesting example is the use of polymer functionalized with phenylboronic acid to selectively capture and release cancer cells that overexpressed sialic acid. ${ }^{80}$ Other work involved the use of imaging agents, 
including quantum dots, ${ }^{81}$ fluorescence probe, ${ }^{82}$ and gold nanoparticles, ${ }^{83,} 84$ functionalized with phenylboronic acid to selectively target and image cancer cells.

It was therefore of interest to examine if metal carbonyl-boronic acid conjugates such as cluster $\mathbf{2 b}-\mathrm{B}(\mathrm{OH})_{2}$ may also be applied to cell-based and, potentially, in vivo applications like the identification of cancer cells via their surface glycans. As a preliminary, however, the cytotoxicity of cluster $\mathbf{2 b}-\mathrm{B}(\mathrm{OH})_{2}$ and other analogues had to be evaluated. This was carried out on the MDA-MB-231 breast cancer cell line via an MTS assay. This metastatic cell line was chosen as it has one of the highest expression of sialic acid among breast cancer. ${ }^{85}$

To our surprise, cluster $\mathbf{2 b}-\mathrm{B}(\mathrm{OH})_{2}$ was found to demonstrate cytotoxicity comparable to that of the positive control, cluster 1 , with $\mathrm{IC}_{50}$ values of $3.8 \pm 0.9$ and $4.0 \pm 0.6 \mu \mathrm{M}$, respectively. ${ }^{86}$ This result is surprising as it has previously been found that alkylthiolato-triosmium carbonyl clusters, which have been employed for bioimaging, exhibited little effect on cell viability. ${ }^{62,87}$ For example, the viability of HL-60 leukemia cell (with respect to control) treated with $30 \mu \mathrm{M}$ of $\left[\mathrm{Os}_{3}(\mathrm{CO})_{10}(\mu-\right.$ $\left.\mathrm{H})\left(\mu-\mathrm{S}\left(\mathrm{CH}_{2}\right)_{10} \mathrm{COO}\right)\right] \mathrm{Na}$ and a phosphatidylcholine analogue were $89 \%$ and $77 \%$, respectively. ${ }^{62}$

To gain an understanding of the reasons behind the cytotoxicity of $\mathbf{2 b}-\mathrm{B}(\mathrm{OH})_{2}$, a number of other phenylthiolato-triosmium carbonyl cluster analogues were synthesized (scheme 3.4).

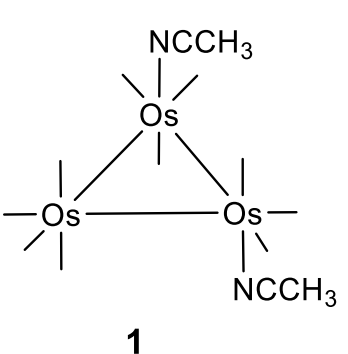

1

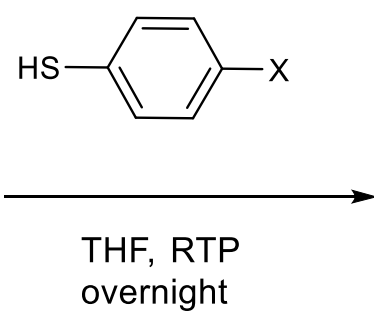

overnight

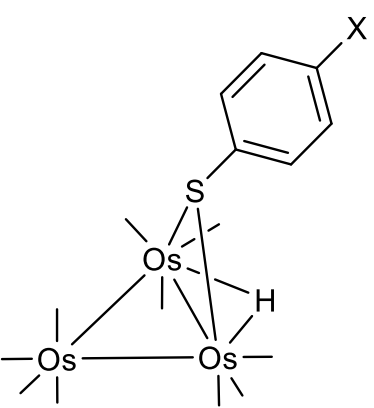

2b- $\mathrm{H}, \mathrm{X}=\mathrm{H}$

2b-OH, $\mathrm{X}=\mathrm{OH}(82 \%)$

2b-COOH, $\mathrm{X}=\mathrm{COOH}(63 \%)$

Scheme 3.4. Synthesis of cluster $\mathbf{2 b -}-\mathrm{H}$, $-\mathrm{OH}$ and $-\mathrm{COOH}$. 

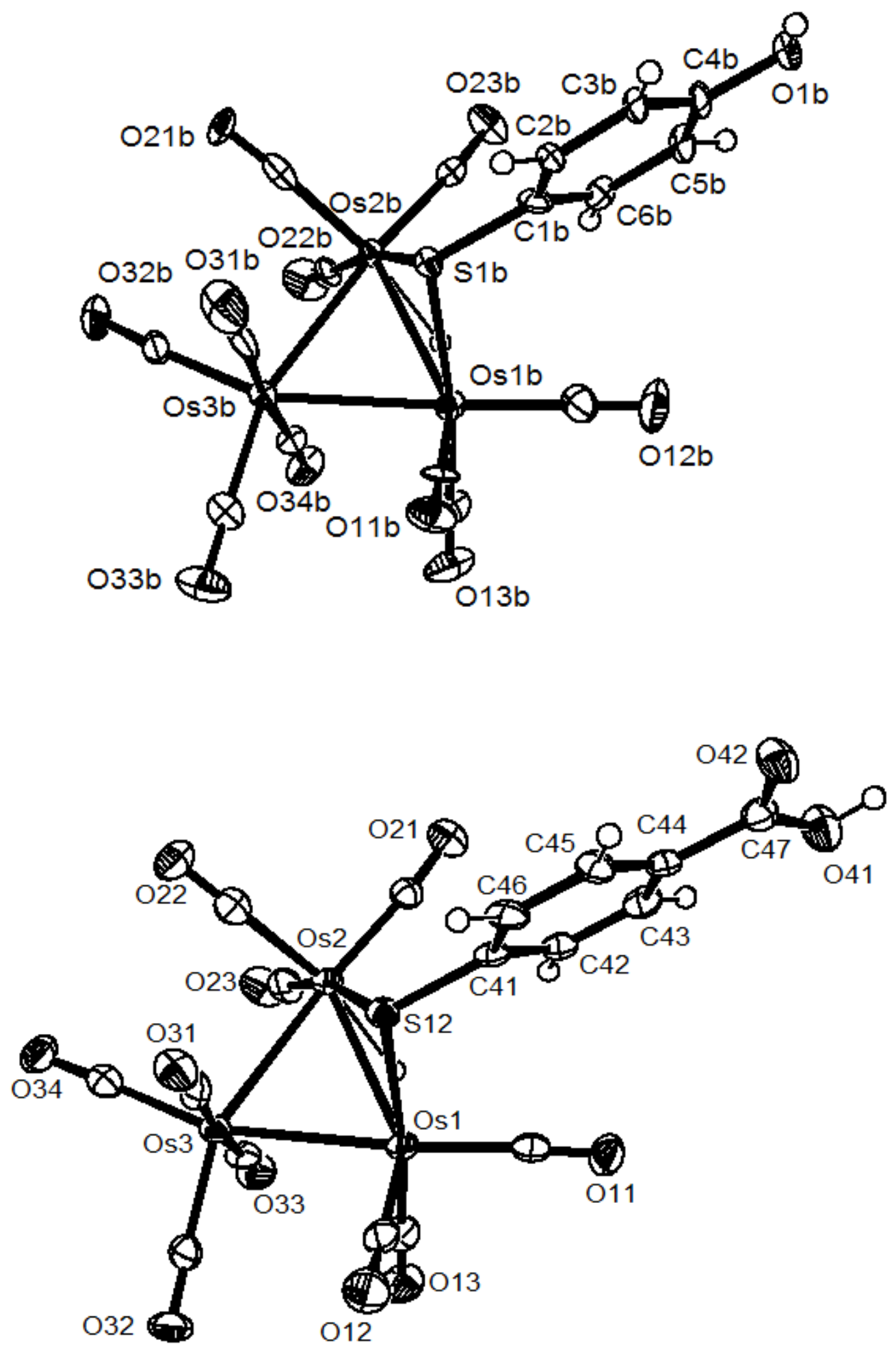

Figure 3.10. ORTEP plot of the molecular structure of $\mathbf{2} \mathbf{b}-\mathrm{OH}$ (top) and $\mathbf{2 b}-\mathrm{COOH}$ (bottom). Thermal ellipsoids are drawn at the $50 \%$ probability level. 
Cluster $\mathbf{2 b}-\mathrm{H}$ was synthesized according to the reported method, ${ }^{88-90}$ and the syntheses of $\mathbf{2} \mathbf{b}-\mathrm{OH}$ and $-\mathrm{COOH}$ were adapted from that. The structures of $\mathbf{2} \mathbf{b}-\mathrm{OH}$ and $-\mathrm{COOH}$ were also confirmed crystallographically (figures 3.10). Cluster $\mathbf{2 b}-\mathrm{B}(\mathrm{OH})_{2}$ showed similar toxicity to the $-\mathrm{OH}$ and $-\mathrm{COOH}$ analogues, while the ligand $\mathrm{BA}$ was found to have little effect on cell viability, even at a high concentration of $50 \mu \mathrm{M}$ (table 3.1 and figure 3.11). The cytotoxicity of cluster $\mathbf{2} \mathbf{b}-\mathrm{H}$ could not be determined as it was found to be highly insoluble in aqueous medium. The differences in cytotoxicity can be attributed to the solubility and not the nature of the functional groups on the phenyl ring. The study also eliminated the ligand BA as the source of toxicity; the triosmium carbonyl cluster, or in combination with the aromatic group, may have been responsible.

Together with earlier work on the alkylthiolato clusters, this indicated that the cytotoxicity of $\mathrm{Os}_{3}(\mathrm{CO})_{10}(\mu-\mathrm{H})(\mu-\mathrm{SR})$ clusters are dependent on the $\mathrm{R}$ groups which may play an important role in modulating the lipophilicity and electronic property of the clusters. It may be speculated that the phenyl group, being more electronwithdrawing than the alkyl group, can result in the sulphur lone pairs less easily donated, thereby forming the $\eta^{1}$-SR more easily; this creates a vacant site on the osmium centre which allows the reaction with biologically relevant targets.

Table 3.1. Inhibition of cell growth on MDA-MB-231 cells under serum-free conditions, after $24 \mathrm{~h}$, as determined by MTS assay.

\begin{tabular}{|c|c|}
\hline Compounds & $\mathbf{I C}_{\mathbf{5 0}} / \boldsymbol{\mu M}$ \\
\hline $\mathbf{1}$ & $4.0 \pm 0.6$ \\
\hline $\mathrm{BA}$ & $>50$ \\
\hline $\mathbf{2 b}-\mathrm{B}(\mathrm{OH})_{2}$ & $3.8 \pm 0.9$ \\
\hline $\mathbf{2 b}-\mathrm{OH}$ & $2.6 \pm 0.4$ \\
\hline $\mathbf{2 b}-\mathrm{COOH}$ & $4.0 \pm 0.4$ \\
\hline
\end{tabular}




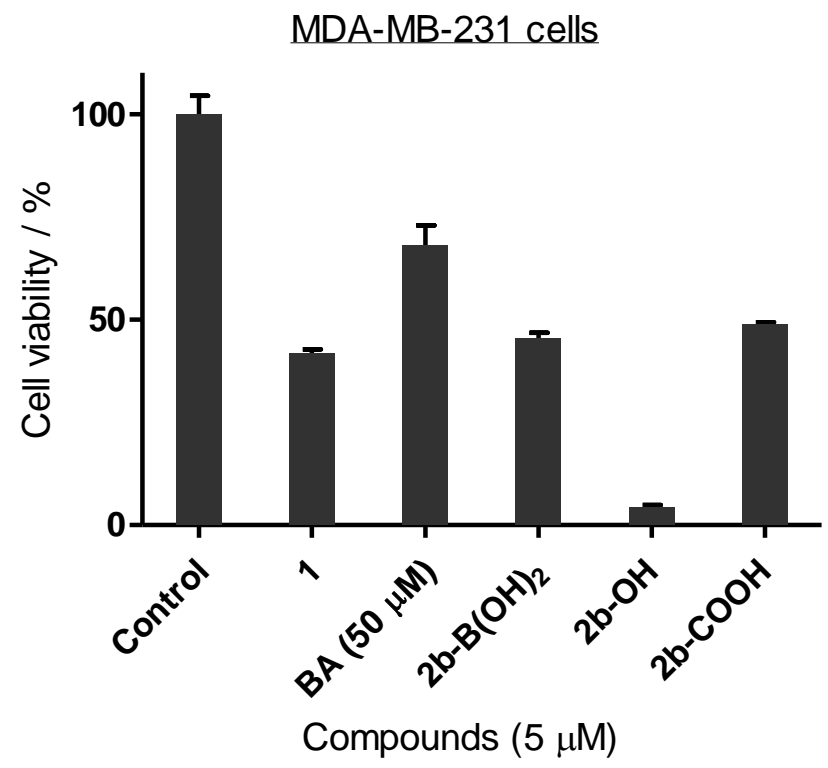

Figure 3.11. Comparison of the antiproliferative activity among the clusters $(5 \mu \mathrm{M})$ and ligand BA $(50 \mu \mathrm{M})$ on MDA-MB-231 cells, using 1 as the positive control. Control: DMSO $(0.5 \%, \mathrm{v} / \mathrm{v})$.

An effective anti-cancer drug is one that selectively targets cancer cells over normal cells; this is important for the reduction of any side effects due to the indiscriminate nature of the drug. To understand the selectivity, $2 \mathbf{b}-\mathrm{B}(\mathrm{OH})_{2}$ and $-\mathrm{OH}$ were further evaluated with the estrogen receptor positive $(\mathrm{ER}+)$ breast cancer cell line MCF-7, as well as on the non-cancerous breast epithelial cell line MCF-10A.

The $\mathrm{IC}_{50}$ values obtained showed that $\mathbf{2 b}-\mathrm{OH}$ exhibited higher toxicity than the $\mathrm{B}(\mathrm{OH})_{2}$ analogue and, together with $\mathbf{1}$, they were more cytotoxic towards MDA-MB231 (ER-) than MCF-7 (ER+) cells (table 3.2). This suggests that the compounds were not acting through the estrogen receptors but via a different target. Unfortunately, all the tested osmium compounds showed higher toxicity towards the normal cells than the cancer cells, with no selectivity observed. The cell death was also verified visually under a bright-field microscope with significant changes to the cell morphologies observed in comparison to the negative control (figure 3.13).

The compounds were also evaluated on the respective cell lines in the presence of serum. Substantial increase in the $\mathrm{IC}_{50}$ for all compounds were observed; this was especially the case for cluster $\mathbf{1}$ (table 3.2). Though the potencies of cluster $\mathbf{2} \mathbf{b}-\mathrm{B}(\mathrm{OH})_{2}$ and $-\mathrm{OH}$ were rather similar to $\mathbf{1}$ under serum-free conditions, they were less susceptible to interference by serum and demonstrated better efficacy than $\mathbf{1}$ in the 
presence of serum; the $\mathrm{IC}_{50}$ of $\mathbf{1}$ increased 5-8 folds in the presence of serum, reflecting its low efficacy in serum conditions (figure 3.12). ${ }^{91}$ The increase can be attributed to the high reactivity of $\mathbf{1}$ towards thiols and carboxylic acid in serum proteins to form the stable $\mu-\mathrm{S}$ and $\mu, \kappa^{2} \mathrm{O}, \mathrm{O}^{\prime}$ clusters respectively. ${ }^{89-92}$ It is precisely this high chemical reactivity which allowed the bioconjugation of triosmium clusters to biomolecules via such functionalities. ${ }^{62}$ Clusters $\mathbf{2 b}-\mathrm{B}(\mathrm{OH})_{2}$ and $-\mathrm{OH}$, which are intrinsically more stable, can be better drug candidates as compared to $\mathbf{1}$.

Table 3.2. Inhibition of cell growth on breast cell lines, after $24 \mathrm{~h}$ in serum and serum-free condition, as determined by MTS assay.

\begin{tabular}{|c|c|c|c|c|c|c|}
\hline \multirow{2}{*}{ Compounds } & \multicolumn{5}{|c|}{ IC $_{\mathbf{5 0}} / \mu \mathrm{M}$} \\
\cline { 2 - 7 } & \multicolumn{2}{|c|}{ MDA-MB-231 } & \multicolumn{2}{c|}{ MCF-7 } & \multicolumn{2}{c|}{ MCF-10A } \\
\cline { 2 - 7 } & $\begin{array}{c}\text { Serum- } \\
\text { free }\end{array}$ & $\begin{array}{c}\text { With } \\
\text { serum }\end{array}$ & $\begin{array}{c}\text { Serum- } \\
\text { free }\end{array}$ & $\begin{array}{c}\text { With } \\
\text { serum }\end{array}$ & $\begin{array}{c}\text { Serum- } \\
\text { free }\end{array}$ & $\begin{array}{c}\text { With } \\
\text { Serum }\end{array}$ \\
\hline $\mathbf{1}$ & $4.0 \pm 0.6$ & $29.2 \pm 0.6$ & $7.9 \pm 1.2$ & $53 \pm 2$ & $2.0 \pm 0.3$ & $19 \pm 4$ \\
\hline $\mathbf{B A}$ & $>50$ & $>100$ & $>50$ & $>100$ & $>50$ & $>100$ \\
\hline $\mathbf{2 b}-\mathrm{B}(\mathrm{OH})_{2}$ & $3.8 \pm 0.9$ & $15 \pm 3$ & $6 \pm 1$ & $24 \pm 1$ & $2.35 \pm 0.09$ & $7 \pm 1$ \\
\hline $\mathbf{2 b}-\mathrm{OH}$ & $2.6 \pm 0.4$ & $6 \pm 1$ & $3.1 \pm 0.8$ & $16.1 \pm 0.6$ & $2.2 \pm 0.5$ & $9.2 \pm 0.1$ \\
\hline
\end{tabular}
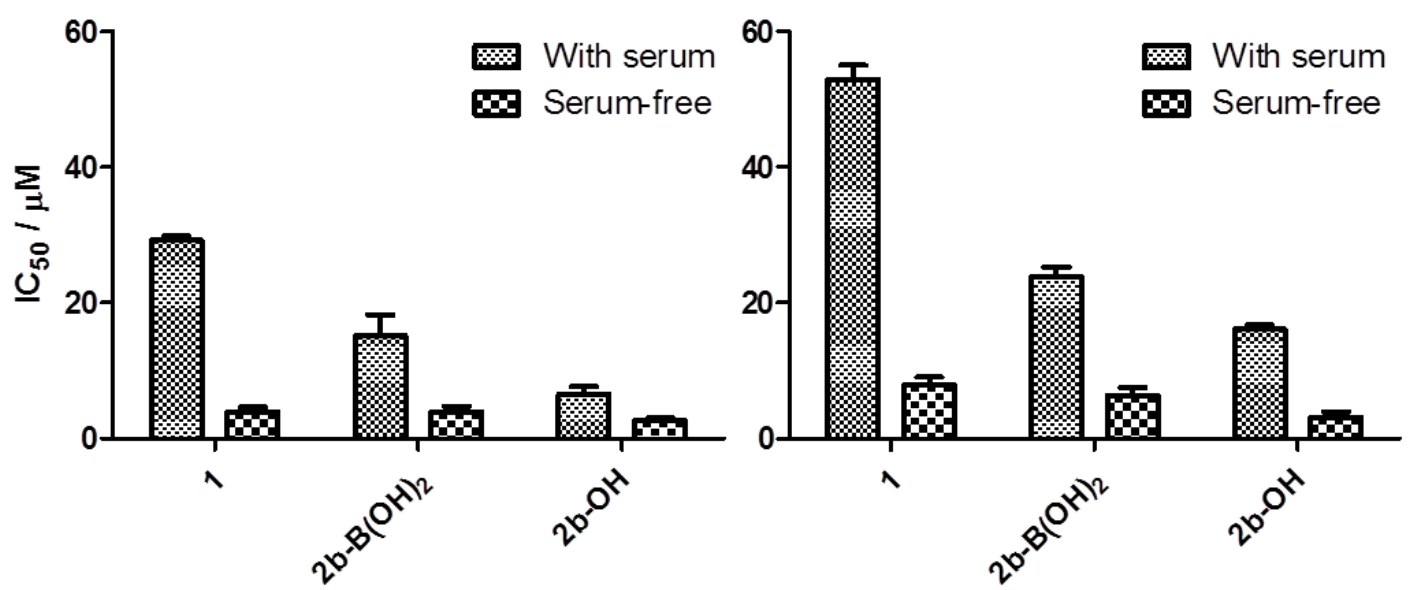

Figure 3.12. Comparison of the $\mathrm{IC}_{50}$ of $\mathbf{1}, \mathbf{2 b}-\mathrm{B}(\mathrm{OH})_{2}$ and $-\mathrm{OH}$ on MDA-MB-231 and MCF-7 cells under serum and serum-free conditions. 


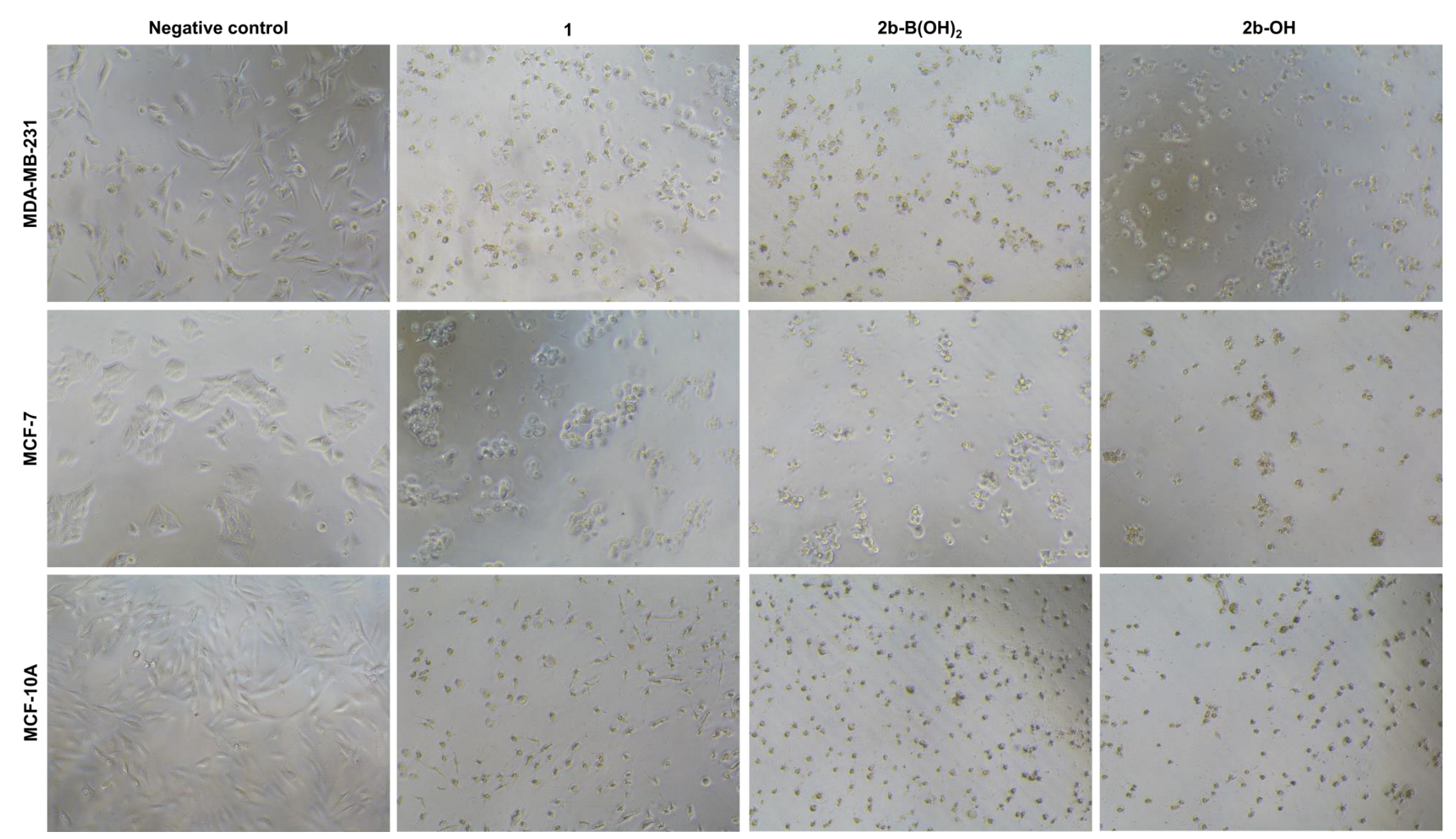

Figure 3.13. Bright-field images of MDA-MB-231, MCF-7 and MCF-10A cells, in serum-free condition, after 24 h incubation with $10 \mu \mathrm{M}$ solutions of $\mathbf{1}$, $\mathbf{2 b}-\mathrm{B}(\mathrm{OH})_{2}$ and $\mathbf{2 b}-\mathrm{OH}$. 


\subsection{Induction of apoptosis by $2 \mathrm{~b}-\mathrm{B}(\mathrm{OH})_{2}$ and $-\mathrm{OH}$}

Cell death can be broadly categorized into either apoptosis or necrosis. ${ }^{93,94}$ Apoptosis, also known as programmed cell death, is an active and controlled process of cellular self-destruction that can be triggered internally or externally, while necrosis is regarded as a form of passive, accidental cell death triggered by external stimuli. Apoptosis is tightly regulated within the cell and plays critical role in cell growth and maintenance of healthy cell population. Alternatively, this form of cell death has been utilized by many for the development of apoptosis-inducing drugs for targeting cancer cells. $^{95,}{ }^{96}$ Some examples include doxorubicin, ${ }^{97}$ palitaxel, ${ }^{98}$ cisplatin, ${ }^{99}$ and more recently osmium carbonyl clusters. ${ }^{86,100}$ Hence, it is of interest to investigate the mode of action of $\mathbf{2} \mathbf{b}-\mathrm{B}(\mathrm{OH})_{2}$ and $-\mathrm{OH}$ whether they triggered cell death via the apoptopic pathway.

\subsubsection{Cell nuclear staining using Hoechst 33342}

Morphological changes in the nuclei are regarded as hallmarks of apoptosis and some of these include shrinkage of the nuclei, chromatin condensation and nuclei fragmentation. ${ }^{101-103}$ These characteristics can be visualized in pre-stained cells via fluorescence microscopy. Cell nuclear staining can be carried out using Hoechst stains (developed by the German company Hoechst AG) - a class of bisbenzimide fluorescent dye widely used as nuclear counterstain. ${ }^{104}$ In particular, the water-soluble and cell mebrane permeable Hoechst 33342, is one of the most frequently used DNA counterstain suitable for cell cycle and apoptosis studies (figure 3.14). ${ }^{105}$

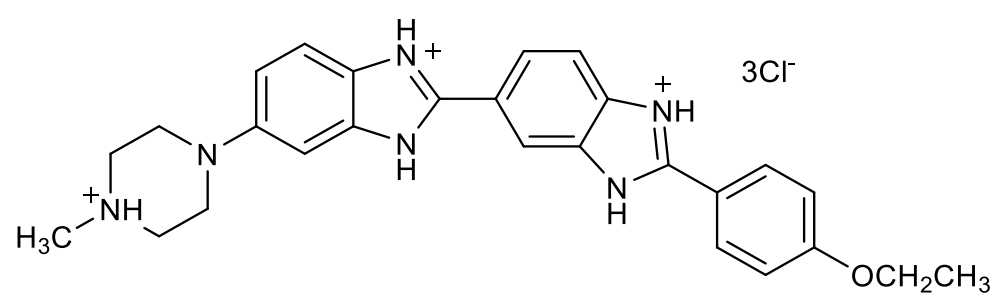

Figure 3.14. Molecular structure of Hoechst 33342.

Live MCF-7 cells treated with $\mathbf{1}, \mathbf{2 b}-\mathrm{B}(\mathrm{OH})_{2}$ and $-\mathrm{OH}$ were stained with Hoechst 33342 and imaged with fluorescence microscopy (figure 3.15). The nuclear morphology of cells treated with $\mathbf{2 b}-\mathrm{B}(\mathrm{OH})_{2}$ and $-\mathrm{OH}$ showed nuclei of reduced size and distorted shape, a sign of early apoptosis. Cells with nuclei showing chromatin 
condensation were observed, similar to that of positive control (cluster 1); in comparison, in the negative control $(0.5 \%$ DMSO, v/v), the nuclei appeared round and intact.

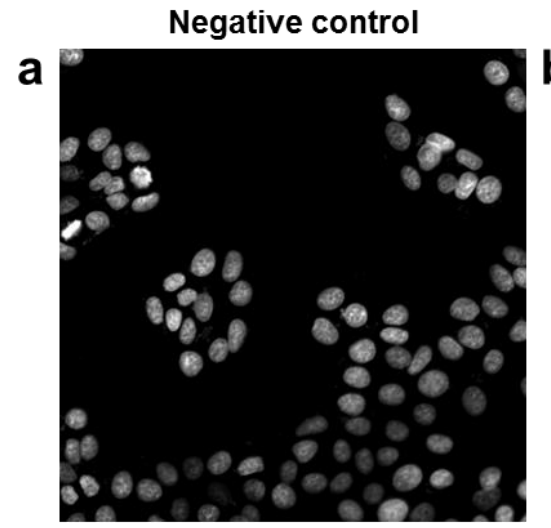

$2 \mathrm{~b}-\mathrm{B}(\mathrm{OH})_{2}(6 \mu \mathrm{M})$

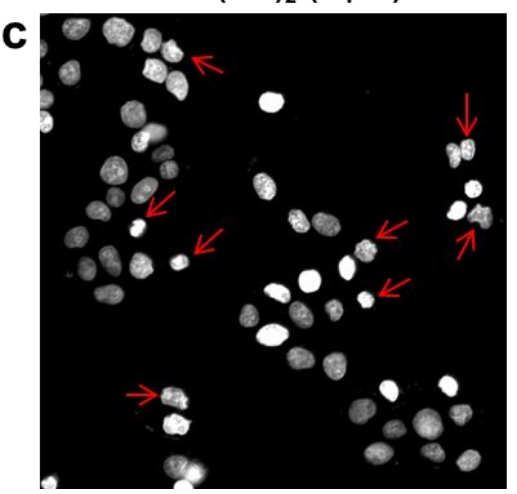

$1(4 \mu \mathrm{M})$

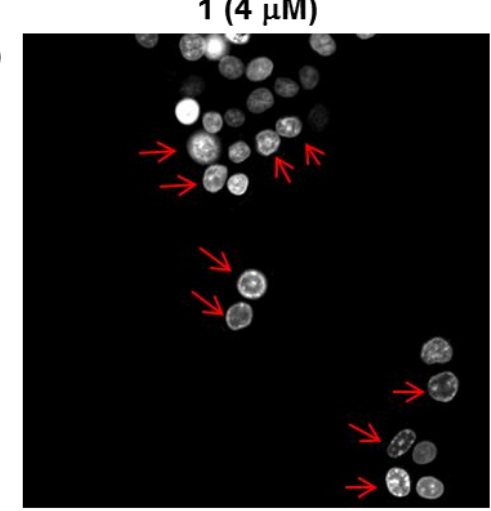

2b-OH (4 $\mu \mathrm{M})$

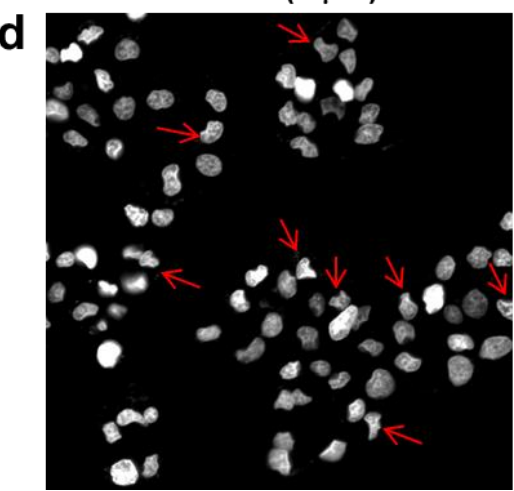

Figure 3.15. Fluorescence images of MCF-7 cells stained with Hoechst 33342 after $20 \mathrm{~h}$ treatment with: a) negative control, 0.5\% DMSO (v/v), b) $1(4 \mu \mathrm{M})$, c) $\mathbf{2 b}-\mathrm{B}(\mathrm{OH})_{2}(6 \mu \mathrm{M})$ and d) $\mathbf{2 b}-\mathrm{OH}(4 \mu \mathrm{M})$. The arrows indicate apoptotic cells.

Changes in the nuclear morphology were quantified through image-based single cell phenotypic analysis of the nuclear area and fluorescence intensity of the cell population. ${ }^{106}$ Typically, the fluorescence images of the cells were processed using an in-house image-processing software (cellXpress) which segments the cells singly and extracts the relevant cellular features for quantification. ${ }^{107}$ This well-established technique has also been used in a high-throughput image-based nephrotoxicity prediction platform for toxic compounds. ${ }^{108}$ Apoptotic cells generally display reduced nuclear area and fluorescence intensity in comparison to normal cells. MCF-7 cells treated with increasing concentrations of both $\mathbf{2 b}-\mathrm{B}(\mathrm{OH})_{2}$ and $\mathbf{2 b}-\mathrm{OH}$ resulted in increased population of cells with smaller nuclear size and lower DNA intensity (lower left quadrant of the contour-scatter plots) in comparison to the negative control (figure 3.16), suggestive of apoptosis. The contour plot of the negative control showed two 
distinct cell populations characterized by the G1 and G2 phases of the cell cycle, respectively (figure 3.16a). Treatment with the clusters resulted in a shift in the cell population to the $\mathrm{G} 2$ phase (top right quadrant) which may suggest $\mathrm{G} 2 / \mathrm{M}$ arrest; the cell cycle was halted and the cells were not able to undergo mitotic cell division.
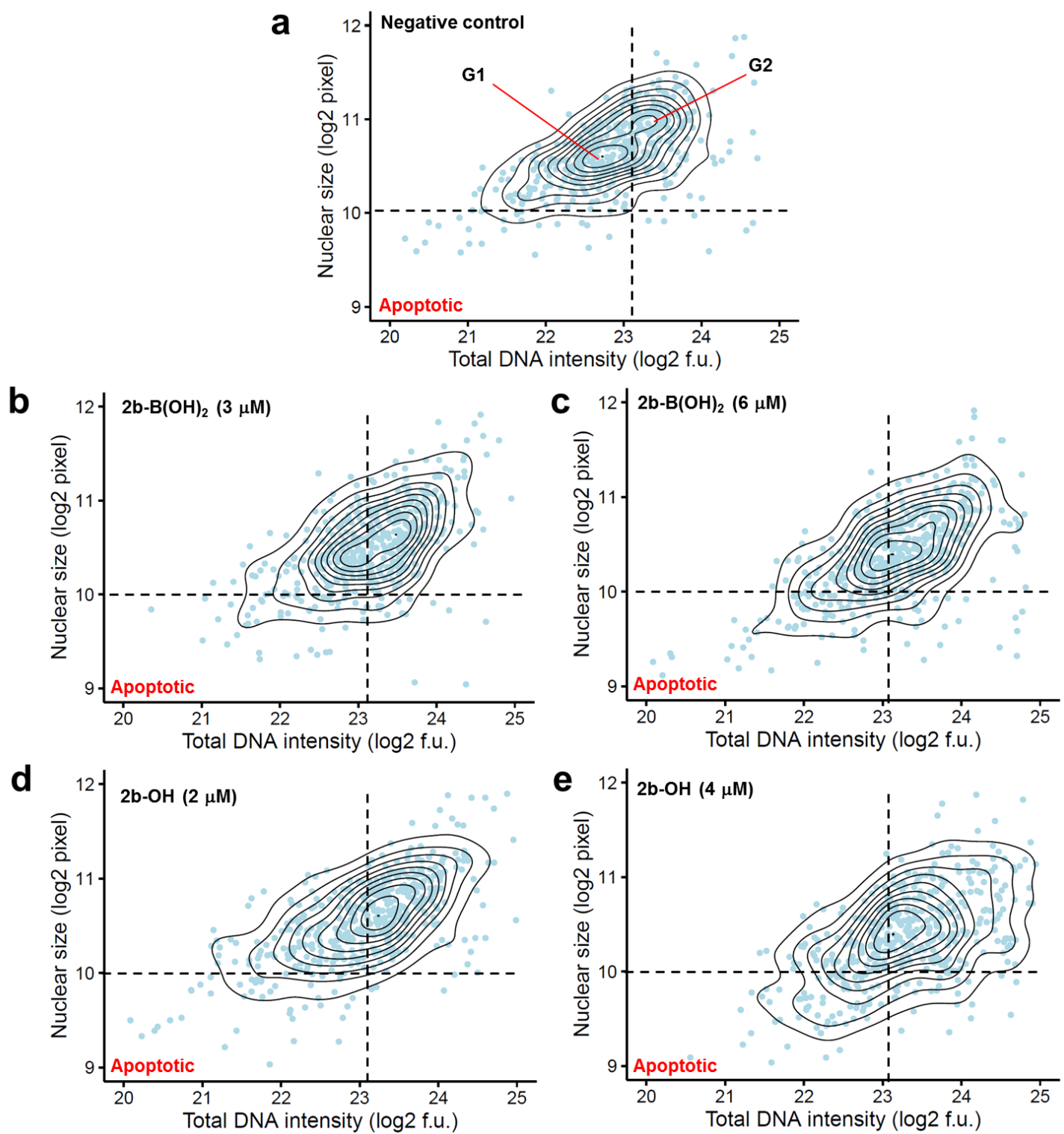

Figure 3.16. Contour-scatter plots showing the nuclear area vs total DNA fluorescence intensity of single MCF-7 cells after 20 h treatment: a) negative control, $0.5 \%$ DMSO (v/v), bc) $\mathbf{2 b}-\mathrm{B}(\mathrm{OH})_{2}$ (3 and $6 \mu \mathrm{M}$ respectively) and d-e) $\mathbf{2 b}-\mathrm{OH}(2$ and $4 \mu \mathrm{M}$ respectively). The dots represent single-cell measurements quantified from the images. 


\subsubsection{Phosphatidylserine detection by Annexin V-FITC conjugate}

Another feature of apoptosis is the externalization of phosphatidylserine (PS) at the cell surface, which is found in the inner side of the membrane of live cells. ${ }^{109,110}$ The exposure of PS on the surface provides recognition sites for macrophages to remove the apoptotic body and also allows the binding of the lipid binding protein, Annexin V, in the presence of $\mathrm{Ca}^{2+}$ ions (figure 3.17). ${ }^{111,112}$ Thus, Annexin V conjugated to a fluorescence probe, fluorescein isothiocyanate (FITC), is commonly used as a label for detecting PS in apoptotic cells. ${ }^{113}$

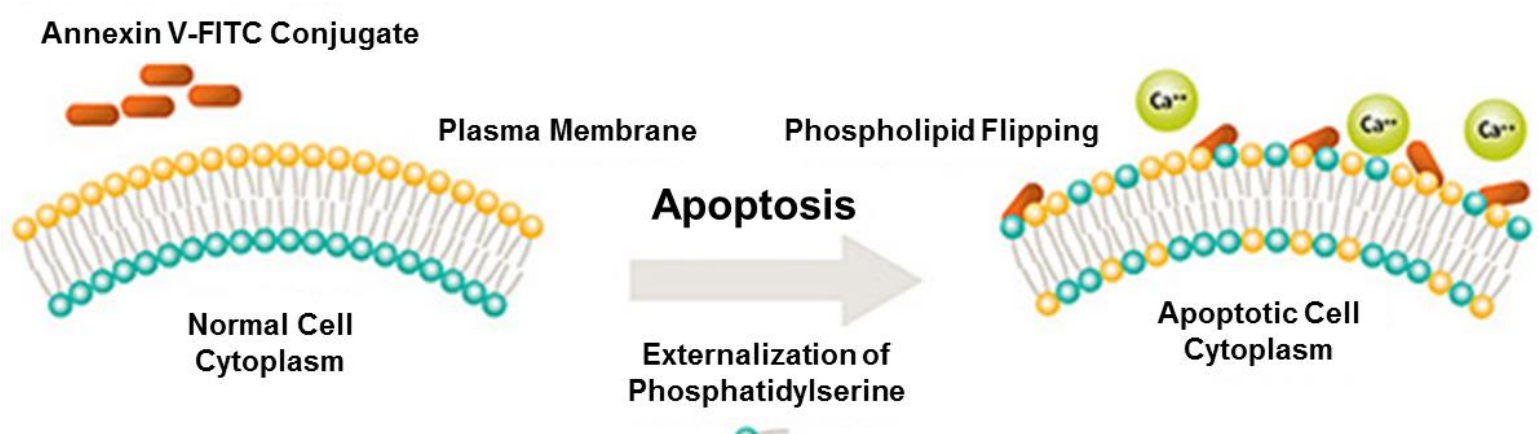

Figure 3.17. Externalization of phosphartidylserine at the plasma membrane of apoptotic cells and detection by Annexin V-FITC conjugate. Adapted from: http://www.bdbiosciences.com/br/instruments/accuri/articles/archive/2015_02/index.jsp. Accessed on 25 Jul 2016.

MCF-7 cells treated with 1, 2b-B $(\mathrm{OH})_{2}$ and $-\mathrm{OH}$ were stained with Hoechst 33342 and Annexin V-FITC and visualized under a fluorescence microscope (figure 3.18). The images showed significant increase in Annexin V-FITC staining (green) in cells treated with $\mathbf{1}, \mathbf{2} \mathbf{b}-\mathrm{B}(\mathrm{OH})_{2}$ and $-\mathrm{OH}$ in comparision to the negative control. The intensity also increased with treatment at higher concentration of $\mathbf{2 b}-\mathbf{B}(\mathrm{OH})_{2}$ and $-\mathrm{OH}$; image-based quantification of the the membrane (non-DNA region) also showed substantial Annexin V-FITC staining with the increase in concentration (figure 3.19). Together with the information obtained from the nuclear staining, these suggest that $\mathbf{2 b}-\mathrm{B}(\mathrm{OH})_{2}$ and $-\mathrm{OH}$ were apoptosis inducing. 


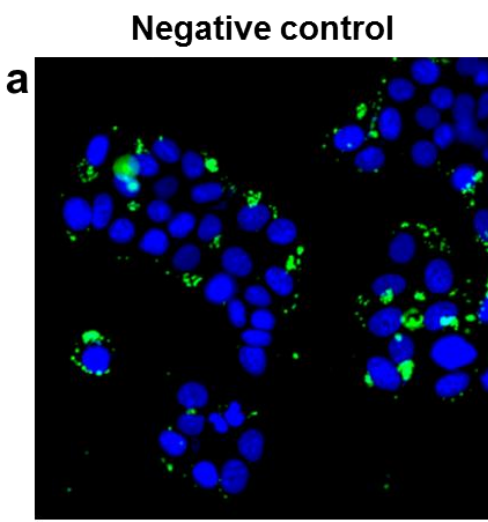

$2 \mathrm{~b}-\mathrm{OH}(2 \mu \mathrm{M})$
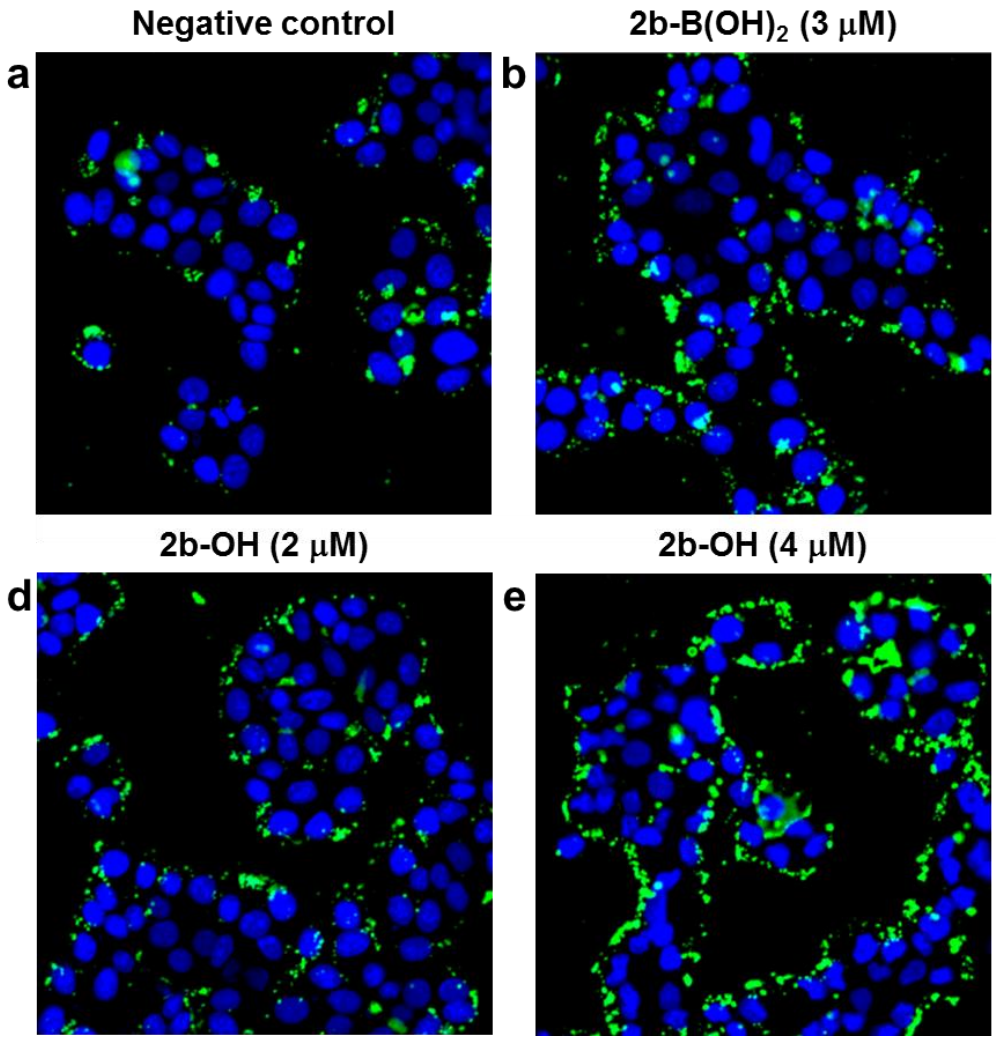

$2 \mathrm{~b}-\mathrm{OH}(4 \mu \mathrm{M})$
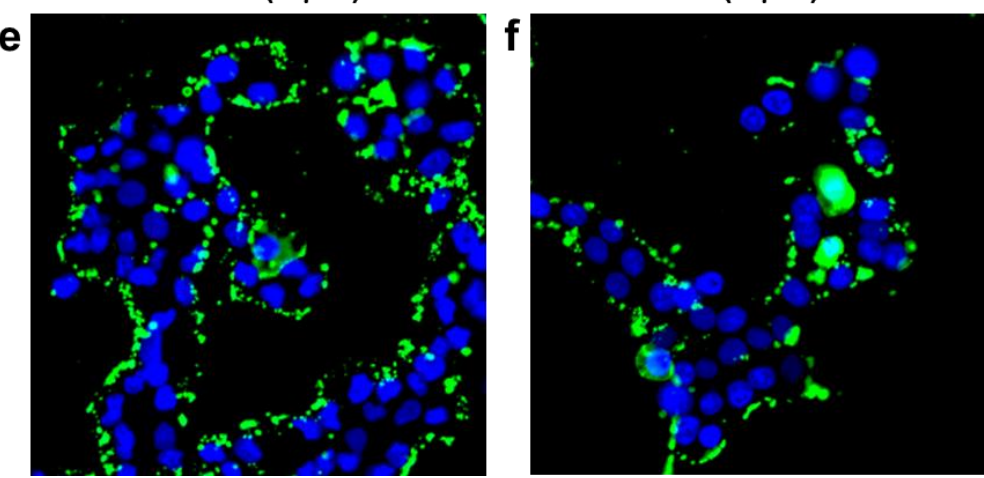

Figure 3.18. Fluorescence images of MCF-7 cells stained with Hoechst 33342 (blue) and Annexin V-FITC (green) after $20 \mathrm{~h}$ treatment with: a) negative control, 0.5\% DMSO (v/v), b-c) $\mathbf{2 b}-\mathrm{B}(\mathrm{OH})_{2}$ (3 and $6 \mu \mathrm{M}$ respectively), d-e) $\mathbf{2 b}-\mathrm{OH}$ (2 and $4 \mu \mathrm{M}$ respectively) and f) $\mathbf{1}(4 \mu \mathrm{M})$.

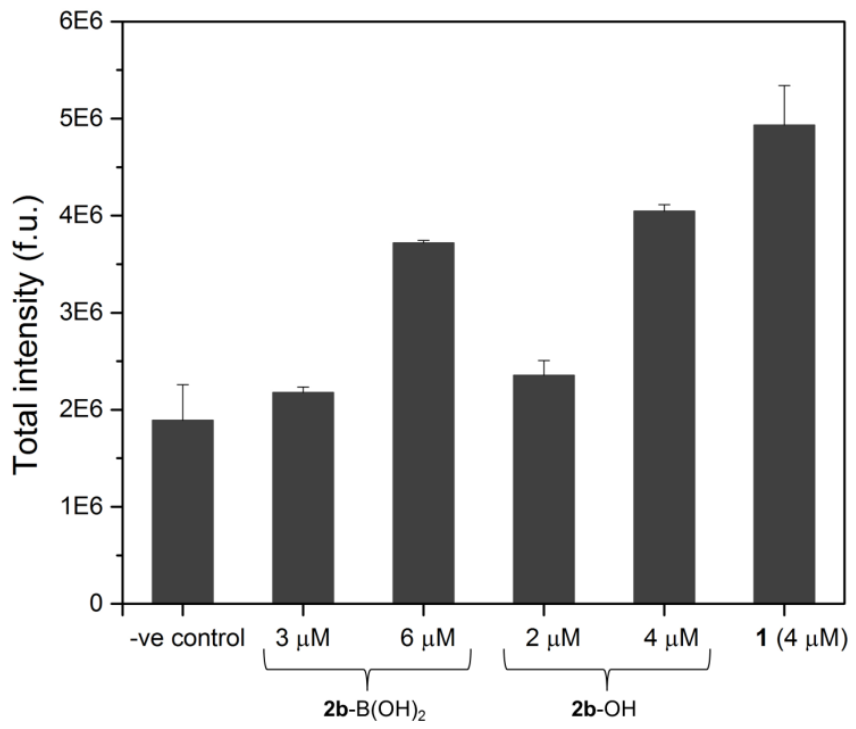

Figure 3.19. Image-based quantification of total fluorescence intensity of Annexin V-FITC from MCF-7 cells treated with various clusters. Negative control, 0.5\% DMSO (v/v). 


\subsection{Chemical oxidation of $2 \mathrm{~b}-\mathrm{B}(\mathrm{OH})_{2}$ to $2 \mathrm{~b}-\mathrm{OH}$ and electrochemical studies}

The study above has shown that $\mathbf{2 b}-\mathrm{B}(\mathrm{OH})_{2},-\mathrm{OH}$ and $-\mathrm{COOH}$ exhibit similar toxicities ( $\mathrm{IC}_{50}$ values). The source of cytotoxicity is likely to be from the phenylthiolato triosmium core, with the substituents on the phenyl ring helping to improve water solubility. Further chemical and electrochemical studies were carried out in attempts to understand the mode of action of these clusters.

The boronic acid functionality of $\mathbf{2} \mathbf{b}-\mathrm{B}(\mathrm{OH})_{2}$, which was intended to interact with sialic acid, can undergo oxidation by $\mathrm{H}_{2} \mathrm{O}_{2}$ to form the hydroxyl group; ${ }^{114}$ this forms the basis for the development of boronic acid or boronate esters-based sensors for $\mathrm{H}_{2} \mathrm{O}_{2}$ sensing (scheme 3.5). ${ }^{115-117}$ The possible chemical oxidation of $\mathbf{2 b}-\mathrm{B}(\mathrm{OH})_{2}$ to $\mathbf{2 b}-\mathrm{OH}$ was thus investigated.

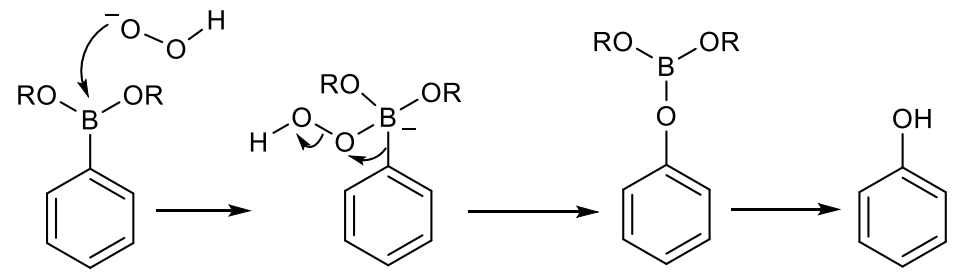

Scheme 3.5. Oxidation of boronic acid or boronate ester by $\mathrm{H}_{2} \mathrm{O}_{2}{ }^{115}$

The conversion of $\mathbf{2 b}-\mathrm{B}(\mathrm{OH})_{2}$ to $\mathbf{2} \mathbf{b}-\mathrm{OH}$ in the presence of $\mathrm{H}_{2} \mathrm{O}_{2}$ and imidazole as base was confirmed by IR spectroscopy (figure 3.20). The shift in the CO vibrational bands to lower wavenumber, in the presence of imidazole, was indicative of the conversion to $\mathbf{2 b}-\mathbf{O H}$, as verified with an authentic sample of $\mathbf{2} \mathbf{b}-\mathrm{OH}$. Monitoring the reaction by ESI-HRMS revealed a decline in the ratio of $\mathbf{2 b}-\mathbf{B}(\mathrm{OH})_{2}(\mathrm{~m} / \mathrm{z}$ 1004) to $\mathbf{2} \mathbf{b}$ $\mathrm{OH}(\mathrm{m} / \mathrm{z}, 976)$ over time (figure 3.21). A control setup without the addition of $\mathrm{H}_{2} \mathrm{O}_{2}$ showed a consistent high ratio of peaks at 1004 to 976 throughout the reaction, indicating little or no loss of $\mathbf{2} \mathbf{b}-\mathrm{B}(\mathrm{OH})_{2}$. 

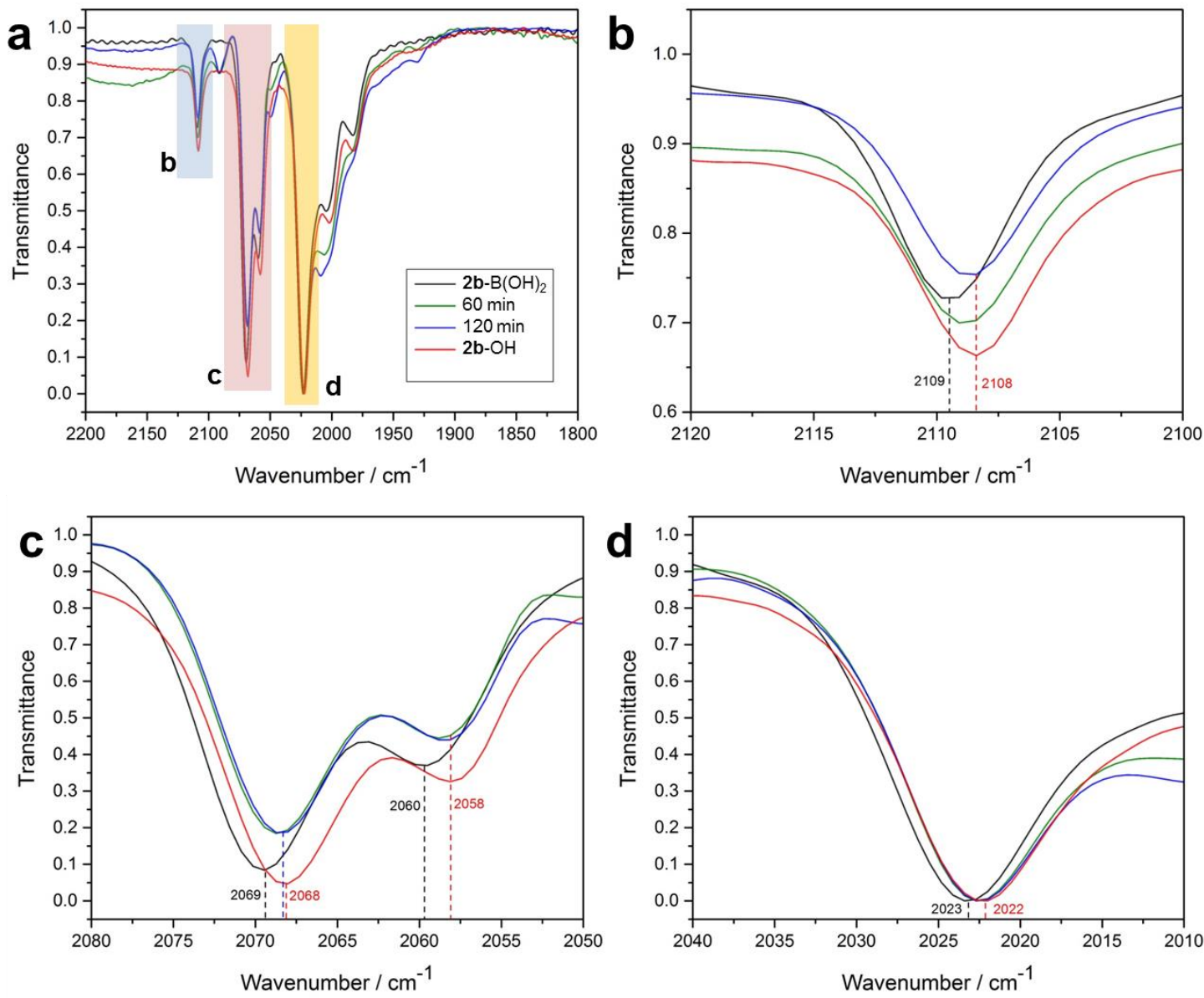

Figure 3.20. a) Overlays of IR spectra of $2 \mathbf{b}-\mathrm{B}(\mathrm{OH})_{2}$ incubated with 5 eq. of $\mathrm{H}_{2} \mathrm{O}_{2}$ and 1 eq. of imidazole in $\mathrm{H}_{2} \mathrm{O}: \mathrm{MeOH}(1: 3, \mathrm{v} / \mathrm{v})$ over time. Authentic spectra of $\mathbf{2 b}-\mathrm{B}(\mathrm{OH})_{2}$ and $-\mathrm{OH}$ were overlaid for comparison. All spectra were taken in $\mathrm{MeOH}$. b-d) Expanded regions of various bands in the spectra. 


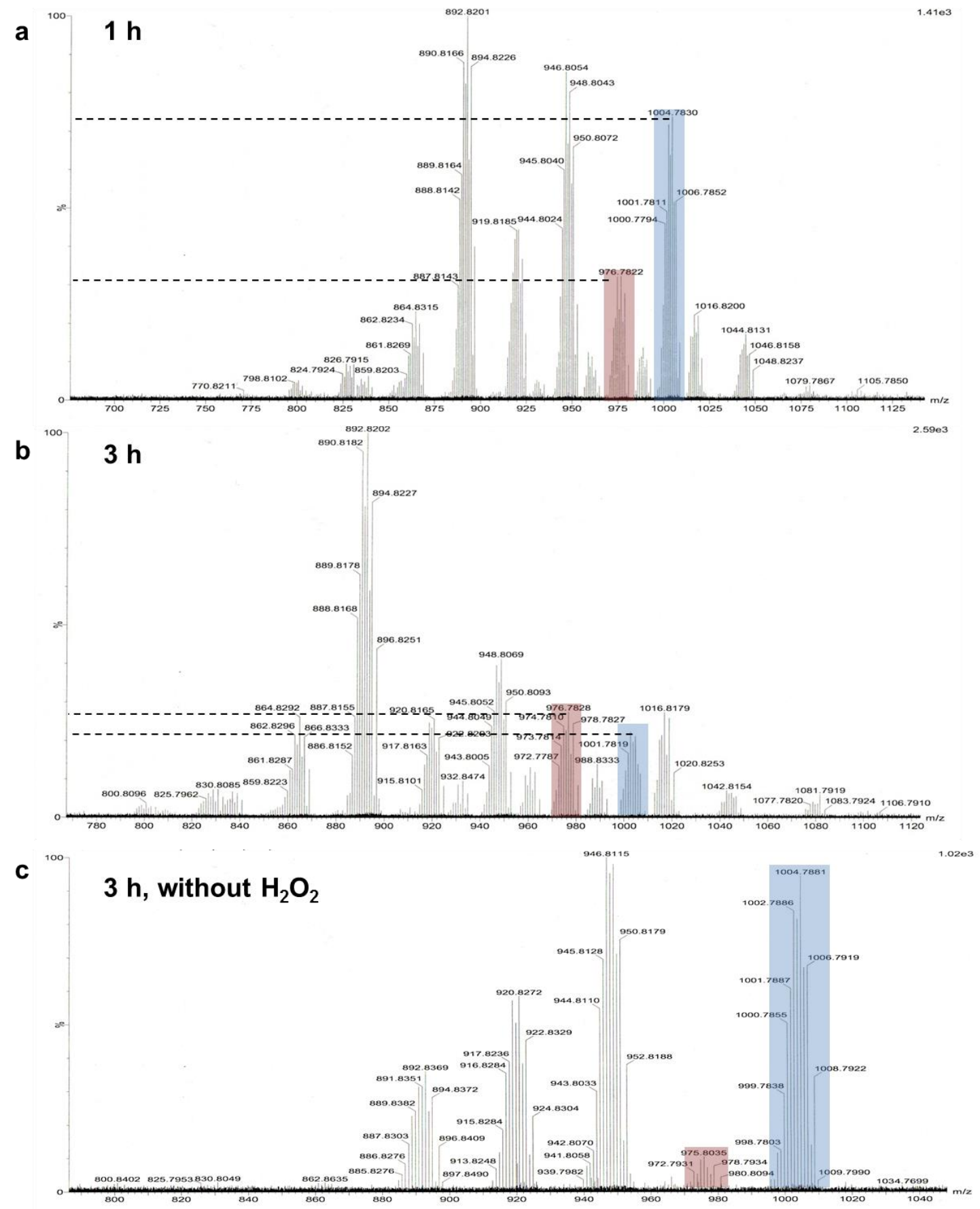

Figure 3.21. ESI-HRMS of $2 \mathbf{b}-\mathrm{B}(\mathrm{OH})_{2}$ incubated with 5 eq. of $\mathrm{H}_{2} \mathrm{O}_{2}$ and 1 eq. of imidazole in $\mathrm{H}_{2} \mathrm{O}: \mathrm{MeOH}\left(1: 3\right.$, v/v) taken at a) $1 \mathrm{~h}$ and b) 3 h. c) ESI-HRMS of $2 \mathbf{b}-\mathrm{B}(\mathrm{OH})_{2}$ incubated with 1 eq. imidazole but without the addition of $\mathrm{H}_{2} \mathrm{O}_{2}$. The $\mathrm{m} / \mathrm{z}$ isotopic pattern corresponding to $\mathbf{2 b}$ $\mathrm{B}(\mathrm{OH})_{2}$ was highlight in blue, while the $\mathbf{2} \mathbf{b}-\mathrm{OH}$ was in red.

Organometallic-based anti-cancer drugs like ferrocifen (ferrocene analogue of tamoxifen) can undergo phenol oxidation as part of its activation process; ferrocenemodulated phenol oxidation to the reactive quinone methide is believed to be 
responsible for their cytotoxicity (scheme 3.6). ${ }^{118-120}$ Cluster $\mathbf{2 b - O H}$ draws similarity with ferrocifen as it contains a phenol moiety which can potentially undergo phenol oxidation. The electrochemical behavior of $\mathbf{2} \mathbf{b}-\mathrm{OH}$ was thus investigated by cyclic voltammetry $(\mathrm{CV})$, using $n \mathrm{Bu}_{4} \mathrm{NPF}_{6}$ as the supporting electrolyte.

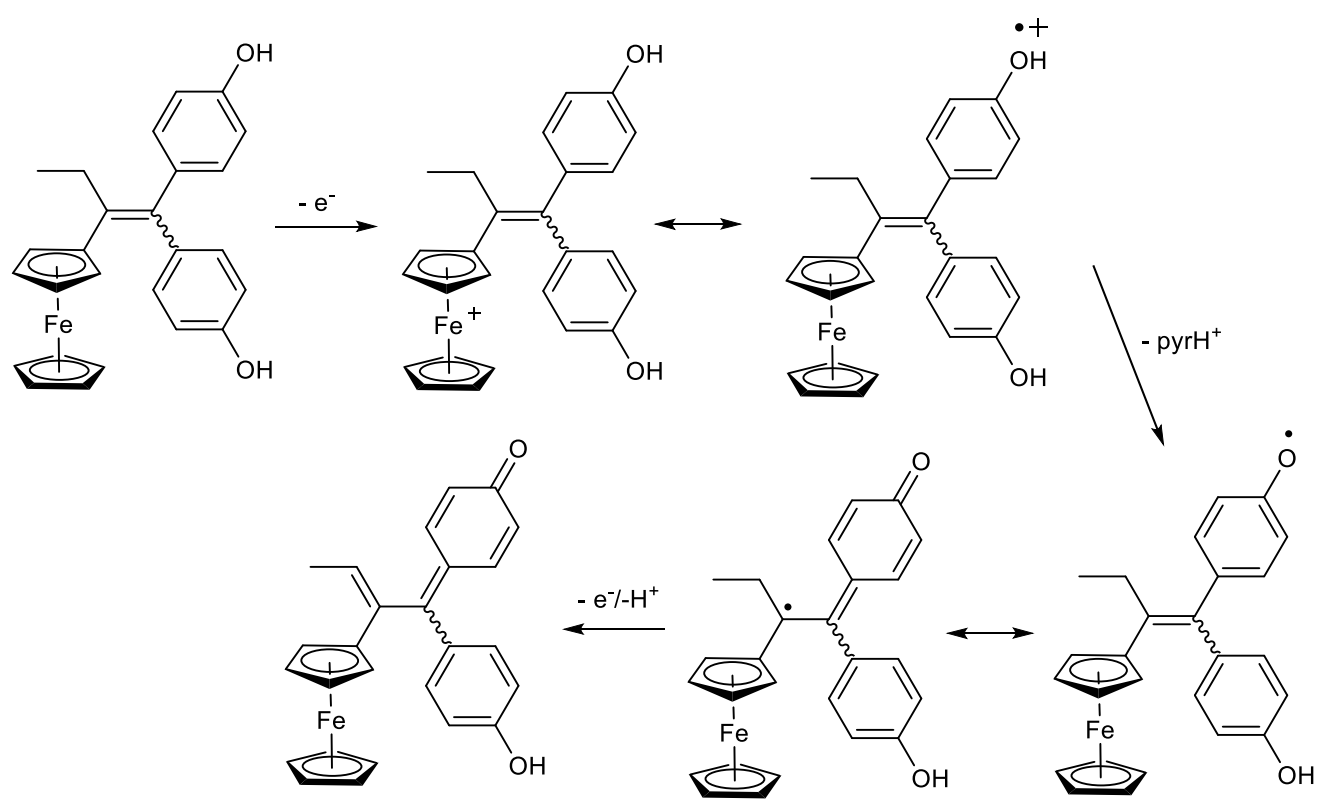

Scheme 3.6. Proposed mechanism for the oxidation and formation of quinone methides from ferrocenyl phenol. ${ }^{118}$

The cyclic voltammogram of $\mathbf{2} \mathbf{b}-\mathrm{OH}$ showed a chemically irreversible oxidation process at $0.80 \mathrm{~V}$ vs. $\mathrm{Fc} / \mathrm{Fc}^{+}$. The $\mathrm{CV}$ of the analogues, $\mathbf{2} \mathbf{b}$ and $\mathbf{2} \mathbf{b}-\mathrm{COOH}$, showed similar chemically irreversible oxidation processes at $0.82 \mathrm{~V}$ and $0.85 \mathrm{~V}$, respectively, under the same conditions (figure 3.22). This implies that oxidation occurred on the triosmium cluster core, since the oxidation potential appears to be independent of the functional groups on the phenyl ring. The oxidized species are unstable, resulting in the corresponding reduction process not being observed when the scan direction is reversed. This is in agreement with other reports that the oxidation of the triosmium pyridyl cluster $\left[\mathrm{Os}_{3}(\mu-\mathrm{H})(\mathrm{CO})_{10}\left(\mu-\mathrm{NC}_{5} \mathrm{H}_{4}\right)\right]$, and analogues, were irreversible; the oxidation was attributed to the cluster and was not affected by substituents on the pyridyl ligand. ${ }^{121}$ 


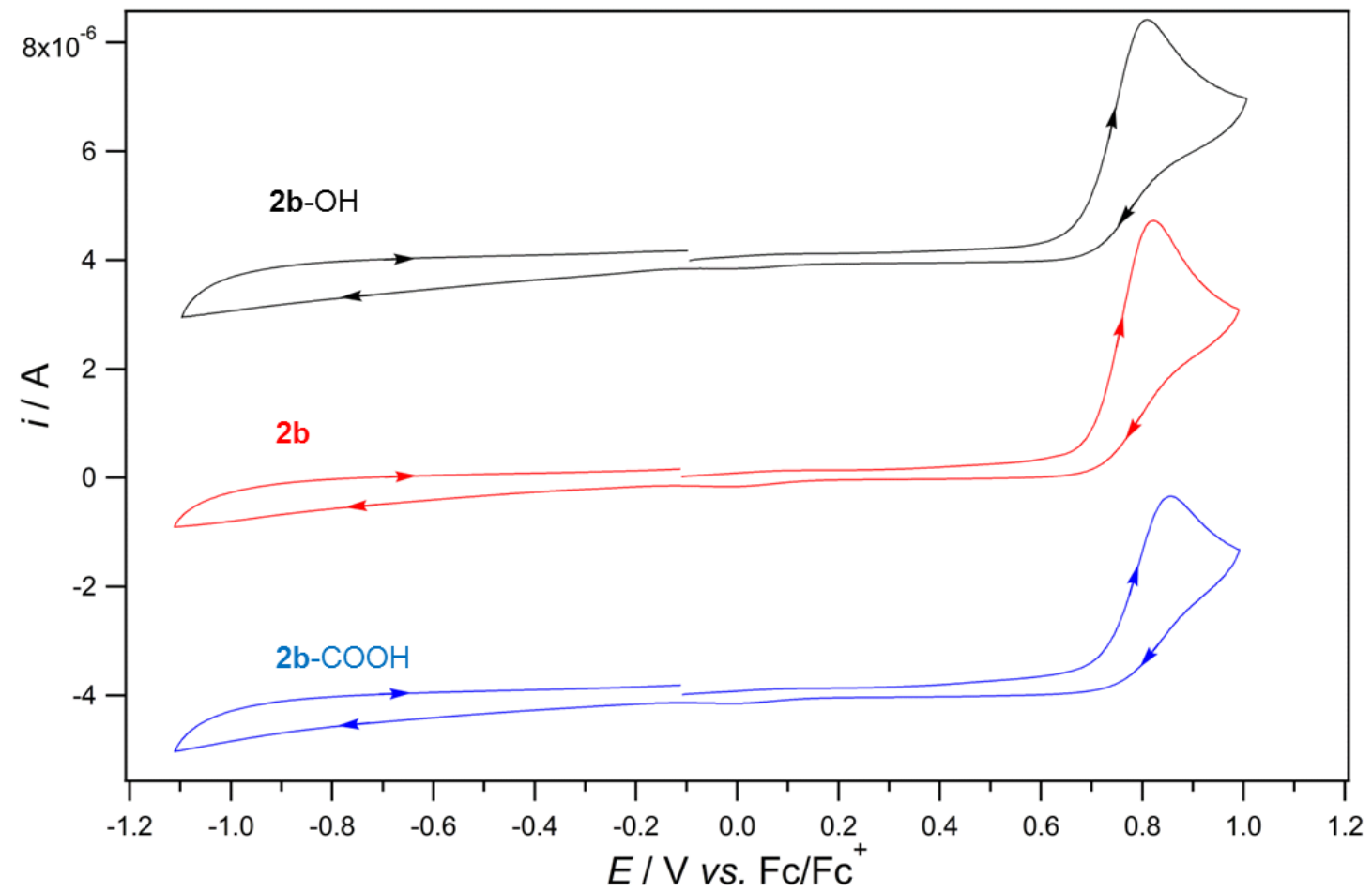

Figure 3.22. Cyclic voltammograms of $2 \mathrm{mM}$ of $\mathbf{2 b}-\mathrm{OH}, \mathbf{2 b}$ and $\mathbf{2 b}-\mathrm{COH}$ in $\mathrm{CH}_{3} \mathrm{CN}$ at $22 \pm$ $0.2{ }^{\circ} \mathrm{C}$ with $0.1 \mathrm{M} \mathrm{nBu} \mathrm{BPF}_{6}$, recorded at a $1 \mathrm{~mm}$ diameter planar glassy carbon electrode at a scan rate of $0.1 \mathrm{~V} \mathrm{~s}^{-1}$ 


\subsection{Conclusion}

A triosmium carbonyl cluster-boronic acid conjugate, $\mathbf{2 b}-\mathrm{B}(\mathrm{OH})_{2}$ was utilized as a SERS probe for selective glucose detection using BMFON as the SERS-active substrate in a sandwich assay. The assay showed good selectivity towards glucose over fructose and galactose. Quantification was carried out using the CO stretching vibration of the conjugate $\mathbf{2 b}-\mathrm{B}(\mathrm{OH})_{2}$, and had a detection range of 0.1-10 mM. This glucose assay exhibited several advantages: (i) no prior purification of the sample was needed, (ii) an extremely low sample volume was required, (iii) there is very high specificity for glucose, and (iv) the spectroscopic handle for glucose quantification was relatively free from interference by other biomolecules. There remain improvements to be made, however, including the use of a cheaper transition metal and making the detection label-free.

Extension of the application of $\mathbf{2} \mathbf{b}-\mathrm{B}(\mathrm{OH})_{2}$ towards cell-based detection was attempted. However, the unexpected discovery that $\mathbf{2} \mathbf{b}-\mathrm{B}(\mathrm{OH})_{2}$ is cytotoxic made it inappropriate for its intended used as an intracellular imaging agent, but potentially suitable as a therapeutic agent. Further investigation on other analogues, 2b-OH and $\mathrm{COOH}$, revealed that the cytotoxicity is independent on the substituents on the phenyl ring, but is probably related to the presence of the aromatic ring. Clusters $\mathbf{2} \mathbf{b}-\mathrm{B}(\mathrm{OH})_{2}$ and $-\mathrm{OH}$ were deduced to induce cell death via the apoptotic pathway. Hence, future improvements can be made in enhancing the selectivity of the clusters and to understand the mode of action in greater details. 


\subsection{Experimental section}

\subsubsection{General methods}

All reactions and manipulations were carried out under an argon atmosphere using standard Schlenk techniques. Solvents that were used for reaction were distilled over the appropriate drying agents under argon before use. Purification of compounds was generally carried out by column chromatography on silica gel, or by preparative thinlayer chromatography (TLC) using $20 \mathrm{~cm}$ x $20 \mathrm{~cm}$ plates pre-coated with silica gel 60 $\mathrm{F}_{254}$ Infrared (IR) spectra were recorded on a Bruker Alpha FT-IR spectrometer. Solution spectra were recorded in DCM solution, unless otherwise stated, in a solution IR cell with $\mathrm{NaCl}$ windows and a path length of $0.1 \mathrm{~mm}$, at a resolution of $2 \mathrm{~cm}^{-1}$. For compounds in $\mathrm{MeOH}$ solution, a solution IR cell with $\mathrm{CaF}_{2}$ windows and a path length of $0.1 \mathrm{~mm}$ was used. ${ }^{1} \mathrm{H}$ and ${ }^{13} \mathrm{C}\left\{{ }^{1} \mathrm{H}\right\}$ NMR spectra were recorded on a Bruker Advance BBFO (400 MHz), JEOL ECA 400 or ECA 400SL (400 MHz) spectrometer, in $\left(\mathrm{CD}_{3}\right)_{2} \mathrm{CO} .{ }^{1} \mathrm{H}$ and ${ }^{13} \mathrm{C}\left\{{ }^{1} \mathrm{H}\right\}$ chemical shifts are referenced to the residual proton and carbon resonance of the solvent. HRMS were recorded in ESI mode on a Waters UPLC-Q-Tof MS mass spectrometer. The Raman spectral measurements were carried out using a Renishaw InVia Raman (UK) microscope with a Peltier cooled CCD detector and an excitation wavelength at $785 \mathrm{~nm}$, where the laser beam was directed to the sample through a $50 \times$ objective lens, which was used to excite the sample and also to collect the return Raman signal. All Raman spectra were processed with the WiRE3.4 software. The laser power at the sample was measured to be $20.8 \mathrm{~mW}$ and the exposure time was set at $10 \mathrm{~s}$, throughout the measurements. Prior to each measurement, the instrument was calibrated against a silicon standard with a Raman peak centered at $520 \mathrm{~cm}^{-1}$.

Cluster $\mathbf{1}$ and $\mathbf{2 b}$ were prepared according to reported procedures with slight modifications. ${ }^{89,122} \mathrm{Os}_{3}(\mathrm{CO})_{12}$ was purchased from Oxkem Ltd, and all other reagents were purchased from other commercial sources and used as supplied without further purification.

\subsubsection{Synthesis of cluster $2 \mathrm{~b}-\mathrm{B}(\mathrm{OH})_{2}$}

Cluster 1 (26 mg, $0.028 \mathrm{mmol}$ ) and 4-mercaptophenylboronic acid (5 mg, $0.034 \mathrm{mmol}$ ) were dissolved in THF (6 mL) and left to stir at room temperature overnight. Solvent was removed in vacuo and the residue was purified by TLC using ethyl acetate:hexane $(1: 1, v / v)$ as eluant to yield one major yellow band as cluster $\mathbf{2 b}-\mathrm{B}(\mathrm{OH})_{2}$. 
Yield: $18 \mathrm{mg}(63 \%)$

$\mathrm{R}_{\mathrm{f}}=0.36$

IR (DCM, $\left.\mathrm{cm}^{-1}\right)$ : $\mathrm{v}_{\mathrm{CO}} 2110 \mathrm{w}, 2069 \mathrm{~s}, 2060 \mathrm{~m}, 2023 \mathrm{~s}, 2001 \mathrm{~m}, 1987 \mathrm{w}$.

${ }^{1} \mathrm{H}$ NMR $\left(400 \mathrm{MHz},\left(\mathrm{CD}_{3}\right)_{2} \mathrm{CO}\right) \delta 7.84(2 \mathrm{H}, \mathrm{d}, J=8.4, \mathrm{Ar}), 7.43(2 \mathrm{H}, \mathrm{d}, J=8.4, \mathrm{Ar})$, $7.32\left(2 \mathrm{H}, \mathrm{s}, \mathrm{B}(\mathrm{OH})_{2}\right),-16.93(1 \mathrm{H}, \mathrm{s}$, OsHOs).

${ }^{13} \mathrm{C}\left\{{ }^{1} \mathrm{H}\right\}$ NMR $\left(100 \mathrm{MHz},\left(\mathrm{CD}_{3}\right)_{2} \mathrm{CO}\right): \delta 182.20,181.57,177.39,175.29,172.74$, $170.64,170.61,148.12,135.17,131.43$.

HRMS-ESI $m / z$ calculated for $\mathrm{C}_{16} \mathrm{H}_{6} \mathrm{BO}_{12} \mathrm{~S}^{190} \mathrm{Os}_{3}[\mathrm{M}-\mathrm{H}]^{-} 1002.8427$, found 1002.8414 .

\subsubsection{Incubation of $2 \mathrm{~b}-\mathrm{B}(\mathrm{OH})_{2}$ with glucose}

Cluster $\mathbf{2 b}-\mathrm{B}(\mathrm{OH})_{2}\left(2 \mathrm{mg}, 2.0 \times 10^{-3} \mathrm{mmol}\right)$ dissolved in $\mathrm{MeOH}(200 \mu \mathrm{L})$ was added to D-glucose $(2 \mathrm{mg}, 0.011 \mathrm{mmol})$ dissolved in $\mathrm{pH} 9.0$ sodium hydroxide solution (200 $\mu \mathrm{L}$ ). The reaction mixture was incubated at room temperature overnight. The mixture was further diluted with MeOH prior to HRMS-ESI analysis.

HRMS-ESI $\mathrm{m} / \mathrm{z}$ calculated for $\mathrm{C}_{22} \mathrm{H}_{16} \mathrm{BO}_{17} \mathrm{~S}^{187} \mathrm{Os}{ }^{192} \mathrm{Os}_{2}[\mathrm{M}]^{-}$1165.8989, found 1165.8999.

\subsubsection{Fabrication of BMFON substrate}

Monodisperse PS colloidal suspension $(\varnothing=384 \mathrm{~nm}, 2.5 \mathrm{wt} \%)$ stored at $4{ }^{\circ} \mathrm{C}$ was purchased from Kisker. During preparation, the PS sphere solution was added with $15 \mathrm{wt} \%$ surfactant sodium dodecyl sulphate (SDS), to composite solution containing $2.36 \mathrm{wt} \%$ PS spheres and $0.85 \mathrm{wt} \%$ SDS. Clean microscope glass slides used as supporting substrate were cut into square pieces $(10 \mathrm{~mm} \times 10 \mathrm{~mm} \times 1 \mathrm{~mm})$. The glass slides were first sonicated in a bath of ethanol for $20 \mathrm{~min}$ before being dried with argon gas. Using the spin-coating method, PS sphere monolayers were fabricated onto the clean glass slides. This was done by dispersing $10 \mu \mathrm{L}$ of the prepared colloidal solution onto the center of a glass slide. Each glass slide was spin coated in the spin coater preset at $2000 \mathrm{rpm}$ for $20 \mathrm{~s}$. Due to the Bragg diffraction given by the closelypacked PS spheres, a green reflective surface was formed straight after. The coated glass slides were then dried in vacuum desiccators overnight at 0.6 pa pressure. The substrates were first coated with Ag (99.999\% purity, JEOL) at thickness that amount to $120 \mathrm{~s}$ of sputtering (JEOL JFC-1600 Auto fine coater) before being sputtered with a 
layer of $\mathrm{Au}(99.999 \%$ purity, JEOL) for $80 \mathrm{~s}$. Each mental layer was deposited at a rate of $1.33 \mathrm{~nm} / \mathrm{s}$.

\subsubsection{Immobilization of 4-mercaptophenylboronic acid on BMFON}

Fabricated BMFON was incubated in 4-mercaptophenylboronic acid ethanolic solution (10 $\mathrm{mM}$ ) for duration of 2 hours. The substrates were then washed thoroughly with pure ethanol and dry in air.

\subsubsection{Detection of saccharides (glucose, fructose and galactose)}

BA-functionalized BMFON was incubated with varied concentration of saccharides ( $20 \mu \mathrm{L})$ for $1 \mathrm{~h}$. The substrate was then washed thrice with water $(3 \times 2 \mathrm{~mL})$ prior to incubation with a solution of $\mathbf{2 b}-\mathrm{B}(\mathrm{OH})_{2}(2 \mathrm{mM})$ for $1 \mathrm{~h}$. The solution was removed and the substrate was washed thrice with water $(3 \times 2 \mathrm{~mL})$. Subsequently, the substrate is placed on a glass slide and under the Raman microscope objective lens for measurements. An excitation wavelength of $785 \mathrm{~nm}$ was used and the exposure time of $10 \mathrm{~s}$ was used throughout the measurement.

\subsubsection{Detection of glucose in clinical urine sample}

Samples used for the study were from clinical specimens, stored at $-20^{\circ} \mathrm{C}$. Specimens were used in accordance to procedures with approval of the local ethics committe (CIRB Re 2011/558/C) and informed consent was given by all patients. In this experiment, urine sample was added a known amount of glucose and was mixed well. BA-functionalized BMFON was incubated with the doped urine for $1 \mathrm{~h}$. The substrate was then washed thrice with water $(3 \times 2 \mathrm{~mL})$ prior to incubation with a solution of $\mathbf{2 b}-\mathrm{B}(\mathrm{OH})_{2}(2 \mathrm{mM})$ for $1 \mathrm{~h}$. The substrate was washed thrice with water $(3 \mathrm{x} 2 \mathrm{~mL})$, prior to SERS measurement.

\subsubsection{Synthesis of cluster $2 \mathrm{~b}-\mathrm{OH}$ and $2 \mathrm{~b}-\mathrm{COOH}$}

In a typical reaction, $1(51 \mathrm{mg}, 0.055 \mathrm{mmol})$ and 1.2 eq. of the respective ligands (4mercaptophenol or 4-mercaptobenzoic acid) were dissolved in THF (6 mL) and left to stir at room temperature overnight. Solvent was removed in vacuo and the residue was purified by TLC to yield the product as the major yellow band. The characterization data is summarized as follow: 


\begin{tabular}{|c|c|c|}
\hline & 2b-OH & 2b-COOH \\
\hline Yield & $44 \mathrm{mg}(82 \%)$ & $35 \mathrm{mg}(63 \%)$ \\
\hline $\mathbf{R}_{\mathbf{f}}$ & 0.48 (DCM as eluant) & 0.54 (ethyl acetate as eluant) \\
\hline $\begin{array}{l}\text { IR }\left(\mathrm{DCM}, \mathrm{cm}^{-1}\right), \\
v_{\mathrm{CO}}\end{array}$ & $\begin{array}{l}2109 \mathrm{w}, 2067 \mathrm{~s}, 2058 \mathrm{~m}, 2022 \mathrm{~s}, \\
1999 \mathrm{~m}, 1985 \mathrm{w} .\end{array}$ & $\begin{array}{l}\text { 2110w, 2070s, 2061m, 2024s, } \\
2003 \mathrm{~m}, 1987 \mathrm{w} .\end{array}$ \\
\hline $\begin{array}{l}{ }^{1} \mathrm{H} \text { NMR, } \\
\left.\left(\mathrm{CD}_{3}\right)_{2} \mathrm{CO}\right)\end{array}$ & $\begin{array}{l}\delta 8.86(1 \mathrm{H}, \mathrm{s}), 7.28(2 \mathrm{H}, \mathrm{d}, J=9.2, \\
\operatorname{Ar}), 6.82(2 \mathrm{H}, \mathrm{d}, J=9.2, \mathrm{Ar}),- \\
16.86(1 \mathrm{H}, \mathrm{s}, \mathrm{OsHOs}) .\end{array}$ & $\begin{array}{l}\delta 8.01(2 \mathrm{H}, \mathrm{d}, J=7.4, \mathrm{Ar}), 7.61(2 \mathrm{H}, \\
\mathrm{d}, J=9.2, \mathrm{Ar}),-16.95(1 \mathrm{H}, \mathrm{s}, \\
\text { OsHOs). }\end{array}$ \\
\hline $\begin{array}{l}{ }^{13} \mathrm{C}\left\{{ }^{1} \mathrm{H}\right\} \mathrm{NMR} \\
(100.46 \mathrm{MHz} \\
\left.\left(\mathrm{CD}_{3}\right)_{2} \mathrm{CO}\right):\end{array}$ & $\begin{array}{l}\delta 182.29,181.73,177.52,175.48, \\
173.01,170.89,170.79,159.27, \\
135.62,133.78,116.30 .\end{array}$ & $\begin{array}{l}\delta 182.19,181.48,177.36,175.20 \\
\text { 172.70, 170.56, 170.45, 166.73, } \\
\text { 151.94, 132.65, 131.93, 130.61. }\end{array}$ \\
\hline HRMS-ESI & $\begin{array}{l}m / z \text { calculated for } \\
\mathrm{C}_{17} \mathrm{H}_{5} \mathrm{O}_{11} \mathrm{~S}^{184} \mathrm{Os}^{186} \mathrm{Os}^{190} \mathrm{Os}[\mathrm{M}-\mathrm{H}]^{-} \\
976.8201 \text {, found } 976.8148 .\end{array}$ & $\begin{array}{l}m / z \text { calculated for } \\
\mathrm{C}_{17} \mathrm{H}_{5} \mathrm{O}_{12} \mathrm{~S}^{189} \mathrm{Os}^{192} \mathrm{Os}_{2}[\mathrm{M}-\mathrm{H}]^{-} \\
1005.8313 \text {, found } 1005.8364 .\end{array}$ \\
\hline
\end{tabular}

\subsubsection{Cell culture}

Experimental cultures of breast (MDA-MB-231, MCF-7 and MCF-10A) were obtained from the American Type Culture Collection (ATCC) and cultured in tissue culture dishes (Corning, NY) at $37^{\circ} \mathrm{C}$ in $5 \% \mathrm{CO}_{2}$ atmosphere. Phosphate-buffered saline (PBS) was obtained from Gibco. Experiments were performed on cells within 20 passages. The breast cancer cells MDA-MB-231 and MCF-7 cells were maintained in Dulbecco's modified Eagle's medium (DMEM; Biowest L0106, France) supplemented with $10 \%$ fetal bovine serum (FBS; Gibco, NY), 1\% L-glutamine (PAA Laboratories, Austria), and $1 \%$ penicillin/streptomycin (HyClone, UT). The MCF-10A cells were maintained in DMEM-F12 medium (Biowest L0093, France) supplemented with 7.5\% FBS (Gibco, NY), epidermal growth factor (Invitrogen), insulin (Sigma Aldrich), hydrocortisone (Sigma Aldrich) and $0.4 \%$ gentamicin (Gibco, $\mathrm{NY}$ ), and $1 \%$ penicillin/streptomycin (HyClone, UT). Bright-field images of the adhered cells were taken using Nikon Eclipse TS100 inverted microscope, with a 10x objective and Nikon digital Sight DS-L3 camera. 


\subsubsection{Cell proliferation assay}

The anti-proliferation activity of the compounds on the various cell lines was screened using a MTS assay modified from one reported previously. ${ }^{86,100}$ Stock solutions of the compounds in sterile-filtered dimethyl sulfoxide (DMSO) were prepared and serial diluted to lower concentrations. For treatment with the compounds, cells were seeded into 96-well plate in serum-supplemented medium at the same initial density of 10000 cells $(80 \mu \mathrm{L})$ per well, and allowed to adhere and grow for $24 \mathrm{~h}$. Sterile water $(110 \mu \mathrm{L})$ was added to the wells at the perimeter to prevent evaporation of the media from the enclosed wells. For serum-free experiments, the cells were allowed to adhere and grow for $18 \mathrm{~h}$, followed by serum-starving in serum-free medium for $6 \mathrm{~h}$ before treatment with the indicated concentrations of compounds in serum-free medium $(0.5 \%$ DMSO) for $24 \mathrm{~h}$. Control cells were treated with vehicle (0.5\% DMSO). To each well, $10 \mu \mathrm{L}$ of MTS reagent (MTS Cell Proliferation Assay Kit, BioVision, CA) was added and then left to incubate in a $37^{\circ} \mathrm{C}$ incubator with $5 \% \mathrm{CO}_{2}$ for $2 \mathrm{~h}$. The absorbance intensities at $490 \mathrm{~nm}$ were then measured and the cell proliferation relative to the control sample was calculated. Each sample was analyzed in triplicates and was corrected with background intensities from same incubation conditions without the cells. $\mathrm{IC}_{50}$ was determined from a sigmoidal dose response (variable slope) equation using the GraphPad Prism 5 software. The values were obtained from at least two separate experiments with the mean value calculated.

\subsubsection{Apoptosis assay}

MCF-7 cells were seeded into 96-well optical-grade glass black plates with transparent bottom (Nunc Inc, IL), pre-coated with fibronectin (0.5\%, Santa Cruz Biotechnology) in serum-supplemented medium at the same initial density of 10000 cells $(80 \mu \mathrm{L})$ per well and allowed to adhere and grow for $18 \mathrm{~h}$. This was followed by serum-starving in serum-free medium for $6 \mathrm{~h}$ before treatment with the indicated concentrations of compounds in serum-free medium (0.5\% DMSO) for $20 \mathrm{~h}$. Control cells were treated with vehicle $(0.5 \%$ DMSO). Three replicates were performed for each treatment condition. Apoptotic/necrotic/healthy cells detection kit (PromoKine, Heidelberg, Germany) was used to identify apoptotic cells. The adhered cells were washed twice with $1 \times$ binding buffer $(2 \times 50 \mu \mathrm{L})$, followed by staining with Hoechst 33342 and 
Annexin V-FITC at room temperature for $15 \mathrm{~min}$. The stained cells were washed twice with $1 \mathrm{x}$ binding buffer $(2 \times 50 \mu \mathrm{L})$ before proceeding to imaging in the buffer $(70 \mu \mathrm{L})$.

\subsubsection{Image acquisition, segmentation and features extraction}

Fluorescence imaging was performed with a 20x objective using the Axio Observer Z1 (Zeiss), with coolSNAP HQ2 (Photometrics, SONY) camera and Definite laser focus (Zeiss) focusing system. Nine sites per well were imaged. The images were saved in 16-bit TIFF format and corrected using the "rolling ball" algorithm implemented in ImageJ (NIH, v1.48) to reduce non-uniform background illuminations. ${ }^{123}$ Cell segmentations and feature measurements were performed using the cellXpress software platform (Bioinformatics Institute, A*STAR, v1.4). ${ }^{107}$

\subsubsection{Chemical oxidation of $2 \mathrm{~b}-\mathrm{B}(\mathrm{OH})_{2}$}

A solution of $\mathbf{2 b}-\mathrm{B}(\mathrm{OH})_{2}(4.4 \mathrm{mg}, 0.0044 \mathrm{mmol})$ dissolved in $\mathrm{MeOH}(3 \mathrm{~mL})$ was added to 1 eq. of imidazole dissolved in deionized water. Hydrogen peroxide solution $(2.2 \mu \mathrm{L}, 30 \%, w / w)$ was added to the mixture and left to incubate at room temperature over $3 \mathrm{~h}$. For analysis by IR spectroscopy, aliquot of the mixture was removed and dried under a stream of nitrogen. The yellow residue was dissolved in $\mathrm{MeOH}$ and analyzed by IR spectroscopy in a $\mathrm{CaF}_{2}$ IR solution cell. For ESI-HRMS analysis, the mixture was diluted with $\mathrm{MeOH}$ prior to analysis.

\subsubsection{Cyclic voltammetry}

Cyclic Voltammetry (CV) experiments were performed with a computer controlled Eco Chemie Autolab PGSTAT302N potentiostat in a three-electrode cell where a $1 \mathrm{~mm}$ diameter planar glassy carbon (GC) disk working working electrode (Cypress Systems) was used together with a Pt auxiliary electrode (Metrohm) and an Ag wire miniature reference electrode (Cypress Systems) which was connected to the test solution via a salt bridge containing $0.5 \mathrm{M} \mathrm{nBu} \mathrm{NPF}_{6}$ in $\mathrm{CH}_{3} \mathrm{CN}$. Accurate potentials were obtained via the addition of ferrocene $(\mathrm{Fc})$ as an internal standard at the end of the measurements. All solutions used for the voltammetric experiments were deoxygenated by purging with argon gas and measurements were performed in a Faraday Cage. 


\subsubsection{X-ray crystallographic analysis of $2 \mathrm{~b}$-boroxine, $-\mathrm{OH}$ and $-\mathrm{COOH}$}

X-ray crystallographic analysis was carried out by Dr Rakesh (CBC, NTU). Diffraction-quality single crystal was obtained by slow evaporation of a toluene solution of $\mathbf{2} \mathbf{b}-\mathrm{B}(\mathrm{OH})_{2}$ and DCM solution of $\mathbf{2 b}-\mathrm{OH}$ and $-\mathrm{COOH}$. The crystal was mounted onto quartz fibers and the diffraction intensity data was collected on a Bruker Kappa diffractometer with the SMART suite of programs, ${ }^{124}$ equipped with a CCD detector and using Mo $\mathrm{K} \alpha$ radiation $(\lambda=0.71073 \AA)$. Data were processed and corrected for Lorentz and polarization effects with SAINT, and for absorption effects with SADABS. ${ }^{125}$ Structural solution and refinement were performed with the SHELXTL suite of programs. ${ }^{126}$ The structure was solved by direct methods to locate the heavy atoms, followed by successive difference maps for the light, non-hydrogen atoms. All non-hydrogen atoms were refined with anisotropic thermal parameters in the final model. The metal hydrides were placed by potential energy calculations using the XHYDEX program, ${ }^{127}$ with fixed isotropic thermal parameters, while organic hydrogen atoms were placed in calculated positions, assigned isotropic thermal parameters and refined with a riding model. The crystallographic data for the structure is summarized in table 3.3 . 
Table 3.3. Crystallographic data for $\mathbf{2 b}$-boroxine, $-\mathrm{OH}$ and $-\mathrm{COOH}$.

\begin{tabular}{|c|c|c|c|}
\hline & 2b-boroxine & 2b-OH & 2b-COOH \\
\hline Empirical formula & $\begin{array}{l}\mathrm{C}_{96} \mathrm{H}_{30} \mathrm{~B}_{6} \mathrm{O}_{66} \mathrm{Os}_{18} \mathrm{~S}_{6} \\
\text { - } 2.8 \mathrm{C}_{7} \mathrm{H}_{8}\end{array}$ & $\begin{array}{l}\mathrm{C}_{64} \mathrm{H}_{24} \mathrm{O}_{44} \mathrm{Os}_{12} \mathrm{~S}_{4} \bullet \\
2.5 \mathrm{CH}_{2} \mathrm{Cl}_{2}\end{array}$ & $\begin{array}{l}\mathrm{C}_{17} \mathrm{H}_{6} \mathrm{O}_{12} \mathrm{Os}_{3} \mathrm{~S} \cdot \\
\mathrm{CH}_{2} \mathrm{Cl}_{2}\end{array}$ \\
\hline Formula weight & 6177.99 & 4119.79 & 1089.80 \\
\hline Temperature (K) & $103(2)$ & $103(2)$ & $153(2)$ \\
\hline Wavelength $(\AA)$ & 0.71073 & 0.71073 & 0.71073 \\
\hline Crystal system & Triclinic & Triclinic & Triclinic \\
\hline Space group & $\mathrm{P} \overline{1}$ & $\mathrm{P} \overline{1}$ & $\mathrm{P} \overline{1}$ \\
\hline$a(\AA)$ & $9.0414(4)$ & $8.4267(6)$ & $8.4854(5)$ \\
\hline$b(\AA)$ & $20.8788(8)$ & $14.1473(9)$ & $12.0178(6)$ \\
\hline$c(\AA)$ & $22.4877(10)$ & $21.5478(13)$ & $12.8840(7)$ \\
\hline$\alpha(\operatorname{deg})$ & $65.347(2)$ & $89.327(3)$ & $81.639(3)$ \\
\hline$\beta(\mathrm{deg})$ & $86.861(3)$ & $87.798(4)$ & $77.714(3)$ \\
\hline$\gamma(\operatorname{deg})$ & $81.045(3)$ & $75.631(4)$ & $76.102(3)$ \\
\hline $\mathrm{V}\left(\AA^{3}\right)$ & $3810.9(3)$ & $2486.6(3)$ & $1240.01(12)$ \\
\hline $\mathrm{Z}$ value & 1 & 1 & 2 \\
\hline$\rho_{\text {calc }}\left(\mathrm{g} / \mathrm{cm}^{3}\right)$ & 2.692 & 2.751 & 2.919 \\
\hline$\mu\left(\mathrm{Mo} \mathrm{K}_{\mathrm{a}}\right)\left(\mathrm{mm}^{-1}\right)$ & 15.103 & 15.561 & 15.692 \\
\hline $\mathrm{F}(000)$ & 2768 & 1841 & 980 \\
\hline Crystal size $\left(\mathrm{mm}^{3}\right)$ & $\begin{array}{l}0.160 \times 0.080 \times \\
0.040\end{array}$ & $\begin{array}{l}0.120 \times 0.100 \times \\
0.060\end{array}$ & $\begin{array}{l}0.420 \times 0.380 \times \\
0.280\end{array}$ \\
\hline $\begin{array}{l}\text { Theta range for data } \\
\text { collection }\end{array}$ & 2.281 to $25.796^{\circ}$ & 2.403 to $26.372^{\circ}$ & 2.270 to $26.372^{\circ}$ \\
\hline Index ranges & $\begin{array}{l}-10<=\mathrm{h}<=10 \\
-22<=\mathrm{k}<=25 \\
0<=\mathrm{l}<=27\end{array}$ & $\begin{array}{l}-12<=\mathrm{h}<=12, \\
-20<=\mathrm{k}<=20, \\
-30<=1<=30\end{array}$ & $\begin{array}{l}-10<=\mathrm{h}<=10 \\
-14<=\mathrm{k}<=15, \\
0<=\mathrm{l}<=16\end{array}$ \\
\hline Reflections collected & 41406 & 55963 & 49433 \\
\hline $\begin{array}{l}\text { Independent } \\
\text { reflections }\end{array}$ & $\begin{array}{l}14383[\mathrm{R}(\text { int })= \\
0.1204]\end{array}$ & $\begin{array}{l}10175[\mathrm{R}(\text { int })= \\
0.1125]\end{array}$ & $\begin{array}{l}5058[\mathrm{R}(\mathrm{int})= \\
0.1026]\end{array}$ \\
\hline $\begin{array}{l}\text { Completeness to } \\
\theta=25.242^{\circ}(\%)\end{array}$ & 99.9 & 99.9 & 99.9 \\
\hline Absorption correction & \multicolumn{3}{|c|}{ Semi-empirical from equivalents } \\
\hline $\begin{array}{l}\text { Max. and min. } \\
\text { transmission }\end{array}$ & 0.58 and 0.20 & 0.46 and 0.16 & 0.0960 and 0.0580 \\
\hline Refinement method & \multicolumn{3}{|c|}{ Full-matrix least-squares on $\mathrm{F}^{2}$} \\
\hline $\begin{array}{l}\text { Data / restraints / } \\
\text { parameters }\end{array}$ & 14383 / $350 / 946$ & 10175 / 9 / 611 & $5058 / 0 / 325$ \\
\hline Goodness-of-fit on $\mathrm{F}^{2}$ & 1.011 & 1.075 & 1.081 \\
\hline $\begin{array}{l}\text { Final R indices } \\
{[\mathrm{I}>2 \operatorname{sigma}(\mathrm{I})]}\end{array}$ & $\begin{array}{l}\mathrm{R} 1=0.0648, w \mathrm{R} 2= \\
0.1591\end{array}$ & $\begin{array}{l}\mathrm{R} 1=0.0557 \\
\mathrm{wR} 2=0.1430\end{array}$ & $\begin{array}{l}\mathrm{R} 1=0.0392 \\
\mathrm{wR} 2=0.1021\end{array}$ \\
\hline $\mathrm{R}$ indices (all data) & $\begin{array}{l}\mathrm{R} 1=0.0912, \mathrm{wR} 2= \\
0.1798\end{array}$ & $\begin{array}{l}\mathrm{R} 1=0.0753 \\
\mathrm{wR} 2=0.1590\end{array}$ & $\begin{array}{l}\mathrm{R} 1=0.0442 \\
\mathrm{wR} 2=0.1054\end{array}$ \\
\hline $\begin{array}{l}\text { Largest diff. peak and } \\
\text { hole }\left(e \bullet \AA^{-3}\right)\end{array}$ & 3.235 and -2.692 & 3.779 and -3.544 & 3.037 and -1.795 \\
\hline
\end{tabular}




\subsection{References}

1. Global report on diabetes. Geneva: World Health Organization; 2016

2. G. Danaei, M. M. Finucane, Y. Lu, G. M. Singh, M. J. Cowan, C. J. Paciorek, J. K. Lin, F. Farzadfar, Y.-H. Khang, G. A. Stevens, M. Rao, M. K. Ali, L. M. Riley, C. A. Robinson and M. Ezzati, Lancet, 2011, 378, 31-40.

3. Definition and diagnosis of diabetes mellitus and intermediate hyperglycemia: report of a WHO/IDF consultation. Geneva: World Health Organization; 2006.

4. A. E. G. Cass, G. Davis, G. D. Francis, H. A. O. Hill, W. J. Aston, I. J. Higgins, E. V. Plotkin, L. D. L. Scott and A. P. F. Turner, Anal. Chem., 1984, 56, 667-671.

5. A. P. F. Turner, Chem. Soc. Rev., 2013, 42, 3184-3196.

6. O. Lyandres, J. M. Yuen, N. C. Shah, R. P. VanDuyne, J. T. Walsh and M. R. Glucksberg, Diabetes Technol. Ther., 2008, 10, 257-265.

7. S. Dong, B. Wang and B. Liu, Biosens. Bioelectron., 1992, 7, 215-222.

8. J. Wang, Chem. Rev., 2008, 108, 814-825.

9. B. D. Cameron, H. W. Gorde, B. Satheesan and G. L. Cote, Diabetes Technol. Ther., 1999, 1, 135-143.

10. R. R. Ansari, S. Böckle and L. Rovati, J. Biomed. Opt., 2004, 9, 103-115.

11. G. L. Cote, M. D. Fox and R. B. Northrop, IEEE Trans. Biomed. Eng., 1992, 39, $752-756$.

12. A. J. Berger, I. Itzkan and M. S. Feld, Spectrochimi. Acta, Part A, 1997, 53, 287-292.

13. A. J. Berger, T.-W. Koo, I. Itzkan, G. Horowitz and M. S. Feld, Appl. Opt., 1999, 38, 2916-2926.

14. J. Lambert, M. Storrie-Lombardi and M. Borchert, IEEE LEOS Newsletter, $1998,12,19-22$.

15. T.-W. Koo, A. J. Berger, I. Itzkan, G. Horowitz and M. S. Feld, IEEE LEOS Newsletter, 1998, 12, 18-19.

16. K. E. Shafer-Peltier, C. L. Haynes, M. R. Glucksberg and R. P. Van Duyne, J. Am. Chem. Soc., 2003, 125, 588-593.

17. D. A. Stuart, C. R. Yonzon, X. Zhang, O. Lyandres, N. C. Shah, M. R. Glucksberg, J. T. Walsh and R. P. Van Duyne, Anal. Chem., 2005, 77, 40134019. 
18. O. Lyandres, N. C. Shah, C. R. Yonzon, J. T. Walsh, M. R. Glucksberg and R. P. Van Duyne, Anal. Chem., 2005, 77, 6134-6139.

19. C. R. Yonzon, C. L. Haynes, X. Zhang, J. T. Walsh and R. P. Van Duyne, Anal. Chem., 2004, 76, 78-85.

20. K. Ma, J. M. Yuen, N. C. Shah, J. T. Walsh, M. R. Glucksberg and R. P. Van Duyne, Anal. Chem., 2011, 83, 9146-9152.

21. J. M. Yuen, N. C. Shah, J. T. Walsh, M. R. Glucksberg and R. P. Van Duyne, Anal. Chem., 2010, 82, 8382-8385.

22. U. S. Dinish, F. C. Yaw, A. Agarwal and M. Olivo, Biosens. Bioelectron., 2011, 26, 1987-1992.

23. X. Zhang, A. V. Whitney, J. Zhao, E. M. Hicks and R. P. Van Duyne, J. Nanosci. Nanotechno., 2006, 6, 1920-1934.

24. K. C. Grabar, K. J. Allison, B. E. Baker, R. M. Bright, K. R. Brown, R. G. Freeman, A. P. Fox, C. D. Keating, M. D. Musick and M. J. Natan, Langmuir, 1996, 12, 2353-2361.

25. N. L. Garrett, P. Vukusic, F. Ogrin, E. Sirotkin, C. P. Winlove and J. Moger, J. Biophotonics, 2009, 2, 157-166.

26. K. A. Stoerzinger, J. Y. Lin and T. W. Odom, Chem. Sci., 2011, 2, 1435-1439.

27. R. A. Tripp, R. A. Dluhy and Y. Zhao, Nano Today, 2008, 3, 31-37.

28. K. Kim, J. W. Lee and K. S. Shin, Analyst, 2013, 138, 2988-2994.

29. J. M. Sylvia, J. A. Janni, J. D. Klein and K. M. Spencer, Anal. Chem., 2000, 72, 5834-5840.

30. D. A. Stuart, K. B. Biggs and R. P. Van Duyne, Analyst, 2006, 131, 568-572.

31. M. Li, S. K. Cushing, J. Zhang, S. Suri, R. Evans, W. P. Petros, L. F. Gibson, D. Ma, Y. Liu and N. Wu, ACS Nano, 2013, 7, 4967-4976.

32. L. Guerrini, E. Pazos, C. Penas, M. E. Vázquez, J. L. Mascareñas and R. A. Alvarez-Puebla, J. Am. Chem. Soc., 2013, 135, 10314-10317.

33. Y. Wen, W. Wang, Z. Zhang, L. Xu, H. Du, X. Zhang and Y. Song, Nanoscale, 2013, 5, 523-526.

34. W. R. Premasiri, D. T. Moir, M. S. Klempner, N. Krieger, G. Jones and L. D. Ziegler, J. Phys. Chem. B, 2005, 109, 312-320.

35. B. Sharma, R. R. Frontiera, A. I. Henry, E. Ringe and R. P. Van Duyne, Mater. Today, 2012, 15, 16-25. 
36. S. Shanmukh, L. Jones, J. Driskell, Y. Zhao, R. Dluhy and R. A. Tripp, Nano Lett., 2006, 6, 2630-2636.

37. Q. Wu, L. Wang, H. Yu, J. Wang and Z. Chen, Chem. Rev., 2011, 111, 78557875 .

38. J. P. Lorand and J. O. Edwards, J. Org. Chem., 1959, 24, 769-774.

39. T. D. James, K. R. A. S. Sandanayake and S. Shinkai, Angewandte Chemie, International Edition in English, 1996, 35, 1910-1922.

40. T. D. James, Top. Curr. Chem., 2007, 107-152.

41. S. D. Bull, M. G. Davidson, J. M. H. van den Elsen, J. S. Fossey, A. T. A. Jenkins, Y.-B. Jiang, Y. Kubo, F. Marken, K. Sakurai, J. Zhao and T. D. James, Acc. Chem. Res., 2013, 46, 312-326.

42. X. Sun and T. D. James, Chem. Rev., 2015, 115, 8001-8037.

43. T. D. James, K. R. A. S. Sandanayake and S. Shinkai, Angew. Chem. Int. Ed. Engl., 1994, 33, 2207-2209.

44. H. Eggert, J. Frederiksen, C. Morin and J. C. Norrild, J. Org. Chem., 1999, 64, 3846-3852.

45. M. P. Nicholls and P. K. C. Paul, Org. Biomol. Chem., 2004, 2, 1434-1441.

46. M. Bielecki, H. Eggert and J. Chr. Norrild, J. Chem. Soc., Perkin Trans. 2, 1999, 449-456.

47. V. V. Karnati, X. Gao, S. Gao, W. Yang, W. Ni, S. Sankar and B. Wang, Bioorg. Med. Chem. Lett., 2002, 12, 3373-3377.

48. W. Yang, H. He and D. G. Drueckhammer, Angew. Chem. Int. Ed., 2001, 40, 1714-1718.

49. V. L. Alexeev, A. C. Sharma, A. V. Goponenko, S. Das, I. K. Lednev, C. S. Wilcox, D. N. Finegold and S. A. Asher, Anal. Chem., 2003, 75, 2316-2323.

50. D. B. Cordes, S. Gamsey and B. Singaram, Angew. Chem. Int. Ed., 2006, 45, 3829-3832.

51. M. M. Ward Muscatello, L. E. Stunja and S. A. Asher, Anal. Chem., 2009, 81, 4978-4986.

52. W. Wu, T. Zhou, A. Berliner, P. Banerjee and S. Zhou, Angew. Chem. Int. Ed., 2010, 49, 6554-6558.

53. C. Ancla, V. Lapeyre, I. Gosse, B. Catargi and V. Ravaine, Langmuir, 2011, 27, 12693-12701. 
54. Y.-J. Huang, W.-J. Ouyang, X. Wu, Z. Li, J. S. Fossey, T. D. James and Y.-B. Jiang, J. Am. Chem. Soc., 2013, 135, 1700-1703.

55. T. D. James, K. R. A. S. Sandanayake, R. Iguchi and S. Shinkai, J. Am. Chem. Soc., 1995, 117, 8982-8987.

56. J. E. Butler, J. Immunoassay, 2000, 21, 165-209.

57. X. Pei, B. Zhang, J. Tang, B. Liu, W. Lai and D. Tang, Anal. Chim. Acta, 2013, 758, $1-18$.

58. C. Y. Fu, K. W. Kho, U. S. Dinish, Z. Y. Koh and O. Malini, J. Raman Spectrosc., 2012, 43, 977-985.

59. D. Goh, U. S. Dinish and M. Olivo, Photonics Global Conference (PGC), 2012.

60. C. Y. Fu, U. S. Dinish, K. W. Kho, W. D. Goh and M. Olivo, Proc. SPIE 8090, Novel Biophotonic Techniques and Applications, 2011.

61. U. S. Dinish, C. Y. Fu, A. Agarwal, P. Thoniyot and M. Olivo, Proc. SPIE 7397, Biosensing II, 2009.

62. K. V. Kong, W. Chew, L. H. K. Lim, W. Y. Fan and W. K. Leong, Bioconjugate Chem., 2007, 18, 1370-1374.

63. A. L. Korich and P. M. Iovine, Dalton Trans., 2010, 39, 1423-1431.

64. J. Liu and J. J. Lavigne, in Boronic Acids: Preparation and Applications in Organic Synthesis, Medicine and Materials (Volume 1 and 2), Second Edition ed. D. G. Hall, Wiley-VCH Verlag GmbH \& Co. KGaA, Weinheim, Germany, 2011, pp. 621-676.

65. M. Mastalerz, Angew. Chem. Int. Ed., 2008, 47, 445-447.

66. T.-H. Chen, W. Kaveevivitchai, N. Bui and O. S. Miljanic, Chem. Commun., 2012, 48, 2855-2857.

67. T. Kawasaki, H. Akanuma and T. Yamanouchi, Diabetes Care, 2002, 25, 353357.

68. T. Kawasaki, N. Ogata, H. Akanuma, T. Sakai, H. Watanabe, K. Ichiyanagi and T. Yamanouchi, Metab., Clin. Exp., 2004, 53, 583-588.

69. C. A. Williams, T. Phillips and I. Macdonald, Metab., Clin. Exp., 1983, 32, 250-256.

70. R. Raman, S. Raguram, G. Venkataraman, J. C. Paulson and R. Sasisekharan, Nat. Methods, 2005, 2, 817-824.

71. S. A. Springer and P. Gagneux, J. Biol. Chem., 2013, 288, 6904-6911. 
72. C. Büll, M. A. Stoel, M. H. den Brok and G. J. Adema, Cancer Res., 2014, 74, 3199-3204.

73. D. H. Dube and C. R. Bertozzi, Nat. Rev. Drug Discovery, 2005, 4, 477-488.

74. A. Varki and R. Schauer, in Essentials of Glycobiology, 2nd edition, eds. A. Varki, R. D. Cummings, J. D. Esko, H. H. Freeze, P. Stanley, C. R. Bertozzi, G. W. Hart and M. E. Etzler., Cold Spring Harbor: Cold Spring Harbor Laboratory Press, NY, 2009.

75. J. Dennis, S. Laferte, C. Waghorne, M. Breitman and R. Kerbel, Science, 1987, 236, 582-585.

76. Y. J. Kim and A. Varki, Glycoconjugate J., 1997, 14, 569-576.

77. T. F. Orntoft and E. M. Vestergaard, Electrophoresis, 1999, 20, 362-371.

78. H. Otsuka, E. Uchimura, H. Koshino, T. Okano and K. Kataoka, J. Am. Chem. Soc., 2003, 125, 3493-3502.

79. K. Djanashvili, L. Frullano and J. A. Peters, Chem. - Eur. J., 2005, 11, 40104018.

80. H. Liu, Y. Li, K. Sun, J. Fan, P. Zhang, J. Meng, S. Wang and L. Jiang, J. Am. Chem. Soc., 2013, 135, 7603-7609.

81. A. Liu, S. Peng, J. C. Soo, M. Kuang, P. Chen and H. Duan, Anal. Chem., 2010, 83, 1124-1130.

82. X.-D. Xu, H. Cheng, W.-H. Chen, S.-X. Cheng, R.-X. Zhuo and X.-Z. Zhang, Sci. Rep., 2013, 3.

83. L. Lin, X. Tian, S. Hong, P. Dai, Q. You, R. Wang, L. Feng, C. Xie, Z.-Q. Tian and X. Chen, Angew. Chem. Int. Ed., 2013, 52, 7266-7271.

84. D. Yin, S. Wang, Y. He, J. Liu, M. Zhou, J. Ouyang, B. Liu, H.-Y. Chen and Z. Liu, Chem. Commun., 2015, 51, 17696-17699.

85. Cui, Lin, Yue, Zhao and Liu, Oncol. Rep., 2011, 25, 1365-1371.

86. K. V. Kong, W. K. Leong, S. P. Ng, T. H. Nguyen and L. H. K. Lim, ChemMedChem, 2008, 3, 1269-1275.

87. K. V. Kong, L.-D. Liao, Z. Lam, N. V. Thakor, W. K. Leong and M. Olivo, Chem. Commun., 2014, 50, 2601-2603.

88. G. R. Crooks, B. F. G. Johnson, J. Lewis and I. G. Williams, J. Chem. Soc. A, 1969, 797-799.

89. E. W. Ainscough, A. M. Brodie, R. K. Coll, A. J. A. Mair and J. M. Waters, J. Organomet. Chem., 1996, 509, 259-264. 
90. C. Li and W. K. Leong, J. Organomet. Chem., 2008, 693, 1292-1300.

91. K. V. Kong, W. K. Leong and L. H. K. Lim, Chem. Res. Toxicol., 2009, 22, 1116-1122.

92. E. W. Ainscough, A. M. Brodie, R. K. Coll, B. A. Coombridge and J. M. Waters, J. Organomet. Chem., 1998, 556, 197-205.

93. S. L. Fink and B. T. Cookson, Infect. Immun., 2005, 73, 1907-1916.

94. B. E. Trump, I. K. Berezesky, S. H. Chang and P. C. Phelps, Toxicol. Pathol., 1997, 25, 82-88.

95. J. A. Hickman, Cancer Metastasis Rev., 1992, 11, 121-139.

96. R. Gerl and D. L. Vaux, Carcinogenesis, 2005, 26, 263-270.

97. S. Wang, E. A. Konorev, S. Kotamraju, J. Joseph, S. Kalivendi and B. Kalyanaraman, J. Biol. Chem., 2004, 279, 25535-25543.

98. T.-H. Wang, H.-S. Wang and Y.-K. Soong, Cancer, 2000, 88, 2619-2628.

99. Z. H. Siddik, Oncogene, 2003, 22, 7265-7279.

100. H. Z. S. Lee, W. K. Leong, S. Top and A. Vessières, ChemMedChem, 2014, 9, $1453-1457$.

101. S. Toné, K. Sugimoto, K. Tanda, T. Suda, K. Uehira, H. Kanouchi, K. Samejima, Y. Minatogawa and W. C. Earnshaw, Exp. Cell Res., 2007, 313, 3635-3644.

102. A. Saraste and K. Pulkki, Cardiovasc. Res., 2000, 45, 528-537.

103. B. S. Cummings, L. P. Wills and R. G. Schnellmann, in Current Protocols in Pharmacology, John Wiley \& Sons, Inc., 2012.

104. S. A. Latt and G. Stetten, J. Histochem. Cytochem., 1976, 24, 24-33.

105. M. J. Lydon, K. D. Keeler and D. B. Thomas, J. Cell. Physiol., 1980, 102, 175181.

106. L.-H. Loo, L. F. Wu and S. J. Altschuler, Nat. Methods, 2007, 4, 445-453.

107. D. Laksameethanasan, R. Z. Tan, G. W.-L. Toh and L.-H. Loo, BMC Bioinf., 2013, 14, 1-12.

108. R. Su, S. Xiong, D. Zink and L.-H. Loo, Arch. Toxicol., 2016, 90, 2793-2808.

109. S. H. Lee, X. W. Meng, K. S. Flatten, D. A. Loegering and S. H. Kaufmann, Cell Death Differ., 2013, 20, 64-76.

110. R. F. A. Zwaal, P. Comfurius and E. M. Bevers, Cell. Mol. Life Sci., 2005, 62, 971-988. 
111. V. A. Fadok, D. R. Voelker, P. A. Campbell, J. J. Cohen, D. L. Bratton and P. M. Henson, J. Immunol., 1992, 148, 2207-2216.

112. G. Koopman, C. Reutelingsperger, G. Kuijten, R. Keehnen, S. Pals and M. van Oers, Blood, 1994, 84, 1415-1420.

113. H. van Genderen, H. Kenis, P. Lux, L. Ungeth, C. Maassen, N. Deckers, J. Narula, L. Hofstra and C. Reutelingsperger, Nat. Protocols, 2006, 1, 363-367.

114. J. Simon, S. Salzbrunn, G. K. Surya Prakash, N. A. Petasis and G. A. Olah, J. Org. Chem., 2001, 66, 633-634.

115. A. R. Lippert, G. C. Van de Bittner and C. J. Chang, Acc. Chem. Res., 2011, 44, 793-804.

116. E. W. Miller, A. E. Albers, A. Pralle, E. Y. Isacoff and C. J. Chang, J. Am. Chem. Soc., 2005, 127, 16652-16659.

117. B. C. Dickinson and C. J. Chang, Nat. Chem. Biol., 2011, 7, 504-511.

118. E. Hillard, A. Vessières, L. Thouin, G. Jaouen and C. Amatore, Angew. Chem. Int. Ed., 2006, 45, 285-290.

119. D. Plażuk, A. Vessières, E. A. Hillard, O. Buriez, E. Labbé, P. Pigeon, M.-A. Plamont, C. Amatore, J. Zakrzewski and G. Jaouen, J. Med. Chem., 2009, 52, 4964-4967.

120. G. Jaouen, A. Vessieres and S. Top, Chem. Soc. Rev., 2015, 44, 8802-8817.

121. W.-Y. Wong and W.-T. Wong, J. Chem. Soc., Dalton Trans., 1996, 3209-3214.

122. J. N. Nicholls, M. D. Vargas, A. J. Deeming and S. E. Kabir, Inorg. Synth., 1990, 28, 232.

123. S. R. Sternberg, Computer, 1983, 16, 22-34.

124. SMART version 5.628; Bruker AXS Inc., Madison, WI, USA, 2001.

125. Sheldrick, G. M. SADABS; University of Göttingen, Göttingen, Germany, 1996.

126. SHELXTL version 5.1; Bruker AXS Inc., Madison, WI, USA, 1997.

127. A. G. Orpen, J. Chem. Soc., Dalton Trans., 1980, 2509-2516. 


\section{Chapter 4: High Nuclearity Metal Carbonyl Clusters as Near-IR Contrast Agents for Photoacoustic In Vivo Imaging}

Photoacoustic (PA) imaging, a hybrid imaging modality which combines optical and acoustic imaging, is emerging as an important tool for deep tissue imaging. The development of various PA contrast agents which serve to improve the contrast for site-specific imaging and disease diagnosis is therefore of great relevance. The first report on a metal carbonyl as a PA contrast agent was by Leong and Olivo et. al.; a water-soluble triosmium carbonyl cluster was used for in vivo imaging of the rat cerebral cortex vasculature. ${ }^{1}$ The highly stable contrast agent allowed the brain vasculature, especially the superior sagittal sinus (SSS), to be imaged at higher photoacoustic contrast compared to their intrinsic contrast or the use of carbon nanotubes. However, a major drawback lies in the incident wavelength of $410 \mathrm{~nm}$; tissue penetration is low at this wavelength and thus an invasive procedure is required to carry out the imaging. A metal carbonyl that absorbs in the near-IR (NIR) range, from 680 to $1000 \mathrm{~nm}$, is therefore more desirable as it will offer deeper tissue penetration and less scattering. This chapter details investigations on the optical and PA properties of NIR-absorbing metal carbonyls, and their application in non-invasive in vivo $\mathrm{PA}$ imaging.

\subsection{Optical properties of group 8 metal carbonyl clusters - low to high nuclearity}

Three ruthenium carbonyl complexes (12'a, 20 and 21, the last as the [PPN] salt; $\left.\mathrm{PPN}=\left[\left(\mathrm{Ph}_{3} \mathrm{P}\right)_{2} \mathrm{~N}\right]^{+}\right)$of low nuclearity $\left(\mathrm{Ru}_{\mathrm{n}}, \mathrm{n}=1,3,6\right)$ were synthesized and identified in accordance to reported methods (figure 4.1a). ${ }^{2-4}$ Their absorption spectra showed strong absorptions in the UV-visible region (figure $4.1 \mathrm{~b}$ ), and they are shifted towards longer wavelengths with an increase in nuclearity. 
a

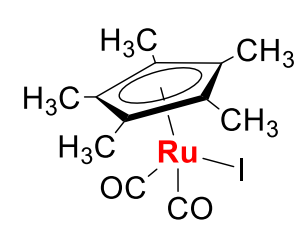

$\mathrm{Cp}{ }^{*} \mathrm{Ru}(\mathrm{CO})_{2} \mathrm{I}$

12 'a

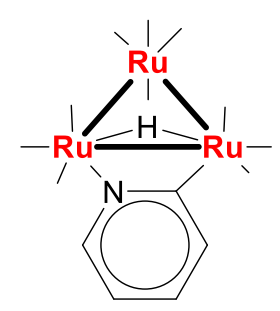

$\mathrm{Ru}_{3}(\mathrm{CO})_{10}(\mu-\mathrm{H})\left(\mu-\mathrm{NC}_{5} \mathrm{H}_{4}\right)$

20

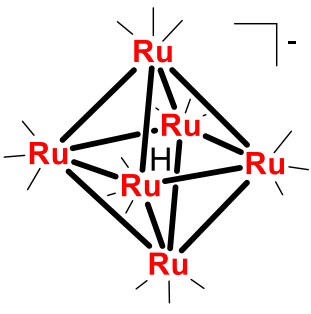

$\left[\mathrm{Ru}_{6}\left(\mu_{6}-\mathrm{H}\right)(\mathrm{CO})_{18}\right]^{-}$

21

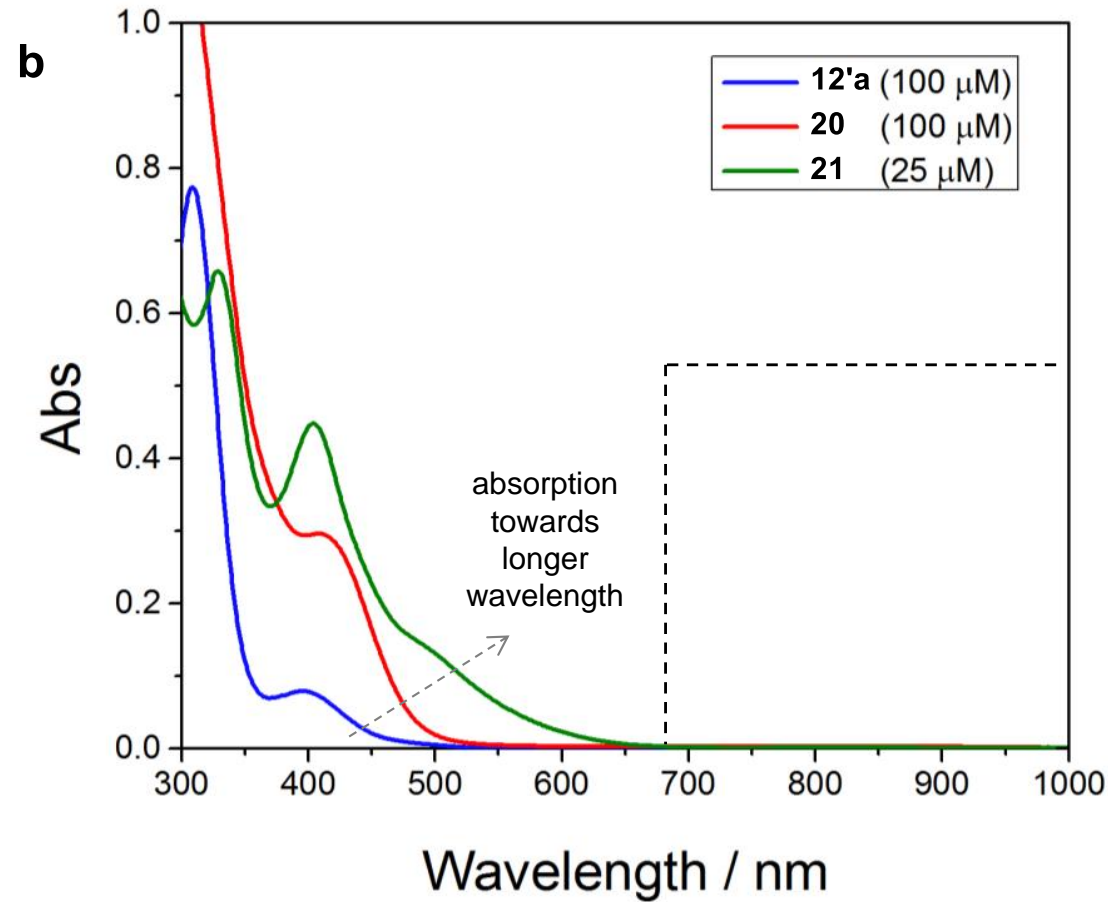

Figure 4.1. a) Molecular structures of low nuclearity ruthenium complexes 12'a, 20 and 21. Counter cation has been omitted for clarity. b) Optical absorption spectra of the complexesin DMSO at their respective concentrations. 
The study was extended to a number of previously reported high nuclearity carbonyl clusters (HNCCs) of ruthenium, 22-25, as well as one of osmium, 26 (24-26 were synthesized as the [PPN] salt). ${ }^{5-8}$ They included homoleptic, as well as carbido, hydrido, and phosphine-substituted derivatives, with different metal core structures (figure 4.2a). All the HNCCs exhibited absorption in the NIR. In particular, there were distinct absorption maxima $\left(\lambda_{\max }\right)$ at 719 and $768 \mathrm{~nm}$ for clusters 22 and 26, respectively; the others showed broad shoulders tapering towards longer wavelengths (figures 5.2). This latter broad and continuous absorption from the UV-visible to the NIR region has been attributed to the large number of "interband" transitions, leading to a high degree of overlap of absorption bands. ${ }^{9}$ These absorptions have been assigned to $\mathrm{M}-\mathrm{CO} \sigma^{*} \leftarrow \pi$, as well as M-M $\sigma^{*} \leftarrow \sigma$ transitions, ${ }^{9,} 10$ and they are correlated to the HOMOLUMO gap. For clusters 22-26, absorption is negligible at $\geq 900 \mathrm{~nm}$, which suggests a HOMO-LUMO gap of $\sim 1.0 \mathrm{eV} .{ }^{10-12}$ Cluster 23 shows the highest molar extinction coefficient for its $\lambda_{\max }$ in the NIR region (appendix A4.1, table 4.1). 
a

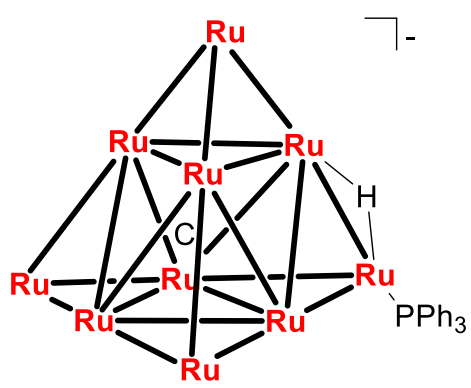

$\left[\mathrm{Ru}_{2}(\mu-\mathrm{H})\left(\mu-\mathrm{NC}_{5} \mathrm{H}_{4}\right)_{2}(\mathrm{CO})_{4}\left(\mathrm{NC}_{5} \mathrm{H}_{5}\right)_{2}\right]$ $\left[\mathrm{Ru}_{10}(\mu-\mathrm{H})\left(\mu_{6}-\mathrm{C}\right)(\mathrm{CO})_{23}\left(\mathrm{PPh}_{3}\right)\right]$

22

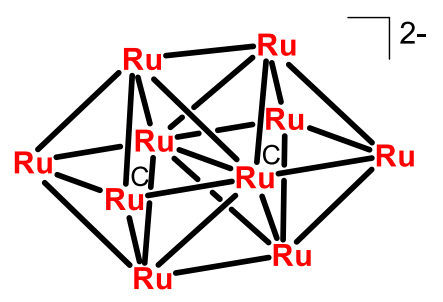

$\left[\mathrm{Ru}_{10}\left(\mu_{6}-\mathrm{C}\right)_{2}(\mathrm{CO})_{24}\right]^{2-}$

24

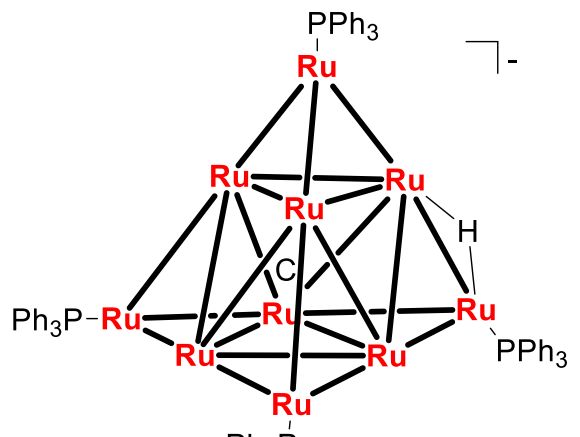

$\mathrm{Ph}_{3} \mathrm{P}$

$\left[\mathrm{Ru}_{2}(\mu-\mathrm{H})\left(\mu-\mathrm{NC}_{5} \mathrm{H}_{4}\right)_{2}(\mathrm{CO})_{4}\left(\mathrm{PPh}_{3}\right)_{2}\right]$

$\left[\mathrm{Ru}_{10}(\mu-\mathrm{H})\left(\mu_{6}-\mathrm{C}\right)(\mathrm{CO})_{20}\left(\mathrm{PPh}_{3}\right)_{4}\right]$

23

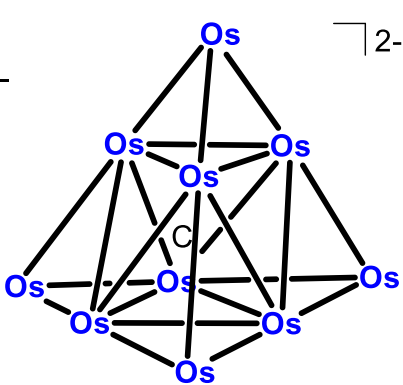

$\left[\mathrm{Os}_{10}\left(\mu_{6}-\mathrm{C}\right)(\mathrm{CO})_{24}\right]^{2-}$

26

b

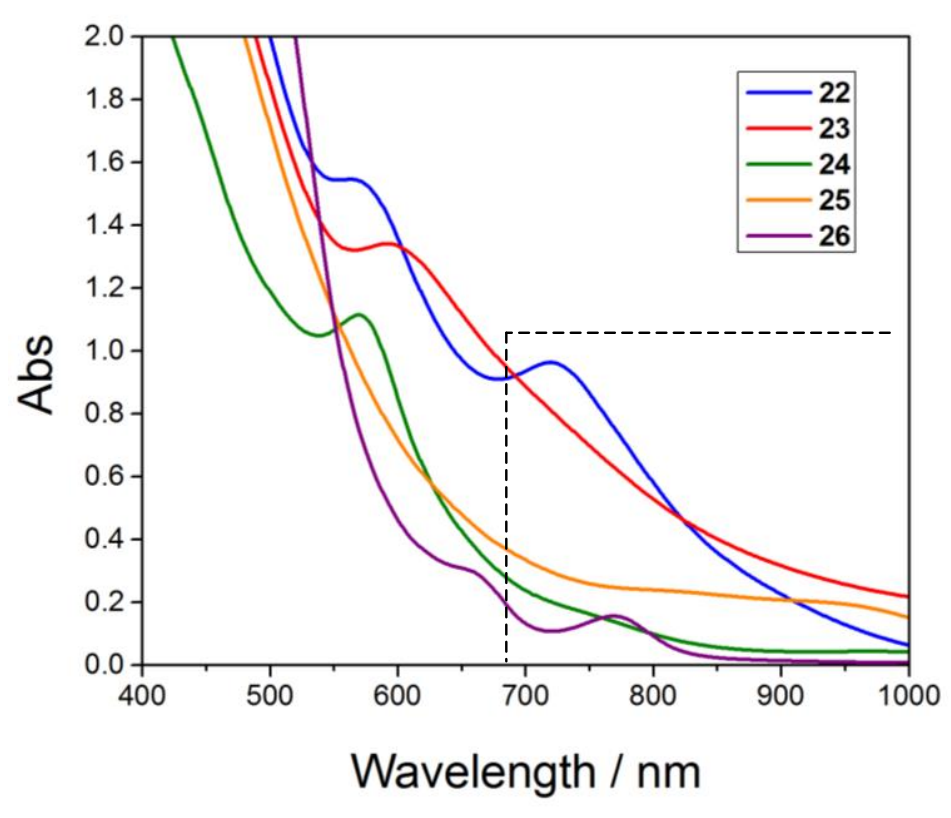

Figure 4.2. a) Molecular structures of HNCC 22-26. Counter cations and CO ligands in the HNCCs have been omitted for clarity. b) Optical absorption spectra of HNCCs in DMSO at $200 \mu \mathrm{M}$. Boxed area represents the NIR region of interest. 


\subsection{Photoacoustic properties of high nuclearity carbonyl clusters 22-26}

The PA activity for the five HNCCs were measured in a phantom, which mimics tissue optical properties. Strong PA signals were observed for all the clusters, with the PA intensity at their corresponding $\lambda_{\max }$ varying linearly with concentration (figure 4.3c). The quantum yields (slope of the graph), a useful indicator of the PA strength, were comparable, with the highest being that for $\mathbf{2 3}$ (figure $4.3 \mathrm{c}$, table 4.1 ). With the exception of 25, the PA spectral intensity followed a similar trend to their optical spectra, indicating little or no decomposition (figure 4.3, appendix A4.2).
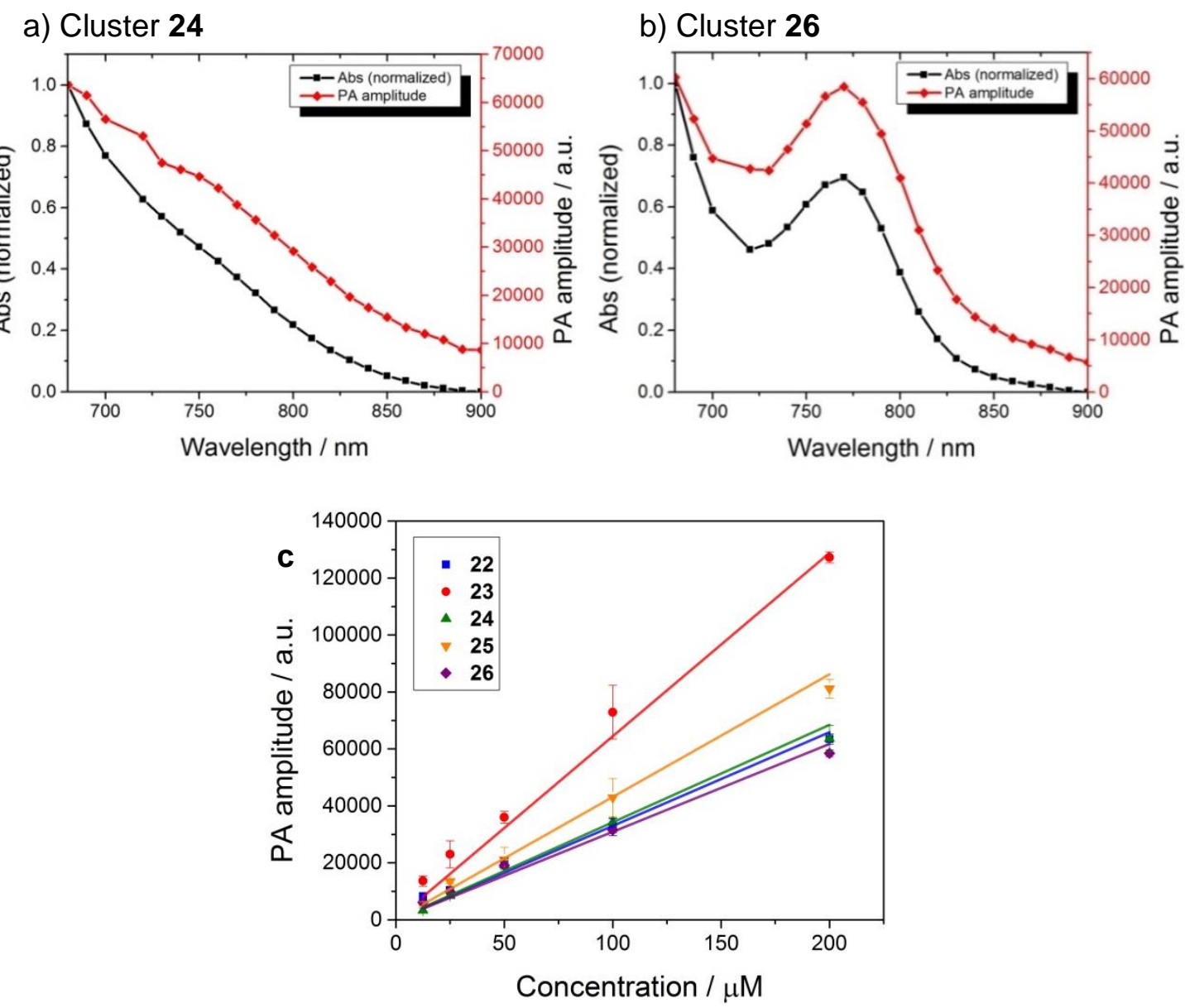

Figure 4.3. i) PA (red) and optical absorption (black) spectra of a) cluster 24 and b) 26. c) Combined plot of PA amplitude as a function of concentrations of 22-26 in DMSO. 


\subsection{Biocompatible sodium salt of cluster 26}

All the ruthenium HNCCs showed moderate stability and decomposed easily in solution over a few days, in contrast to the more stable osmium cluster 26. The $\mathrm{PPN}^{+}$ counterion, a common cation for stabilizing the HNCCs and other organometallic complexes, was evaluated against an oral squamous cell carcinoma (OSCC) cell line in an MTS assay. It exhibited very high cytotoxicity with an $\mathrm{IC}_{50}$ value of $1.1 \pm 0.4 \mu \mathrm{M}$ (figure 4.4, appendix A4.3). To increase biocompatibility, the synthesis of $\left[\mathrm{Os}_{10}\left(\mu_{6^{-}}\right.\right.$ $\left.\mathrm{C})(\mathrm{CO})_{24}\right]^{2-}$ was modified to afford the sodium salt $\mathrm{Na}_{2} .26$ (Scheme 4.1). ${ }^{8}$ This had a significantly lower toxicity, with an $\mathrm{IC}_{50}$ of $83 \pm 2 \mu \mathrm{M}$ (figure 4.5 , appendix $\mathrm{A} 4.4$ ).

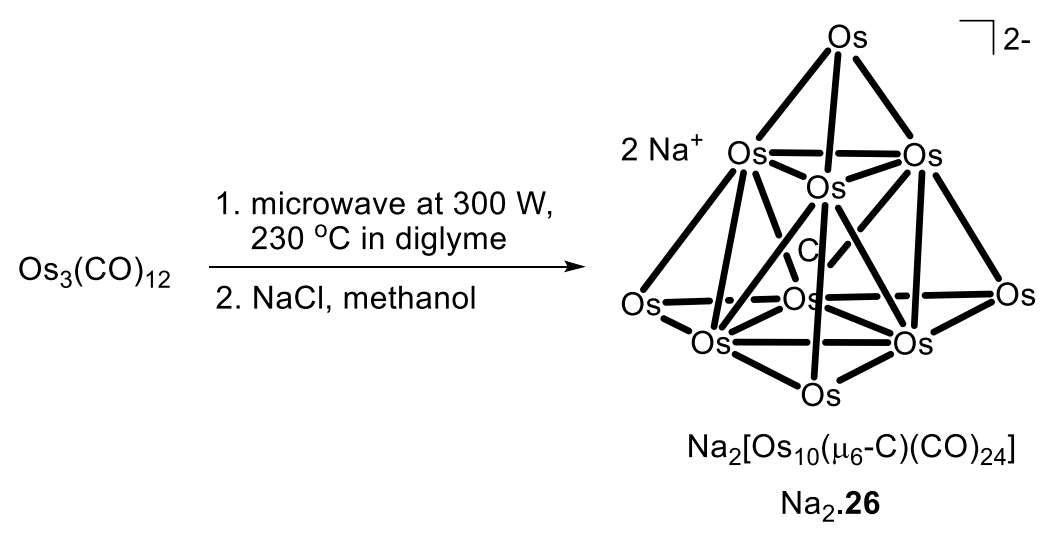

Scheme 4.1. Microwave-assisted synthesis of cluster $\mathrm{Na}_{2}$.26. $\mathrm{CO}$ ligands are omitted for clarity.
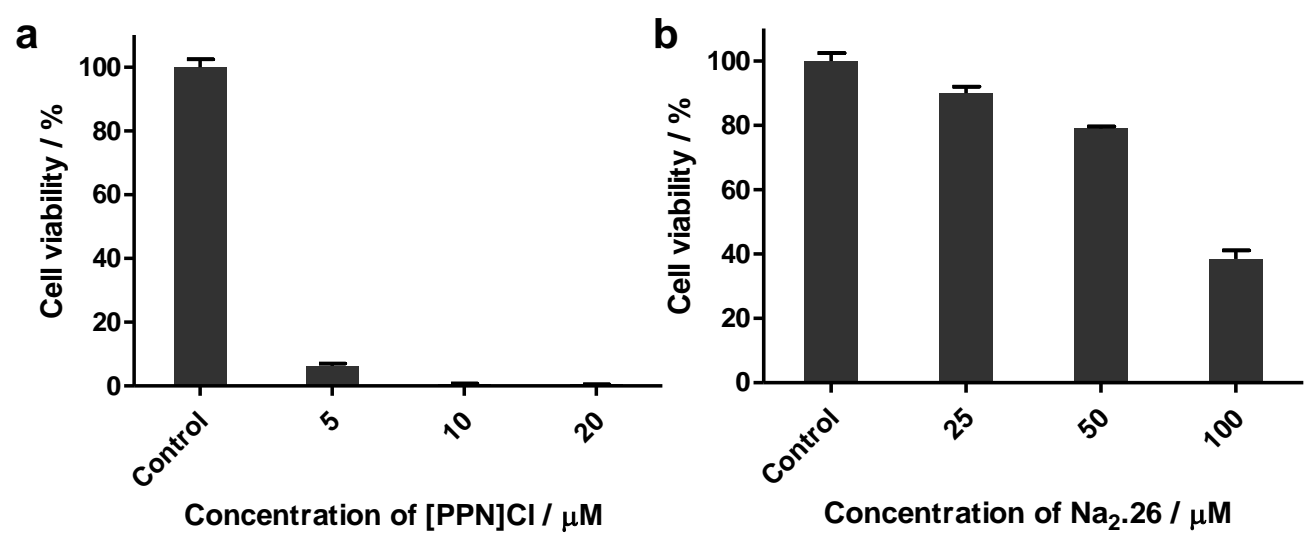

Figure 4.4. Cell viability (MTS) assay of a) $[\mathrm{PPN}] \mathrm{Cl}$ and b) $\mathrm{Na}_{2} . \mathbf{2 6}$ on OSCC cells, using $1 \%$ DMSO as vehicle.

The molar extinction coefficient (at the $\lambda_{\max }$ ) of $\mathrm{Na}_{2} . \mathbf{2 6}$ in three different solvent compositions were found to be similar: (1) DMSO, (2) 10\% DMSO in phosphate buffer saline (PBS) (v/v), and (3) 10\% DMSO in Dulbecco's modified Eagle medium (DMEM) containing 10\% fetal bovine serum (FBS), 1\% L-glutamine and 1\% 
penicillin/ streptomycin (v/v) (appendix A4.5). This was, however, not reflected in the PA response as the signal amplitude was higher in DMSO than in the aqueous biological media (figure 4.5, appendix A4.6, table 4.1). That a stronger PA signal is found in organic solvents than in aqueous media has been reported for a number of contrast agents, and attributed to differences in the Grüneisen parameter. ${ }^{13,14}$ This multi-component parameter, which describes the solvent environment, is temperaturedependent and governs the thermoelasticity of materials, a key contributor towards the PA effect. ${ }^{15-17}$ Different solvent compositions result in different Grüneisen parameters which will affect the amplitude of the PA signal. Nevertheless, the PA signal of $\mathrm{Na}_{2} . \mathbf{2 6}$ in modified DMEM was sufficiently strong and remained detectable down to $12.5 \mu \mathrm{M}$.
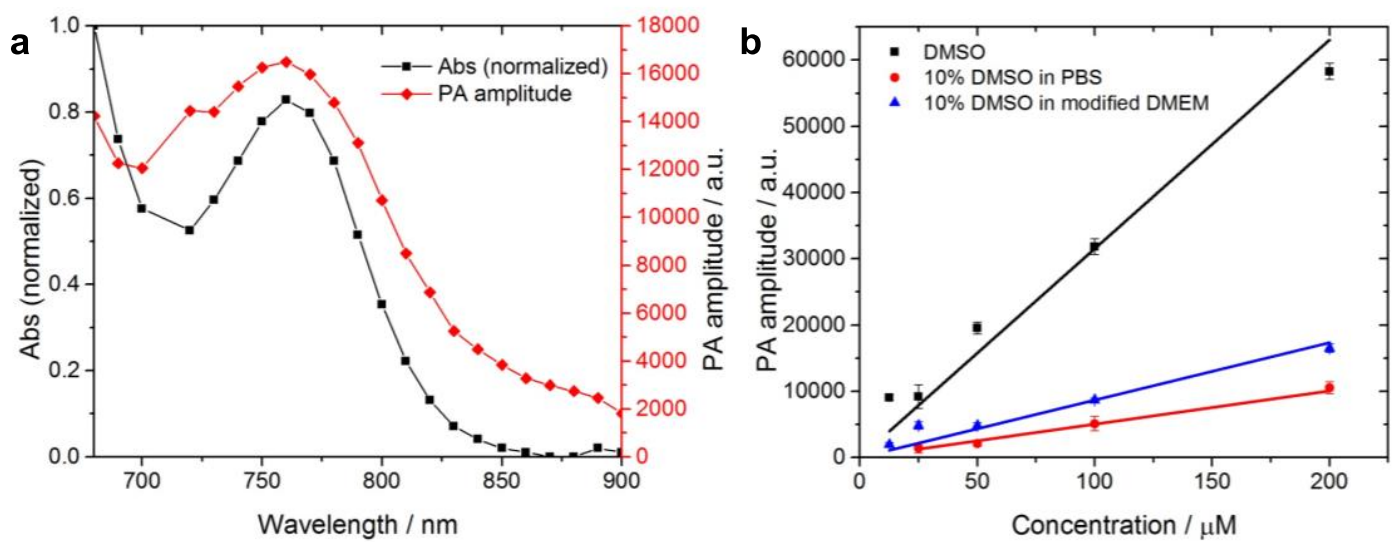

c

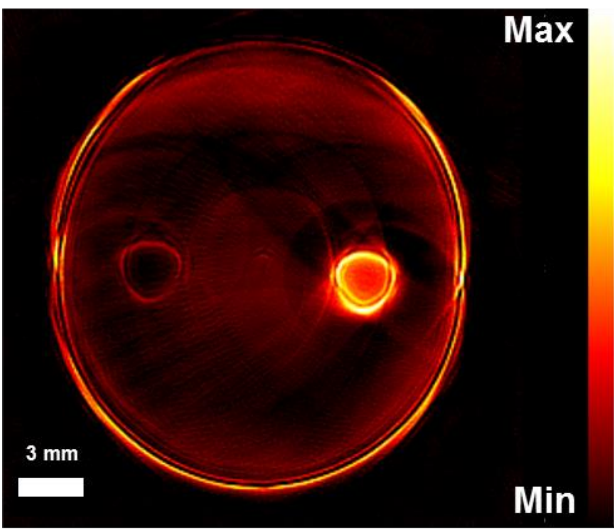

Figure 4.5 a) Optical absorption (black) and PA spectra (red) of $\mathrm{Na}_{2} .26(200 \mu \mathrm{M}, 10 \%$ DMSO in modified DMEM). b) PA response of $\mathrm{Na}_{2} .26$ as a function of concentration in three different solvent compositions. c) Single wavelength PA image of the phantom at $760 \mathrm{~nm}$. The right channel contains a solution of $\mathrm{Na}_{2} .26(200 \mu \mathrm{M}, 10 \%$ DMSO in modified DMEM), while the left channel contains only the DMSO in medium.

The salt $\mathrm{Na}_{2} .26$ exhibited good chemical and PA stability in 10\% DMSO in modified DMEM; the absorbance at $\lambda_{\max }=760 \mathrm{~nm}$ remained consistent, and the 
CO vibrations at 2034 and $1988 \mathrm{~cm}^{-1}$ remained unchanged with consistent peak integrals, over a 7-day period (figure 4.6a-c). The salt incubated in modified DMEM for various time points (10 min, 1, 3, 5 and 7 days) were subjected to gel electrophoresis. The gel showed a similar dark red band for all time-points, indicating little or no decomposition (figure 4.6d). Furthermore, the solution was stable to irradiation at $760 \mathrm{~nm}$ over a period of $120 \mathrm{~min}$; the CO peaks remain unchanged with consistent peak integrals (appendix A4.7).
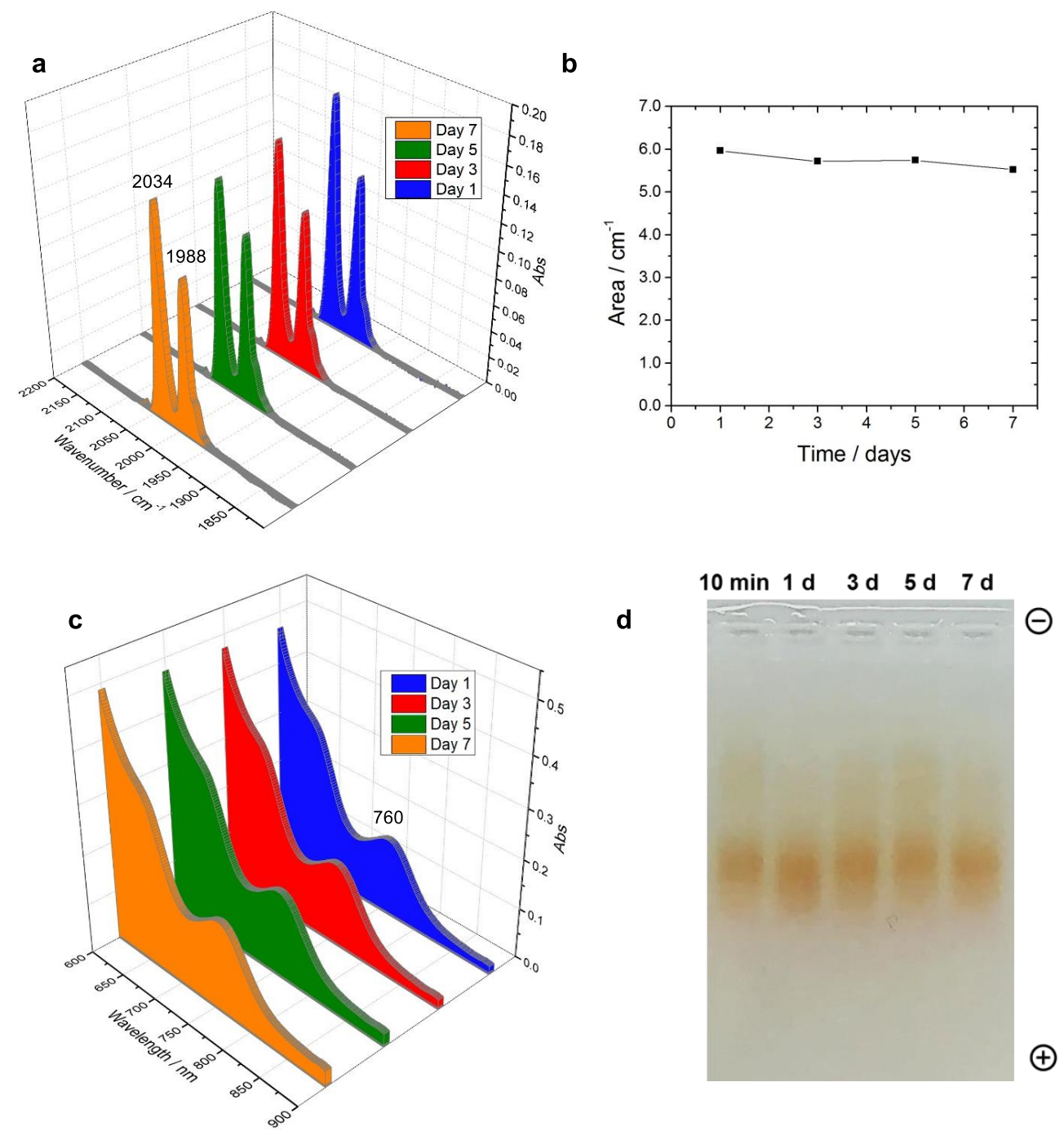

Figure 4.6. Time course study of a solution of $\mathrm{Na}_{2} . \mathbf{2 6}(200 \mu \mathrm{M})$ in $10 \%$ DMSO in modified DMEM, over a period of 7 days: a) FTIR spectra ( $v C O)$ obtained from the dried samples, $b$ ) integrals of the $\mathrm{vCO}$ absorbance, c) optical absorption spectra, d) electrophoresis gel. 
In a comparative study with indocyanine green (ICG), we found that the latter has low PA stability although it has better optical and PA properties (appendix A4.9, table 4.1); the PA signal decreased sharply upon irradiation at $790 \mathrm{~nm}$, and a similar decline was observed in its optical spectrum (figure 4.7, appendix A4.8). ICG is an organic tricarbocyanine NIR dye which has been approved by the U.S. Food and Drug Administration for clinical use in angiography, oncology and lymphatic vasculature imaging. ${ }^{18,} 19$ Furthermore, there have also been many reports on its use in PA imaging, including through development into nano-sized constructs. ${ }^{\mathbf{2 0 - 2 3}}$
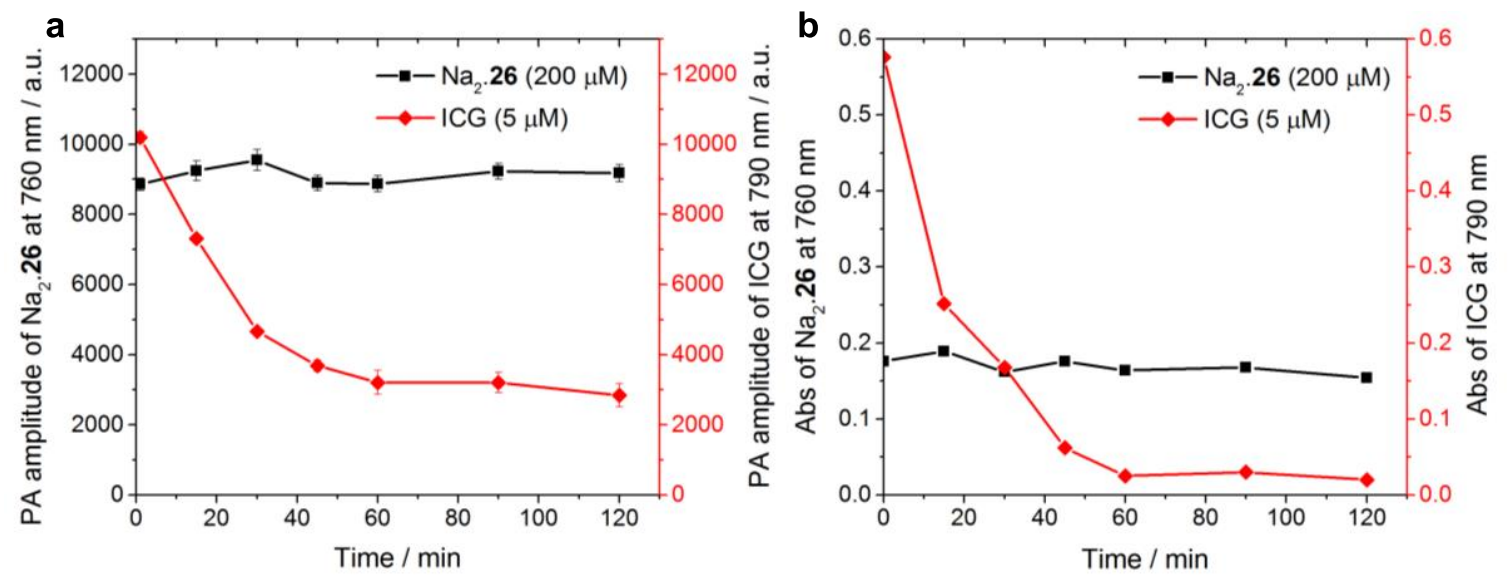

Figure 4.7. Time course studies of $\mathrm{Na}_{2} .26$ and ICG over 120 min with irradiation at the respective $\lambda_{\max }$ : a) PA amplitude as a function of time, b) optical absorbance as a function of time. 
Table 4.1. Optical absorption and PA properties of clusters 22-26 and indocyanine green (ICG).

\begin{tabular}{|c|c|c|c|c|}
\hline Entry & Compounds & $\lambda_{\max } / \mathbf{n m}^{[\mathrm{a}]}$ & $\boldsymbol{\varepsilon} / \mathbf{M}^{-\mathbf{1}} \mathbf{c m}^{-1}$ & PA quantum yield \\
\hline $1^{[\mathrm{b}]}$ & $\mathbf{2 2}$ & 719 & $4.52 \times 10^{3}$ & $329( \pm 20)$ \\
\hline $2^{[\mathrm{b}]}$ & $\mathbf{2 3}$ & $680^{[\mathrm{f}]}$ & $4.81 \times 10^{3}$ & $644( \pm 19)$ \\
\hline $3^{[\mathrm{b}]}$ & $\mathbf{2 4}^{[\mathrm{e}]}$ & $680^{[\mathrm{f}]}$ & $1.46 \times 10^{3}$ & $342( \pm 12)$ \\
\hline $4^{[\mathrm{b}]}$ & $\mathbf{2 5}^{[\mathrm{e}]}$ & $680^{[\mathrm{f}]}$ & $1.87 \times 10^{3}$ & $431( \pm 24)$ \\
\hline $5^{[\mathrm{b}]}$ & $\mathbf{2 6}^{[\mathrm{e}]}$ & 768 & $7.75 \times 10^{2}$ & $308( \pm 15)$ \\
\hline $6^{[\mathrm{b}]}$ & $\mathrm{Na}_{2} . \mathbf{2 6}$ & 770 & $9.08 \times 10^{2}$ & $315( \pm 35)$ \\
\hline $7^{[\mathrm{c}]}$ & $\mathrm{Na}_{2} . \mathbf{2 6}$ & 727 & $9.69 \times 10^{2}$ & $50( \pm 3)$ \\
\hline $8^{[\mathrm{d}]}$ & $\mathrm{Na}_{2} . \mathbf{2 6}$ & 760 & $8.41 \times 10^{2}$ & $87( \pm 5)$ \\
\hline $9^{[\mathrm{d}]}$ & $\mathrm{ICG}$ & 789 & $1.14 \times 10^{5}$ & $5097( \pm 157)$ \\
\hline
\end{tabular}

[a] Within the NIR region of 680-900 $\mathrm{nm}$.

[b] In DMSO.

[c] In 10\% DMSO in PBS (v/v).

[d] In 10\% DMSO in DMEM containing 10\% FBS, 1\% L-glutamine and 1\% penicillin/ streptomycin (v/v).

[e] As [PPN] salt

[f] Absorption spectra showed only a broad shoulder tapering towards longer wavelengths in the $680-900 \mathrm{~nm}$ region, so $680 \mathrm{~nm}$ was chosen as it gave the highest absorption in the region. 


\subsection{Photoacoustic in vivo imaging and biodistribution of 26}

The potential of $\mathrm{Na}_{2} . \mathbf{2 6}$ as an exogenous contrast agent was assessed on three mice with OSCC xenografts; they were injected intravenously through the tail vein with a solution of $\mathrm{Na}_{2} .26$ in $10 \%$ DMSO in heparinized saline $(200 \mu \mathrm{L}, 500 \mu \mathrm{M})$, giving an effective oncentration of approximately $50 \mu \mathrm{M}$ upon dilution with blood in the animal. Abdominal scans were made before, and up to $24 \mathrm{~h}$ after, the injection using real-time multispectral optoacoustic tomography (MSOT). ${ }^{24}$ In MSOT, the specimen is irradiated with light pulses of different wavelengths. The PA signals generated are collected to afford reconstructed ultrasound images, and spectral unmixing to identify the spectral signatures of molecules of interest present. ${ }^{25}$

The use of $\mathrm{Na}_{2} . \mathbf{2 6}$ as the contrast agent for whole body imaging was achieved as evident from the increase in PA contrast in various major abdominal organs, therefore, allowing them to be visualized and outlined easily. MSOT reconstructed images of the mice showed an increase in PA contrast in the liver, spleen and kidneys up to the first hour; by up to 4-, 3- and more than 2-folds, respectively (figure 4.8-4.9). This was reversed by the third hour, however, and almost reached baseline at $6 \mathrm{~h}$, suggesting fast clearance or breakdown of $\mathrm{Na}_{2} .26$ in the body. As expected, there was no significant accumulation in the tumor in comparison to other tissues as $\mathrm{Na}_{2} . \mathbf{2 6}$ does not bear any tumor targeting ligand, although the tumor-to-muscle ratio of the PA signal intensity showed an apparent 5-fold contrast at $3 \mathrm{~h}$ followed by a gradual decrease at 6 h (appendix A4.10). 
a
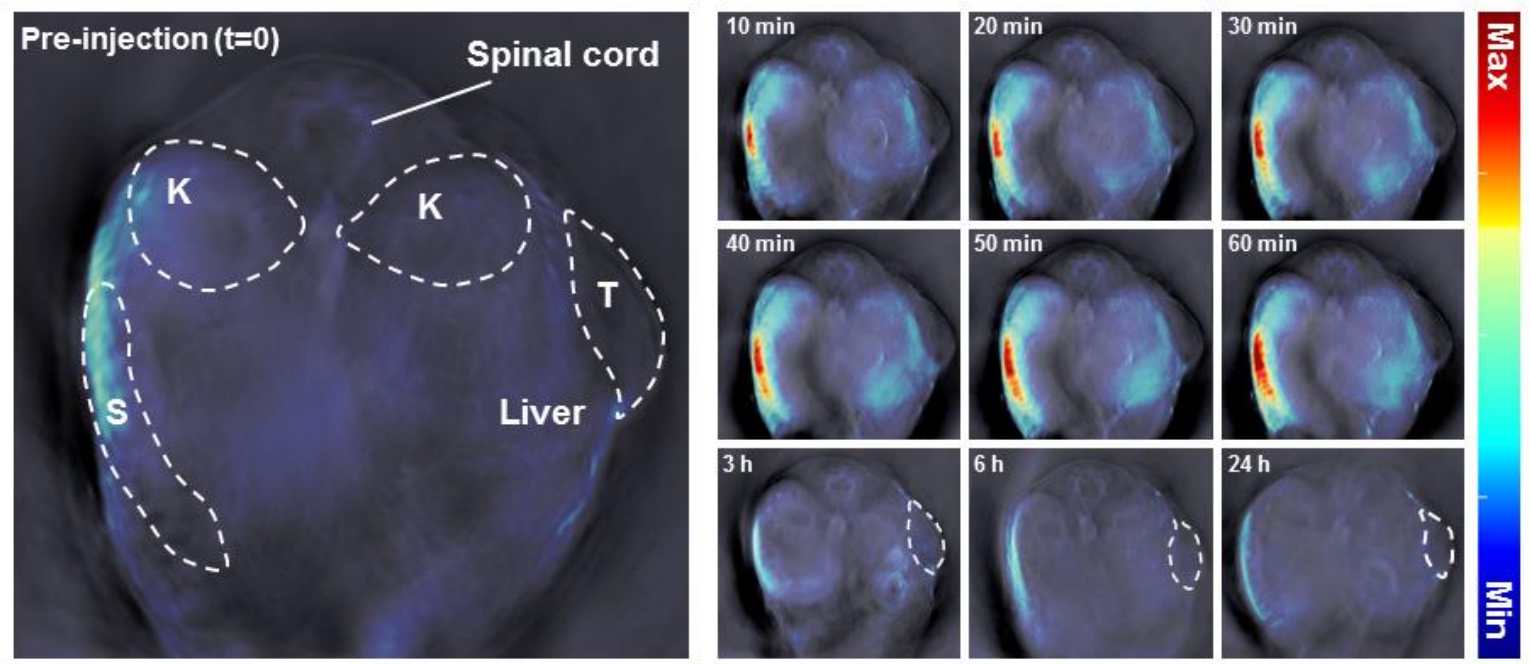

b
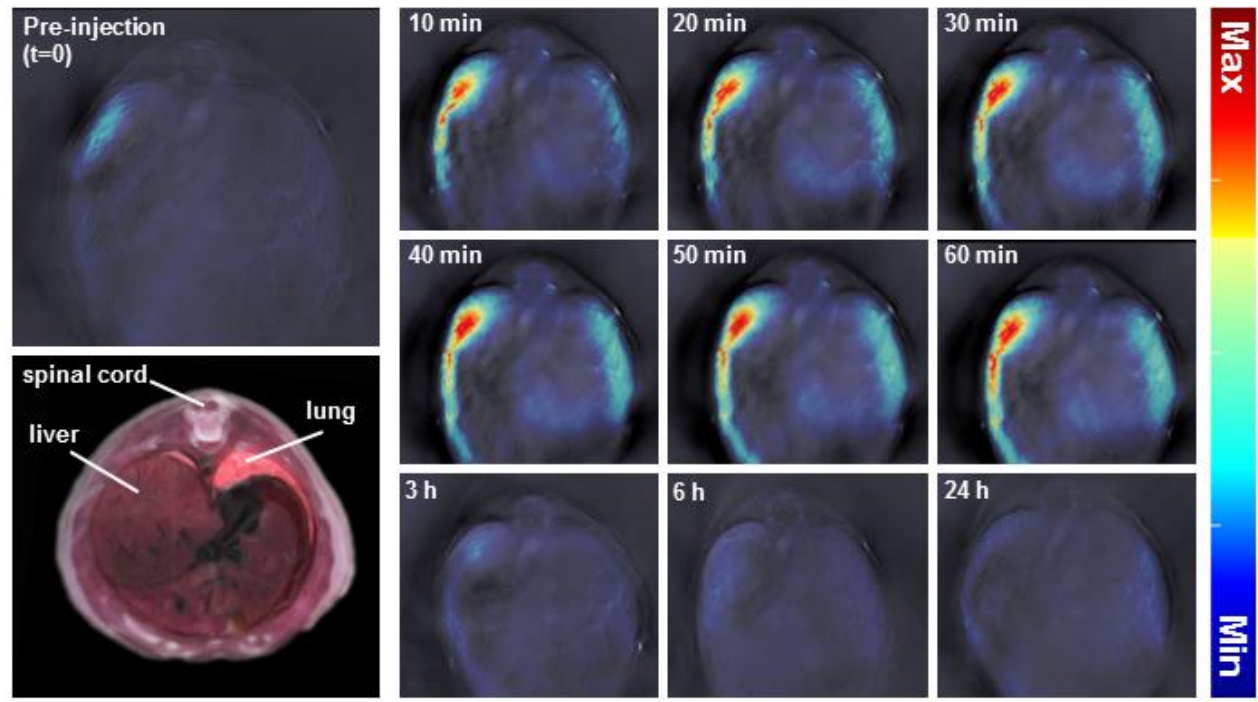

Figure 4.8. In vivo PA imaging of mouse OSCC xenograft model using $\mathrm{Na}_{2} . \mathbf{2 6}(200 \mu \mathrm{L}, 500$ $\mu \mathrm{M})$ as a contrast agent. a) PA signal from $\mathrm{Na}_{2} .26$ across a single slice of the mouse abdomen showing part of the liver, spleen (S), kidneys $(\mathrm{K})$ and tumor $(\mathrm{T})$ before and after injection up to $24 \mathrm{~h}$. All PA images are overlaid on their corresponding anatomy images at $800 \mathrm{~nm}$. b) PA signal from $\mathrm{Na}_{2} . \mathbf{2 6}$ across a single slice of the mouse abdomen showing a greater portion of the liver before and after injection up to $24 \mathrm{~h}$. All PA images are overlaid on their corresponding anatomy images at $800 \mathrm{~nm}$. Bottom left corner shows the reference anatomy of a single abdominal slice depicting in particular, the liver of a frozen sectioned mouse, as obtained elsewhere. ${ }^{26}$ 

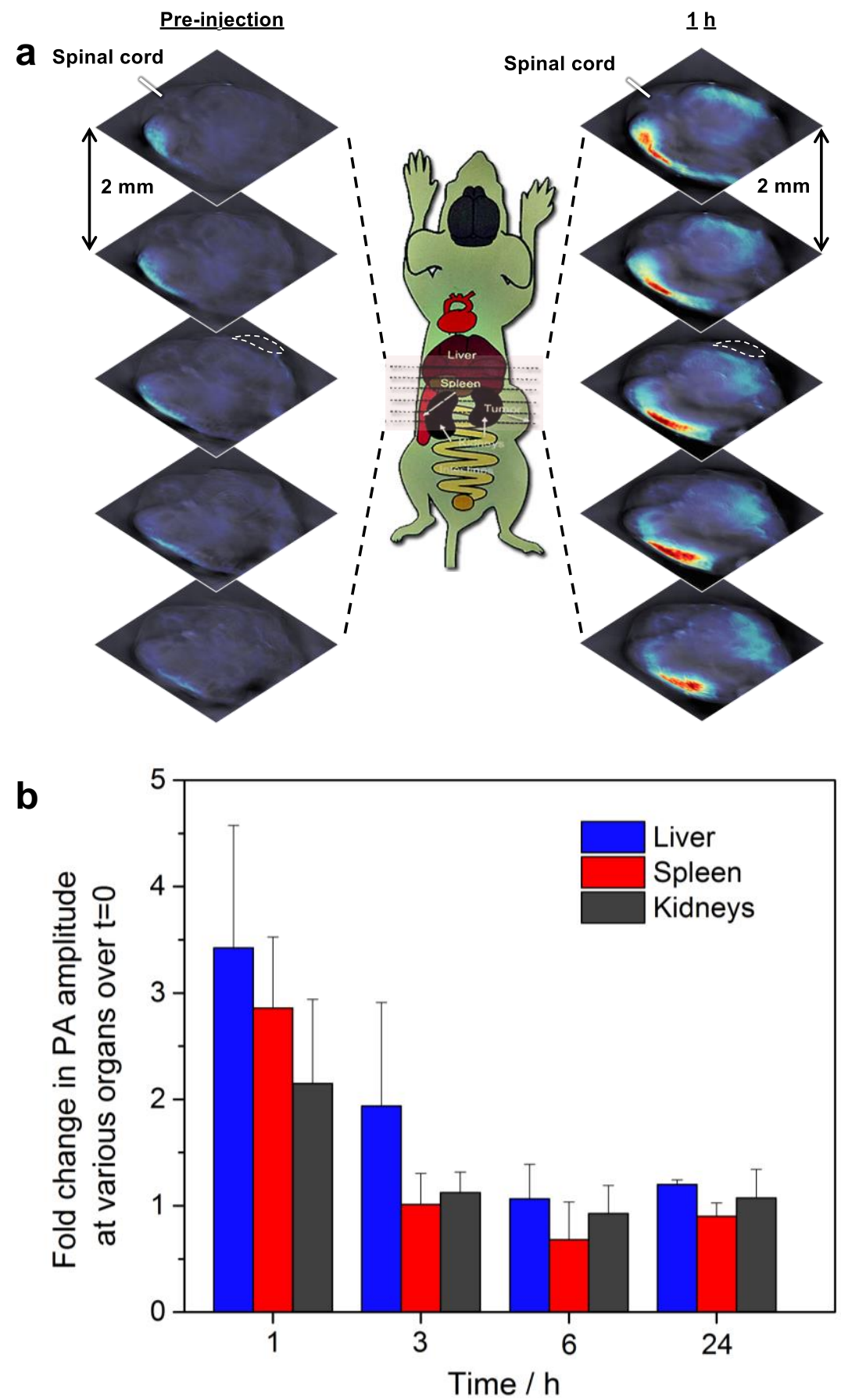

Figure 4.9. In vivo PA imaging of mouse OSCC xenograft model using $\mathrm{Na}_{2} . \mathbf{2 6}(200 \mu \mathrm{L}, 500$ $\mu \mathrm{M})$ as a contrast agent. a) Transverse sections of the mouse abdomen, at $2 \mathrm{~mm}$ interval, before and at $1 \mathrm{~h}$ after the injection of $\mathrm{Na}_{2} .26$. b) Fold change in the PA intensity in the liver, spleen and kidneys over that at $\mathrm{t}=0$. 
The biodistribution of $\mathrm{Na}_{2} . \mathbf{2 6}$ inferred from the MSOT experiment has been corroborated by an analysis of the osmium contents through inductively coupled plasma mass spectrometry (ICP-MS), after the mice were sacrificed at $24 \mathrm{~h}$ and $96 \mathrm{~h}$. Comparison of the values obtained at $24 \mathrm{~h}$ and $96 \mathrm{~h}$ reveal that accumulation of osmium exists even after $96 \mathrm{~h}$, mostly in the liver, spleen and kidneys, while other organs showed a significant drop in osmium content. The amount of osmium per tissue mass is significantly higher in the liver, spleen, lungs and kidneys than that in the control mice; it is highest in the liver, followed by the spleen (figure 4.10, appendix A4.11). This is similar to what has been found with the more extensively studied gold nanoparticles; ${ }^{27-29}$ while there have been variations across the studies, in most of the cases, accumulation has been observed to be highest in the liver, followed by tumor, spleen or kidneys.

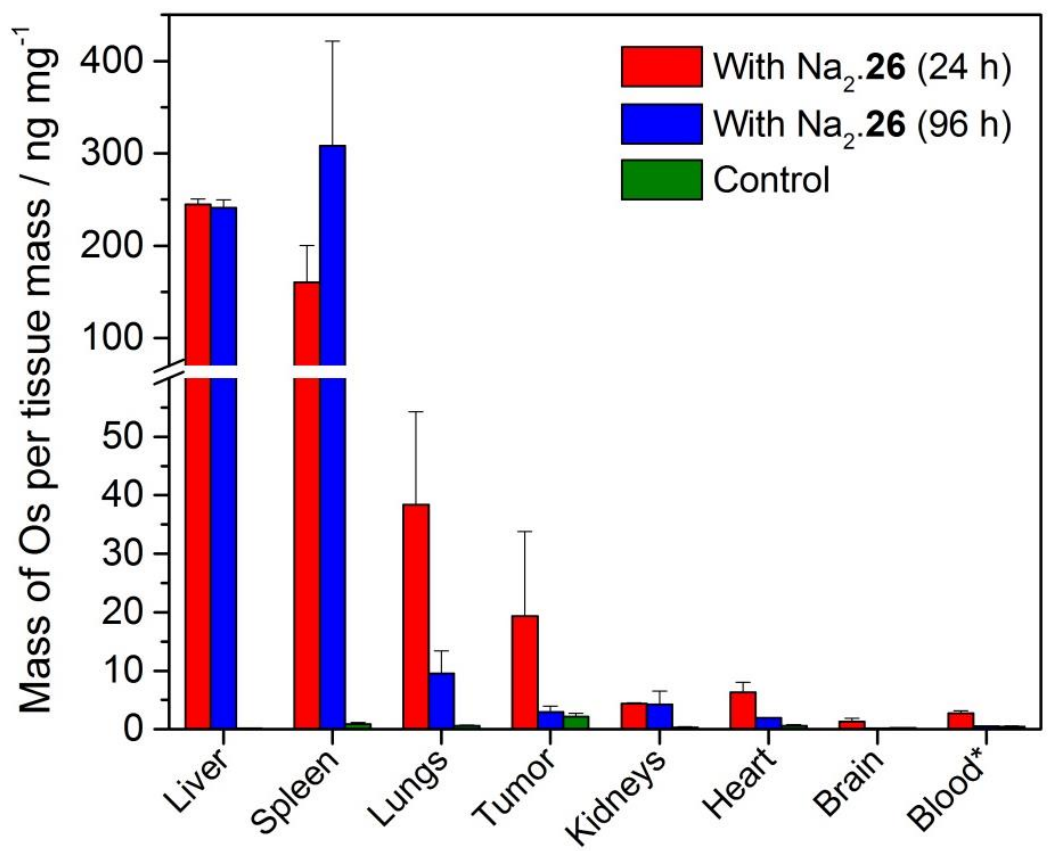

Figure 4.10. ICP-MS analysis of the amount of Os per tissue mass in various major organs of mice, $24 \mathrm{~h}$ and $96 \mathrm{~h}$ after injection of $\mathrm{Na}_{2} . \mathbf{2 6}$, with that of control samples. Error bars represent standard error of the mean, $\mathrm{n}=3$ for samples with $\mathrm{Na}_{2} .26(24 \mathrm{~h})$ and $\mathrm{n}=2$ for samples with $\mathrm{Na}_{2} .26$ (96 h) and control samples. ${ }^{*}$ Represented as mass of Os per fluid volume / $\mathrm{ng} \mu \mathrm{L}^{-1}$.

To further study the effect of the accumulation of the osmium metal in the various organs (liver, spleen, liver, lungs and heart), the mice were sacrificed at the $24 \mathrm{~h}$ and $96 \mathrm{~h}$ and the tissue morphology of the organs analyzed by histopathology. 
Hematoxylin and eosin $(\mathrm{H} \& \mathrm{E})$ staining of liver and spleen tissues displayed no obvious damage between the samples treated with $\mathrm{Na}_{2} . \mathbf{2 6}$ and that of control (figure 4.11), despite the higher accumulation of osmium content. Furthermore, no visible abnormalities were observed in the tissue of kidneys, heart and lungs (appendix A4.12). The condition and weight of the mice were also monitored over $24 \mathrm{~h}$ and $96 \mathrm{~h}$. The mice administered with cluster $\mathrm{Na}_{2} . \mathbf{2 6}$ were well-conditioned and showed no sign of weight loss after $96 \mathrm{~h}$ (appendix A4.13). However, the deposition of osmium metal in the liver and spleen is still of concern which requires a long-term study in the future.

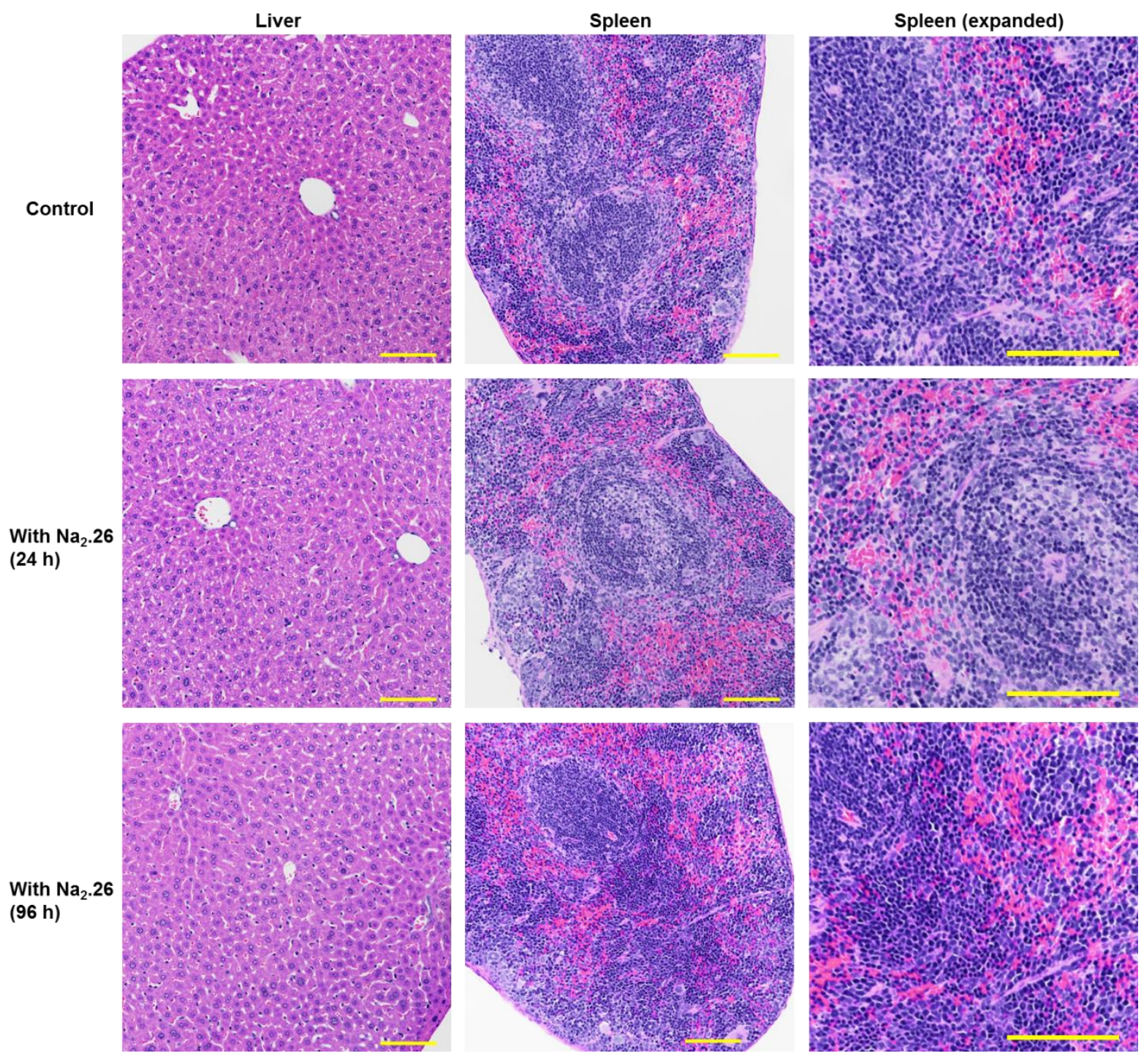

Figure 4.11. Histopathology ( $\mathrm{H} \& \mathrm{E}$ staining) of liver and spleen tissues of mice, $24 \mathrm{~h}$ and 96 $\mathrm{h}$ after intravenous injection of $\mathrm{Na}_{2} . \mathbf{2 6}$, with that of control samples. Scale bar $=100 \mu \mathrm{m}$. 


\subsection{Development of biocompatible HNCC via polymer encapsulation}

The moderate water solubility of the HNCCs, however, necessitated the use of a high concentration of DMSO for delivery into the biological system. This is a concern as DMSO is cytotoxic. One possible strategy to address this is to encapsulate the HNCCs in a micelle. Amphiphilic diblock copolymers like polystyrene-blockpoly(acrylic acid) (PS- $b$-PAA) can form water soluble and biocompatible micelles which have been used to encapsulate a wide range of materials like gold nanoparticles, ${ }^{30,31}$ carbon nanotubes, ${ }^{32}$ iron oxide nanoparticles, ${ }^{33}, 34$ and cadmium sulfide quantum dots. ${ }^{35}$

Encapsulation by PS- $b$-PAA can be carried out via either a kinetic or a thermodynamic approach (figure 4.12). The kinetic approach is suitable for polymers with shorter PAA chains, like $\mathrm{PS}_{250}-b-\mathrm{PAA}_{13}$, and involves the slow addition of a nonsolvent to a solution of the polymer. ${ }^{30}$ This induces self-assembly of the polymer, with controlled exclusion of polymer from the solution. Polymers with longer PAA chains, like $\mathrm{PS}_{154}-b$-PAA 49 , can self-assemble into micelles on heating followed by slow cooling in the presence of hydrophobic nanostructures. This thermodynamic approach involves allowing the micelles to form homogeneously as the critical micelle concentration (CMC) is gradually reached on cooling. ${ }^{31}$

\section{Thermodynamic approach}

- Use of longer PAA chain, like $\mathrm{PS}_{154}-b-\mathrm{PAA}_{49}$

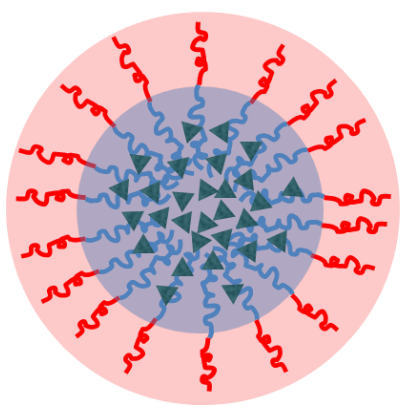

Heat, followed

by slow cooling

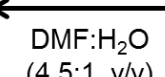

DMF: $\mathrm{H}_{2} \mathrm{O}$
$(4.5: 1, \mathrm{v} / \mathrm{v})$

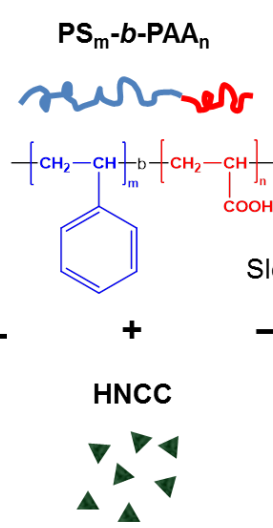

Kinetic approach

- Use of short PAA chain, like $\mathrm{PS}_{144}-b-\mathrm{PAA}_{22}$

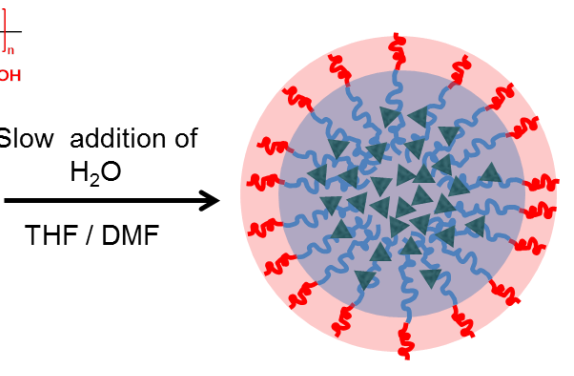

Figure 4.12. Graphical representation of the encapsulation of HNCC by PS- $b$-PAA via the thermodynamic and kinetic approach. 
An attempt at the thermodynamic approach was made with the polymer $\mathrm{PS}_{154}-b$ $\mathrm{PAA}_{49}$ which contains long PAA chains. To enhance interaction with the polystyrene block of the polymer, cluster 23 was used; this tetrahedral-shaped cluster has $\mathrm{PPh}_{3}$ ligands substituted at the four apices of the metal core. The cluster and the polymer were dissolved in DMF: water $(4.5: 1, \mathrm{v} / \mathrm{v})$, heated at $110{ }^{\circ} \mathrm{C}$ for $15 \mathrm{~min}$, and then allowed to slowly cool to room temperature in the oil bath. The sample was then diluted with a large amount of water to de-swell the polymer. A black precipitate, a sign of cluster decomposition, was observed. With a shorter heating time ( $5 \mathrm{~min})$, TEM images of the sample showed electron-rich spherical particles surrounded by a dense network of the polymer (figure 4.13). However, no well-defined encapsulated particles were observed. Characterization of the polymer-bound clusters by ATR-FTIR showed that the $\mathrm{CO}$ vibrational frequencies were shifted, indicating that the cluster has changed (figure 4.14). The frequency shifts were greater with the longer heating time (15 min), pointing to thermal instability of the cluster.

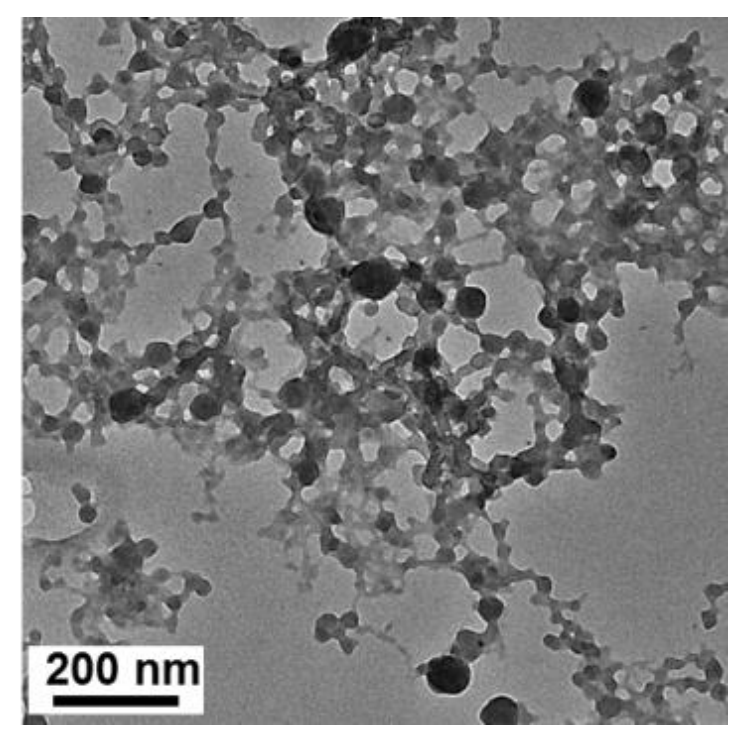

Figure 4.13. TEM images of $\mathrm{PS}_{154}-b-\mathrm{PAA}_{49}$ encapsulation of 23 heated at $110{ }^{\circ} \mathrm{C}$ for $5 \mathrm{~min}$. 


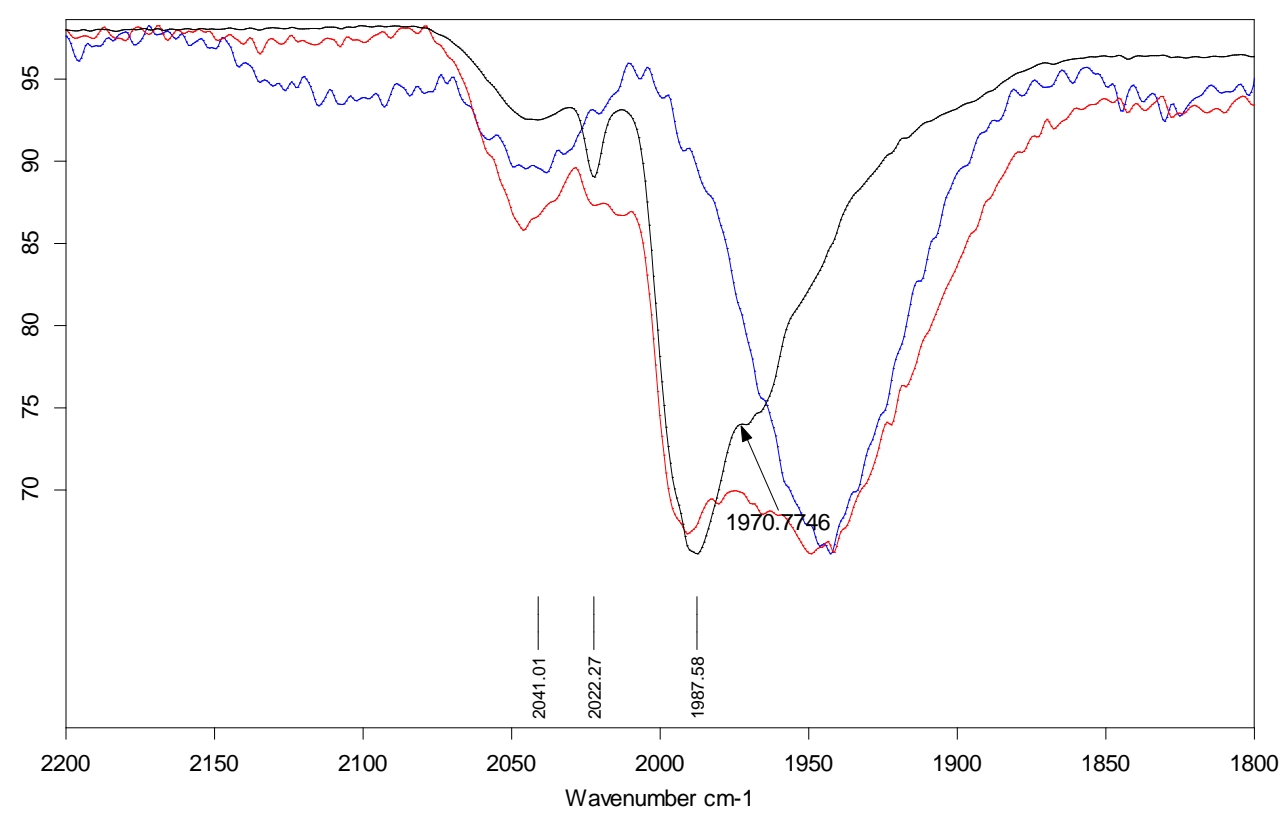

Figure 4.14. ATR-FTIR spectra of polymer bound cluster 23 heated at $110{ }^{\circ} \mathrm{C}$ for $5 \mathrm{~min}$ (red) and 15 min (blue) respectively, in comparison with cluster $\mathbf{2 3}$ (black).

Encapsulation of cluster $\mathbf{2 3}$ via the kinetic approach was investigated with $\mathrm{PS}_{144}-b$ $\mathrm{PAA}_{22}$, a polymer with the shorter PAA chain. A fixed amount of the cluster $(1 \mathrm{mg})$ with different amounts of the polymer (from 0.48 to $0.08 \mathrm{mg}$ ) were dissolved in DMF, and then water was slowly added. A higher amount of polymer $(0.48 \mathrm{mg})$ was required to achieve homogeneity as lower concentrations resulted in precipitation of the cluster on standing overnight (figure 4.15). The TEM images showed dark spherical structures in a dense network of polymer (figure 4.16). However, there were no observable changes in the spherical structures with the polymer concentration.

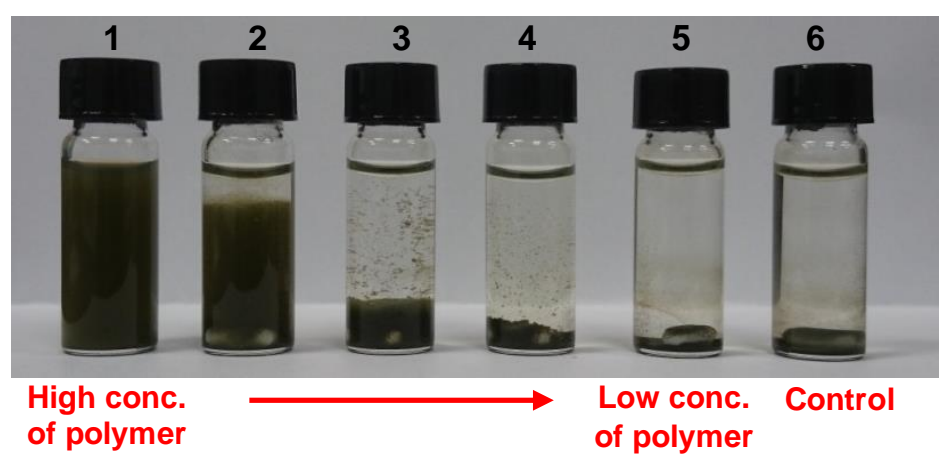

Figure 4.15. Photograph of cluster 23 incubated with various amount of polymer $(0.48,0.40$, $0.24,0.16$ and $0.08 \mathrm{mg}$ from samples 1 to 5 respectively). Control sample contains no polymer. 

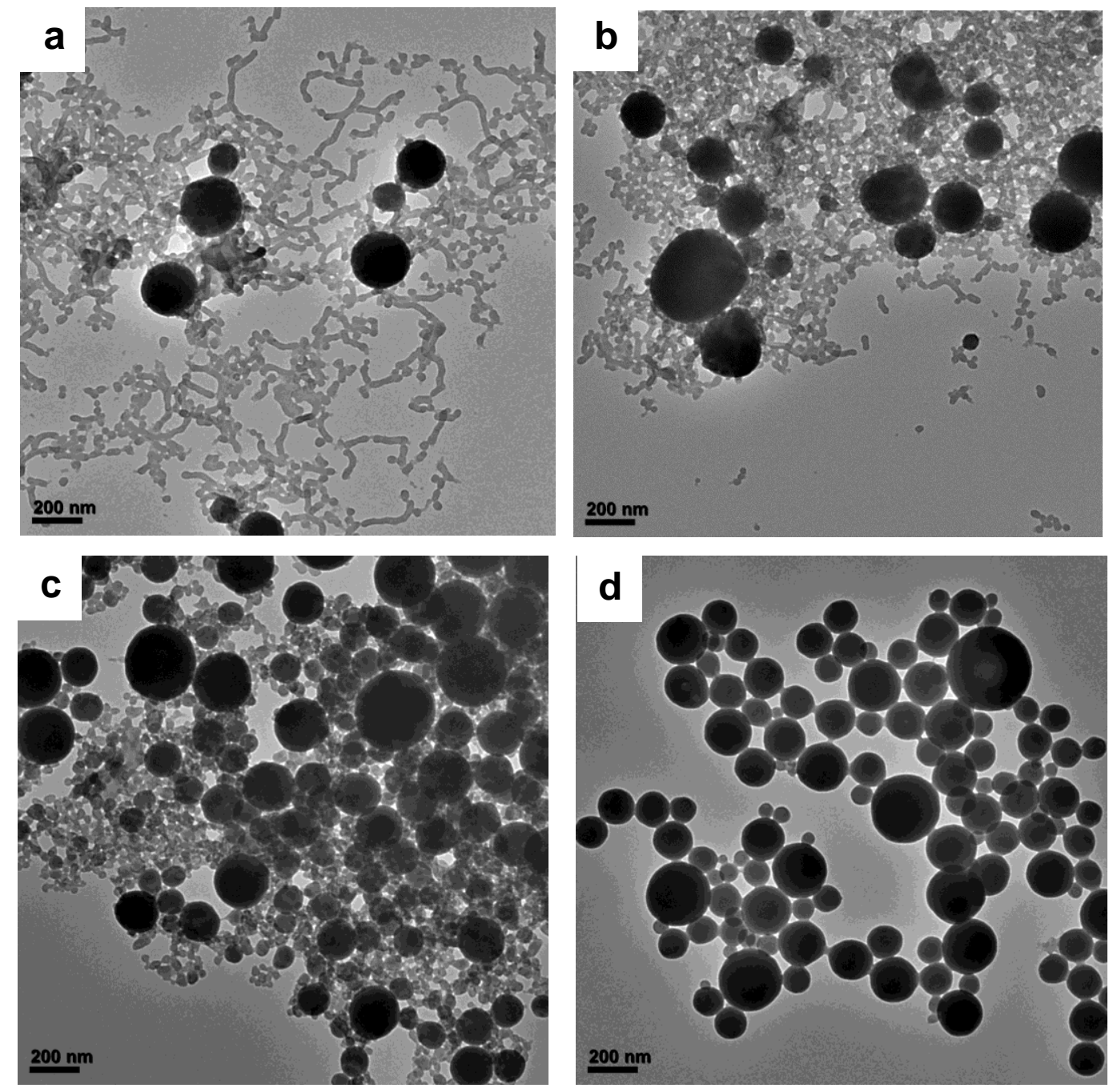

Figure 4.16. TEM images of (a-c) samples 1,2 and 5, and d) control sample.

The homogeneous mixture of sample 1, with $0.48 \mathrm{mg}$ of polymer, was characterized by ATR-IR and the CO vibrational signals were in good agreement with that of cluster $\mathbf{2 3}$ (figure 4.17). This showed that the addition of polymer to cluster $\mathbf{2 3}$ did not affect the integrity of cluster, which would otherwise have shown up as changes in the $\mathrm{CO}$ vibrational frequencies. Nevertheless, precipitation occurred upon standing overnight. An attempt was made to stabilize the micelles with amines; this is known to fix and stabilize the micelles by crosslinking the carboxylic ends. ${ }^{30,34}$ The homogeneous mixture was first dialyzed overnight against deionized water. The sequential addition of 1-ethyl-3-(3-dimethylaminopropyl)carbodiimide (EDC), followed by 2,2-(ethylenedioxy)bis (ethylamine) and $\mathrm{NH}_{2}$-PEG-COOH, led to precipitation after incubating for $2 \mathrm{~h}$; the micelles could not be stabilized. 


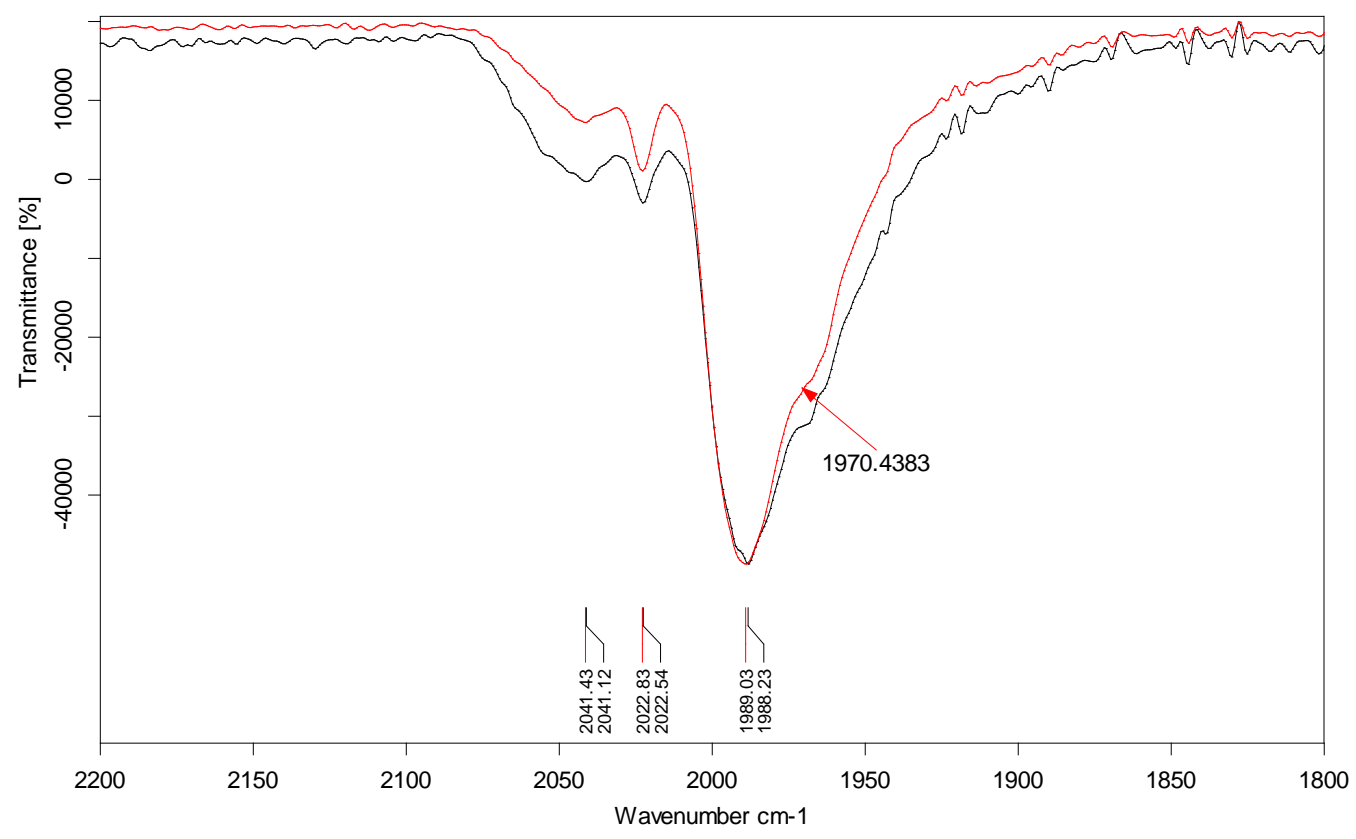

Figure 4.17. IR spectra of cluster 23 (red) and the cluster with polymer (black).

\subsection{Conclusion}

In this chapter, the cluster nuclearity was shown to be a useful parameter for fine-tuning the absorption wavelength. The $\operatorname{HNCC}\left[\mathrm{Os}_{10}\left(\mu_{6}-\mathrm{C}\right)(\mathrm{CO})_{24}\right]^{2-}, \mathbf{2 6}$, was shown to exhibit good PA properties in the NIR region and was applied successfully in PA imaging of live mice. Although no significant pathological changes were observed in the major organs, the accumulation of osmium metal in the liver and spleen is of concern and would require a more comprehensive, long-term study. Attempts to make the HNCCs more biocompatible through delivery as polymer encapsulated micelles were unsuccessful. Nevertheless, these results have demonstrated that HNCCs are reliable NIR contrast agents with potential use in deep tissue imaging, although further improvements to their biocompatibility and performance will be desirable. 


\subsection{Experimental section}

\subsubsection{General methods}

All reactions and manipulations were carried out under an argon atmosphere using standard Schlenk techniques. Solvents that were used for reaction were distilled over the appropriate drying agents under argon before use. Purification of compounds was generally carried out by column chromatography on silica gel, or by preparative thinlayer chromatography (TLC) using $20 \mathrm{~cm}$ x $20 \mathrm{~cm}$ plates pre-coated with silica gel 60 $\mathrm{F}_{254}$ Infrared (IR) spectra were recorded on a Bruker Alpha FT-IR spectrometer. Solution spectra were recorded in DCM solution, unless otherwise stated, in a solution IR cell with $\mathrm{NaCl}$ windows and a path length of $0.1 \mathrm{~mm}$, at a resolution of $2 \mathrm{~cm}^{-1}$. Alternatively, IR spectra of aqueous samples were recorded by drop casting the mixture $(10 \mu \mathrm{L})$ onto a $\mathrm{CaF}_{2}$ disc or onto the ZnSe ATR attachment, followed by drying under a stream of nitrogen before recording on the spectrometer. Optical absorption spectra were recorded using a Hitachi U-2900 double-beam spectrophotometer using quartz cuvette with path length of $10 \mathrm{~mm}$. For samples of smaller volume $(100 \mu \mathrm{L})$, the optical absorption spectra were recorded using a Beckman Coulter DU 730 spectrometer. HRMS were recorded in ESI mode on a Waters UPLC-Q-Tof MS mass spectrometer.

Copper specimen grids (300 mesh) with formvar/carbon support film were purchased from Beijing XXBR Technology Co. TEM images were collected from a JEM-1400 (JEOL) Transmission Electron Microscopy operated at $100 \mathrm{kV}$. TEM grids were treated with oxygen plasma in a Harrick® plasma cleaner/sterilizer for 1 min to improve the surface hydrophilicity. A sample solution $(10 \mu \mathrm{L})$ was dropped onto the hydrophilic side of the TEM grid. Filter paper was used to wick off the excess solution on the TEM grid, which was then left to dry in air for $5 \mathrm{~min}$.

Compounds 11'a, 20-26 were synthesized and identified according to reported methods with slight modifications. ${ }^{2-8}$ Their respective IR spectra can be found in the appendix A4.14-5.21. $\mathrm{Os}_{3}(\mathrm{CO})_{12}$ and $\mathrm{Ru}_{3}(\mathrm{CO})_{12}$ were purchased from Oxkem Ltd. $[\mathrm{PPN}] \mathrm{Cl}$ and indocyanine green (ICG) were purchased from Sigma Aldrich. Amphiphilic diblock copolymer polystyrene-block-poly(acrylic acid) ( $\mathrm{PS}_{154}-b$-PAA 49 , $M_{n}=16000$ for the polystyrene block and $M_{n}=3500$ for the poly(acrylic acid) block, $\mathrm{M}_{\mathrm{w}} / \mathrm{M}_{\mathrm{n}}=1.15 ; \mathrm{PS}_{144}-b-\mathrm{PAA}_{22}, \mathrm{M}_{\mathrm{n}}=15000$ for the polystyrene block and $\mathrm{Mn}=1600$ for the poly(acrylic acid) block, $\mathrm{M}_{\mathrm{w}} / \mathrm{M}_{\mathrm{n}}=1.11$ ) was purchased from Polymer Source, 
Inc. All other chemical reagents were purchased from other commercial sources and used without further purification.

\subsubsection{Synthesis of cluster $\mathrm{Na}_{2} .26$}

The synthesis was adapted from the microwave-assisted synthesis of cluster PPN salt of 26. ${ }^{8}$ A Discover-SP microwave reactor (2450 MHz, CEM Corp., Matthews, NC) and thick-walled $35 \mathrm{~mL}$ glass vessel with Teflon-lined caps (CEM Corp., Matthews, NC) were used. In a typical reaction, a $35 \mathrm{~mL}$ microwave vessel was charged with $\mathrm{Os}_{3}(\mathrm{CO})_{12}(150 \mathrm{mg}, 0.1654 \mathrm{mmol})$ and diglyme $(7 \mathrm{~mL})$. The mixture was irradiated with $300 \mathrm{~W}$ with high stirring rate, till the temperature reached $230{ }^{\circ} \mathrm{C}$. The power was subsequently reduced to $100 \mathrm{~W}$ for additional heating for $10 \mathrm{~min}$. The reaction mixture was cooled to $50{ }^{\circ} \mathrm{C}$ and vented, before irradiated at $300 \mathrm{~W}$ for $4 \mathrm{~min}$. The cooling, venting and irradiation at $300 \mathrm{~W}$ for $4 \mathrm{~min}$ was repeated once. The solvent was removed by evaporation over a hot water bath and the residue was extracted with acetone $(20 \mathrm{~mL})$ and filtered over a thin layer of celite. The solvent was removed and the residue dissolved in $\mathrm{MeOH}(10 \mathrm{~mL}) . \mathrm{NaCl}(20 \mathrm{mg}, 0.34 \mathrm{mmol})$ was added to the solution and stirred at $60{ }^{\circ} \mathrm{C}$ for $1 \mathrm{~h}$. Solvent was removed and the residue was purified by TLC using acetone-hexane (3:2, v/v) as eluant to give cluster $\mathbf{N a} \mathbf{a}_{\mathbf{2}} \mathbf{2 6}$ as a dark red band.

Yield: $33 \mathrm{mg}(24 \%)$

$\mathrm{R}_{\mathrm{f}}=0.23$

IR (acetonitrile, $\mathrm{cm}^{-1}$ ): $v_{\mathrm{CO}} 2038 \mathrm{~s}, 1990 \mathrm{~s}$. (see appendix 5.21, lit: 2038s, 1990s) ${ }^{36}$

HRMS-ESI: $m / z$ 1294.0946 [M] ${ }^{2-}$ (see appendix A4.22 for isotopic pattern)

\subsubsection{Cell culture and cell viability assay}

Experimental cultures of oral squamous cell carcinoma (OSCC) cell line were obtained from the American Type Culture Collection (ATCC) and cultured in tissue culture dishes (Nunc Inc., IL). The cells were maintained in Dulbecco's modified Eagle's medium (DMEM; Biowest, France) supplemented with $10 \%$ fetal bovine serum (FBS; Gibco $\left.^{\circledR}, \quad \mathrm{NY}\right), \quad 1 \%$ L-glutamine $\quad$ (PAA Laboratories, Austria), and $1 \%$ penicillin/streptomycin (HyClone, UT) at $37^{\circ} \mathrm{C}$ in $5 \% \mathrm{CO}_{2}$ atmosphere. Phosphatebuffered saline (PBS) was obtained from PAA Laboratories. Stock solutions of cluster $\mathrm{Na}_{2} .26$ and $[\mathrm{PPN}] \mathrm{Cl}$ in sterile-filtered dimethyl sulfoxide (DMSO) were prepared and 
serial diluted to lower concentrations. For treatment with the compounds, cells were seeded in wells within 96-well plate in growth medium at the same initial density of 10 000 cells per well, and allowed to adhere and grow for $24 \mathrm{~h}$. They were serum-starved in serum-free DMEM for $24 \mathrm{~h}$, followed by treatment with the indicated concentrations of compounds in serum-free DMEM (1\% DMSO) for $24 \mathrm{~h}$. Control cells were treated with vehicle (1\% DMSO). To each well, $10 \mu \mathrm{L}$ of MTS reagent (MTS Cell Proliferation Assay Kit, BioVision, CA) was added and then left to incubate in a $37^{\circ} \mathrm{C}$ incubator with $5 \% \mathrm{CO}_{2}$ for $2 \mathrm{~h}$. The absorbance intensities at 490 $\mathrm{nm}$ were then measured and the cell proliferation relative to the control sample was calculated. Each sample was analyzed in triplicates and was corrected with background intensities from same incubation conditions without the cells. $\mathrm{IC}_{50}$ was determined from three separate experiments and the mean value calculated.

\subsubsection{Gel electrophoresis}

Agarose gel (1\%, UltraPure ${ }^{\mathrm{TM}}$ Agarose, Invitrogen) was cast with and immersed in 0.5 $\mathrm{x}$ TAE (Tris-acetate-EDTA buffer, prepared from $50 \mathrm{x}$ stock solution, $\mathrm{pH} 8.0,1^{\text {st }}$ BASE). Cluster $\mathrm{Na}_{2} . \mathbf{2 6}$, incubated in $10 \%$ DMSO in modified DMEM (30 $\left.\mu \mathrm{L}\right)$ for different duration (10 $\min , 1,3,5$ and 7 days), was loaded into the respective wells of the gel and electrophoresed in a horizontal electrophoresis system (Wide Mini-sub® cell GT Basic System, Biorad, electrode spacing of $15 \mathrm{~cm}$ ) at $150 \mathrm{~V}$ for $30 \mathrm{~min}$.

\subsubsection{Multispectral optoacoustic tomography (MSOT) experimental parameters and protocol}

All phantom and in vivo mouse imaging experiments were performed using a real-time MSOT imaging system; inVision 128 (ithera Medical GmbH, Germany). ${ }^{24}$ Optical excitation was provided by an optical parametric oscillator with a tunable NIR wavelength range from 680 to $900 \mathrm{~nm}$, which was in turn pumped by a Q-switched $\mathrm{Nd}$ :YAG laser with a pulse duration of $10 \mathrm{~ns}$ and repetition rate of $10 \mathrm{~Hz}$. Light was delivered by a fiber bundle divided into 10 output arms to illuminate the sample from multiple angles around the imaging plane. PA signals were acquired using a 128element concave transducer array spanning a circular arc of $270^{\circ}$. This transducer array had a central frequency of $5 \mathrm{MHz}$, which provided a transverse spatial resolution in the range of $150-200 \mu \mathrm{m}$. One transverse image slice was acquired from each laser 
pulse, resulting in a frame rate of $10 \mathrm{~Hz}$. During image acquisition, the sample was translated through the transducer array along its axis across the volume region of interest (ROI), in order to capture the corresponding transverse image slices.

\subsubsection{Evaluation of PA activity of clusters 22-26 in phantom model}

The polyurethane phantom is cylindrical in shape with a diameter of $2 \mathrm{~cm}$; it is specially designed to mimic the shape, size, and optical properties of the mouse. In addition, it has two inner cylindrical channels, each with an inner diameter of $3 \mathrm{~mm}$, one for holding the control medium and the other for holding the dissolved contrast agent in the same medium. The control medium and contrast agent suspension $(170 \mu \mathrm{L}$ each) were pipetted into the respective channels of the phantom, and placed in the MSOT imaging chamber using a phantom holder. For data acquisition, an ROI of multiple transverse slices with a step size of $1 \mathrm{~mm}$ was set up across the channel portion which contained the probe and control, applied excitation wavelengths from 680 to $900 \mathrm{~nm}$ with an interval of $10 \mathrm{~nm}$ for each transverse slice, and recorded the averaged PA signals from 10 frames for each wavelength and position. The acquired images were reconstructed using model based approach and PA signals were quantified using ViewMSOT at the respective $\lambda_{\max }$ of each probe from the

reconstructed images. ${ }^{37}$ The PA amplitude of the probe was determined by subtracting the values of the channel containing the control from that of the probe, and the mean was calculated from 10 different transverse slices.

\subsubsection{In vivo longitudinal monitoring of probe biodistribution in mouse xenograft model}

Dr Ghayathri (SBIC, A*STAR) prepared the mouse xenograft model and performed the animal imaging experiments with my assistance. All animal experiments were performed in compliance with the protocol \#120774 and \#151085 approved by the Institutional Animal Care and Use Committee, Agency for Science, Technology and Research (A*STAR). Xenograft models were established on three Balb/C nude mice by injecting subcutaneously into the right flank of each mouse, a cell suspension $(0.1$ $\mathrm{mL}$ ) containing $5 \times 10^{6}$ oral squamous carcinoma cells (OSCC) and matrigel (BD Biosciences, San Jose, CA) in a 1:1 volume ratio. When the tumor volume reached a palpable size, a solution of $\mathrm{Na}_{2} .26(200 \mu \mathrm{L}, 500 \mu \mathrm{M})$ in $10 \%$ DMSO in heparinized 
saline (v/v) was injected intravenously through the tail vein of each mouse anaesthetized under isoflurane. Ultrasound gel was applied on the mouse skin surface for in vivo imaging, and measurements were recorded in temperature-controlled water medium for good acoustic coupling. An animal holder with a thin polyethylene membrane was used to prevent direct contact between the mouse and the water. Seven laser excitation wavelengths $(680,700,730,750,810,850$, and $900 \mathrm{~nm})$ were chosen for multispectral imaging on the basis of the absorption maxima and minima of the contrast agent, and oxy- and deoxyhemoglobin. PA signals were acquired across multiple transverse slices from the liver to the lower abdomen region inclusive of the tumor at a step size of $0.5 \mathrm{~mm}$ and averaged over 10 frames for each wavelength.

\subsubsection{Image processing and analysis for in vivo experiments}

Images acquired from the in vivo experiments were reconstructed using direct back projection approach for offline analysis. After image reconstruction, spectral unmixing was performed to resolve individual components from different chromophores in the system. For each pixel, the method fits the total measured optoacoustic spectrum to the known absorption spectra of the individual chromophores, based on least-squares linear regression. Images were exported out from ViewMSOT and further analyzed using Fiji (version 2.0.0), an open source image-processing package. ${ }^{38}$ Regions of Interest (ROIs) were chosen from liver, spleen, kidney and tumor and quantified for multispectrally unmixed PA signals before and after probe injection. PA signals at all time-points were normalized against that of the pre-injection scan and expressed as fold change over the same.

\subsubsection{Microwave acid digestion and ICP-MS analysis of osmium content of tissue samples}

ICP-MS analysis was carried out by Dr Bahareh (CBC, NTU). The mice were euthanized after 24 and $96 \mathrm{~h}$ respectively, the organs extracted, washed with deionized water and snap-freezed in liquid nitrogen for future use. The selected organs were freeze-dried over a period of five days and the dried masses measured, prior to microwave acid digestion. A programmable Milestone microwave system (ETHOS EZ, Italy) was used to digest and prepare the samples for ICP analysis. Samples were placed inside the Teflon microwave vessels and $12 \mathrm{~mL} \mathrm{HCl} \mathrm{(37 \% ,} \mathrm{ultrapure} \mathrm{grade,} \mathrm{J.}$ T. Baker, Canada) were added. Ultra-pure water with $18.2 \mathrm{M} \Omega \bullet \mathrm{cm}$ resistivity was used 
(ELGA, PURLAB Option - Q, UK) for sample and standard preparations. Using the microwave radiation (Power $1100 \mathrm{~W}$ ) the temperature was ramped to $185^{\circ} \mathrm{C}$ in $20 \mathrm{~min}$ and then held at that temperature for $15 \mathrm{~min}$. The vessels were opened after cooling down to room temperature for several hours, to prevent any sample loss. The extracts were diluted with ultrapure water, filtered through $0.2 \mu \mathrm{m}$ syringe filters, and carefully transferred to ICP sample vials for elemental analysis. Samples were analyzed using an Agilent 7700 series ICP-MS (Japan) equipped with a third generation He reaction / collision cell (ORS3) to minimize interference. An external calibration curve for osmium was constructed, before each analytical run, from serial dilutions of an osmium standard for ICP-MS (1000 $\mathrm{mgL}^{-1}$, Inorganic Venture, Christiansburg, VA, USA) with high purity deionized water.

\subsubsection{Histopathology of organ tissues of mice}

Six mice were intravenously injected with cluster $\mathrm{Na}_{2} .26(200 \mu \mathrm{L}, 500 \mu \mathrm{M}, 10 \%$ DMSO in saline) and euthanized after $24 \mathrm{~h}$ and $96 \mathrm{~h}$ (three mice at each time point) respectively. For the control sample, four mice were injected with 10\% DMSO in saline instead. The mice were anaesthetized using ketamine $(150 \mathrm{mg} / \mathrm{mL})$ and xylazine $(10 \mathrm{mg} / \mathrm{kg})$ and euthanized by cervical dislocation to collect various organs for histopathology analysis. All organs were immediately fixed in $10 \%$ neutral buffered formalin (Sigma) and submitted to Advanced Molecular Pathology Laboratory (AMPL), IMCB for histopathology evaluation. The pathology report was prepared by an experienced pathologist. All bright field images were captured using the Nikon Ni-E upright microscope, with Plan Apo 20x DIC M N2 objective and Nikon DS-Ri2 camera. The images were viewed using NIS-Elements software.

\subsubsection{Attempted polymer encapsulation of cluster 23 via the thermodynamic approach}

In a typical reaction, $\mathrm{PS}_{154}-b$-PAA 60 solution $\left(10 \mu \mathrm{L}, 8 \mathrm{mg} \mathrm{mL}^{-1}\right.$ in DMF) was added to a solution of cluster $23(1 \mathrm{mg})$ dissolved in DMF $(790 \mu \mathrm{L})$. Deionized water $(180$ $\mu \mathrm{L}$ ) was then added and the mixture was vortexed for $10 \mathrm{~s}$. The mixture was heated in a pre-heated oil bath at $110{ }^{\circ} \mathrm{C}$ for 5 or $15 \mathrm{~min}$, before it was slowly cooled to room temperature in the oil bath. A portion of the sample $(100 \mu \mathrm{L})$ was topped up with deionized water $(1400 \mu \mathrm{L})$, mixed well and centrifuged at $6000 \mathrm{~g}$ for $10 \mathrm{~min}$. The 
supernatant was removed and the pellet was dispersed in deionized water $(100 \mu \mathrm{L})$ before proceeding to TEM analysis. For ATR-FTIR analysis, the pellet was dispersed in $\mathrm{MeOH}$ and was dropped onto the ATR sample stage and air-dried, before proceeding with IR analysis.

\subsubsection{Attempted polymer encapsulation of cluster 23 via the kinetic approach}

In a typical reaction, $\mathrm{PS}_{144}-b-\mathrm{PAA}_{22}$ solution $\left(60 \mu \mathrm{L}, 8 \mathrm{mg} \mathrm{mL}^{-1}\right.$ in DMF) was added to a solution of cluster $23\left(400 \mu \mathrm{L}, 1 \mathrm{mg} \mathrm{mL}^{-1}\right.$ in DMF). The amount of polymer was varied with the use of $10-60 \mu \mathrm{L}$ of the $\mathrm{PS}_{144}-b-\mathrm{PAA}_{22}$ solution. The volume of the mixture was topped up to $500 \mu \mathrm{L}$ with $\mathrm{DMF}$ and vortexed briefly. The dark green solution was stirred vigorously while deionized water $(3 \mathrm{~mL})$ was added using a syringe pump at a rate of $0.05 \mathrm{~mL} / \mathrm{min}$. The mixture was left to stir for another $1 \mathrm{~h}$. The carboxylic acid groups were first activated by the addition of EDC solution (13 $\mu \mathrm{L}, \quad 40 \mathrm{mM}$ ) for $30 \mathrm{~min}$, before the sequential addition of 2,2'(ethylenedioxy)bis(ethylamine) solution $(20 \mu \mathrm{L}, 10 \mathrm{mM})$ and $\mathrm{NH}_{2}$-PEG-COOH solution $(13 \mu \mathrm{L}, 40 \mathrm{mM})$. The mixture was stirred at room temperature for $2 \mathrm{~h}$ and precipitation was observed.

In another attempt, after the addition of water using the syringe pump, the mixture was dialyzed against deionized water using a cellulose membrane (Spectra Por 7 dialysis membrane, 15000 Da MWCO) overnight to remove DMF and other small organic compounds. Unfortunately, precipitation was observed in the tubing, indicating the instability of the micelles. 


\subsection{References}

1. K. V. Kong, L.-D. Liao, Z. Lam, N. V. Thakor, W. K. Leong and M. Olivo, Chem. Commun., 2014, 50, 2601-2603.

2. G. O. Nelson and C. E. Sumner, Organometallics, 1986, 5, 1983-1990.

3. M. P. Cifuentes, M. G. Humphrey, J. R. Shapley and K. Lee, Inorg. Synth., 2007, 287-293.

4. C. R. Eady, P. F. Jackson, B. F. G. Johnson, J. Lewis, M. C. Malatesta, M. McPartlin and W. J. H. Nelson, J. Chem. Soc., Dalton Trans., 1980, 383-392.

5. M. P. Cifuentes, M. G. Humphrey, B. W. Skelton and A. H. White, Organometallics, 1995, 14, 1536-1538.

6. P. J. Bailey, M. A. Beswick, B. F. G. Johnson, J. Lewis, M. McPartlin, P. R. Raithby and M. C. R. de Arellano, J. Chem. Soc., Dalton Trans., 1996, 35153520.

7. M. I. Bruce, N. N. Zaitseva, B. W. Skelton and A. H. White, J. Chem. Soc., Dalton Trans., 2002, 3879-3885.

8. K. D. Johnson and G. L. Powell, J. Organomet. Chem., 2008, 693, 1712-1715.

9. M. P. Cifuentes, M. G. Humphrey, J. E. McGrady, P. J. Smith, R. Stranger, K. S. Murray and B. Moubaraki, J. Am. Chem. Soc., 1997, 119, 2647-2655.

10. S. R. Drake, B. F. G. Johnson, J. Lewis and R. G. Woolley, Inorg. Chem., 1987, 26, 3952-3956.

11. R. E. Benfield, in Physics and Chemistry of Metal Cluster Compounds, ed. L. J. De Jongh, Springer Netherlands, 1994, vol. 18, pp. 249-275.

12. C. Femoni, M. C. Iapalucci, F. Kaswalder, G. Longoni and S. Zacchini, Coord. Chem. Rev., 2006, 250, 1580-1604.

13. Y.-S. Chen, W. Frey, S. Aglyamov and S. Emelianov, Small, 2012, 8, 47-52.

14. Y.-S. Chen, W. Frey, S. Kim, P. Kruizinga, K. Homan and S. Emelianov, Nano Lett., 2011, 11, 348-354.

15. M. Xu and L. V. Wang, Rev. Sci. Instrum., 2006, 77, 041101.

16. E. Petrova, S. Ermilov, R. Su, V. Nadvoretskiy, A. Conjusteau and A. Oraevsky, Opt. Express, 2013, 21, 25077-25090.

17. B. Cox, J. G. Laufer, S. R. Arridge and P. C. Beard, J. Biomed. Opt., 2012, 17, 0612021-06120222.

18. J. T. Alander, I. Kaartinen, A. Laakso, T. Pätilä, T. Spillmann, V. V. Tuchin, M. Venermo and P. Välisuo, Int. J. Biomed. Imaging, 2012, 2012, 26. 
19. M. V. Marshall, J. C. Rasmussen, I. C. Tan, M. B. Aldrich, K. E. Adams, X. Wang, C. E. Fife, E. A. Maus, L. A. Smith and E. M. Sevick-Muraca, Open Surg. Oncol. J., 2010, 2, 12-25.

20. X. Wang, G. Ku, M. A. Wegiel, D. J. Bornhop, G. Stoica and L. V. Wang, Opt. Lett., 2004, 29, 730-732.

21. C. Kim, K. H. Song, F. Gao and L. V. Wang, Radiology, 2010, 255, 442-450.

22. A. Hannah, G. Luke, K. Wilson, K. Homan and S. Emelianov, ACS Nano, $2014,8,250-259$.

23. H. Wang, C. Liu, X. Gong, D. Hu, R. Lin, Z. Sheng, C. Zheng, M. Yan, J. Chen, L. Cai and L. Song, Nanoscale, 2014, 6, 14270-14279.

24. A. Buehler, E. Herzog, D. Razansky and V. Ntziachristos, Opt. Lett., 2010, 35, 2475-2477.

25. V. Ntziachristos and D. Razansky, Chem. Rev., 2010, 110, 2783-2794.

26. N. C. Burton, M. Patel, S. Morscher, W. H. P. Driessen, J. Claussen, N. Beziere, T. Jetzfellner, A. Taruttis, D. Razansky, B. Bednar and V. Ntziachristos, NeuroImage, 2013, 65, 522-528.

27. W. H. De Jong, W. I. Hagens, P. Krystek, M. C. Burger, A. J. A. M. Sips and R. E. Geertsma, Biomaterials, 2008, 29, 1912-1919.

28. M. Semmler-Behnke, W. G. Kreyling, J. Lipka, S. Fertsch, A. Wenk, S. Takenaka, G. Schmid and W. Brandau, Small, 2008, 4, 2108-2111.

29. N. Khlebtsov and L. Dykman, Chem. Soc. Rev., 2011, 40, 1647-1671.

30. Y. Kang and T. A. Taton, Angew. Chem. Int. Ed., 2005, 44, 409-412.

31. H. Y. Chen, S. Abraham, J. Mendenhall, S. C. Delamarre, K. Smith, I. Kim and C. A. Batt, ChemPhysChem, 2008, 9, 388-392.

32. Y. Kang and T. A. Taton, J. Am. Chem. Soc., 2003, 125, 5650-5651.

33. D. Niu, Y. Li, X. Qiao, L. Li, W. Zhao, H. Chen, Q. Zhao, Z. Ma and J. Shi, Chem. Commun., 2008, 4463-4465.

34. B.-S. Kim, J.-M. Qiu, J.-P. Wang and T. A. Taton, Nano Lett., 2005, 5, 19871991.

35. C. W. Wang and M. G. Moffitt, Langmuir, 2005, 21, 2465-2473.

36. E. Cariati, C. Dragonetti, E. Lucenti and D. Roberto, Inorg. Synth., 2004, 34, 218-225.

37. A. Rosenthal, D. Razansky and V. Ntziachristos, IEEE Trans. Med. Imag., 2010, 29, 1275-1285. 
38. J. Schindelin, I. Arganda-Carreras, E. Frise, V. Kaynig, M. Longair, T. Pietzsch, S. Preibisch, C. Rueden, S. Saalfeld, B. Schmid, J.-Y. Tinevez, D. J. White, V. Hartenstein, K. Eliceiri, P. Tomancak and A. Cardona, Nat. Methods, 2012, 9, 676-682. 


\section{Chapter 5: Conclusion}

In this work, the application of transition metal carbonyls in bio-sensing and imaging via two optical techniques, namely surface-enhanced Raman spectroscopy (SERS) and photoacoustic (PA) imaging, were examined (figure 5.1).

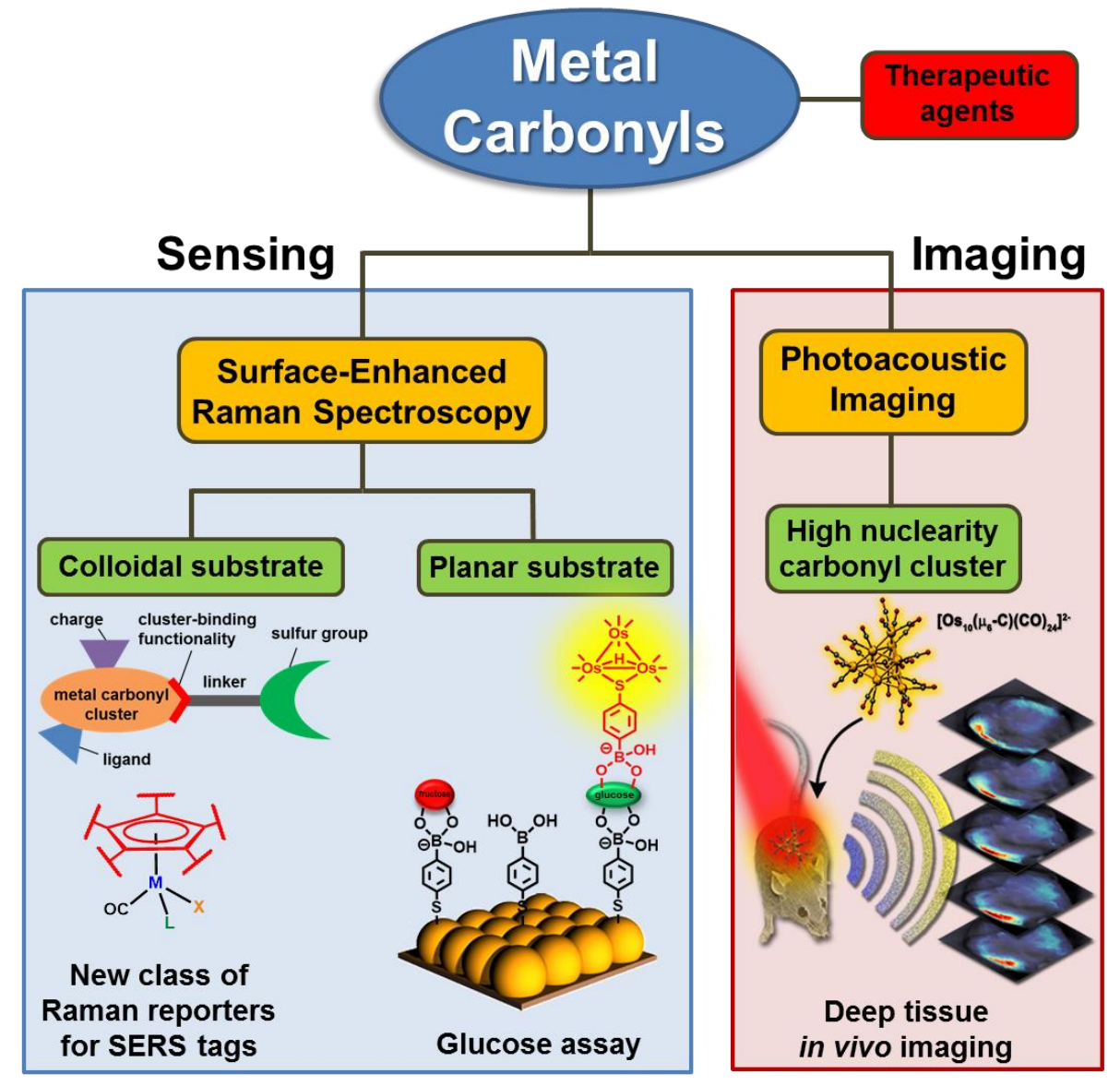

Figure 5.1. Summary of the work investigated in this thesis.

Two designs of metal carbonyl-based Raman reporters were examined for potential use in multiplexing - one based on transition metal carbonyl clusters and another on mononuclear half-sandwich metal carbonyls complexes. SERS multiplexing applications require non-overlapping signals, and changes to the ligand sphere and the charge (for cluster complexes) were found to be effective for tuning of the $\mathrm{CO}$ vibrational stretches over a wide frequency range. It was observed, however, that signal enhancement was dependent on the extent of aggregation and often led to large variations in the SERS enhancement. Vigorous study in developing controlled 
aggregates involving metal carbonyls would therefore be required to determine the conditions needed to produce highly reproducible SERS tags. Nevertheless, incubation of the metal carbonyls with AuNS, followed by centrifugation and re-dispersion, was found to be convenient for qualitative screening of suitable metal carbonyls. Alternatively, the use of colloidal substrates with higher SERS enhancements can be employed, for example, gold nanostars and nanoflowers. ${ }^{1,2}$ Further functionalization of the SERS tags with ligands for cell-labelling or for the detection of biomolecules of interest would be the next logical extension. Another would be in the study of the COreleasing process of $\mathrm{CO}$-releasing molecules (CORMs) and to monitor their fate in cellular applications.

The extension of metal carbonyl-based SERS reporters to a planar substrate eliminated the problem of aggregation associated with colloidal substrates, thereby ensuring reproducibility. Coupling boronic acid chemistry to metal carbonyl led to a selective glucose assay based on the conjugate, $\mathbf{2 b}-\mathrm{B}(\mathrm{OH})_{2}$. This sandwich-based approach proved to be highly effective and may be extended to the detection of other biomolecules via the binding affinity between a binding group and the molecule, for example, through antigen-antibody and enzyme-substrate affinity (figure 5.2). A future worthwhile improvement would be replacement of the transition metal with a cheaper metal.
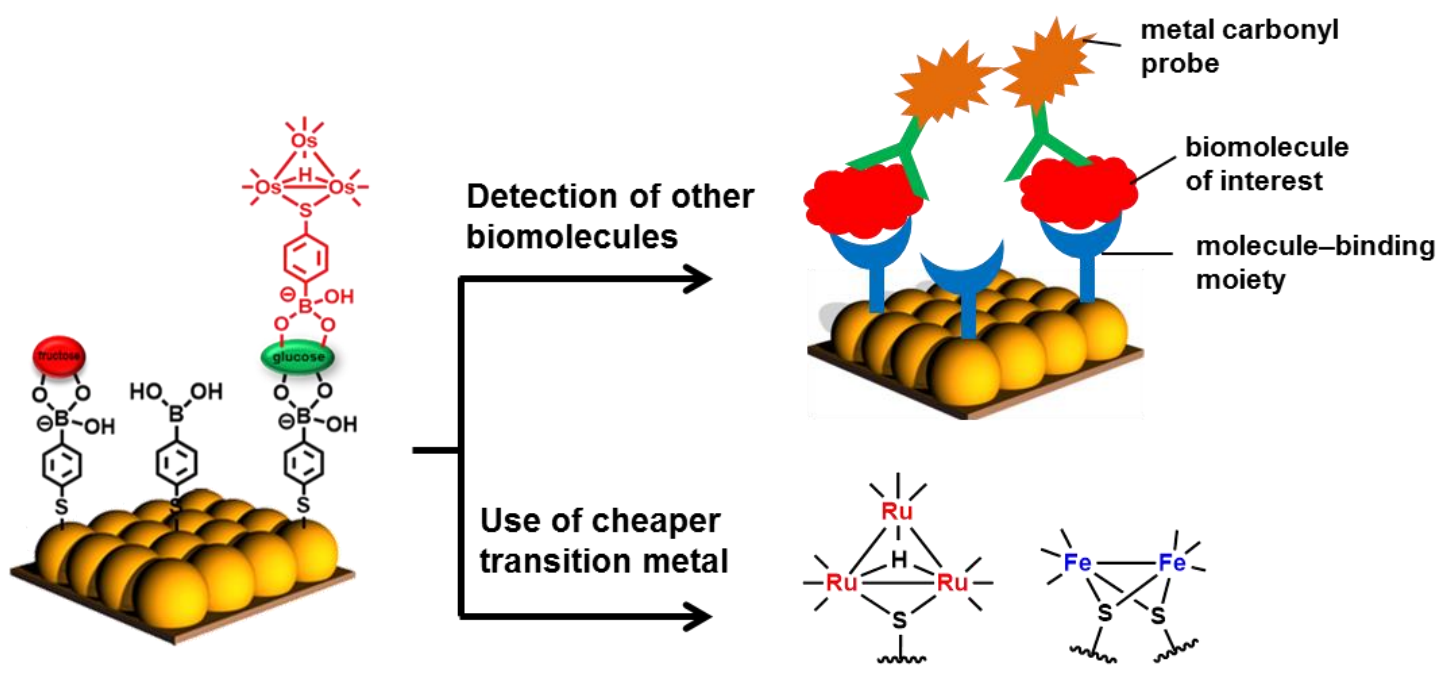

Figure 5.2. Future work for translating the glucose assay to the detection of other biomolecules and for the use of cheaper transition metals as SERS probes. 
Transition metal carbonyl clusters were also demonstrated to be useful as contrast agents in PA imaging through their electronic spectral properties. The electronic spectra of these clusters showed optical absorption due to transitions relating to the cluster's metal core, and increasing the cluster nuclearity shifted the absorption into the NIR region. The HNCC $\left[\mathrm{Os}_{10}\left(\mu_{6}-\mathrm{C}\right)(\mathrm{CO})_{24}\right]^{2-}, \mathbf{2 6}$, was shown to exhibit good PA properties in the NIR region, and its sodium salt was applied successfully for the whole body PA imaging of live mice. The need for improvement on the PA performance, biocompatibility and selectivity remain, however. Improvements to the NIR absorption, and hence the PA activity, of the HNCCs may be sought through a further increase in the cluster nuclearity, or through the use of conjugated ligands to enhance the NIR absorption. Improvements to the biocompatibility and selectivity may be carried out via functionalization of the HNCC with suitable bioligands that target the site of interest. Using nano-sized polymers, carbon nano-structures or mesoporous silica nanoparticles, as carriers for the HNCCs may also enhance biocompatibility and selectivity. Nevertheless, a comprehensive, long-term in vivo toxicity study will be required to better understand the effect of such clusters in biological systems.

Given the success of SERS sensing and PA imaging, it may also be worthwhile to investigate the use of metal carbonyls for multimodal imaging, which is, the use of more than one imaging technique simultaneously. Multimodal imaging has tremendous potential in clinical applications and can provide complementary information that is valuable at both cellular and molecular levels. ${ }^{3,4}$ SERS-PA dual modal sensing is possible with the development of transition metal carbonyl-based SERS-PA active probes. For instance, the conjugation of HNCCs onto gold colloids can allow both the intrinsic NIR PA activity of the HNCCs and the enhanced CO vibrational frequency to serve as a dual handle, for both imaging and sensing.

The discovery of the cytotoxic nature of the metal carbonyl-boronic acid conjugate, 2b- $\mathrm{B}(\mathrm{OH})_{2}$, was quite unexpected as the alkylthiolato analogues were much less cytotoxic; that $\mathbf{2 b}-\mathrm{B}(\mathrm{OH})_{2}$ was apoptosis-inducing was confirmed. It is now important to verify the role of the thiolate substituent in affecting the cytotoxicity. Examples of clusters that can be tested on to validate this point are the $\mathrm{Os}_{3}(\mathrm{CO})_{10}(\mu-\mathrm{H})(\mu$ $\mathrm{SCH}_{2} \mathrm{C}_{6} \mathrm{H}_{4} \mathrm{X}$ ) clusters, where $\mathrm{X}=-\mathrm{B}(\mathrm{OH})_{2},-\mathrm{COOH}$ and $-\mathrm{OH}$ (figure 5.3). Establishing this can allow us to modulate such clusters for their intended purposes accurately (for imaging or therapy) by virtue of the R groups. This could, for instance, 
lead to improvements in selectivity towards cancer cells - the functional groups ($\mathrm{B}(\mathrm{OH})_{2},-\mathrm{OH}$ and $\left.-\mathrm{COOH}\right)$ can provide convenient conjugation points for ligands that target cancer cells (figure 5.3). In contrast to some of the cytotoxic osmium carbonyl clusters reported thus far, $\mathbf{2 b}$ represent yet another class of clusters with anti-cancer potential and are more amenable to modification. ${ }^{5-8}$

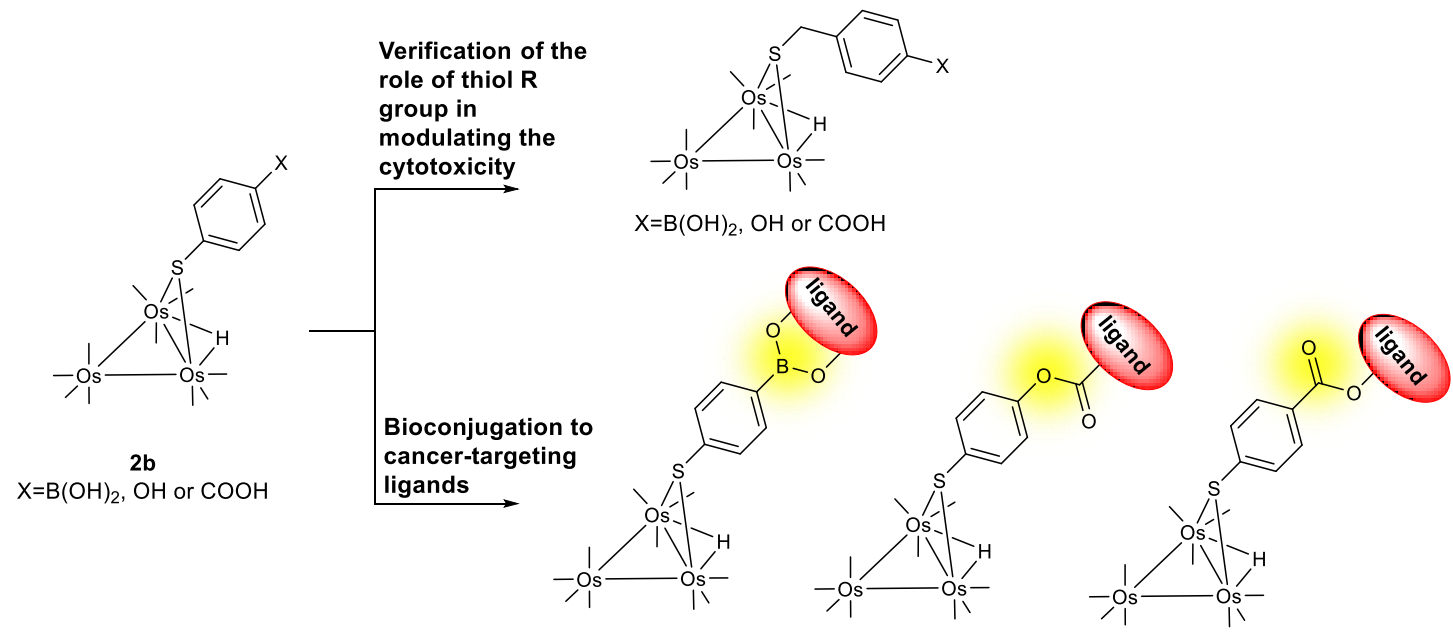

Figure 5.3. Future work for the validation of the role of the $\mathrm{R}$ group in modulating the cytotoxicity of the phenylthiolatio clusters $\mathbf{2 b}$ and bioconjugation of $\mathbf{2 b}$ to cancer-targeting ligands via the $\mathrm{X}$ group.

We have demonstrated through the work in this thesis that transition metal carbonyls, in particular, clusters have great potential for two relatively new optical imaging modalities, viz., SERS and PA imaging. These modalities have attracted much attention over the years as functional and robust tools in biology. The applications of metal carbonyls in these two techniques are still in its infancy, and the outlook is positive, with many more examples sure to emerge. 


\section{$\underline{\text { References }}$}

1. H. Yuan, C. G. Khoury, H. Hwang, C. M. Wilson, G. A. Grant and T. Vo-Dinh, Nanotechnology, 2012, 23, 075102.

2. J. Xie, Q. Zhang, J. Y. Lee and D. I. C. Wang, ACS Nano, 2008, 2, 2473-2480.

3. U. S. Dinish, Z. Song, C. J. H. Ho, G. Balasundaram, A. B. E. Attia, X. Lu, B. Z. Tang, B. Liu and M. Olivo, Adv. Funct. Mater., 2015, 25, 2316-2325.

4. L. Martí-Bonmatí, R. Sopena, P. Bartumeus and P. Sopena, Contrast Media Mol. Imaging, 2010, 5, 180-189.

5. K. V. Kong, W. K. Leong, S. P. Ng, T. H. Nguyen and L. H. K. Lim, ChemMedChem, 2008, 3, 1269-1275.

6. K. V. Kong, W. K. Leong and L. H. K. Lim, Chem. Res. Toxicol., 2009, 22, 1116-1122.

7. K. V. Kong, W. K. Leong and L. H. K. Lim, J. Organomet. Chem., 2009, 694, 834-839.

8. H. Z. S. Lee, W. K. Leong, S. Top and A. Vessières, ChemMedChem, 2014, 9, 1453-1457. 


\section{Appendix}

\section{List of contents}

\section{Chapter 2: Surface-Enhanced Raman Spectroscopy (SERS) of Metal Carbonyls on Gold Nanospheres}

1. Figure A2.1. IR spectrum ( $\left.v_{\mathrm{CO}}\right)$ of $\mathbf{2 a}$ in DCM.

2. Figure A2.2. IR spectrum ( $\left.v_{\mathrm{CO}}\right)$ of $\mathbf{3 b}$ in DCM.

3. Figure A2.3. IR spectrum $\left(v_{\mathrm{CO}}\right)$ of $\mathbf{4}$ in DCM.

4. Figure A2.4. IR spectrum $\left(v_{\mathrm{CO}}\right)$ of 6 in DCM.

5. Figure A2.5. IR spectrum $\left(v_{\mathrm{CO}}\right)$ of $\mathbf{6} \cdot \mathrm{H}^{+}$in DCM.

6. Figure A2.6. IR spectrum $\left(v_{\mathrm{CO}}\right)$ of 7a in DCM.

7. Figure A2.7. IR spectrum ( $\left.v_{\mathrm{CO}}\right)$ of $\mathbf{7 b}$ in DCM.

8. Figure A2.8. IR spectrum $\left(v_{\mathrm{CO}}\right)$ of $8 \mathbf{a}$ in DCM.

9. Figure A2.9. IR spectrum ( $\left.v_{\mathrm{CO}}\right)$ of $9 \mathbf{a}$ in DCM.

10. Figure A2.10. IR spectrum $\left(v_{\mathrm{CO}}\right)$ of 10a in DCM.

11. Figure A2.11. IR spectrum $\left(v_{\mathrm{CO}}\right)$ of $\mathbf{1 0 b}$ in THF.

12. Figure A2.12. IR spectrum $\left(v_{\mathrm{CO}}\right)$ of $\mathbf{1 0 c}$ in THF.

13. Figure A2.13. IR spectrum $\left(v_{\mathrm{CO}}\right)$ of 11a in DCM.

14. Figure A2.14. IR spectrum ( $\left.v_{\mathrm{CO}}\right)$ of 12a in DCM.

15. Figure A2.15. IR spectrum ( $\left.v_{\mathrm{CO}}\right)$ of 12'a in DCM.

16. Figure A2.16. IR spectrum ( $\left.v_{\mathrm{CO}}\right)$ of 13a in DCM.

17. Figure A2.17. ${ }^{1} \mathrm{H}$ NMR spectrum of $\mathbf{3 b}$ in $\mathrm{CDCl}_{3}$.

18. Figure A2.18. ${ }^{13} \mathrm{C}\left\{{ }^{1} \mathrm{H}\right\}$ NMR spectrum of $\mathbf{3 b}$ in $\mathrm{CDCl}_{3}$.

19. Figure A2.19. ${ }^{1} \mathrm{H}$ NMR spectrum of 6 in $\mathrm{CDCl}_{3}$.

20. Figure A2.20. COSY NMR spectrum of 6 in $\mathrm{CDCl}_{3}$.

21. Figure A2.21. ${ }^{31} \mathrm{P}\left\{{ }^{1} \mathrm{H}\right\}$ NMR spectrum of 6 in $\mathrm{CDCl}_{3}$.

22. Figure A2.22. a) ESI-HRMS of 6 (top) with calculated isotopic pattern (bottom). b) ESI-HRMS single mass analysis of 6.

23. Figure A2.23. ${ }^{1} \mathrm{H}$ NMR spectrum of $6 . \mathrm{H}^{+}$in $\mathrm{CDCl}_{3}$.

24. Figure A2.24. COSY NMR spectrum of $6 \cdot \mathrm{H}^{+}$in $\mathrm{CDCl}_{3}$.

25. Figure A2.25. ${ }^{31} \mathrm{P}\left\{{ }^{1} \mathrm{H}\right\}$ NMR spectrum of $6 . \mathrm{H}^{+}$in $\mathrm{CDCl}_{3}$.

26. Figure A2.26. a) ESI-HRMS of 6. $\mathrm{H}^{+}$(top) with calculated isotopic pattern (bottom). b) ESI-HRMS single mass analysis of $6 . \mathrm{H}^{+}$.

27. Figure A2.27. a) IR spectrum $\left(v_{\mathrm{CO}}\right)$ of $\mathrm{CpW}(\mathrm{CO})_{3} \mathrm{X}$ in $\mathrm{DCM}$ and b) SERS spectra of respective complexes with AuNS, where $\mathrm{X}=\mathrm{I}$ (blue), $\mathrm{Br}$ (red) and $\mathrm{Cl}$ (black), in DCM. 


\section{Chapter 3: Metal Carbonyl-Boronic Acid Conjuagte - a SERS Probe for Glucose Detection and its Cytotoxicity}

28. Figure A3.1. ${ }^{1} \mathrm{H}$ NMR spectrum of $\mathbf{2 b}-\mathrm{B}(\mathrm{OH})_{2}$ in $\left(\mathrm{CD}_{3}\right)_{2} \mathrm{CO}$.

29. Figure A3.2. ${ }^{1} \mathrm{H}$ NMR spectrum of $\mathbf{2 b}-\mathrm{OH}$ in $\left(\mathrm{CD}_{3}\right)_{2} \mathrm{CO}$.

30. Figure A3.3. ${ }^{1} \mathrm{H}$ NMR spectrum of $\mathbf{2 b}-\mathrm{COOH}$ in $\left(\mathrm{CD}_{3}\right)_{2} \mathrm{CO}$.

31. Figure A3.4. ${ }^{13} \mathrm{C}\left\{{ }^{1} \mathrm{H}\right\}$ NMR spectrum of $\mathbf{2 b}-\mathrm{B}(\mathrm{OH})_{2}$ in $\left(\mathrm{CD}_{3}\right)_{2} \mathrm{CO}$.

32. Figure A3.5. ${ }^{13} \mathrm{C}\left\{{ }^{1} \mathrm{H}\right\}$ NMR spectrum of $\mathbf{2 b}-\mathrm{OH}$ in $\left(\mathrm{CD}_{3}\right)_{2} \mathrm{CO}$.

33. Figure A3.6. ${ }^{13} \mathrm{C}\left\{{ }^{1} \mathrm{H}\right\}$ NMR spectrum of $2 \mathbf{b}-\mathrm{COOH}$ in $\left(\mathrm{CD}_{3}\right)_{2} \mathrm{CO}$.

34. Figure A3.7. IR spectrum $\left(v_{\mathrm{CO}}\right)$ of $\mathbf{1}$ in DCM.

35. Figure A3.8. IR spectrum $\left(v_{\mathrm{CO}}\right)$ of $\mathbf{2 b}-\mathrm{B}(\mathrm{OH})_{2}$ in DCM.

36. Figure A3.9. IR spectrum $\left(v_{\mathrm{CO}}\right)$ of $\mathbf{2 b}-\mathrm{H}$ in DCM.

37. Figure A3.10. IR spectrum ( $\left.v_{\mathrm{CO}}\right)$ of $\mathbf{2 b}-\mathrm{OH}$ in DCM.

38. Figure A3.11. IR spectrum $\left(v_{\mathrm{CO}}\right)$ of $\mathbf{2 b}-\mathrm{COOH}$ in DCM.

39. Figure A3.12. ESI-HRMS of $\mathbf{2 b}-\mathrm{B}(\mathrm{OH})_{2}$.

40. Figure A3.13. a) ESI-HRMS of $\mathbf{2 b}-\mathrm{B}(\mathrm{OH})_{2}$ (top) with calculated isotopic pattern (bottom). b) ESIHRMS single mass analysis of $\mathbf{2 b}-\mathrm{B}(\mathrm{OH})_{2}$.

41. Figure A3.14. a) ESI-HRMS of glucose adduct of $\mathbf{2} \mathbf{b}-\mathrm{B}(\mathrm{OH})_{2}$ (top) with calculated isotopic pattern (bottom). b) ESI-HRMS single mass analysis of the adduct.

42. Figure A3.15. a) ESI-HRMS of 2b-OH (top) with calculated isotopic pattern (bottom). b) ESI-HRMS single mass analysis of $\mathbf{2 b}-\mathrm{OH}$.

43. Figure A3.16. a) ESI-HRMS of $\mathbf{2 b}-\mathrm{COOH}$ (top) with calculated isotopic pattern (bottom). b) ESIHRMS single mass analysis of $\mathbf{2 b}-\mathrm{COOH}$.

44. Figure A3.17. Dose-response curves of a) $\mathbf{1}$, b) $\mathbf{2 b}-\mathrm{B}(\mathrm{OH})_{2}$, c) $\mathbf{2 b}-\mathrm{OH}$ and d) $\mathbf{2 b}-\mathrm{COOH}$ on MDA-MB231 cells, after $24 \mathrm{~h}$, in serum-free condition.

45. Figure A3.18. Dose-response curves of a) $\mathbf{1}$, b) $2 \mathbf{b}-\mathrm{B}(\mathrm{OH})_{2}$ and c) $2 \mathbf{b}-\mathrm{OH}$ on MCF-7 cells, after 24 h, in serum-free condition.

46. Figure A3.19. Dose-response curves of a) 1, b) $2 \mathbf{b}-\mathrm{B}(\mathrm{OH})_{2}$ and c) $2 \mathbf{b}-\mathrm{OH}$ on MCF-10A cells, after 24 $\mathrm{h}$, in serum-free condition.

47. Figure A3.21. Dose-response curves of a) $\mathbf{1}$, b) $\mathbf{2} \mathbf{b}-\mathrm{B}(\mathrm{OH})_{2}$ and c) $\mathbf{2 b}-\mathrm{OH}$ on $\mathrm{MCf}-7$ cells, after $24 \mathrm{~h}$, in serum condition.

48. Figure A3.22. Dose-response curves of a) $\mathbf{1}$, b) $\mathbf{2} \mathbf{b}-\mathrm{B}(\mathrm{OH})_{2}$ and c) $\mathbf{2} \mathbf{b}-\mathrm{OH}$ on MCF-10A cells, after 24 $\mathrm{h}$, in serum condition. 


\section{Chapter 4: High Nuclearity Metal Carbonyl Clusters as Near-IR}

\section{Contrast Agents for Photoacoustic In Vivo Imaging}

49. Figure A4.1. Optical absorption spectra of clusters $22-[\mathrm{PPN}]_{2} .26$ at various concentrations (in DMSO) and plots of optical absorbance (at respective $\lambda_{\max }$ ) as a function of concentration.

50. Figure A4.2. i) PA (red) and optical absorption (black) spectra, ii) PA amplitude as a function of concentration of cluster $\mathbf{2 2}-[\mathrm{PPN}]_{2} . \mathbf{2 6}$.

51. Figure A4.3. Cell viability assay of $[\mathrm{PPN}] \mathrm{Cl}$ on $\mathrm{OSCC}$ cells and calculation of $\mathrm{IC}_{50}$ from three replicates.

52. Figure A4.4. Cell viability assay of $\mathrm{Na}_{2} .26$ on OSCC cells and calculation of $\mathrm{IC}_{50}$ from three replicates.

53. Figure A4.5. Optical absorption spectra of $\mathrm{Na}_{2} .26$ at various concentrations and plot of $\lambda_{\max }$ as a function of concentration of $\mathrm{Na}_{2} .26$ in a) DMSO, b) $10 \%$ DMSO in PBS (v/v), and c) $10 \%$ DMSO in modified DMEM (v/v).

54. Figure A4.6. PA, optical absorption spectra and plot of PA amplitude (at respective $\lambda_{\max }$ ) as a function of concentration of $\mathrm{Na}_{2} .26$ in a) DMSO, b) $10 \%$ DMSO in PBS (v/v), and c) $10 \%$ DMSO in modified $\operatorname{DMEM}(\mathrm{v} / \mathrm{v})$.

55. Figure A4.7. Irradiation of a solution of $\mathrm{Na}_{2} . \mathbf{2 6}(200 \mu \mathrm{M})$ in $10 \% \mathrm{DMSO}$ in modified DMEM at 760 $\mathrm{nm}$ for various time intervals: a) FTIR spectra $\left(v_{\mathrm{CO}}\right)$ obtained from the dried samples, and b) integrals of the $v_{\mathrm{CO}}$ absorbance.

56. Figure A4.8. a) Optical absorption spectra of ICG of various concentrations, b) plot of absorbance of ICG at $\lambda_{\max =} 790 \mathrm{~nm}$ as

57. Figure A4.9. a) Optical absorption spectra of ICG of various concentrations, b) plot of absorbance of ICG at $\lambda_{\max } 790 \mathrm{~nm}$ as a function of concentration, c) PA (red) and optical absorption (black) spectra of ICG, d) plot of PA amplitude at $\lambda_{\max } 790 \mathrm{~nm}$ as a function of concentration of ICG in $10 \%$ DMSO in modified DMEM (v/v).

58. Figure A4.10. a) Enlarged images of the tumors at various time point with the PA intensities normalized (top). Fold change in PA intensity in the tumor over that at $t=0$ in the tumor (bottom). b) Tumor-to-muscle ratio of PA amplitude at various time points up till $24 \mathrm{~h}$.

59. Figure A4.11. ICP-MS analysis of the percentage retention of injected dose (\% ID) of $\mathrm{Na}_{2} .26$ per organs, $24 \mathrm{~h}$ after injection. Error bars represent standard error of the mean, $\mathrm{n}=3$.

60. Figure A4.12. Histopathology (H \& E staining) of kidneys, heart and lungs tissues of mice, $24 \mathrm{~h}$ and $96 \mathrm{~h}$ after intravenous injection of $\mathrm{Na}_{2} . \mathbf{2 6}$, with that of control samples.

61. Figure A4.13. Percentage weight change of control mice and mice injected with $\mathrm{Na}_{2} .26$ up till $24 \mathrm{~h}$ and $96 \mathrm{~h}$ with respect to initial weight. Error bars represent standard error of the mean, $\mathrm{n}=3$ for samples with 9 and $n=2$ for control samples.

62. Figure A4.14. IR spectrum ( $v_{\mathrm{CO}}$ ) of 12'a in DCM.

63. Figure A4.15. IR spectrum $\left(v_{\mathrm{CO}}\right)$ of cluster 20 in hexane.

64. Figure A4.16. IR spectrum ( $v_{\mathrm{CO}}$ ) of cluster [PPN].21 in DCM.

65. Figure A4.17. IR spectrum ( $\left.v_{\mathrm{CO}}\right)$ of cluster 22 in DCM.

66. Figure A4.18. IR spectrum $\left(v_{\mathrm{CO}}\right)$ of cluster 23 in DCM. 
67. Figure A4.19. IR spectrum ( $\left.v_{\mathrm{CO}}\right)$ of cluster $[\mathrm{PPN}]_{2} .24$ in DCM.

68. Figure A4.20. IR spectrum $\left(v_{\mathrm{CO}}\right)$ of cluster $[\mathrm{PPN}]_{3} .25$ in DCM.

69. Figure A4.21. IR spectrum $\left(v_{\mathrm{CO}}\right)$ of cluster $[\mathrm{PPN}]_{2} .26$ in DCM.

70. Figure A4.22. IR spectrum ( $\left.v_{\mathrm{CO}}\right)$ of cluster $\mathrm{Na}_{2} .26$ in acetonitrile.

71. Figure A4.23. HRMS-ESI spectrum of cluster $\mathrm{Na}_{2} .26$ with the calculated isotopic pattern.

72. Table A4.1. ICP-MS analysis of Os content in various organs $24 \mathrm{~h}$ after injection of $\mathrm{Na}_{2} . \mathbf{2 6}$.

73. Table A4.2. ICP-MS analysis of Os content in various organs $96 \mathrm{~h}$ after injection of $\mathrm{Na}_{2} . \mathbf{2 6}$.

74. Table A4.3. ICP-MS analysis of background Os content in various organs in control animals (without the introduction of $\mathrm{Na}_{2} . \mathbf{2 6}$ ).

75. Table A4.4. Calculation of the percentage retention of injected dose (\% ID) of $\mathrm{Na}_{2} .26$ in various organ tissues.

76. References 
Chapter 2: Surface-Enhanced Raman Spectroscopy (SERS) of Metal Carbonyls on Gold Nanospheres

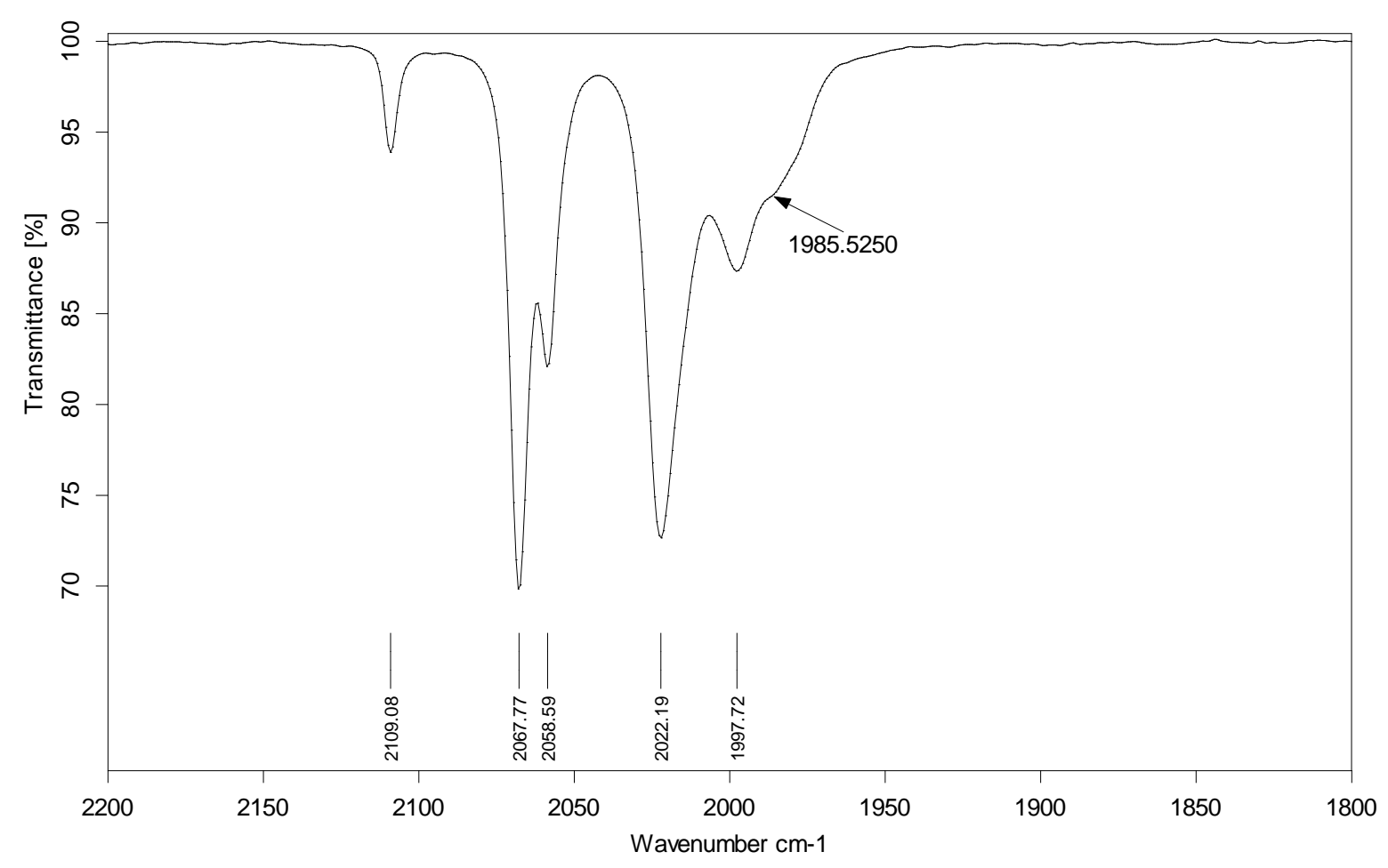

Figure A2.1. IR spectrum ( $\left.v_{\mathrm{CO}}\right)$ of $\mathbf{2 a}$ in DCM.

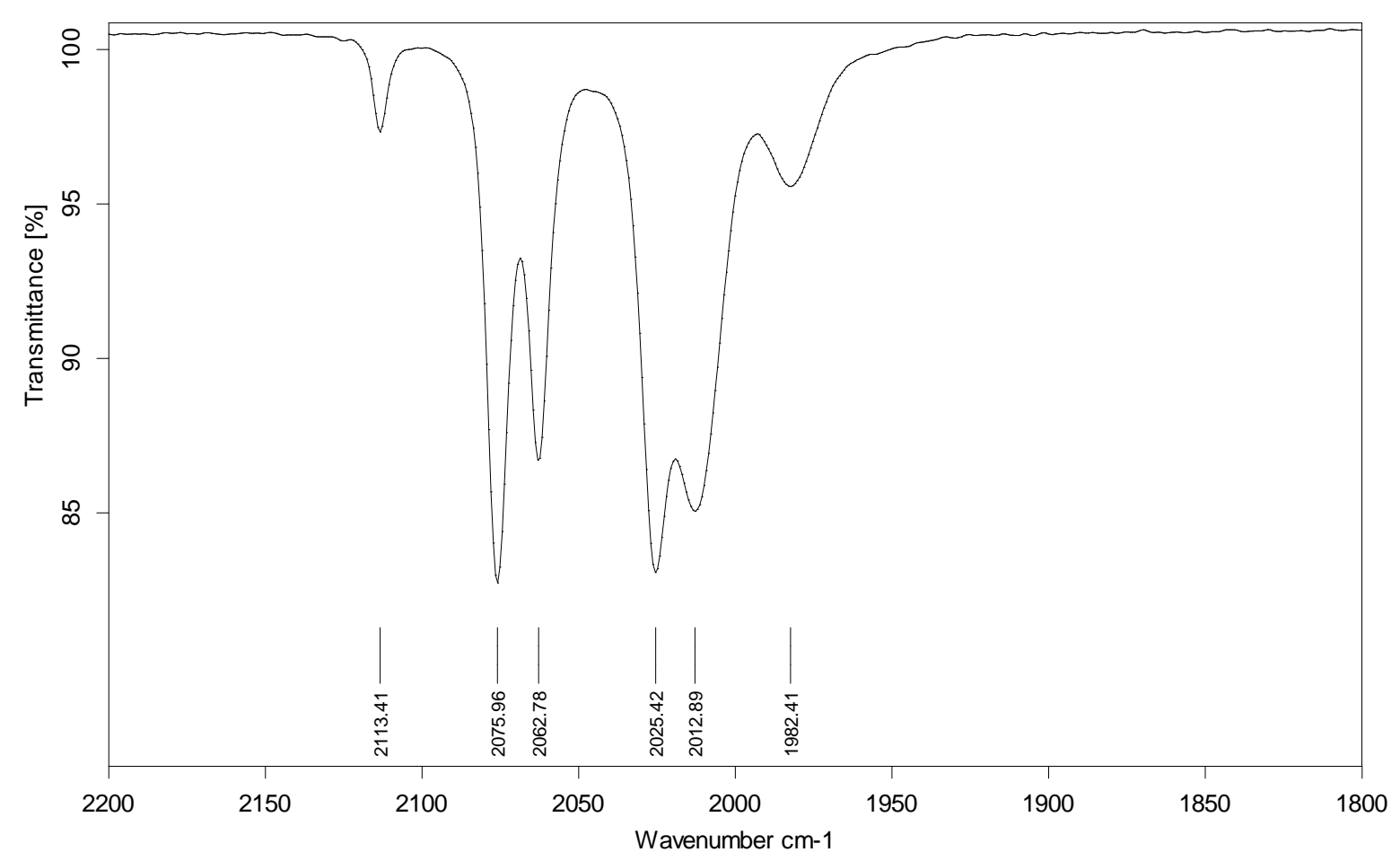

Figure A2.2. IR spectrum $\left(v_{\mathrm{CO}}\right)$ of $\mathbf{3 b}$ in DCM. 


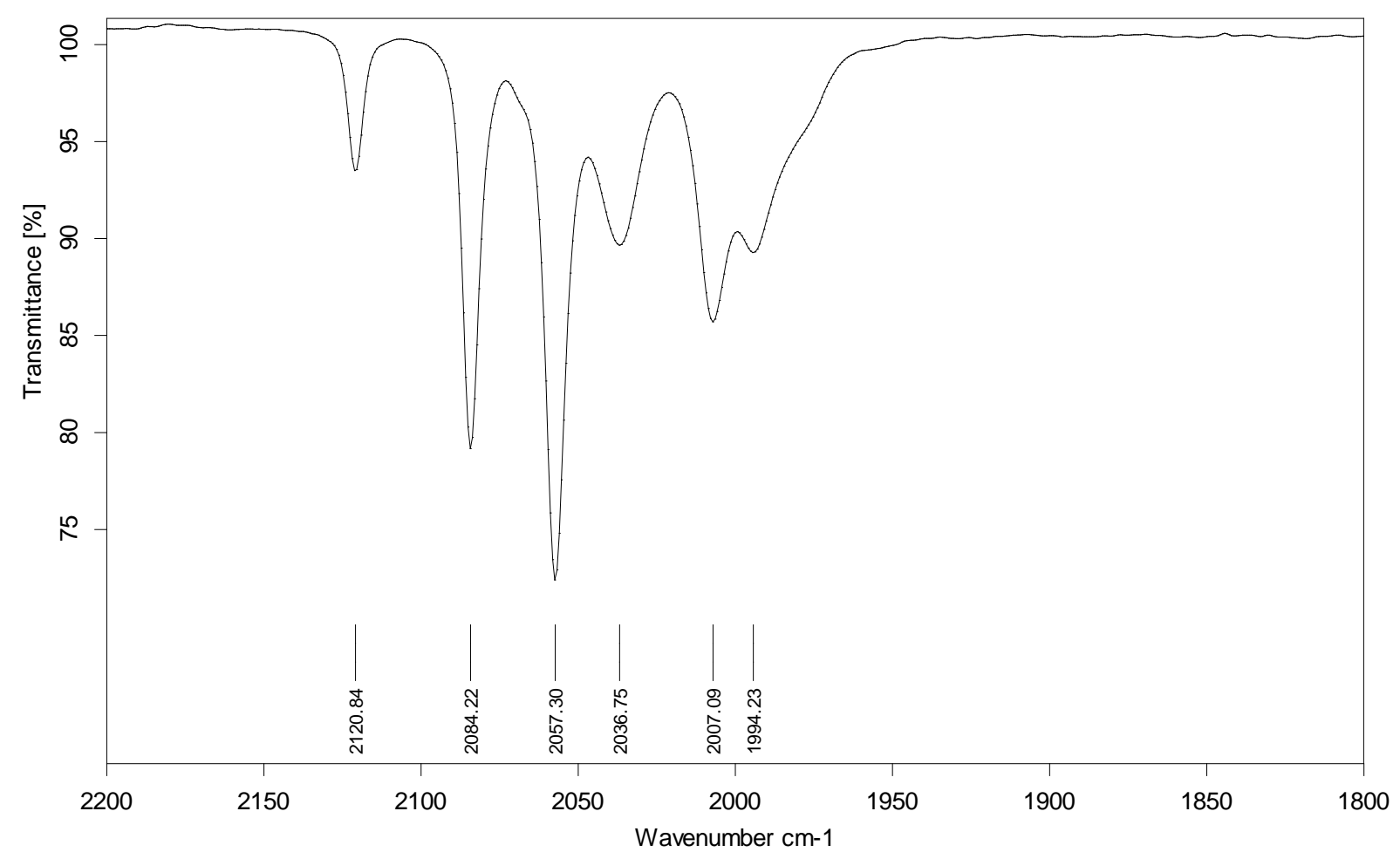

Figure A2.3. IR spectrum ( $\left.v_{\mathrm{CO}}\right)$ of 4 in DCM.

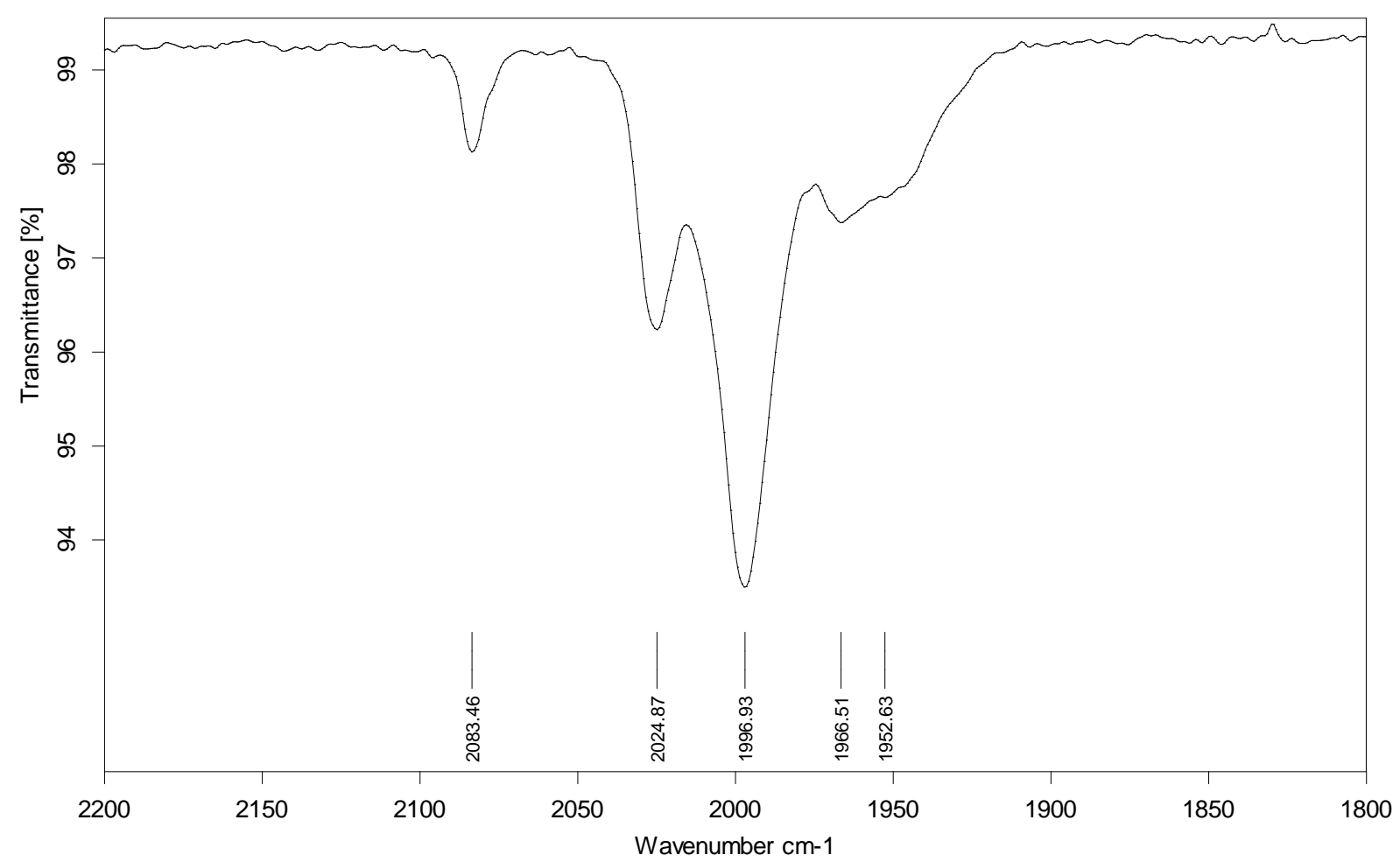

Figure A2.4. IR spectrum $\left(v_{\mathrm{CO}}\right)$ of 6 in DCM. 


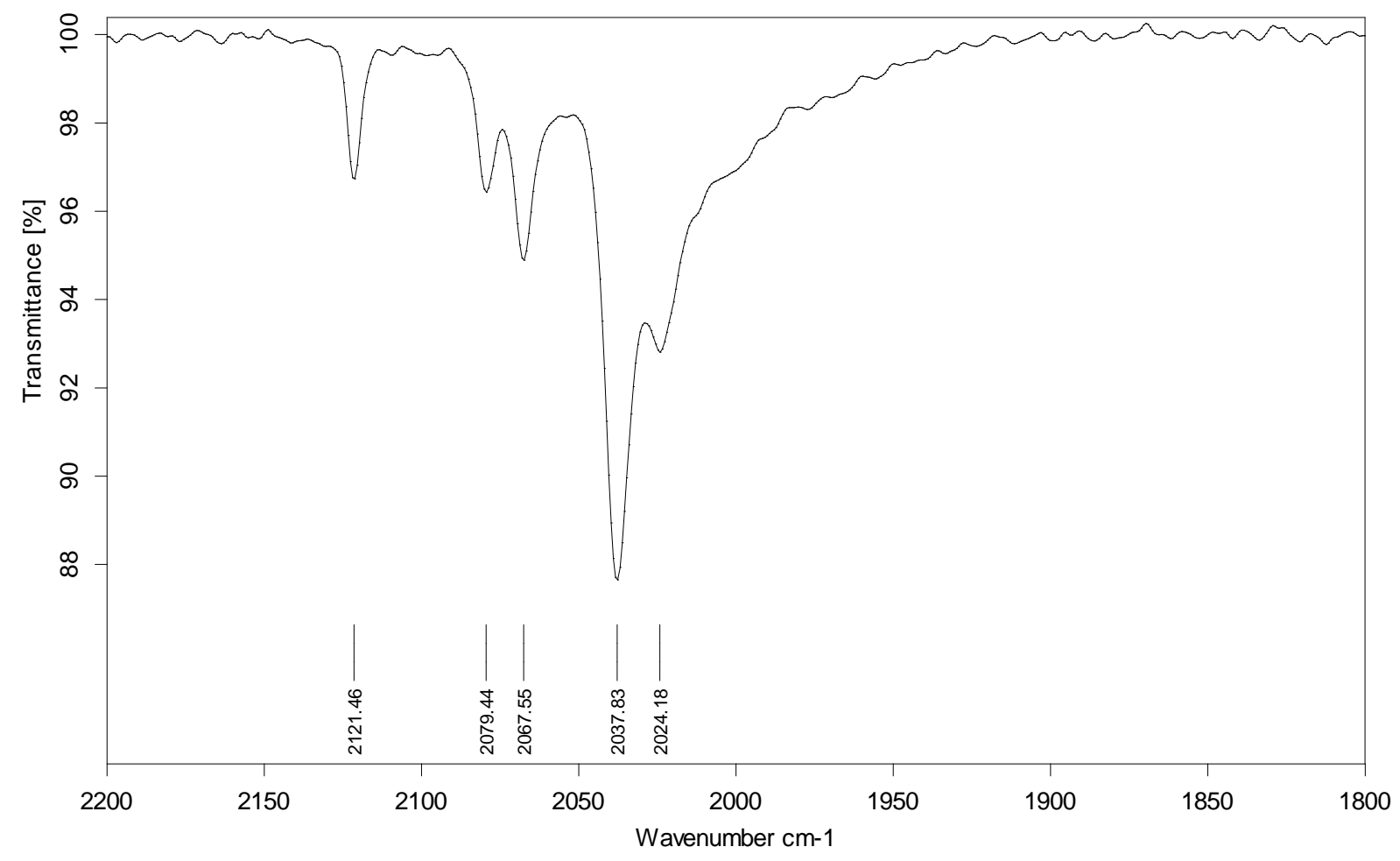

Figure A2.5. IR spectrum ( $\left.v_{\mathrm{CO}}\right)$ of $6 . \mathrm{H}^{+}$in DCM.

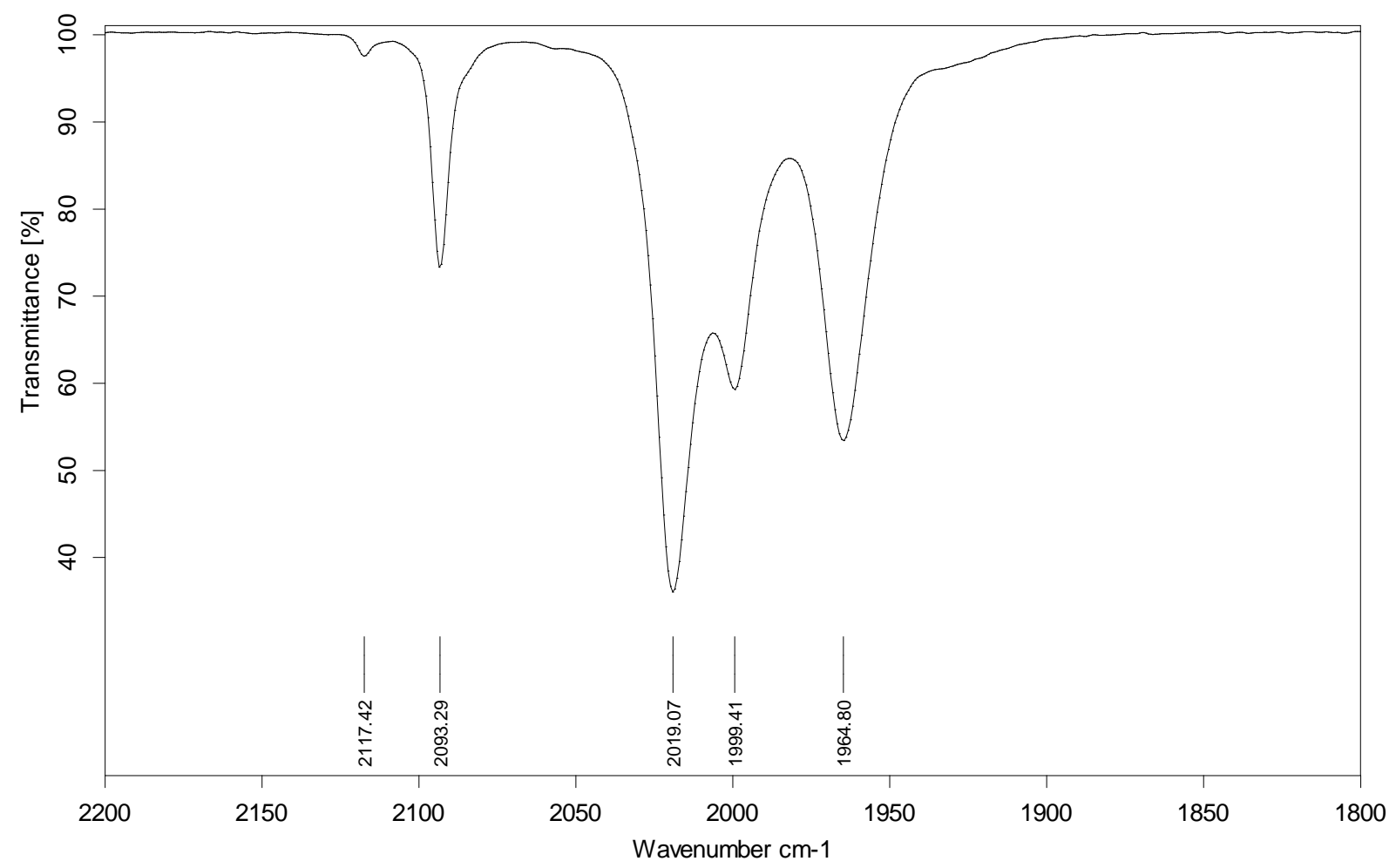

Figure A2.6. IR spectrum ( $\left.v_{\mathrm{CO}}\right)$ of $\mathbf{7 a}$ in DCM. 


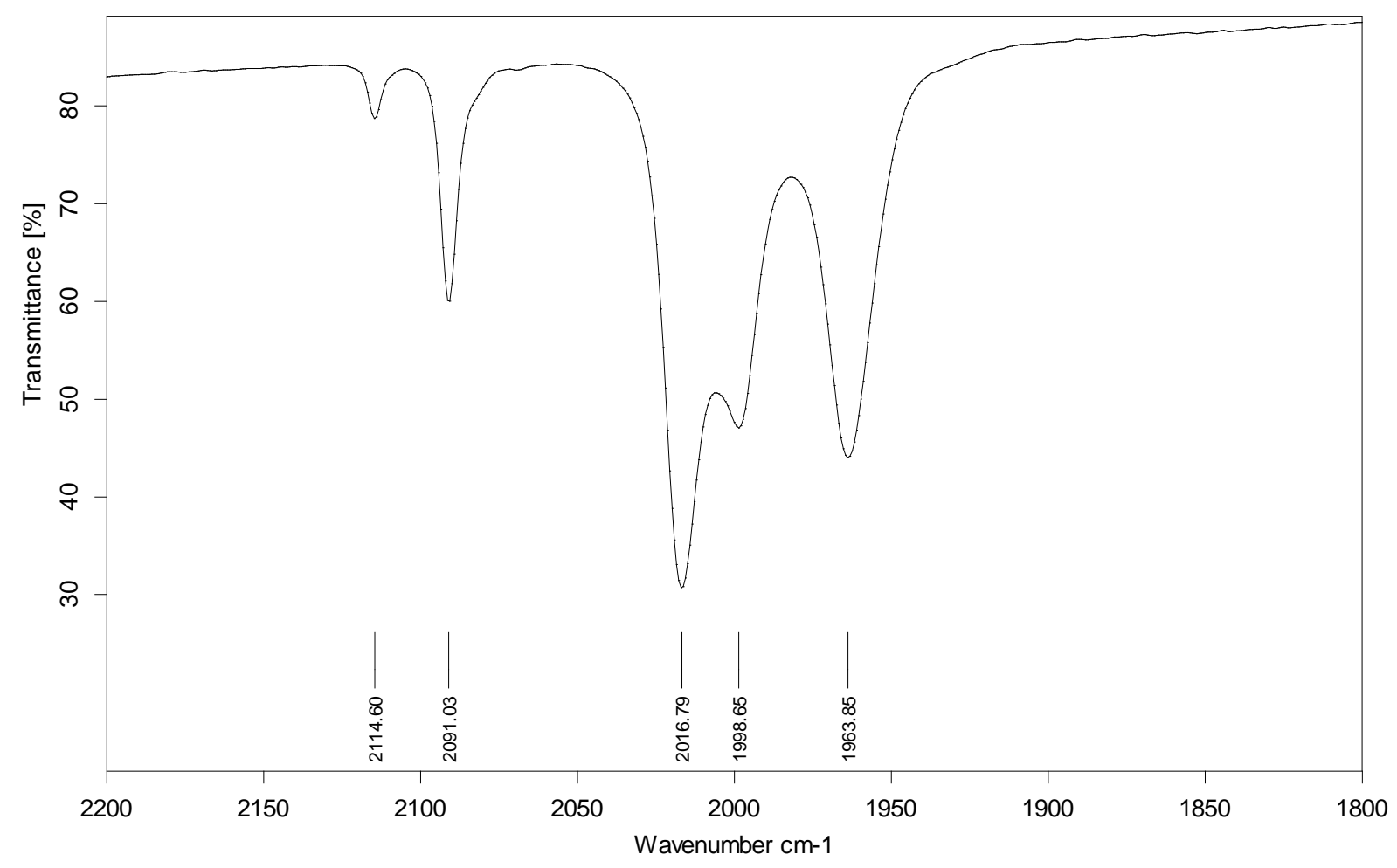

Figure A2.7. IR spectrum $\left(v_{\mathrm{CO}}\right)$ of $\mathbf{7 b}$ in DCM.

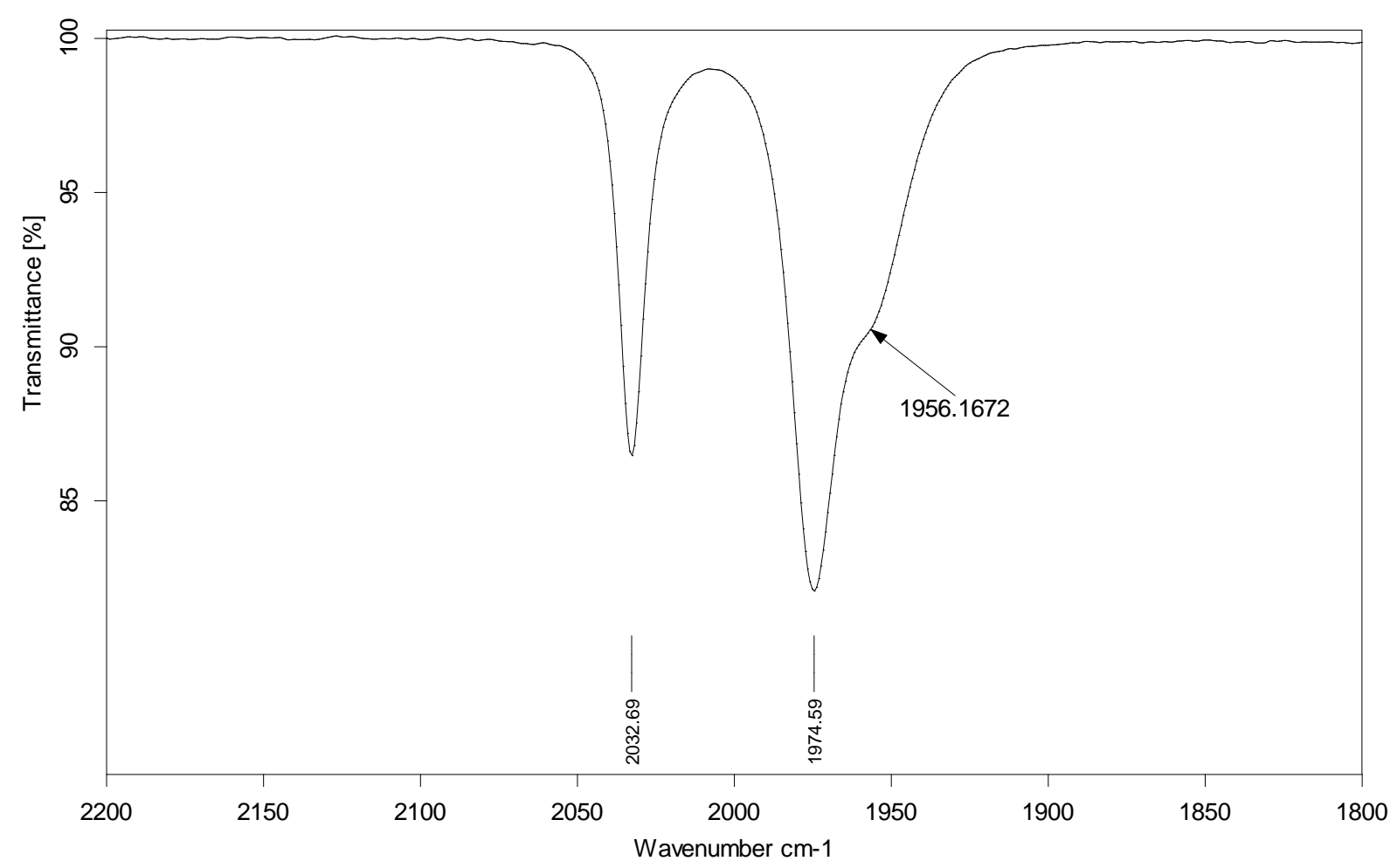

Figure A2.8. IR spectrum ( $\left.v_{\mathrm{CO}}\right)$ of $\mathbf{8 a}$ in DCM. 


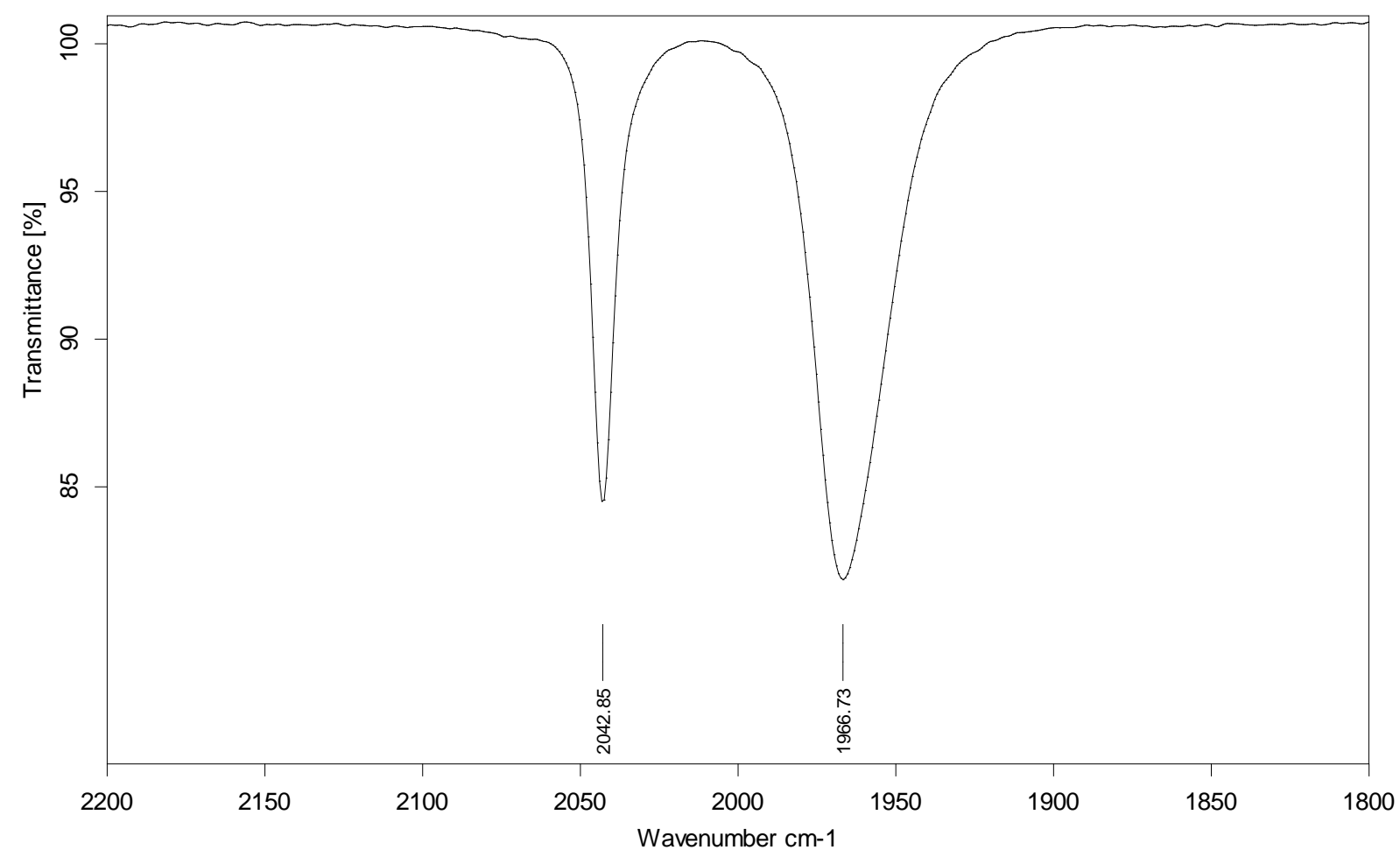

Figure A2.9. IR spectrum $\left(v_{\mathrm{CO}}\right)$ of $9 \mathbf{a}$ in DCM.

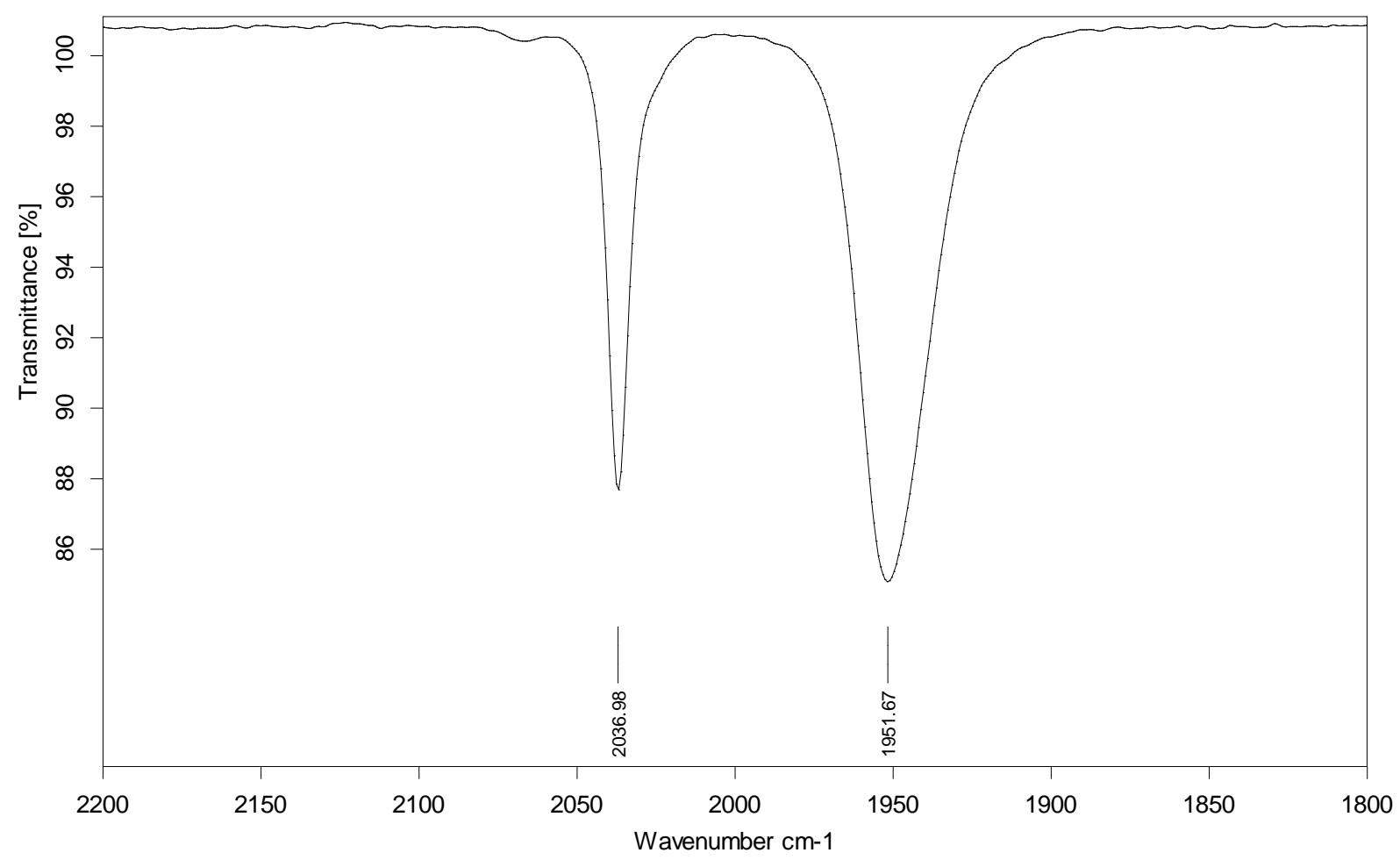

Figure A2.10. IR spectrum ( $\left.v_{\mathrm{CO}}\right)$ of 10a in DCM. 


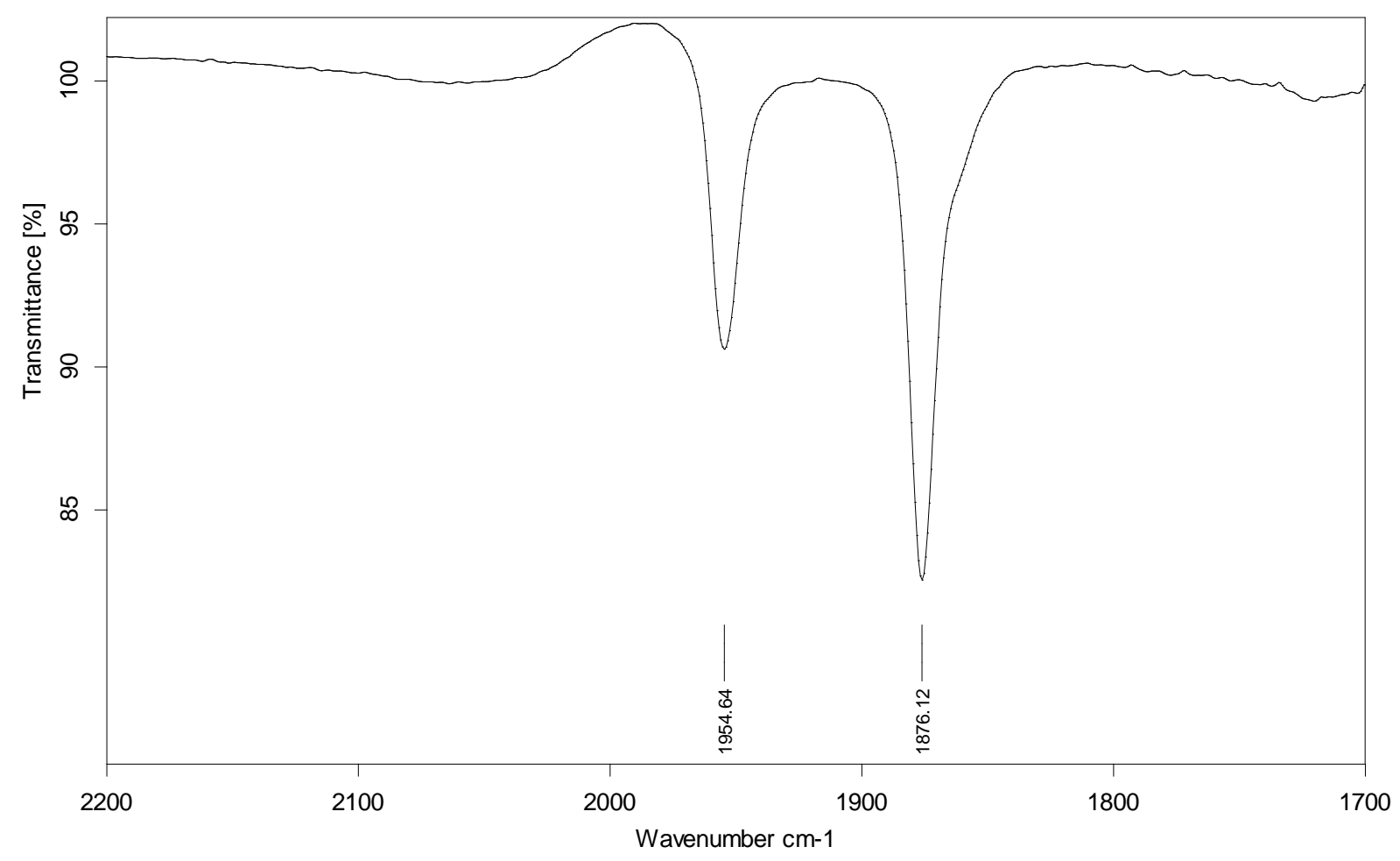

Figure A2.11. IR spectrum ( $\left.v_{\mathrm{CO}}\right)$ of $\mathbf{1 0 b}$ in THF.

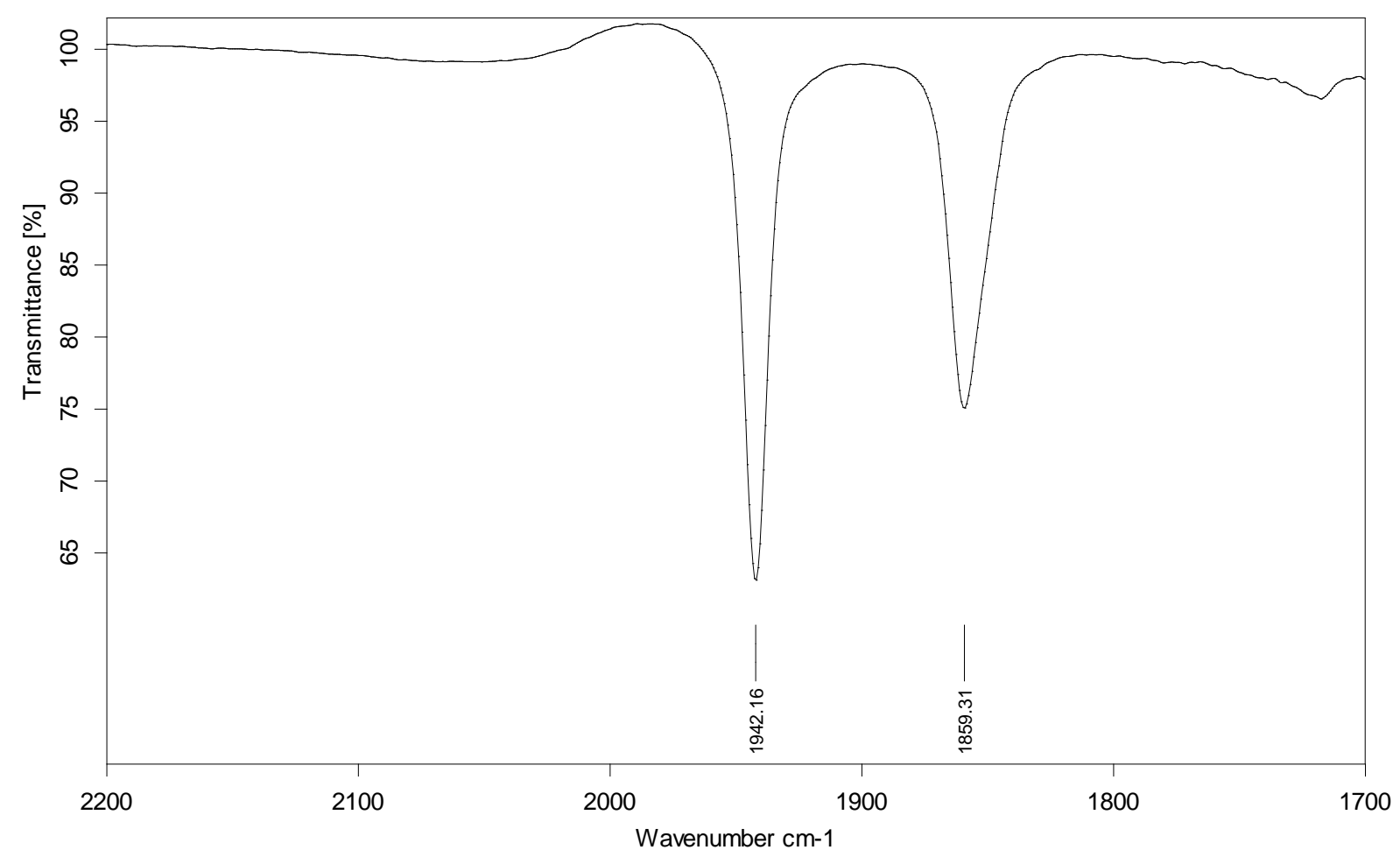

Figure A2.12. IR spectrum $\left(v_{\mathrm{CO}}\right)$ of $10 \mathrm{c}$ in THF. 


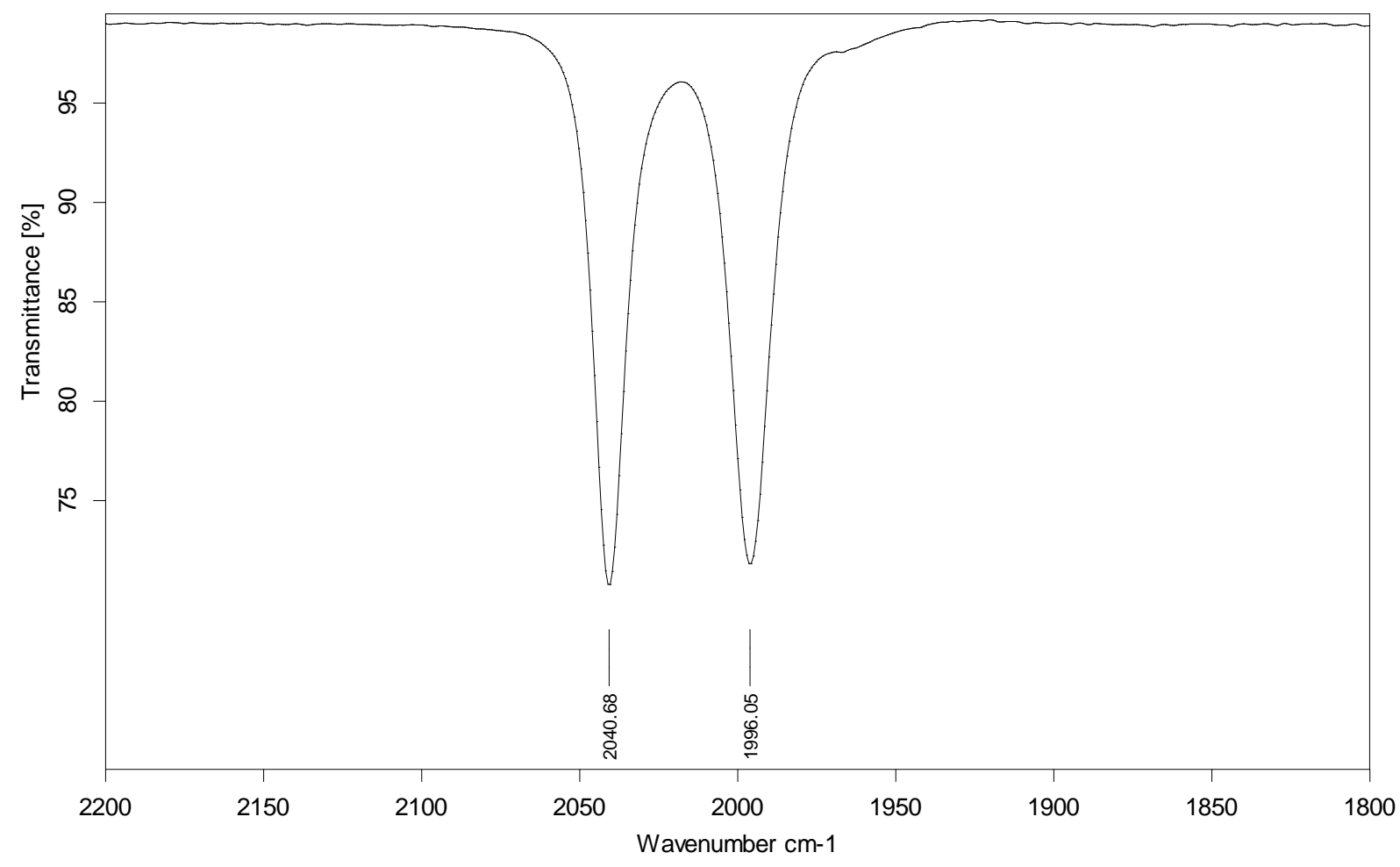

Figure A2.13. IR spectrum ( $\left.v_{\mathrm{CO}}\right)$ of 11a in DCM.

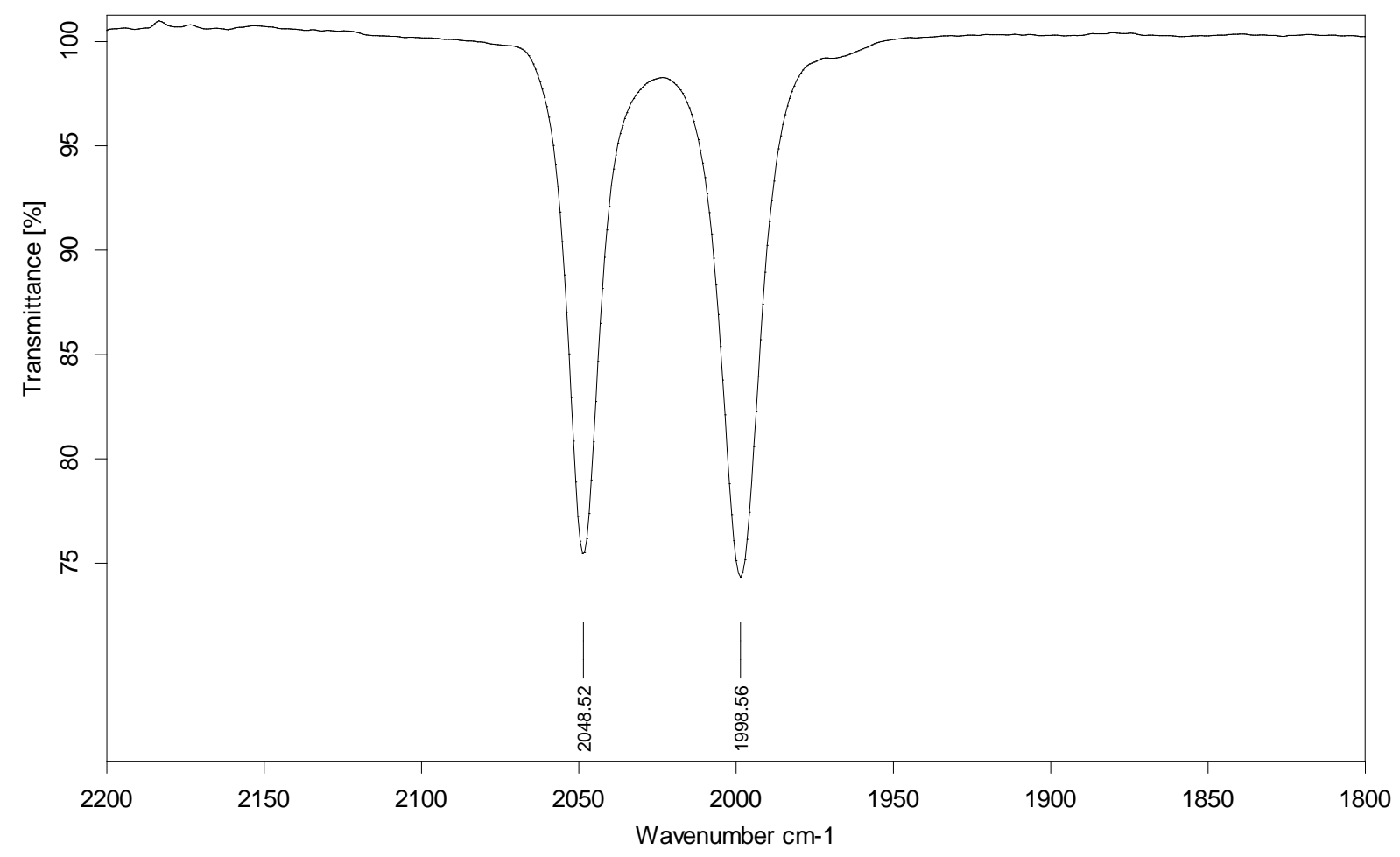

Figure A2.14. IR spectrum ( $\left.v_{\mathrm{CO}}\right)$ of $\mathbf{1 2 a}$ in DCM. 


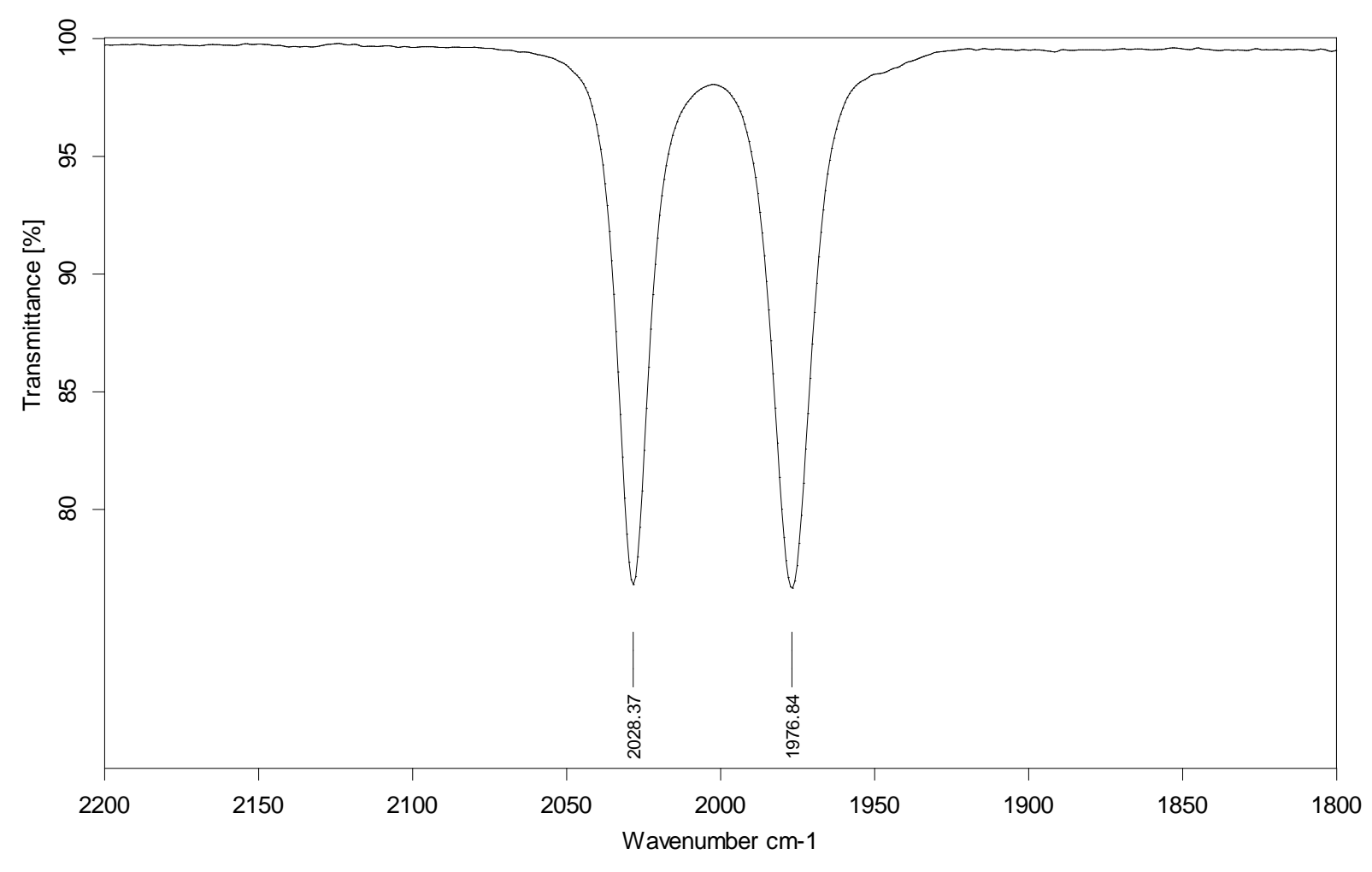

Figure A2.15. IR spectrum ( $\left.v_{\mathrm{CO}}\right)$ of 12'a in DCM.

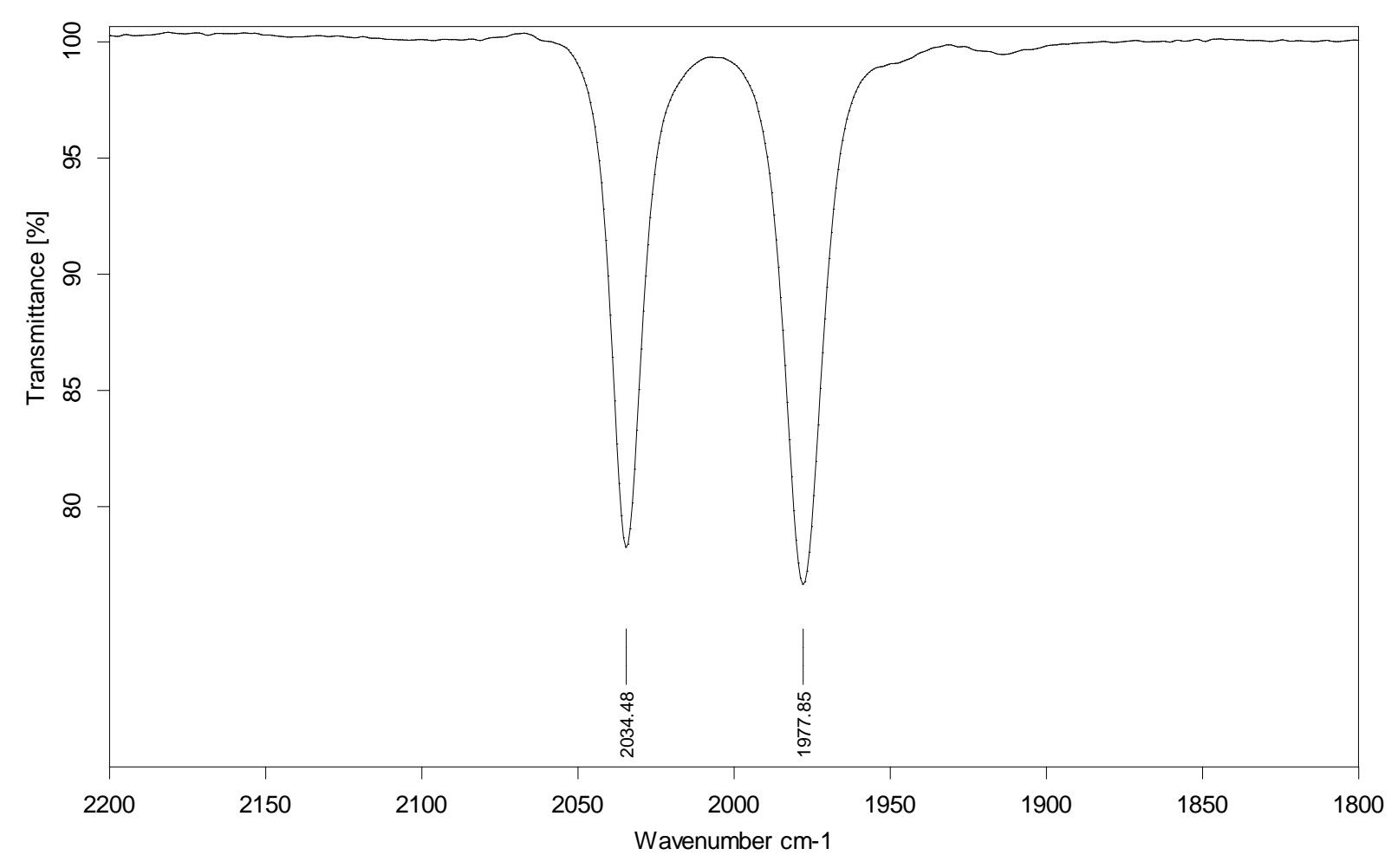

Figure A2.16. IR spectrum ( $\left.v_{\mathrm{CO}}\right)$ of 13a in DCM. 


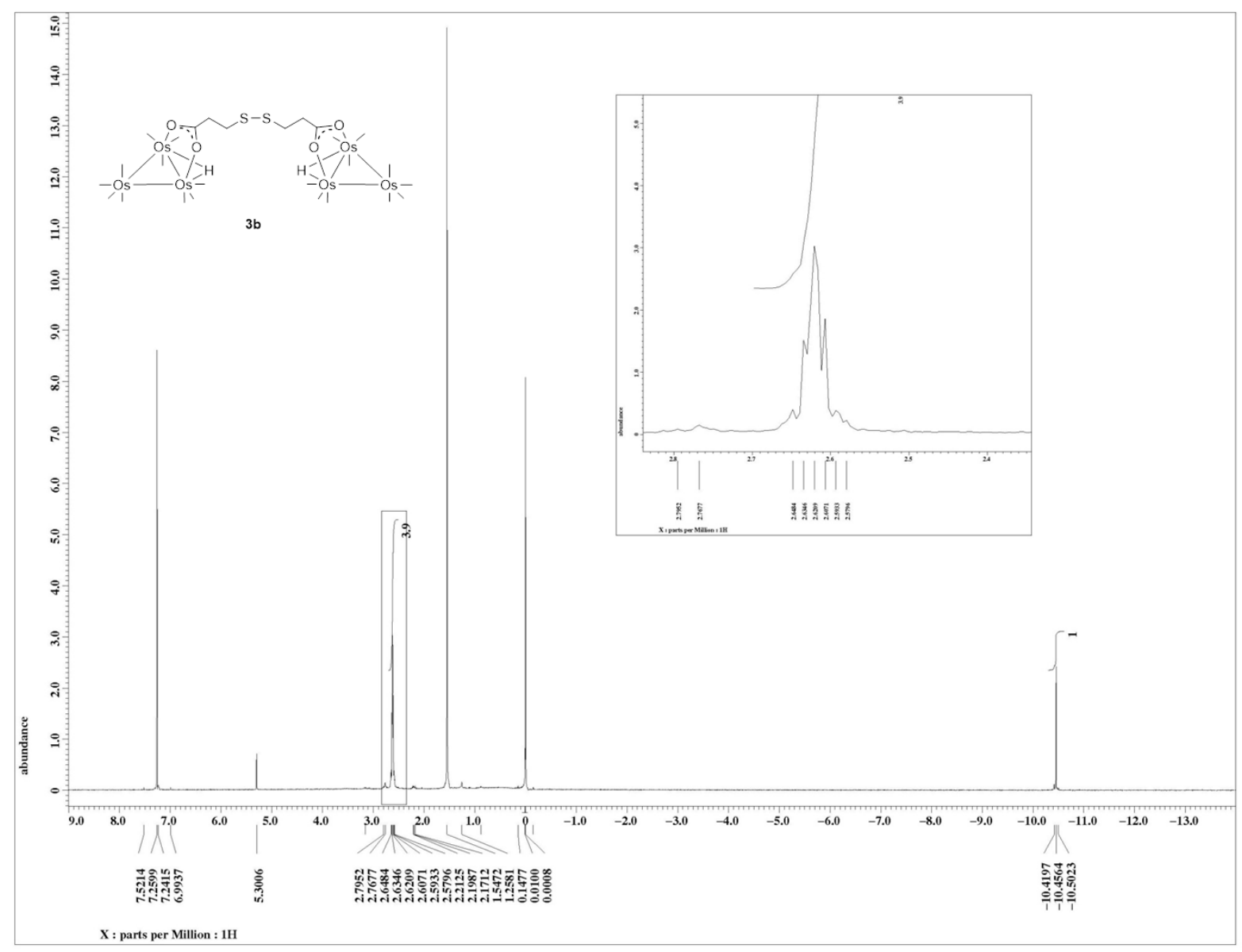

Figure A2.17. ${ }^{1} \mathrm{H}$ NMR spectrum of $\mathbf{3 b}$ in $\mathrm{CDCl}_{3}$. 


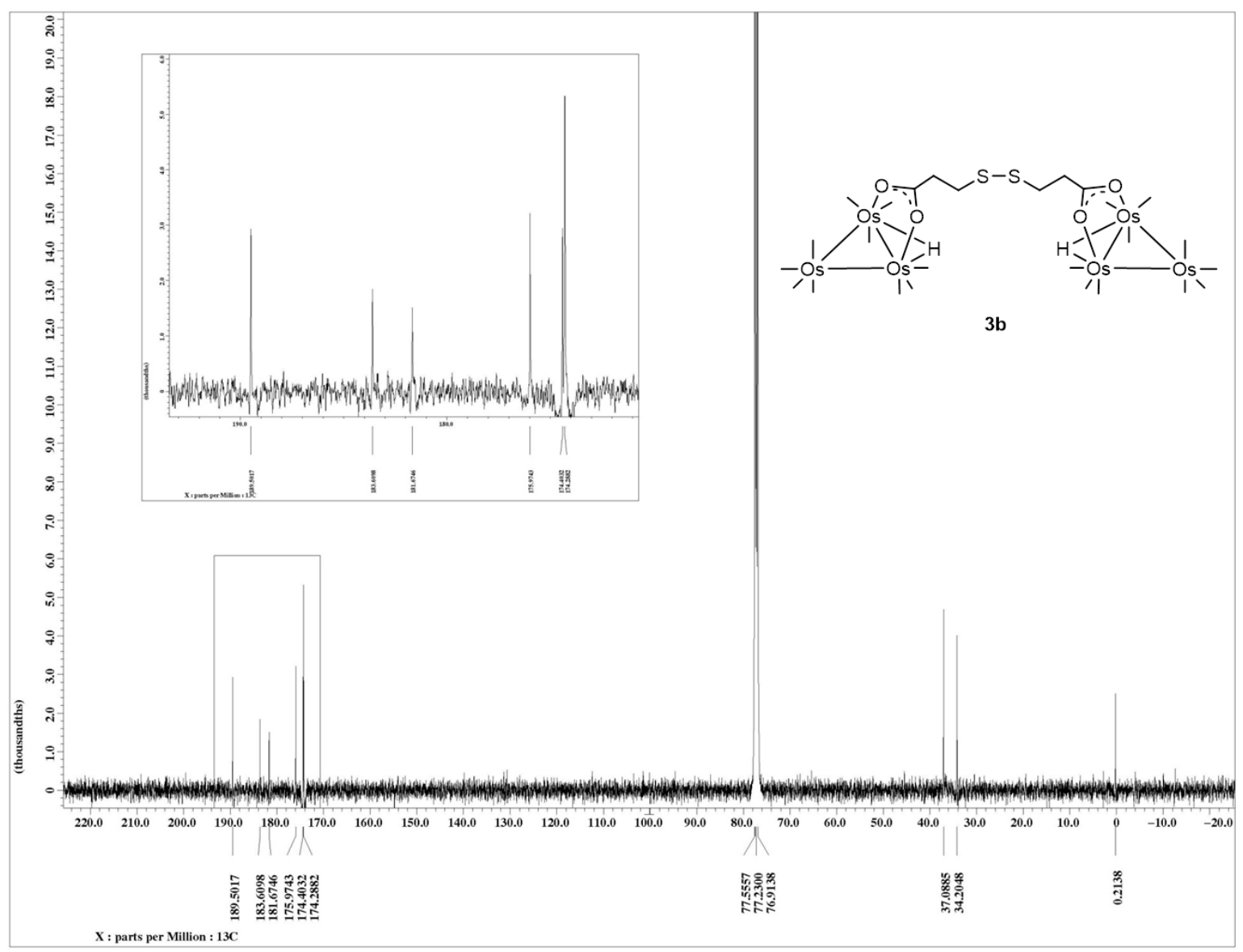

Figure A2.18. ${ }^{13} \mathrm{C}\left\{{ }^{1} \mathrm{H}\right\}$ NMR spectrum of $\mathbf{3 b}$ in $\mathrm{CDCl}_{3}$. 


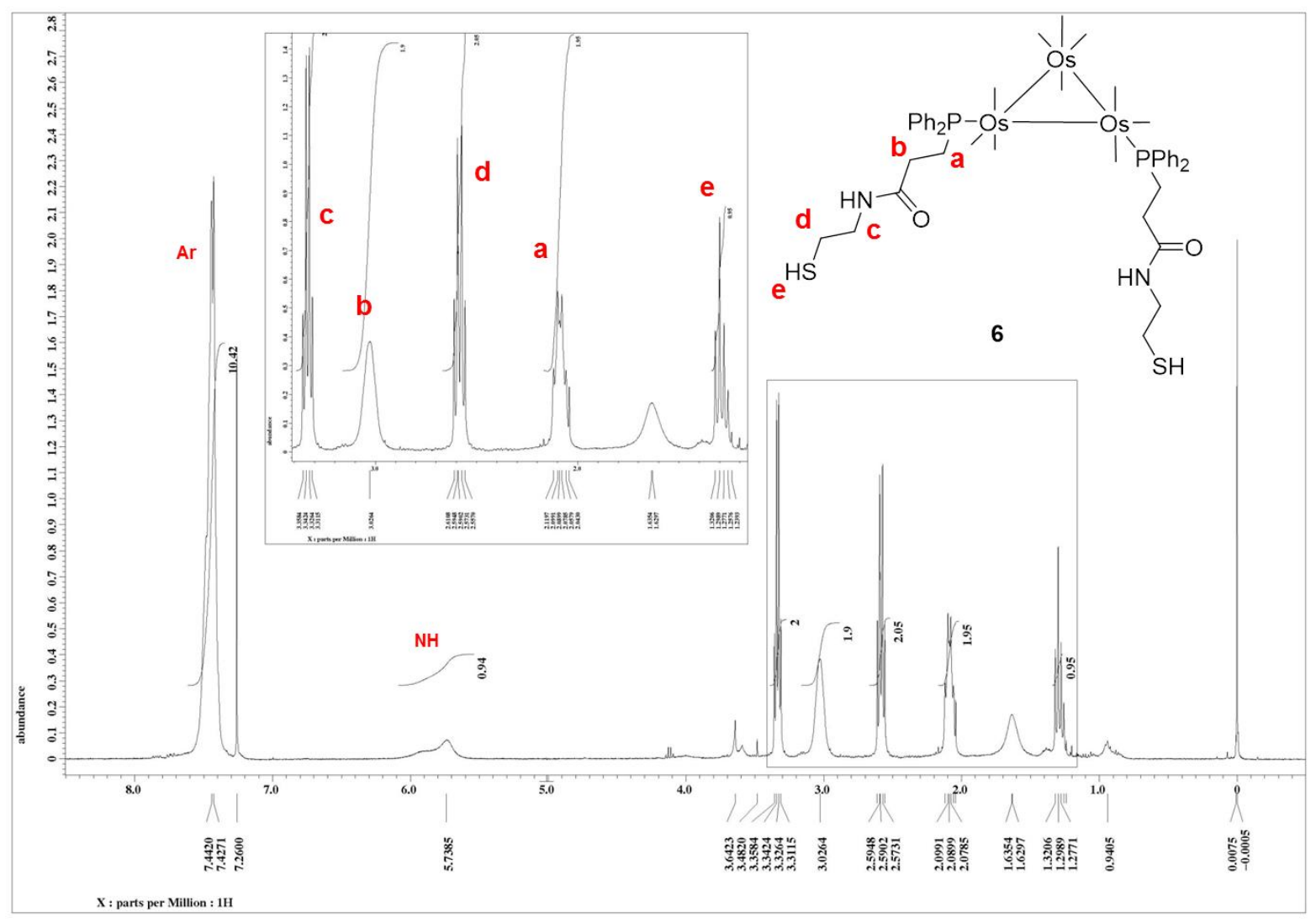

Figure A2.19. ${ }^{1} \mathrm{H}$ NMR spectrum of 6 in $\mathrm{CDCl}_{3}$. 


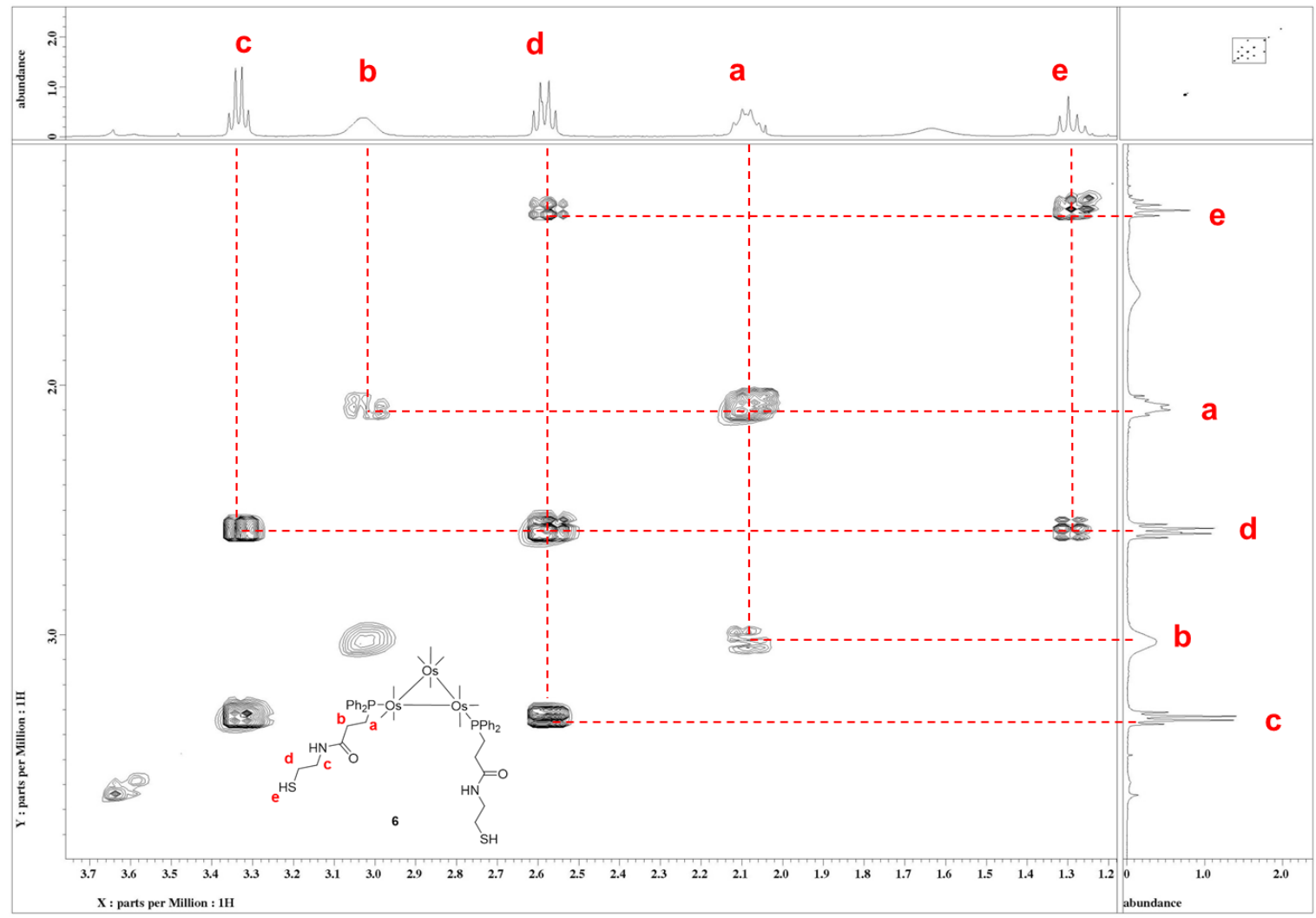

Figure A2.20. COSY NMR spectrum of 6 in $\mathrm{CDCl}_{3}$. 


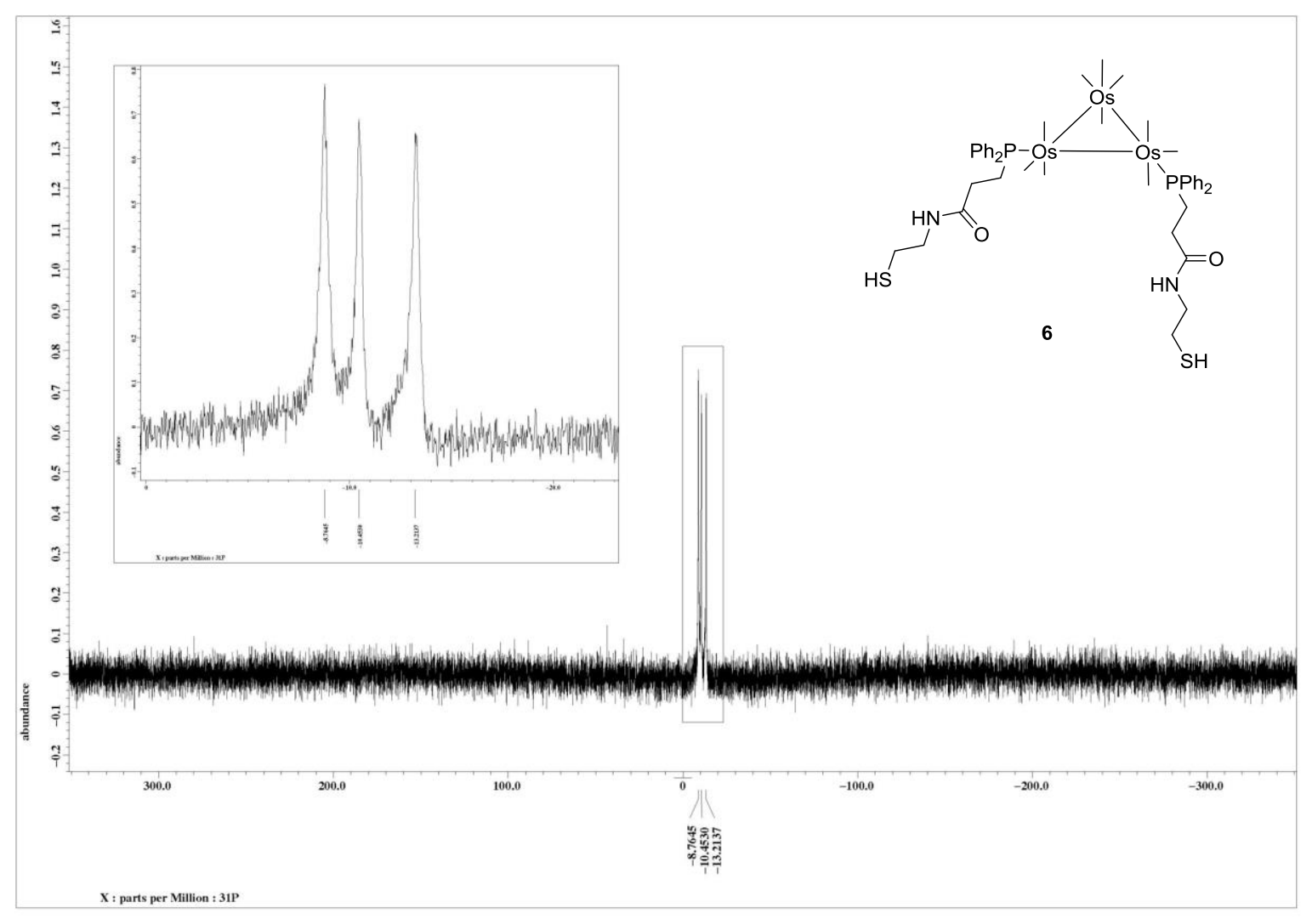

Figure A2.21. ${ }^{31} \mathrm{P}\left\{{ }^{1} \mathrm{H}\right\}$ NMR spectrum of 6 in $\mathrm{CDCl}_{3}$.

17 
$\mathrm{C} 44 \mathrm{H} 40 \mathrm{~N} 2 \mathrm{O} 12 \mathrm{Os} 3$

a $\mathrm{ZY}-2803(0.082) \mathrm{Cm}(1: 11)$

100
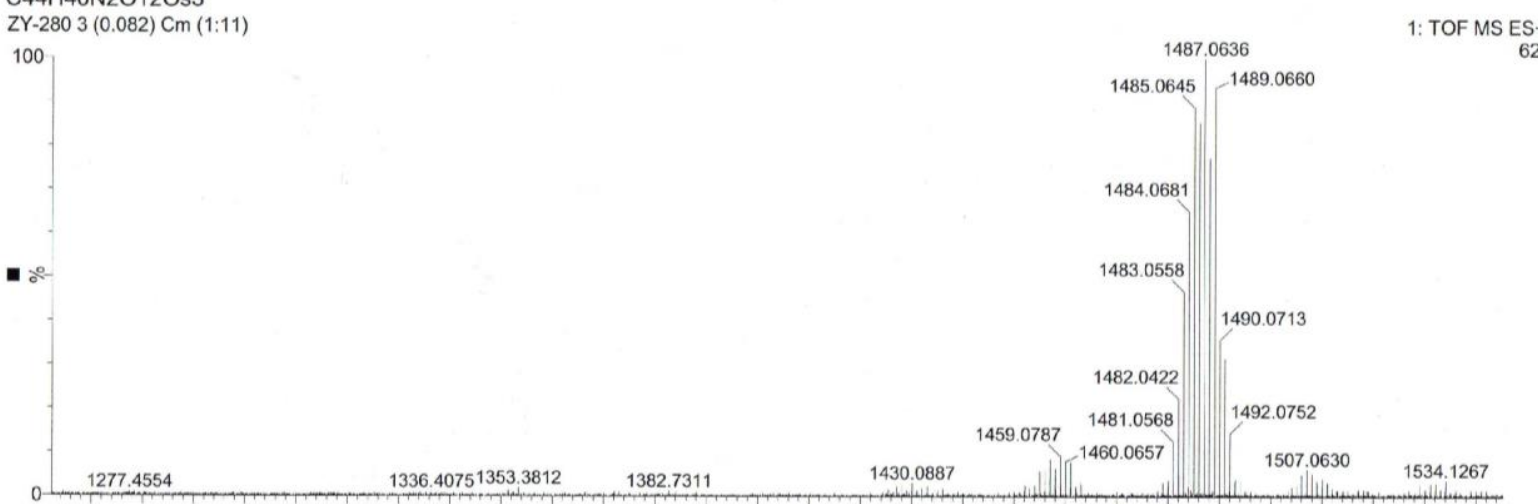

ZY-280 (0.045) Is $(1.00,1.00)$ C44H41N2O12Os3P2S2 100
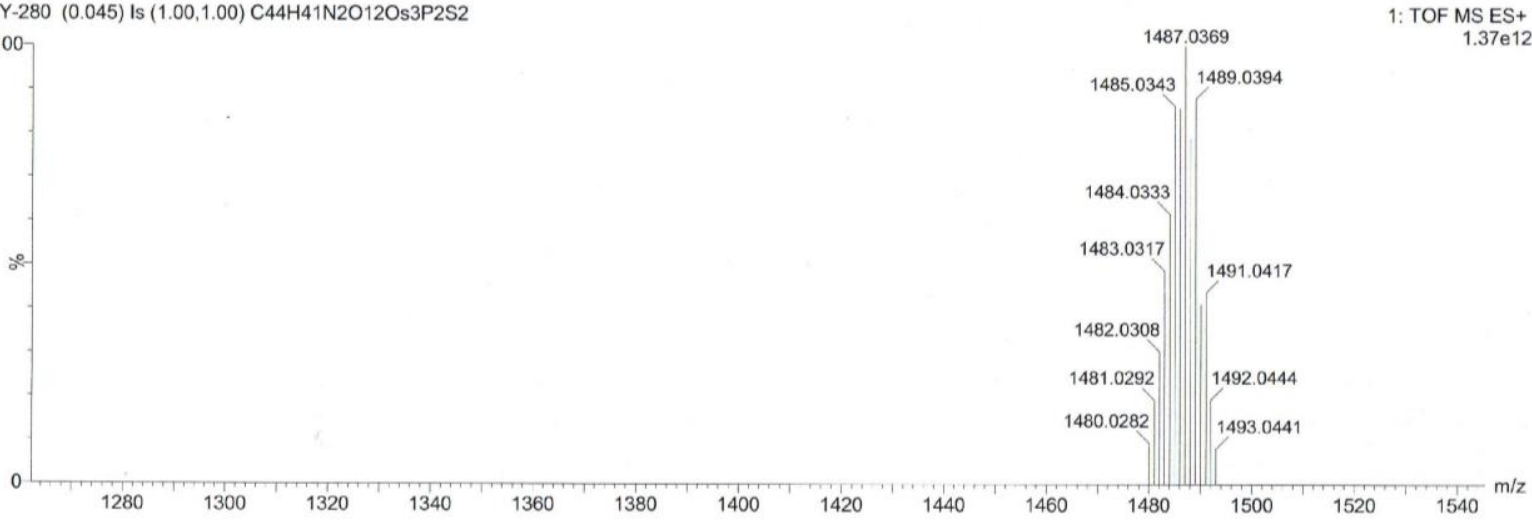

b

Elemental Composition Report

Page 1

Single Mass Analysis

T.0 PPM / DBE: $\min =-1.5, \max =50.0$

Element prediction: Of

Number of isotope peaks used for $\mathrm{i}-\mathrm{FIT}=3$

Monoisotopic Mass, Even Electron lons

Win limits (up to 50 closest results for each mass)

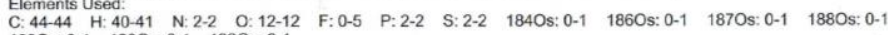
1890s: 0-1 1900s: 0-1 1920s: 0-1

C $44 H 40 N 2 O 12 O s 3$
ZY-280 11(0.259)

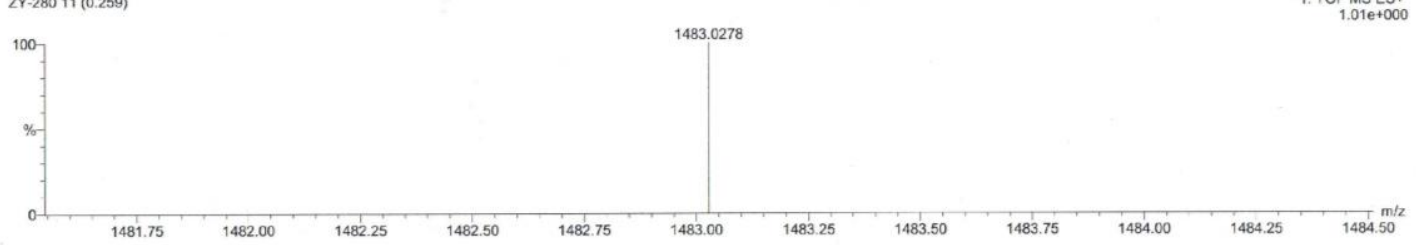

$\begin{array}{llr}\text { Minimum: } & 5.0 \quad 5.0 & -1.5 \\ \text { Maximum: } & 50.0\end{array}$

Mass Calc. Mass . mDa PPM DRE i-PIT i-PIT (Norm) Formula

$\begin{array}{llllllllllllllll}14883.0278 & 1483.0314 & -3.6 & -2.4 & 29.5 & 14.4 & 0.7 & \mathrm{C} 44 & \mathrm{H} 41 & \mathrm{~N} 2 & \mathrm{O} 12 & \mathrm{P} 2 & \mathrm{~S} 2 & 1860 \mathrm{~s} & 1900 \mathrm{~s} & 1920 \mathrm{~s} \\ & 1483.0330 & -5.2 & -3.5 & 29.5 & 14.4 & 0.7 & \mathrm{C} 44 & \mathrm{H} 41 & \mathrm{~N} 2 & \mathrm{O} 12 & \mathrm{P} 2 & \mathrm{~S} 2 & 1870 \mathrm{~s} & 1890 \mathrm{~s} & 1920 \mathrm{~s}\end{array}$

Figure A2.22. a) ESI-HRMS of 6 (top) with calculated isotopic pattern (bottom). b) ESI-

HRMS single mass analysis of $\mathbf{6}$. 


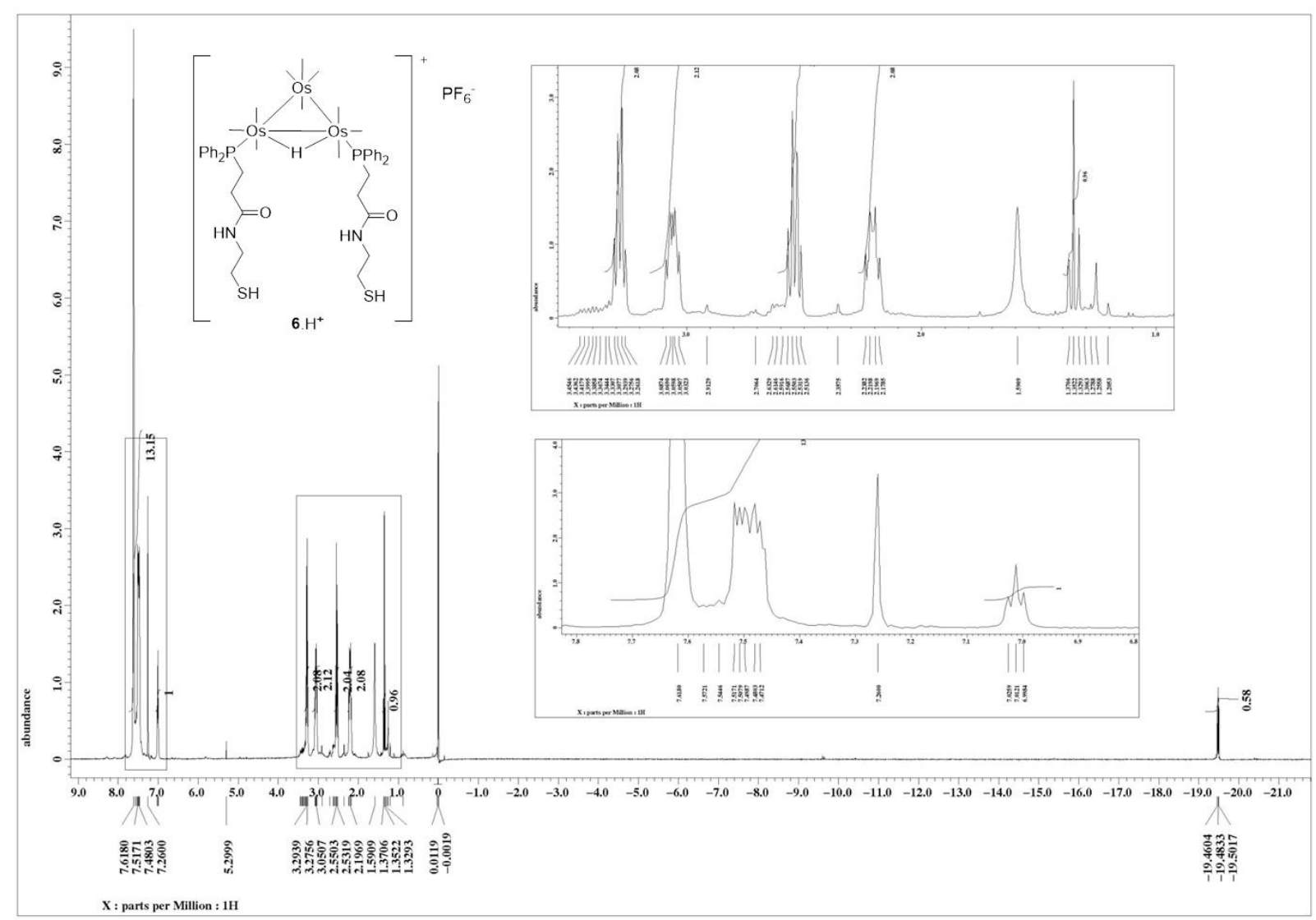

Figure A2.23. ${ }^{1} \mathrm{H}$ NMR spectrum of $6 . \mathrm{H}^{+}$in $\mathrm{CDCl}_{3}$. 


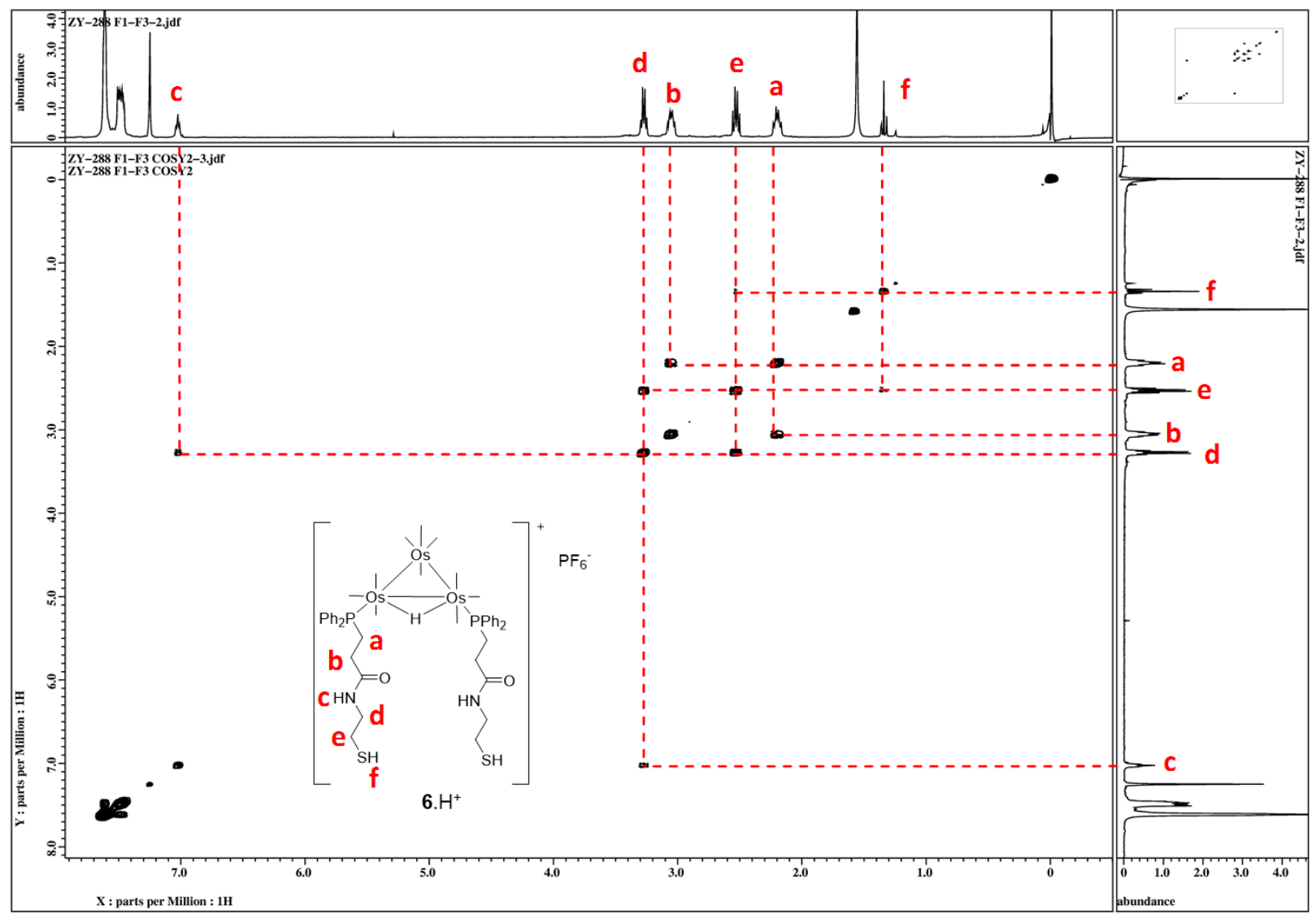

Figure A2.24. COSY NMR spectrum of $\mathbf{6} \cdot \mathrm{H}^{+}$in $\mathrm{CDCl}_{3}$. 


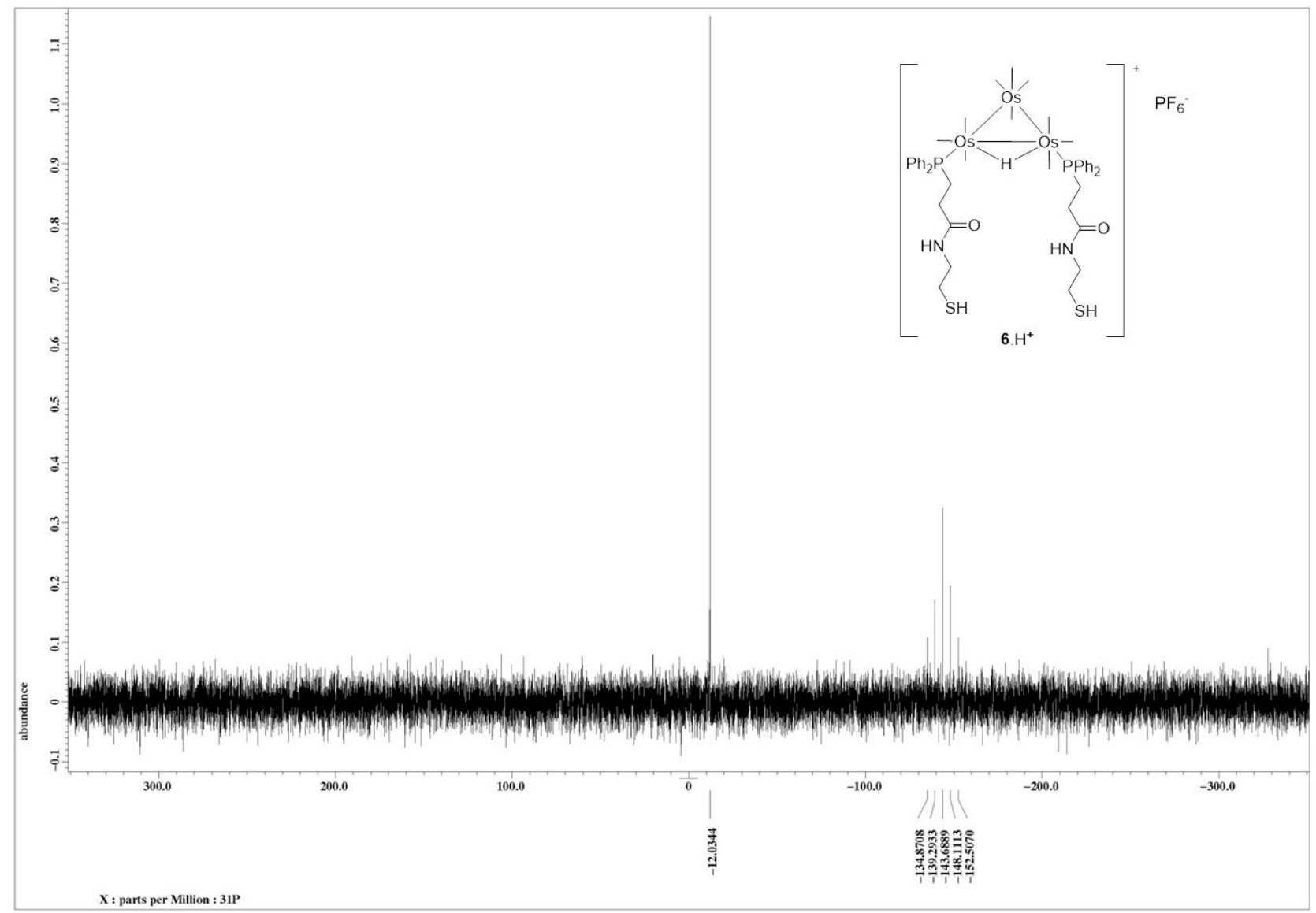

Figure A2.25. ${ }^{31} \mathrm{P}\left\{{ }^{1} \mathrm{H}\right\} \mathrm{NMR}$ spectrum of $6 . \mathrm{H}^{+}$in $\mathrm{CDCl}_{3}$. 


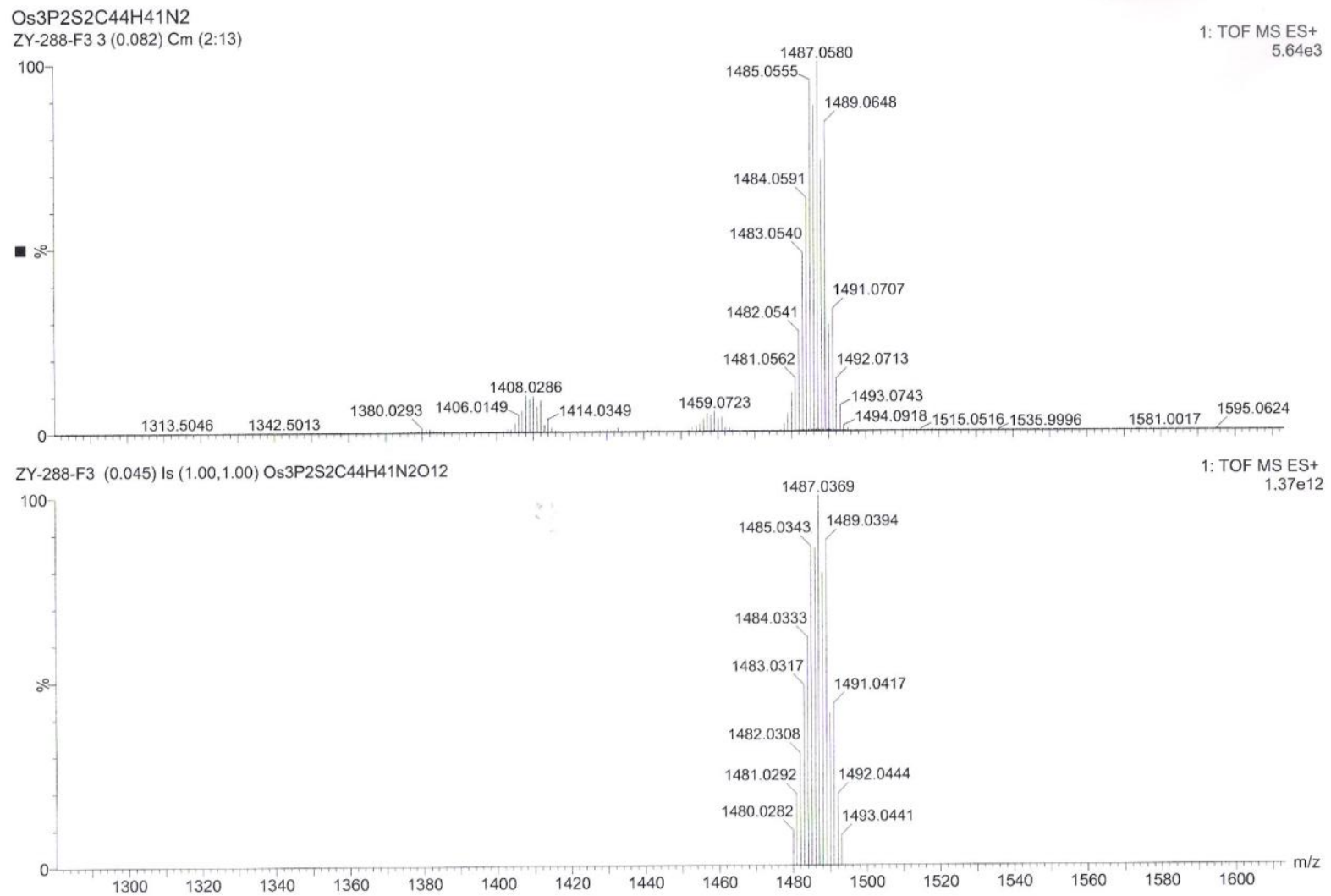

Elemental Composition Report

Page 1

b

Single Mass Analysis

Tolerance $=5.0$ PPM / DBE: $\min =-1.5, \max =50.0$

Element prediction: O

Number of isotope peaks used for $\mathrm{i}-\mathrm{FIT}=3$

Monoisotopic Mass, Even Electron lons

1349 formula(e) evaluated with 3 results within limits (up to 50 closest results for each mass)

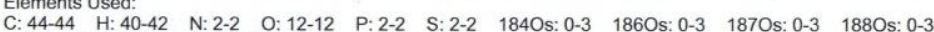

1890s: 0-3 1900s: 0-3 1920s: $0-3$

Os $3 P 2 S 2 C 44 H 41 N 2$
$Z Y-288-F 315(0.332)$

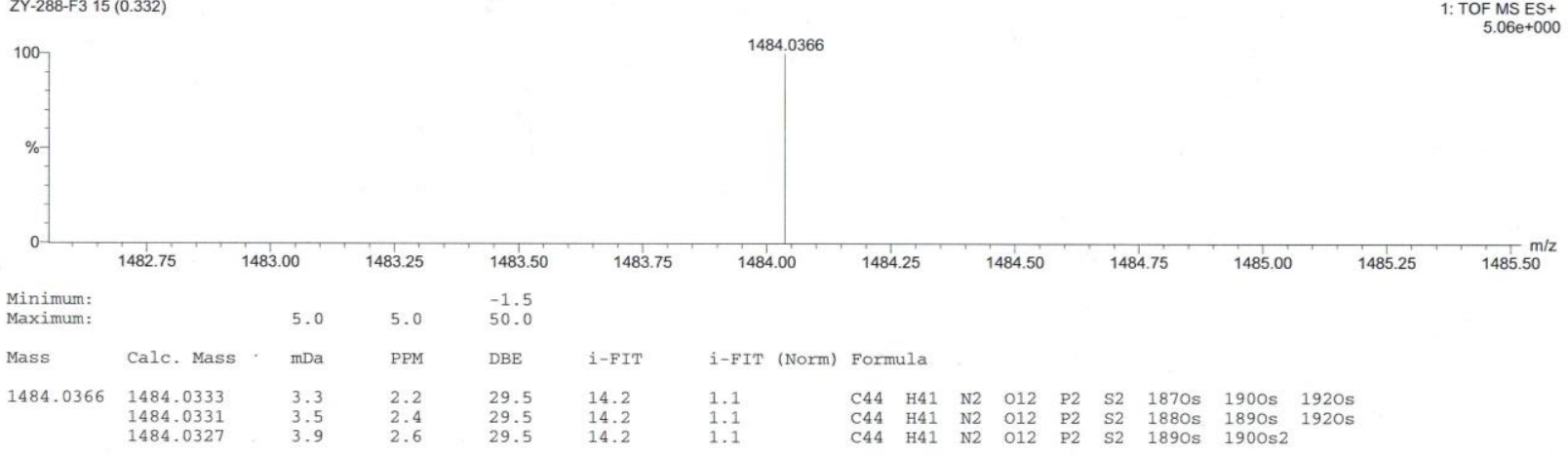

Figure A2.26. a) ESI-HRMS of $6 . \mathrm{H}^{+}$(top) with calculated isotopic pattern (bottom). b) ESIHRMS single mass analysis of $\mathbf{6} . \mathrm{H}^{+}$ 

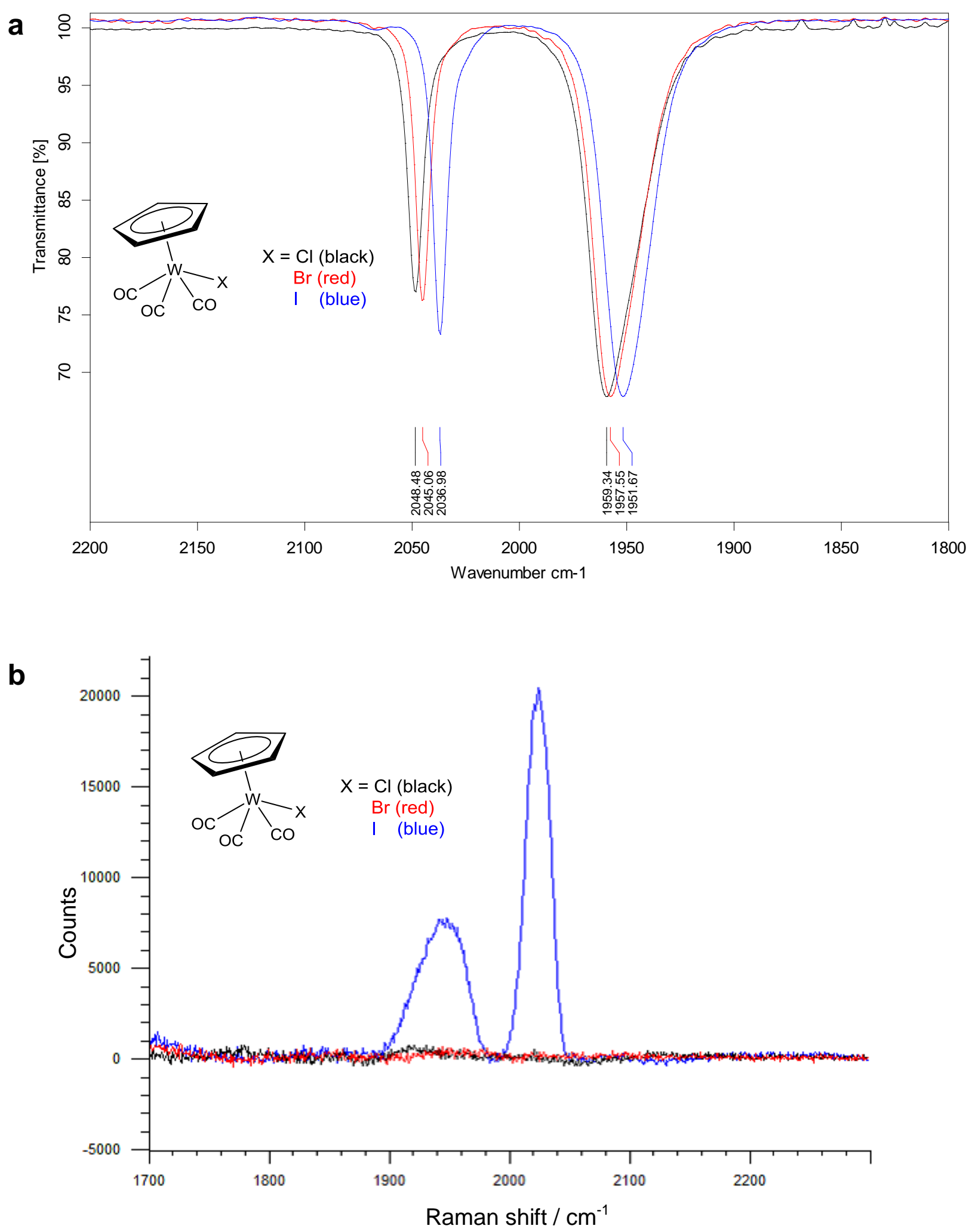

Figure A2.27. a) IR spectrum $\left(v_{\mathrm{CO}}\right)$ of $\mathrm{CpW}(\mathrm{CO})_{3} \mathrm{X}$ in DCM and b) SERS spectra of respective complexes with AuNS, where $\mathrm{X}=\mathrm{Cl}$ (black), $\mathrm{Br}$ (red) and $\mathrm{I}$ (blue), in DCM. 
Chapter 3: Metal Carbonyl-Boronic Acid Conjuagte - a SERS Probe for Glucose Detection and its Cytotoxicity

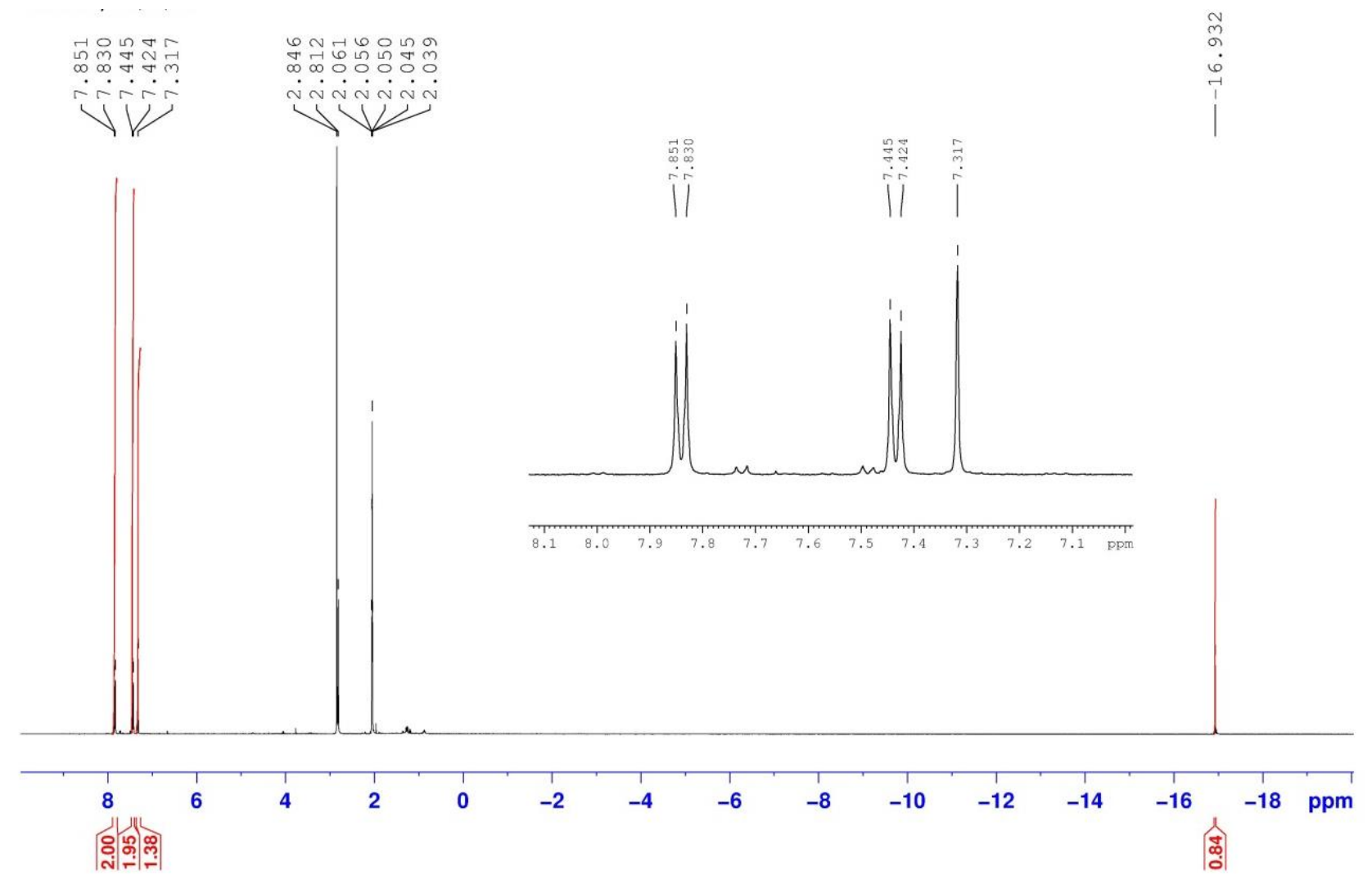

Figure A3.1. ${ }^{1} \mathrm{H}$ NMR spectrum of $\mathbf{2 b}-\mathrm{B}(\mathrm{OH})_{2}$ in $\left(\mathrm{CD}_{3}\right)_{2} \mathrm{CO}$. 


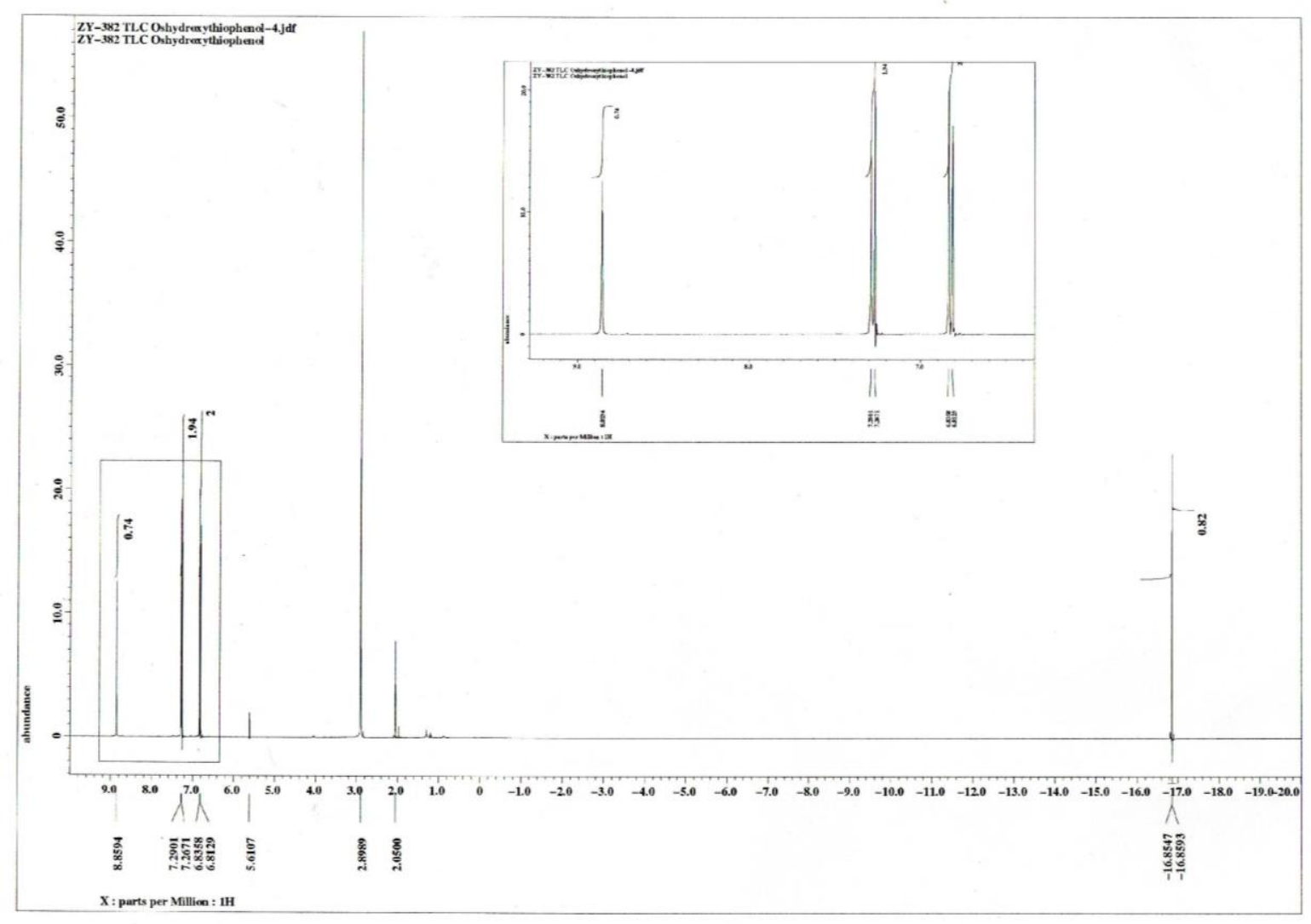

Figure A3.2. ${ }^{1} \mathrm{H}$ NMR spectrum of $\mathbf{2 b}-\mathrm{OH}$ in $\left(\mathrm{CD}_{3}\right)_{2} \mathrm{CO}$. 


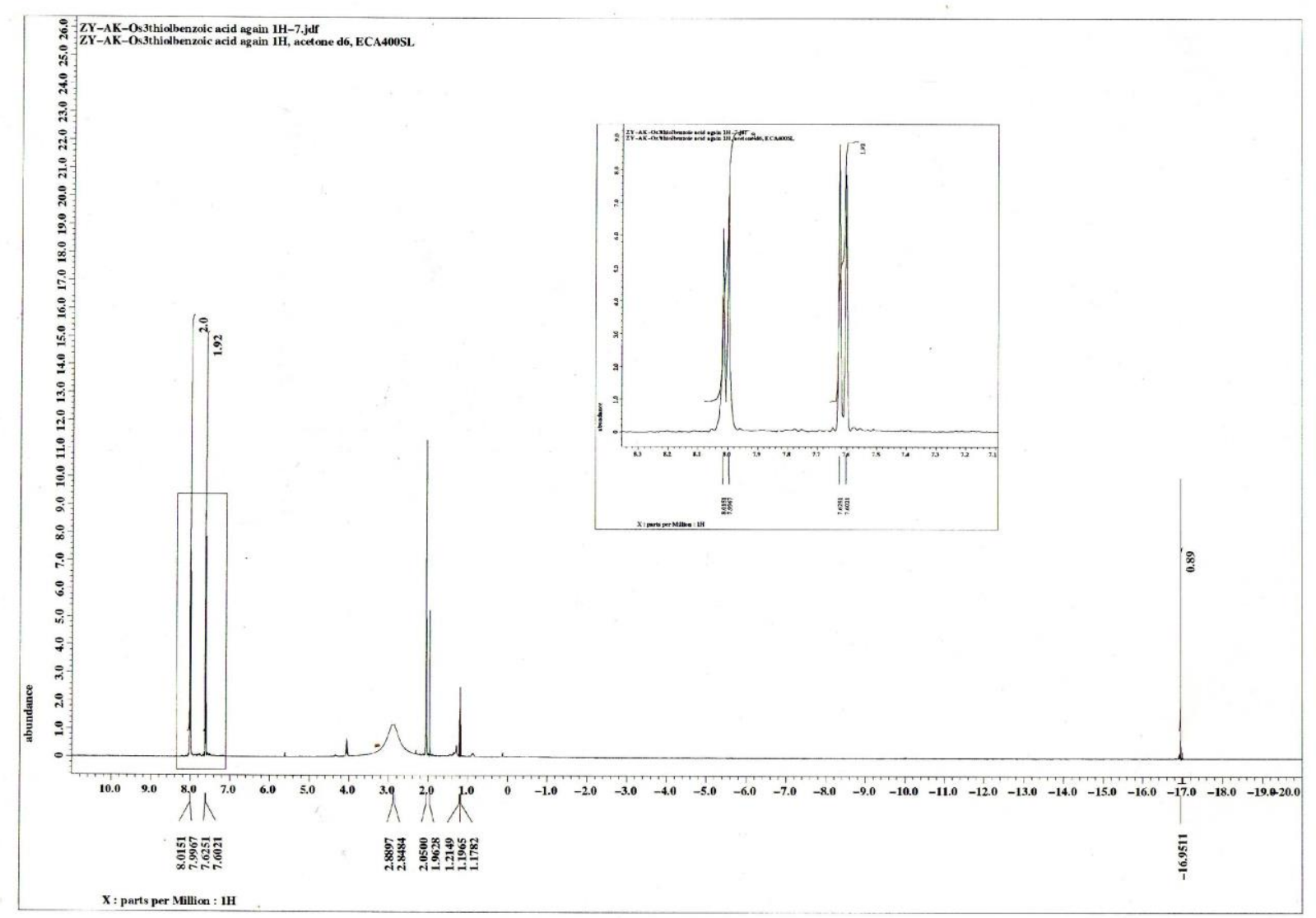

Figure A3.3. ${ }^{1} \mathrm{H}$ NMR spectrum of $\mathbf{2 b - C O O H}$ in $\left(\mathrm{CD}_{3}\right)_{2} \mathrm{CO}$. 


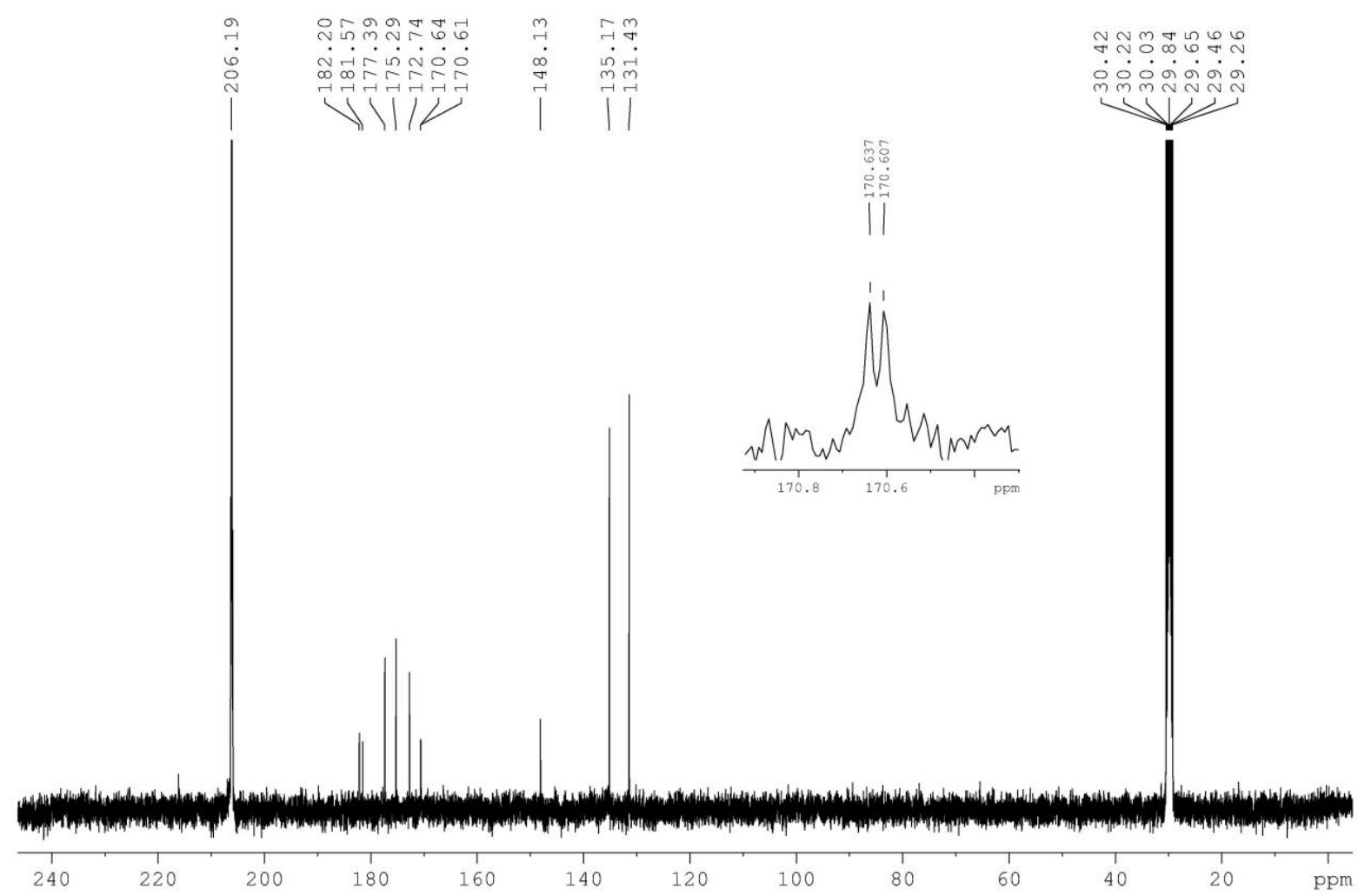

Figure A3.4. ${ }^{13} \mathrm{C}\{1 \mathrm{H}\}$ NMR spectrum of $\mathbf{2 b}-\mathrm{B}(\mathrm{OH})_{2}$ in $\left(\mathrm{CD}_{3}\right)_{2} \mathrm{CO}$. 


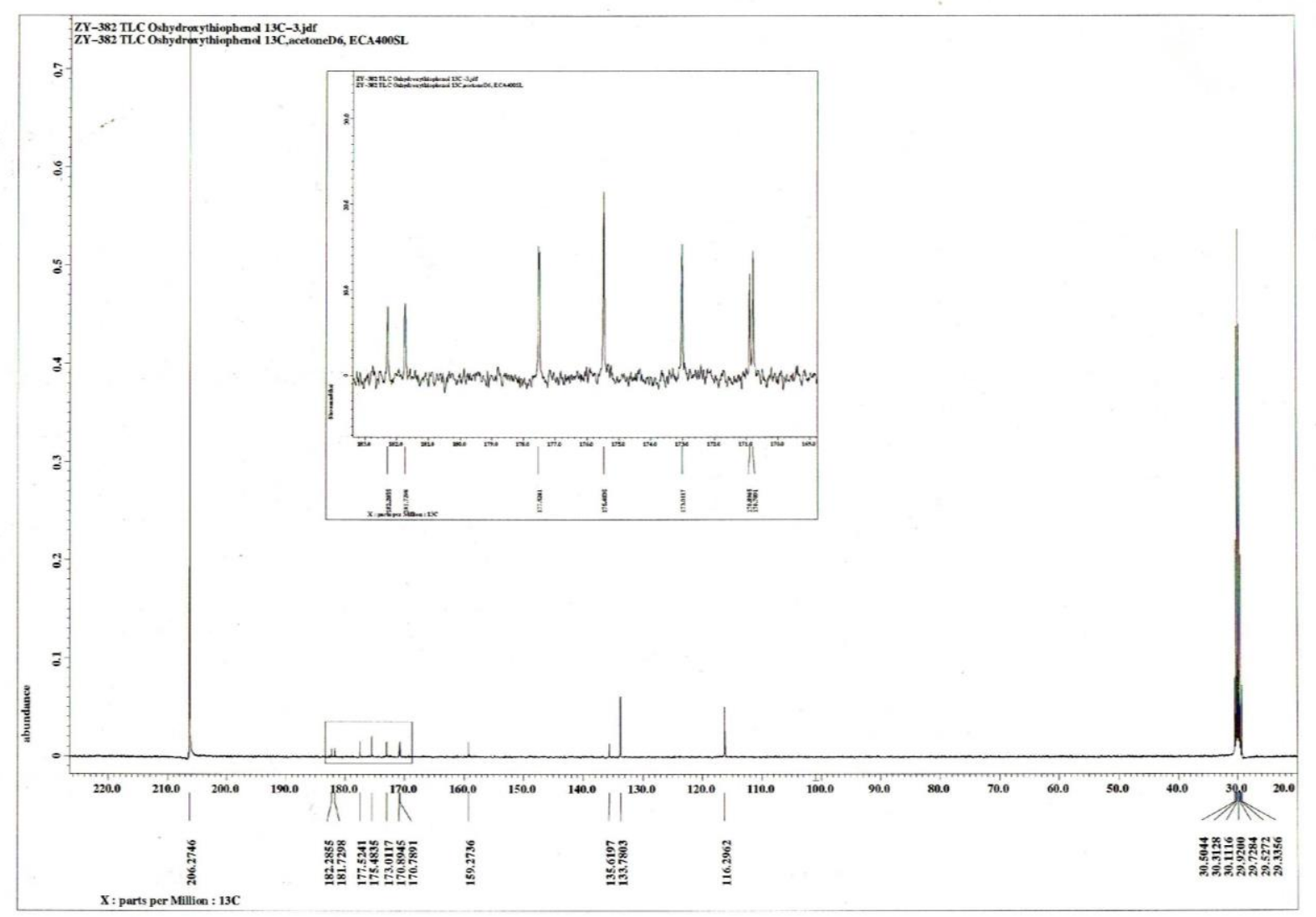

Figure A3.5. ${ }^{13} \mathrm{C}\left\{{ }^{1} \mathrm{H}\right\}$ NMR spectrum of $\mathbf{2 b}-\mathrm{OH}$ in $\left(\mathrm{CD}_{3}\right)_{2} \mathrm{CO}$. 


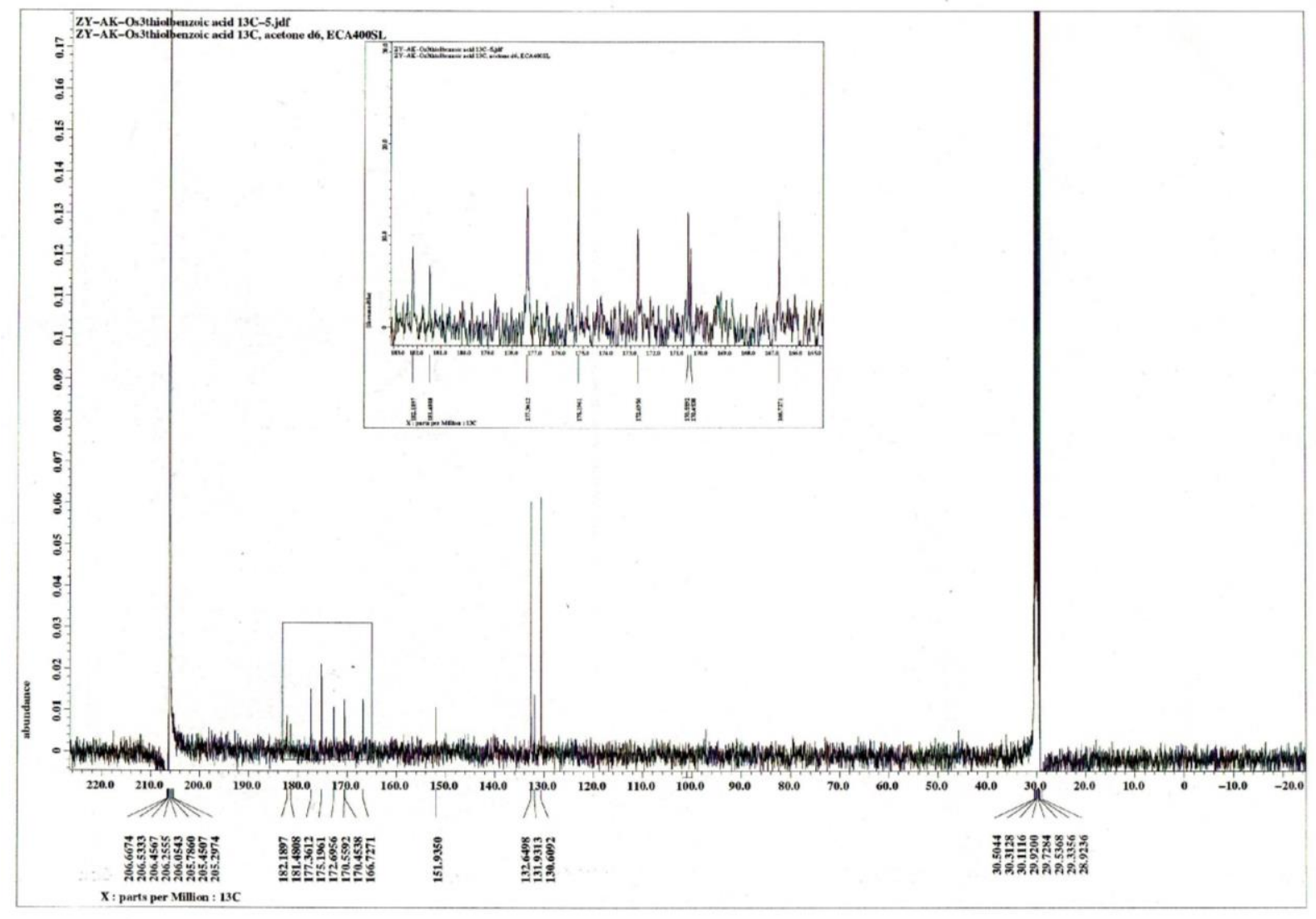

Figure A3.6. ${ }^{13} \mathrm{C}\left\{{ }^{1} \mathrm{H}\right\}$ NMR spectrum of $\mathbf{2 b}-\mathrm{COOH}$ in $\left(\mathrm{CD}_{3}\right)_{2} \mathrm{CO}$.

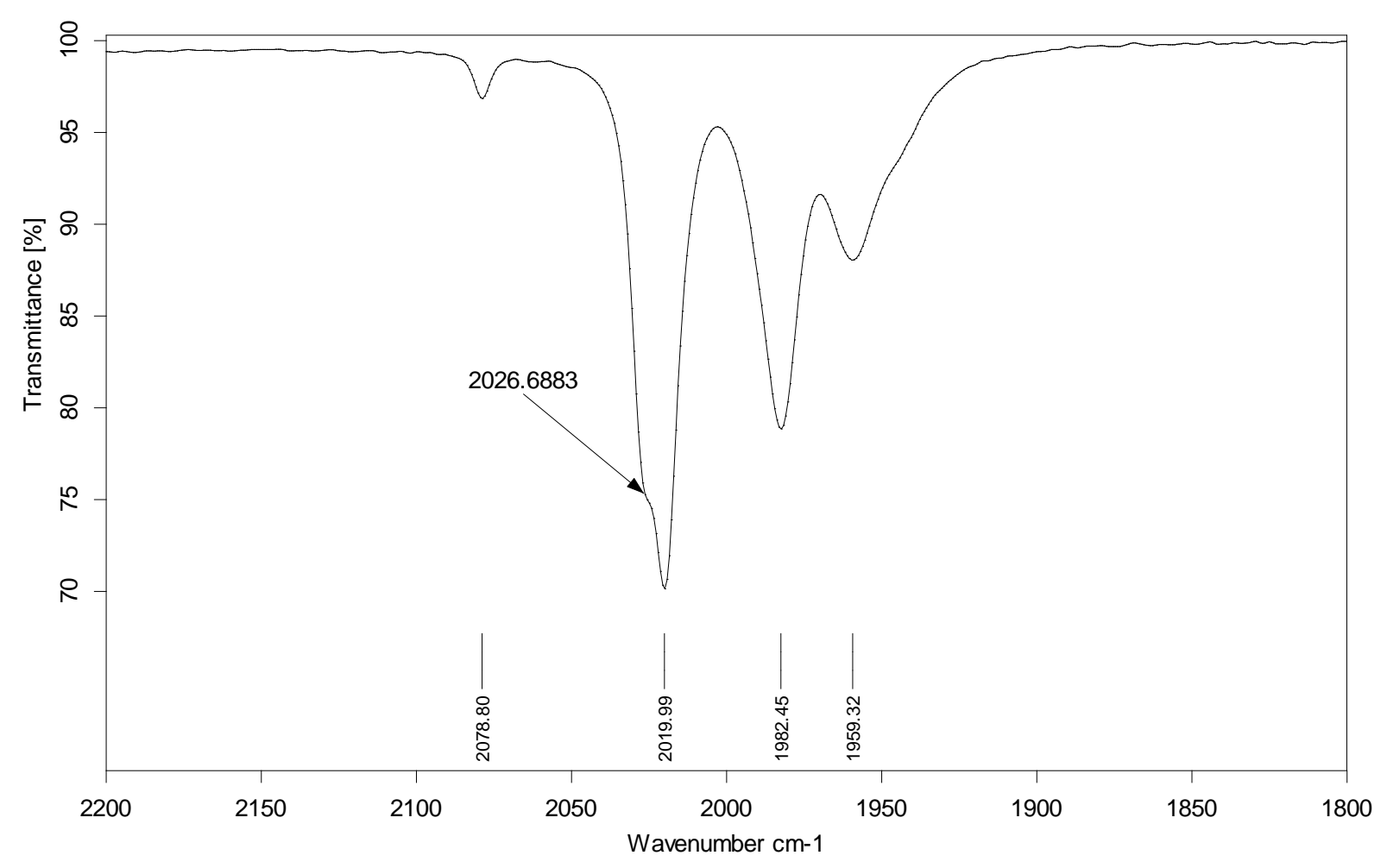

Figure A3.7. IR spectrum ( $\left.v_{\mathrm{CO}}\right)$ of 1 in DCM. 


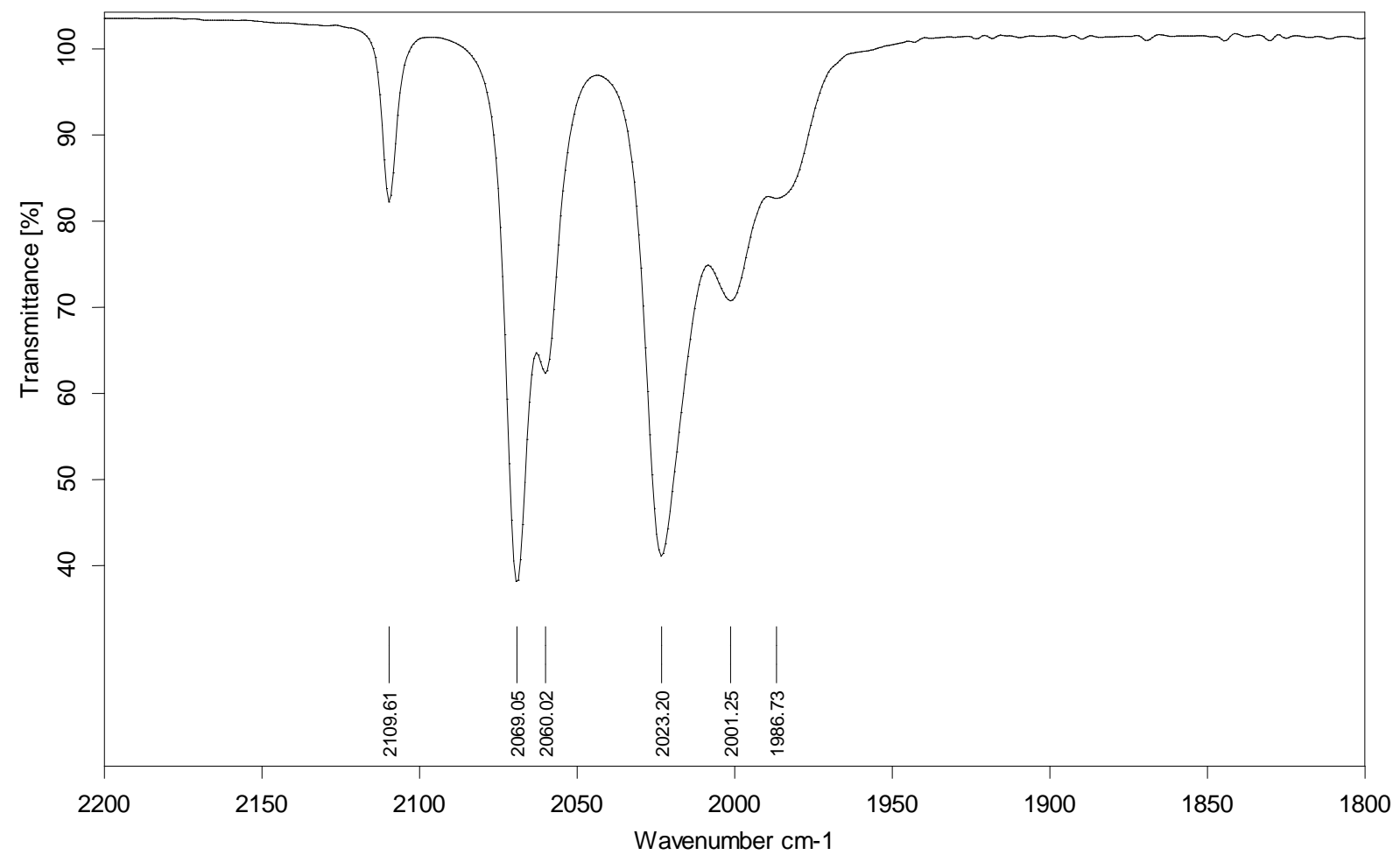

Figure A3.8. IR spectrum $\left(v_{\mathrm{CO}}\right)$ of $\mathbf{2 b}-\mathrm{B}(\mathrm{OH})_{2}$ in DCM.

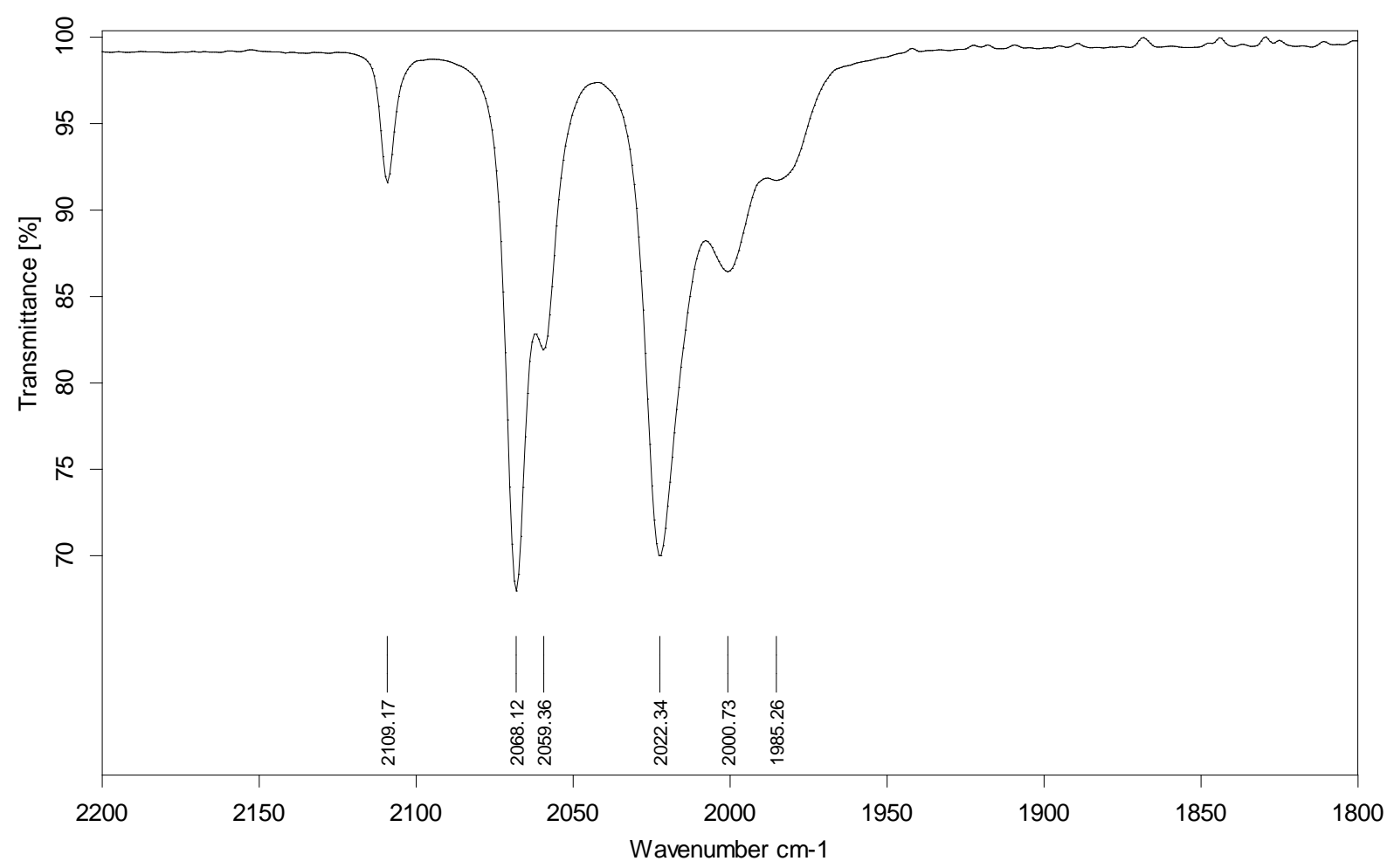

Figure A3.9. IR spectrum $\left(v_{\mathrm{CO}}\right)$ of $\mathbf{2 b}-\mathrm{H}$ in DCM. 


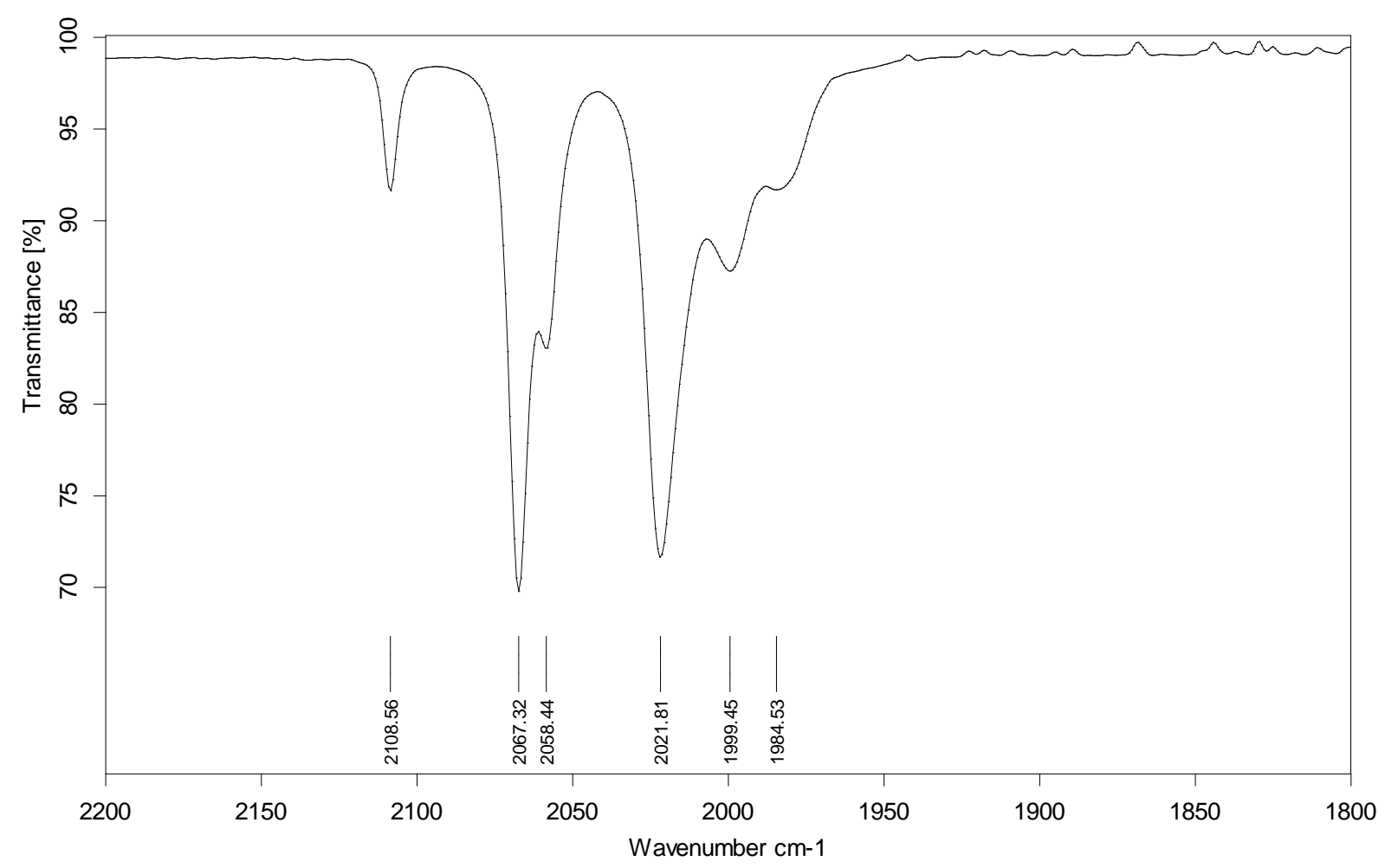

Figure A3.10. IR spectrum ( $\left.v_{\mathrm{CO}}\right)$ of $\mathbf{2 b}-\mathrm{OH}$ in DCM.

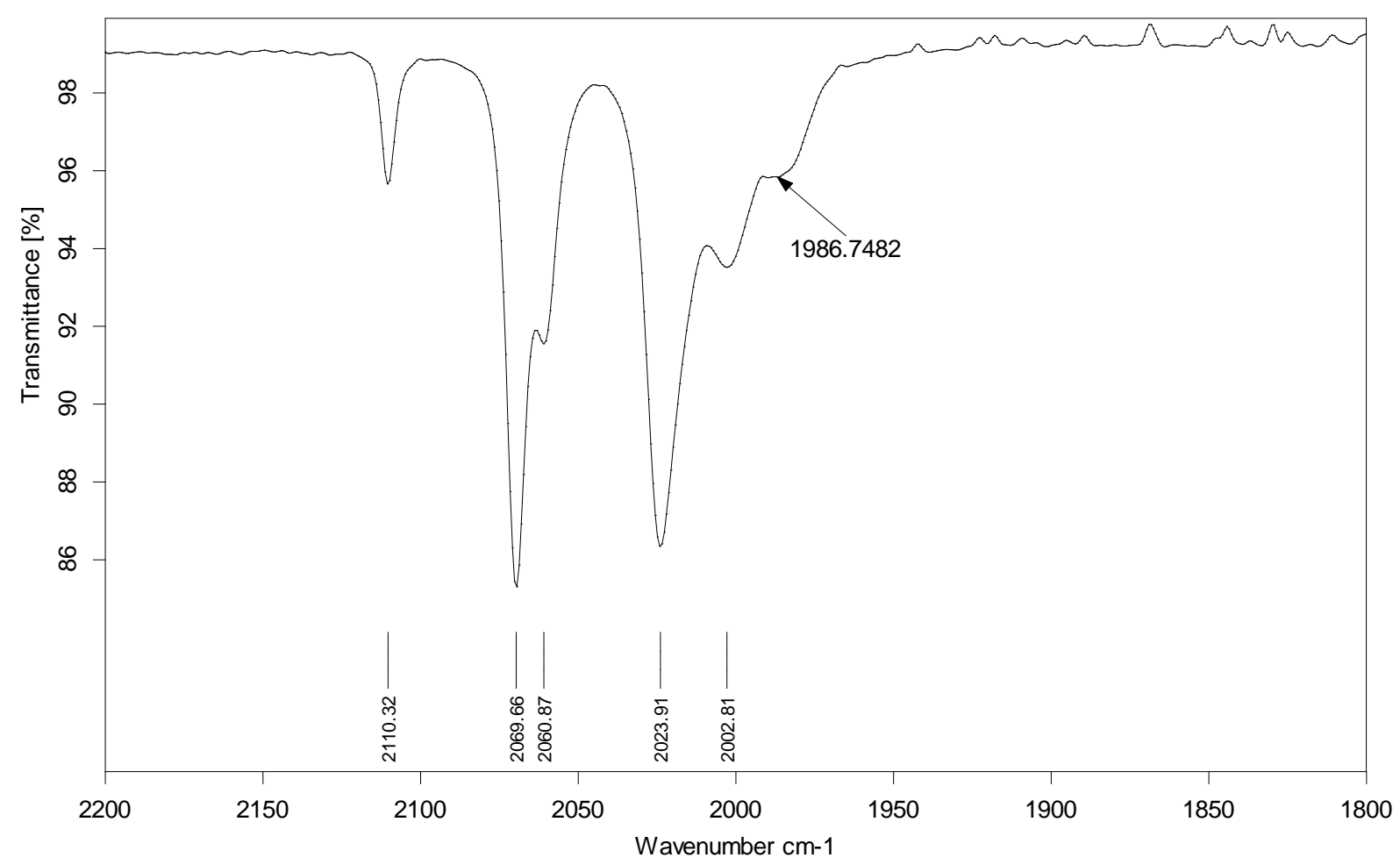

Figure A3.11. IR spectrum ( $\left.v_{\mathrm{CO}}\right)$ of $\mathbf{2 b}-\mathrm{COOH}$ in DCM. 


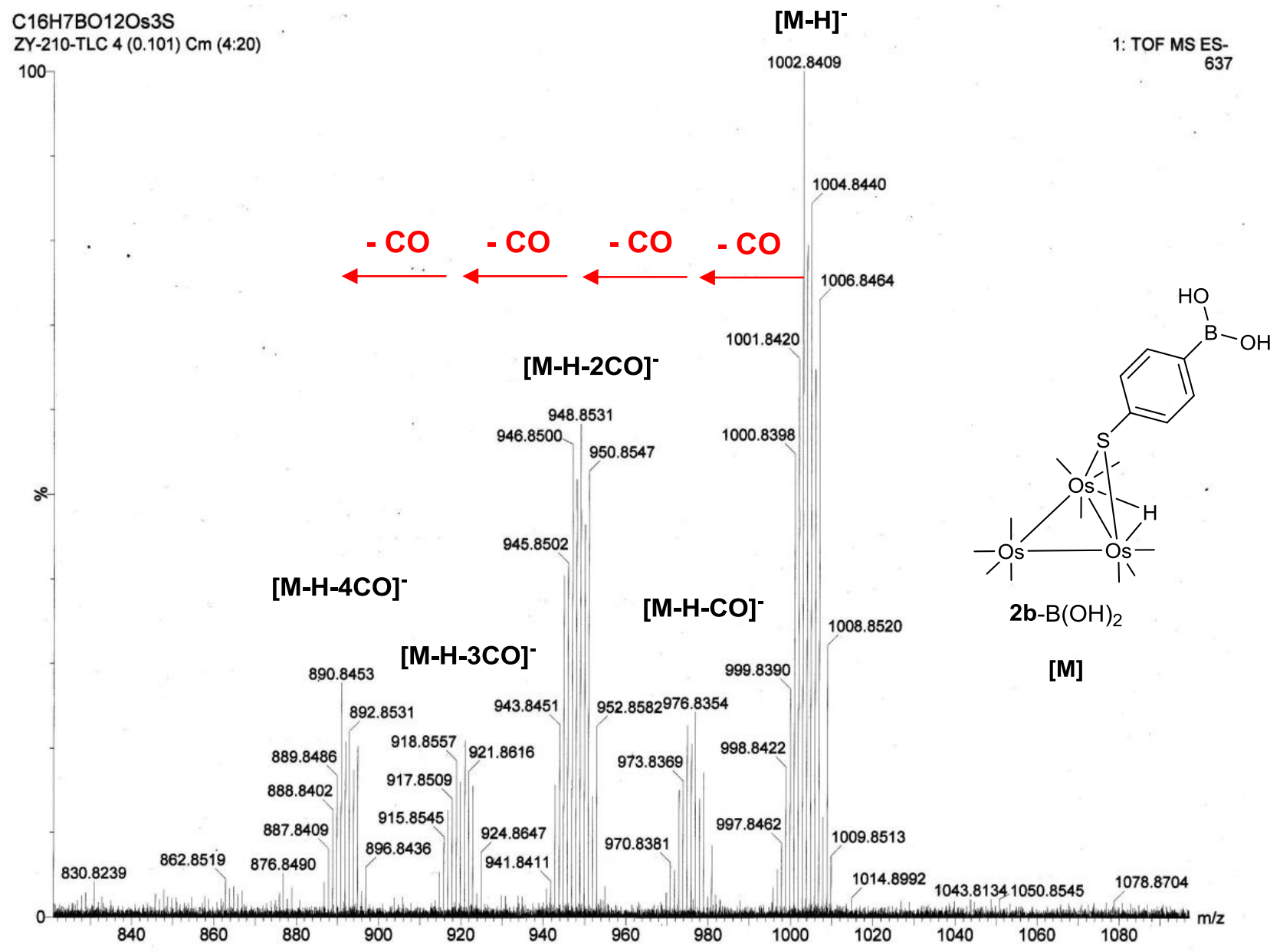

Figure A3.12. ESI-HRMS of $\mathbf{2 b}-\mathrm{B}(\mathrm{OH})_{2}$. 
a

C16H7BO12Os3S

ZY-210-TLC $3(0.083) \mathrm{Cm}(1: 8)$

100

$\sin (-10)$

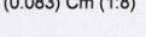

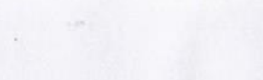

$(1002841$

1002.8414
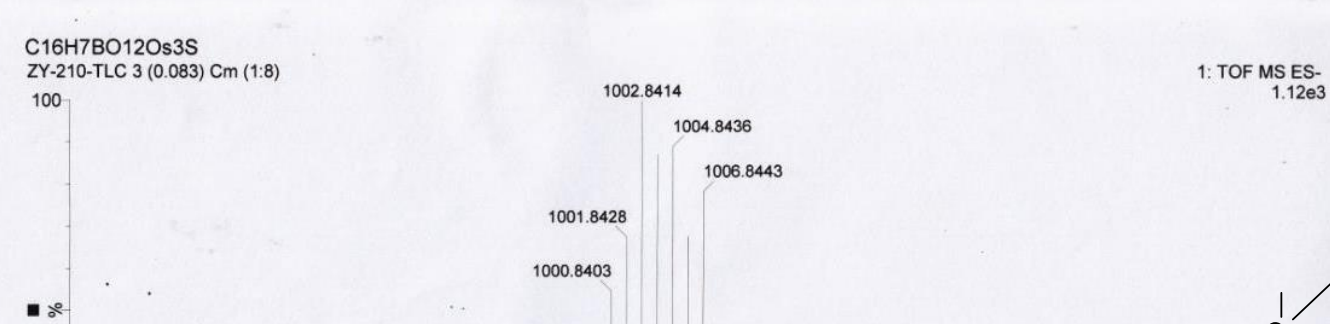

- ะ

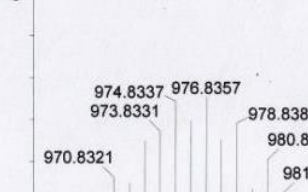

980.8370
981.8441

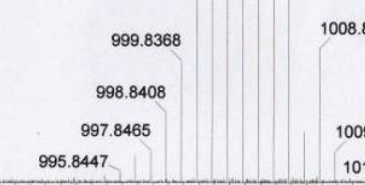

1008.8499

1009.8568

$1.12 \mathrm{e} 3$

Y-210-TLC $(0.045)$ Is $(1.00,1.00)$ C16H6BO12Os3S

ZY-2

100

$10028447^{1004.8469}$

1012.92981014.8992 1023.8687

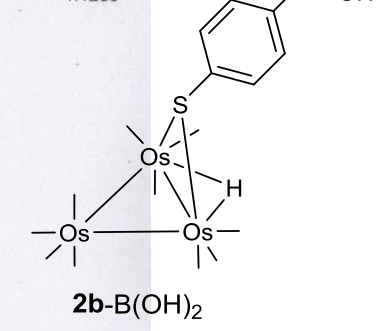

\section{[M]}

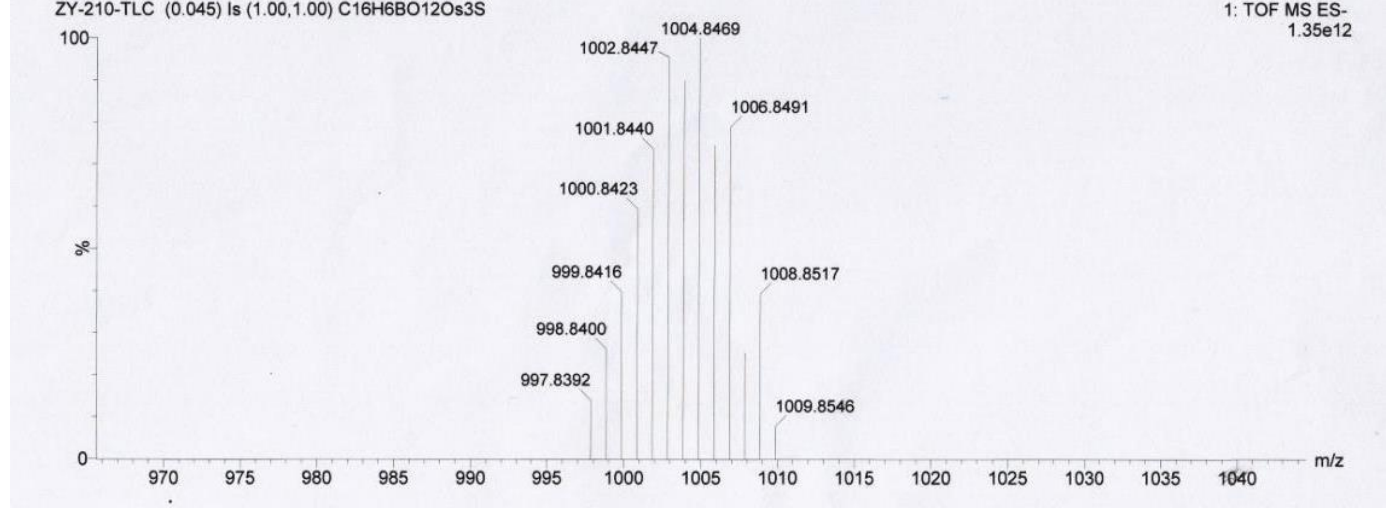

b Elemental Composition Report

Page 1

Single Mass Analysis

Tolerance $=1.5$ PPM I DBE: $\min =-1.5, \max =50.0$

Number of isotope peaks used for $\mathrm{i}-\mathrm{FIT}=3$

Monoisotopic Mass, Even Electron Ions

Monoisotopic Mass, Even Electron lons
12118 formula(e) evaluated with 1 results within limits (up to 50 closest results for each mass)

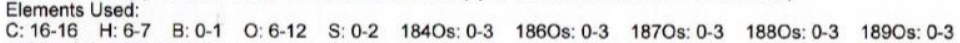

1900s: 0-3 1920s: 0-3

C16H78012Os3S

ZY-210-TLC $3(0.083) \mathrm{Cm}(1: 8)$

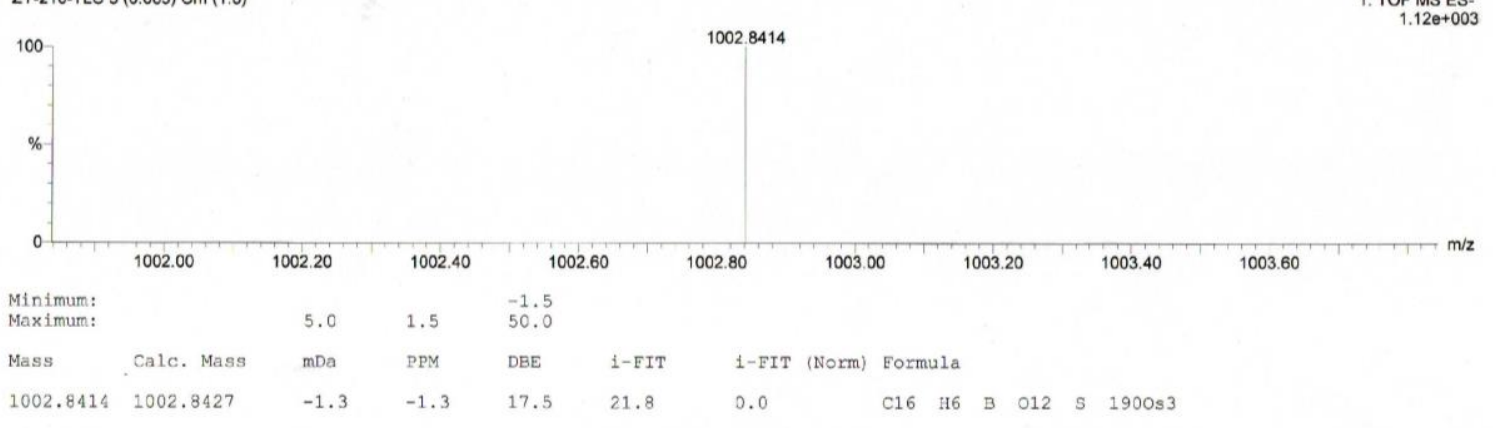

Figure A3.13. a) ESI-HRMS of $\mathbf{2 b}-\mathrm{B}(\mathrm{OH})_{2}$ (top) with calculated isotopic pattern (bottom). b) ESI-HRMS single mass analysis of $\mathbf{2} \mathbf{b}-\mathrm{B}(\mathrm{OH})_{2}$. 
a $\mathrm{C} 16 \mathrm{H} 7 \mathrm{BO} 12 \mathrm{Os} 3 \mathrm{~S}$

ZY-GLUCOSE $3(0.082) \mathrm{Cm}(1: 8)$

100

$\begin{array}{ll}\text { [M] }^{-} & \dot{\mathrm{OH}}_{1166.9033} \\ 1: \text { TOF MS ES- }\end{array}$

$[\mathrm{M}-\mathrm{OH}]^{-}$

1164.9028

$(108000$

- 1.

\begin{tabular}{c|c|}
1147.8970 & \\
1145.8928 & 1163.9022 \\
& 1162.8986
\end{tabular} \mid$\quad 1168.9076$

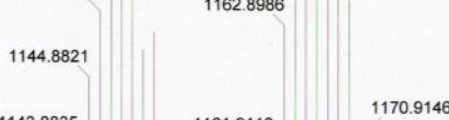

\begin{tabular}{l|l|l|}
\hline 1143.8835 & 1161.9113 & 1170.914 \\
\hline
\end{tabular}

\begin{tabular}{l||||lll}
1141.8800 & 1171.8922 & 1185.9022 & 1196.9075
\end{tabular}

ZY-GLUCOSE $(0.045)$ Is $(1.00,1.00)$ C22H16BO17Os $3 S$ 100

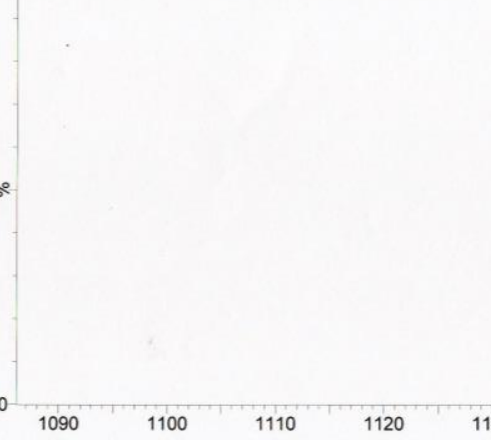

1166.8999

1: TOF MS ES-

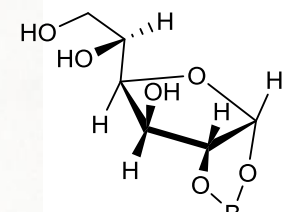

O'

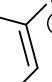

(1.34)

|| 1168.9022

1162.8953

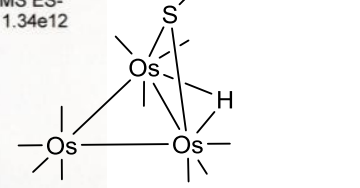

$[\mathrm{M}]^{-}$

\begin{tabular}{l||l|l}
161.8944 & 1170.9047
\end{tabular}

1160.8929

1159.8921

1171.9076

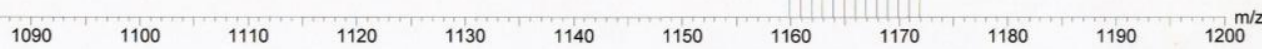

b Elemental Composition Report

Page 1

Single Mass Analysis

Tolerance $=1.0$ PPM / DBE: $\min =-1.5, \max =50.0$

Element prediction: Of

Number of isotope peaks used for $\mathrm{i}-\mathrm{FIT}=3$

Monoisotopic Mass, Even Electron lons

19839 formula(e) evaluated with 1 results within limits (up to 50 closest results for each mass)

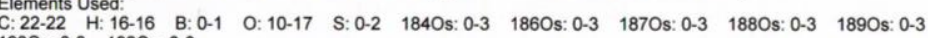

1900s: 0-3 1920s: 0-3

C16H7BO12Os3s

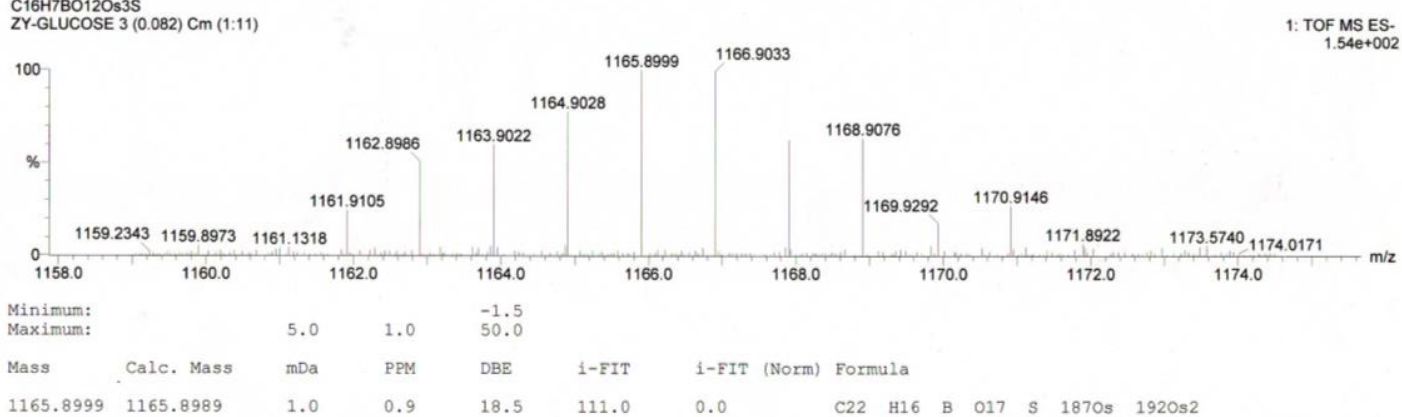

Figure A3.14. a) ESI-HRMS of glucose adduct of $\mathbf{2 b}-\mathrm{B}(\mathrm{OH})_{2}$ (top) with calculated isotopic pattern (bottom). b) ESI-HRMS single mass analysis of the adduct. 
$\mathrm{C} 16 \mathrm{H} 6 \mathrm{O} 110 \mathrm{Os} 3 \mathrm{~S}$

$\mathrm{ZY}-\mathrm{OsOH} 4(0.101) \mathrm{Cm}(1: 13)$
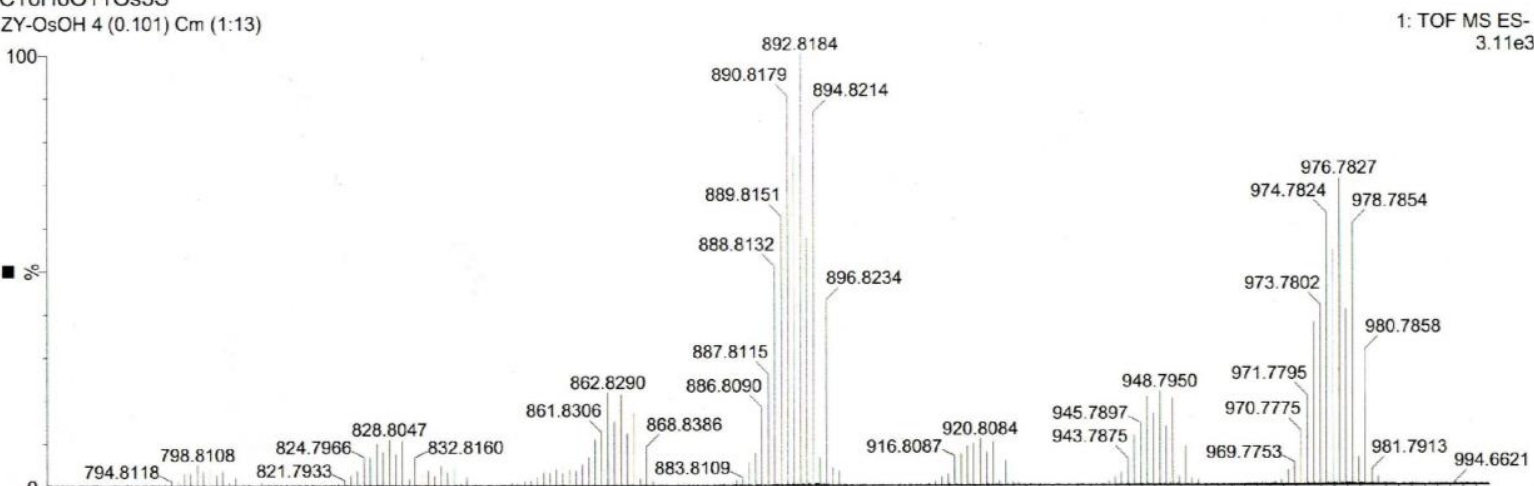

ZY-OsOH $(0.045)$ Is $(1.00,1.00) \mathrm{C} 16 \mathrm{H} 5011 \mathrm{Os} 3 \mathrm{~S}$

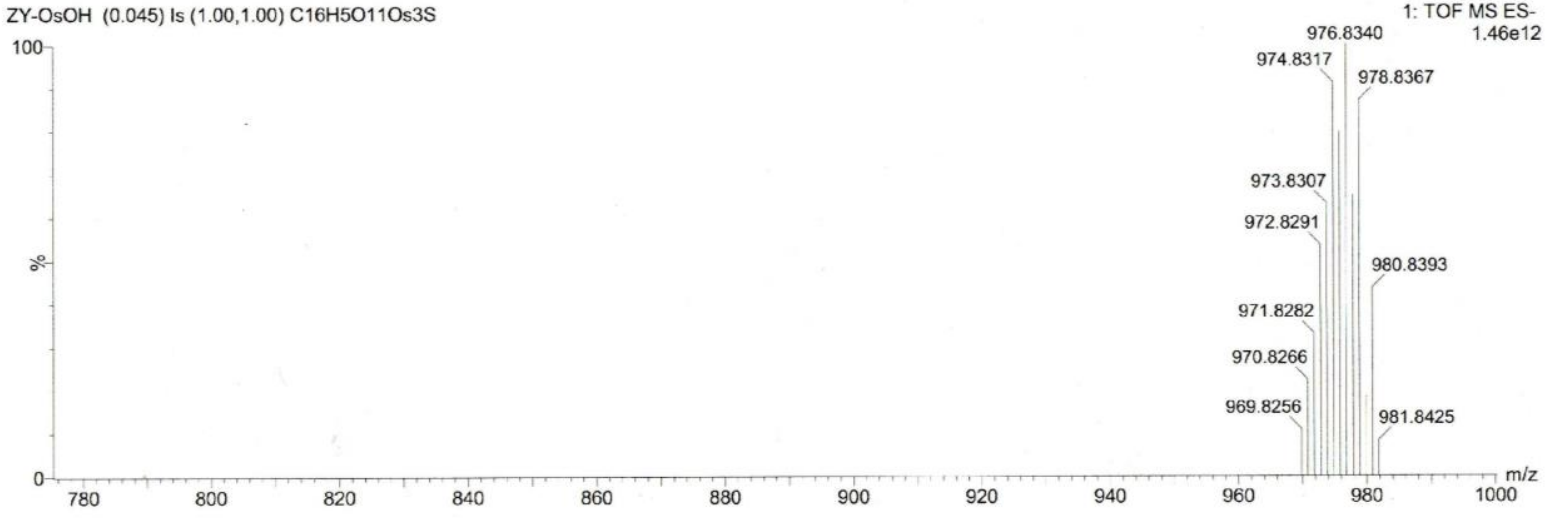

b Elemental Composition Report

Single Mass Analysis

Tolerance $=10.0$ PPM / DBE: $\min =-1.5, \max =50.0$

Element prediction: Off

Number of isotope peaks used for $\mathrm{i}-\mathrm{FIT}=2$

Monoisotopic Mass, Even Electron Ions

255 formula(e) evaluated with 2 results within limits (up to 50 closest results for each mass)

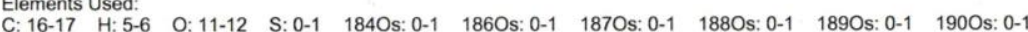

1920s: $0-1$

1920s: 0-1

C16H6O110s3S

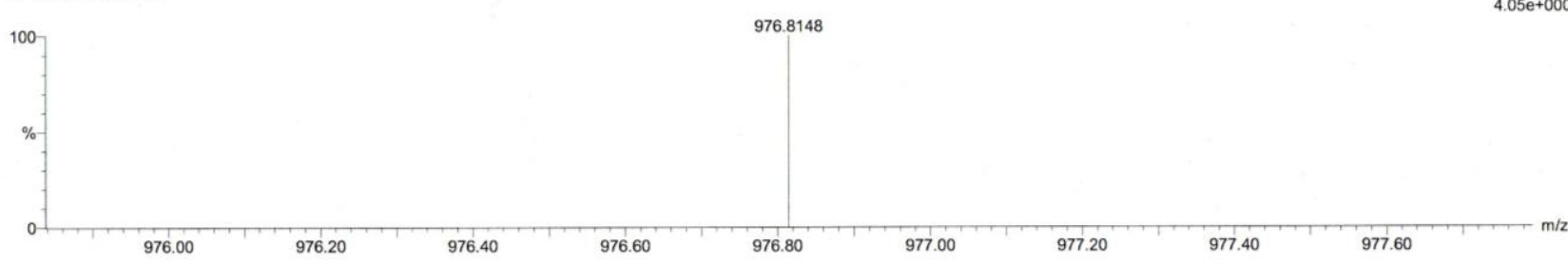

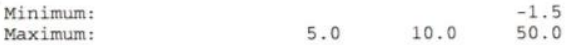

Mass Calc. Mass mDa PPM DBE i-FIT i-FIT (Norm) Formula

\begin{tabular}{llllllllllllll}
\multirow{2}{9}{976.8148} & 976.8201 & -5.3 & -5.4 & 18.5 & 11.6 & 0.7 & C17 & H5 & O11 & S & $1840 \mathrm{~S}$ & $1860 \mathrm{~s}$ & $1900 \mathrm{~s}$ \\
& 976.8217 & -6.9 & -7.1 & 18.5 & 11.6 & 0.7 & C17 & H5 & O11 & S & $1840 \mathrm{~S}$ & $1870 \mathrm{~S}$ & $1890 \mathrm{~s}$
\end{tabular}

Figure A3.15. a) ESI-HRMS of 2b-OH (top) with calculated isotopic pattern (bottom). b) ESI-HRMS single mass analysis of $\mathbf{2} \mathbf{b}-\mathrm{OH}$. 
a $\mathrm{C} 17 \mathrm{H} 6 \mathrm{O} 12 \mathrm{Os} 3 \mathrm{~S}$

ZY-OsCOOH $4(0.101) \mathrm{Cm}(1: 12)$

100

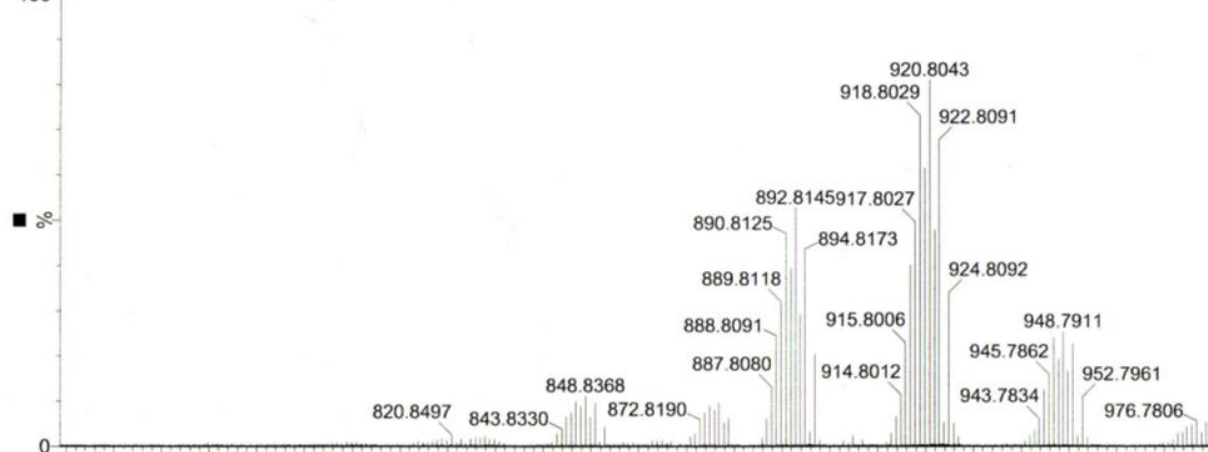

$\mathrm{ZY}-\mathrm{OsCOOH}(0.045)$ Is $(1.00,1.00) \mathrm{C} 17 \mathrm{H} 5012 \mathrm{Os} 3 \mathrm{~S}$

100

b

Elemental Composition Report

Single Mass Analysis

Tolerance $=10.0$ PPM / DBE: $\min =-1.5, \max =50.0$

Element prediction: Of

Number of isotope peaks used for $\mathrm{i}-\mathrm{FIT}=2$

Monoisotopic Mass, Even Electron lons

492 formula(e) evaluated with 1 results within limits (up to 50 closest results for each mass)

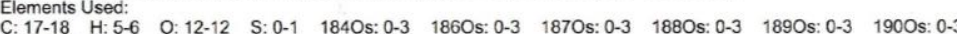

1920s: 0-3

$\mathrm{C} 17 \mathrm{H} 6 \mathrm{O} 12 \mathrm{Os} 3 \mathrm{~S}$
$\mathrm{ZY}-\mathrm{OsCOOH} 12(0.277)$

$100-1005.8364$

$\%$

$\%$

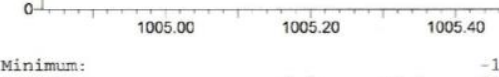

$\begin{array}{lll}\text { Minimum: } & 5.0 \quad 10.0 \quad 50.0 \\ \text { Maximum: } & 5.0\end{array}$

Mass Calc. Mass mDa PFM DBE i-FIT i-FIT (Norm) Formula

$\begin{array}{llllllllllllll}1005.8364 & 1005.8313 & 5.1 & 5.1 & 18.5 & 12.0 & 0.0 & \text { C17 } & \text { H5 } & 012 & \mathrm{~S} & 1890 \mathrm{~s} & 1920 \mathrm{~s} 2\end{array}$

Figure A3.16. a) ESI-HRMS of 2b-COOH (top) with calculated isotopic pattern (bottom). b) ESI-HRMS single mass analysis of $\mathbf{2} \mathbf{b}-\mathrm{COOH}$.

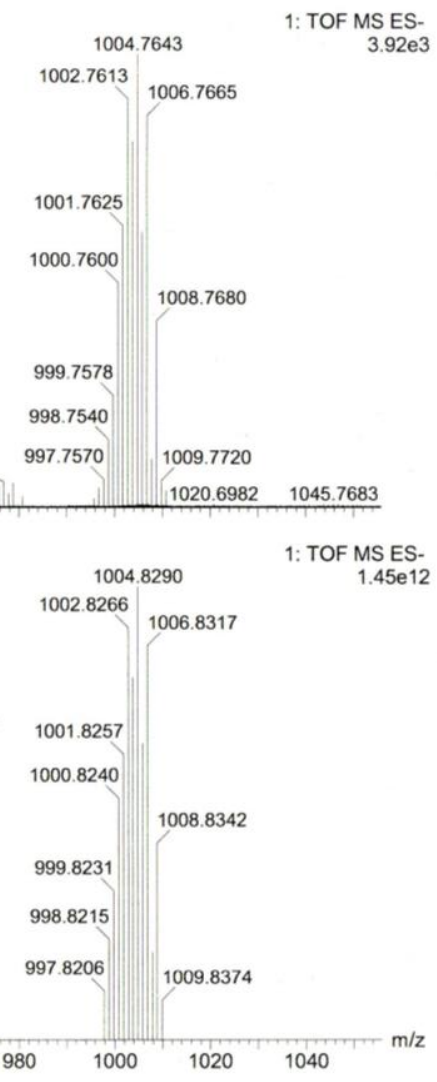

Page 1
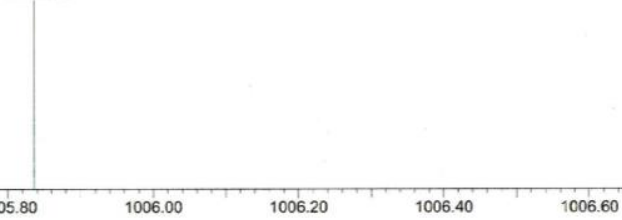
a

Dose-response curve of 1 on MDA-MB-231 cells in serum-free condition

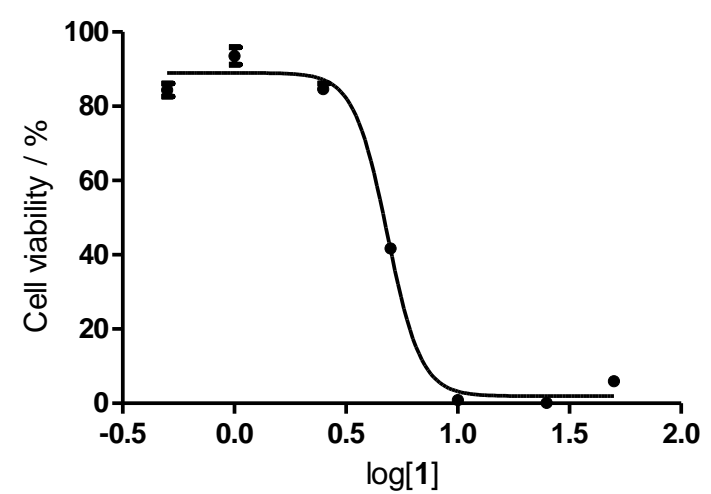

log(inhibitor) vs. response -- Variable slope

\begin{tabular}{|l|c|l|c}
\hline \multicolumn{2}{|c|}{ Best-fit values } & \multicolumn{2}{c}{ 95\% Confidence Intervals } \\
\hline Bottom & 1.938 & Bottom & -1.288 to 5.163 \\
\hline Top & 88.98 & Top & 85.28 to 92.68 \\
\hline LogIC50 & 0.6851 & LogIC50 & 0.6631 to 0.7071 \\
\hline HillSlope & -5.809 & HillSlope & -10.31 to -1.311 \\
\hline IC50 & 4.843 & IC50 & 4.604 to 5.094 \\
\hline Span & 87.04 & Span & 81.75 to 92.34 \\
\hline
\end{tabular}

\begin{tabular}{|l|c|l|c|}
\hline \multicolumn{2}{|c|}{ Std. Error } & \multicolumn{2}{c|}{ Goodness of Fit } \\
\hline Bottom & 1.514 & Degrees of Freedom & 15 \\
\hline Top & 1.737 & $\mathrm{R}^{2}$ & 0.9915 \\
\hline LogIC50 & 0.01032 & Absolute Sum of Squares & 249.9 \\
\hline HillSlope & 2.111 & Sy.x & 4.082 \\
\hline Span & 2.485 & Number of points analyzed & 19 \\
\hline
\end{tabular}

b Dose-response curve of $\mathbf{2 b}-\mathrm{B}(\mathrm{OH})_{2}$ on MDA-MB-231 cells in serum-free condition

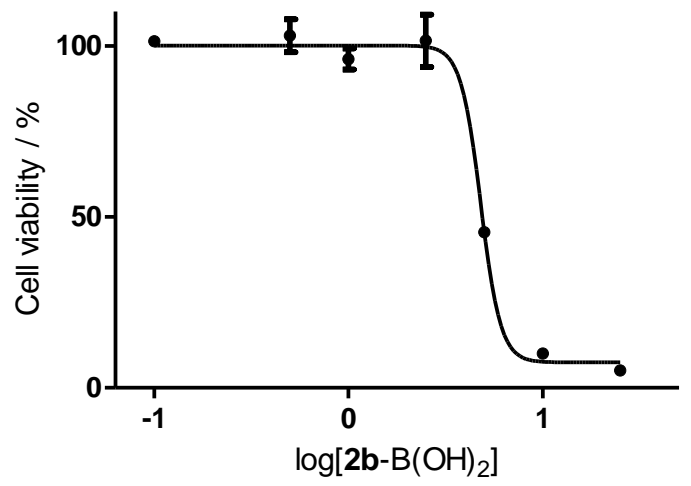

log(inhibitor) vs. response -- Variable slope

\begin{tabular}{|l|c|l|c}
\hline \multicolumn{3}{|c|}{ Best-fit values } & \multicolumn{2}{c}{ 95\% Confidence Intervals } \\
\hline Bottom & 7.438 & Bottom & 2.647 to 12.23 \\
\hline Top & 100.1 & Top & 96.12 to 104.1 \\
\hline LogIC50 & 0.6805 & LogIC50 & 0.6172 to 0.7437 \\
\hline HillSlope & -8.377 & HillSlope & -36.64 to 19.89 \\
\hline IC50 & 4.792 & IC50 & 4.142 to 5.543 \\
\hline Span & 92.65 & Span & 85.93 to 99.37 \\
\hline \multicolumn{3}{|c}{ Goodness of Fit } \\
\hline \multicolumn{3}{|c|}{ Std. Error } & \multicolumn{2}{c}{14} \\
\hline Bottom & 2.233 & Degrees of Freedom & 0.989 \\
\hline Top & 1.85 & $R^{2}$ & 349.4 \\
\hline LogIC50 & 0.02949 & Absolute Sum of Squares & 4.996 \\
\hline HillSlope & 13.18 & Sy.x & 18 \\
\hline Span & 3.133 & Number of points analyzed & \\
\hline
\end{tabular}

C

Dose-response curve of $\mathbf{2} \mathbf{b}-\mathrm{OH}$ on MDA-MB-231 cells in serum-free condition

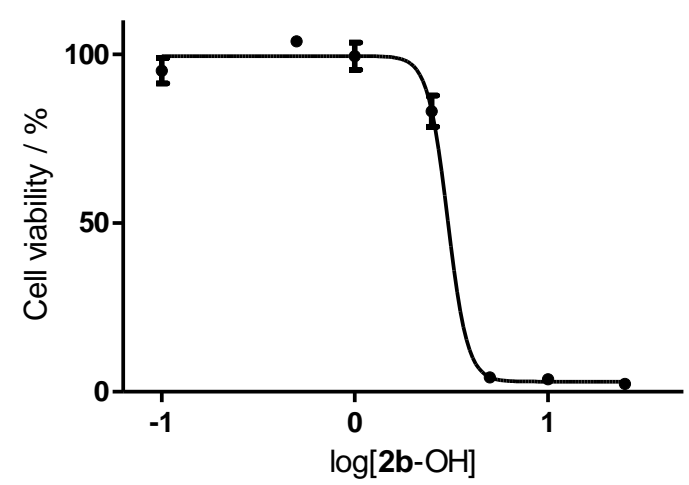

\begin{tabular}{|c|c|c|c|}
\hline \multicolumn{4}{|c|}{ log(inhibitor) vs. response -- Variable slope } \\
\hline \multicolumn{2}{|c|}{ Best-fit values } & \multicolumn{2}{|c|}{ 95\% Confidence Intervals } \\
\hline Bottom & 3.012 & Bottom & -1.974 to 7.997 \\
\hline Top & 99.44 & Top & 95.68 to 103.2 \\
\hline LogIC50 & 0.4793 & LogIC50 & 0.3933 to 0.5654 \\
\hline HillSlope & -8.495 & HillSlope & -17.02 to $0.0319 ?$ \\
\hline IC50 & 3.015 & IC50 & 2.473 to 3.676 \\
\hline Span & 96.43 & Span & 90.17 to 102.7 \\
\hline \multicolumn{2}{|c|}{ Std. Error } & \multicolumn{2}{|c|}{ Goodness of Fit } \\
\hline Bottom & 2.288 & Degrees of Freedom & 12 \\
\hline Top & 1.725 & $\mathrm{R}^{2}$ & 0.9927 \\
\hline LogIC50 & 0.03949 & Absolute Sum of Squares & 249.8 \\
\hline HillSlope & 3.913 & Sy.x & 4.563 \\
\hline Span & 2.872 & Number of points analyzed & 16 \\
\hline
\end{tabular}

Figure A3.17. Dose-response curves of a) 1, b) 2b-B $(\mathrm{OH})_{2}$, c) 2b-OH on MDA-MB-231 cells, after $24 \mathrm{~h}$, in serum-free condition. 
d

Dose-response curve of $\mathbf{2 b}-\mathrm{COOH}$ on MDA-MB-231 cells in serum-free condition

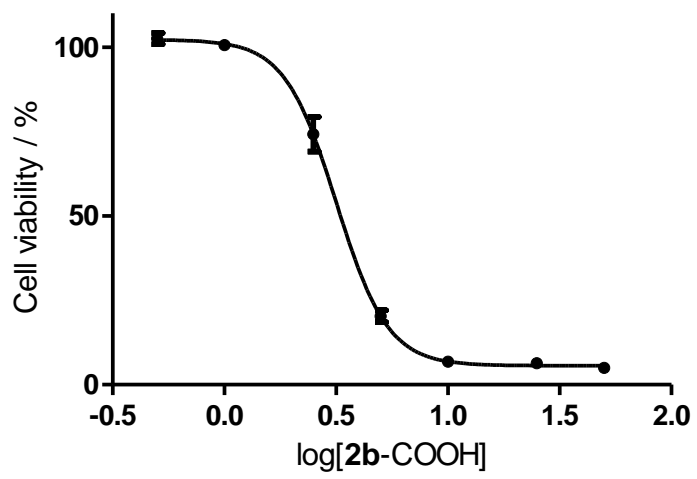

log(inhibitor) vs. response -- Variable slope

\begin{tabular}{|l|c|l|c}
\hline \multicolumn{2}{|c|}{ Best-fit values } & \multicolumn{2}{c}{ 95\% Confidence Intervals } \\
\hline Bottom & 5.63 & Bottom & 3.738 to 7.522 \\
\hline Top & 102.2 & Top & 99.89 to 104.5 \\
\hline LogIC50 & 0.5009 & LogIC50 & 0.4804 to 0.5214 \\
\hline HillSlope & -3.764 & HillSlope & -4.259 to -3.269 \\
\hline IC50 & 3.169 & IC50 & 3.023 to 3.322 \\
\hline Span & 96.59 & Span & 93.50 to 99.68 \\
\hline
\end{tabular}

\begin{tabular}{|l|c|l|c|}
\hline \multicolumn{2}{|c|}{ Std. Error } & \multicolumn{2}{c}{ Goodness of Fit } \\
\hline Bottom & 0.8924 & Degrees of Freedom & 16 \\
\hline Top & 1.097 & $\mathrm{R}^{2}$ & 0.9972 \\
\hline LoglC50 & 0.00966 & Absolute Sum of Squares & 101.2 \\
\hline HillSlope & 0.2334 & Sy.x & 2.515 \\
\hline Span & 1.458 & Number of points analyzed & 20 \\
\hline
\end{tabular}

Figure A3.17 (cont'd). Dose-response curve of d) 2b-COOH on MDA-MB-231 cells, after $24 \mathrm{~h}$, in serum-free condition. 
Dose-response curve of 1 on MCF- 7 cells in serum-free condition

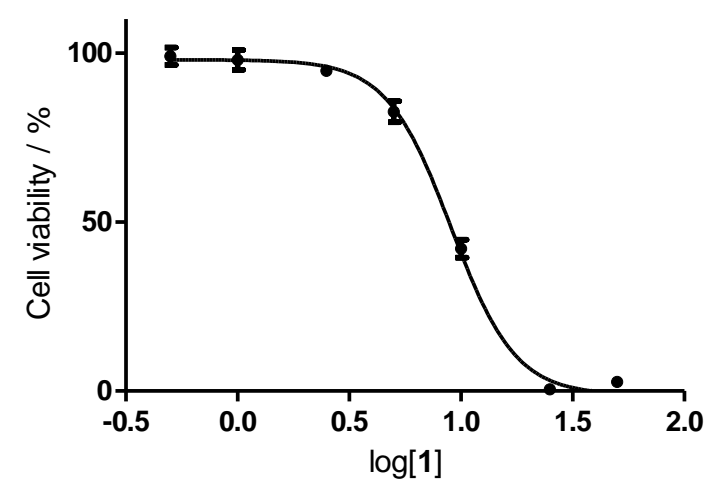

b

Dose-response curve of $\mathbf{2 b}-\mathrm{B}(\mathrm{OH})_{2}$ on MCF-7 cells in serum-free condition

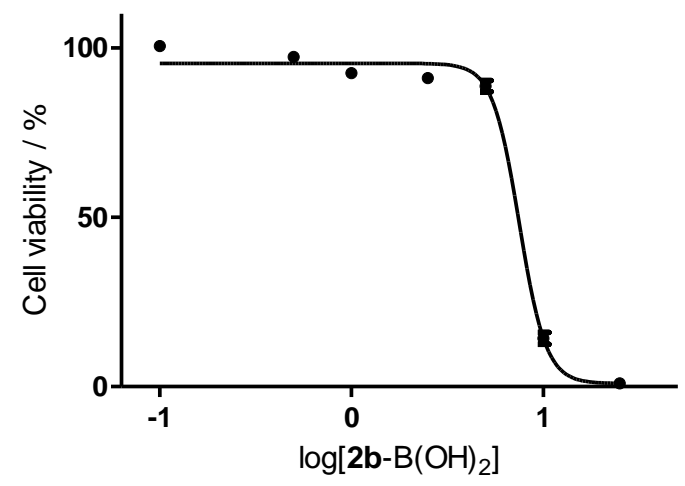

C

Dose-response curve of $\mathbf{2 b - O H}$ on MCF- 7 cells in serum-free condition

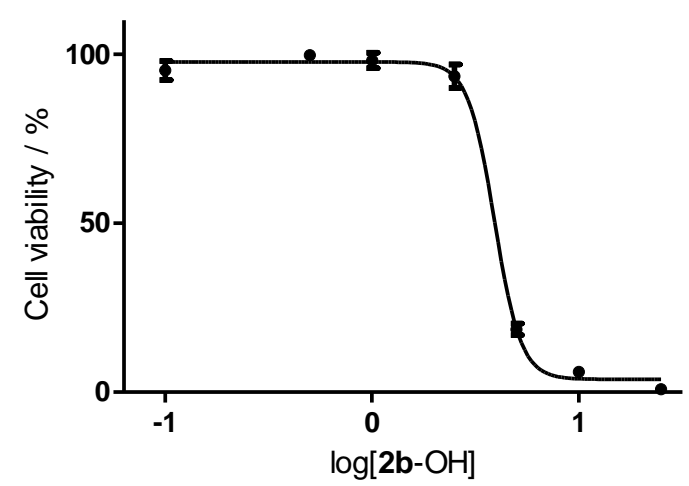

log(inhibitor) vs. response -- Variable slope

\begin{tabular}{|l|c|l|c|}
\hline \multicolumn{2}{|c|}{ Best-fit values } & \multicolumn{2}{c|}{ 95\% Confidence Intervals } \\
\hline Bottom & -1.222 & Bottom & -6.091 to 3.647 \\
\hline Top & 97.99 & Top & 94.89 to 101.1 \\
\hline LogIC50 & 0.9558 & LogIC50 & 0.9183 to 0.9933 \\
\hline HillSlope & -3.016 & HillSlope & -3.800 to -2.233 \\
\hline IC50 & 9.033 & IC50 & 8.286 to 9.847 \\
\hline Span & 99.21 & Span & 92.98 to 105.4 \\
\hline \multicolumn{3}{|c|}{ Goodness of Fit } \\
\hline \multicolumn{3}{|c|}{ Std. Error } & \multicolumn{2}{|c|}{13} \\
\hline Bottom & 2.254 & Degrees of Freedom & 0.9939 \\
\hline Top & 1.438 & R $^{2}$ & 184.2 \\
\hline LogIC50 & 0.01735 & Absolute Sum of Squares & 3.765 \\
\hline HillSlope & 0.3627 & Sy.x & 17 \\
\hline Span & 2.884 & Number of points analyzed & \\
\hline
\end{tabular}

\begin{tabular}{|l|c|l|c|}
\hline \multicolumn{3}{|c|}{ log(inhibitor) vs. response -- Variable slope } \\
\hline \multicolumn{1}{|c|}{ Best-fit values } & \multicolumn{2}{c|}{ 95\% Confidence Intervals } \\
\hline Bottom & 0.9207 & Bottom & -3.699 to 5.540 \\
\hline Top & 95.4 & Top & 93.09 to 97.70 \\
\hline LogIC50 & 0.8752 & LoglC50 & 0.8379 to 0.9124 \\
\hline HillSlope & -6.264 & HillSlope & -7.850 to -4.678 \\
\hline IC50 & 7.502 & IC50 & 6.886 to 8.174 \\
\hline Span & 94.48 & Span & 89.30 to 99.66 \\
\hline \multicolumn{3}{|c|}{ Goodness of Fit } \\
\hline \multicolumn{3}{|c|}{ Std. Error } & \multicolumn{3}{c|}{15} \\
\hline Bottom & 2.168 & Degrees of Freedom & 0.9929 \\
\hline Top & 1.082 & R ${ }^{2}$ & 207.8 \\
\hline LogIC50 & 0.01748 & Absolute Sum of Squares & 3.722 \\
\hline HillSlope & 0.7443 & Sy.x & 19 \\
\hline Span & 2.43 & Number of points analyzed & \\
\hline
\end{tabular}

\begin{tabular}{|l|c|l|c|}
\hline \multicolumn{3}{|c|}{ log(inhibitor) vs. response -- Variable slope } \\
\hline \multicolumn{1}{|c|}{ Best-fit values } & \multicolumn{2}{c|}{ 95\% Confidence Intervals } \\
\hline Bottom & 3.81 & Bottom & 0.04107 to 7.578 \\
\hline Top & 97.69 & Top & 94.91 to 100.5 \\
\hline LogIC50 & 0.5919 & LogIC50 & 0.5554 to 0.6283 \\
\hline HillSlope & -6.79 & HillSlope & -8.802 to -4.779 \\
\hline IC50 & 3.907 & IC50 & 3.593 to 4.249 \\
\hline Span & 93.88 & Span & 89.16 to 98.59 \\
\hline \multicolumn{3}{|c|}{ Goodness of Fit } \\
\hline \multicolumn{2}{|c|}{ Std. Error } & \multicolumn{2}{c|}{16} \\
\hline Bottom & 1.778 & Degrees of Freedom & 0.9934 \\
\hline Top & 1.309 & R 2 & 246 \\
\hline LogIC50 & 0.01719 & Absolute Sum of Squares & 3.921 \\
\hline HillSlope & 0.9488 & Sy.x & 20 \\
\hline Span & 2.224 & Number of points analyzed & \\
\hline
\end{tabular}

Figure A3.18. Dose-response curves of a) $\mathbf{1}$, b) $\mathbf{2 b}-\mathrm{B}(\mathrm{OH})_{2}$ and c) $\mathbf{2 b}-\mathrm{OH}$ on MCF-7 cells, after $24 \mathrm{~h}$, in serum-free condition. 
a

Dose-response curve of 1 on MCF-10A cells in serum-free condition

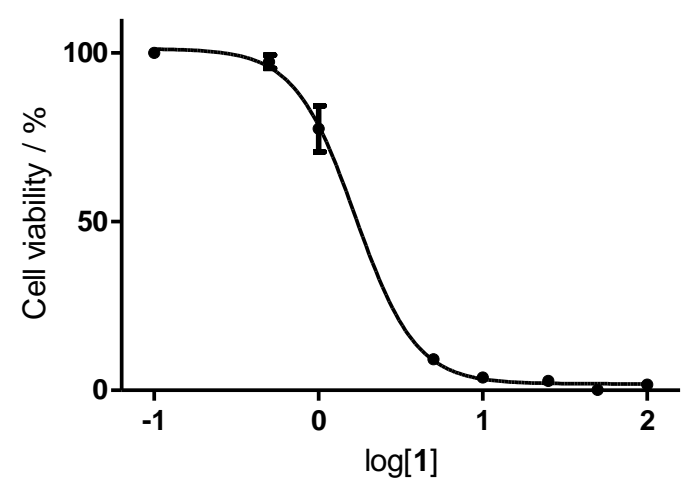

b

Dose-response curve of $\mathbf{2 b}-\mathrm{B}(\mathrm{OH})_{2}$ on MCF-10A cells in serum-free condition

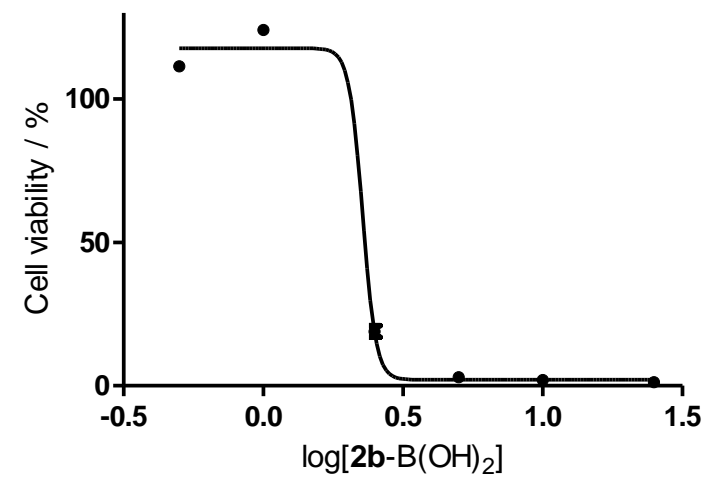

C

Dose-response curve of $\mathbf{2 b}-\mathrm{OH}$ on MCF-10A cells in serum-free condition

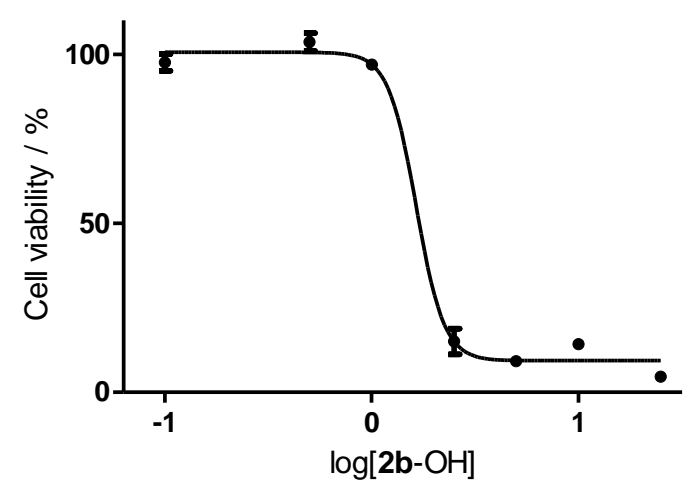

\begin{tabular}{|l|c|l|c|}
\hline \multicolumn{3}{|c|}{ log(inhibitor) vs. response - Variable slope } \\
\hline \multicolumn{1}{|c|}{ Best-fit values } & \multicolumn{2}{c|}{ 95\% Confidence Intervals } \\
\hline Bottom & 1.902 & Bottom & -0.08518 to 3.889 \\
\hline Top & 101.2 & Top & 98.08 to 104.3 \\
\hline LogIC50 & 0.2232 & LoglC50 & 0.1706 to 0.2759 \\
\hline HillSlope & -2.359 & HillSlope & -2.759 to -1.959 \\
\hline IC50 & 1.672 & IC50 & 1.481 to 1.887 \\
\hline Span & 99.28 & Span & 95.41 to 103.2 \\
\hline \multicolumn{3}{|c|}{ Goodness of Fit } \\
\hline \multicolumn{2}{|c|}{ Std. Error } & \multicolumn{3}{c|}{} \\
\hline Bottom & 0.9458 & Degrees of Freedom & 18 \\
\hline Top & 1.477 & R $^{2}$ & 0.9965 \\
\hline LogIC50 & 0.02506 & Absolute Sum of Squares & 146.9 \\
\hline HillSlope & 0.1902 & Sy.x & 2.857 \\
\hline Span & 1.843 & Number of points analyzed & 22 \\
\hline
\end{tabular}

\begin{tabular}{|l|c|l|c|}
\hline \multicolumn{3}{|c|}{ Iog(inhibitor) vs. response -- Variable slope } \\
\hline \multicolumn{1}{|c|}{ Best-fit values } & \multicolumn{2}{c|}{ 95\% Confidence Intervals } \\
\hline Bottom & 2.087 & Bottom & -1.779 to 5.952 \\
\hline Top & 117.7 & Top & 113.0 to 122.4 \\
\hline LogIC50 & $\sim 0.3554$ & LogIC50 & (Very wide) \\
\hline HillSlope & $\sim-18.06$ & HillSlope & (Very wide) \\
\hline IC50 & $\sim 2.267$ & IC50 & (Very wide) \\
\hline Span & 115.6 & Span & 108.8 to 122.4 \\
\hline \multicolumn{3}{|c|}{ Goodness of Fit } \\
\hline \multicolumn{2}{|c|}{ Std. Error } & \multicolumn{3}{c|}{} \\
\hline Bottom & 1.79 & Degrees of Freedom & 13 \\
\hline Top & 2.186 & R ${ }^{2}$ & 0.9944 \\
\hline LogIC50 & $\sim 126.4$ & Absolute Sum of Squares & 280.6 \\
\hline HillSlope & $\sim 53658$ & Sy.x & 4.646 \\
\hline Span & 3.152 & Number of points analyzed & 17 \\
\hline
\end{tabular}

\begin{tabular}{|l|c|l|c|}
\hline \multicolumn{3}{|c|}{ log(inhibitor) vs. response -- Variable slope } \\
\hline \multicolumn{1}{|c|}{ Best-fit values } & \multicolumn{2}{c|}{ 95\% Confidence Intervals } \\
\hline Bottom & 9.357 & Bottom & 6.190 to 12.52 \\
\hline Top & 100.6 & Top & 95.82 to 105.4 \\
\hline LogIC50 & 0.2152 & LoglC50 & 0.1258 to 0.3047 \\
\hline HillSlope & -6.444 & HillSlope & -9.394 to -3.493 \\
\hline IC50 & 1.641 & IC50 & 1.336 to 2.017 \\
\hline Span & 91.23 & Span & 85.48 to 96.97 \\
\hline \multicolumn{3}{|c|}{ Goodness of Fit } \\
\hline \multicolumn{3}{|c|}{ Std. Error } & \multicolumn{3}{c|}{13} \\
\hline Bottom & 1.466 & Degrees of Freedom & 0.992 \\
\hline Top & 2.206 & R $^{2}$ & 247.7 \\
\hline LogIC50 & 0.04142 & Absolute Sum of Squares & 4.365 \\
\hline HillSlope & 1.366 & Sy.x & 17 \\
\hline Span & 2.66 & Number of points analyzed & \\
\hline
\end{tabular}

Figure A3.19. Dose-response curves of a) $\mathbf{1}$, b) $\mathbf{2 b}-\mathrm{B}(\mathrm{OH})_{2}$ and c) $\mathbf{2 b}-\mathrm{OH}$ on $\mathrm{MCF}-10 \mathrm{~A}$ cells, after $24 \mathrm{~h}$, in serum-free condition. 
Dose-response curve of 1 on MDA-MB-231 cells in serum condition

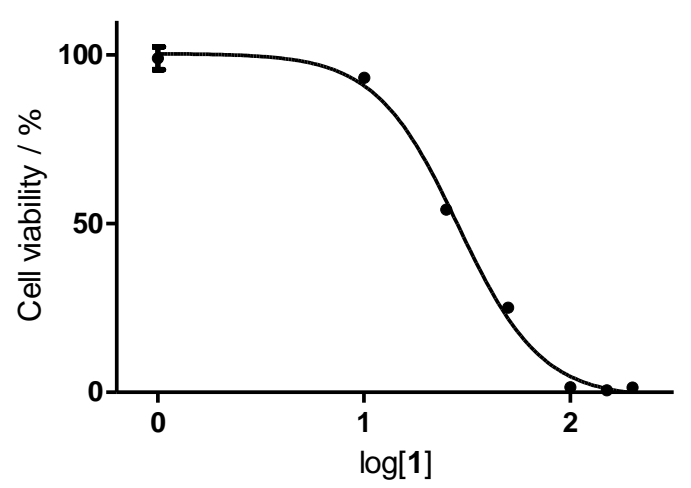

b

Dose-response curve of $\mathbf{2 b}-\mathrm{B}(\mathrm{OH})_{2}$ on MDA-MB-231 cells in serum condition

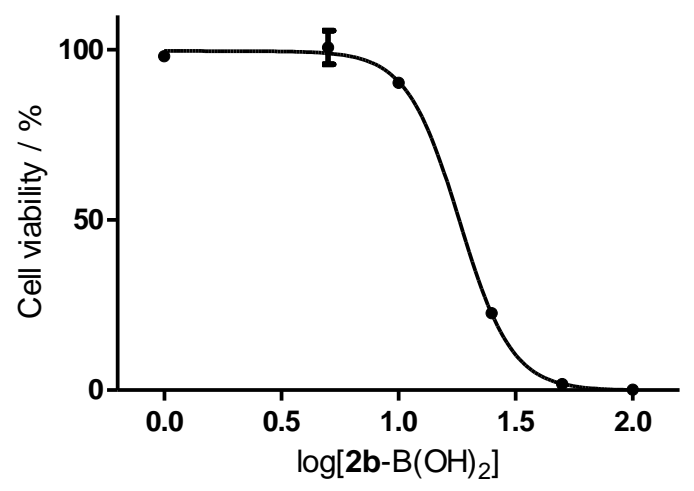

C

Dose-reponse curve of $\mathbf{2 b}$-OH on MDA-MB-231 cells in serum condition

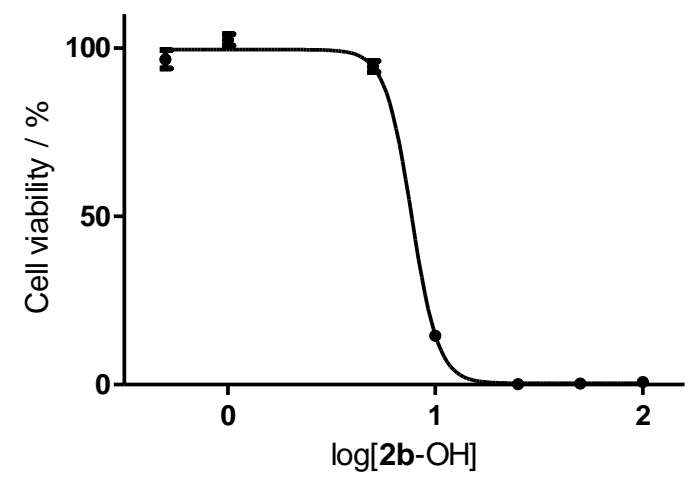

\begin{tabular}{|l|c|l|c|}
\hline \multicolumn{3}{|c|}{ log(inhibitor) vs. response - Variable slope } \\
\hline \multicolumn{1}{|c|}{ Best-fit values } & \multicolumn{2}{c|}{ 95\% Confidence Intervals } \\
\hline Bottom & 0.571 & Bottom & -1.963 to 3.105 \\
\hline Top & 104.7 & Top & 102.1 to 107.3 \\
\hline LogIC50 & 1.475 & LogIC50 & 1.451 to 1.499 \\
\hline HillSlope & -2.744 & HillSlope & -3.112 to -2.377 \\
\hline IC50 & 29.84 & IC50 & 28.25 to 31.53 \\
\hline Span & 104.2 & Span & 100.2 to 108.1 \\
\hline \multicolumn{3}{|c|}{ Goodness of Fit } \\
\hline \multicolumn{2}{|c|}{ Std. Error } & \multicolumn{2}{|c|}{14} \\
\hline Bottom & 1.181 & Degrees of Freedom & 0.9976 \\
\hline Top & 1.215 & $R^{2}$ & 85.06 \\
\hline LogIC50 & 0.01113 & Absolute Sum of Squares & 2.465 \\
\hline HillSlope & 0.1714 & Sy.x & 18 \\
\hline Span & 1.841 & Number of points analyzed & \\
\hline
\end{tabular}

\begin{tabular}{|l|c|l|c|}
\hline \multicolumn{3}{|c|}{ log(inhibitor) vs. response - Variable slope } \\
\hline \multicolumn{1}{|c|}{ Best-fit values } & \multicolumn{2}{c|}{ 95\% Confidence Intervals } \\
\hline Bottom & -0.0985 & Bottom & -3.830 to 3.633 \\
\hline Top & 99.56 & Top & 95.98 to 103.1 \\
\hline LoglC50 & 1.26 & LoglC50 & 1.219 to 1.302 \\
\hline HillSlope & -3.87 & HillSlope & -4.841 to -2.900 \\
\hline IC50 & 18.21 & IC50 & 16.57 to 20.02 \\
\hline Span & 99.65 & Span & 94.23 to 105.1 \\
\hline \multicolumn{3}{|c|}{ Goodness of Fit } \\
\hline \multicolumn{3}{|c|}{ Std. Error } & \multicolumn{3}{|c|}{13} \\
\hline Bottom & 1.727 & Degrees of Freedom & 0.9946 \\
\hline Top & 1.656 & $R^{2}$ & 186.3 \\
\hline LogIC50 & 0.01903 & Absolute Sum of Squares & 3.786 \\
\hline HillSlope & 0.4493 & Sy.x & 17 \\
\hline Span & 2.513 & Number of points analyzed & \\
\hline
\end{tabular}

\begin{tabular}{|l|c|l|c|}
\hline \multicolumn{3}{|c|}{ log(inhibitor) vs. response - Variable slope } \\
\hline \multicolumn{1}{|c|}{ Best-fit values } & \multicolumn{2}{c|}{ 95\% Confidence Intervals } \\
\hline Bottom & 0.3788 & Bottom & -2.342 to 3.099 \\
\hline Top & 99.49 & Top & 96.77 to 102.2 \\
\hline LogIC50 & 0.8857 & LogIC50 & 0.8573 to 0.9141 \\
\hline HillSlope & -6.805 & HillSlope & -8.268 to -5.341 \\
\hline IC50 & 7.686 & IC50 & 7.199 to 8.206 \\
\hline Span & 99.11 & Span & 95.26 to 103.0 \\
\hline \multicolumn{3}{|c|}{ Goodness of Fit } \\
\hline \multicolumn{3}{|c|}{ Std. Error } & \multicolumn{3}{|c|}{14} \\
\hline Bottom & 1.268 & Degrees of Freedom & 0.9966 \\
\hline Top & 1.266 & $\mathrm{R}^{2}$ & 134.7 \\
\hline LogIC50 & 0.01325 & Absolute Sum of Squares & 3.101 \\
\hline HillSlope & 0.6822 & Sy.x & 18 \\
\hline Span & 1.795 & Number of points analyzed & \\
\hline
\end{tabular}

Figure A3.20. Dose-response curves of a) 1, b) $\mathbf{2 b}-\mathrm{B}(\mathrm{OH})_{2}$ and c) $\mathbf{2 b}-\mathrm{OH}$ on MDA-MB-231 cells, after $24 \mathrm{~h}$, in serum condition. 
a

Dose-reponse curve of 1 on MCF- 7 cells in serum condition

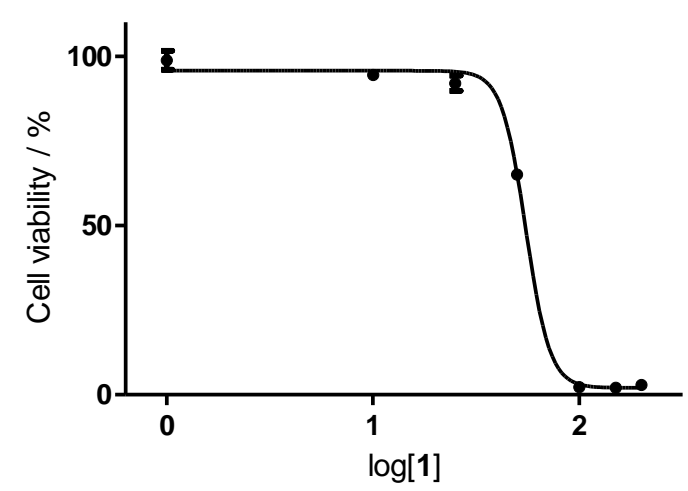

b

Dose-reponse curve of $\mathbf{2 b}-\mathrm{B}(\mathrm{OH})_{2}$ on MCF-7 cells in serum condition

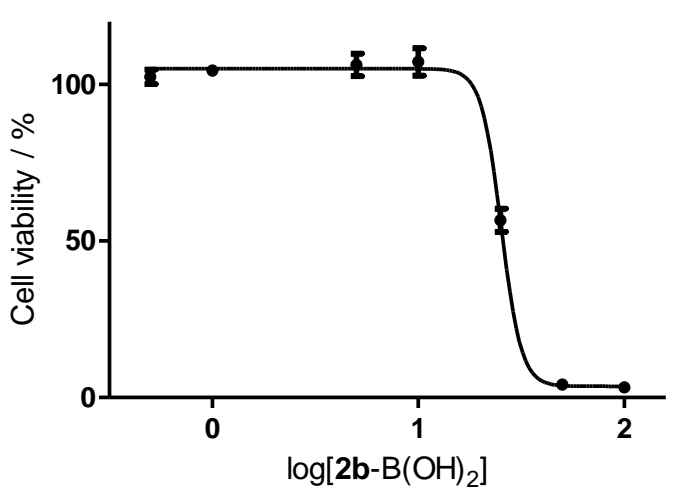

C

Dose-reponse curve of $\mathbf{2 b}-\mathrm{OH}$ on MCF- 7 cells in serum condition

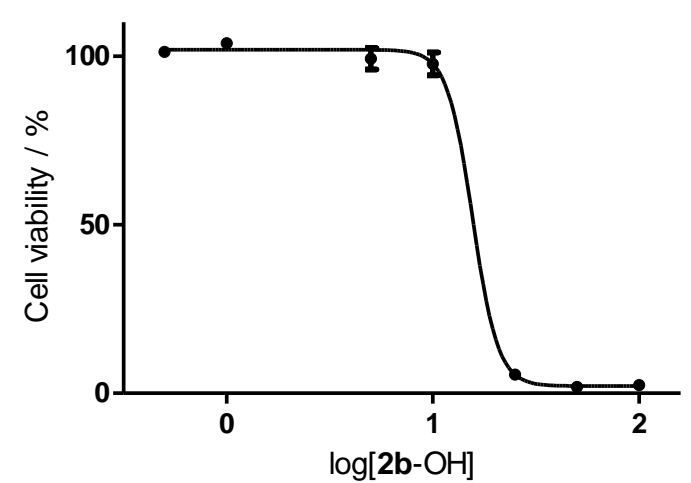

\begin{tabular}{|l|c|l|c|}
\hline \multicolumn{4}{|c|}{ log(inhibitor) vs. response - Variable slope } \\
\hline \multicolumn{1}{|c|}{ Best-fit values } & \multicolumn{2}{c|}{ 95\% Confidence Intervals } \\
\hline Bottom & 2.029 & Bottom & -1.538 to 5.596 \\
\hline Top & 95.71 & Top & 93.06 to 98.36 \\
\hline LoglC50 & 1.741 & LoglC50 & 1.692 to 1.789 \\
\hline HillSlope & -7.475 & HillSlope & -15.67 to 0.7169 \\
\hline IC50 & 55.03 & IC50 & 49.26 to 61.49 \\
\hline Span & 93.68 & Span & 89.04 to 98.32 \\
\hline \multicolumn{3}{|c|}{ Goodness of Fit } \\
\hline \multicolumn{3}{|c|}{ Std. Error } & \multicolumn{2}{|c|}{} \\
\hline Bottom & 1.637 & Degrees of Freedom & 12 \\
\hline Top & 1.216 & $R^{2}$ & 0.9958 \\
\hline LoglC50 & 0.0221 & Absolute Sum of Squares & 120 \\
\hline HillSlope & 3.759 & Sy.x & 3.163 \\
\hline Span & 2.129 & Number of points analyzed & 16 \\
\hline
\end{tabular}

\begin{tabular}{|l|c|l|c|}
\hline \multicolumn{3}{|c|}{ log(inhibitor) vs. response -- Variable slope } \\
\hline \multicolumn{1}{|c|}{ Best-fit values } & \multicolumn{2}{c|}{ 95\% Confidence Intervals } \\
\hline Bottom & 3.597 & Bottom & -1.843 to 9.038 \\
\hline Top & 105 & Top & 102.0 to 108.1 \\
\hline LogIC50 & 1.402 & LoglC50 & 1.372 to 1.433 \\
\hline HillSlope & -8.972 & HillSlope & -58.76 to 40.82 \\
\hline IC50 & 25.26 & IC50 & 23.56 to 27.08 \\
\hline Span & 101.4 & Span & 95.02 to 107.9 \\
\hline \multicolumn{3}{|c|}{ Goodness of Fit } \\
\hline \multicolumn{3}{|c|}{ Std. Error } & \multicolumn{2}{|c|}{15} \\
\hline Bottom & 2.553 & Degrees of Freedom & 0.9924 \\
\hline Top & 1.413 & $R^{2}$ & 295.4 \\
\hline LogIC50 & 0.01415 & Absolute Sum of Squares & 4.438 \\
\hline HillSlope & 23.36 & Sy.x & 19 \\
\hline Span & 3.015 & Number of points analyzed & \\
\hline
\end{tabular}

\begin{tabular}{|l|c|l|c|}
\hline \multicolumn{4}{|c|}{ log(inhibitor) vs. response - Variable slope } \\
\hline \multicolumn{2}{|c|}{ Best-fit values } & \multicolumn{2}{c|}{ 95\% Confidence Intervals } \\
\hline Bottom & 2.139 & Bottom & -0.5311 to 4.810 \\
\hline Top & 101.9 & Top & 99.22 to 104.6 \\
\hline LogIC50 & 1.192 & LoglC50 & 1.137 to 1.247 \\
\hline HillSlope & -7.047 & HillSlope & -8.976 to -5.119 \\
\hline IC50 & 15.56 & IC50 & 13.70 to 17.67 \\
\hline Span & 99.75 & Span & 95.97 to 103.5 \\
\hline \multicolumn{3}{|c|}{ Goodness of Fit } \\
\hline \multicolumn{3}{|c|}{ Std. Error } & \multicolumn{2}{|c|}{14} \\
\hline Bottom & 1.245 & Degrees of Freedom & 0.997 \\
\hline Top & 1.242 & $\mathrm{R}^{2}$ & 128.4 \\
\hline LogIC50 & 0.02574 & Absolute Sum of Squares & 3.028 \\
\hline HillSlope & 0.8991 & Sy.x & 18 \\
\hline Span & 1.763 & Number of points analyzed & \\
\hline
\end{tabular}

Figure A3.21. Dose-response curves of a) $\mathbf{1}$, b) $\mathbf{2} \mathbf{b}-\mathrm{B}(\mathrm{OH})_{2}$ and c) $\mathbf{2 b}-\mathrm{OH}$ on $\mathrm{MCF}-7$ cells, after $24 \mathrm{~h}$, in serum condition. 
a

Dose-response curve of 1 on MCF-10A cells in serum condition

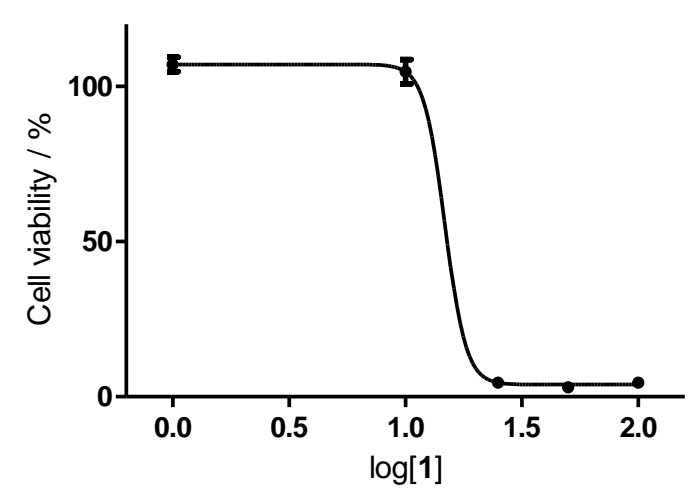

b

Dose-response curve of $\mathbf{2 b}-\mathrm{B}(\mathrm{OH})_{2}$ on MCF-10A cells in serum condition

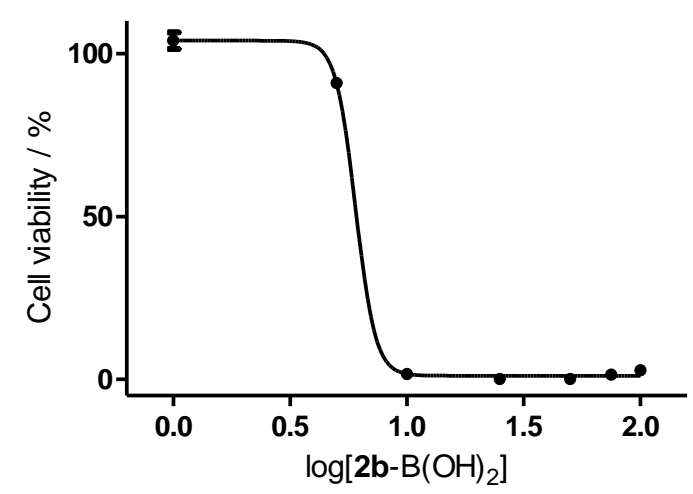

C

Dose-response curve of $\mathbf{2 b}-\mathrm{OH}$ on MCF-10A cells in serum condition

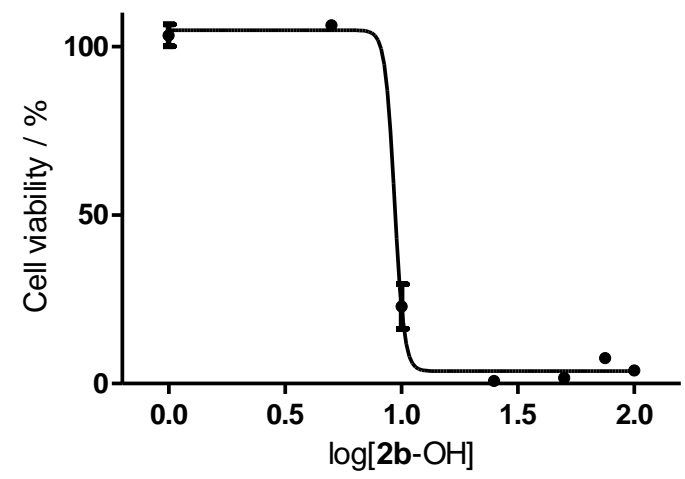

log(inhibitor) vs. response - Variable slope

\begin{tabular}{|l|c|l|c|}
\hline \multicolumn{2}{|c|}{ Best-fit values } & \multicolumn{2}{c|}{ 95\% Confidence Intervals } \\
\hline Bottom & 3.904 & Bottom & 0.3116 to 7.496 \\
\hline Top & 107.1 & Top & 102.5 to 111.7 \\
\hline LogIC50 & 1.17 & LogIC50 & 0.9829 to 1.357 \\
\hline HillSlope & -9.599 & HillSlope & -19.72 to 0.5246 \\
\hline IC50 & 14.79 & IC50 & 9.615 to 22.75 \\
\hline Span & 103.2 & Span & 97.33 to 109.1 \\
\hline
\end{tabular}

Span

\begin{tabular}{|l|c|l|c|}
\hline \multicolumn{2}{|c|}{ Std. Error } & \multicolumn{2}{c|}{ Goodness of Fit } \\
\hline Bottom & 1.612 & Degrees of Freedom & 10 \\
\hline Top & 2.08 & $\mathrm{R}^{2}$ & 0.9964 \\
\hline LogIC50 & 0.08392 & Absolute Sum of Squares & 129.7 \\
\hline HillSlope & 4.544 & Sy.x & 3.602 \\
\hline Span & 2.632 & Number of points analyzed & 10 \\
\hline
\end{tabular}

\begin{tabular}{|l|c|l|c|}
\hline \multicolumn{3}{|c|}{ log(inhibitor) vs. response - Variable slope } \\
\hline \multicolumn{1}{|c|}{ Best-fit values } & \multicolumn{2}{c|}{ 95\% Confidence Intervals } \\
\hline Bottom & 1.123 & Bottom & -0.02876 to 2.276 \\
\hline Top & 104 & Top & 101.1 to 106.8 \\
\hline LogIC50 & 0.7808 & LogIC50 & 0.7182 to 0.8434 \\
\hline HillSlope & -10.28 & HillSlope & -18.02 to -2.537 \\
\hline IC50 & 6.037 & IC50 & 5.226 to 6.972 \\
\hline Span & 102.8 & Span & 99.80 to 105.9 \\
\hline \multicolumn{3}{|c|}{ Goodness of Fit } \\
\hline \multicolumn{3}{|c|}{ Std. Error } & \multicolumn{3}{c|}{14} \\
\hline Bottom & 0.5372 & Degrees of Freedom & 0.9983 \\
\hline Top & 1.316 & $\mathrm{R}^{2}$ & 48.47 \\
\hline LogIC50 & 0.02918 & Absolute Sum of Squares & 1.861 \\
\hline HillSlope & 3.608 & Sy.x & 18 \\
\hline Span & 1.421 & Number of points analyzed & \\
\hline
\end{tabular}

log(inhibitor) vs. response -- Variable slope

\begin{tabular}{|l|c|l|c|}
\hline \multicolumn{2}{|c|}{ Best-fit values } & \multicolumn{2}{c|}{ 95\% Confidence Intervals } \\
\hline Bottom & 3.715 & Bottom & 1.041 to 6.389 \\
\hline Top & 104.8 & Top & 99.68 to 109.9 \\
\hline LogIC50 & $\sim 0.9706$ & LogIC50 & (Very wide) \\
\hline HillSlope & $\sim-21.48$ & HillSlope & (Very wide) \\
\hline IC50 & $\sim 9.346$ & IC50 & (Very wide) \\
\hline Span & 101.1 & Span & 95.31 to 106.9 \\
\hline
\end{tabular}

\begin{tabular}{|l|c|l|c|}
\hline \multicolumn{2}{|c|}{ Std. Error } & \multicolumn{2}{c|}{ Goodness of Fit } \\
\hline Bottom & 1.255 & Degrees of Freedom & 15 \\
\hline Top & 2.403 & $\mathrm{R}^{2}$ & 0.9936 \\
\hline LogIC50 & $\sim 45.45$ & Absolute Sum of Squares & 259.8 \\
\hline HillSlope & $\sim 33249$ & Sy.x & 4.162 \\
\hline Span & 2.711 & Number of points analyzed & 19 \\
\hline
\end{tabular}

Figure A3.22. Dose-response curves of a) $\mathbf{1}$, b) $\mathbf{2 b}-\mathrm{B}(\mathrm{OH})_{2}$ and c) $\mathbf{2 b}-\mathrm{OH}$ on $\mathrm{MCF}-10 \mathrm{~A}$ cells, after $24 \mathrm{~h}$, in serum condition. 


\section{Chapter 4: High Nuclearity Metal Carbonyl Clusters as Near-IR Contrast}

\section{Agents for Photoacoustic In Vivo Imaging}

a) Cluster 22
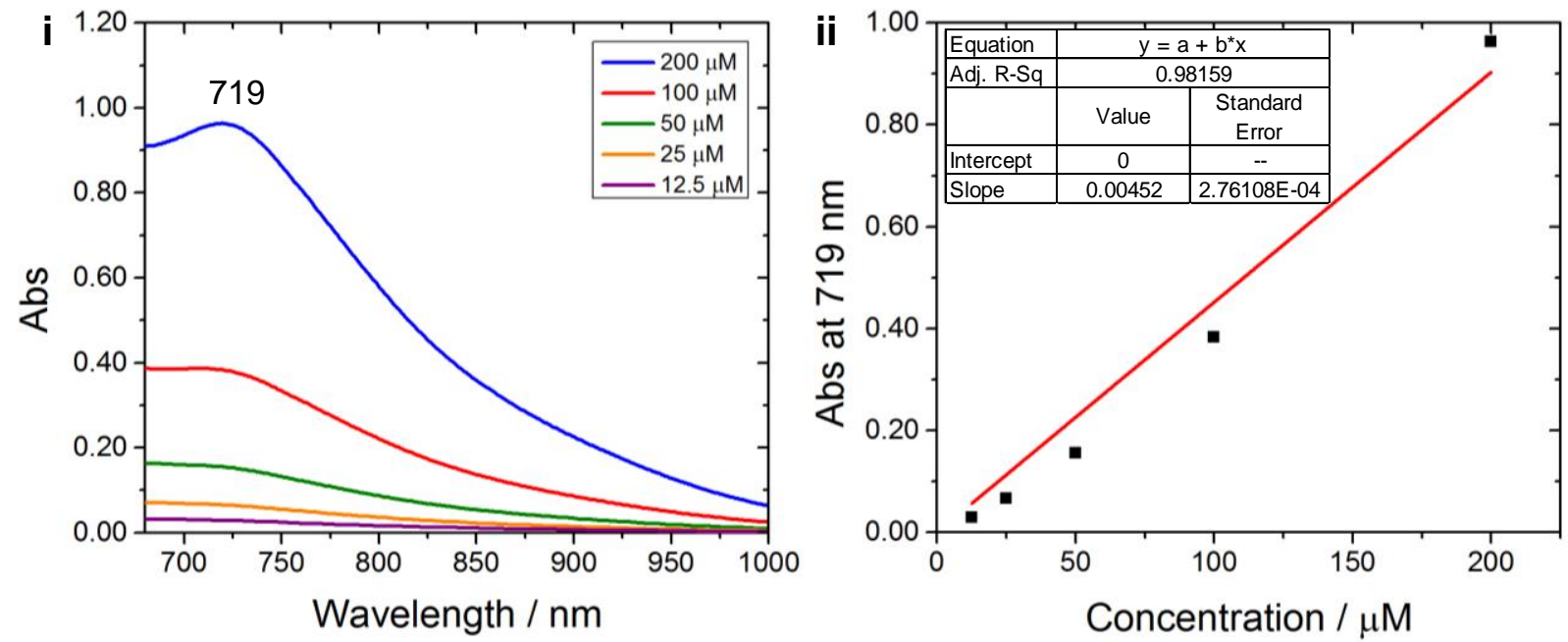

b) Cluster 23
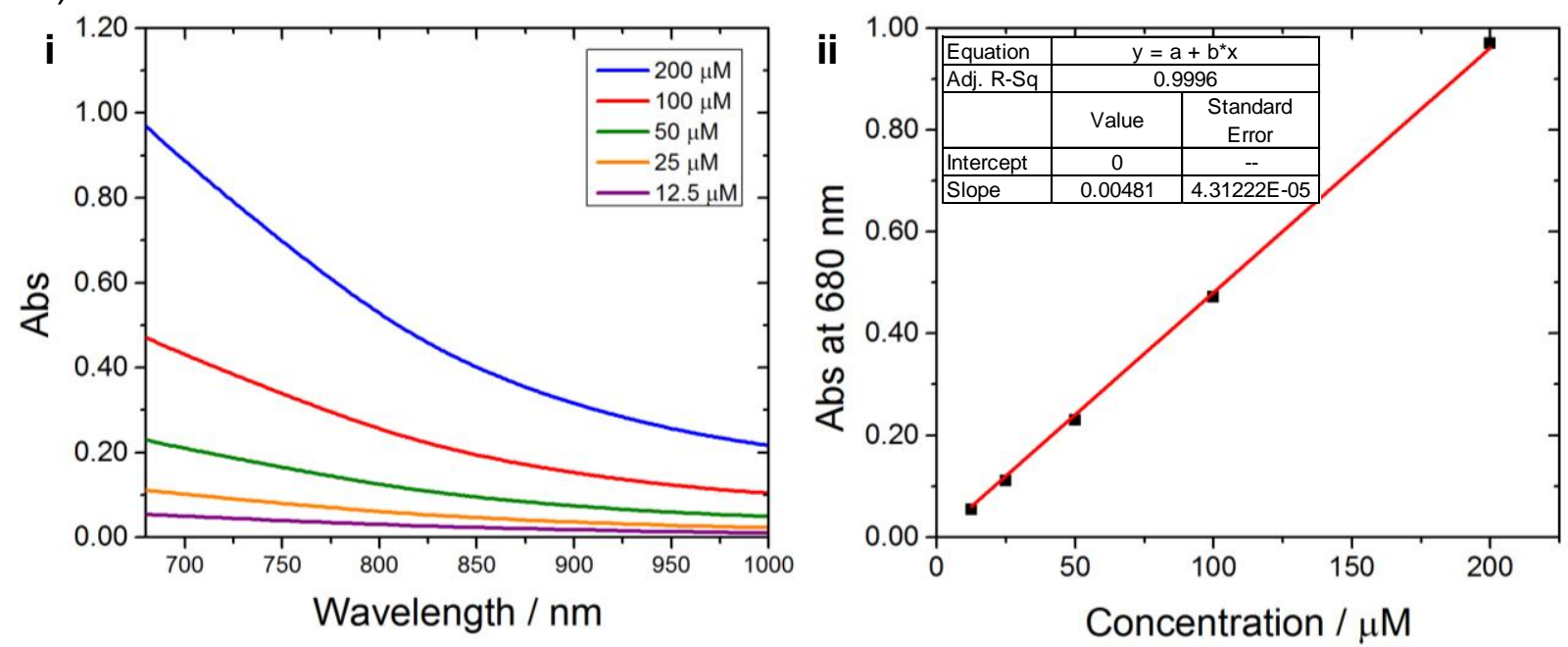

Figure A4.1. i) Optical absorption spectra of various concentrations (in DMSO), ii) plot of optical absorbance (at respective $\lambda_{\max }$ ) as a function of concentrations of cluster a) 22 and b) 23 and. 
c) Cluster $[\mathrm{PPN}]_{2} .24$
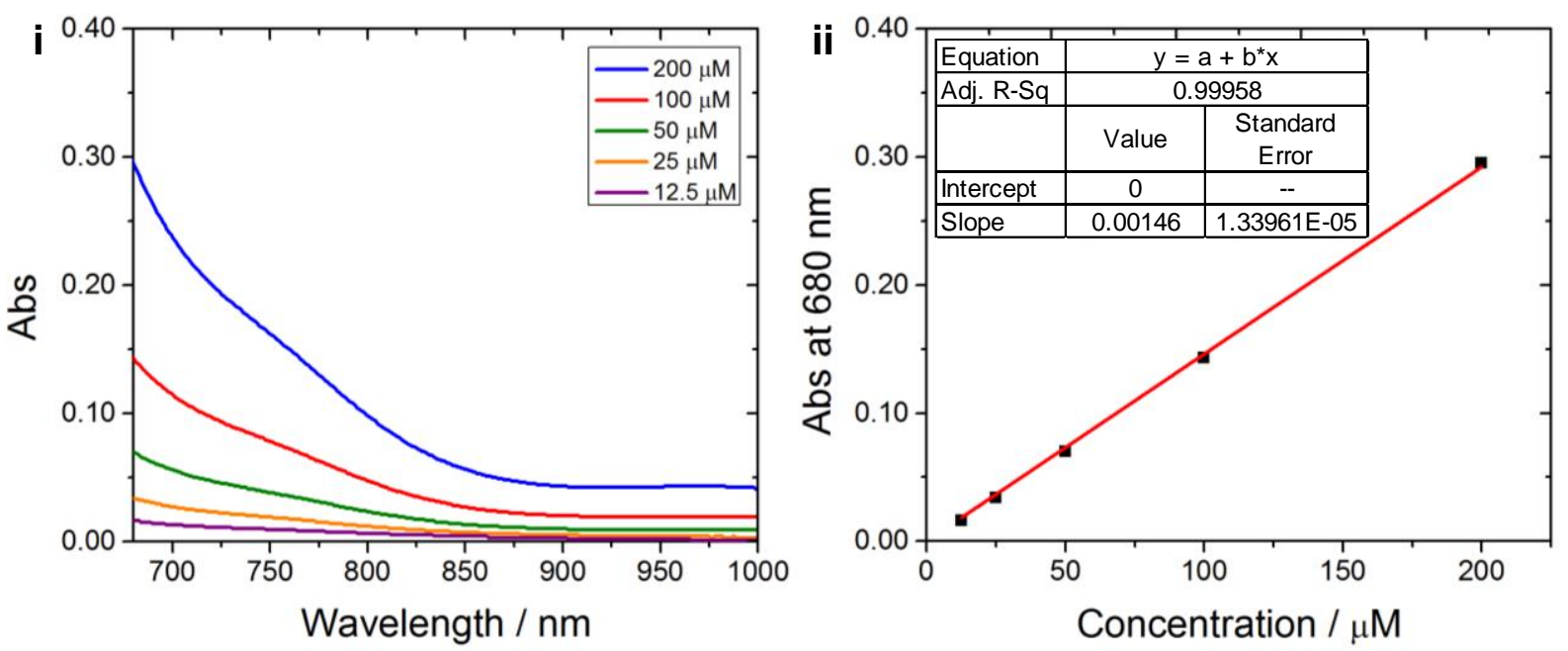

d) Cluster $[\mathrm{PPN}]_{3} .25$
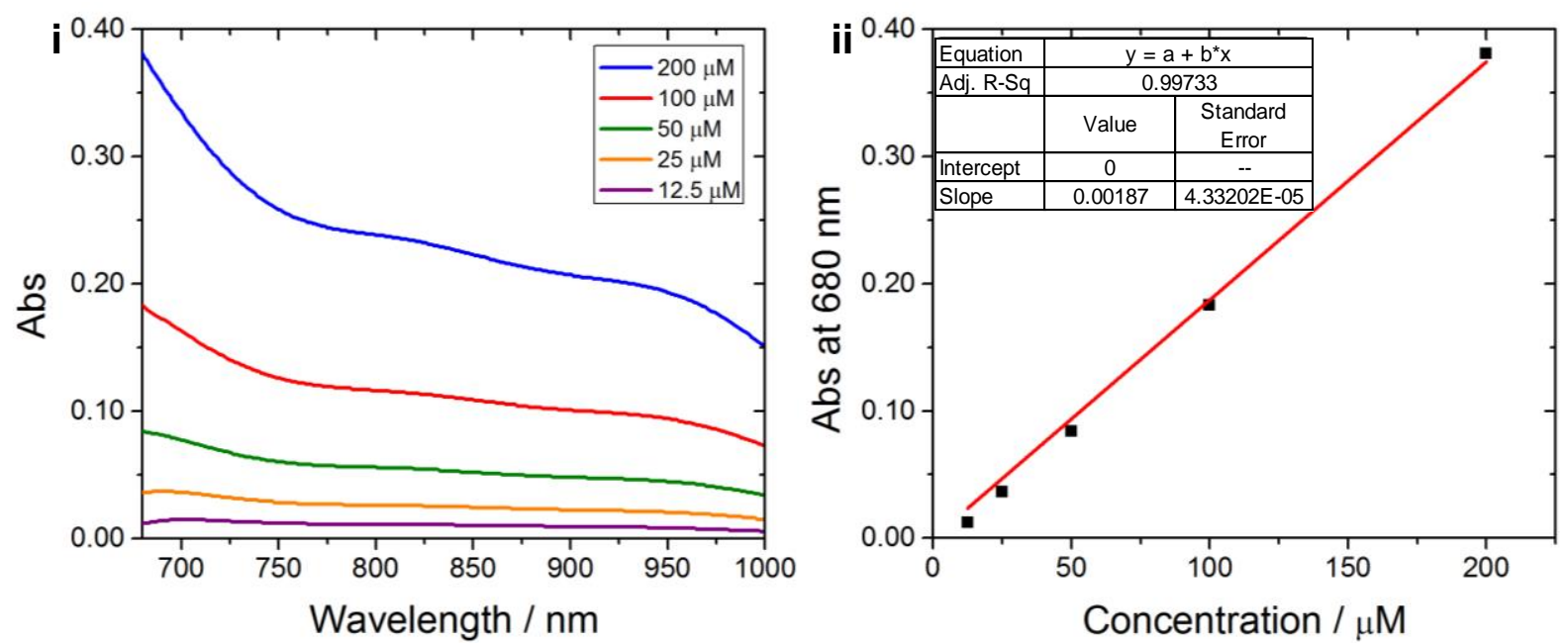

e) Cluster $[\mathrm{PPN}]_{2} .26$
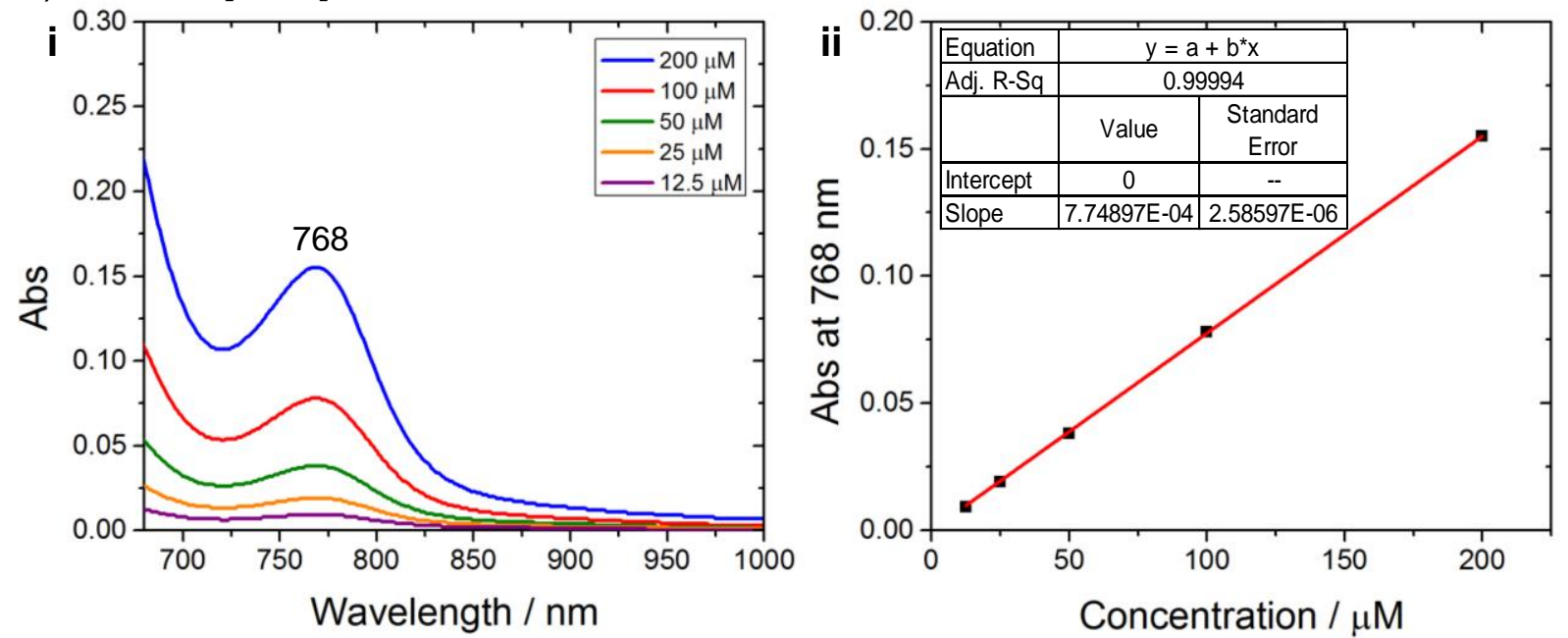

Figure A4.1 (cont'd). i) Optical absorption spectra of various concentrations (in DMSO), ii) plot of optical absorbance (at respective $\lambda_{\max }$ ) as a function of concentrations of cluster c) $[\mathrm{PPN}]_{2} . \mathbf{2 4}, \mathrm{d}$ ) $[\mathrm{PPN}]_{3} .25$ and e) $[\mathrm{PPN}]_{2} .26$. 

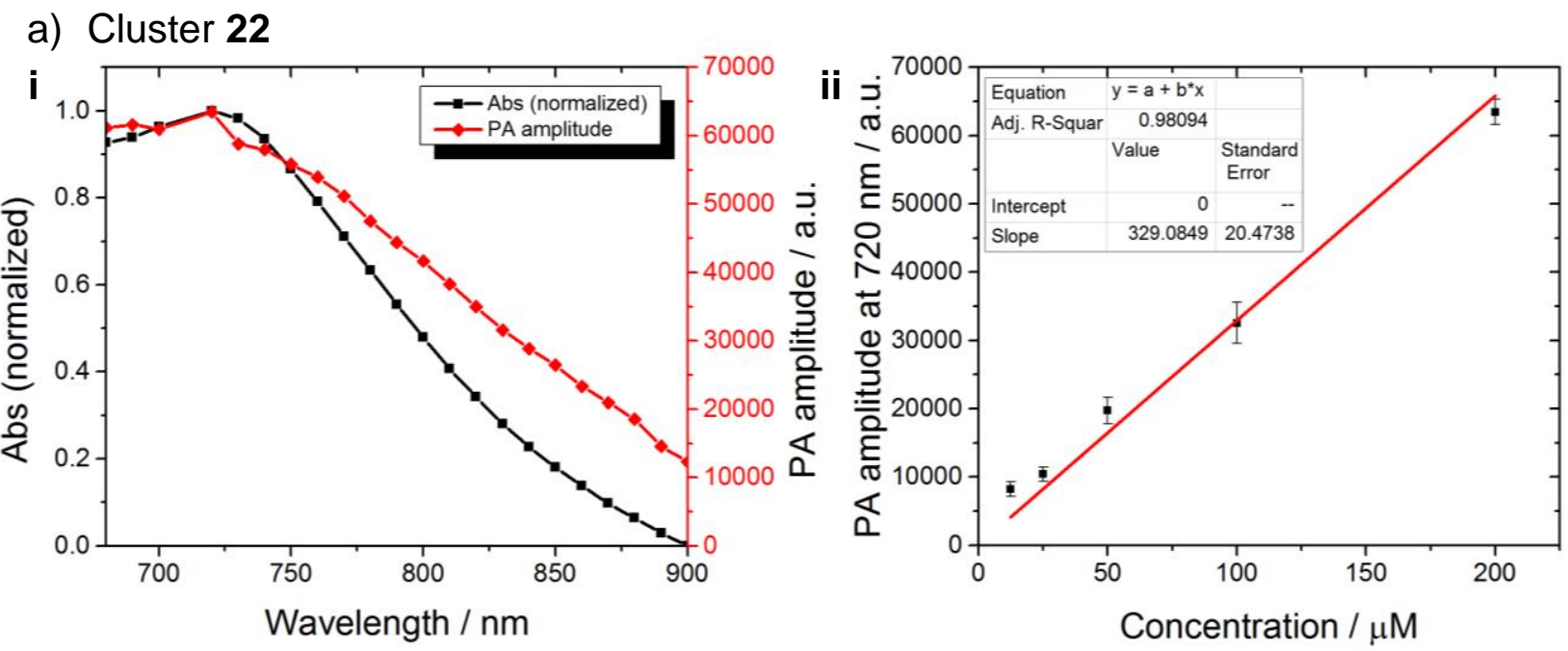

b) Cluster 23
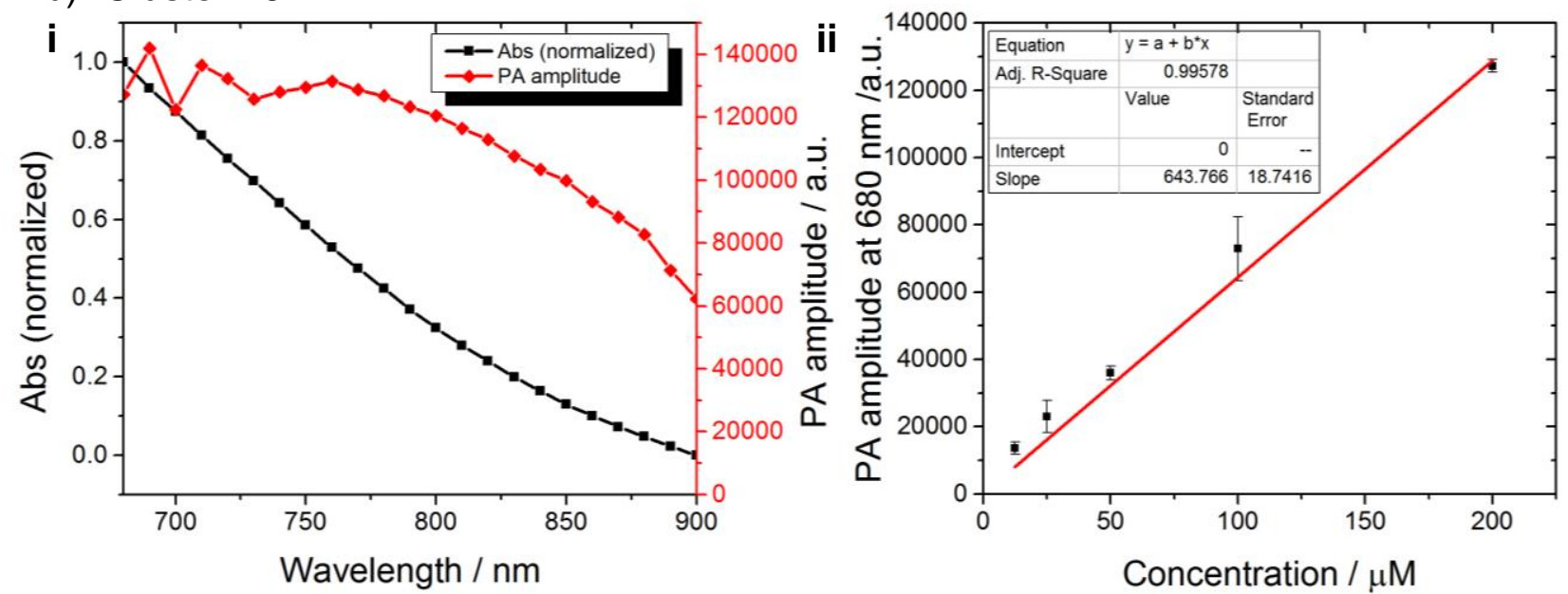

c) Cluster $[\mathrm{PPN}]_{2} .24$
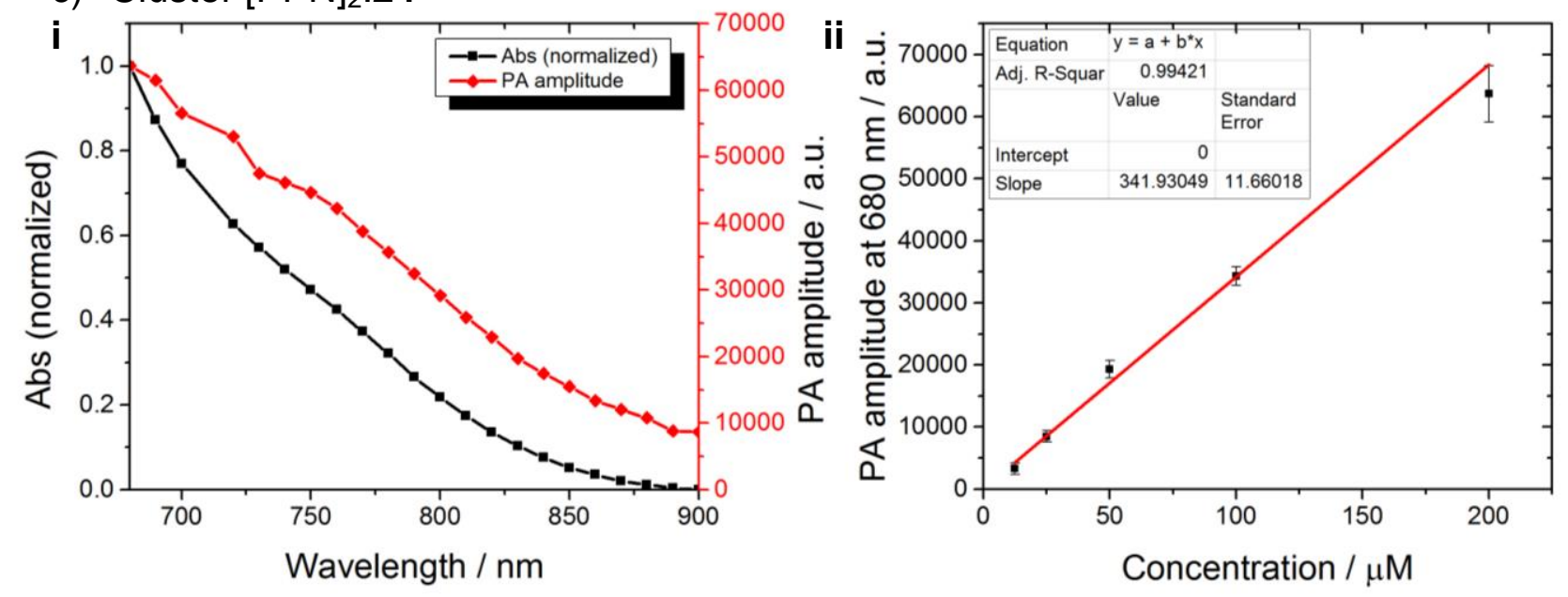

Figure A4.2. i) PA (red) and optical absorption (black) spectra, ii) PA amplitude as a function of concentration of cluster a) 22, b) 23 and c) $\left[\mathrm{PPN}_{2} .24\right.$. 
d) Cluster $[\mathrm{PPN}]_{3} .25$

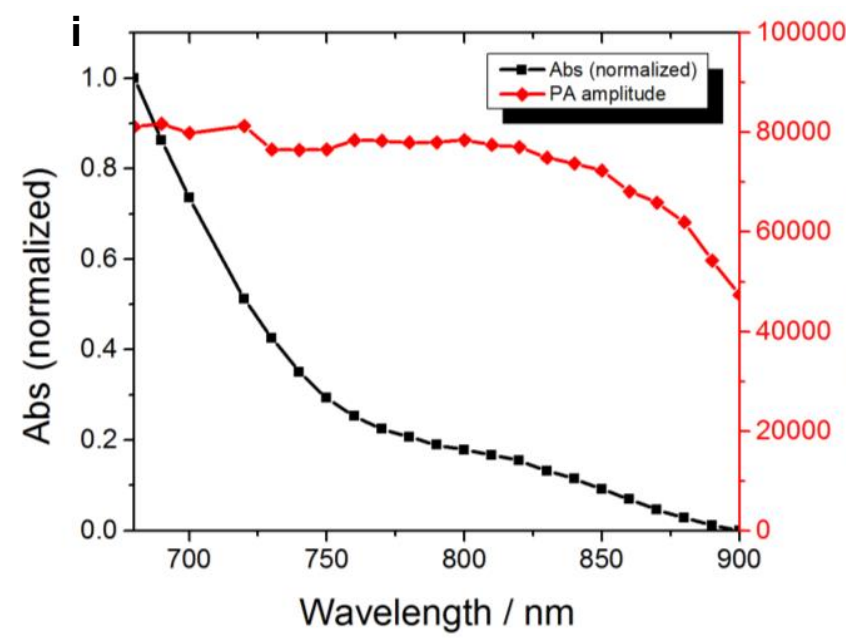

e) Cluster $[\mathrm{PPN}]_{2} .26$

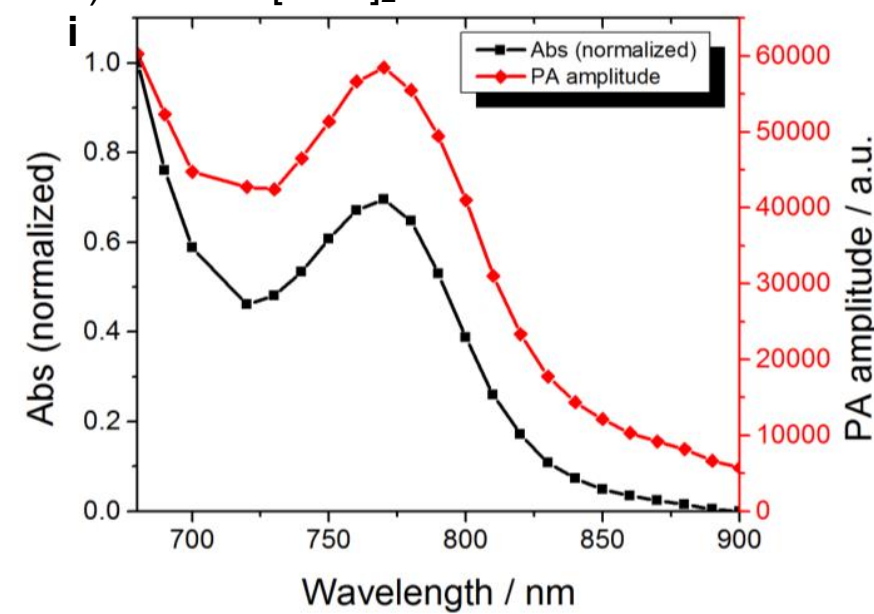

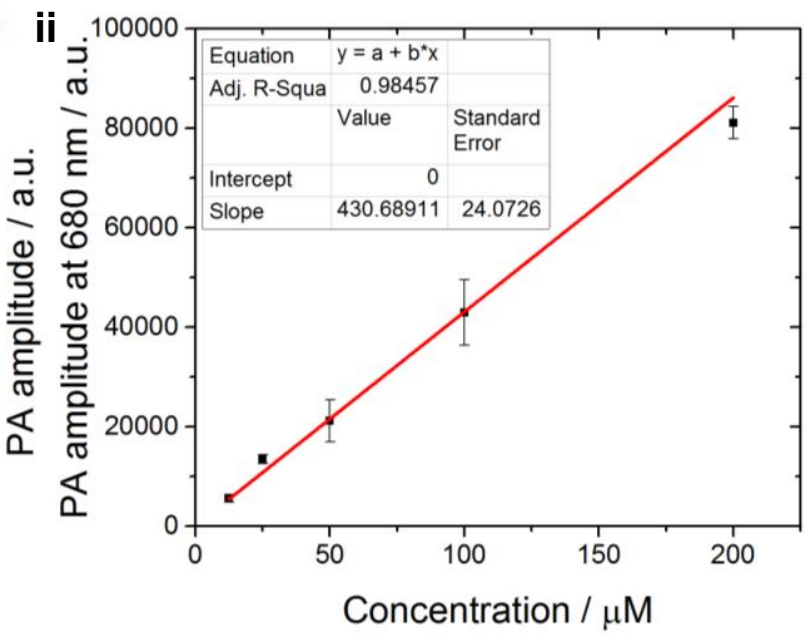

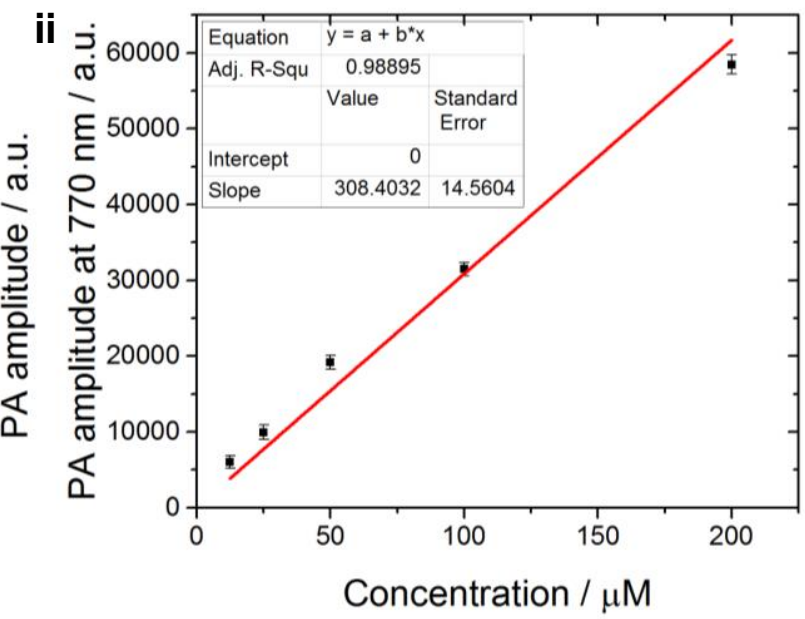

Figure A4.2 (cont'd). i) PA (red) and optical absorption (black) spectra, ii) plot of PA amplitude as a function of concentration of cluster d) $[\mathrm{PPN}]_{3} .25$ and e) $[\mathrm{PPN}]_{2} .26$. 
b)

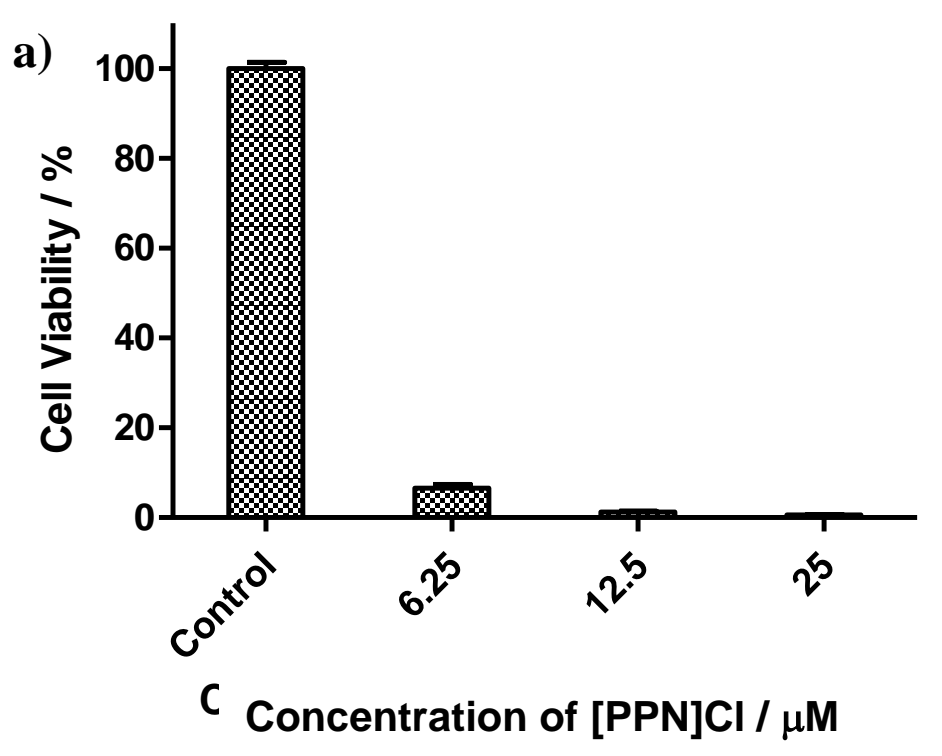

Replicate 1 - MTS assay of [PPN]Cl on OSCC cells

\begin{tabular}{|c|c|c|c|c|c|c|}
\hline $\begin{array}{c}\text { Concentration (C) / } \\
\boldsymbol{\mu M}\end{array}$ & Cell viability (V)/\% & $\mathbf{1 0 0 - Y}$ & $\mathbf{X = l n} \mathbf{C}$ & $\mathbf{Y = l n}[\mathbf{V} /(\mathbf{1 0 0}-\mathbf{V})]$ & $\mathbf{Y}_{\mathbf{0}}$ & $\mathbf{X}_{\mathbf{0}}$ \\
\hline 25.0 & 0.60 & 99.40 & 3.22 & -5.103 & 0 & 0.3773 \\
\hline 12.5 & 1.21 & 98.79 & 2.53 & -4.406 & $\mathbf{I C}_{\mathbf{5 0}}=$ & $\mathbf{1 . 4 6}$ \\
\cline { 1 - 5 } & 6.55 & 93.45 & 1.84 & -2.657 & &
\end{tabular}

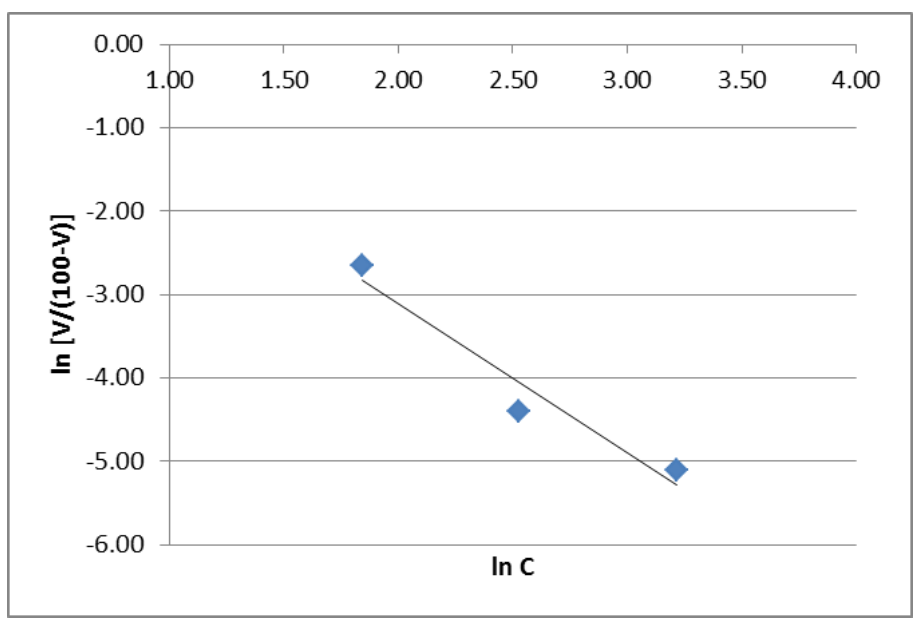

\begin{tabular}{|c|c|}
\hline-0.5304402 & 0.3773 \\
0.1336792 & 0.5593 \\
0.9402810 & 0.2382 \\
15.7450903 & 1 \\
0.8931749 & 0.0567 \\
\hline
\end{tabular}

Figure A4.3. a) First replicate of cell viability assay of $[\mathrm{PPN}] \mathrm{Cl}$ on OSCC cells, using $1 \%$ DMSO as vehicle, b) calculation of $\mathrm{IC}_{50}$. 


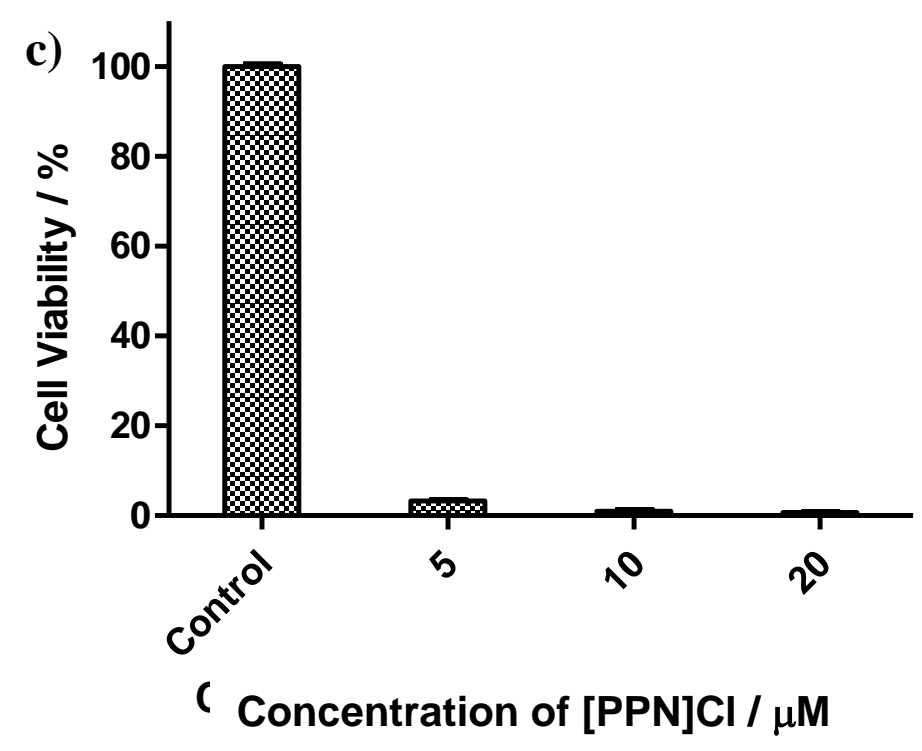

d)

Replicate 2 - MTS assay of [PPN]Cl on OSCC cells

\begin{tabular}{|c|c|c|c|c|c|c|}
\hline $\begin{array}{c}\text { Concentration }(\mathbf{C}) / \\
\boldsymbol{\mu M}\end{array}$ & Cell viability (V) / \% & $\mathbf{1 0 0 - V}$ & $\mathbf{X = l n} \mathbf{C}$ & $\mathbf{Y = l n}[\mathbf{V} /(\mathbf{1 0 0}-\mathbf{V})]$ & $\mathbf{Y}_{\mathbf{0}}$ & $\mathbf{X}_{\mathbf{0}}$ \\
\hline 20.0 & 0.72 & 99.28 & 3.00 & -4.923 & 0 & -1.1710 \\
\hline 10.0 & 0.96 & 99.04 & 2.30 & -4.635 & $\mathbf{I C}_{\mathbf{5 0}}=$ & $\mathbf{0 . 3 1}$ \\
\hline 5.0 & 3.24 & 96.76 & 1.61 & -3.397 & \multicolumn{2}{c}{} \\
\cline { 1 - 5 }
\end{tabular}

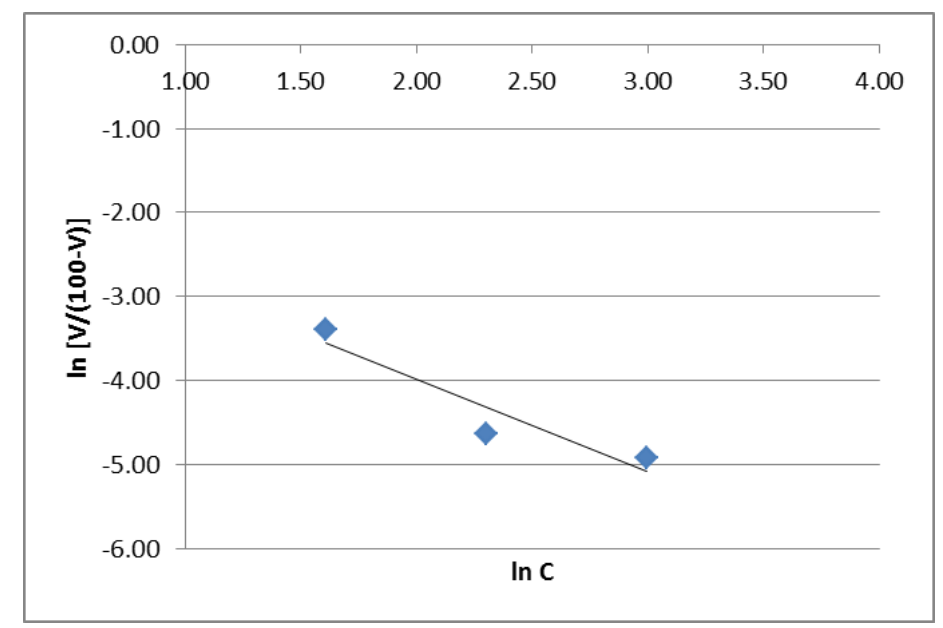

\begin{tabular}{|c|c|}
\hline-0.8043229 & -1.1710 \\
0.2890845 & 1.2630 \\
0.8855999 & 0.3316 \\
7.7412536 & 1 \\
0.8509783 & 0.1099 \\
\hline
\end{tabular}

Figure A4.3 (cont'd). c) Second replicate of cell viability assay of [PPN]Cl on OSCC cells, using $1 \%$ DMSO as vehicle, d) calculation of $\mathrm{IC}_{50}$. 


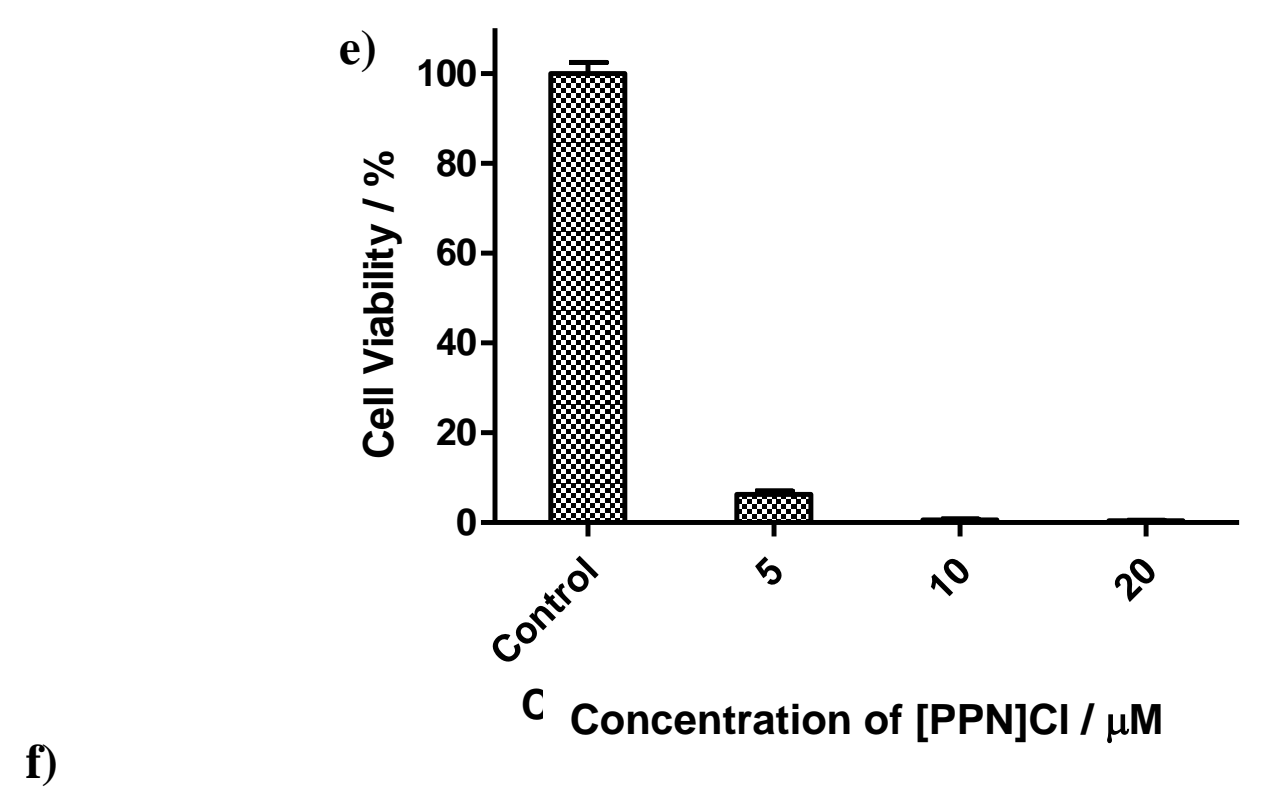

Replicate 3 - MTS assay of [PPN]CI on OSCC cells

\begin{tabular}{|c|c|c|c|c|c|c|}
\hline $\begin{array}{c}\text { Concentration }(\mathbf{C}) / \\
\boldsymbol{\mu M}\end{array}$ & Cell viability (V) / \% & $\mathbf{1 0 0 - V}$ & $\mathbf{X}=\mathbf{l n} \mathbf{C}$ & $\mathbf{Y =} \mathbf{l n}[\mathbf{V} /(\mathbf{1 0 0}-\mathbf{V})]$ & $\mathbf{Y}_{\mathbf{0}}$ & $\mathbf{X}_{\mathbf{0}}$ \\
\hline 20.0 & 0.40 & 99.60 & 3.00 & -5.511 & 0 & 0.4231 \\
\hline 10.0 & 0.62 & 99.38 & 2.30 & -5.083 & $\mathbf{I C}_{\mathbf{5 0}}=$ & $\mathbf{1 . 5 3}$ \\
\hline 5.0 & 6.32 & 93.68 & 1.61 & -2.697 & &
\end{tabular}

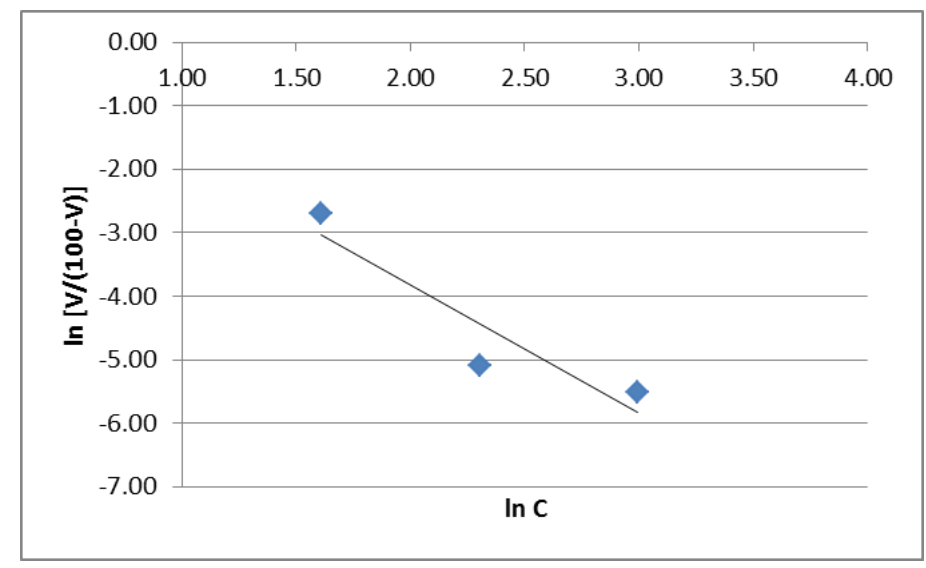

\begin{tabular}{|c|c|}
\hline-0.4242441 & 0.4231 \\
0.1703612 & 0.7836 \\
0.8611382 & 0.3653 \\
6.2014057 & 1 \\
0.8274729 & 0.1334 \\
\hline
\end{tabular}

\begin{tabular}{|c|c|}
\hline Mean $\mathbf{I C}_{\mathbf{5 0}}$ & $\underline{\mathbf{1 . 1 0}}$ \\
\hline Standard error & 0.40 \\
\hline
\end{tabular}

Figure A4.3 (cont'd). e) Third replicate of cell viability assay of [PPN]Cl on OSCC cells, using $1 \%$ DMSO as vehicle, f) calculation of $\mathrm{IC}_{50}$ and the mean value from three replicates. 


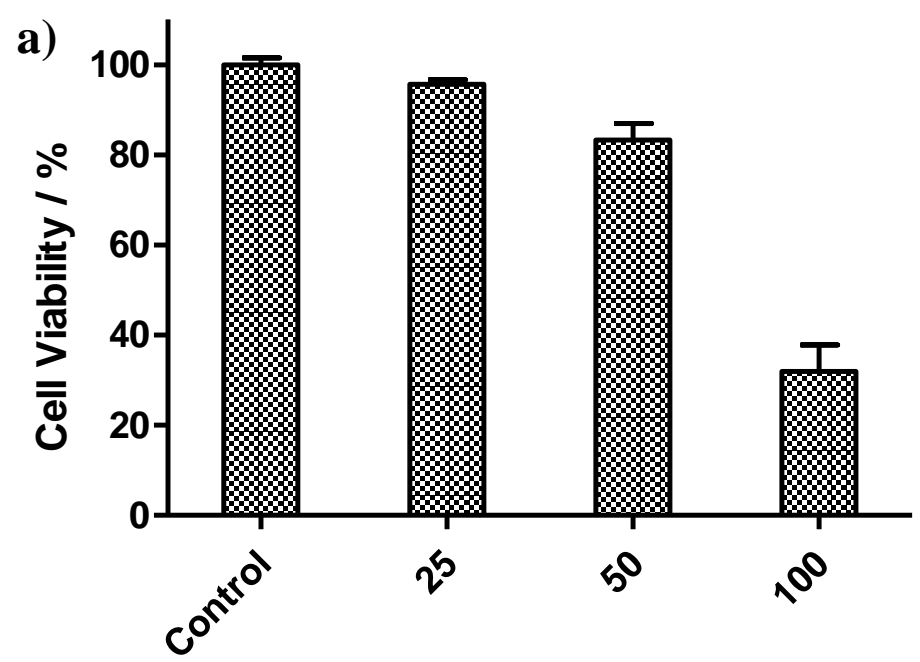

Concentration of $\mathrm{Na}_{2} .26 / \mu \mathrm{M}$

b)

Replicate 1 - MTS assay of $\mathrm{Na}_{2}\left[\mathrm{Os}_{10}\left(\mu_{6}-\mathrm{C}\right)(\mathrm{CO})_{24}\right]\left(\mathrm{Na}_{2} .26\right)$ on OSCC cells

\begin{tabular}{|c|c|c|c|c|c|c|}
\hline $\begin{array}{c}\text { Concentration (C) / } \\
\boldsymbol{\mu M}\end{array}$ & Cell viability (V)/\% & $\mathbf{1 0 0 - V}$ & $\mathbf{X = l n} \mathbf{C}$ & $\mathbf{Y = l n}[\mathbf{V} /(\mathbf{1 0 0}-\mathrm{V})]$ & $\mathbf{Y}_{\mathbf{0}}$ & $\mathbf{X}_{\mathbf{0}}$ \\
\hline 100.0 & 31.91 & 68.09 & 4.61 & -0.758 & 0 & 4.3773 \\
\hline 50.0 & 83.32 & 16.68 & 3.91 & 1.609 & $\mathbf{I C}_{\mathbf{5 0}}=$ & $\mathbf{7 9 . 6 2}$ \\
\hline 25.0 & 95.69 & 4.31 & 3.22 & 3.099 & &
\end{tabular}

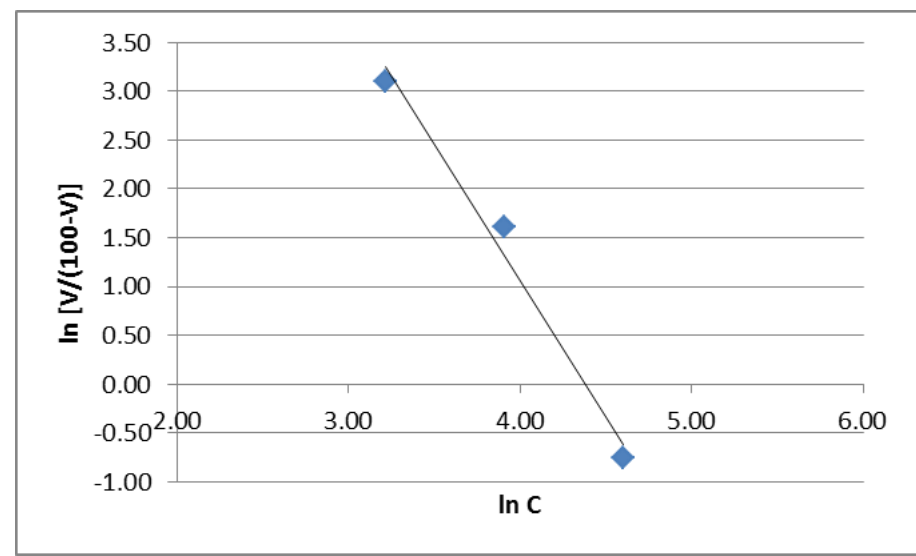

\begin{tabular}{|c|c|}
\hline-0.3533478 & 4.3773 \\
0.0463439 & 0.0956 \\
0.9830888 & 0.1275 \\
58.1325284 & 1 \\
0.9446560 & 0.0163 \\
\hline
\end{tabular}

Figure A4.4. a) First replicate of cell viability assay of $\mathrm{Na}_{2}\left[\mathrm{Os}_{10}\left(\mu_{6}-\mathrm{C}\right)(\mathrm{CO})_{24}\right]\left(\mathrm{Na}_{2} .26\right)$ on OSCC cells, using $1 \%$ DMSO as vehicle, b) calculation of $\mathrm{IC}_{50}$. 


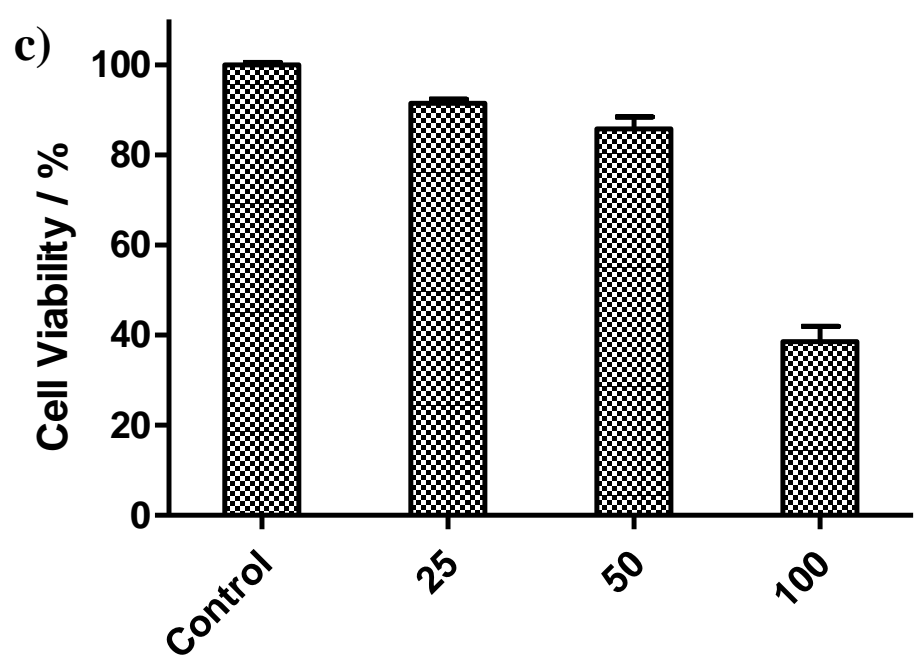

Concentration of $\mathrm{Na}_{2} .26 / \mu \mathrm{M}$

d)

Replicate 2 - MTS assay of $\mathrm{Na}_{2}\left[\mathrm{Os}_{10}\left(\mu_{6}-\mathrm{C}\right)(\mathrm{CO})_{24}\right]\left(\mathrm{Na}_{2} .26\right)$ on OSCC cells

\begin{tabular}{|c|c|c|c|c|c|r|}
\hline $\begin{array}{c}\text { Concentration }(\mathbf{C}) / \\
\boldsymbol{\mu M}\end{array}$ & Cell viability $(\mathbf{V}) / \%$ & $\mathbf{1 0 0}-\mathbf{V}$ & $\mathbf{X}=\mathbf{l n} \mathbf{C}$ & $\mathbf{Y}=\ln [\mathbf{V} /(\mathbf{1 0 0}-\mathbf{V})]$ & $\mathbf{Y}_{\mathbf{0}}$ & $\mathbf{X}_{\mathbf{0}}$ \\
\hline 100.0 & 38.58 & 61.42 & 4.61 & -0.465 & 0 & 4.4513 \\
\hline 50.0 & 85.77 & 14.23 & 3.91 & 1.796 & $\mathbf{I C}_{50}=$ & $\mathbf{8 5 . 7 3}$ \\
\hline 25.0 & 91.41 & 8.59 & 3.22 & 2.364 & \multicolumn{2}{|r}{} \\
\cline { 1 - 5 } &
\end{tabular}

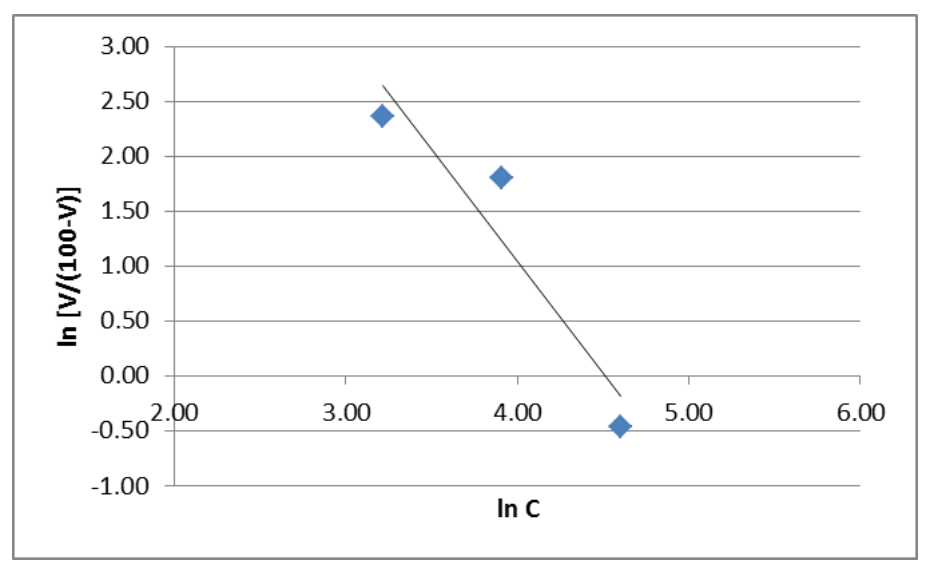

\begin{tabular}{|c|c|}
\hline-0.4377199 & 4.4513 \\
0.1513021 & 0.2625 \\
0.8932715 & 0.3202 \\
8.3695677 & 1 \\
0.8583500 & 0.1026 \\
\hline
\end{tabular}

Figure A4.4 (cont'd). c) Second replicate of cell viability assay of $\mathrm{Na}_{2}\left[\mathrm{Os}_{10}\left(\mu_{6}-\mathrm{C}\right)(\mathrm{CO})_{24}\right]$ $\left(\mathrm{Na}_{2} .26\right)$ on OSCC cells, using $1 \%$ DMSO as vehicle, d) calculation of $\mathrm{IC}_{50}$. 


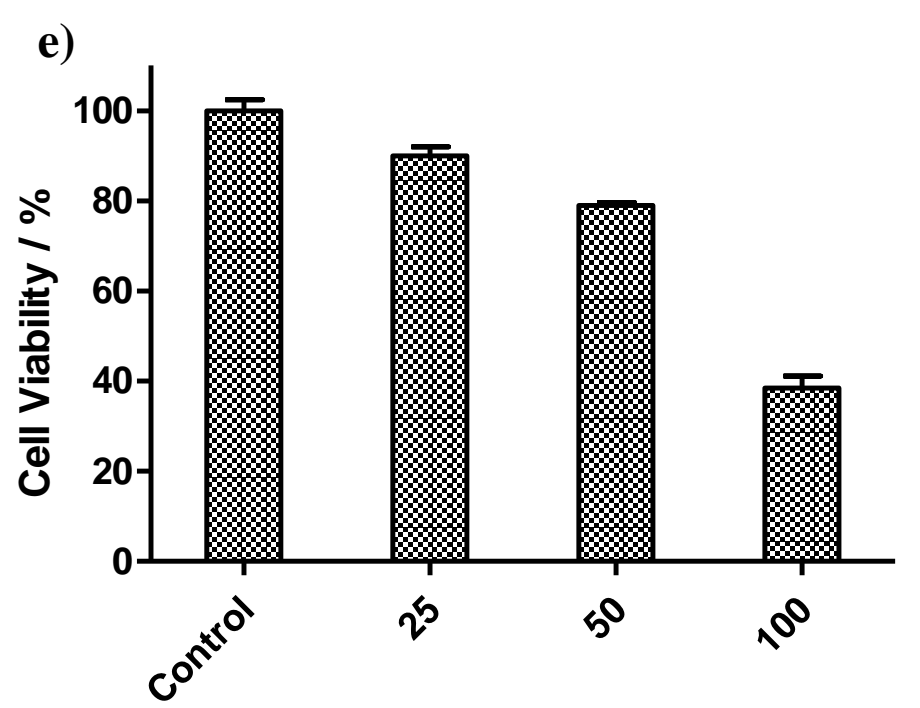

Concentration of $\mathrm{Na}_{2} .26 / \mu \mathrm{M}$

f)

Replicate 3 - MTS assay of $\mathrm{Na}_{2}\left[\mathrm{Os}_{10}\left(\mu_{6}-\mathrm{C}\right)(\mathrm{CO})_{24}\right]\left(\mathrm{Na}_{2} .26\right)$ on OSCC cells

\begin{tabular}{|c|c|c|c|c|c|c|}
\hline $\begin{array}{c}\text { Concentration }(\mathrm{C}) / \\
\mu \mathrm{M}\end{array}$ & Cell viability $(\mathrm{V}) / \%$ & $100-\mathrm{V}$ & $X=\ln C$ & $Y=\ln [V /(100-V)]$ & $\mathbf{Y}_{\mathbf{0}}$ & $\mathbf{X}_{\mathbf{0}}$ \\
\hline 100.0 & 38.47 & 61.53 & 4.61 & -0.470 & 0 & 4.4205 \\
\hline 50.0 & 78.99 & 21.01 & 3.91 & 1.324 & $\mathrm{IC}_{50}=$ & 83.14 \\
\hline 25.0 & 89.95 & 10.05 & 3.22 & 2.192 & & \\
\hline
\end{tabular}

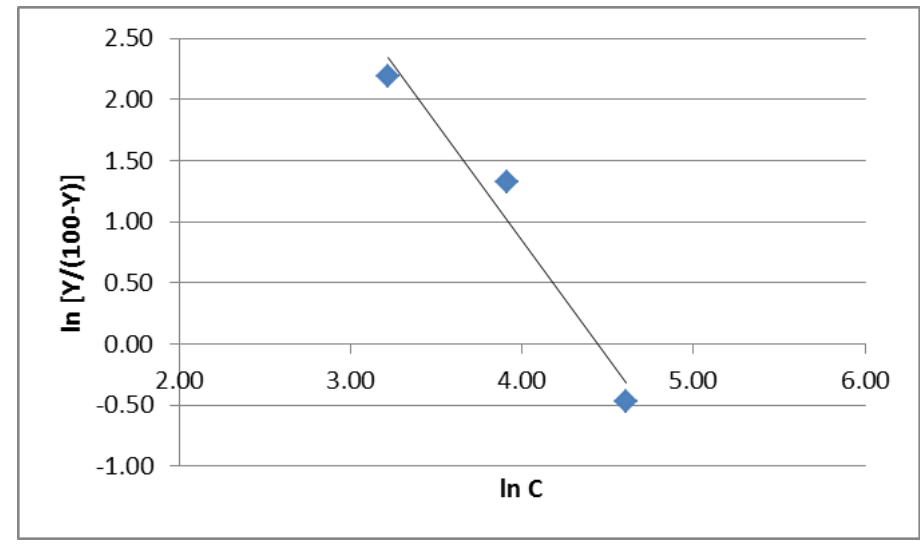

\begin{tabular}{|c|c|}
\hline-0.5006497 & 4.4205 \\
0.1005357 & 0.1511 \\
0.9612382 & 0.1930 \\
24.7986260 & 1 \\
0.9236596 & 0.0372 \\
\hline
\end{tabular}

\begin{tabular}{|c|c|}
\hline Mean $\mathbf{I C}_{\mathbf{5 0}}$ & $\underline{\mathbf{8 2 . 8 3}}$ \\
\hline Standard error & 1.77 \\
\hline
\end{tabular}

Figure A4.4 (cont'd). e) Third replicate of cell viability assay of $\mathrm{Na}_{2}\left[\mathrm{Os}_{10}\left(\mu_{6}-\mathrm{C}\right)(\mathrm{CO})_{24}\right]$ $\left(\mathrm{Na}_{2} .26\right)$ on OSCC cells, using $1 \%$ DMSO as vehicle, f) calculation of $\mathrm{IC}_{50}$ and the mean value from three replicates. 
a) DMSO

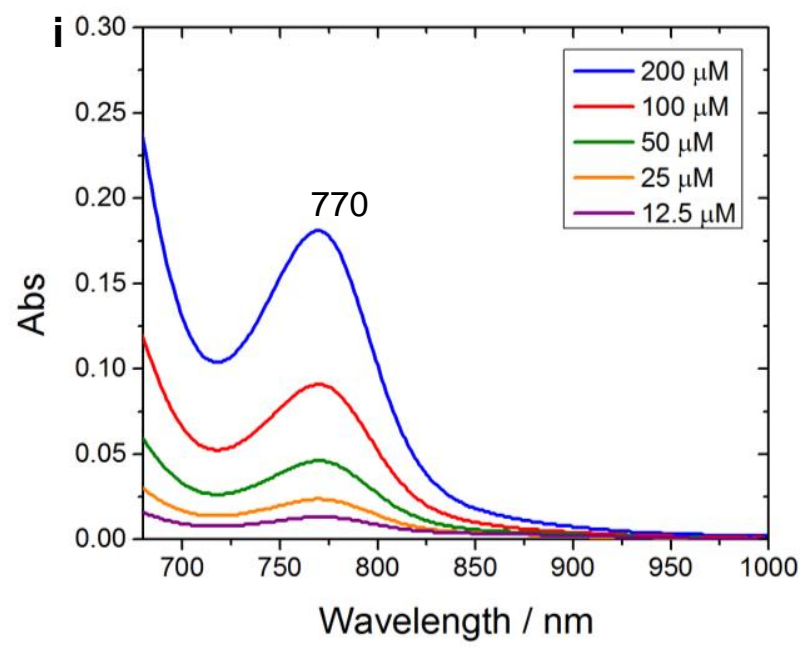

b) $10 \%$ DMSO in PBS

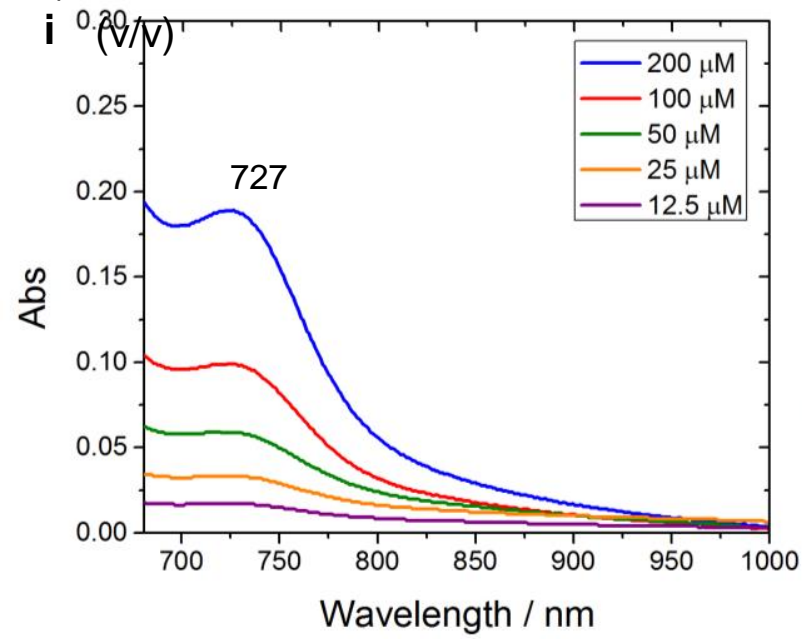

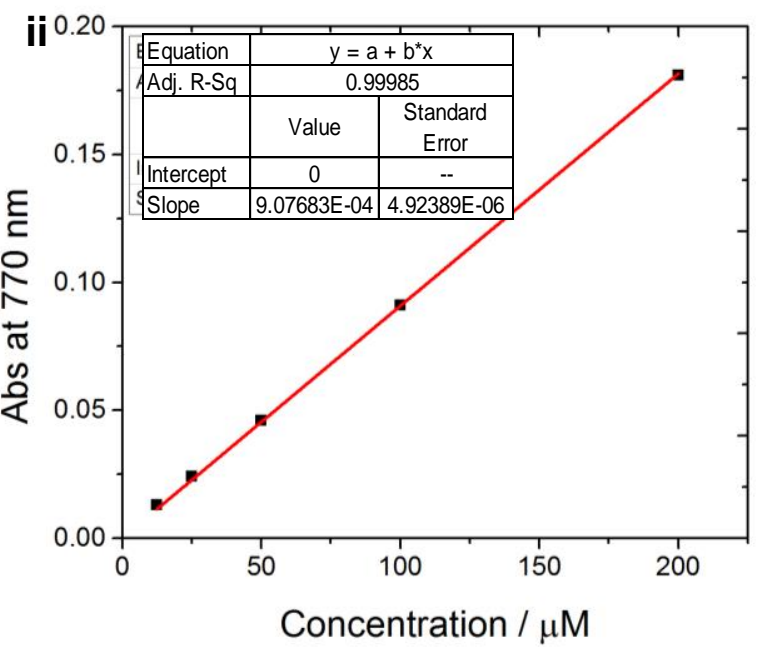

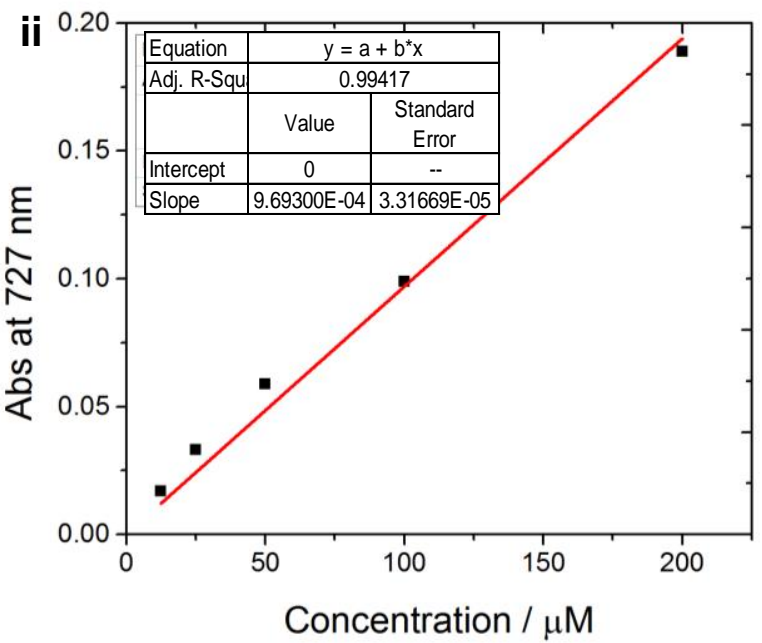

c) $10 \%$ DMSO in DMEM with 10\% FBS, 1\% L-glutamine and penicillin/streptomycin (v/v)
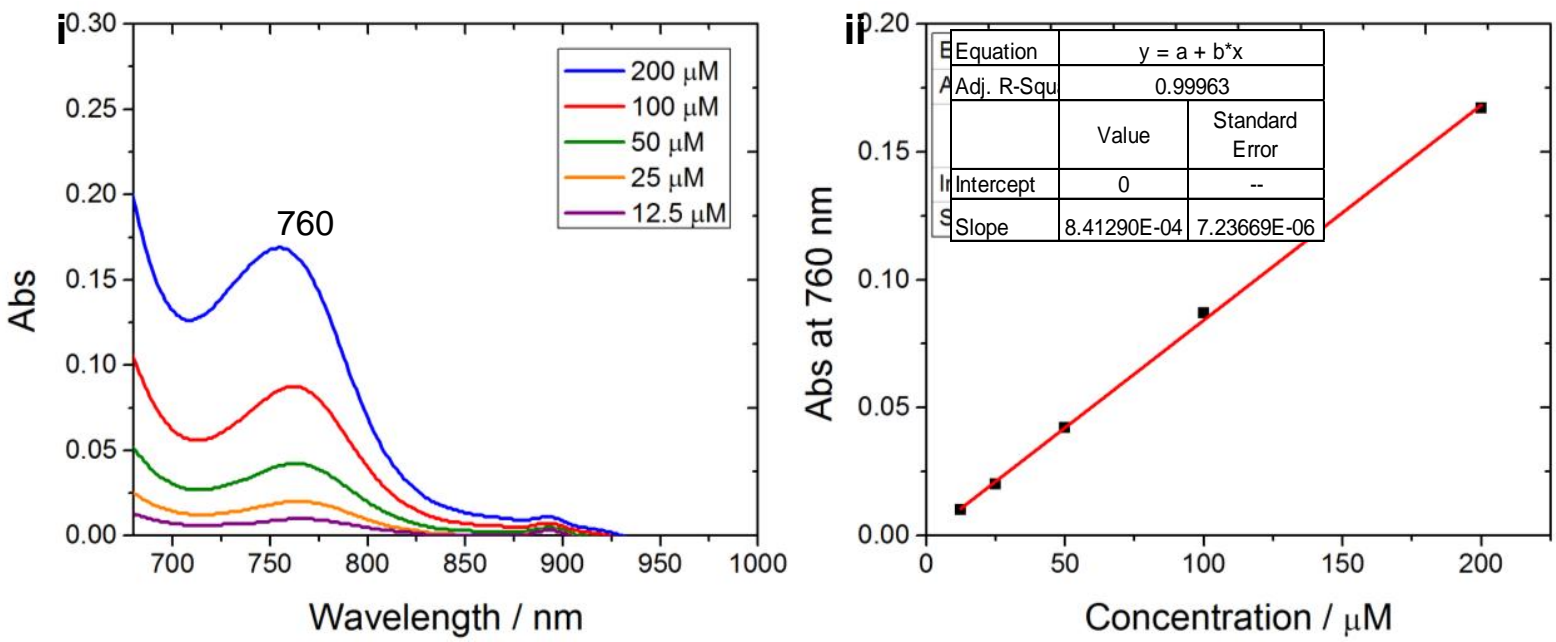

Figure A4.5. i) Optical absorption spectra of $\mathrm{Na}_{2} .26$ of various concentrations and ii) plot of $\lambda_{\max }$ as a function of concentration of $\mathrm{Na}_{2} .26$ in a) DMSO, b) $10 \%$ DMSO in PBS (v/v), and c) $10 \%$ DMSO in modified DMEM (v/v). 
a) DMSO

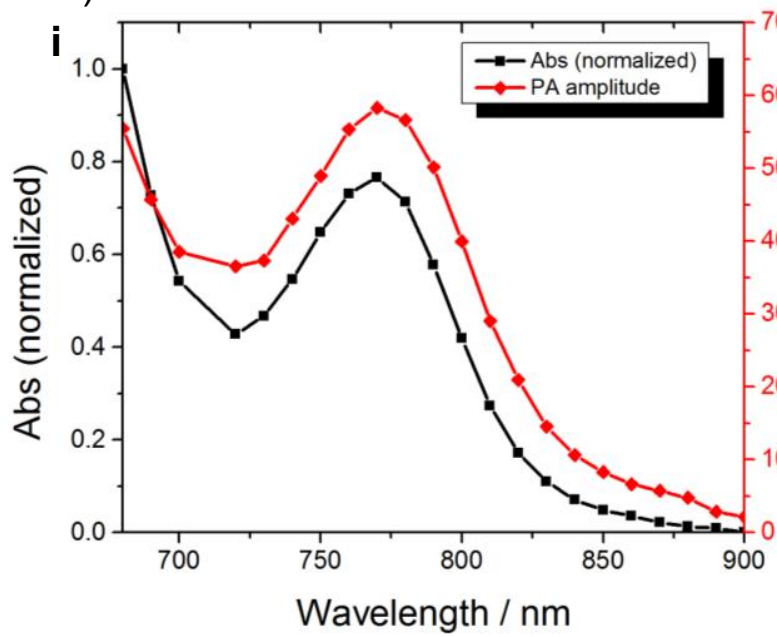

b) $10 \%$ DMSO in PBS
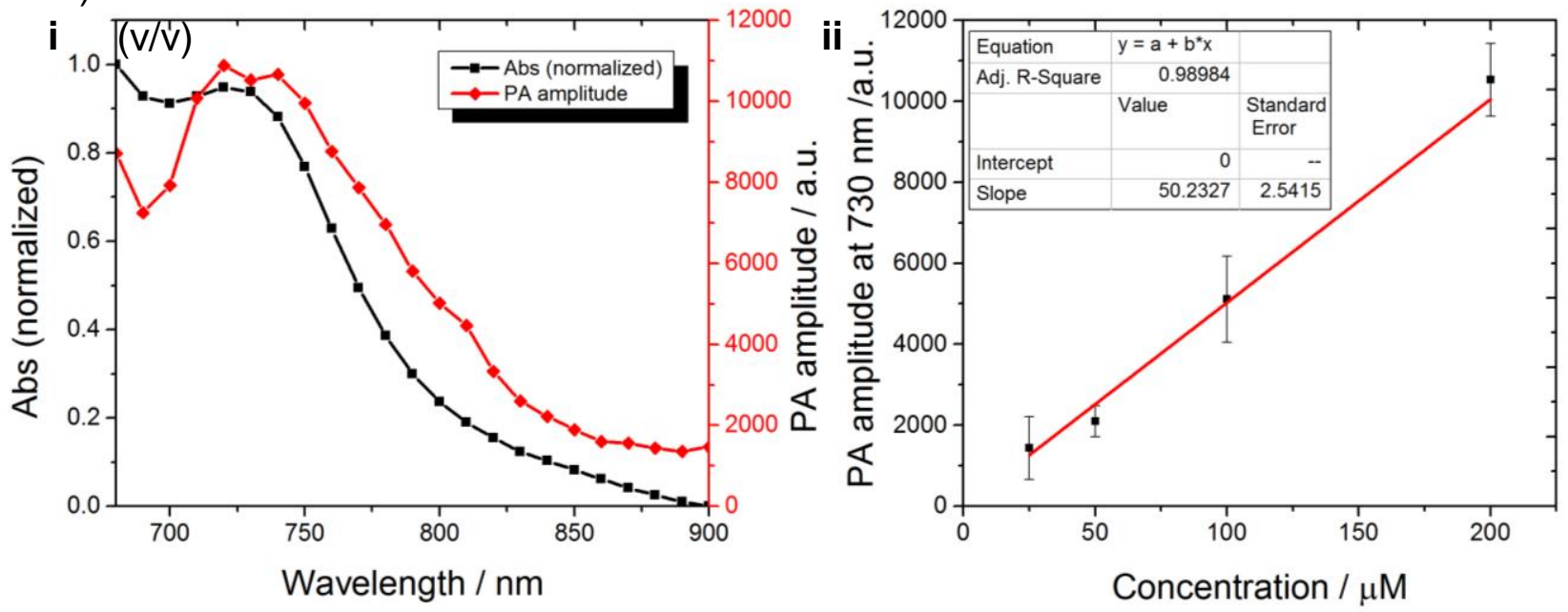

c) $10 \%$ DMSO in DMEM with 10\% FBS, 1\% L-glutamine and penicillin/streptomycin (v/v)
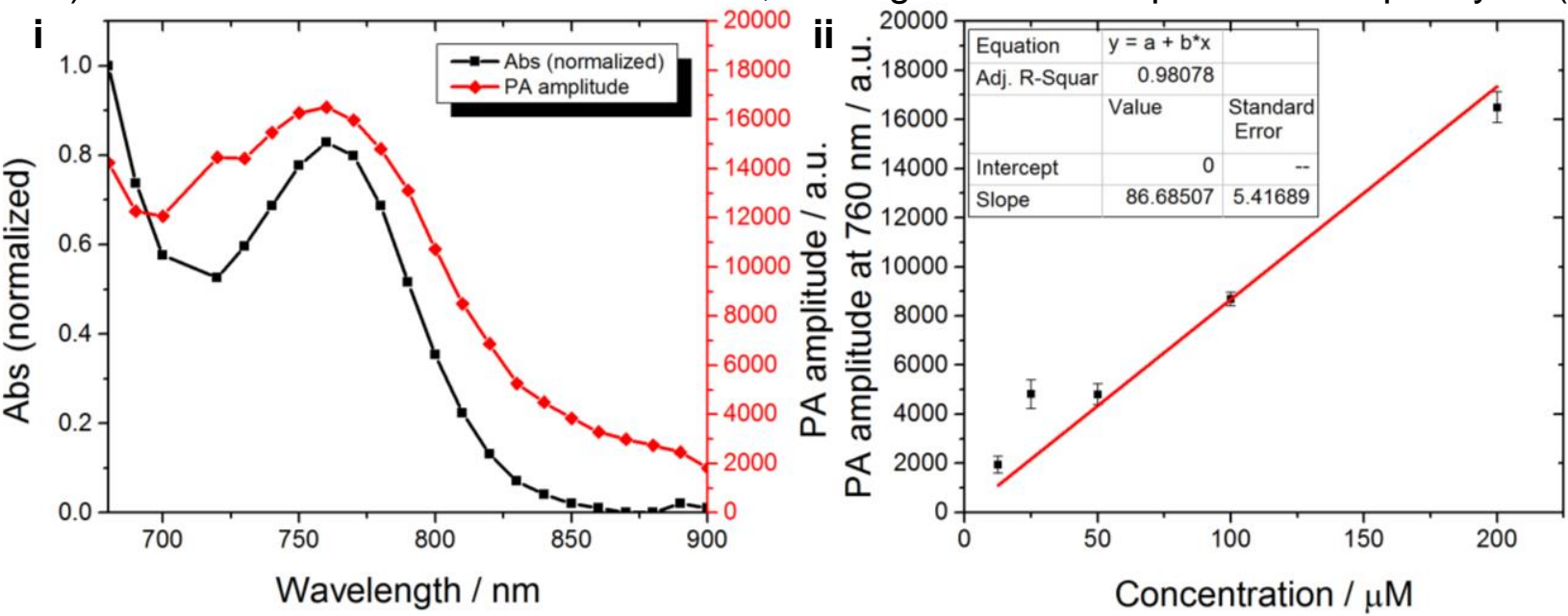

Figure A4.6. i) PA (red) and optical absorption (black) spectra of $\mathrm{Na}_{2} .26$, ii) plot of PA amplitude (at respective $\lambda_{\max }$ ) as a function of concentration of $\mathrm{Na}_{2} .26$ in a) DMSO, b) $10 \%$ DMSO in PBS (v/v), and c) 10\% DMSO in modified DMEM (v/v). 


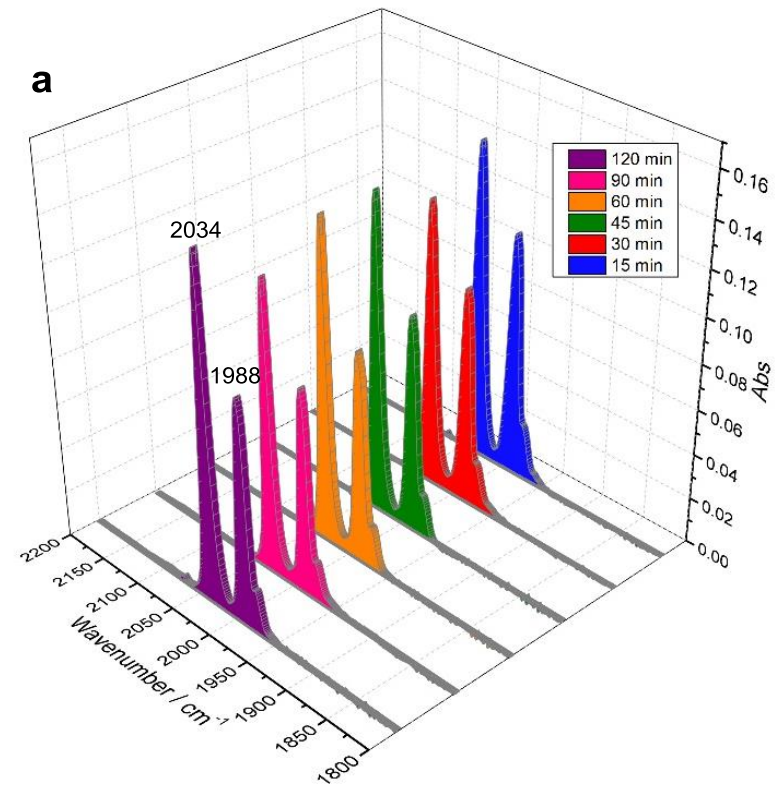

\section{b}

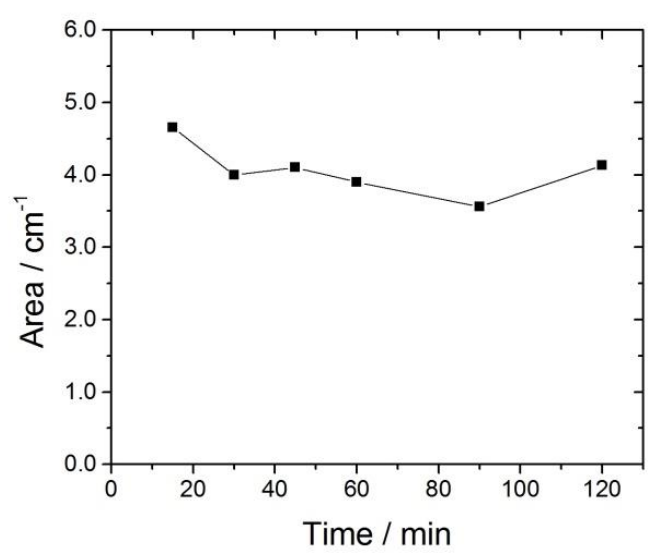

Figure A4.7. Irradiation of a solution of $\mathrm{Na}_{2} .26(200 \mu \mathrm{M})$ in $10 \%$ DMSO in modified DMEM at $760 \mathrm{~nm}$ for various time intervals: a) FTIR spectra $\left(v_{\mathrm{CO}}\right)$ obtained from the dried samples, and b) integrals of the $v_{\mathrm{CO}}$ absorbance.
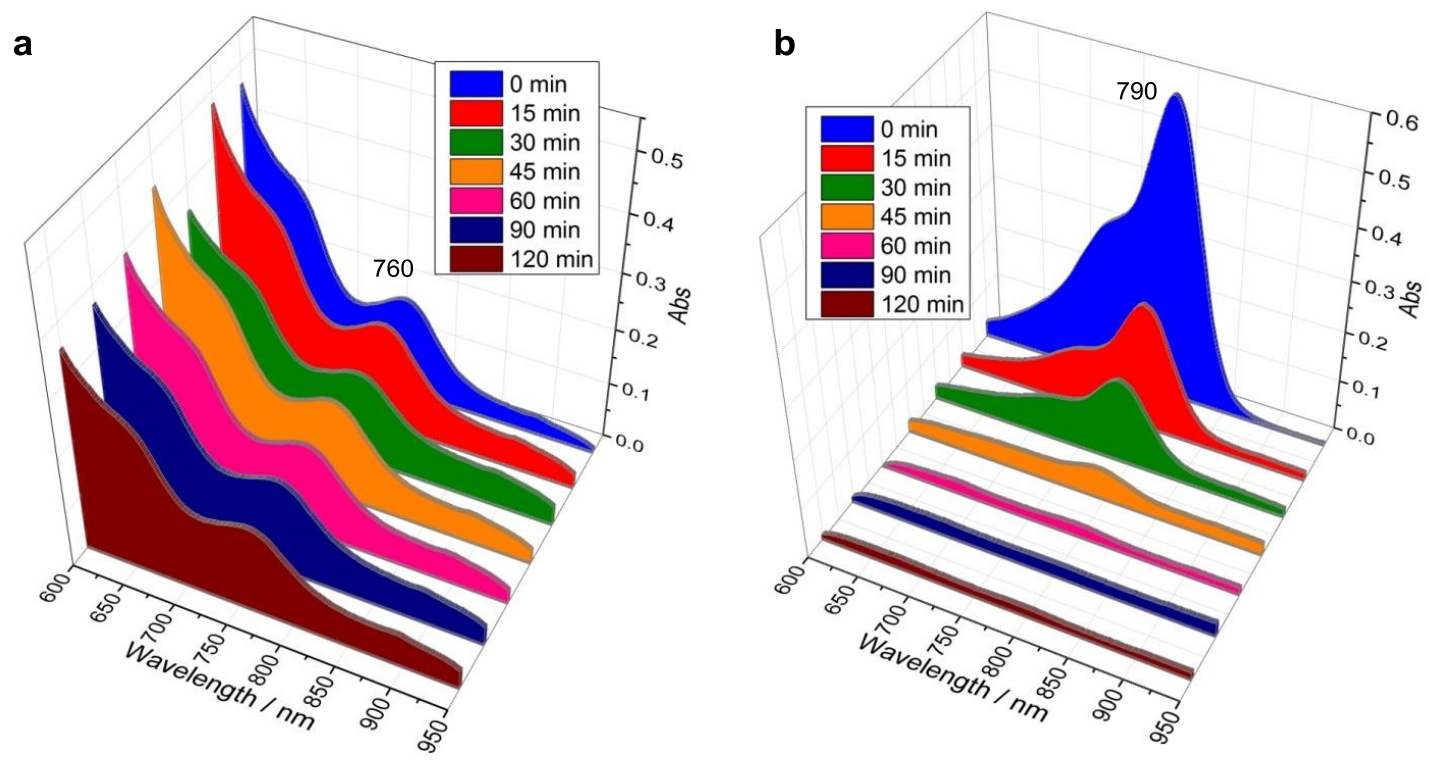

Figure A4.8. Time course studies of $\mathrm{Na}_{2} .26$ and ICG over 120 min with irradiation at the respective $\lambda_{\max }$ : a) optical absorption spectra of $\mathrm{Na}_{2} .26$ and d) ICG. 

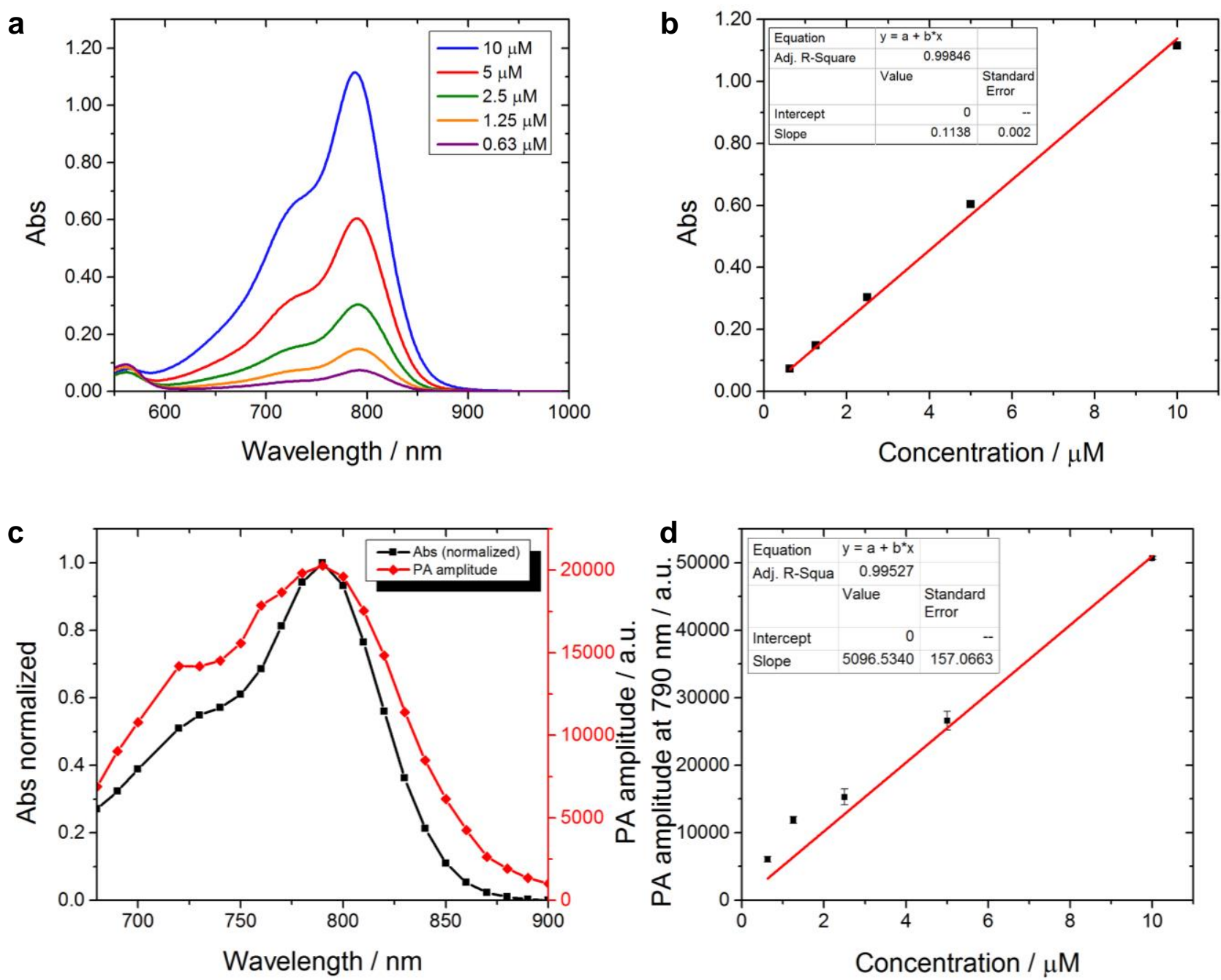

Figure A4.9. a) Optical absorption spectra of ICG of various concentrations, b) plot of absorbance of ICG at $\lambda_{\max }=790 \mathrm{~nm}$ as a function of concentration, c) PA (red) and optical absorption (black) spectra of ICG, d) plot of PA amplitude at $\lambda_{\max }=790 \mathrm{~nm}$ as a function of concentration of ICG in 10\% DMSO in modified DMEM (v/v). 

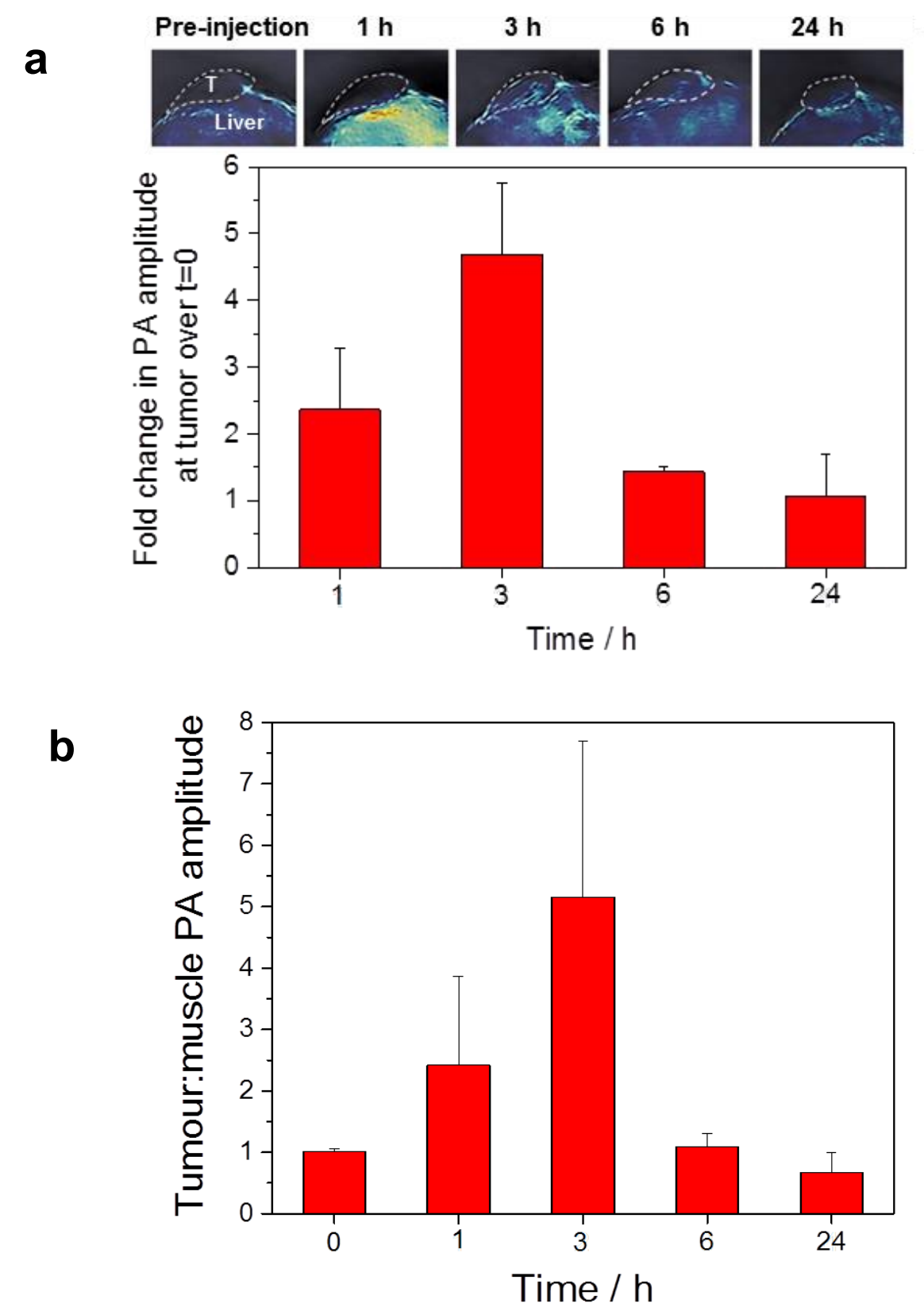

Figure A4.10. a) Enlarged images of the tumors at various time point with the PA intensities normalized (top). Fold change in PA intensity in the tumor over that at $t=0$ in the tumor (bottom). Error bars represent standard error of the mean, $n=3$. b) Tumor-to-muscle ratio of PA amplitude at various time points up till $24 \mathrm{~h}$. Error bars represent standard error of the mean, $\mathrm{n}=3$. 


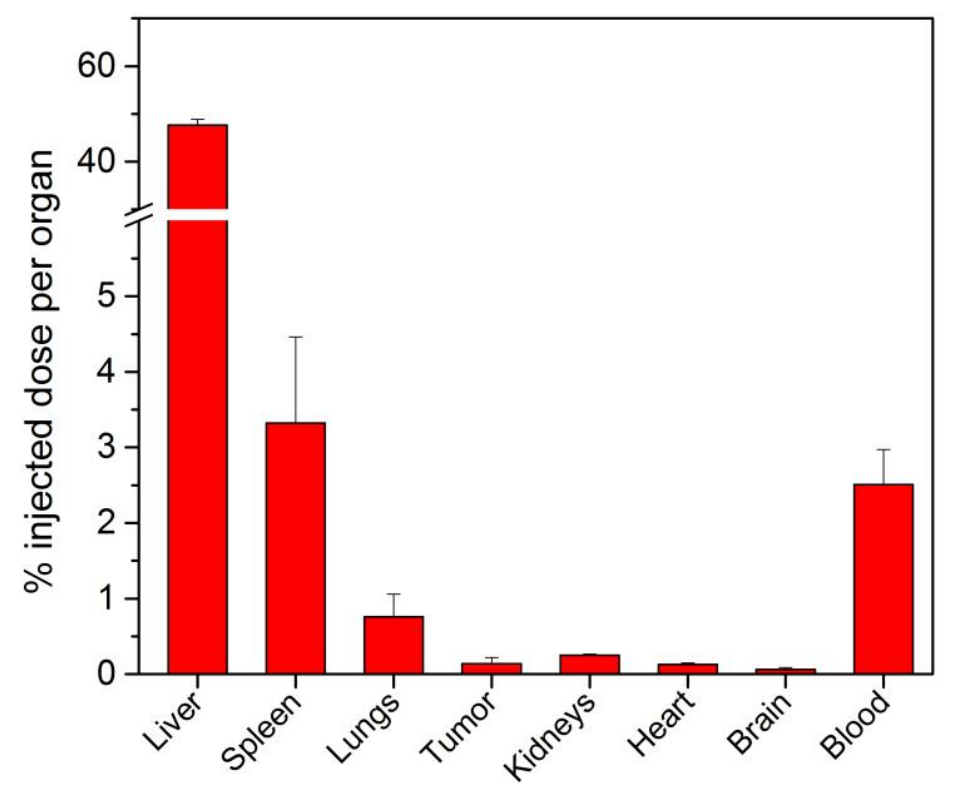

Figure A4.11. ICP-MS analysis of the percentage retention of injected dose (\% ID) of $\mathrm{Na}_{2} . \mathbf{2 6}$ per organs, $24 \mathrm{~h}$ after injection. Error bars represent standard error of the mean, $\mathrm{n}=3$. 


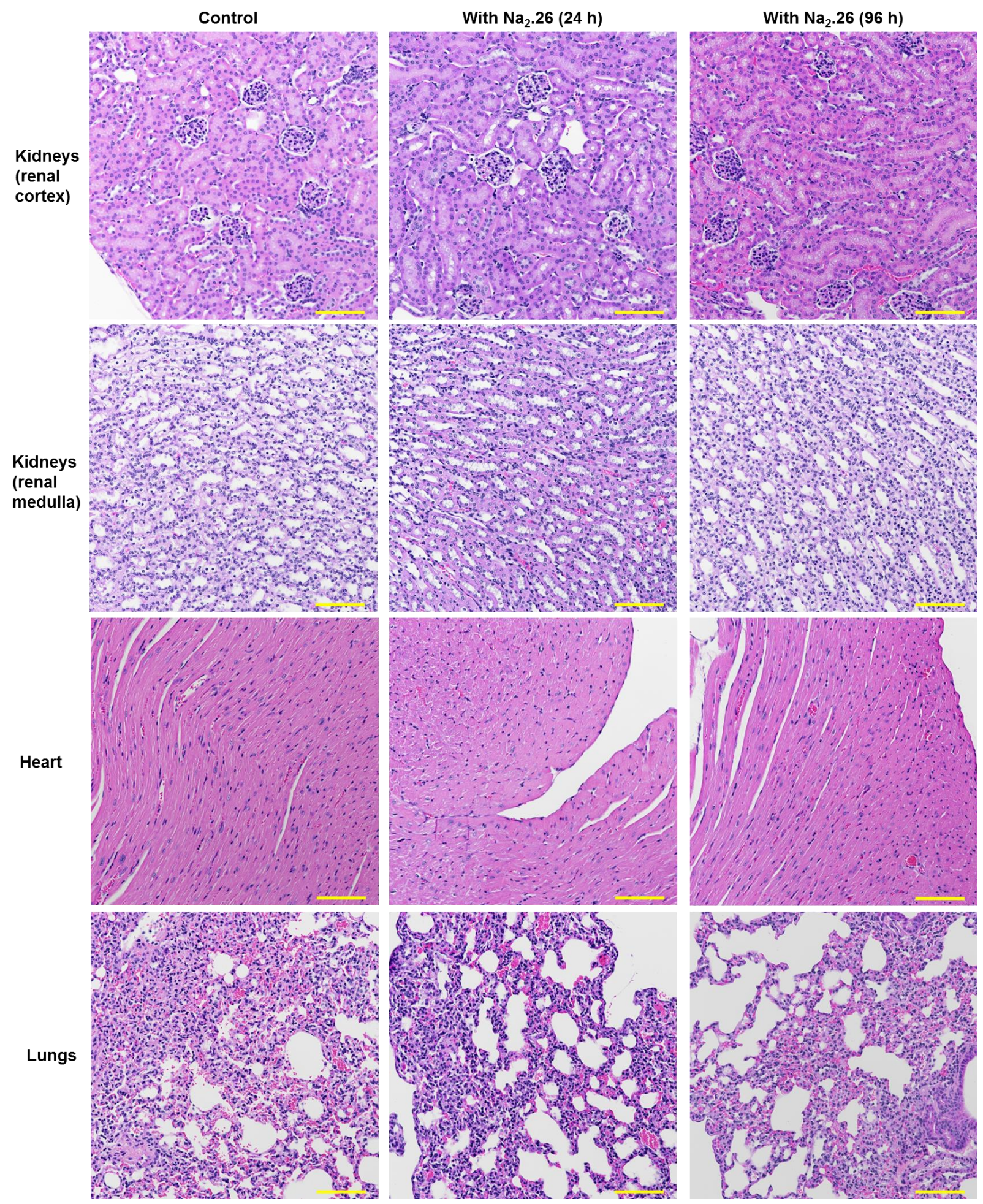

Figure A4.12. Histopathology (H \& E staining) of kidneys, heart and lungs tissues of mice, $24 \mathrm{~h}$ and $96 \mathrm{~h}$ after intravenous injection of $\mathrm{Na}_{2} . \mathbf{2 6}$, with that of control samples. Scale bar $=$ $100 \mu \mathrm{m}$. 


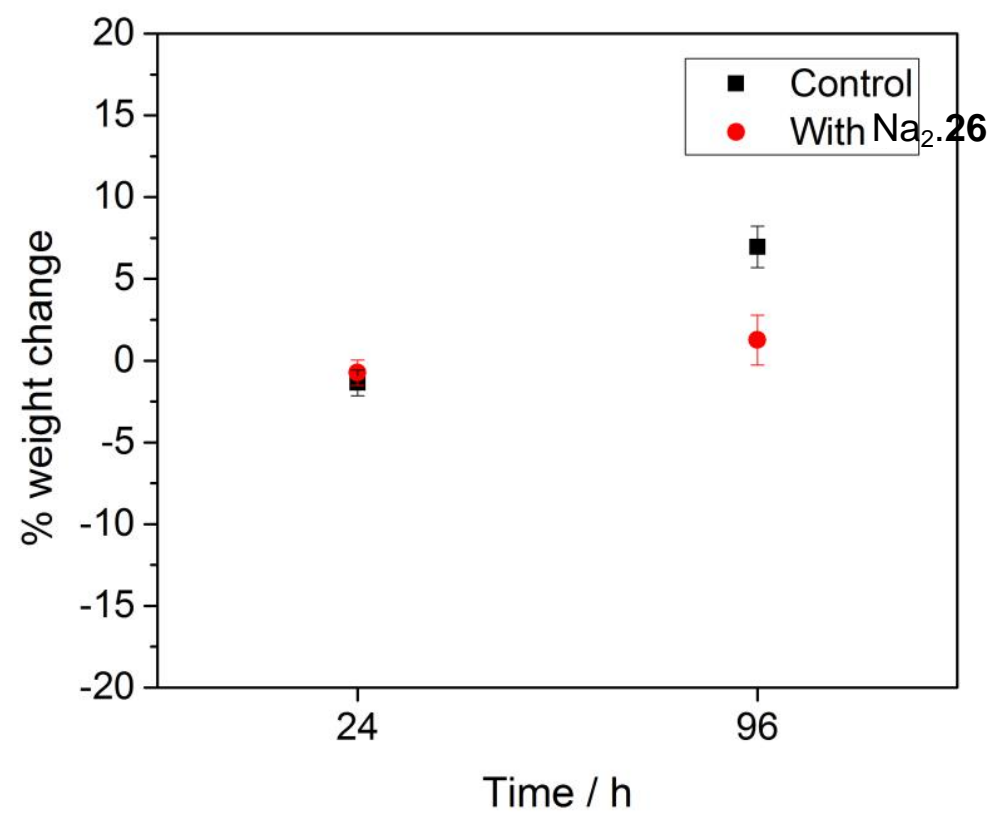

Figure A4.13. Percentage weight change of control mice and mice injected with $\mathrm{Na}_{2} .26$ up till $24 \mathrm{~h}$ and $96 \mathrm{~h}$ with respect to initial weight. Error bars represent standard error of the mean, $\mathrm{n}=3$ for samples with $\mathrm{Na}_{2} .26$ and $\mathrm{n}=2$ for control samples. 


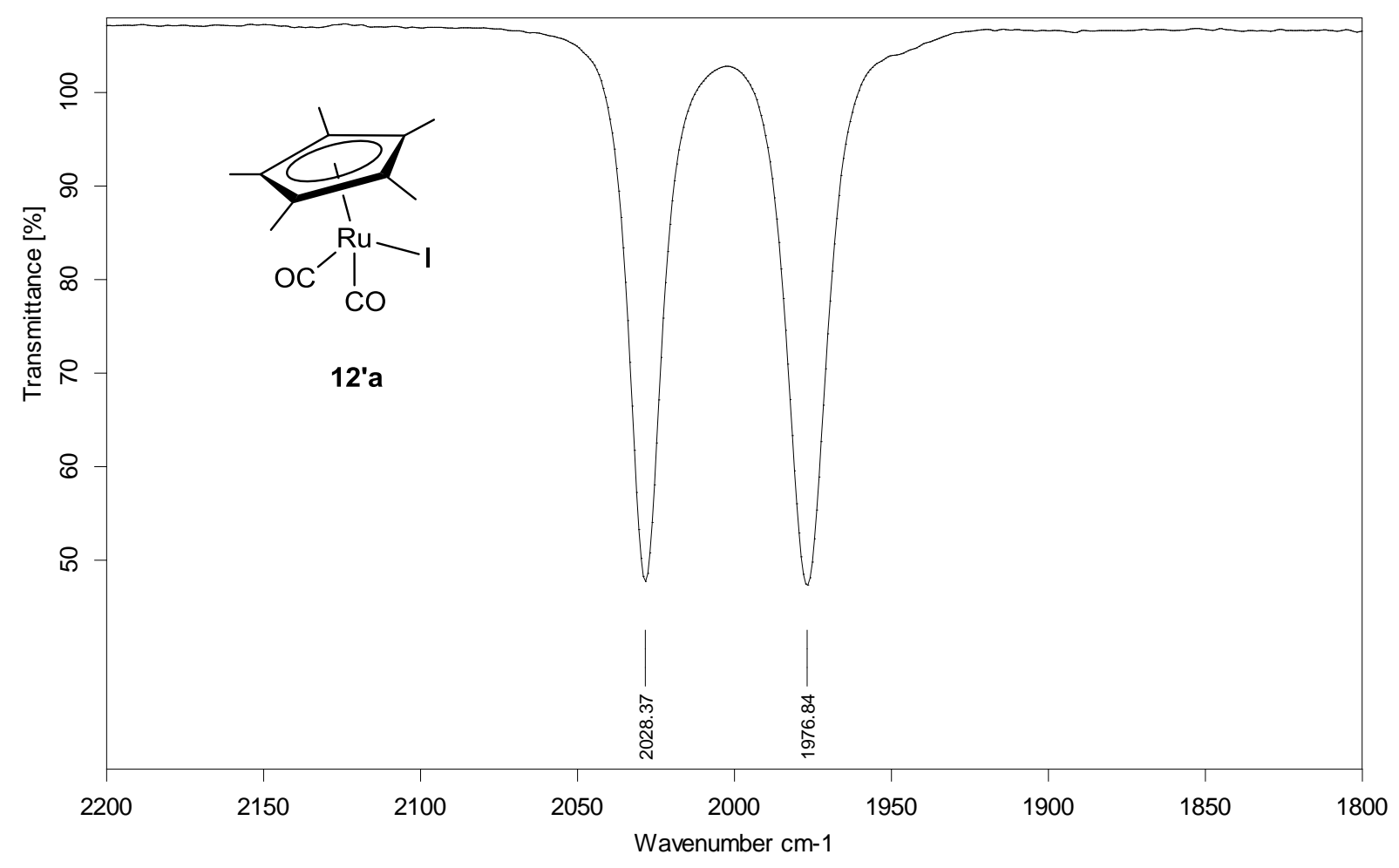

Figure A4.14. IR spectrum ( $\left.v_{\mathrm{CO}}\right)$ of 12'a in DCM.

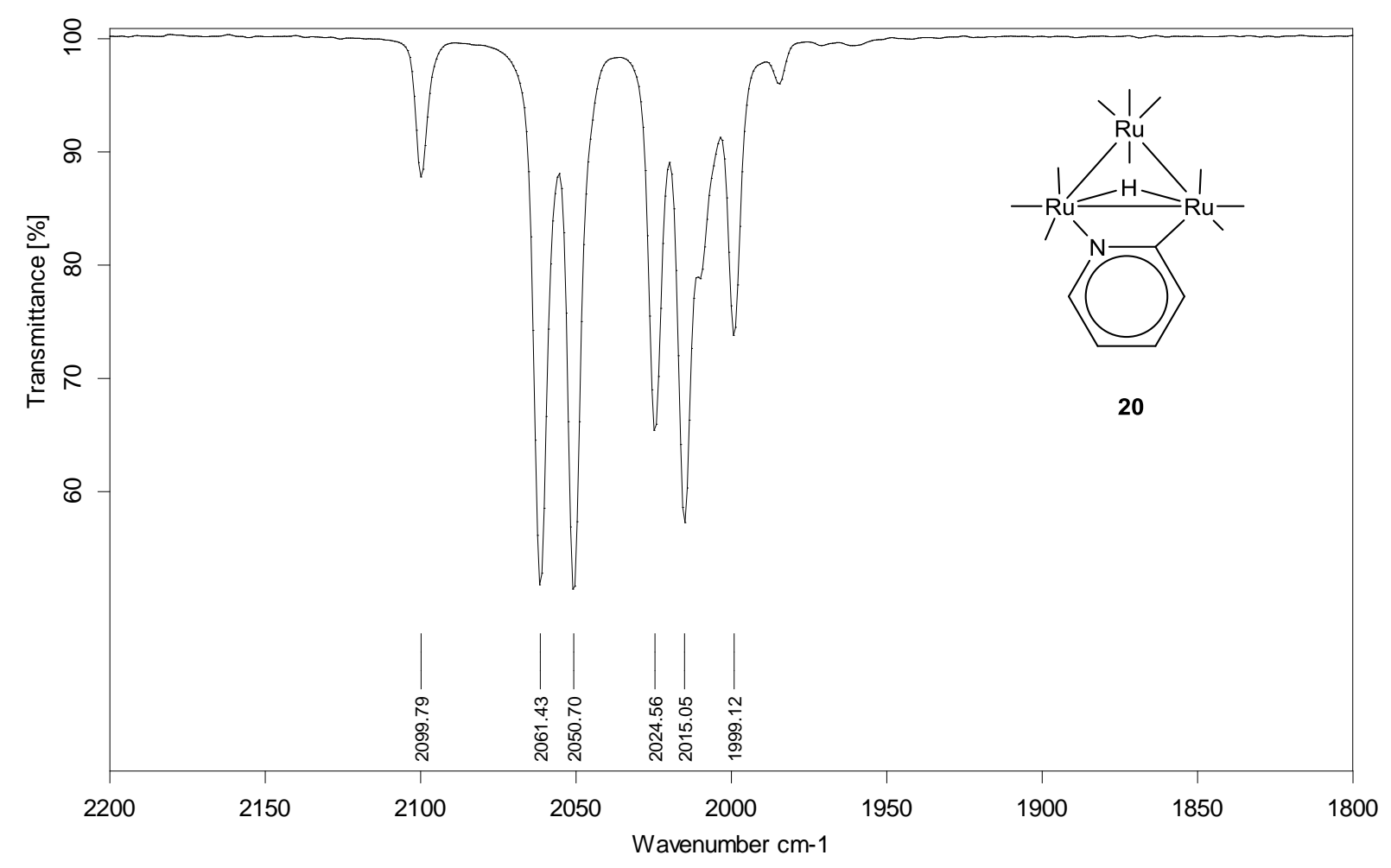

Figure A4.15. IR spectrum ( $\left.v_{\mathrm{CO}}\right)$ of cluster 20 in hexane. 


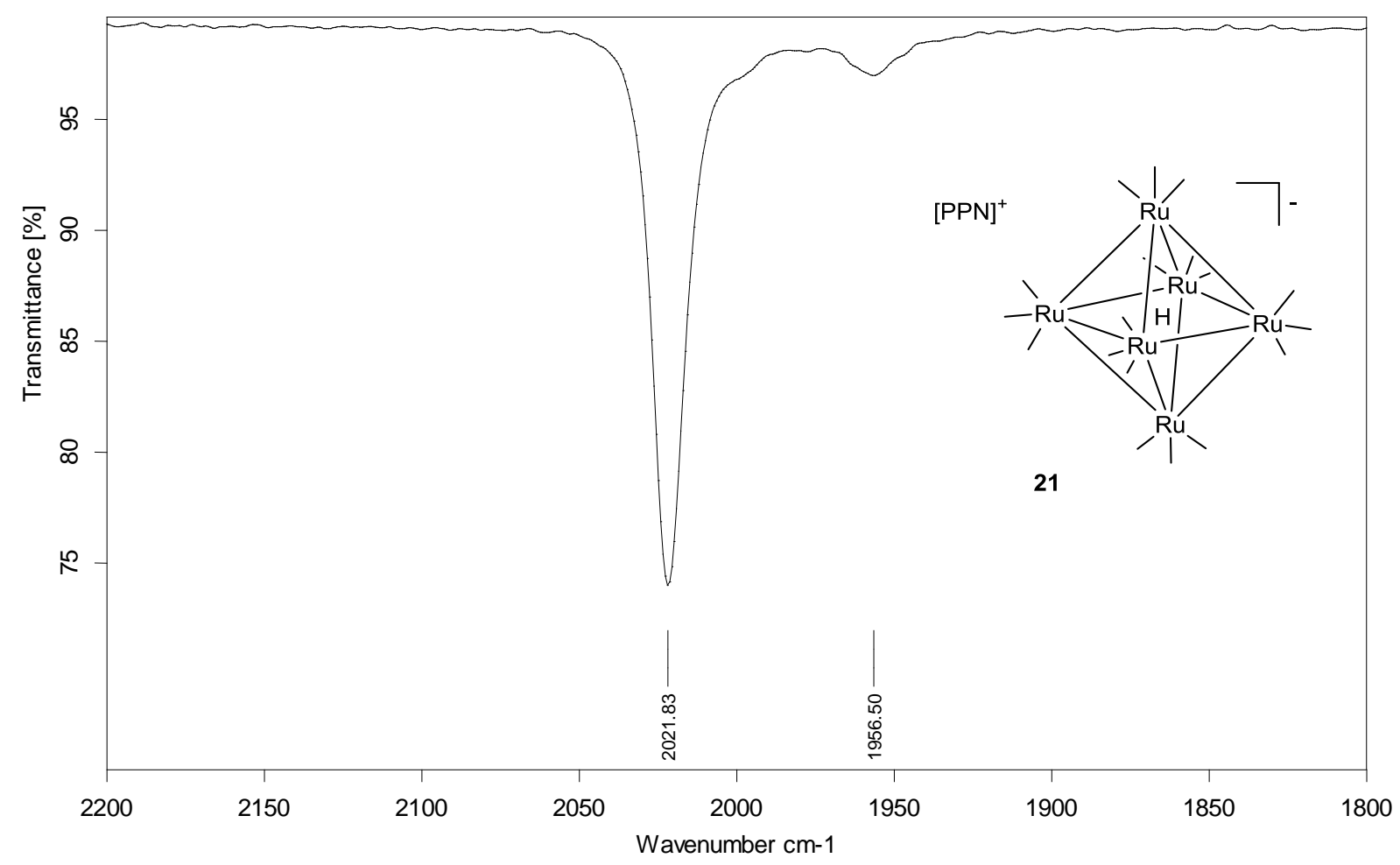

Figure A4.16. IR spectrum ( $\left.v_{\mathrm{CO}}\right)$ of cluster 21 in DCM.

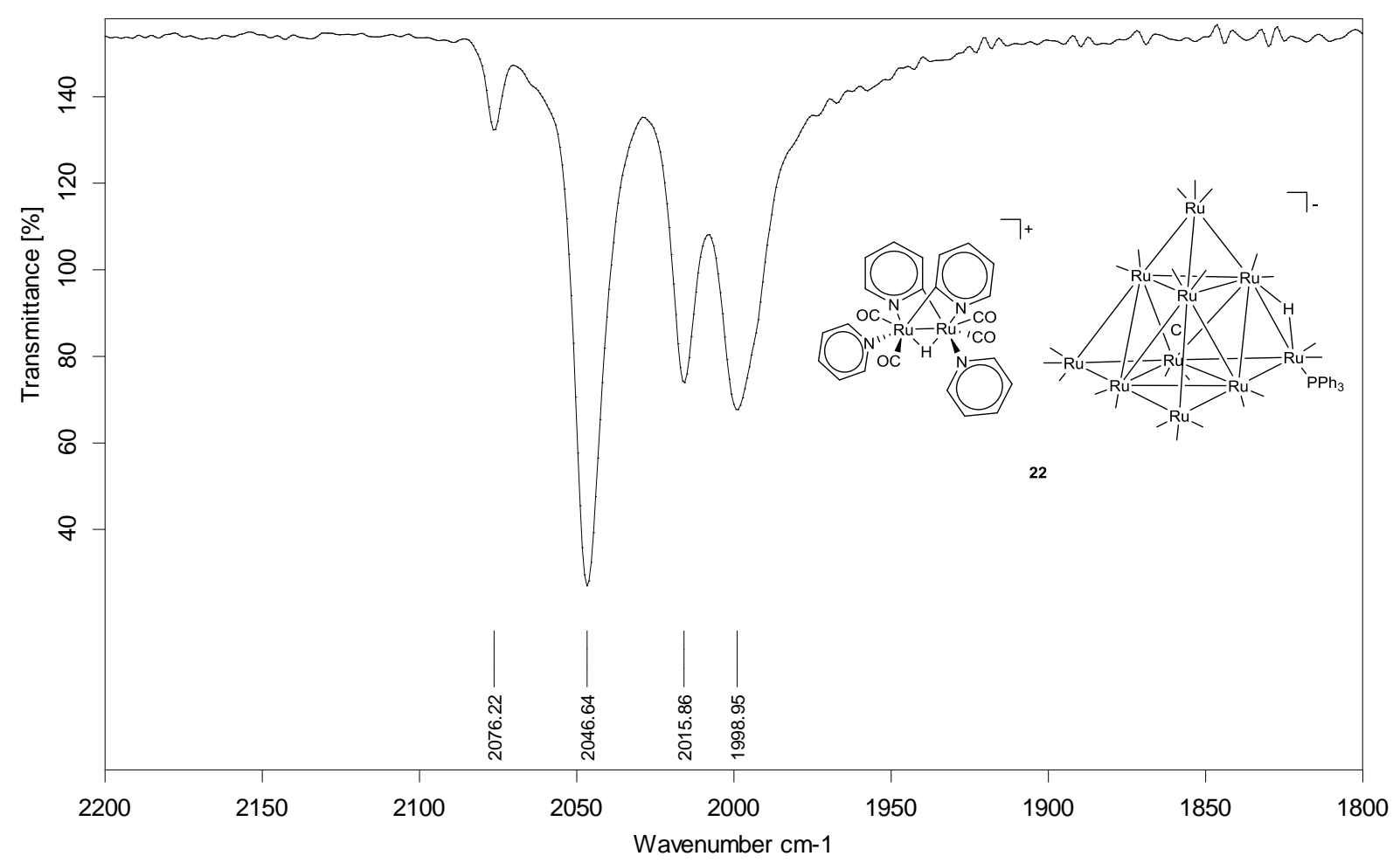

Figure A4.17. IR spectrum ( $\left.v_{\mathrm{CO}}\right)$ of cluster 22 in DCM. 


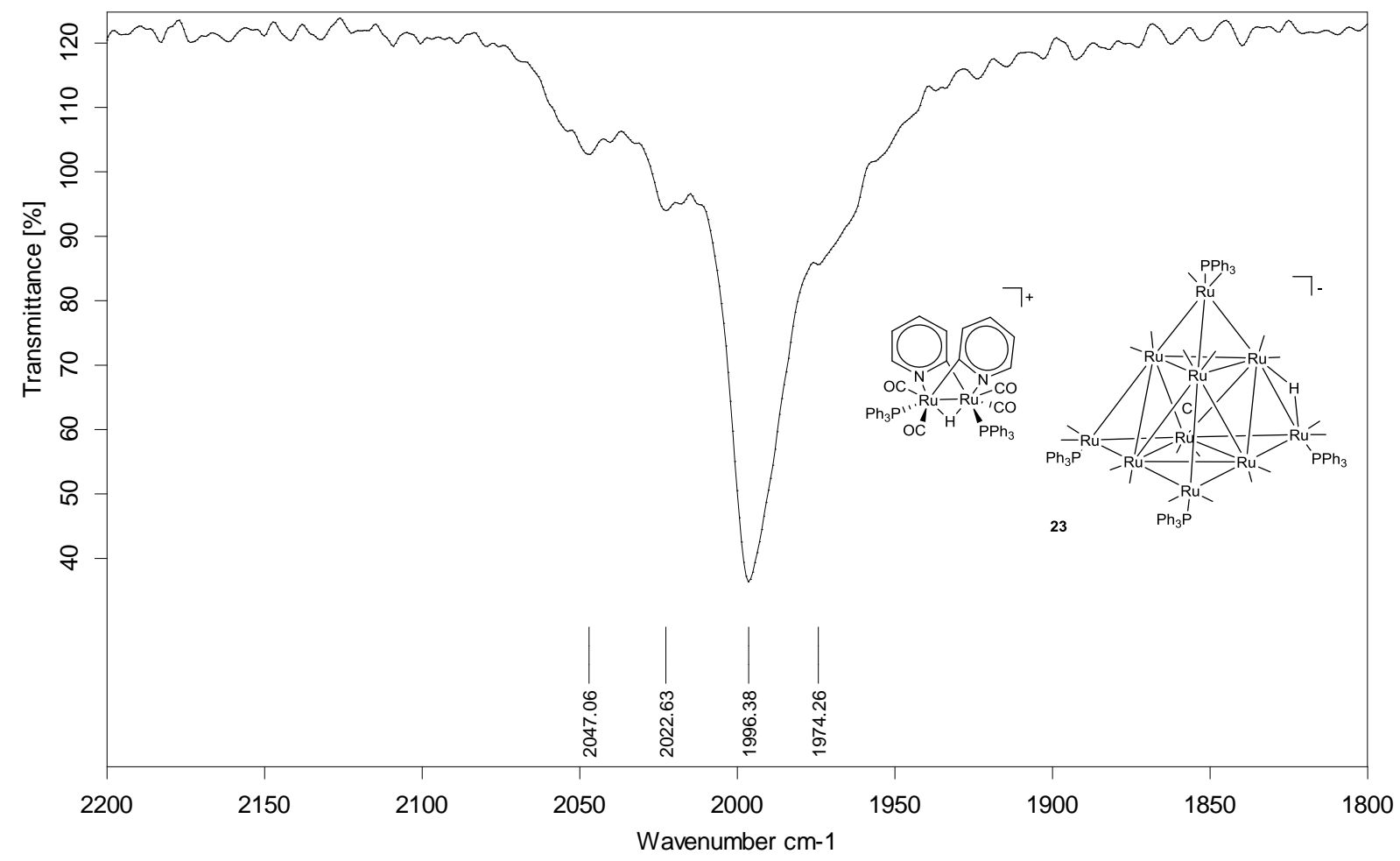

Figure A4.18. IR spectrum ( $\left.v_{\mathrm{CO}}\right)$ of cluster 23 in DCM.

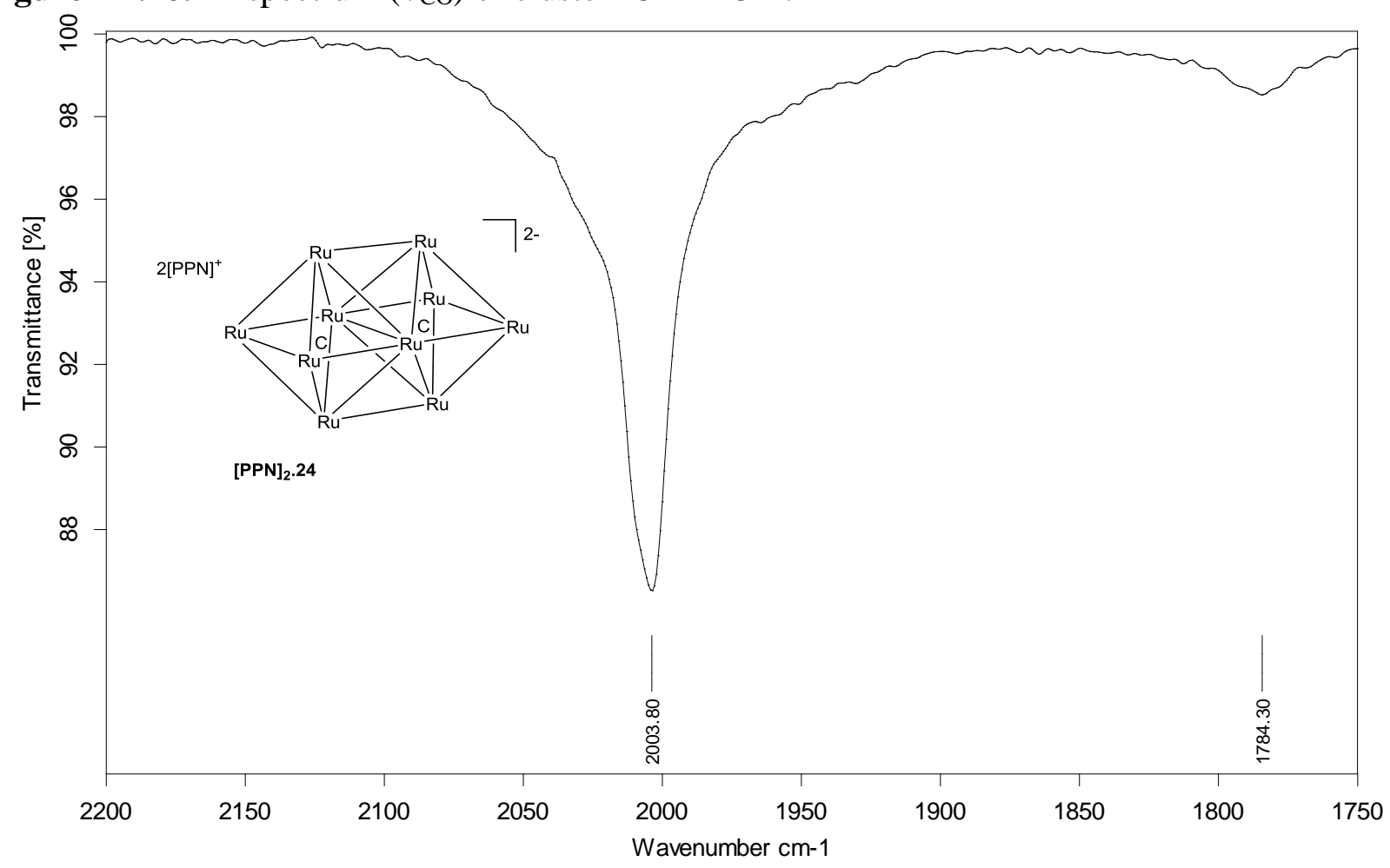

Figure A4.19. IR spectrum ( $\left.v_{\mathrm{CO}}\right)$ of cluster 24 in DCM. 


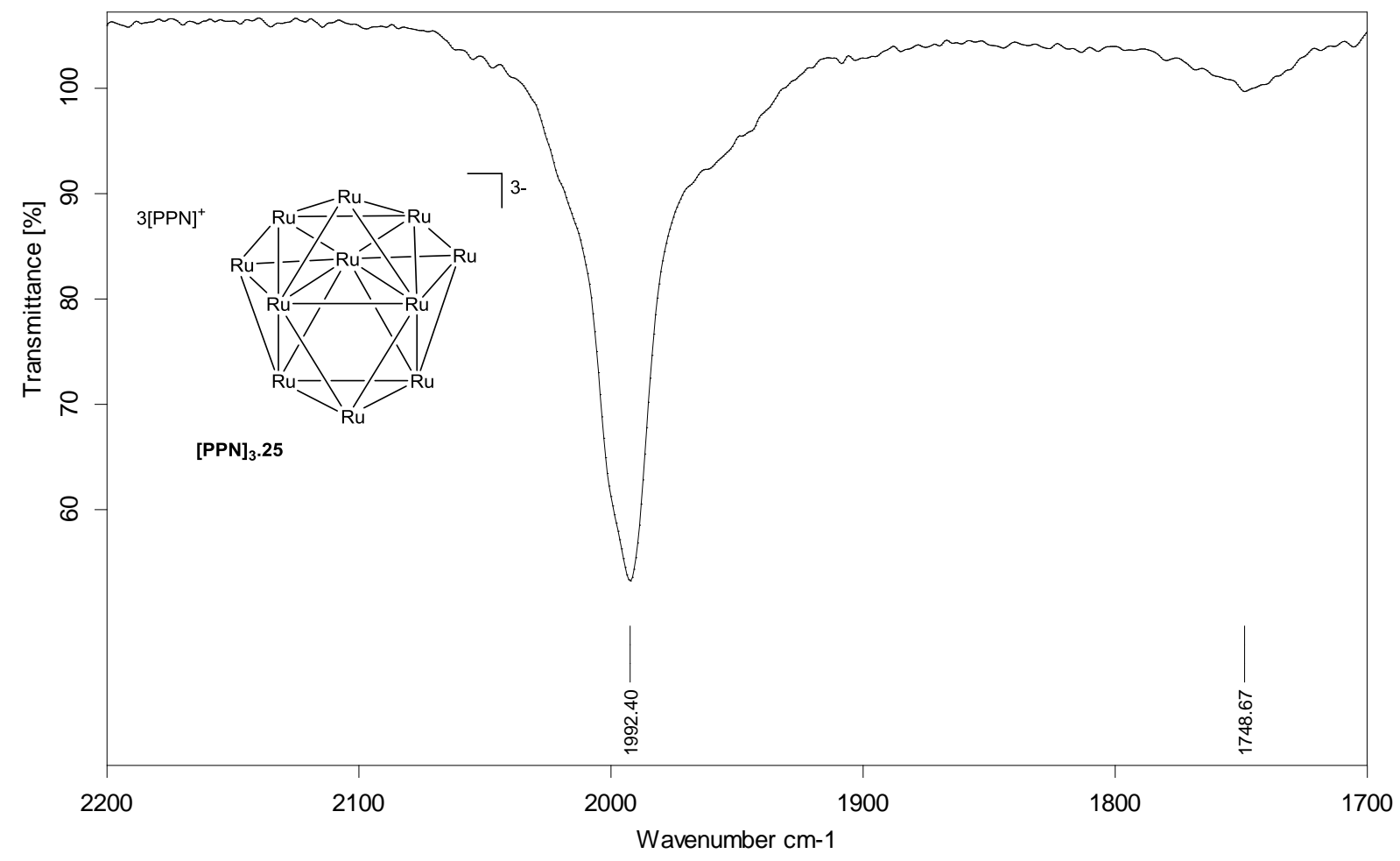

Figure A4.20. IR spectrum ( $\left.v_{\mathrm{CO}}\right)$ of cluster 25 in DCM.

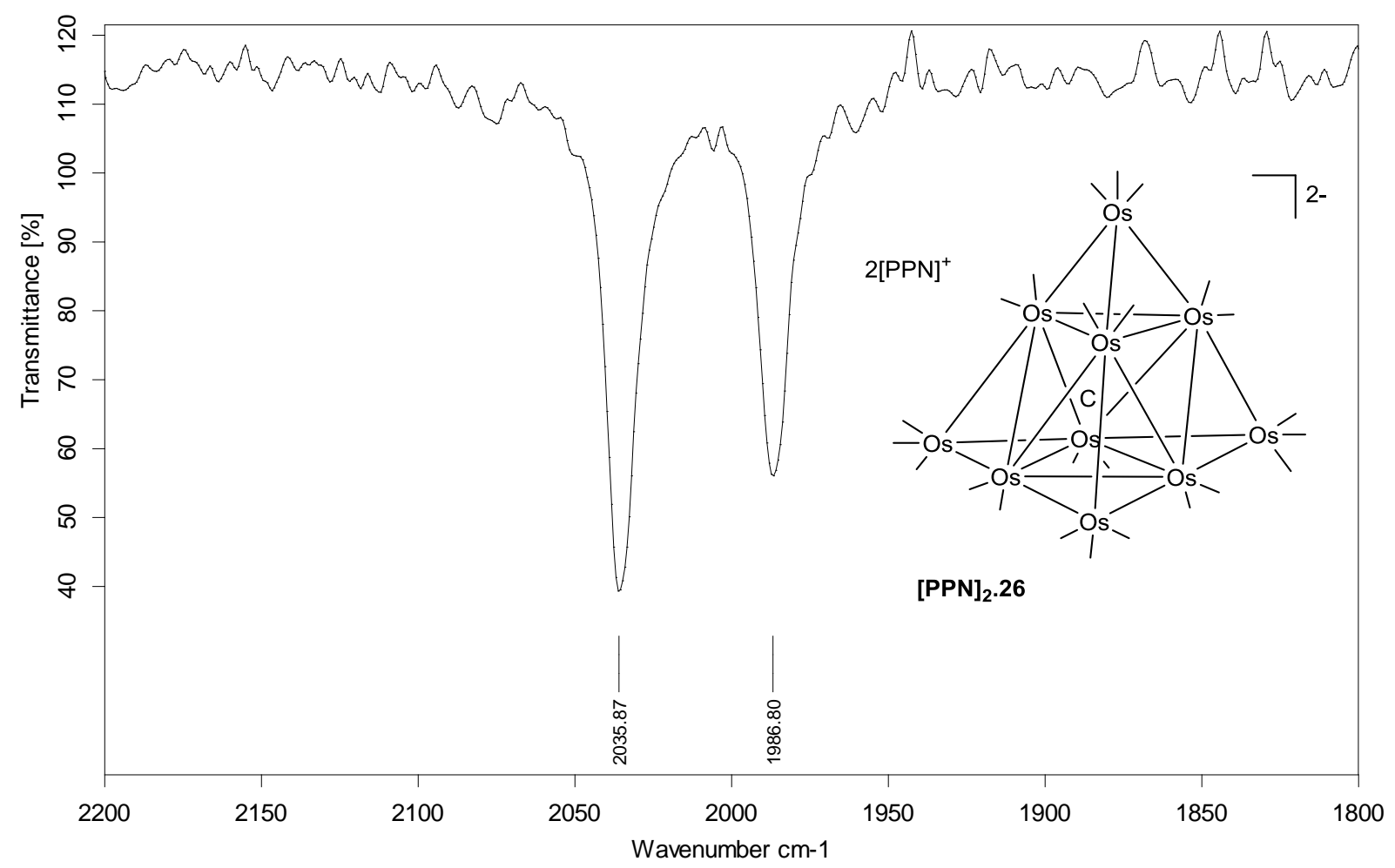

Figure A4.21. IR spectrum ( $\left.v_{\mathrm{CO}}\right)$ of cluster 26 in DCM. 


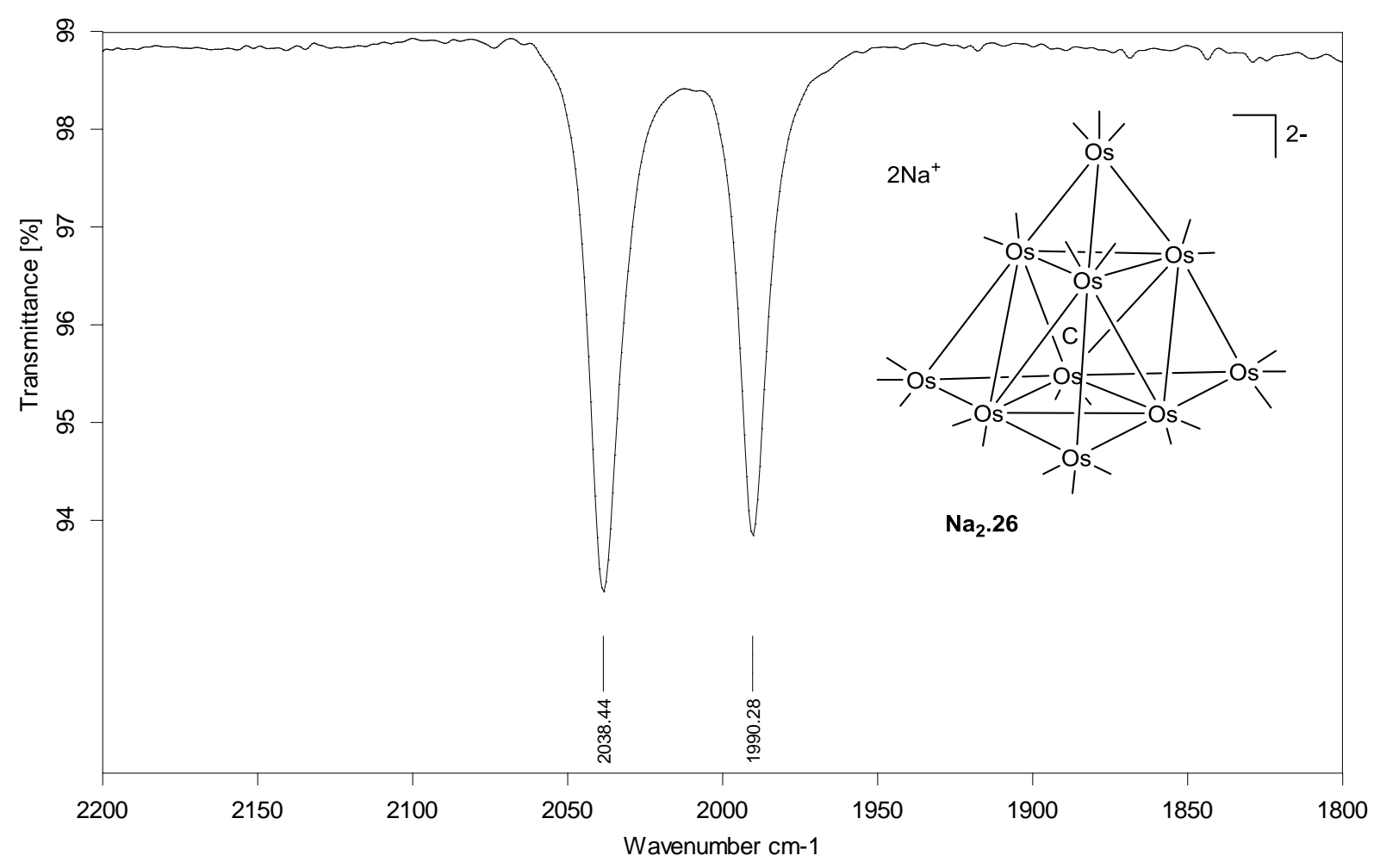

Figure A4.22. IR spectrum $\left(v_{\mathrm{CO}}\right)$ of cluster $\mathrm{Na}_{2} . \mathbf{2 6}$ in acetonitrile. 


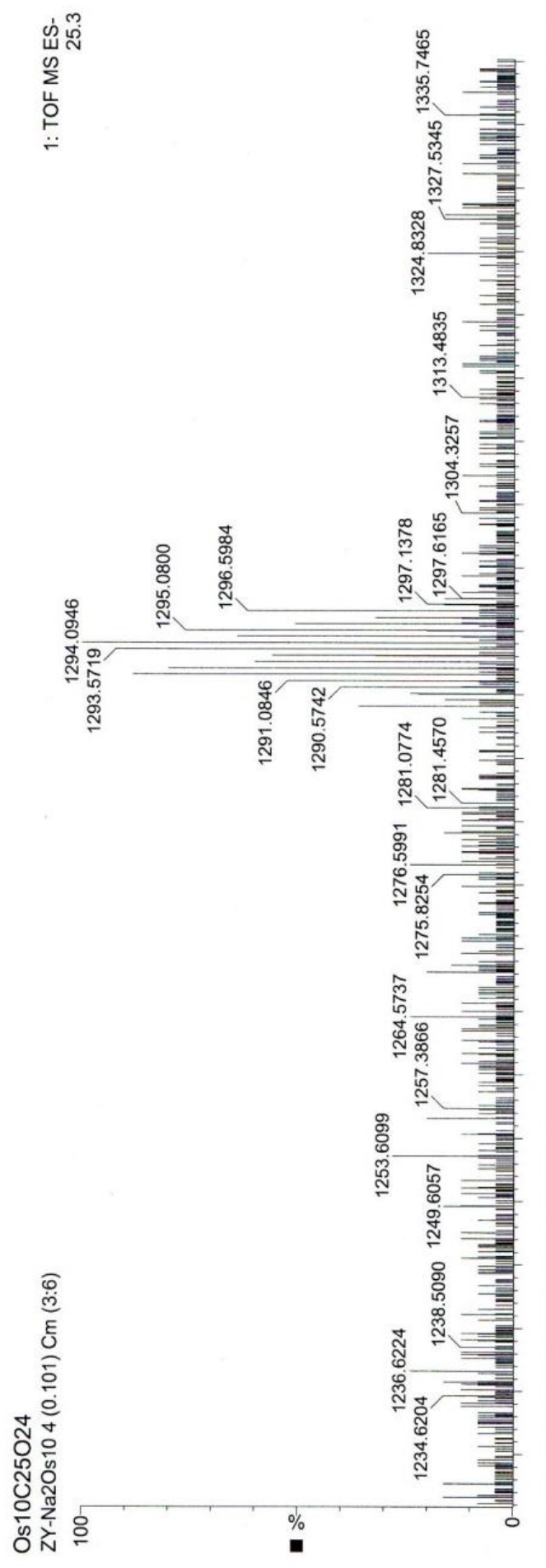

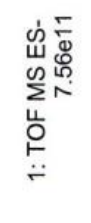

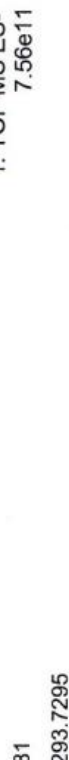

空

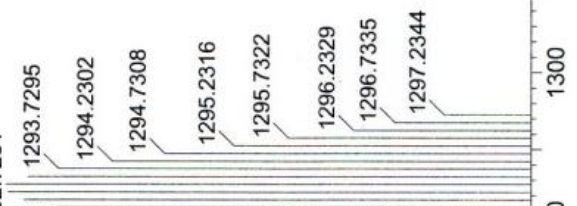

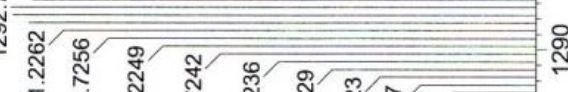

ํㅗㄴ

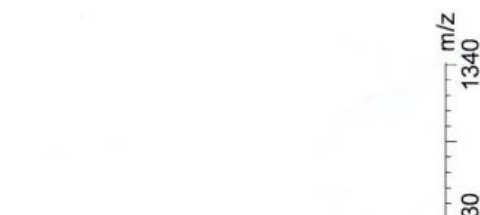

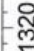

음

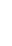

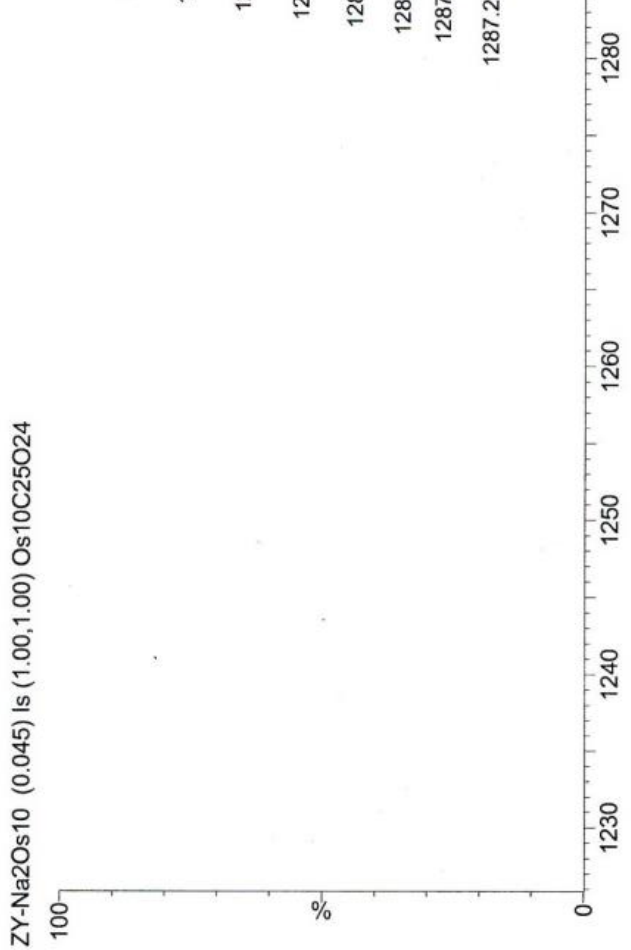

Figure A4.22. HRMS-ESI spectrum of cluster $\mathrm{Na}_{2} .26$ (top). The calculated isotopic pattern is as shown at the bottom. 
Table A4.1. ICP-MS analysis of Os in various organs of animals $24 \mathrm{~h}$ after injection of $\mathrm{Na}_{2} .26$.

a) Animal 1 (mass $=23.1 \mathrm{~g}$ )

\begin{tabular}{lcccc} 
Tissue & $\begin{array}{c}\text { Tissue mass } \\
\text { (dried) / } \mathbf{~ m g}\end{array}$ & $\begin{array}{c}\text { Elemental } \\
\text { concentration of } \\
\text { Os / ppb }\end{array}$ & $\begin{array}{c}\text { Mass of Os } \\
/ \mathbf{\mu g}\end{array}$ & $\begin{array}{c}\text { Mass of Os per } \\
\text { tissue mass / } \\
\text { ng } \mathbf{~ m g}^{-1}\end{array}$ \\
\hline Liver & 363.28 & 859.50 & 85.95 & 236.6 \\
Spleen & 44.20 & 105.81 & 10.58 & 239.4 \\
Lungs & 36.07 & 10.47 & 1.05 & 29.0 \\
Tumor & 11.82 & 5.70 & 0.57 & 48.2 \\
Kidneys & 114.53 & 4.82 & 0.48 & 4.2 \\
Heart & 33.09 & 1.32 & 0.32 & 9.7 \\
Brain & 91.27 & 2.16 & 0.22 & 2.4 \\
Blood $(\mathbf{5 0} \boldsymbol{\mu L})$ & N. A. & 1.79 & 0.18 & $3.6^{*}$ \\
\hline
\end{tabular}

*Represented as mass of Os per fluid volume / $\mathrm{ng} \mu \mathrm{L}^{-1}$

b) Animal $2($ mass $=22.3 \mathrm{~g})$

\begin{tabular}{|c|c|c|c|c|}
\hline Tissue & $\begin{array}{l}\text { Tissue mass } \\
\text { (dried) / mg }\end{array}$ & $\begin{array}{c}\text { Elemental } \\
\text { concentration of } \\
\text { Os / ppb }\end{array}$ & $\begin{array}{c}\text { Mass of Os } \\
\qquad / \boldsymbol{\mu g}\end{array}$ & $\begin{array}{l}\text { Mass of Os per } \\
\text { tissue mass / } \\
\quad \mathrm{ng} \mathrm{mg}^{-1}\end{array}$ \\
\hline Liver & 387.20 & 932.75 & 93.28 & 240.9 \\
\hline Spleen & 38.01 & 48.55 & 4.86 & 127.7 \\
\hline Lungs & 41.43 & 7.01 & 0.70 & 16.9 \\
\hline Tumor & 27.03 & 1.25 & 0.13 & 4.6 \\
\hline Kidneys & 117.58 & 5.19 & 0.52 & 4.4 \\
\hline Heart & 44.31 & 1.76 & 0.18 & 4.0 \\
\hline Brain & 94.73 & 1.01 & 0.10 & 1.1 \\
\hline Blood $(50 \mu \mathrm{L})$ & N. A. & 1.14 & 0.11 & $2.3^{*}$ \\
\hline
\end{tabular}

*Represented as mass of Os per fluid volume / ng $\mu \mathrm{L}^{-1}$ 
c) Animal 3 (mass $=21.0 \mathrm{~g})$

\begin{tabular}{lcccc} 
Tissue & $\begin{array}{c}\text { Tissue mass } \\
\text { (dried) / mg }\end{array}$ & $\begin{array}{c}\text { Elemental } \\
\text { concentration of } \\
\text { Os / ppb }\end{array}$ & $\begin{array}{c}\text { Mass of Os } \\
/ \mathbf{\mu g}\end{array}$ & $\begin{array}{c}\text { Mass of Os per } \\
\text { tissue mass / } \\
\text { ng } \mathbf{~ m g}^{-1}\end{array}$ \\
\hline Liver & 361.55 & 927.50 & 92.75 & 256.5 \\
Spleen & 30.86 & 35.42 & 3.54 & 114.8 \\
Lungs & 37.22 & 25.79 & 2.58 & 69.3 \\
Tumor & 17.55 & 0.90 & 0.09 & 5.1 \\
Kidneys & 91.76 & 4.18 & 0.42 & 4.6 \\
Heart & 44.35 & 2.32 & 0.23 & 5.2 \\
Brain & 87.63 & 0.41 & 0.04 & 0.5 \\
Blood $(\mathbf{5 0} \boldsymbol{\mu L})$ & N. A. & 1.14 & 0.11 & $2.3^{*}$ \\
\hline
\end{tabular}

*Represented as mass of Os per fluid volume / $\mathrm{ng} \mu \mathrm{L}^{-1}$

d) Mean values

\begin{tabular}{lc}
\hline \multirow{2}{*}{ Tissue } & Mass of Os per tissue mass $/ \mathbf{~ n g ~ m}^{-1}$ \\
\cline { 2 - 2 } & Mean ( \pm SE of mean) \\
\hline Liver & $245 \pm 6$ \\
Spleen & $161 \pm 40$ \\
Lungs & $38 \pm 16$ \\
Tumor & $19 \pm 14$ \\
Kidneys & $4.4 \pm 0.1$ \\
Heart & $6.3 \pm 3.0$ \\
Brain & $1.3 \pm 0.6$ \\
Blood* & $2.7 \pm 0.4^{*}$ \\
\hline *Represented as mass of Os per fluid volume $/ \mathrm{ng} \mu \mathrm{L}^{-1}$
\end{tabular}


Table A4.2. ICP-MS analysis of Os in various organs of animals $96 \mathrm{~h}$ after injection of $\mathrm{Na}_{2} .26$.

a) Animal 1 (mass $=20.1 \mathrm{~g})$

\begin{tabular}{lcccc}
\hline Tissue & $\begin{array}{c}\text { Tissue mass } \\
\text { (dried) / mg }\end{array}$ & $\begin{array}{c}\text { Elemental } \\
\text { concentration of } \\
\text { Os / ppb }\end{array}$ & $\begin{array}{c}\text { Mass of Os } \\
/ \boldsymbol{\mu g}\end{array}$ & $\begin{array}{c}\text { Mass of Os per } \\
\text { tissue mass / } \\
\mathbf{n g ~ m g}^{-1}\end{array}$ \\
\hline Liver & 311.45 & 777.31 & 77.73 & 249.6 \\
Spleen & 29.94 & 126.17 & 12.62 & 421.4 \\
Lungs & 26.56 & 3.56 & 0.36 & 13.4 \\
Tumor & 9.44 & 0.37 & 0.04 & 3.9 \\
Kidneys & 94.69 & 1.79 & 0.18 & 1.9 \\
Heart & 25.73 & 0.51 & 0.05 & 2.0 \\
Brain & 91.07 & 0.13 & 0.01 & 0.1 \\
Blood $(\mathbf{5 0} \boldsymbol{\mu L})$ & N. A. & 0.28 & 0.03 & 0.6 \\
\hline
\end{tabular}

*Represented as mass of Os per fluid volume / $\mathrm{ng} \mu \mathrm{L}^{-1}$

b) Animal $2($ mass $=20.0 \mathrm{~g})$

\begin{tabular}{lcccc}
\hline Tissue & $\begin{array}{c}\text { Tissue mass } \\
(\mathbf{d r i e d}) / \mathbf{~ m g}\end{array}$ & $\begin{array}{c}\text { Elemental } \\
\text { concentration of } \\
\text { Os / ppb }\end{array}$ & $\begin{array}{c}\text { Mass of Os } \\
/ \boldsymbol{\mu g}\end{array}$ & $\begin{array}{c}\text { Mass of Os per } \\
\text { tissue mass / } \\
\mathbf{n g ~ m g ~}^{-1}\end{array}$ \\
\hline Liver & 314.38 & 732.42 & 73.24 & 233.0 \\
Spleen & 28.74 & 55.96 & 5.60 & 194.7 \\
Lungs & 31.75 & 1.82 & 0.18 & 5.7 \\
Tumor & 24.82 & 0.51 & 0.05 & 2.1 \\
Kidneys & 99.23 & 6.51 & 0.65 & 6.6 \\
Heart & 22.41 & 0.42 & 0.04 & 1.9 \\
Brain & 83.46 & 0.16 & 0.02 & 0.2 \\
Blood $(\mathbf{5 0} \boldsymbol{\mu L})$ & N. A. & 0.23 & 0.02 & 0.5 \\
\hline
\end{tabular}

*Represented as mass of Os per fluid volume / $\mathrm{ng} \mu \mathrm{L}^{-1}$

c) Mean values

\begin{tabular}{lc}
\hline & Mass of Os per tissue mass $/ \mathbf{~ n g ~ m g ~}^{-\mathbf{1}}$ \\
\cline { 2 - 3 } Tissue & Mean $(\mathbf{E}$ SE of mean) \\
\hline Liver & $241 \pm 8$ \\
Spleen & $308 \pm 113$ \\
Lungs & $10 \pm 4$ \\
Tumor & $3.0 \pm 0.9$ \\
Kidneys & $4 \pm 2$ \\
Heart & $1.93 \pm 0.05$ \\
Brain & $0.17 \pm 0.02$ \\
Blood* & $0.51 \pm 0.05^{*}$ \\
\hline *Represented as mass of Os per fluid volume $/ \mathrm{ng}^{-1} \mathrm{~L}^{-1}$
\end{tabular}


Table A4.3. ICP-MS analysis of background Os content in various organs in control animals (without the introduction of $\mathrm{Na}_{2} .26$ ).

a) Animal 1 (mass $=21.8 \mathrm{~g}$ )

\begin{tabular}{lcccc}
\hline Tissue & $\begin{array}{c}\text { Tissue mass } \\
\text { (dried) / } \mathbf{~ m g}\end{array}$ & $\begin{array}{c}\text { Elemental } \\
\text { concentration of } \\
\text { Os / ppb }\end{array}$ & $\begin{array}{c}\text { Mass of Os } \\
\text { / } \boldsymbol{\mu g}\end{array}$ & $\begin{array}{c}\text { Mass of Os per } \\
\text { tissue mass / } \\
\mathbf{n g ~ m g}^{-1}\end{array}$ \\
\hline Liver & 341.14 & 0.84 & 0.08 & 0.25 \\
Spleen & 33.86 & 0.39 & 0.04 & 1.15 \\
Lungs & 30.33 & 0.23 & 0.02 & 0.76 \\
Tumor & 13.15 & 0.22 & 0.02 & 1.67 \\
Kidneys & 103.46 & 0.46 & 0.05 & 0.44 \\
Heart & 36.89 & 0.31 & 0.03 & 0.84 \\
Brain & 90.78 & 0.28 & 0.03 & 0.31 \\
Blood $(\mathbf{5 0} \boldsymbol{\mu L})$ & N. A. & 0.29 & 0.03 & 0.58 \\
\hline
\end{tabular}

*Represented as mass of Os per fluid volume / $\mathrm{ng} \mu \mathrm{L}^{-1}$

b) Animal $2($ mass $=21.0 \mathrm{~g})$

\begin{tabular}{lcccc}
\hline Tissue & $\begin{array}{c}\text { Tissue mass } \\
(\mathbf{d r i e d}) / \mathbf{~ m g}\end{array}$ & $\begin{array}{c}\text { Elemental } \\
\text { concentration of } \\
\text { Os / ppb }\end{array}$ & $\begin{array}{c}\text { Mass of Os } \\
/ \boldsymbol{\mu g}\end{array}$ & $\begin{array}{c}\text { Mass of Os per } \\
\text { tissue mass / } \\
\mathbf{n g ~ m g ~}^{-1}\end{array}$ \\
\hline Liver & 309.90 & 0.17 & 0.02 & 0.05 \\
Spleen & 24.06 & 0.13 & 0.01 & 0.54 \\
Lungs & 27.97 & 0.11 & 0.01 & 0.39 \\
Tumor & 5.89 & 0.16 & 0.02 & 2.72 \\
Kidneys & 102.63 & 0.15 & 0.02 & 0.15 \\
Heart & 37.17 & 0.12 & 0.01 & 0.32 \\
Brain & 88.43 & 0.13 & 0.01 & 0.15 \\
Blood $(\mathbf{5 0} \boldsymbol{\mu L})$ & N. A. & 0.14 & 0.01 & 0.28 \\
\hline
\end{tabular}

*Represented as mass of Os per fluid volume / $\mathrm{ng} \mu \mathrm{L}^{-1}$

c) Mean values

\begin{tabular}{|c|c|}
\hline \multirow{2}{*}{ Tissue } & Mass of Os per tissue mass / $\mathrm{ng} \mathrm{mg}^{-1}$ \\
\hline & Mean $( \pm S E$ of mean $)$ \\
\hline Liver & $0.15 \pm 0.1$ \\
\hline Spleen & $0.8 \pm 0.3$ \\
\hline Lungs & $0.6 \pm 0.2$ \\
\hline Tumor & $2.2 \pm 0.5$ \\
\hline Kidneys & $0.3 \pm 0.1$ \\
\hline Heart & $0.6 \pm 0.3$ \\
\hline Brain & $0.23 \pm 0.08$ \\
\hline Blood* & $0.4 \pm 0.2 *$ \\
\hline
\end{tabular}


Table A4.4. Calculation of the retention of Os in various organ tissues.

a) Calculation of mass of Os administered into each animal

Percentage mass of Os in $\mathrm{Na}_{2} . \mathbf{2 6}=\mathbf{7 2 . 2 6 \%}$

No. of moles of $\mathrm{Na}_{2} .26$ administered into the animal $=200 \mu \mathrm{L}$ x $500 \mu \mathrm{M}=0.100 \mu \mathrm{mol}$

Mass of $\mathrm{Na}_{2} .26$ administered into each animal $=0.100 \mu \mathrm{mol} \times 2632.53 \mathrm{~g} \mathrm{~mol}^{-1}=263.25 \mu \mathrm{g}$

Mass of Os administered into each animal $\left(\mathrm{M}_{\mathrm{T}}\right)=(72.26 / 100) \times 263.25=\underline{\mathbf{1 9 0 . 2 3} \boldsymbol{\mu g}}$

b) Estimation of the amount of Os in blood based on projected total blood volume

The blood volume of mouse was estimated to be 79 (77-80) $\mu \mathrm{L} \mathrm{g}^{-1}{ }^{1,2}$ Hence, this value was used for the calculation of blood volume:

Estimated blood volume of animal $1(\mathrm{~A} 1)=79 \mu \mathrm{L} \mathrm{g}^{-1} \times 23.1 \mathrm{~g}=1825 \mu \mathrm{L}$

$\therefore$ Mass of Os in blood of $\mathrm{A} 1=(0.179 \mu \mathrm{g} / 50 \mu \mathrm{L}) \times 1825 \mu \mathrm{L}=\underline{\mathbf{6 . 5 3} \mu \mathbf{g}}$

Estimated blood volume of animal $2(\mathrm{~A} 2)=79 \mu \mathrm{L} \mathrm{g}^{-1} \times 22.3 \mathrm{~g}=1762 \mu \mathrm{L}$

$\therefore$ Mass of Os in blood of A2 $=(0.114 \mu \mathrm{g} / 50 \mu \mathrm{L}) \times 1762 \mu \mathrm{L}=\underline{\mathbf{4 . 0 2} \mu \mathbf{g}}$

Estimated blood volume of animal $3(\mathrm{~A} 3)=79 \mu \mathrm{L} \mathrm{g}^{-1} \times 21.0 \mathrm{~g}=1659 \mu \mathrm{L}$

$\therefore$ Mass of Os in blood of A3 $=(0.114 \mu \mathrm{g} / 50 \mu \mathrm{L}) \times 1659 \mu \mathrm{L}=\underline{\mathbf{3 . 7 8} \mu \mathbf{g}}$

\begin{tabular}{|c|c|c|c|c|c|c|c|}
\hline \multirow{2}{*}{ Tissue } & \multicolumn{3}{|c|}{ Mass of Os $\left(M_{0}\right) / \mu g$} & \multicolumn{4}{|c|}{$\begin{array}{l}\text { Percentage retention of Os in the tissue } \\
\qquad\left(\mathbf{M}_{\mathrm{O}} / \mathbf{M}_{\mathrm{T}} \times 100\right) / \%\end{array}$} \\
\hline & A1 & $\mathbf{A} 2$ & A3 & A1 & A2 & A3 & $\begin{array}{c}\text { Mean }( \pm \mathrm{SE} \\
\text { of mean }\end{array}$ \\
\hline Liver & 85.95 & 93.28 & 92.75 & 45.18 & 49.03 & 48.76 & $47.66 \pm 1.24$ \\
\hline Spleen & 10.58 & 4.86 & 3.54 & 5.56 & 2.55 & 1.86 & $3.33 \pm 1.14$ \\
\hline Lungs & 1.05 & 0.70 & 2.58 & 0.55 & 0.37 & 1.36 & $0.76 \pm 0.30$ \\
\hline Tumor & 0.57 & 0.13 & 0.09 & 0.30 & 0.07 & 0.05 & $0.14 \pm 0.08$ \\
\hline Kidneys & 0.48 & 0.52 & 0.42 & 0.25 & 0.27 & 0.22 & $0.25 \pm 0.02$ \\
\hline Heart & 0.32 & 0.18 & 0.23 & 0.17 & 0.09 & 0.12 & $0.13 \pm 0.02$ \\
\hline Brain & 0.22 & 0.10 & 0.04 & 0.11 & 0.05 & 0.02 & $0.06 \pm 0.03$ \\
\hline Blood $^{\wedge}$ & 6.53 & 4.02 & 3.78 & 3.43 & 2.11 & 1.99 & $2.51 \pm 0.46$ \\
\hline
\end{tabular}

${ }^{\wedge}$ Estimation based on total blood volume of animals 


\section{References}

1. B. M. Mitruka and H. M. Rawnsley, Clinical Biochemical and Hematological Reference Values in Normal Experimental Animals and Normal Humans, Masson Publishing, New York, USA, 1981.

2. J. E. Harkness and J. E. Wagner, The Biology and Medicine of Rabbits and Rodents, Lea and Febiger, Philadelphia, USA, 1989. 